\title{
Field Assessment of Air Barriers at the Building Envelope Systems Test (BEST) Laboratory - Phase 1
}

September 2011

Prepared by

Diana Hun

Andre Desjarlais

Phil Childs Jerry Atchley

Achilles Karagiozis 


\section{DOCUMENT AVAILABILITY}

Reports produced after January 1, 1996, are generally available free via the U.S. Department of Energy (DOE) Information Bridge.

Web site http://www.osti.gov/bridge

Reports produced before January 1,1996, may be purchased by members of the public from the following source.

National Technical Information Service

5285 Port Royal Road

Springfield, VA 22161

Telephone 703-605-6000 (1-800-553-6847)

TDD 703-487-4639

Fax 703-605-6900

E-mailinfo@ntis.gov

Web site http://www.ntis.gov/support/ordernowabout.htm

Reports are available to DOE employees, DOE contractors, Energy Technology Data Exchange (ETDE) representatives, and International Nuclear Information System (INIS) representatives from the following source.

Office of Scientific and Technical Information

P.O. Box 62

Oak Ridge, TN 37831

Telephone 865-576-8401

Fax 865-576-5728

E-mail reports@osti.gov

Web site http://www.osti.gov/contact.html

This report was prepared as an account of work sponsored by an agency of the United States Government. Neither the United States Government nor any agency thereof, nor any of their employees, makes any warranty, express or implied, or assumes any legal liability or responsibility for the accuracy, completeness, or usefulness of any information, apparatus, product, or process disclosed, or represents that its use would not infringe privately owned rights. Reference herein to any specific commercial product, process, or service by trade name, trademark, manufacturer, or otherwise, does not necessarily constitute or imply its endorsement, recommendation, or favoring by the United States Government or any agency thereof. The views and opinions of authors expressed herein do not necessarily state or reflect those of the United States Government or any agency thereof. 


\title{
Field Assessment of Air Barriers at the Building Envelope Systems Test (BEST) Laboratory Phase 1
}

\author{
Diana Hun \\ Andre Desjarlais \\ Phil Childs \\ Jerry Atchley \\ Achilles Karagiozis
}

Date Published: September 2011

Prepared by

OAK RIDGE NATIONAL LABORATORY

Oak Ridge, Tennessee 37831-6283

managed by

UT-BATTELLE, LLC

for the

U.S. DEPARTMENT OF ENERGY

under contract DE-AC05-00OR22725 


\section{TABLE OF CONTENTS}

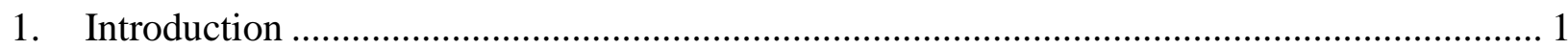

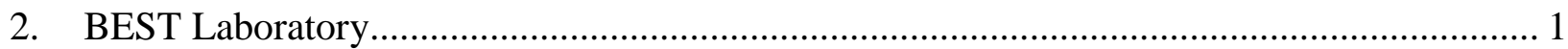

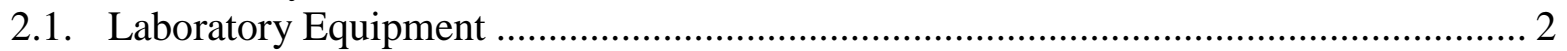

3. Panel Material and Sensor Layout................................................................................ 3

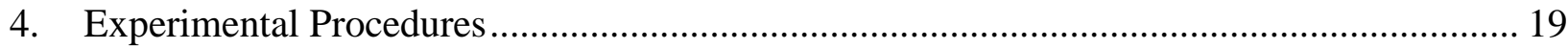

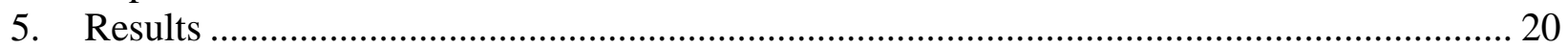

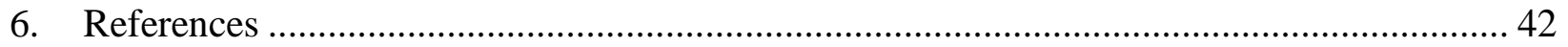

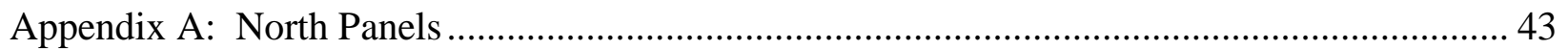

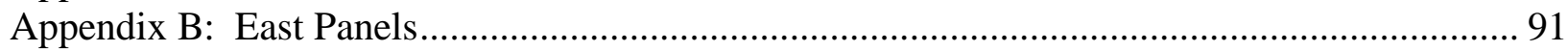

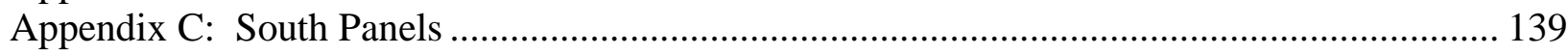

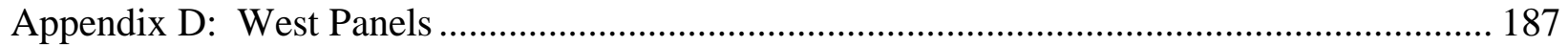

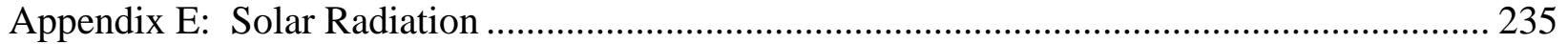

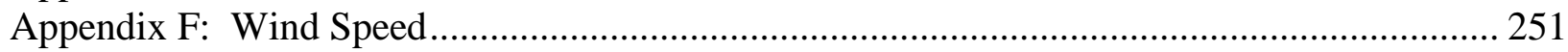

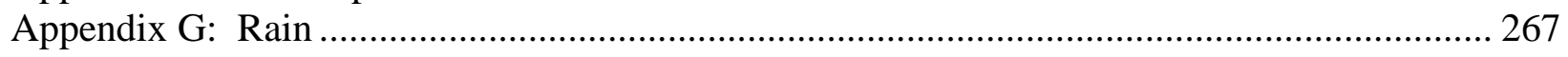

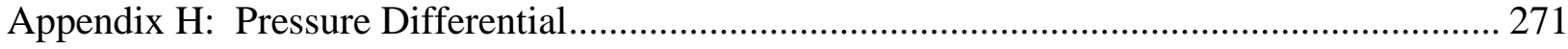




\section{Field Assessment of Air Barriers at the Building Envelope Systems Testing (BEST) Laboratory Phase 1}

\section{Introduction}

The Buildings Technologies Research and Integration Center (BTRIC) at the Oak Ridge National Laboratory (ORNL) performed field evaluations of 24 wall panels with different types of air barriers that are commonly used in residential and commercial construction. Field assessments were conducted from September 2009 until August 2010 at the Building Envelope Systems Test (BEST) laboratory, which is located at Syracuse University, Syracuse, NY. This project is a cooperative research among ORNL, the Air Barrier Association of America (ABAA), Syracuse University, and the New York State Energy Research and Development Authority (NYSERDA).

The 12-month data presented in this report is intended to be used to evaluate the energy and moisture performance of wall panels as individual specimens. ORNL measurements complement air leakage rates reported by Syracuse University for each of the panels (Pradhan et al. 2011). The data should not be used to compare the performance of different air barrier types because the panels were not designed for this purpose. Multiple factors varied among specimens such as the building materials used and their layout, as well as the location of the panel in the test hut. Furthermore, the installation of the air barrier assemblies was performed by representatives from 11 manufacturers, which introduced discrepancies in workmanship.

\section{BEST Laboratory}

The BEST lab is a two-story facility where up to 34 wall panels can be exposed to controlled indoor conditions (i.e., temperature) and the outdoor environment (Figure 1). The laboratory is equipped with a weather station that monitors temperature, relative humidity, solar radiation, rain accumulation, wind speed and direction, and atmospheric pressure. 


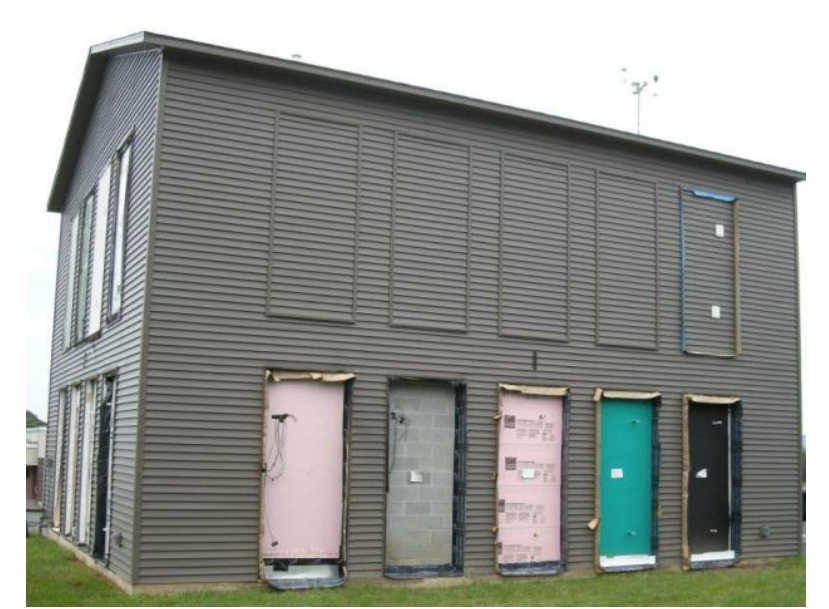

Figure 1. Building Envelope Systems Testing (BEST) lab.

\subsection{Laboratory Equipment}

Laboratory monitoring instruments include:

1. Temperature: Honeywell/Fenwal 192-103LET-A01 thermistor ( $\pm 0.2 \%$ accuracy).

2. Relative humidity: Honeywell HIH-4000 Series $( \pm 3.5 \%$ accuracy and $\pm 0.5 \%$ repeatability).

3. Dataloggers: Campbell Scientific CR1000.

4. Multiplexers: Campbell Scientific AM16/32.

5. Pressure: Energy Conservatory Automated Performance Testing system (resolution: 0.1 Pa; accuracy: $\pm 1 \%$ of reading or twice the resolution, whichever is greater)

Weather station instruments are:

1. Temperature: Campbell Scientific CS215 (accuracy: $\pm 0.3{ }^{\circ} \mathrm{C}$ at $25^{\circ} \mathrm{C} ; \pm 0.4{ }^{\circ} \mathrm{C}$ over $25^{\circ} \mathrm{C}$ to $40{ }^{\circ} \mathrm{C} ; \pm 0.9{ }^{\circ} \mathrm{C}$ over $40{ }^{\circ} \mathrm{C}$ to $70{ }^{\circ} \mathrm{C}$ ).

2. Relative humidity: Campbell Scientific CS215 (accuracy at $25^{\circ} \mathrm{C}: \pm 2 \%$ over $10 \%$ to $90 \% \mathrm{RH}$, and $\pm 4 \%$ over $90 \%$ to $100 \% \mathrm{RH})$.

3. Wind speed: Gill Windsonic $1( \pm 2 \%$ accuracy at $12 \mathrm{~m} / \mathrm{s})$.

4. Wind direction: Gill Windsonic $1\left( \pm 3^{\circ}\right.$ accuracy at $\left.20 \mathrm{~m} / \mathrm{s}\right)$.

5. Rainfall: Texas Electronics TE525WS ( $\pm 1 \%$ accuracy at up to $2.54 \mathrm{~cm} / \mathrm{hr}$ ).

6. Total solar radiation flux: Hukseflux LP02.

7. Solar radiation on vertical surfaces: Campbell Scientific LI200X pyranometer. 
8. Atmospheric pressure: Vaisala CS106 (accuracy: $\pm 0.3 \mathrm{mb}$ at $20{ }^{\circ} \mathrm{C} ; \pm 0.6 \mathrm{mb}$ over 20 ${ }^{\circ} \mathrm{C}$ to $40{ }^{\circ} \mathrm{C} ; \pm 1.5 \mathrm{mb}$ over $40{ }^{\circ} \mathrm{C}$ to $60{ }^{\circ} \mathrm{C}$ ).

Monitoring instruments within wall panels:

1. Temperature: Honeywell/Fenwal 192-103LET-A01 thermistor ( $\pm 0.2 \%$ accuracy).

When installed in conjunction with a relative humidity sensor and in a location where the instruments could get wet (e.g., behind the cladding), the sensors were protected with a membrane that was impermeable to water and had a high water vapor permeability.

2. Relative humidity: Honeywell HIH-4000 Series $( \pm 3.5 \%$ accuracy and $\pm 0.5 \%$ repeatability). When installed in a location where the instruments could get wet (e.g., behind the cladding), the sensors were protected with a membrane that was impermeable to water and had a high water vapor permeability.

3. Heat flux: Concept Engineering Model F-002-4.

\section{Panel Material and Sensor Layout}

Twenty-four panels were monitored in Phase 1 at the BEST lab. Figure 2 illustrates the arrangement of the panels in the building as well as the air barrier type utilized in them. The materials that were used in each of the panels are described in Table 1 and in more detail in Figures 3 to 24 .
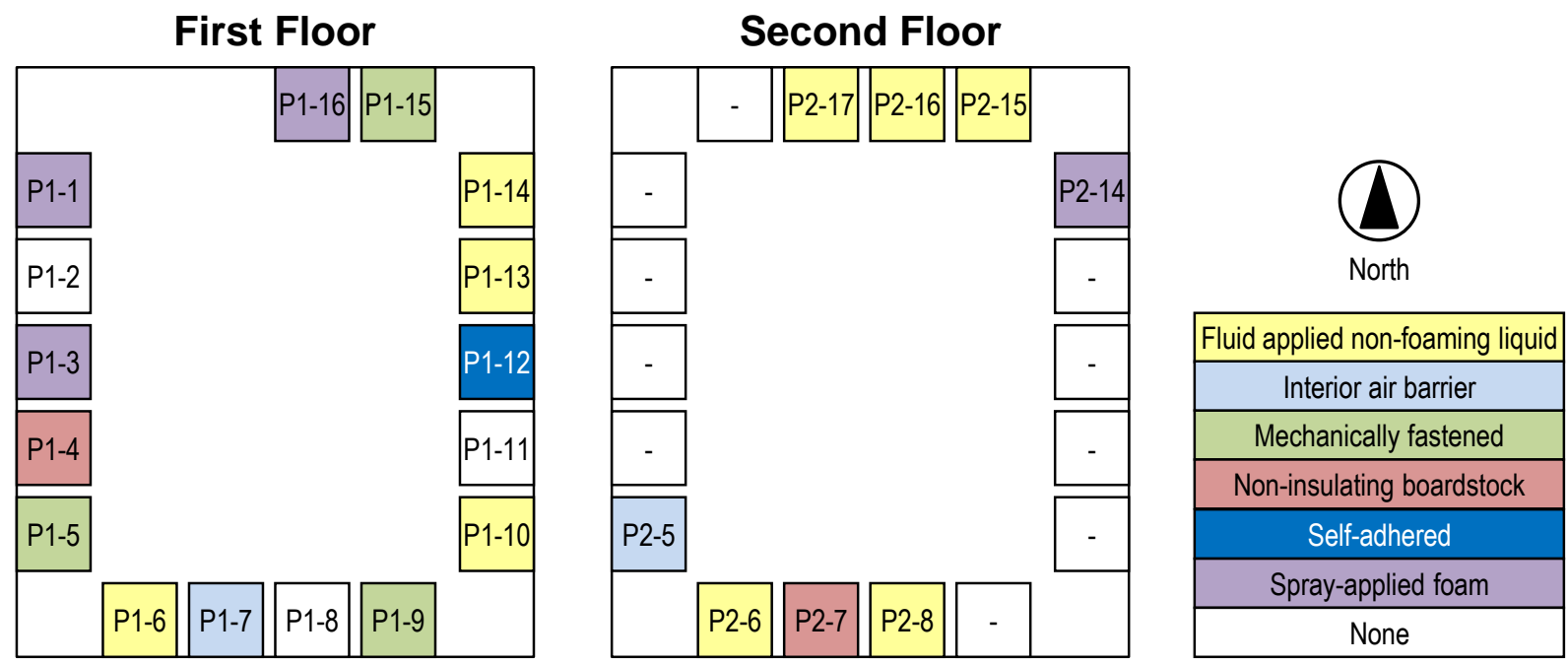

Figure 2. Panel layout at the BEST lab. The figure also illustrates the air barrier types utilized in the panels. 
Table 1. Building materials and sensor layout in wall panels ${ }^{\mathrm{a}}$.

\begin{tabular}{|c|c|c|c|c|c|c|c|}
\hline Panel & $I D^{b}$ & Air Barrier Type & Cladding & Structural Framing & Exterior Sheathing & Insulation & $\begin{array}{l}\text { Sensor } \\
\text { Layoutc }\end{array}$ \\
\hline P1-1 & $\mathrm{AB} 13$ & Spray-applied foam & Brick & 8" CMU & - & $2 \frac{1}{2} "$ Spray foam & C \\
\hline P1-2 & - & No air barrier (control) & - & 8" split-faced CMU & - & - & $\mathrm{D}$ \\
\hline P1-3 & AB27 & Spray-applied foam & Vinyl siding & $2 \times 6$ wood studs & OSB & Spray foam & B \\
\hline P1-4 & AB16 & Non-insulating boardstock & Stone & $2 \times 6$ wood studs & OSB & R-21 Faced fiberglass & $A$ \\
\hline P1-5 & AB19 & Mechanically fastened membrane & Stone & $6 "$ steel studs & Gypsum w/ fiberglass mat & R-21 Faced fiberglass & $A$ \\
\hline P1-6 & AB9 & Fluid-applied non-foaming liquid & Brick & 6" steel studs & Gypsum w/ fiberglass mat & R-10 XPS rigid foam & A \\
\hline P1-7 & AB23 & Interior air barrier & Brick & $6 "$ steel studs & Gypsum w/ fiberglass mat & R-21 Unfaced fiberglass and 1" XPS rigid insulation & A \\
\hline P1-8 & - & No air barrier (control) & Brick & $6 "$ steel studs & Gypsum w/ fiberglass mat & R-21 Faced fiberglass & B \\
\hline P1-9 & AB6 & Mechanically fastened membrane & Brick & 6" steel studs & Gypsum w/ fiberglass mat & R-21 Faced fiberglass & B \\
\hline P1-10 & AB24 & Fluid-applied non-foaming liquid & Brick & 8" CMU & - & R-10 XPS Rigid foam & C \\
\hline P1-11 & - & No air barrier (control) & Brick & 8" CMU & - & - & C \\
\hline P1-12 & AB20 & Self-adhered membrane & Brick & $6 "$ steel studs & Gypsum w/ fiberglass mat & R-10 XPS Rigid foam & A \\
\hline $\mathrm{P} 1-13$ & AB21 & Fluid-applied non-foaming liquid & Brick & 6" steel studs & Gypsum w/ fiberglass mat & R-21 Faced fiberglass & A \\
\hline P1-14 & $\mathrm{AB} 2$ & Fluid-applied non-foaming liquid & Brick & $6 "$ steel studs & Gypsum w/ fiberglass mat & R-21 Faced fiberglass & $A$ \\
\hline P1-15 & AB25 & Mechanically fastened membrane & Thin brick & 6" steel studs & Gypsum w/ fiberglass mat & R-21 Faced fiberglass & A \\
\hline P1-16 & AB13 & Spray-applied foam & Brick & $6 "$ steel studs & Gypsum w/ fiberglass mat & $2 \frac{1}{2} "$ "Spray foam & $A$ \\
\hline $\mathrm{P} 2-5$ & AB23 & Interior air barrier & Fiber cement siding & $2 \times 6$ wood studs & - & R-21 Unfaced fiberglass & B \\
\hline P2-6 & AB10 & Fluid-applied non-foaming liquid & Metal panel & $6 "$ steel studs & Gypsum w/ fiberglass mat & R-21 Faced fiberglass & A \\
\hline P2-7 & $A B 16$ & Non-insulating boardstock & Fiber cement siding & $2 \times 6$ wood studs & OSB & R-21 Faced fiberglass & B \\
\hline P2-8 & $\mathrm{AB} 7$ & Fluid-applied non-foaming liquid & Stucco & $6 "$ steel studs & Gypsum w/ fiberglass mat & R-21 Faced fiberglass & A \\
\hline$P 2-14$ & AB26 & Spray-applied foam & Vinyl siding & $2 \times 6$ wood studs & - & Spray foam & A \\
\hline P2-15 & AB4 & Fluid-applied non-foaming liquid & Fiber cement siding & $2 \times 6$ wood studs & OSB & R-21 Faced fiberglass & $A$ \\
\hline$P 2-16$ & - & No air barrier (control) & Stucco & $6 "$ steel studs & Gypsum w/ fiberglass mat & R-21 EPS rigid foam & B \\
\hline $\mathrm{P} 2-17$ & AB8-2 & Fluid-applied non-foaming liquid & Stucco & 6" steel studs & Gypsum w/ fiberglass mat & R-21 EPS rigid foam & B \\
\hline
\end{tabular}

a. All panels, except P1-2, had drywall with latex paint on their interior side. In P1-2, the interior side of the CMU was coated with latex paint.

b. Panel ID used in the report "Air Leakage Measurement of 25 Wall Assemblies with Different Types of Air Barriers" (Pradhan et al. 2011).

c. Sensor layouts are depicted in Figures 25 through 28. 


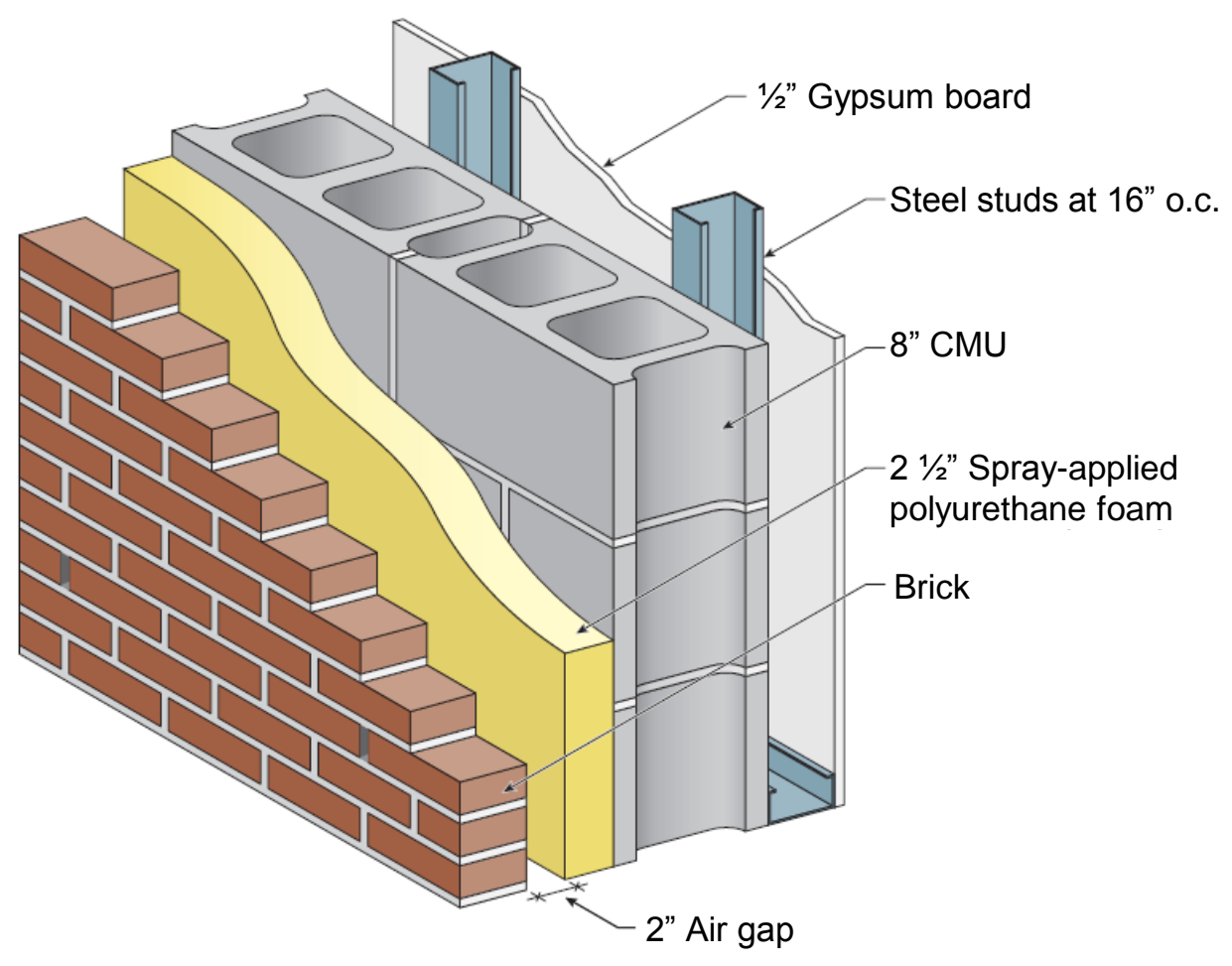

Figure 3. Material layout in panel P1-1.

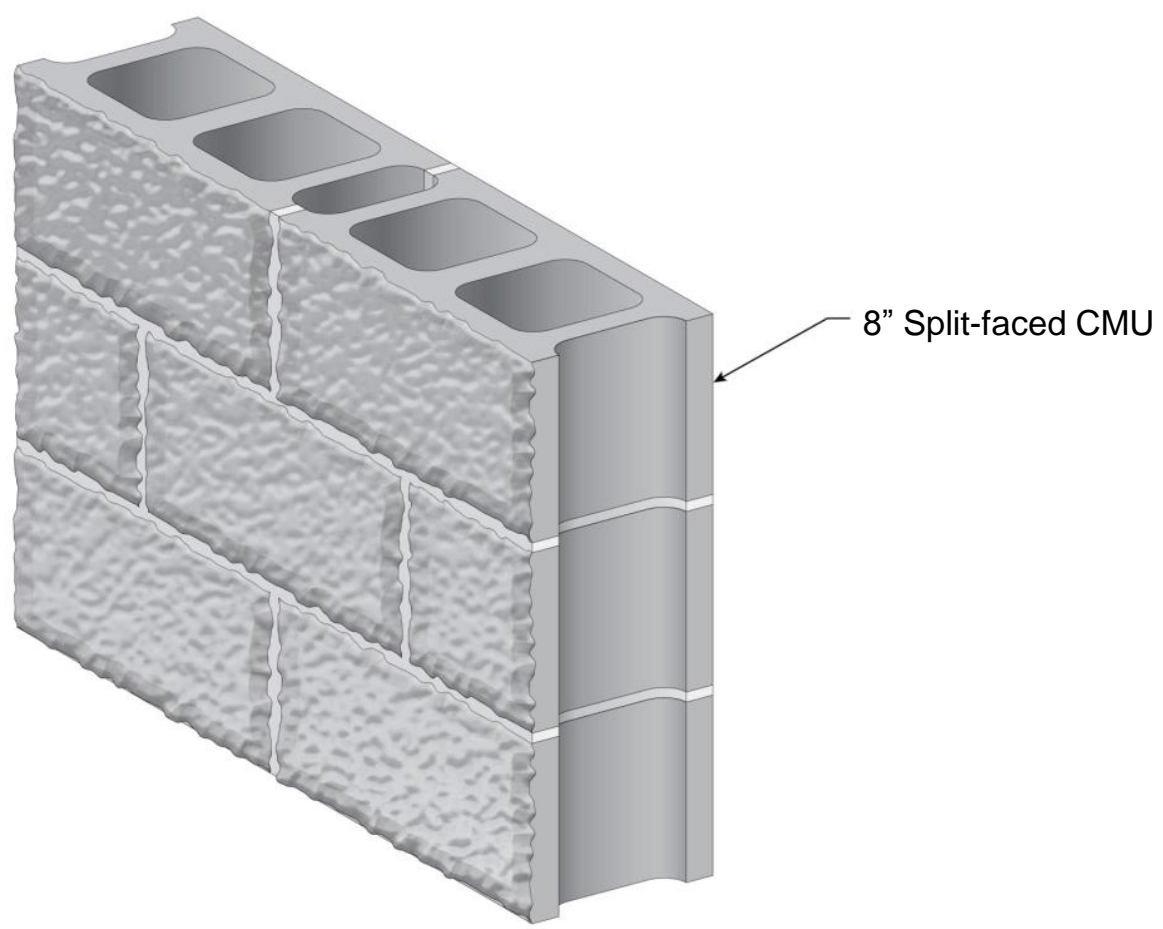

Figure 4. Material layout in panel P1-2 (control). 


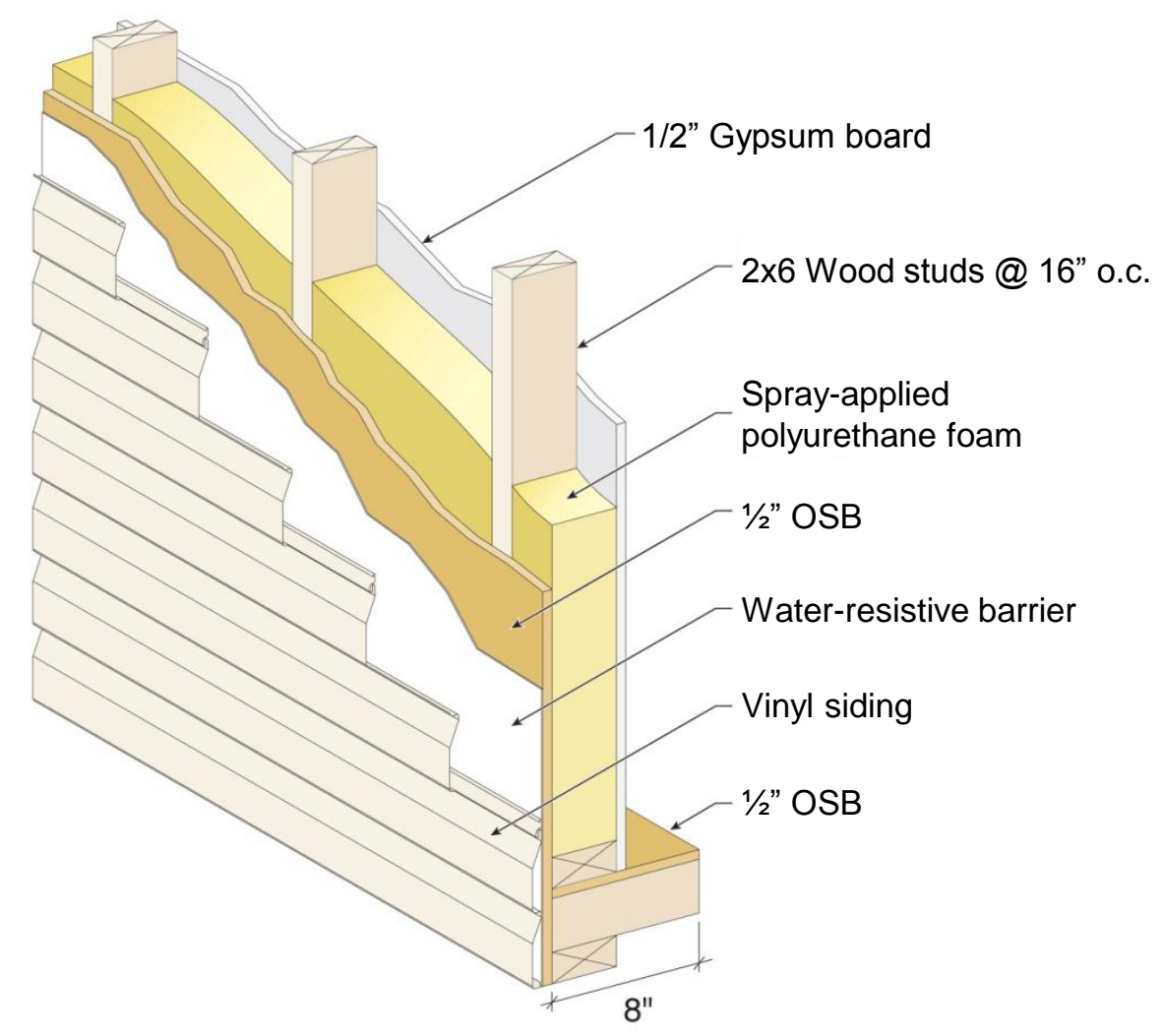

Figure 5. Material layout in panels P1-3 and P2-14.

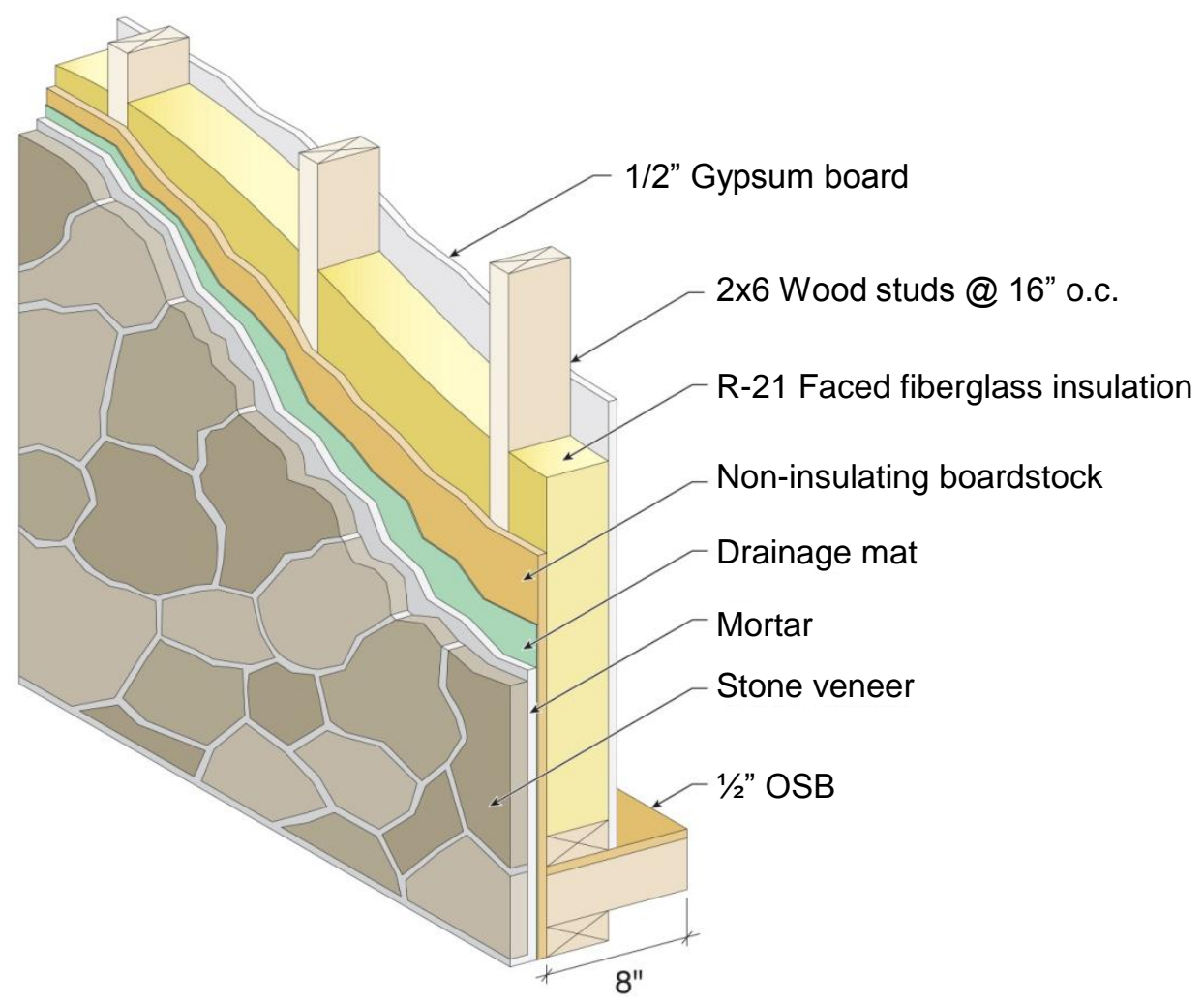

Figure 6. Material layout in panel P1-4 


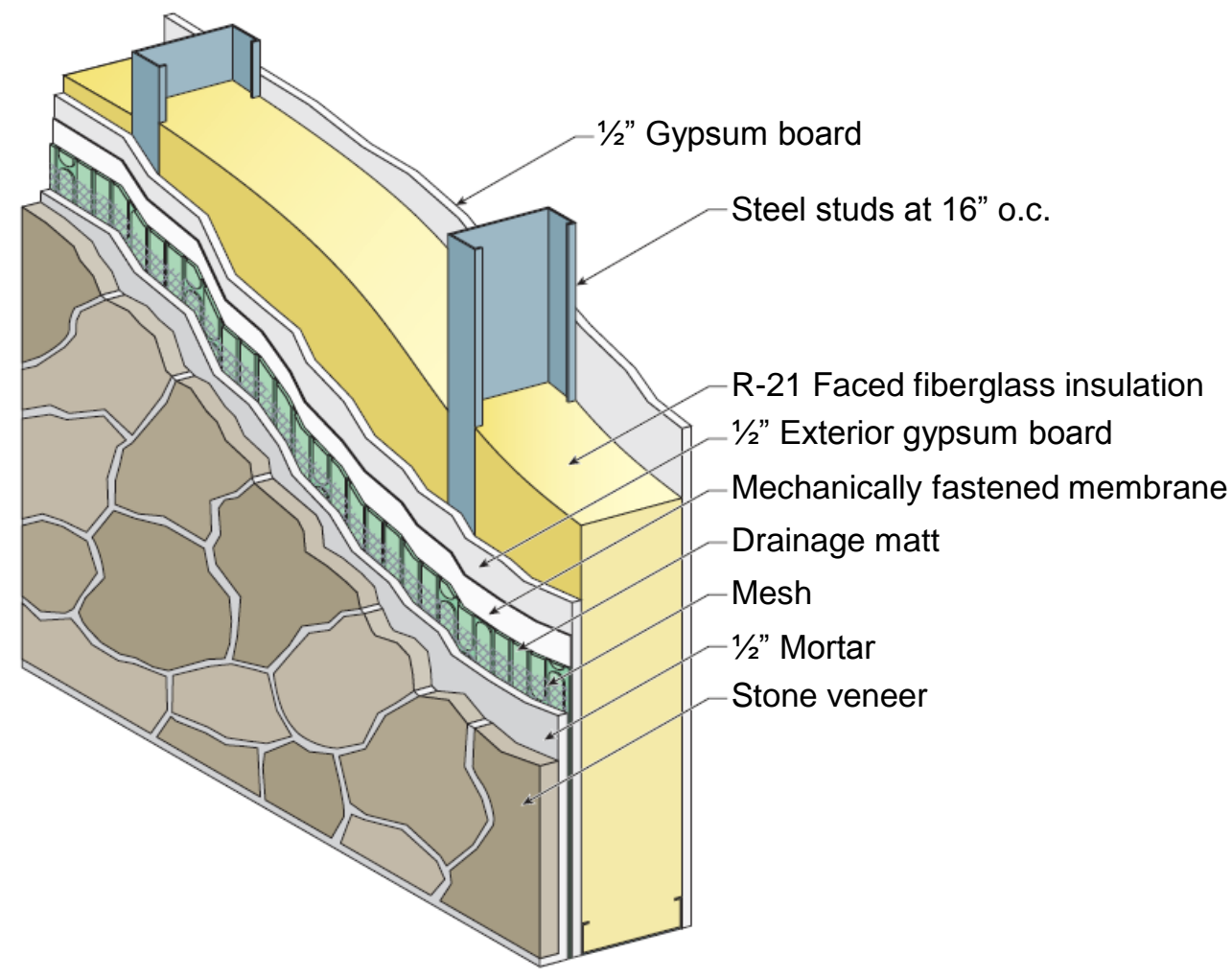

Figure 7. Material layout in panel P1-5.

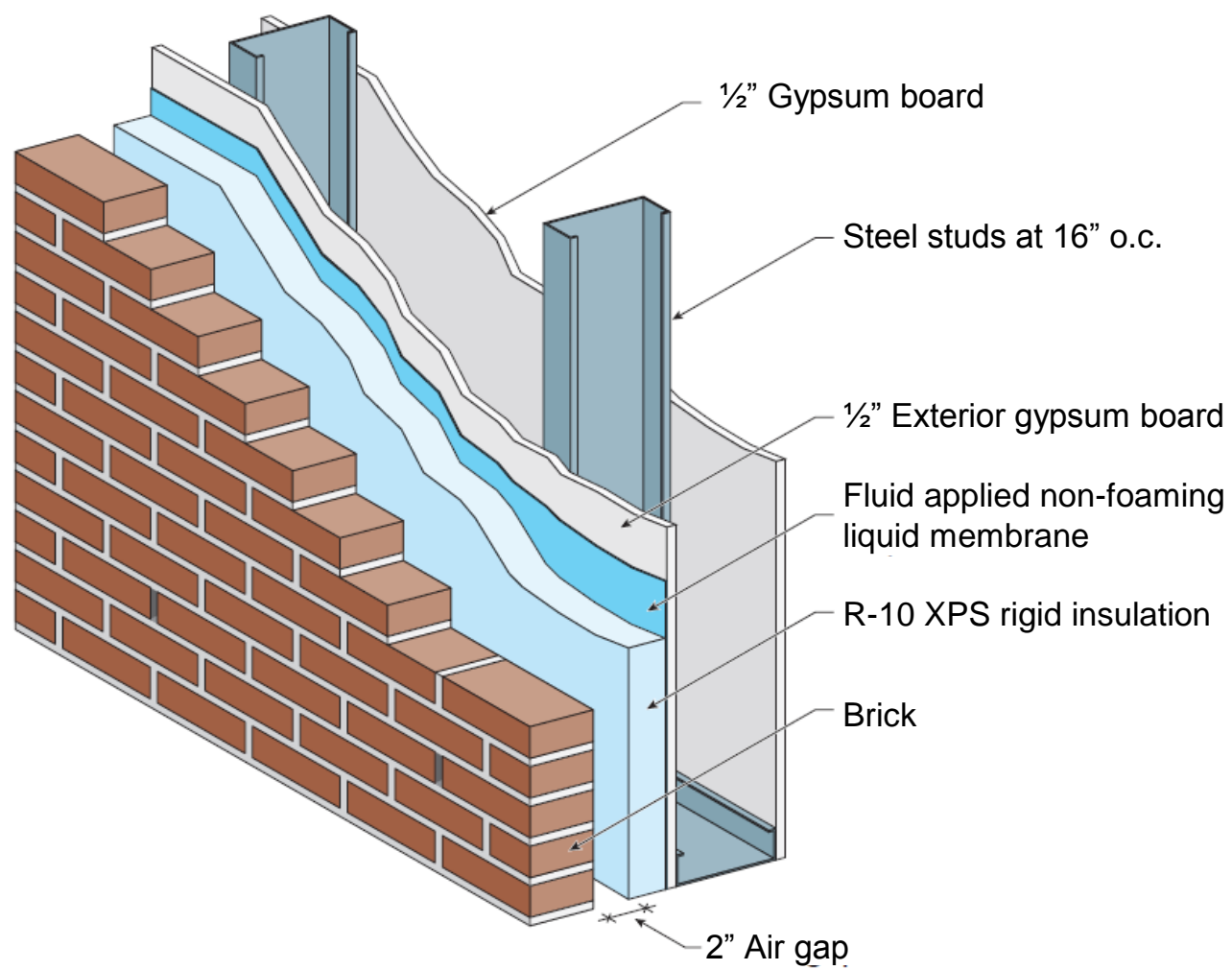

Figure 8. Material layout in panel P1-6. 


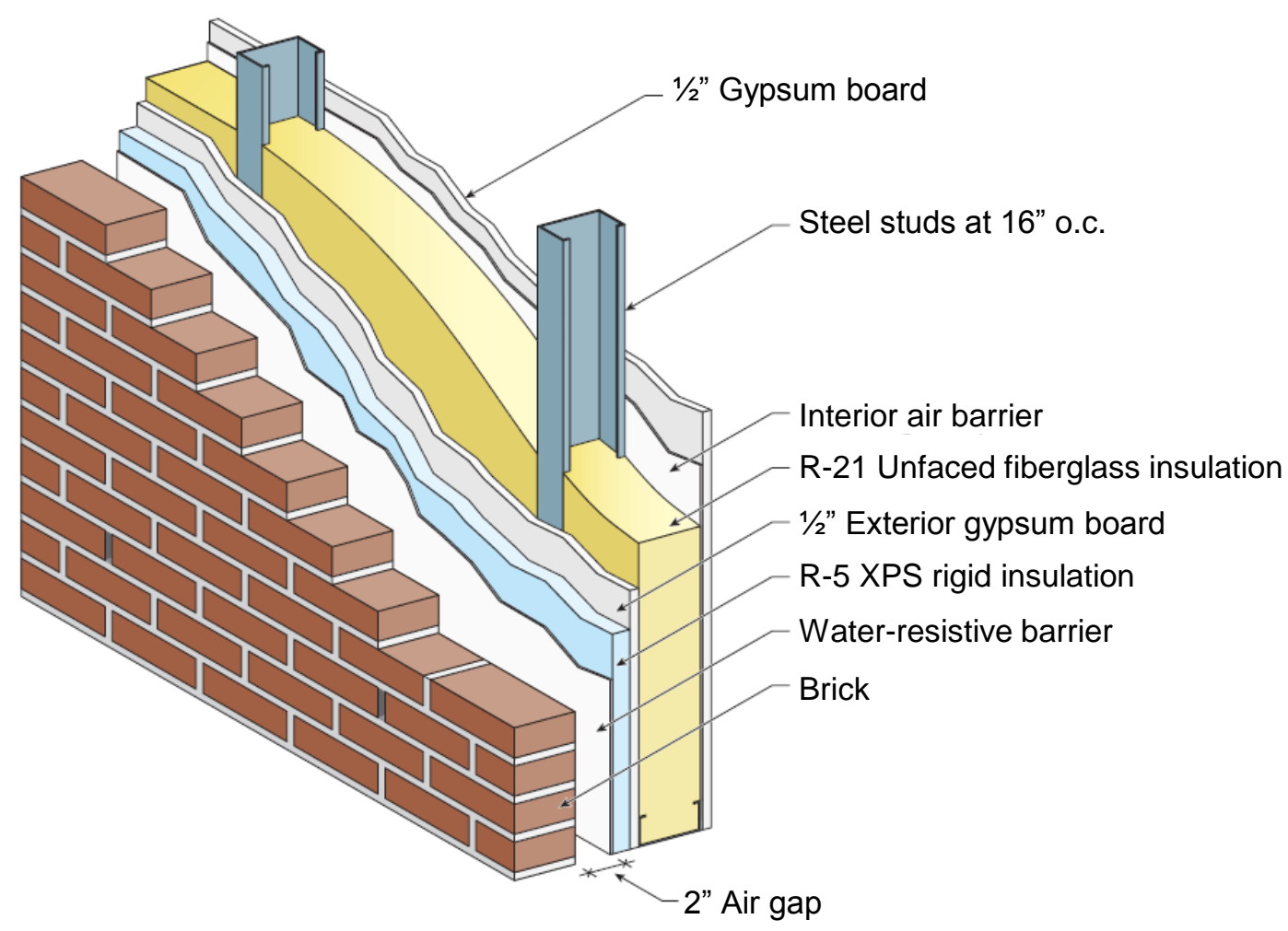

Figure 9. Material layout in panel P1-7.

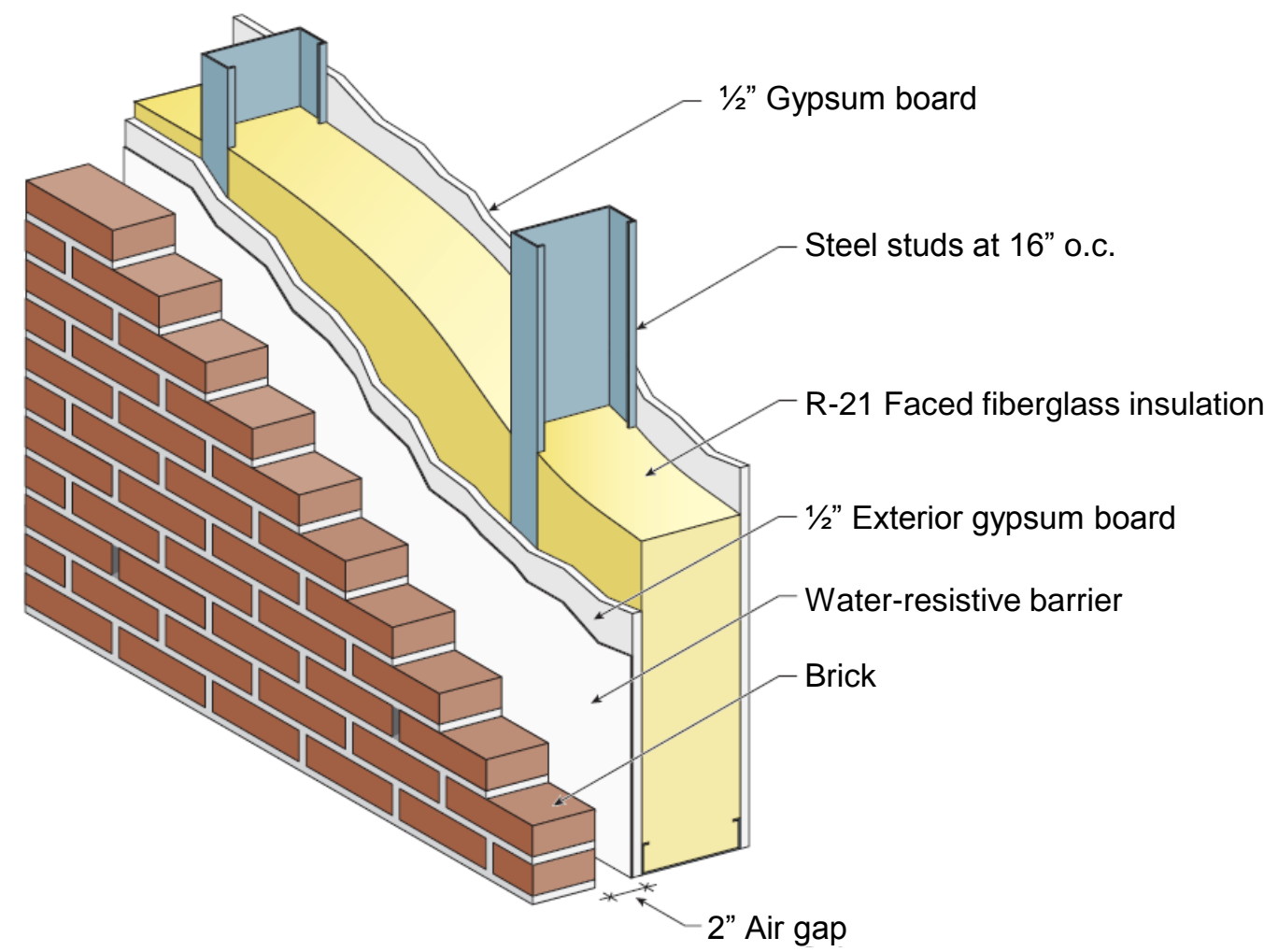

Figure 10. Material layout in panel P1-8 (control). 


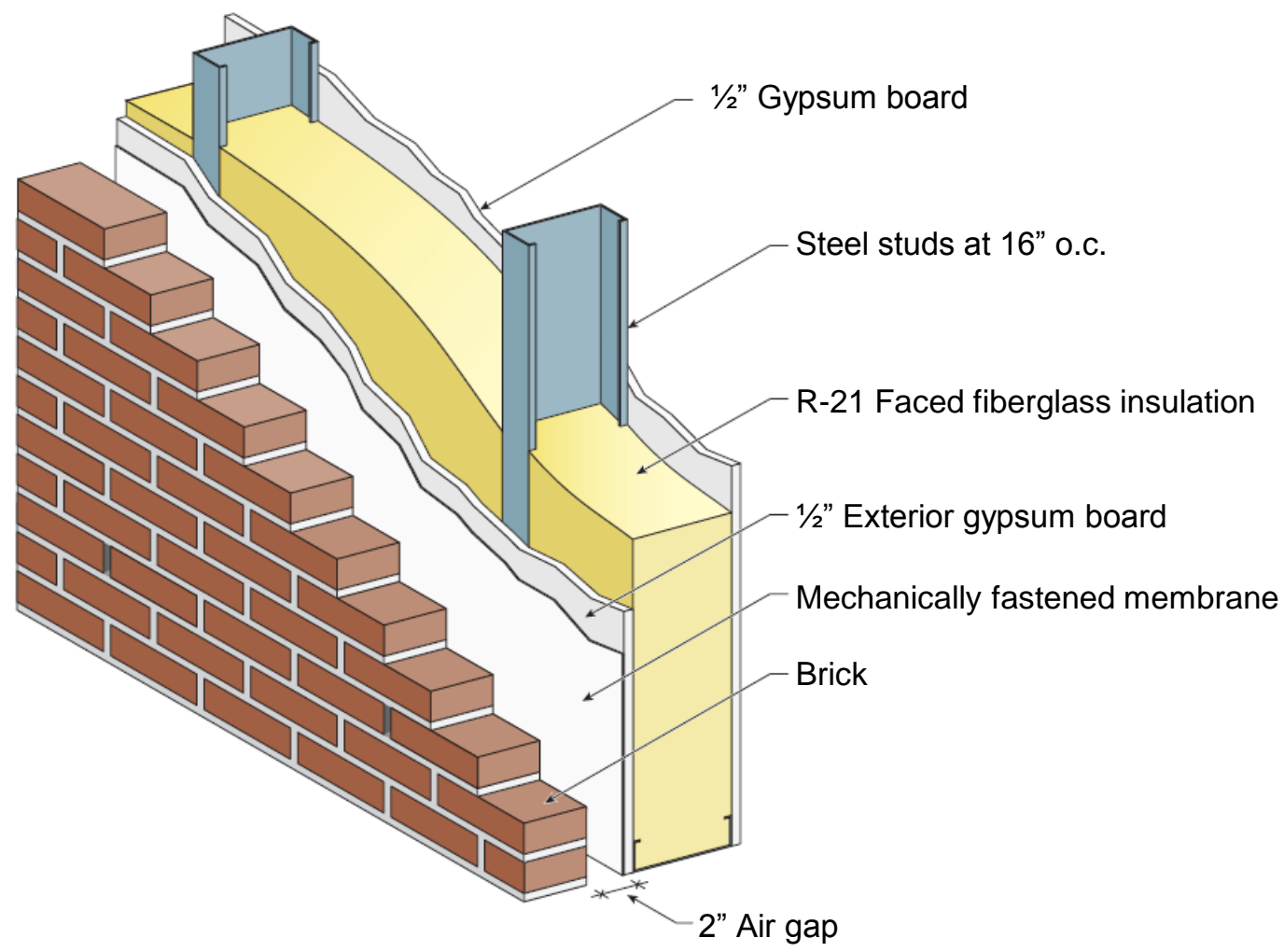

Figure 11. Material layout in panel P1-9.

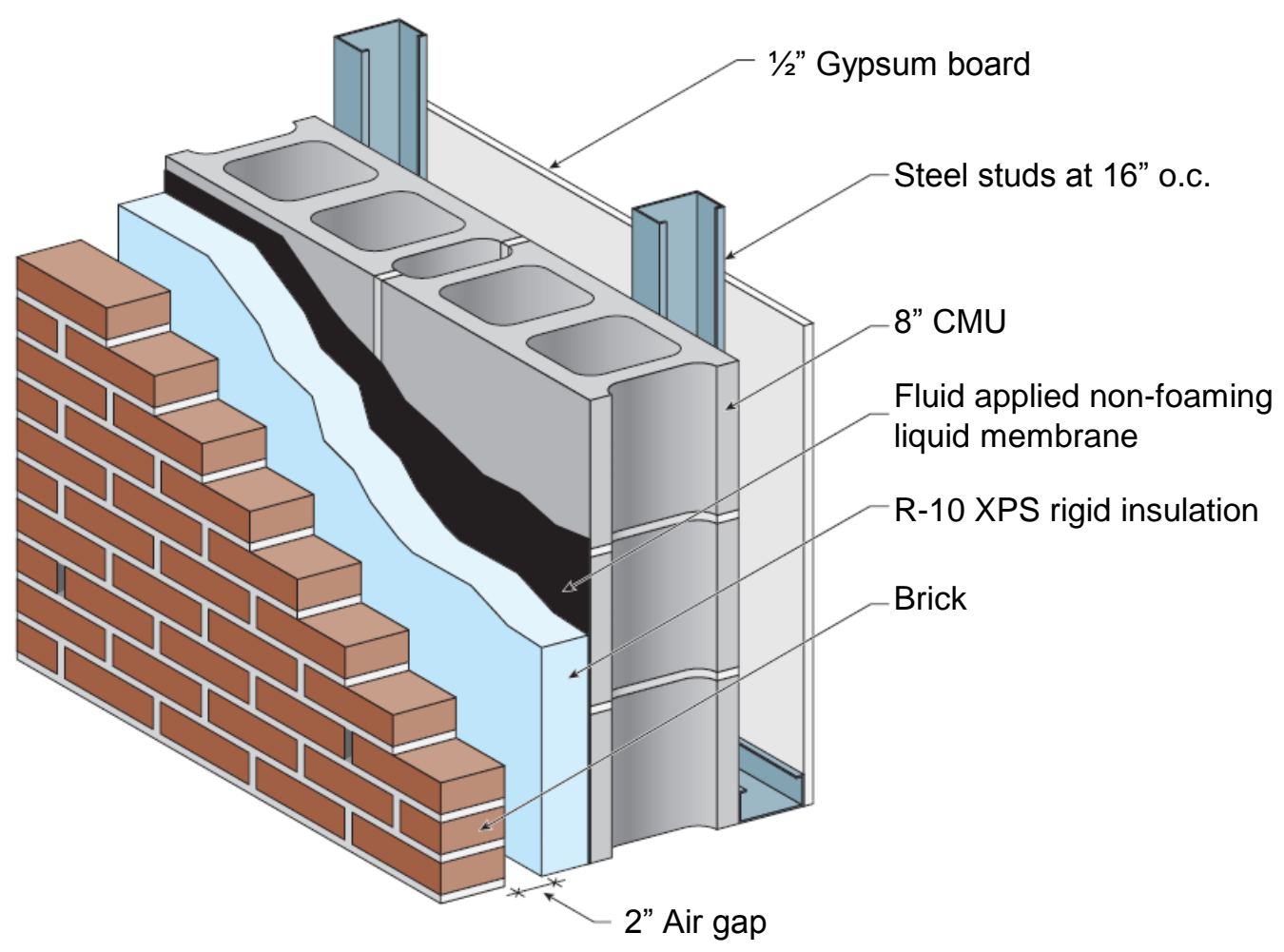

Figure 12. Material layout in panel P1-10. 


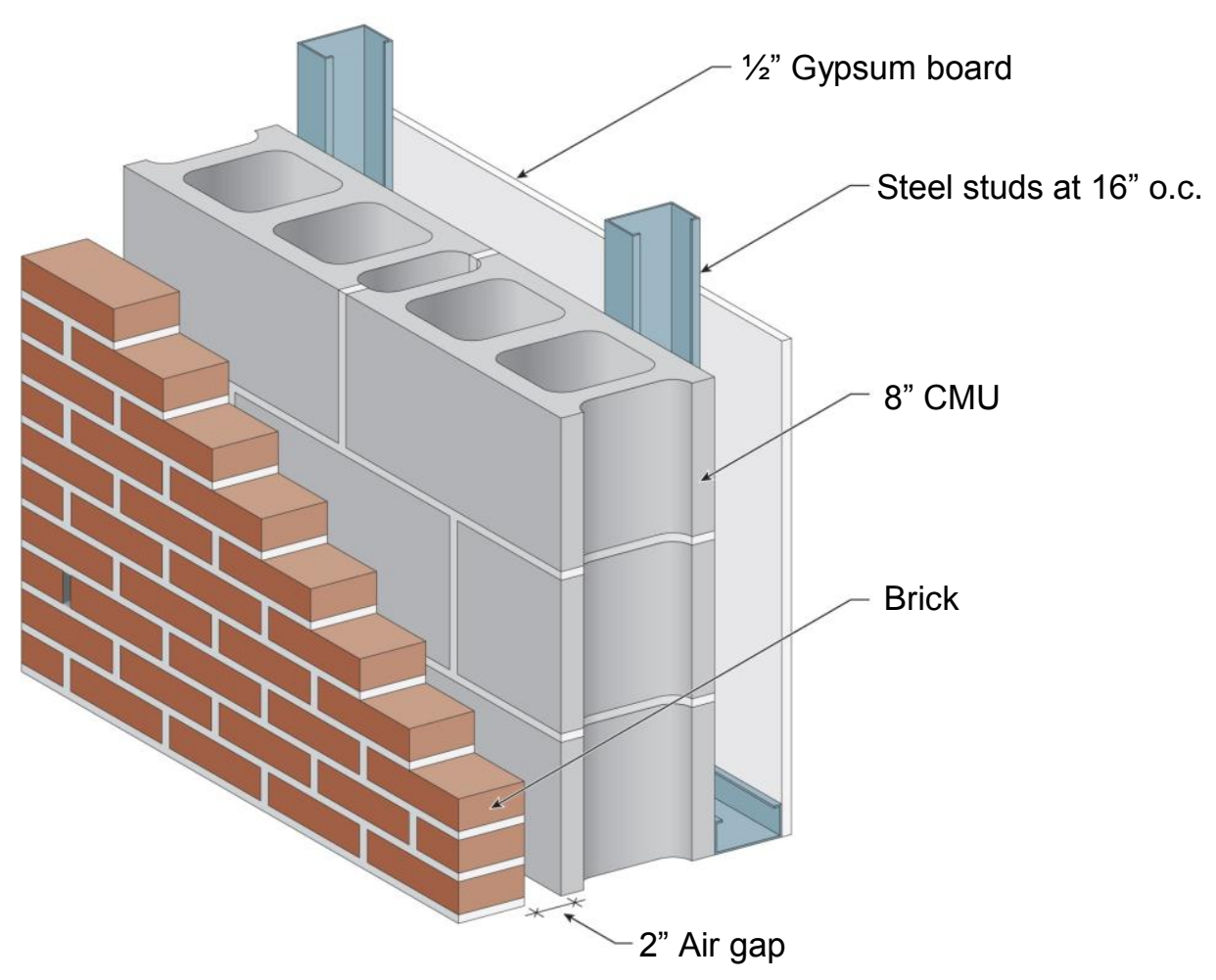

Figure 13. Material layout in panel P1-11 (control).

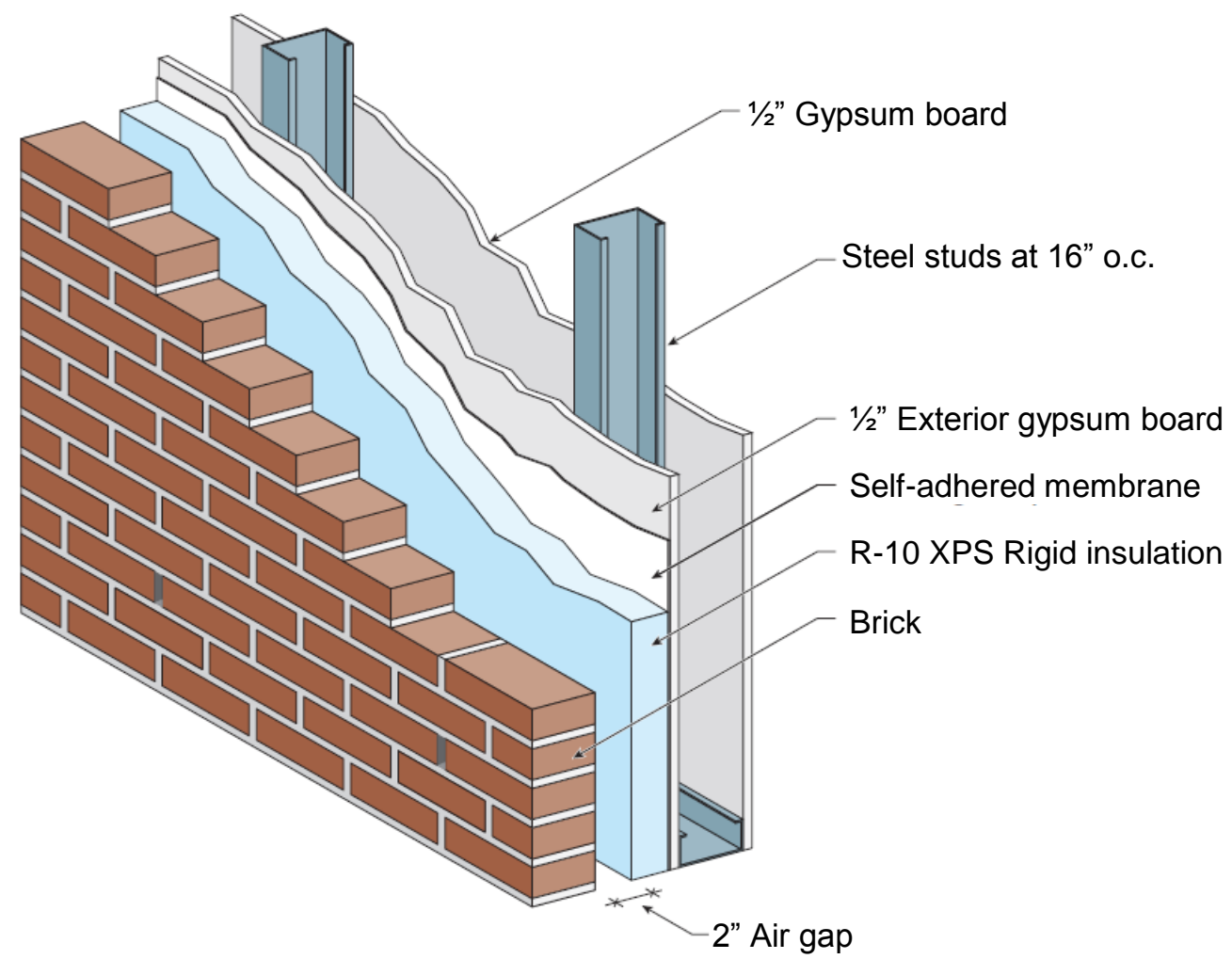

Figure 14. Material layout in panel P1-12. 


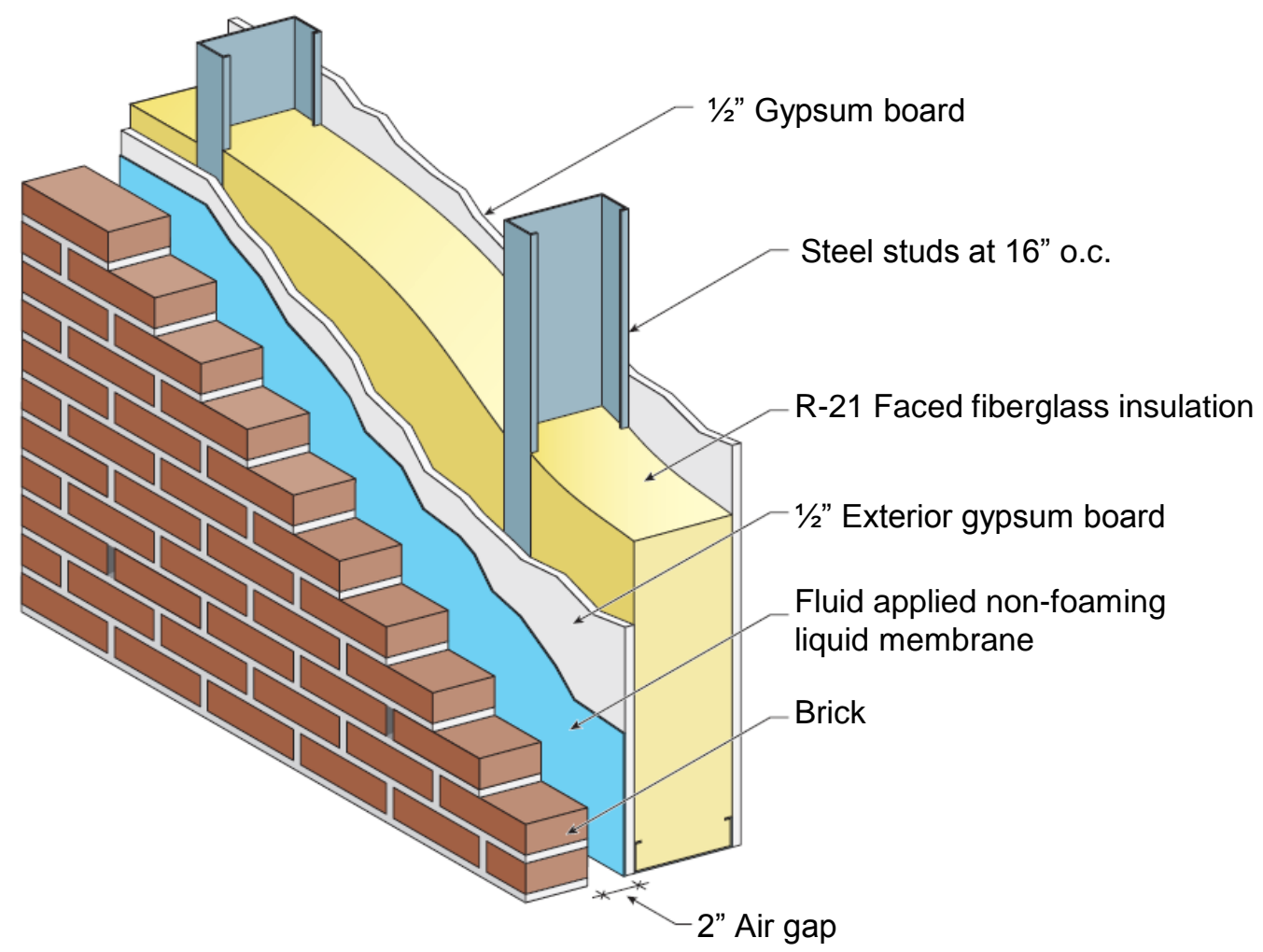

Figure 15. Material layout in panels P1-13 and P1-14.

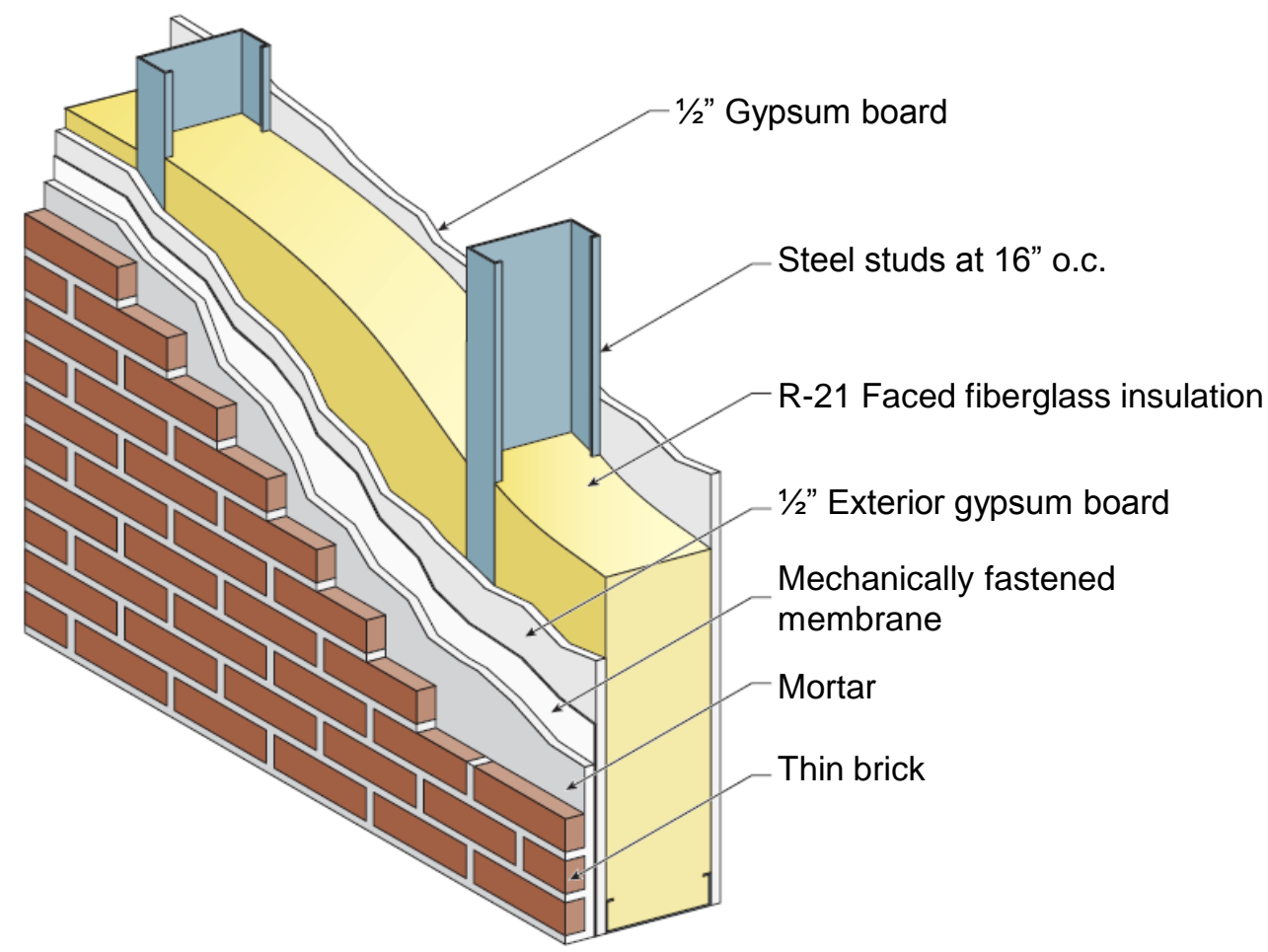

Figure 16. Material layout in panel P1-15. 


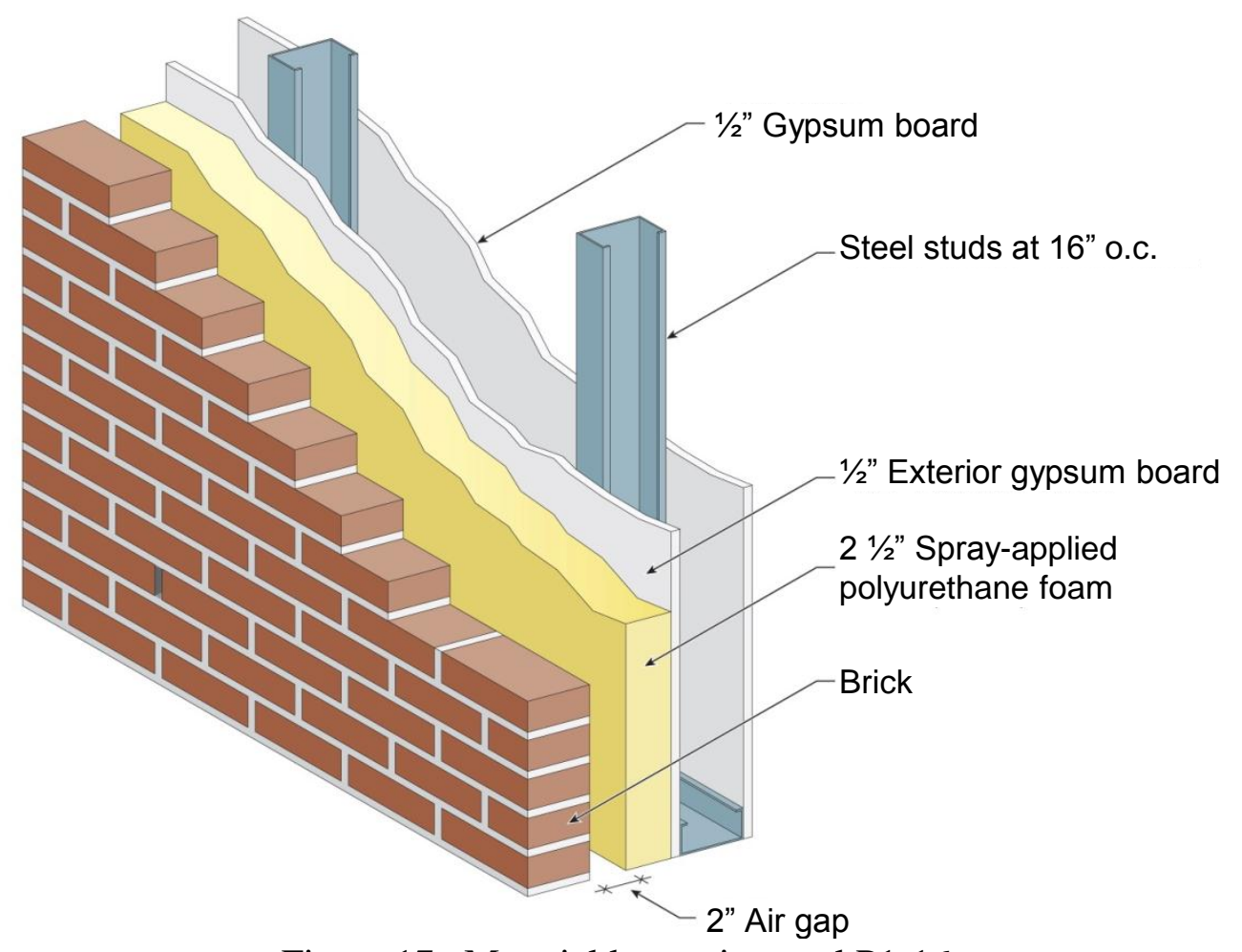

Figure 17. Material layout in panel P1-16.

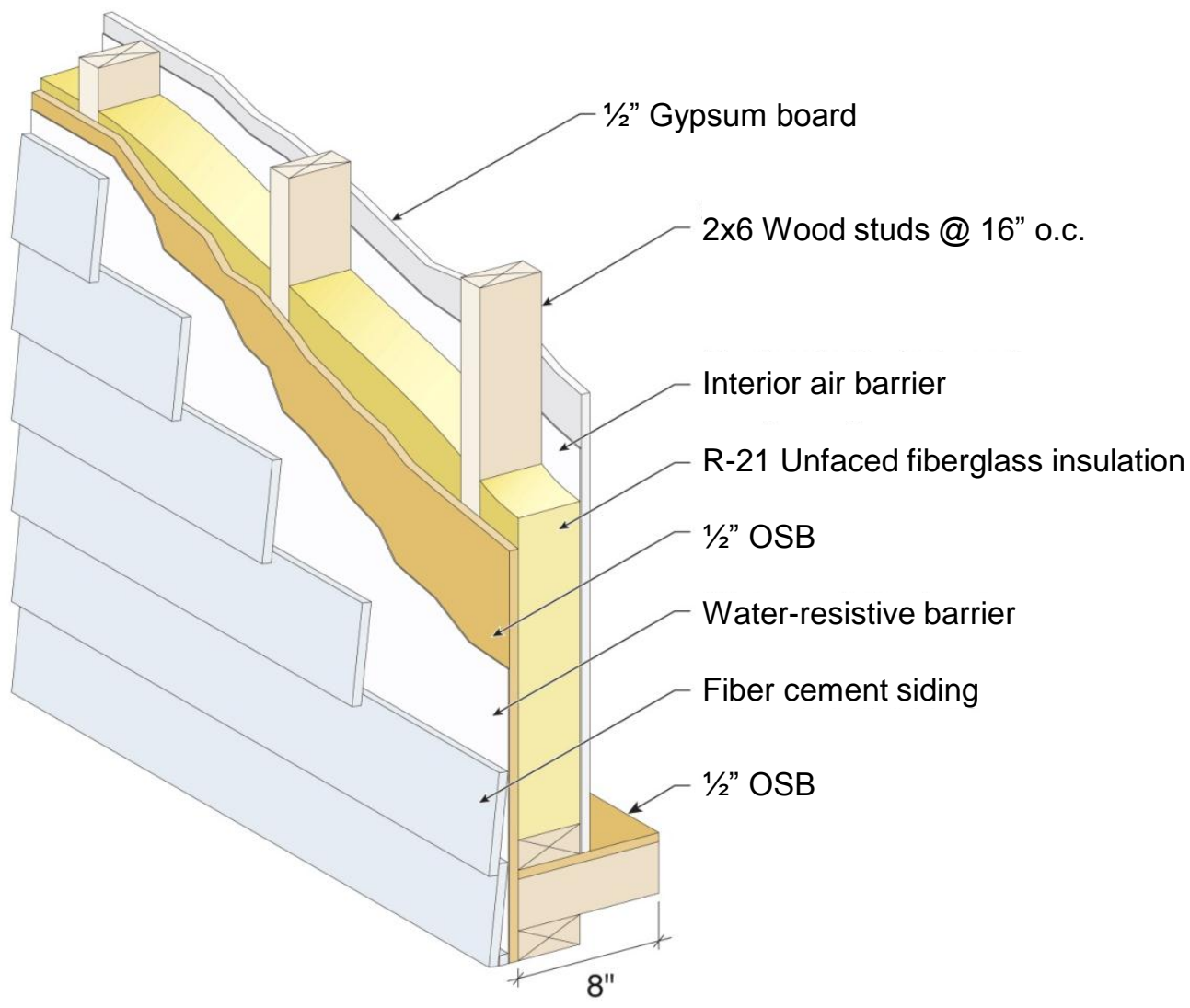

Figure 18. Material layout in panel P2-5. 


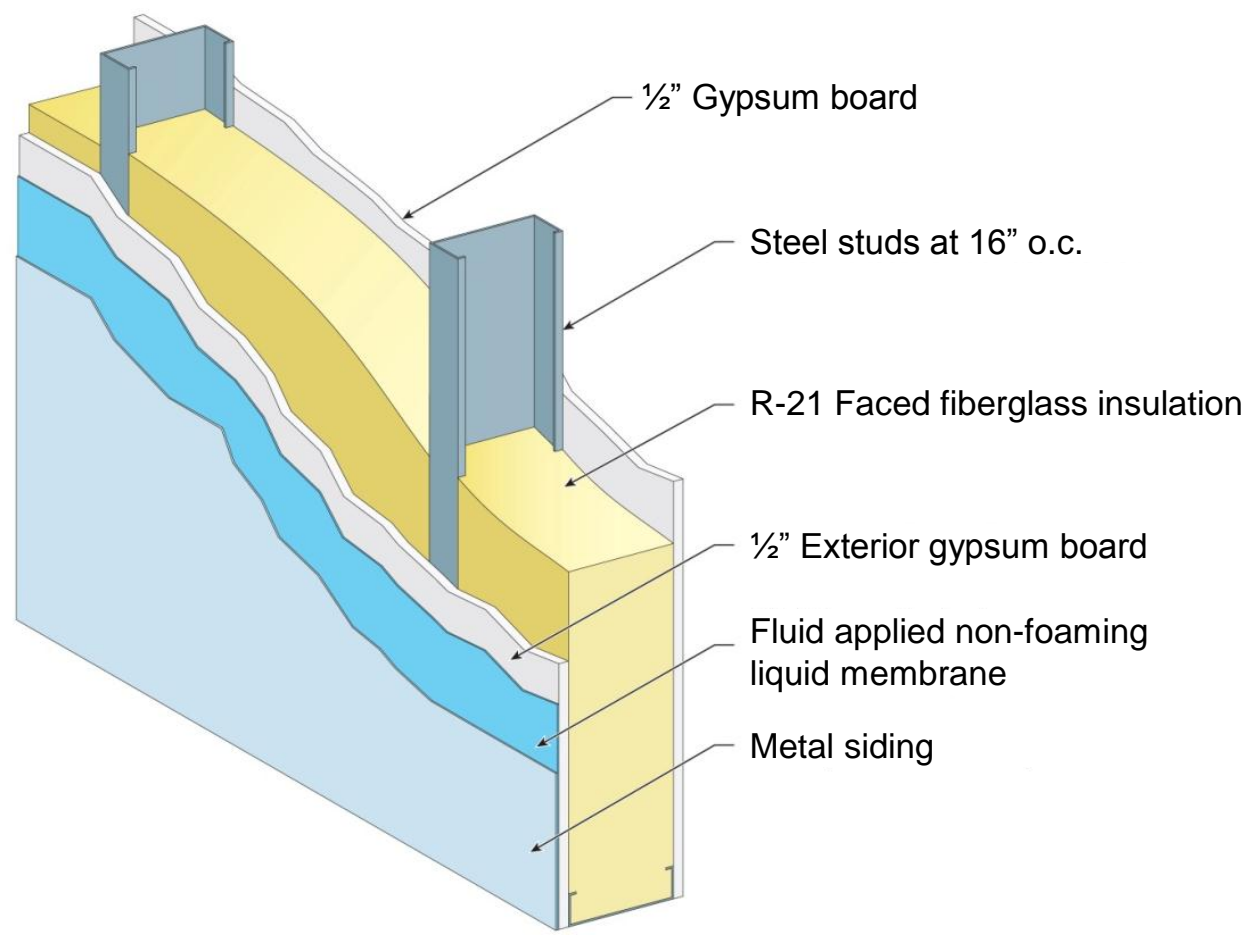

Figure 19. Material layout in panel P2-6.

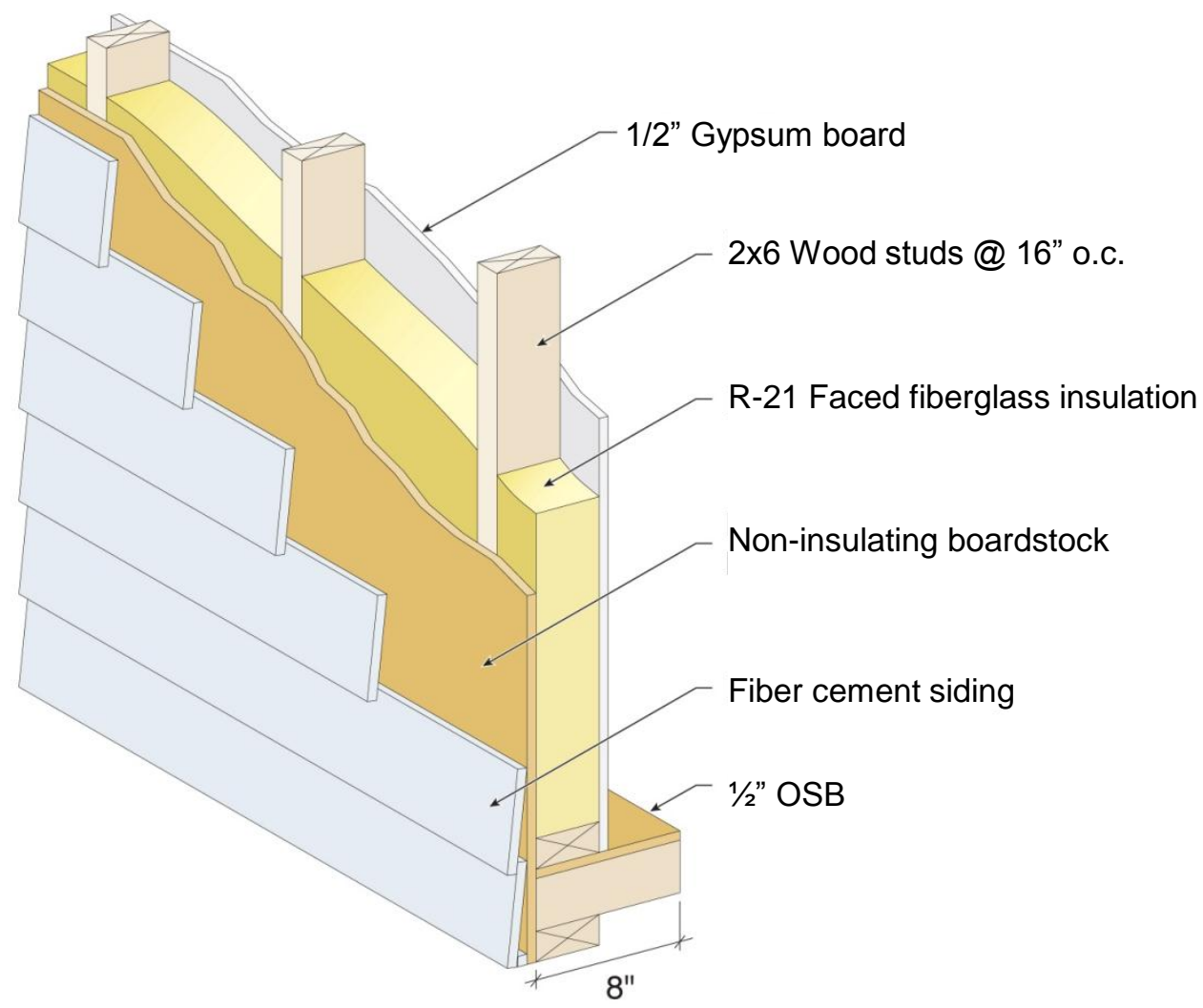

Figure 20. Material layout in panel P2-7. 


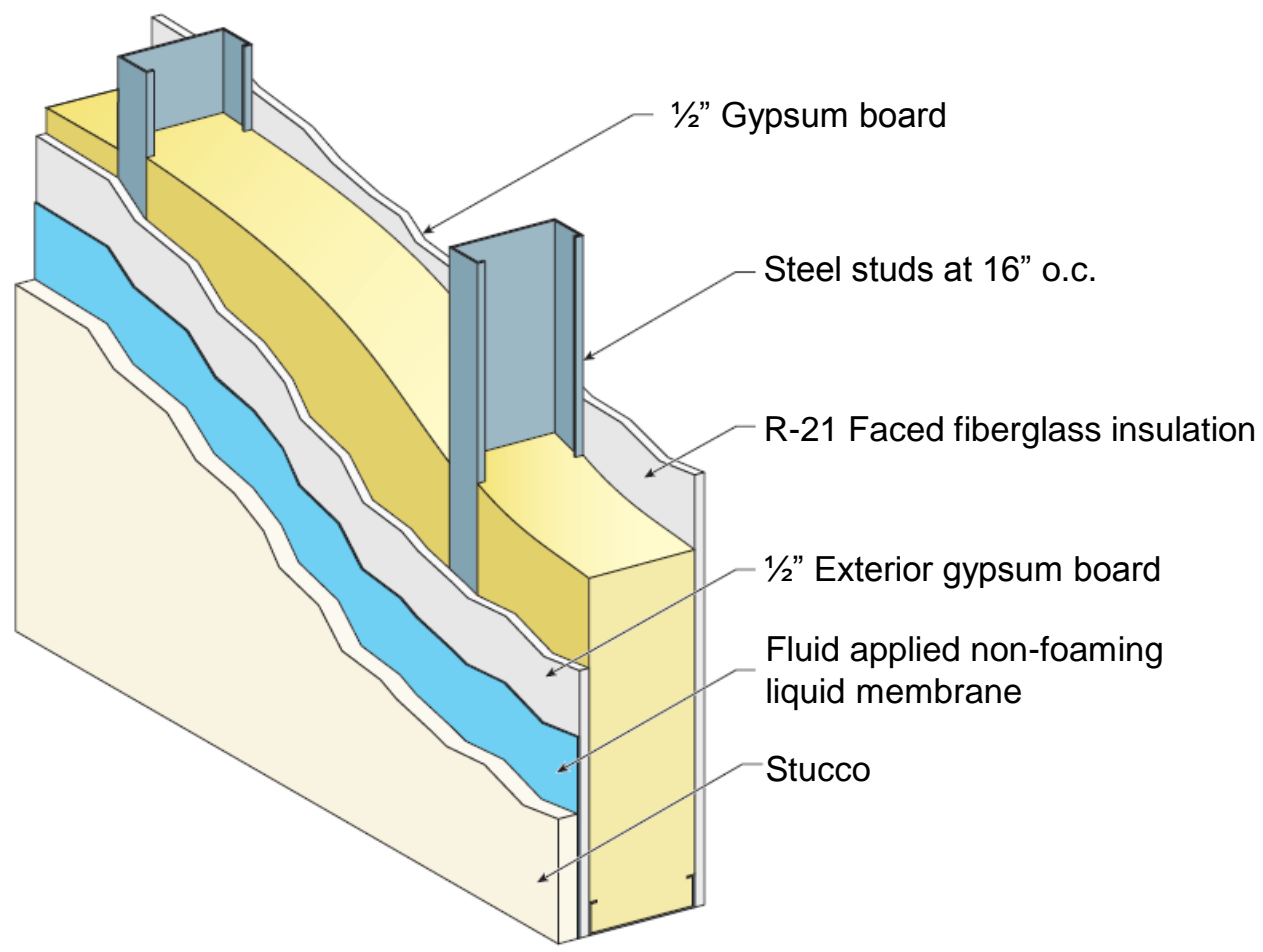

Figure 21. Material layout in panel P2-8.

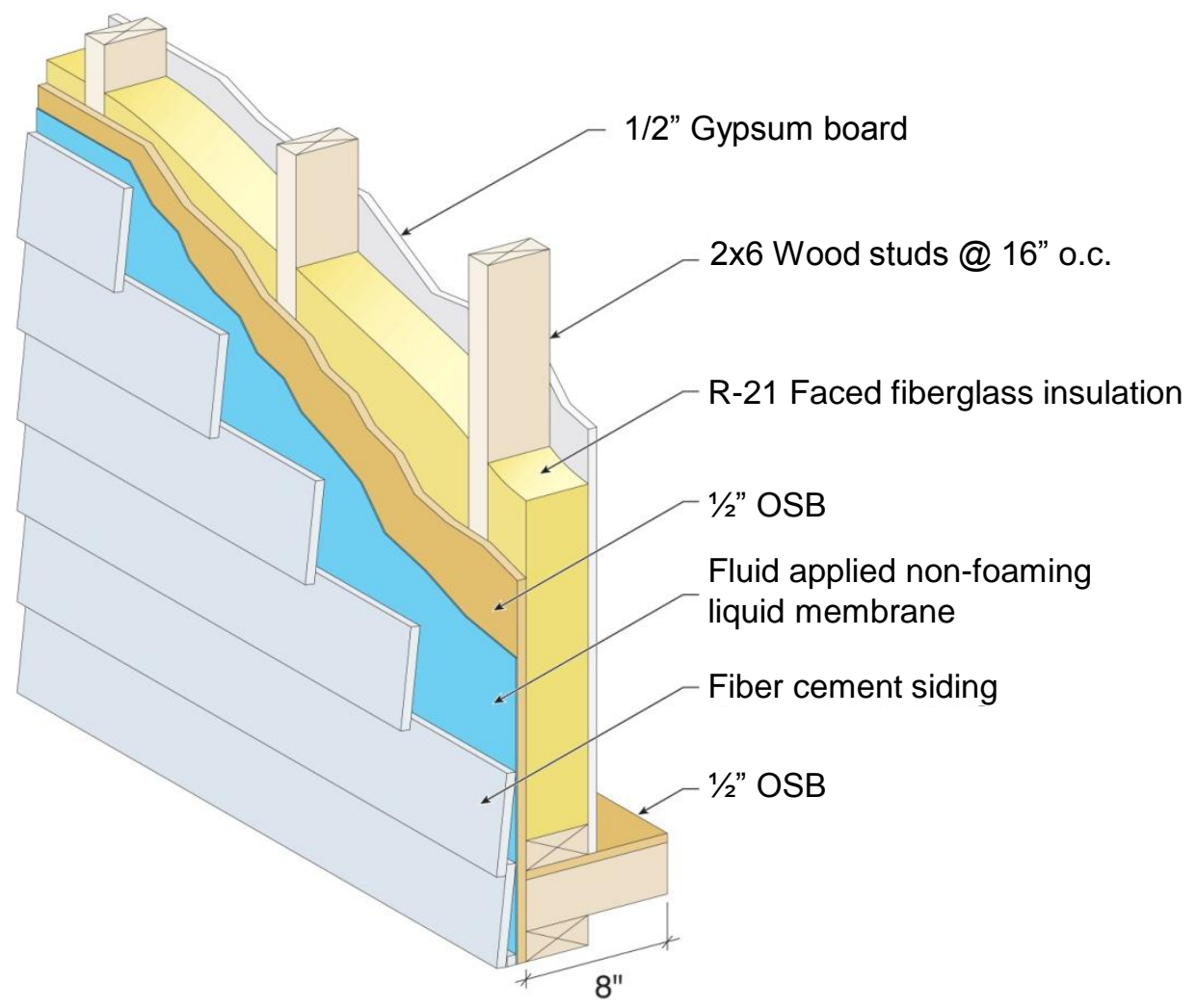

Figure 22. Material layout in panel P2-15. 


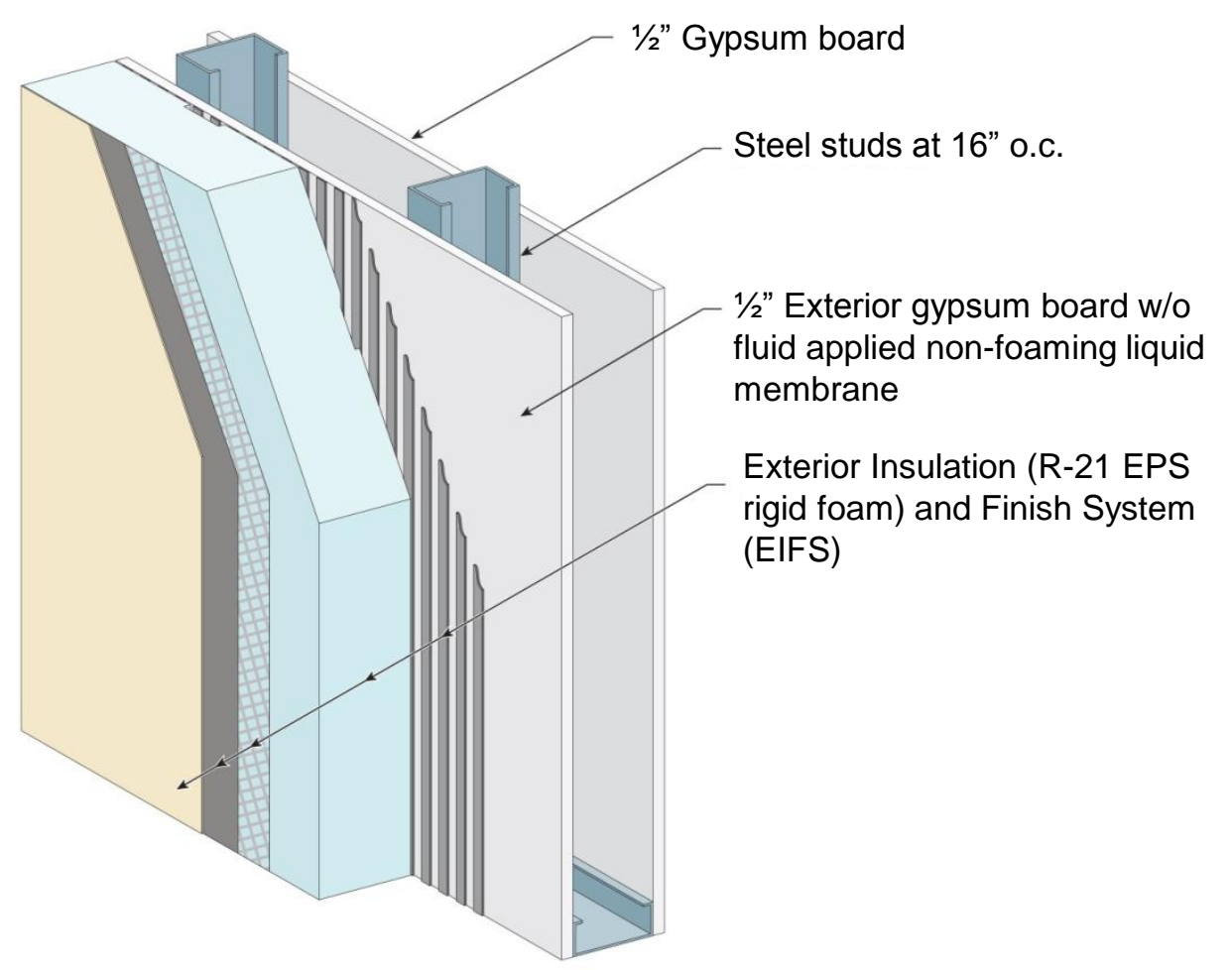

Figure 23. Material layout in panel P2-16 (control).

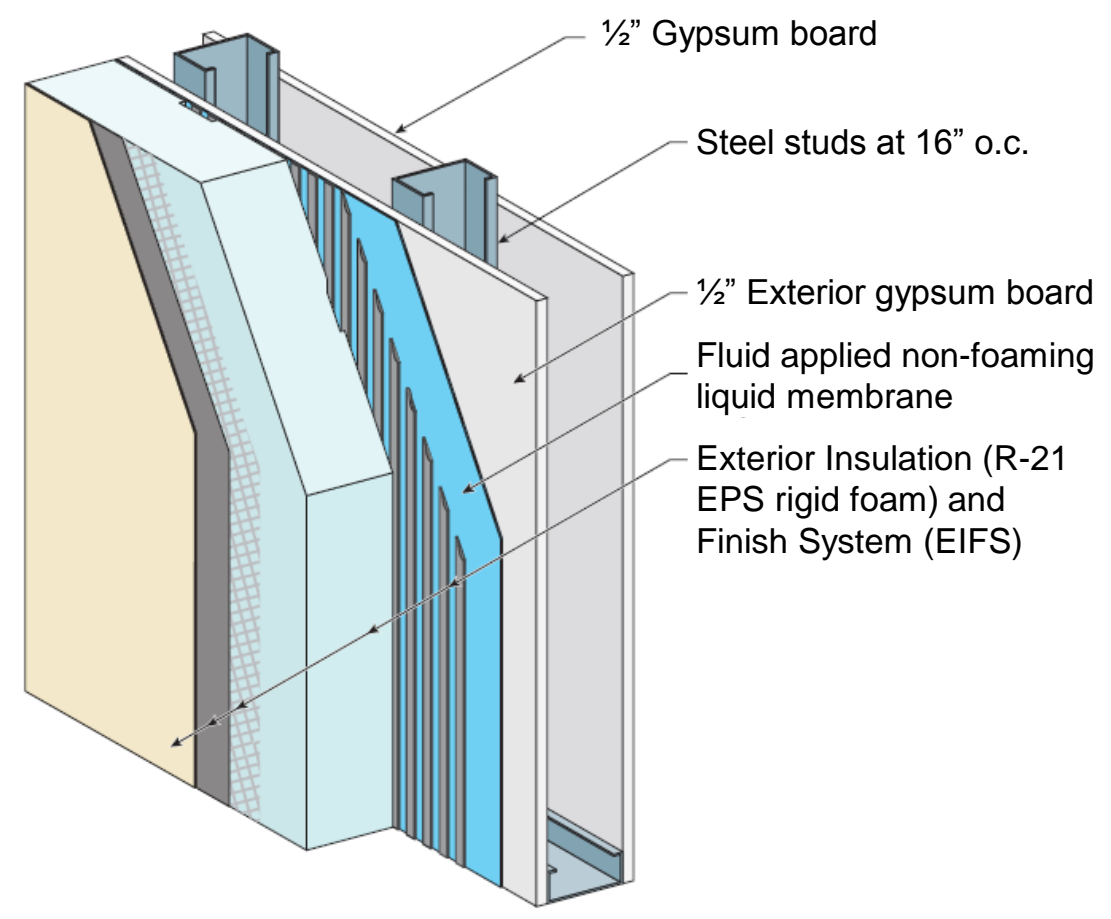

Figure 24. Material layout in panel P2-17. 
The four layouts that were used with the temperature, relative humidity and heat flux sensors are illustrated in Figures 25 through 28. In these figures, "exterior" indicates that the instrument was on the outdoor side of the material, while "interior" implies that the sensor was on the indoor side of the member. The sensor layout used in each of the panels is described in Table 1.

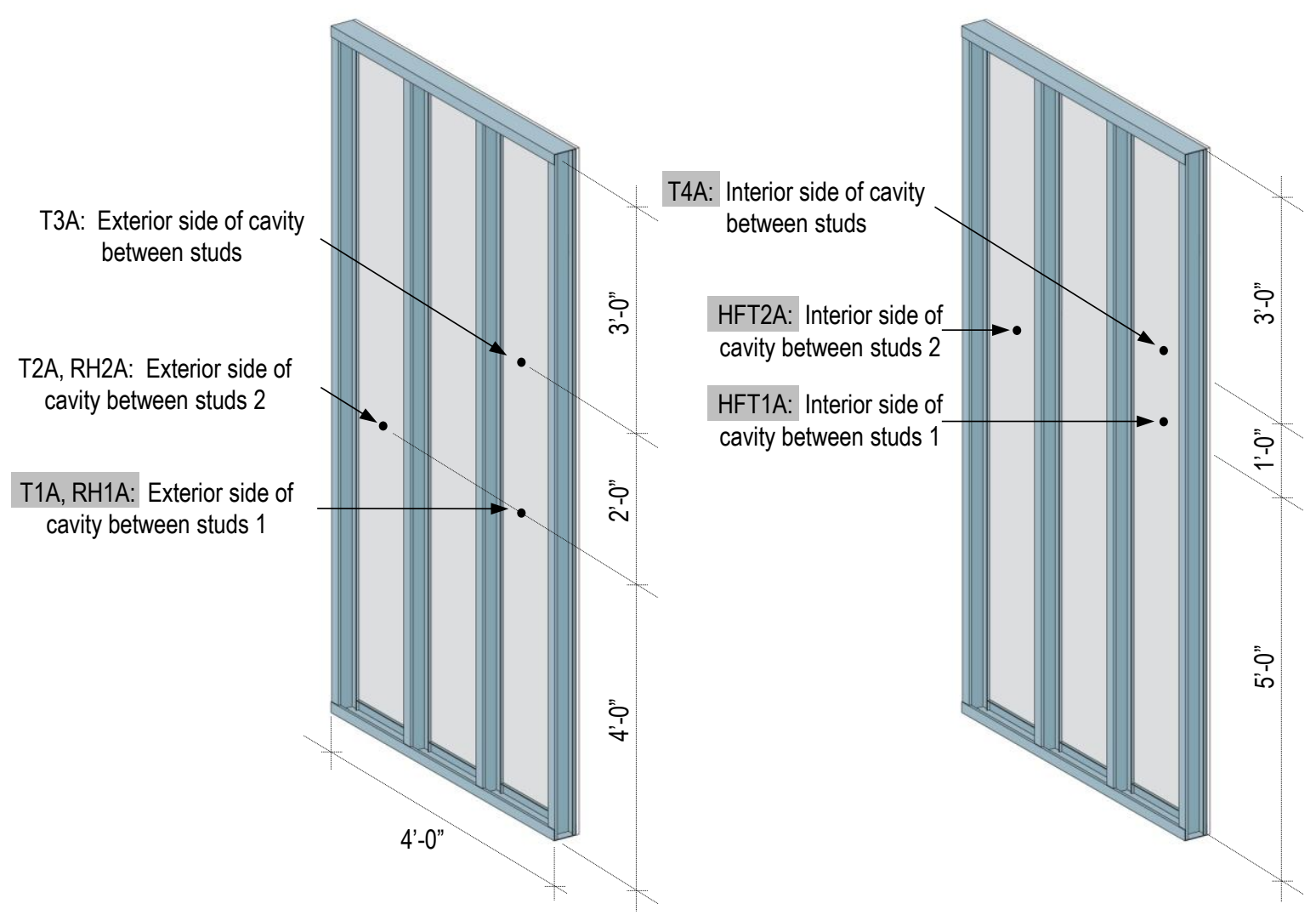

Figure 25. Sensor layout A.

Notes:

1. Data from sensors highlighted in gray are summarized in Tables 2 to 6 and are plotted in Appendixes A through D.

2. Sensor location

a. "Exterior" indicates that the sensor was on the outdoor side of the material.

b. "Interior" implies that the sensor was on the indoor side of the member.

3. Cavity insulation is not show for clarity.

4. Abbreviations: HFT: heat flux transducer; RH: relative humidity sensors; T: temperature sensors. 


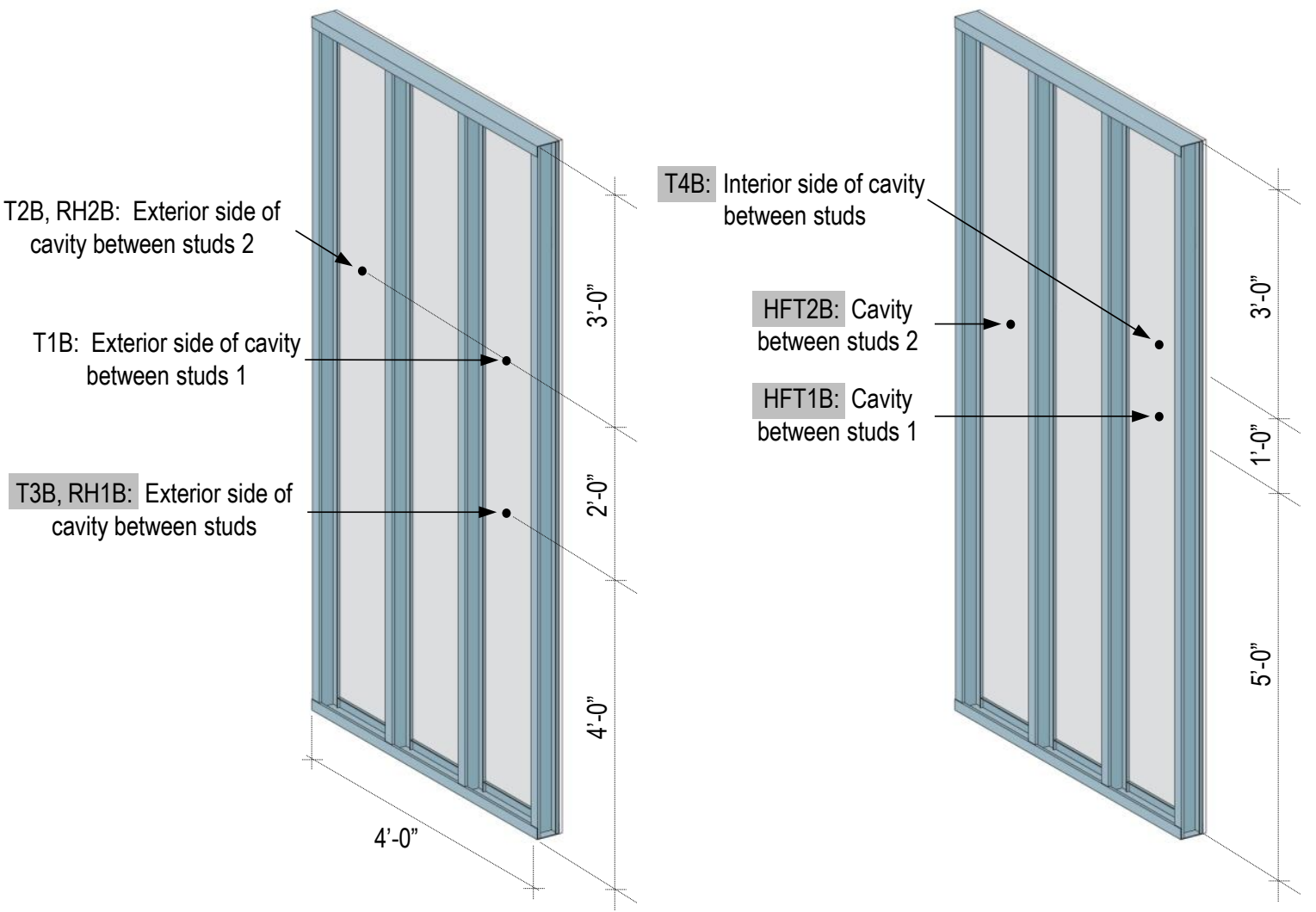

Figure 26. Sensor layout B.

Notes:

1. Data from sensors highlighted in gray are summarized in Tables 2 to 6 and are plotted in Appendixes A through D.

2. Sensor location

a. "Exterior" indicates that the sensor was on the outdoor side of the material.

b. "Interior" implies that the sensor was on the indoor side of the member.

3. Cavity insulation is not show for clarity.

4. Abbreviations: HFT: heat flux transducer; RH: relative humidity sensors; T: temperature sensors. 

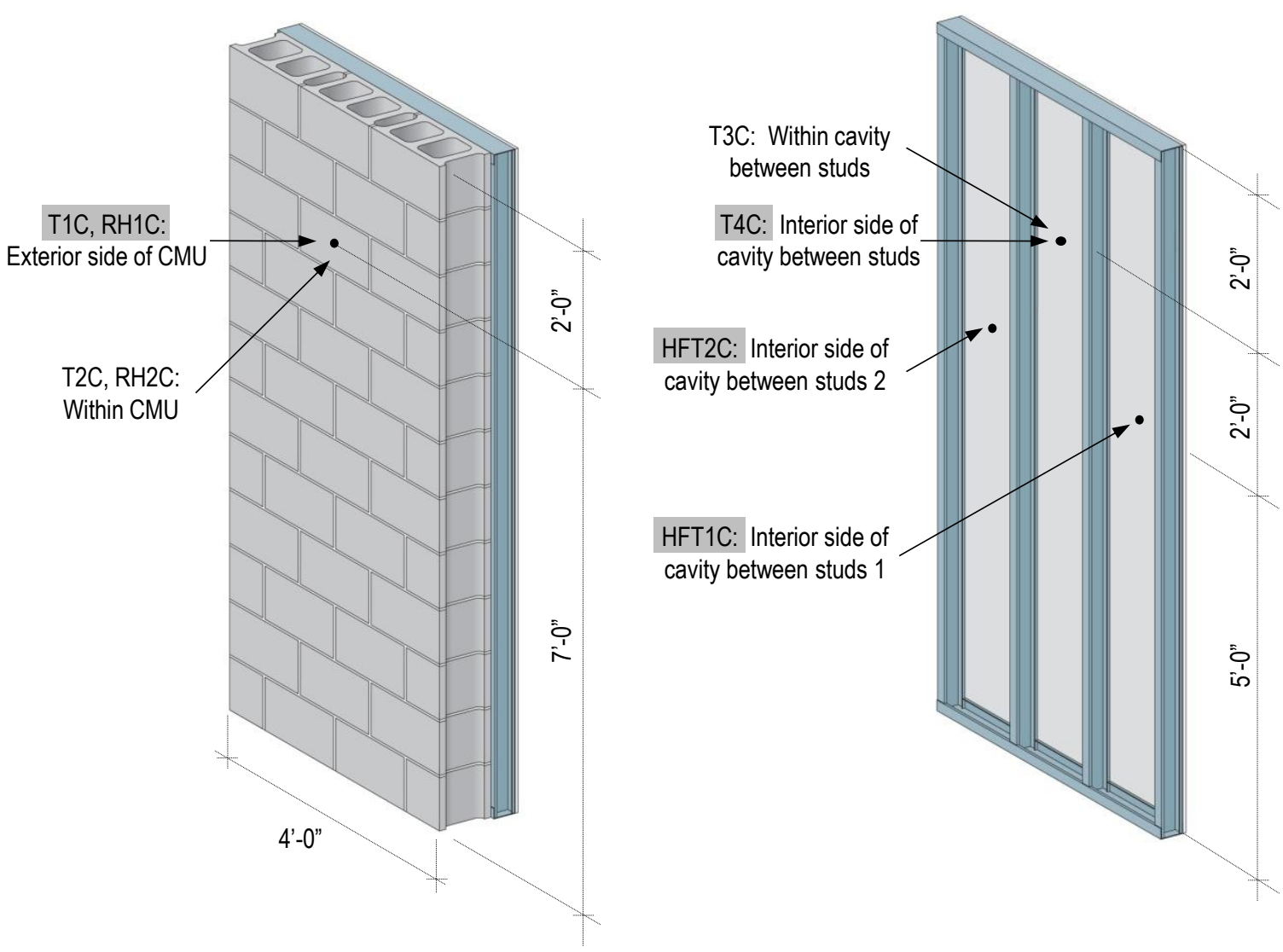

Figure 27. Sensor layout C.

Notes:

1. Data from sensors highlighted in gray are summarized in Tables 2 to 6 and are plotted in Appendixes A through D.

2. Sensor location

a. "Exterior" indicates that the sensor was on the outdoor side of the material.

b. "Interior" implies that the sensor was on the indoor side of the member.

c. Sensors within the CMU and stud cavity were located at mid-depth.

3. Abbreviations: HFT: heat flux transducer; RH: relative humidity sensors; T: temperature sensors. 

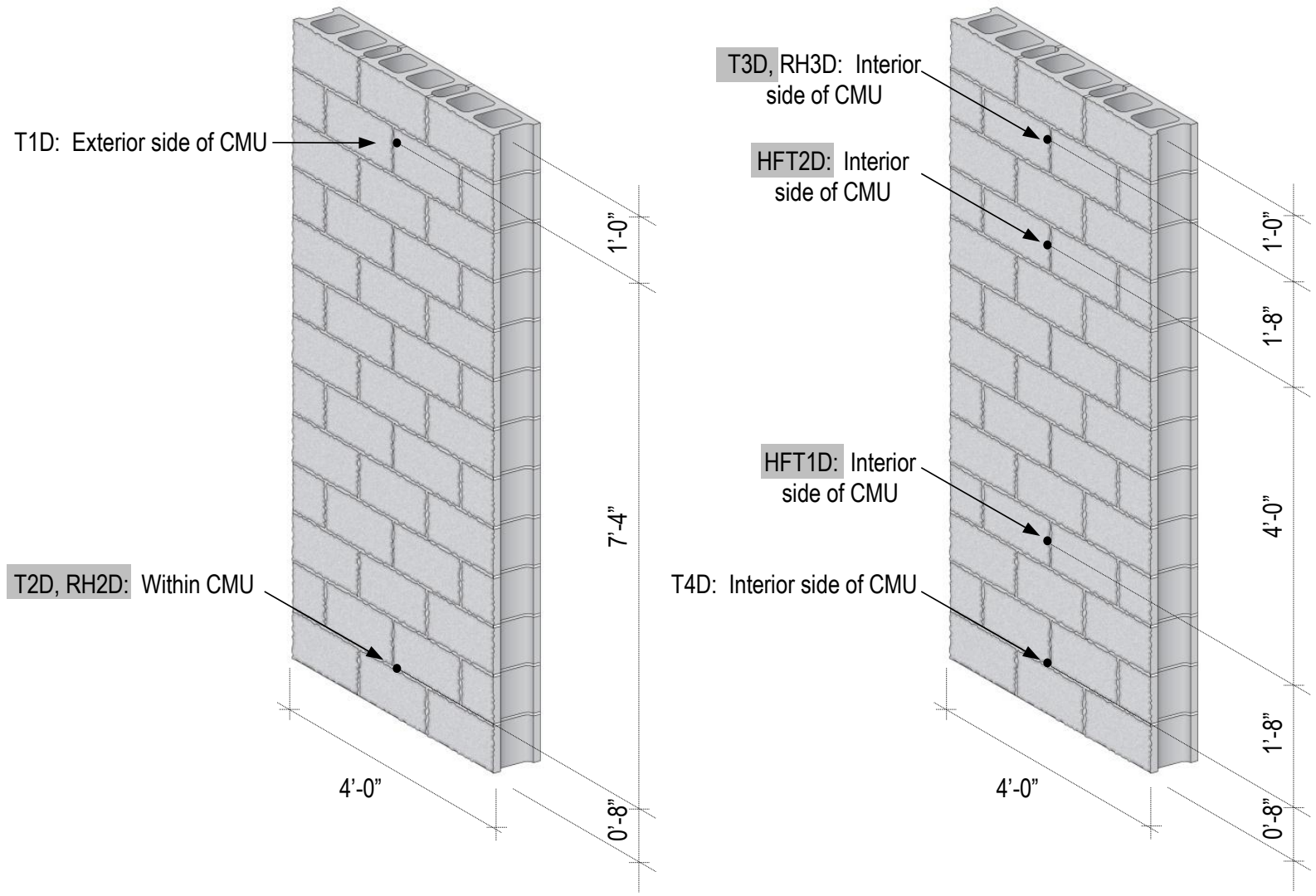

Figure 28. Sensor layout D

Notes:

1. Data from sensors highlighted in gray are summarized in Tables 2 to 6 and are plotted in Appendixes A through D.

2. Sensor location

a. "Exterior" indicates that the sensor was on the outdoor side of the material.

b. "Interior" implies that the sensor was on the indoor side of the member.

c. Sensors within the CMU and stud cavity were located at mid-depth.

d. Sensors shown as triangles were located on the interior side of the CMU.

3. Abbreviations: HFT: heat flux transducer; RH: relative humidity sensors; $\mathrm{T}$ : temperature sensors.

\section{Experimental Procedures}

Data from all sensors, except from pressure monitors, were scanned every minute and are reported as hourly averages. Pressure measurements were collected and are reported every minute. Temperature and relative humidity measurements collected indoors and from the specimens were utilized to estimate water vapor pressure based on equations from the 2005 ASHRAE Handbook - Fundamentals (ASHRAE 2005). Heat flux data from the two transducers were averaged and used to calculate cooling and heating loads. 


\section{Results}

Monthly data from temperature, relative humidity and heat flux sensors that are highlighted in gray in Figures 25 through 28 were grouped based on panel orientation and are summarized in Tables 2 to 6 . These tables also include indoor and outdoor measurements. Figures 29 through 36 show the monthly contribution to the cooling and heating loads from each of the panels. Additionally, Figures 37 and 38 indicate their respective annual contributions. Note that these heating and cooling load estimates were obtained from a section of the test panel; consequently, these are not representative of the heat transfer throughout the entire specimen. Monthly and hourly data for all of these variables are plotted in Appendixes A through D. Monthly total solar insolation on each side of the test hut is summarized in Table 7. Monthly average wind speeds are shown in Table 8. Rain accumulation per month is described in Table 9. Weekly and monthly differences in pressure between outdoors and indoors (reference) for each side of the building are shown in Table 10. Missing pressure measurements were due to equipment malfunction; more specifically, taps were clogged from the outside by insects. Pressure data and outdoor environmental measurements are plotted in Appendixes E to $\mathrm{H}$. Spreadsheets with the aforementioned data will be available online. 
Table 2. Monthly temperature $\left({ }^{\circ} \mathrm{C}\right)$ at exterior side of stud cavity. Refer to Figures 25 through 28 for sensor location.

\begin{tabular}{|c|c|c|c|c|c|c|c|c|c|c|c|c|c|c|c|c|c|c|c|c|c|c|c|c|c|c|c|c|}
\hline \multirow{3}{*}{ Month } & \multirow{3}{*}{$\begin{array}{l}\text { Descriptive } \\
\text { Statistics }\end{array}$} & \multirow{3}{*}{$\underset{1 \mathrm{st} f \mid}{\ln }$} & \multirow{3}{*}{$\underset{2^{\text {nd fl }}}{\ln }$} & \multirow{3}{*}{ Out } & \multicolumn{5}{|c|}{ North Panels } & \multicolumn{6}{|c|}{ East Panels } & \multicolumn{7}{|c|}{ South Panels } & \multicolumn{6}{|c|}{ West Panels } \\
\hline & & & & & P1-15 & $\mathrm{P} 1-16$ & P2-15 & P2-16 & P2-17 & P1-10 & P1-11 & $\mathrm{P} 1-12$ & P1-13 & P1-14 & P2-14 & P1-6 & P1-7 & P1-8 & P1-9 & P2-6 & P2-7 & P2-8 & P1-1 & P1-2 & P1-3 & P1-4 & P1-5 & $\mathrm{P} 2-5$ \\
\hline & & & & & $\begin{array}{l}\text { Layout A } \\
\text { T1A }\end{array}$ & $\begin{array}{l}\text { Layout A } \\
\text { T1A }\end{array}$ & $\begin{array}{c}\text { Layout A } \\
\text { T1A }\end{array}$ & $\begin{array}{c}\text { Layout } B \\
T 3 B\end{array}$ & $\begin{array}{c}\text { Layout B } \\
\text { T3B }\end{array}$ & $\begin{array}{l}\text { Layout C } \\
\text { T1C }\end{array}$ & $\begin{array}{l}\text { Layout C } \\
\text { T1C }\end{array}$ & Layout A & Layout A & Layout A & $\begin{array}{l}\text { Layout A } \\
\end{array}$ & Layout A & Layout A & $\begin{array}{l}\text { Layout B } \\
T 3 B\end{array}$ & Layout B & Layout A & Layout $B$ & Layout $\mathrm{A}$ & Layout C & Layout D & Layout B & Layout $A$ & Layout A & $\begin{array}{l}\text { Layout B } \\
\text { T3B }\end{array}$ \\
\hline 09 & ean & .5 & 1.8 & NA & 16.1 & 20.7 & 16.4 & 21.0 & 21.0 & 21.3 & 20.9 & 2 & 2 & 19.8 & 19 & 21.9 & 22.3 & 22.8 & 22.4 & 20.1 & 19.9 & 17.8 & 21.3 & 20.0 & 19.8 & 20.5 & 20.6 & 18.8 \\
\hline & SD & & 1. & $N_{1}$ & & 1.24 & 5.07 & 1. & 1. & 17 & 3.56 & & & 5.17 & 7.19 & 1.74 & 3.19 & 7.37 & 7.09 & 9.65 & 7.95 & 6.66 & 1.26 & 4.67 & 7.75 & 3.73 & 7.81 & 7.34 \\
\hline & ptile & & $\pi s$ & 118 & & 18.5 & 8.00 & 18.6 & 18.5 & .5 & 15.2 & 19.2 & 11.7 & 11.8 & 9.34 & 19.5 & 17.5 & 12.5 & 12.7 & 8.41 & 9.12 & 8.33 & 19.3 & 13.1 & 9.92 & 9.05 & 10.1 & 8.31 \\
\hline & Median & 1.5 & 21.6 & NA & 5.8 & 20.7 & 16.3 & 20.8 & 20.8 & 21.2 & 20.7 & 21.0 & .4 & 19.3 & 17.9 & 21.8 & 22.0 & 21.1 & 20.8 & 17.0 & 17.9 & 16.6 & 21.3 & 19.3 & 18.0 & 18.6 & 18.9 & 17.6 \\
\hline & $95^{\text {th }}$ ptile & 3.1 & 24.2 & & 6 & 22.8 & 24.9 & 23.4 & 23.5 & 23.2 & 26.5 & 23.4 & 29.4 & 28.8 & 33.5 & 25.1 & 28.0 & 36.4 & 35.9 & 39.9 & 35.5 & 30.1 & 23.3 & 28.8 & 35.5 & 38.3 & 35.7 & 33.0 \\
\hline ct 09 & ean & & & & & 19.7 & 13.1 & 19.9 & 11.4 & 7 & 16.0 & 19.8 & 8 & 12.6 & 12.4 & 20.4 & 18.6 & 15.6 & 15.6 & 13.1 & 13.1 & 11.4 & 19.5 & 14.5 & 12.8 & 12.1 & 12.7 & 11.3 \\
\hline & & & & & & 0.55 & 7.60 & 0.62 & 5.2 & & 2.01 & 0 & & 3.79 & 5.30 & 1.15 & 2.69 & 6.43 & 6.05 & 7.60 & 6.22 & 5.26 & & 3.20 & 5.04 & 5.73 & 5.05 & 4.95 \\
\hline & $5^{\text {th }}$ ptile & 0.9 & 21.0 & $\mathrm{~N}$ & 37 & 18.8 & 2.75 & 18.8 & 18.8 & 7 & 12.4 & 18.7 & 54 & 6.33 & 4.07 & 18.9 & 15.0 & 7.58 & 8.06 & 3.48 & 4.40 & 3.18 & 18.5 & 9.75 & 5.38 & 3.78 & 5.19 & 3.44 \\
\hline & $\mathrm{N}$ & 0 & 21 & & 2 & 19.7 & 10.5 & & 19 & 17 & 16.0 & 198 & & 12.5 & 12.1 & 20.1 & 18.1 & 14.2 & 14.2 & 11.8 & 12.2 & 11. & 9.6 & 14.2 & 2.4 & 1.6 & 2.3 & 11.2 \\
\hline & & & & IV & .8 & 20.5 & & 20.9 & 20.8 & . & 19.4 & 8 & & 9.4 & 22.0 & 22.9 & 24.3 & 29.8 & 29.3 & 37.2 & 27.1 & 21 & & 20.4 & 21.9 & 22.8 & 22.3 & 19.5 \\
\hline Nov 09 & an & 21.2 & 21.4 & 601 & & 19.2 & 7.09 & 19.4 & 19.4 & 9.2 & 14.6 & 19.4 & & 10.2 & $7-7$ & 20.0 & 17.7 & 13.8 & 13.8 & 10.6 & 10.9 & 8.93 & 18.9 & 12.4 & 10.0 & 9.00 & 9.78 & 8.25 \\
\hline & & & & & & 0.57 & & & 0. & & 2.09 & & & 3.96 & 5.47 & 1. & 2.98 & 7.11 & 6.67 & 8.3 & 7.10 & 5. & & 3.22 & 05 & .71 & 5.07 & 5.27 \\
\hline & & 20.8 & & & & 18.3 & -0.63 & & 18 & & 11.4 & 18 & & & 1.66 & 18.4 & 13.7 & 5.08 & 5.93 & 0.69 & 1.81 & 0. & & 7.61 & 2.49 & .49 & 2.12 & -0.07 \\
\hline & Median & 2 & 21.3 & & 3 & 19.2 & 7.58 & 19.5 & 19.4 & .2 & 14.6 & 19.4 & 1 & 0.4 & 9.54 & 19.8 & 17.3 & 12.3 & 12.4 & 904 & 9.86 & 8.5 & 19.0 & 12.5 & 9.93 & .86 & 9.72 & 8.37 \\
\hline & 95 & 21. & & 7 & 13.6 & 20.1 & 14.0 & 2 & 20 & .3 & 18.3 & 0 & 17 & 17.1 & 20 & 2 & 23 & 29.1 & 27.9 & 29.0 & 26.7 & 20 & 0.0 & 18.1 & 19.3 & 19.4 & 18.8 & 17.6 \\
\hline 6 & 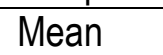 & & & & & & & & & & & & & & 1. & & & & & 1 & 2.4 & 1 & & & & .74 & .0 & 0.31 \\
\hline & & & & & & 0.84 & & & 5 & & & & & & & & 2.58 & & & 7.60 & & & & 3.48 & 77 & 5.27 & 4.83 & 5.23 \\
\hline & & & 21 & & 41 & 16.7 & -9 & & 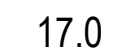 & & 5 & 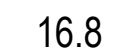 & -3 & -4.85 & -6 . & & 9.29 & -2.39 & -1.20 & -7.09 & -6.32 & -7. & & 0.66 & -5.28 & -7.94 & 5.87 & -8.39 \\
\hline & & 2 & 2 & & 54 & 18.1 & -0.09 & 18 & $1 \varepsilon$ & & & & & 2.47 & 1. & & 13 & 4.56 & 5. & 1.5 & 2.1 & 0. & & 6.81 & 2.93 & 0.97 & 2.32 & 0.61 \\
\hline & h ntilo & & & & & 19.2 & 7.70 & 19.6 & 19 & 9 & 14.2 & 19 & & 102 & & 200 & 17.9 & 15.5 & 15.0 & 14.7 & 13.7 & 10 & 18.7 & 12.1 & 10.5 & 9.7 & 10.1 & 8.56 \\
\hline 10 & & & & & & & & & & & 8.90 & & & & -0 . & & & 4.01 & 4.61 & 1 & 0.67 & & 7.1 & 6.26 & 37 & -0.70 & 0.68 & -1.45 \\
\hline & & & & & 5. & 00 & & & 5. & & 2 & & & & 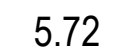 & 1.2 & 3.2 & 6.83 & 6. & 7.60 & $6 . \varepsilon$ & 5 & & 4.04 & 5. & 6.19 & 5.58 & 5.97 \\
\hline & $5^{\text {th }}$ & 20 & & & .8 & 16.0 & -1 & 1 & 16 & & 4.91 & 1 & & -7 & -9. & & 7.97 & -4.02 & -3.25 & -10.1 & -9.28 & -10 & & -1.21 & -7.59 & -10.7 & 8.49 & -11.2 \\
\hline & $50^{t}$ & & & & & & & & & & & & & & -0 & & & & & -0.19 & 0.4 & -0 & & 6.71 & 1.55 & -0.49 & 0.75 & -1.27 \\
\hline & $95^{\text {th }}$ & & 21 & & 6.46 & 8.8 & 6 & 19.1 & 15 & 3.4 & 12.8 & & & 8.61 & 0 & 20. & 19. & 17 & j.J & 12 & 12 & 10 & 18.3 & 12.7 & 10.4 & 10.1 & 10.6 & 8.40 \\
\hline Feb 10 & 1 & & & & & 17 & & 1 & $1 \varepsilon$ & & & & & & 1 & & 1 & 4 & 5.30 & 1 & 1 & 0 & & 7 & 2.73 & 0.85 & 04 & 0.03 \\
\hline & & & & & & & & & & & & & & & & & & & & & & & & & & & & \\
\hline & & & & & & & -7. & & & & & & & & -5 & & 1 & -1.28 & & $-\pi$ & -4.77 & -6 & & 3. & -3.10 & -6.08 & 89 & -6.37 \\
\hline & & 2 & & & -0 & 18.0 & & 1 & $1 \varepsilon$ & & J & 1 & & & 1. & 18 & 12.8 & 4.26 & 4. & 0.7 & $1 . t$ & 0. & 3 & 7.19 & 2.54 & .64 & 1.86 & -0.08 \\
\hline & & & & & & & & & & & & & & & & & & & & & & & & & 77 & 01 & 30 & 6.62 \\
\hline 10 & & & & & & & & & & & & & & & & & & & & & & & & 12.80 & 9.68 & 8.85 & 62 & 7.55 \\
\hline & 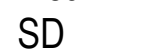 & & & & & 1.27 & & 1 & 5 & & 2.87 & & & & & & 3.57 & 7.95 & 7.5 & 7.60 & 7. & & & 4.48 & 6.31 & 7.41 & 6.60 & 6.16 \\
\hline & & & & & & & & & & & & & & & & & & & & & & & & & & 81 & 0.76 & -1.18 \\
\hline & & & & & & & & & & & & & & & & & & & & & & & & 12.43 & & 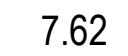 & 5 & 601 \\
\hline & $95^{\text {th }} \mathrm{pt}$ & 2 & & 1 & 12.0 & 22.0 & $T_{1}$ & 22.7 & 2 & 22.2 & 20.2 & 2 & & 2 & 2 & 24.3 & 25.3 & 30.9 & 30.4 & 32.1 & 2 & 2 & 21.9 & 22.1 & 23.6 & 25.5 & 23.5 & 20.2 \\
\hline 10 & ean & & & & & & & & & & & & & & & & & & & & & & & & & & 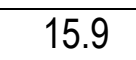 & 13.7 \\
\hline & & & & & & & & & & & & & & & & & & & & & & & & & 72 & 66 & .97 & 7.42 \\
\hline & $5^{\text {th }}$ ptile & 17 & 17 & 2. & 2.97 & 15.9 & 3. & 15 & 15 & 10.6 & 12.5 & 1 & & 6. & 4. & 16.6 & 13.8 & 7.56 & 7.78 & 3.78 & 4.8 & 3. & 0.3 & 9.90 & 5.82 & 4.28 & 5.32 & 3.80 \\
\hline & & 21. & 21 & 10 & 11.1 & 20.0 & & 20 & 20 & 20 & 18 & & & 1 & 15 & 21 & 20 & $1 /$ & 1 & 12 & 13 & 12 & 20.0 & 16.7 & 14. & 13.7 & 14.3 & 12.6 \\
\hline & $95^{\text {th }}$ ptile & 28.3 & 28.9 & 23.3 & 22.9 & 26.6 & 22.9 & 27.6 & 27.6 & 26.7 & 26.2 & 27.5 & 27.3 & 26.9 & 31. & 27.9 & 28.1 & 32.1 & 31.9 & 34.8 & 30.9 & 26.2 & 26.4 & 27.3 & 31.0 & 33.4 & 31.5 & 28.2 \\
\hline
\end{tabular}


Table 2. Continuation.

North Panels

East Panels

South Panels

West Panels

\begin{tabular}{|c|c|c|c|c|c|c|c|c|c|c|c|c|c|c|c|c|c|c|c|c|c|c|c|c|c|c|c|c|}
\hline \multirow{3}{*}{ Month } & \multirow{3}{*}{$\begin{array}{l}\text { Descriptive } \\
\text { Statistics }\end{array}$} & \multirow{3}{*}{$\underset{1 \mathrm{st} f \mid}{\ln }$} & \multirow{3}{*}{$\underset{2^{\text {nd }} \mathrm{ln}}{\text { fl }}$} & \multirow{3}{*}{ Out } & \multicolumn{5}{|c|}{ North Panels } & \multicolumn{6}{|c|}{ East Panels } & \multicolumn{7}{|c|}{ South Panels } & \multicolumn{6}{|c|}{ West Panels } \\
\hline & & & & & P1-15 & P1-16 & P2-15 & P2-16 & P2-17 & P1-10 & P1-11 & P1-12 & P1-13 & P1-14 & P2-14 & P1-6 & P1-7 & P1-8 & P1-9 & $\mathrm{P} 2-6$ & P2-7 & P2-8 & P1-1 & P1-2 & P1-3 & $\mathrm{P} 1-4$ & P1-5 & $\mathrm{P} 2-5$ \\
\hline & & & & & $\begin{array}{c}\text { Layout } \mathrm{A} \\
\text { T1A }\end{array}$ & $\begin{array}{c}\text { Layout A } \\
\text { T1A }\end{array}$ & $\begin{array}{c}\text { Layout A } \\
\text { T1A }\end{array}$ & $\begin{array}{l}\text { Layout B } \\
\text { T3B }\end{array}$ & $\begin{array}{c}\text { Layout B } \\
\text { T3B }\end{array}$ & $\begin{array}{l}\text { Layout C } \\
\text { T1C }\end{array}$ & $\begin{array}{l}\text { Layout C } \\
\text { T1C }\end{array}$ & $\begin{array}{l}\text { Layout A } \\
\text { T1A A }\end{array}$ & $\begin{array}{c}\text { Layout A } \\
\text { T1A }\end{array}$ & $\begin{array}{c}\text { Layout } \mathrm{A} \\
\mathrm{T} 1 \mathrm{~A}\end{array}$ & $\begin{array}{c}\text { Layout A } \\
\text { T1A }\end{array}$ & $\begin{array}{l}\text { Layout } \mathrm{A} \\
\text { T1A }\end{array}$ & $\begin{array}{l}\text { Layout } A \\
T 1 A\end{array}$ & $\begin{array}{l}\text { Layout B } \\
\text { T3B }\end{array}$ & $\begin{array}{c}\text { Layout B } \\
\text { T3B }\end{array}$ & $\begin{array}{c}\text { Layout A } \\
\text { T1A }\end{array}$ & $\begin{array}{c}\text { Layout B } \\
\text { T3B }\end{array}$ & $\begin{array}{c}\text { Layout } \mathrm{A} \\
\mathrm{T} 1 \mathrm{~A}\end{array}$ & $\begin{array}{l}\text { Layout C } \\
\text { T1C }\end{array}$ & $\begin{array}{c}\text { Layout D } \\
\text { T2D }\end{array}$ & $\begin{array}{l}\text { Layout B } \\
\text { T3B }\end{array}$ & $\begin{array}{l}\text { Layout A } \\
\text { T1A }\end{array}$ & $\begin{array}{l}\text { Layout A } \\
\text { T1A A }\end{array}$ & $\begin{array}{c}\text { Layout B } \\
\text { T3B }\end{array}$ \\
\hline lay 10 & Mean & 21.5 & 21.7 & 16.5 & 17.4 & 20.8 & 13.1 & 21.1 & 11.4 & 21.4 & 21.4 & 21.2 & 20.8 & 20.8 & 20.9 & 21.2 & 21.8 & 22.7 & 22.7 & 21.7 & 21.2 & 20.2 & 21.5 & 21.8 & 23.0 & 23.7 & 23.4 & 21.9 \\
\hline & SD & 1.77 & 2.12 & 7.18 & 7.53 & 2.31 & 7.60 & 2.58 & 5.26 & 2.46 & 4.77 & 2.36 & 7.58 & 7.65 & 9.77 & 1.91 & 2.50 & 4.95 & 4.93 & 6.59 & 5.29 & 5.08 & 1.82 & 4.23 & 7.03 & 7.78 & 7.02 & 6.24 \\
\hline & $5^{\text {th }}$ ptile & 17.5 & 17.0 & 3.20 & 3.90 & 15.8 & 4.15 & 15.5 & 15.5 & 16.3 & 12.3 & 16.2 & 7.65 & 7.52 & 5.56 & 18.3 & 17.3 & 14.6 & 14.6 & 11.8 & 12.8 & 11.7 & 18.7 & 15.6 & 13.8 & 13.2 & 13.9 & 12.6 \\
\hline & Median & 22.3 & 22.5 & 17.0 & 17.0 & 21.7 & 17.5 & 21.8 & 21.9 & 22.1 & 22.0 & 21.9 & 20.5 & 20.5 & 19.5 & 21.7 & 22.0 & 22.3 & 22.2 & 20.6 & 20.7 & 19.9 & 22.0 & 21.2 & 21.6 & 22.0 & 22.0 & 21.0 \\
\hline & $95^{\text {th }}$ ptile & 23.0 & 23.8 & 27.5 & 29.5 & 23.2 & 29.7 & 24.1 & 24.1 & 24.1 & 28.8 & 24.0 & 33.9 & 34.0 & 38.8 & 24.0 & 25.8 & 31.4 & 31.3 & 34.0 & 30.8 & 28.8 & 23.8 & 30.0 & 37.6 & 39.2 & 37.2 & 34.0 \\
\hline Jun 10 & Mean & 20.5 & 21.1 & 19.4 & 20.7 & 20.6 & 20.5 & 20.9 & 21.0 & 21.2 & 22.2 & 20.7 & 22.5 & 22.8 & 23.0 & 23.5 & 24.9 & 27.3 & 27.2 & 13.1 & 25.7 & 11.4 & 23.9 & 25.2 & 27.3 & 28.3 & 27.9 & 26.3 \\
\hline & SD & 2.06 & 2.04 & 4.29 & 5.13 & 1.94 & 4.85 & 2.04 & 2.04 & 1.83 & 2.91 & 2.06 & 5.11 & 5.16 & 6.96 & 1.08 & 2.26 & 5.45 & 5.46 & 7.60 & 6.01 & 5.26 & 0.90 & 4.46 & 7.99 & 8.69 & 8.01 & 6.98 \\
\hline & $5^{\text {th }}$ ptile & 17.6 & 18.1 & 12.0 & 11.9 & 17.8 & 12.2 & 17.7 & 17.7 & 18.4 & 16.9 & 17.5 & 14.2 & 14.4 & 12.8 & 21.8 & 21.3 & 18.9 & 18.9 & 15.5 & 16.4 & 15.0 & 22.0 & 19.2 & 17.3 & 17.4 & 17.8 & 16.6 \\
\hline & Median & 22.1 & 22.2 & 19.3 & 20.5 & 21.4 & 20.2 & 21.5 & 21.6 & 21.7 & 22.4 & 21.5 & 22.1 & 22.3 & 22.1 & 23.4 & 24.8 & 26.5 & 26.4 & 24.5 & 24.6 & 23.7 & 23.9 & 24.2 & 25.1 & 25.8 & 25.7 & 24.8 \\
\hline & $95^{\text {th }}$ ptile & 22.5 & 23.5 & 26.4 & 29.0 & 23.2 & 28.4 & 23.8 & 23.9 & 23.6 & 26.5 & 23.4 & 31.3 & 31.7 & 35.9 & 25.3 & 28.7 & 36.6 & 36.6 & 39.7 & 36.2 & 33.5 & 25.4 & 33.9 & 44.4 & 46.3 & 44.1 & 40.4 \\
\hline Jul 10 & Mean & 22.4 & 23.2 & 23.6 & 24.4 & 22.7 & 13.1 & 23.2 & $\begin{array}{l}11.4 \\
\end{array}$ & 23.6 & 25.7 & 22.9 & 27.0 & 27.3 & 27.7 & 23.3 & 24.4 & 25.9 & 26.1 & 13.1 & 24.3 & 11.4 & 23.2 & 23.6 & 25.0 & 25.6 & 25.4 & 23.8 \\
\hline & SD & 0.16 & 0.64 & 4.26 & 5.33 & 0.72 & 7.60 & 0.99 & 5.26 & 0.82 & 2.84 & 0.91 & 5.47 & 5.49 & 7.76 & 1.18 & 2.42 & 5.64 & 5.87 & 7.60 & 6.28 & 5.26 & 0.78 & 3.99 & 7.21 & 7.84 & 7.11 & 6.25 \\
\hline & $5^{\text {th }}$ ptile & 22.1 & 22.3 & 16.3 & 15.8 & 21.5 & 16.3 & 21.6 & 21.7 & 22.2 & 21.1 & 21.5 & 18.7 & 18.9 & 17.1 & 21.7 & 20.7 & 18.0 & 18.2 & 14.5 & 15.7 & 14.4 & 21.8 & 18.3 & 16.2 & 16.1 & 16.6 & 15.3 \\
\hline & Median & 22.4 & 23.1 & 23.3 & 24.2 & 22.7 & 24.1 & 23.2 & 23.2 & 23.6 & 25.6 & 22.8 & 26.2 & 26.6 & 26.4 & 23.1 & 24.1 & 24.9 & 24.9 & 22.8 & 23.0 & 22.0 & 23.2 & 22.8 & 23.2 & 23.6 & 23.6 & 22.8 \\
\hline & 95th ptile & 22.6 & 24.3 & 30.4 & 33.5 & 24.0 & 33.3 & 25.0 & 25.1 & 25.2 & 30.9 & 24.4 & 36.3 & 36.6 & 41.9 & 25.6 & 28.6 & 36.9 & 37.1 & 41.4 & 36.7 & 33.3 & 24.5 & 31.8 & 40.7 & 42.1 & 40.1 & 37.0 \\
\hline$g 10$ & Mean & 22.4 & 22.9 & 21.7 & 22.0 & 22.4 & 13.1 & 22.8 & 11.4 & 232 & 244 & 22.6 & 24.8 & 24.9 & 24.9 & 21. & 21.8 & 22.7 & 22.7 & 21.7 & 21.2 & 20.2 & 21.5 & 21 & 23.0 & 23.7 & 23.4 & 21.9 \\
\hline & SD & 0.16 & 0.73 & 3.96 & 4.68 & 0.67 & 7.60 & 1.04 & 5.26 & 0.74 & 2.54 & 0.86 & 4.95 & 4.89 & 6.82 & 1.91 & 2.50 & 4.95 & 4.93 & 6.59 & 5.29 & 5.08 & 1.82 & 4.23 & 7.03 & 7.78 & 7.02 & 6.24 \\
\hline & $5^{\text {th }} \mathrm{ptile}$ & 22.1 & 22.1 & 15.3 & 14.2 & 21.3 & 14.3 & 21.3 & 21.3 & 21.8 & 20.2 & 21.4 & 17.4 & 17.6 & 15.5 & 18.3 & 17.3 & 14.6 & 14.6 & 11.8 & 12.8 & 11.7 & 18.7 & 15.6 & 13.8 & 13.2 & 13.9 & 12.6 \\
\hline & Median & 22.4 & 22.7 & 21.3 & 21.8 & 22.3 & 21.9 & 22.7 & 22.7 & 23.2 & 24.3 & 22.5 & 24.2 & 24.3 & 23.6 & 21.7 & 22.0 & 22.3 & 22.2 & 20.6 & 20.7 & 19.9 & 22.0 & 21.2 & 21.6 & 22.0 & 22.0 & 21.0 \\
\hline & $95^{\text {th }}$ ptile & 22.7 & 24.5 & 28.3 & 29.8 & 23.5 & 30.0 & 24.8 & 24.9 & 24.4 & 28.9 & 24.2 & 33.8 & 33.6 & 38.0 & 24.0 & 25.8 & 31.4 & 31.3 & 34.0 & 30.8 & 28.8 & 23.8 & 30.0 & 37.6 & 39.2 & 37.2 & 34.0 \\
\hline
\end{tabular}

Notes: Layouts A, B, C and D refer to the location of the sensors per Figures 25 through 28

Abbreviations: NA, not available; ptile, percentile; SD, standard deviation. 
Table 3. Monthly temperature $\left({ }^{\circ} \mathrm{C}\right)$ at interior side of stud cavity. Refer to Figures 25 through 28 for sensor location.

\begin{tabular}{|c|c|c|c|c|c|c|c|c|c|c|c|c|c|c|c|c|c|c|c|c|c|c|c|c|c|c|c|c|}
\hline \multirow{4}{*}{ Month } & \multirow{4}{*}{$\begin{array}{l}\text { Descriptive } \\
\text { Statistics }\end{array}$} & \multirow{4}{*}{$\ln _{1 \text { st fl }}$} & \multirow{4}{*}{$\begin{array}{l}\ln \\
2^{\text {ndfl }}\end{array}$} & \multirow{4}{*}{ Out } & \multicolumn{5}{|c|}{ North Panels } & \multicolumn{6}{|c|}{ East Panels } & \multicolumn{7}{|c|}{ South Panels } & \multicolumn{6}{|c|}{ West Panels } \\
\hline & & & & & P1-15 & P1-16 & P2-15 & P2-16 & P2-17 & $1-10$ & $1-11$ & P1-12 & $\mathrm{P} 1-13$ & $\mid-14$ & P2-14 & P1-6 & P1-7 & P1-8 & P1-9 & P2-6 & P2-7 & P2-8 & P1-1 & P1-2 & P1-3 & P1-4 & P1-5 & P2-5 \\
\hline & & & & & $\Delta$ & & L & Layout B & Layout B & Layout C & Layout C & $\begin{array}{l}\text { Layout A } \\
\end{array}$ & Layout A & Layout A & Layout A & Layout A & Layout A & Layout B & Layout B & Layout A & Layout B & Layout A & Layout C & Layout D & Layout B & Layout A & Layout $A$ & ayout B \\
\hline & & & & & T4A & T4A & T4A & $\mathrm{T} 4 \mathrm{~B}$ & T4B & $\mathrm{T} 4 \mathrm{C}$ & $\mathrm{T} 4 \mathrm{C}$ & T4A & T4A & T4A & T4A & T4A & T4A & T4B & T4B & T4A & T4C & T4A & T4C & $\mathrm{T} 3 \mathrm{D}$ & T4B & T4A & T4A & T4B \\
\hline \multirow[t]{5}{*}{ Sep 09} & ean & 21.5 & 21.8 & $\mathrm{NA}$ & 20.9 & 21.0 & 16.6 & 21.0 & 21.0 & 21.5 & 21.4 & 21.4 & 21.4 & 21.3 & 21.5 & 22.0 & 21.8 & 21.7 & 21.7 & 21.6 & 21.6 & 21.3 & 21.5 & 21.4 & 21.3 & 21.6 & 21.7 & 21.4 \\
\hline & & & 1.45 & NA & 1.22 & 1.17 & 4.84 & 1.56 & 1.56 & 1.03 & 1.58 & 1.22 & 1.18 & 1.16 & 1.53 & 1.45 & 1.29 & 1.41 & 1.36 & 1.96 & 1.67 & 1.80 & 1.07 & 3.51 & 1.33 & 1.46 & 1.43 & 1.74 \\
\hline & $5^{\text {th }}$ ptile & 19.6 & 19.5 & NA & 18.6 & 18.8 & 8.4 & 18.6 & 18.6 & 19.6 & 19.1 & 19.5 & 19.5 & 19.5 & 19.2 & 19.9 & 19.8 & 19.5 & 19.5 & 18.9 & 19.2 & 18.7 & 19.4 & 16.8 & 19.1 & 19.3 & 19.5 & 18.9 \\
\hline & Median & 21.5 & 21.6 & NA & 20.8 & 20.9 & 16.4 & 20.8 & 20.9 & 21.3 & 21.2 & 21.2 & 21.3 & 21.2 & 21.2 & 21.8 & 21.6 & 21.5 & 21.5 & 21.4 & 21.4 & 21.1 & 21.4 & 20.8 & 21.2 & 21.3 & 21.4 & 21.2 \\
\hline & ptile & 3.1 & 12 & & 2.9 & 22.9 & 24.6 & 23.5 & 23.6 & 23.1 & 24. & 23.3 & 23.2 & 231 & 23.9 & 24.6 & 24.0 & 24.1 & 24.1 & 25.4 & 24.5 & 24.5 & 23.1 & 28.2 & 23.7 & 24.3 & 24.3 & 24.5 \\
\hline \multirow[t]{5}{*}{ Oct 09} & lean & 21.3 & 21.4 & $\bar{N}$ & 20.3 & 20.4 & 20.4 & 20.3 & 20.2 & 20.8 & 19.6 & 20.7 & 21.2 & 20 & 20.6 & 21.0 & 21.3 & 21.1 & 21.0 & 20.4 & 20.6 & 20.2 & 20.7 & 18.1 & 20.6 & 20.9 & 20.9 & 20.3 \\
\hline & S & & & & & .32 & 1.16 & 0.48 & 0. & 0.24 & 0.6 & 0.37 & 0.33 & 0.27 & 0.5 & $0.7^{\prime}$ & 0.45 & 0.66 & 0.60 & 1.16 & 0.72 & 0.89 & 0.25 & 1.90 & 0.42 & 0.46 & 0.45 & 0.64 \\
\hline & ptile & & & & & .9 & 9.7 & 100 & 19. & .4 & 18 & 20.1 & 20.7 & 20.6 & 19.7 & 20.2 & 20.8 & 20.3 & 20.4 & 19.1 & 19.6 & 18 & & 15.6 & 20.0 & 20.4 & 20.3 & 19.4 \\
\hline & Median & 3 & 1.4 & $\mathrm{~N}$ & 3 & 20.4 & 20.5 & 20.3 & 20.0 & 20.8 & 19.7 & 20.7 & 21.1 & 20.9 & 20.6 & 20.9 & 21.1 & 20.9 & 20.9 & 20.1 & 20.5 & 20.1 & 20.7 & 17.9 & 20.6 & 20.9 & 20.9 & 20.3 \\
\hline & 95 & 0 & 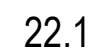 & TV & 21 & 20.9 & 21.3 & 21.1 & 20.9 & 21.1 & 20.7 & 21.4 & 21.7 & 21 & 21.6 & 22.6 & 22.2 & 22.6 & 22.4 & 23.3 & 22.2 & 22.0 & 21.1 & 21.6 & 21.3 & 21.7 & 21.7 & 21.4 \\
\hline \multirow[t]{5}{*}{ Nov 09} & & & & & & & & & & & & & & & 2 & 20.8 & 2 & 20.9 & 2 & 26 & 20.4 & 19 & & 17.0 & 20.4 & 20.7 & 20.7 & 20.0 \\
\hline & & & & & & 0.35 & 0 & U & 0 & 24 & & 0.38 & 0. & 0 & 0. & 0.76 & 0.47 & 0.72 & 0.66 & 1.19 & 0.80 & 0.97 & 0.25 & 1.80 & 0.41 & 0.41 & 0.44 & 0.68 \\
\hline & & 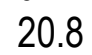 & 21.0 & & & 19.6 & 19.3 & 19.1 & 18 & & 1 & 19.9 & (1) & 20 & 19 & 19.9 & 20.7 & 20.1 & 20.2 & 18.8 & 19.3 & 18. & & 14.5 & 19.8 & 20.3 & 20.1 & 19.0 \\
\hline & & 21.2 & 2 & & & & & 00 & $1 s$ & & 19 & & 21.0 & 20 & 20 & 20.7 & 21.1 & 20.8 & $\angle U$ & 19.9 & 20 & 19 & & 17.0 & 20.3 & 20.7 & 20.7 & 20.0 \\
\hline & $95^{\text {th }} \mathrm{pti}$ & .6 & 21.9 & 14.7 & 20.7 & 20.7 & 21.0 & 20.8 & 20.6 & 21.1 & 20 & 21.1 & 21.6 & 21 & 21.3 & 22.4 & 22.2 & 22.5 & 22.3 & 22.8 & 21.9 & 21.7 & 20.9 & 20.3 & 21.1 & 21.5 & 21.5 & 21.2 \\
\hline \multirow[t]{5}{*}{ Dec 09} & - & & & & & 11 & 201 & 40 & 200 & 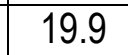 & & 198 & 2010 & & 19.6 & 10 & 20.6 & 20.1 & 20.1 & 20.4 & 19.5 & 20.2 & 198 & 14.1 & 19.7 & 20.3 & 20.1 & 19.1 \\
\hline & & & & & & & & & & & & & & & 0 & & 0. & 0. & $4 c$ & 1.1 & & 0. & & 1.74 & 0.42 & .29 & 0.45 & 0.63 \\
\hline & $5^{t}$ & 7 & 21.0 & & & 18.5 & 1 & & 1 & 4 & 16 & 19.3 & 2( & 20 & 18 & 19.2 & 20.2 & 19.4 & 19.6 & 18.1 & 18.6 & 17 &.$\angle$ & 11.2 & 19.0 & 19.9 & 19.3 & 18.0 \\
\hline & & & 2 & & 15 & 106 & 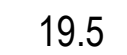 & 1 & $1 \varepsilon$ & & & & 21 & & 19 & 19.8 & 20 & 20.0 & 20.1 & 19.0 & 19 & 18 & & 14.2 & 19.7 & 0.3 & 20.2 & 19.1 \\
\hline & & 21.3 & & & 2 & 2 & 2 & 20 & 19 & & $1 \varepsilon$ & 2 & 2 & 20 & 20 & 20. & 21 & 21.2 & 21.0 & 20 & 20 & 20 & 20.4 & 16.8 & 20.3 & 20.8 & 20.8 & 20.1 \\
\hline Jan 10 & Mean & & & & & 19.0 & & 1 & 2 & & & 16 & & & 10 & 1 & 2 & 19 & 1 & 2 & 15 & 20 & 1 & 15.3 & 19.8 & 1 & 20.2 & 18.6 \\
\hline & & & & & & & & & & & & & & & & & & & & 1. & & & & 1. & & & .48 & 0.70 \\
\hline & $5^{\text {th }}$ & & & & & & & & & & & & & & 18 & & 2 & 19 & $19.3^{3}$ & 17 & $1 \varepsilon$ & 17 & & 11.4 & 19.0 & 9.7 & 19.3 & 17.4 \\
\hline & $50^{t}$ & 21.4 & 20.9 & & & 1 & 1 & 1 & $1 \varepsilon$ & 1 & 1 & 19.6 & & 20 & 10 & 19.6 & 20 & 19.8 & 19.8 & 18 & 18. & $1 \varepsilon$ & .7 & 15.4 & 19.8 & 20.1 & 20.2 & 18.5 \\
\hline & & & & & & & & & & & & & & & 2 & & & & & 20 & 20 & 1 & & 18.2 & & 0.7 & 21.0 & 19.9 \\
\hline 10 & & & & & & & 19. & 19 & 18 & & & 19 & & & 19. & 19. & & 20. & 20. & 1 & 19 & 1 & & 15 & 19.9 & 20.3 & 20.2 & 18.9 \\
\hline & $S$ & & & & & & & & 0 & & & 0 & & & 0. & & 0. & 0.47 & 0.42 & 0.68 & 0.51 & 0. & 0.2 & 1.23 & 0.31 & 0.30 & 0.33 & 0.54 \\
\hline & & & & & & & & & & & & & & & & & & & & & & & & & & & & 181 \\
\hline & & & & & & & & & 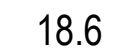 & & & & & & 15 & & 2 & 2 & & 1 & 15 & $1 \varepsilon$ & & 15.6 & 19 & 2 & 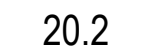 & 18.9 \\
\hline & $5^{\mathrm{m}} \mathrm{pti}$ & 2 & 21 & & 20.0 & 1 & 20.0 & 20 & 19.5 & 20.2 & 1 & 20.2 & & & 20 & 20.7 & 2 & 20.8 & 20.7 & 20.0 & 20.2 & 19 & 20.3 & 18.1 & 20.4 & 20.8 & 20.8 & 19.8 \\
\hline r10 & & & & & & & & & & & & & & & & & & & & & & & & & & & & 20.6 \\
\hline & & & & & & & & & & & & & & & & & & & & & & 0 & & 2.38 & 1. & y & 10 & 142 \\
\hline & $5^{\text {th }}$ ptile & 21.0 & 20.9 & -3 & 18.5 & 18.8 & $1 s$ & 19.1 & 18 & 20.1 & 1 & 19.6 & & & 19 & 19.8 & 20 & 19.9 & 19.8 & 18.7 & 19 & 18 & 19.9 & 15.3 & 19.8 & 20.0 & 20.1 & 19.0 \\
\hline & & 21.6 & & & & & & & & & & & & & & & 2 & & & & & & & 18.0 & & 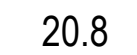 & 20.9 & 20.2 \\
\hline & & & & & & & & & & & & & & & & & & & & & & & & 23.2 & 23. & 23.8 & 23.9 & 23.6 \\
\hline Apr 10 & Mean & 2 & 22 & 11 & 21.1 & 21.3 & 2 & 21 & 21 & 22.0 & 2 & 2 & 2 & & 21 & 22.2 & 22 & 22.1 & 22.0 & 21 & 2 & 2 & 21.9 & 20.6 & 21.8 & 22.1 & 22.2 & 21.5 \\
\hline & & & 3 & 6. & & 3.65 & 3.8 & 3. & 3 & & & 3 & & & & & 3. & 3. & $\mathrm{~J}$ & 3. & 3. & 3. & & 4.08 & 3.59 & 3.64 & 3.58 & 3.95 \\
\hline & & & & & & 16 & & 1 & 1 & & & 1 & & & 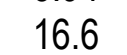 & & 17 & 17 & & 16 & & 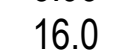 & 17.0 & 14.5 & 17.0 & 17.2 & 17.4 & 16.1 \\
\hline & Median & 21 & 21. & 10. & 20.3 & 20.5 & 20.8 & 20.6 & 20 & 21.4 & 20.9 & 21.1 & 2 & 21 & 21 & 21.5 & 21.4 & 21.3 & 21.3 & 2 & 21.0 & 2 & 21.3 & 20.1 & 21.0 & 21.3 & 21.5 & 20.8 \\
\hline & $95^{\text {th }}$ ptile & 28.3 & 28.9 & 23.3 & 26.9 & 27.3 & 28.1 & 27.9 & 27.7 & 27.6 & 27.3 & 27.8 & 28.1 & 27.8 & 28.4 & 28.1 & 28.3 & 28.2 & 28.0 & 28.4 & 28.3 & 28.0 & 27.5 & 27.5 & 27.7 & 28.0 & 28.0 & 28.1 \\
\hline
\end{tabular}


Table 3. Continuation.

North Panels

East Panels

South Panels

West Panels

\begin{tabular}{|c|c|c|c|c|c|c|c|c|c|c|c|c|c|c|c|c|c|c|c|c|c|c|c|c|c|c|c|c|}
\hline \multirow{3}{*}{ Month } & \multirow{4}{*}{$\begin{array}{l}\text { Descriptive } \\
\text { Statistics }\end{array}$} & \multirow{3}{*}{$\underset{1 \mathrm{st} f \mid}{\ln }$} & \multirow{4}{*}{$\begin{array}{c}\ln \\
2^{\text {nd f }} \mid\end{array}$} & \multirow{4}{*}{ Out } & \multicolumn{5}{|c|}{ Panels } & \multicolumn{6}{|c|}{ East Panels } & \multicolumn{7}{|c|}{ South Panels } & \multicolumn{6}{|c|}{ Vest Panels } \\
\hline & & & & & P1-15 & P1-16 & P2-15 & P2-16 & P2-17 & P1-10 & P1-11 & P1-12 & P1-13 & P1-14 & P2-14 & P1-6 & P1-7 & P1-8 & P1-9 & P2-6 & P2-7 & P2-8 & P1-1 & P1-2 & P1-3 & P1-4 & P1-5 & P2-5 \\
\hline & & & & & Layout A & Layout A & $\begin{array}{l}\text { Layout A } \\
\end{array}$ & Layout B & Layout B & Lavou & ayout C & $\begin{array}{l}\text { Layou } \\
\end{array}$ & $\begin{array}{l}\text { Layout A } \\
\text {. }\end{array}$ & Lavout $\mathrm{A}$ & ayout A & Layou & Layout A & avout $B$ & Layol & 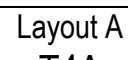 & Iavo & 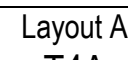 & & - & & avout & (2) & Iavout $B$ \\
\hline May 10 & & & & & $A$ & T4A & T4A & T4B & AB & T4C & T4C & T4A & T4A & T4A & A & T4A & T4A & T4B & T4B & T4A & T4C & T4A & T. & T3D & T4B & 4 & 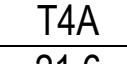 & ? \\
\hline & SD & 177 & 212 & 7.0 & 206 & 2.12 & 1.4 & 21.2 & 20.4 & $\begin{array}{l}21.4 \\
108\end{array}$ & 261 & 210 & $\begin{array}{l}21.4 \\
109\end{array}$ & $\begin{array}{l}1.4 \\
2.03\end{array}$ & 21.0 & 206 & 201 & 213 & $\begin{array}{l}21.4 \\
2.17\end{array}$ & 20.4 & 21.4 & 0.2 & 21.4 & 286 & 217 & $\begin{array}{l}21.4 \\
2.21\end{array}$ & 21.0 & 267 \\
\hline & $5^{\text {th }}$ ptile & 17.5 & 17.0 & 3.20 & 16.1 & 16.2 & 16.3 & 16.0 & 15.7 & 17.0 & 16.0 & 16.7 & 17.0 & 17.0 & 16.3 & 16.8 & 17.1 & 16.7 & 16.7 & 15.8 & 16.1 & 15.7 & 17.0 & 14.2 & 16.7 & 16.8 & 17.1 & 15.8 \\
\hline & Median & 22.3 & 22.5 & 17.0 & 21.6 & 21.8 & 22.1 & 22.0 & 21.9 & 22.4 & 22.4 & 22.1 & 22.2 & 22.2 & 22.2 & 22.4 & 22.4 & 22.3 & 22.2 & 22.0 & 22.1 & 21.8 & 22.5 & 21.6 & 22.1 & 22.0 & 22.4 & 22.0 \\
\hline & $95^{\text {th }}$ ptile & 23.0 & 23.8 & 27.5 & 23.5 & 23.0 & 23.8 & 23.9 & 24.2 & 23.1 & 24.5 & 23.6 & 23.3 & 23.5 & 24.4 & 24.2 & 23.6 & 23.7 & 23.8 & 25.1 & 24.5 & 24.6 & 23.2 & 28.2 & 23.9 & 24.5 & 24.2 & 24.8 \\
\hline Jun 10 & Mean & 20.5 & 21.1 & 19.4 & 20.5 & 20.5 & 20.9 & 21.0 & 21.1 & 20.8 & 21.2 & 20.6 & 20.5 & 20.7 & 21.2 & 21.1 & 21.0 & 20.8 & 20.8 & 21.2 & 21.1 & 21.0 & 21.0 & 21.8 & 20.8 & 20.8 & 21.0 & 21.1 \\
\hline & SD & 2.06 & 2.04 & 4.29 & 2.01 & 1.96 & 2.01 & 2.01 & 2.01 & 1.90 & 1.99 & 2.06 & 2.11 & 2.05 & 2.06 & 1.87 & 1.83 & 1.90 & 1.93 & 2.18 & 2.05 & 2.11 & 1.90 & 2.92 & 1.99 & 1.99 & 1.96 & 2.11 \\
\hline & $5^{\text {th }}$ ptile & 17.6 & 18.1 & 12.0 & 17.4 & 17.8 & 17.7 & 17.8 & 17.8 & 18.2 & 17.9 & 17.6 & 17.5 & 17.7 & 17.8 & 18.3 & 18.3 & 18.0 & 17.9 & 17.6 & 17.8 & 17.6 & 18.4 & 17.3 & 17.8 & 17.8 & 18.0 & 17.7 \\
\hline & Median & 22.1 & 22.2 & 19.3 & 21.4 & 21.6 & 21.7 & 21.7 & 21.6 & 22.0 & 21.9 & 21.8 & 21.9 & 21.9 & 21.9 & 22.0 & 22.1 & 21.9 & 21.8 & 21.6 & 21.7 & 21.5 & 22.1 & 21.6 & 21.7 & 21.8 & 22.0 & 21.7 \\
\hline & $95^{\text {th }}$ ptile & 22.5 & 23.5 & 26.4 & 23.2 & 22.9 & 23.4 & 23.7 & 24.0 & 22.8 & 23.8 & 23.1 & 22.9 & 23.1 & 24.1 & 23.5 & 23.1 & 23.1 & 23.1 & 24.5 & 24.0 & 24.1 & 23.0 & 27.1 & 23.4 & 23.5 & 23.5 & 24.2 \\
\hline Jul 10 & Mean & 22.4 & 23.2 & 23.6 & 22.6 & 22.6 & 20.4 & 23.2 & 20.2 & 22.8 & 22.7 & 22.7 & 22.5 & 22.8 & 23.6 & 23.3 & 22.9 & 22.8 & 22.8 & 20.4 & 23.5 & 20.2 & 23.1 & 24.6 & 23.0 & 23.0 & 23.1 & 23.5 \\
\hline & SD & 0.16 & 0.64 & 4.26 & 0.81 & 0.55 & 1.16 & 0.91 & 0.89 & 0.38 & 0.37 & 0.70 & 0.50 & 0.69 & 1.04 & 0.79 & 0.51 & 0.58 & 0.64 & 1.16 & 1.09 & 0.89 & 0.43 & 2.91 & 0.79 & 1.13 & 0.83 & 1.26 \\
\hline & $5^{\text {th }}$ ptile & 22.1 & 22.3 & 16.3 & 21.4 & 21.7 & 21.8 & 21.8 & 21.7 & 22.2 & 22.1 & 21.8 & 21.8 & 21.9 & 22.0 & 22.1 & 22.1 & 21.9 & 21.9 & 21.6 & 21.8 & 21.4 & 22.2 & 20.9 & 21.9 & 21.8 & 22.1 & 21.8 \\
\hline & Median & 22.4 & 23.1 & 23.3 & 22.5 & 22.5 & 23.0 & 23.2 & 23.3 & 22.8 & 22.6 & 22.6 & 22.4 & 22.6 & 23.5 & 23.2 & 22.9 & 22.7 & 22.8 & 23.5 & 23.4 & 23.3 & 23.1 & 23.8 & 22.8 & 22.6 & 22.8 & 23.4 \\
\hline & 95th ptile & 22.6 & 24.3 & 30.4 & 24.2 & 23.6 & 24.6 & 24.9 & 25.3 & 23.6 & 23.4 & 24.0 & 23.4 & 24.1 & 25.4 & 24.6 & 23.8 & 23.8 & 24.0 & 26.2 & 25.4 & 25.7 & 23.8 & 30.5 & 24.5 & 25.5 & 24.7 & 25.9 \\
\hline g 10 & Mean & 22.4 & 22.9 & 21.7 & 22.3 & 22.3 & 20.4 & 22.9 & 20.2 & 22.7 & 22.6 & 22.6 & 22.4 & 22 & 23.1 & 23. & 22.8 & 22.7 & 22.8 & 20.4 & 23.1 & 20.2 & 22.8 & 23.7 & 22.7 & 22.8 & 22.9 & 23.1 \\
\hline & SD & 0.16 & 0.73 & 3.96 & 0.67 & 0.52 & 1.16 & 1.00 & 0.89 & 0.35 & 0.31 & 0.64 & 0.47 & 0.57 & 1.14 & 0.85 & 0.53 & 0.58 & 0.69 & 1.16 & 1.18 & 0.89 & 0.41 & 2.67 & 0.73 & 1.00 & 0.66 & 1.26 \\
\hline & $5^{\text {th }}$ ptile & 22.1 & 22.1 & 15.3 & 21.3 & 21.5 & 21.5 & 21.6 & 21.4 & 22.1 & 22.1 & 21.7 & 21.8 & 21.8 & 21.6 & 22.0 & 22.1 & 21.9 & 21.9 & 21.3 & 21.6 & 21.2 & 22.1 & 20.5 & 21.8 & 21.7 & 22.1 & 21.4 \\
\hline & Median & 22.4 & 22.7 & 21.3 & 22.2 & 22.2 & 22.6 & 22.7 & 22.8 & 22.7 & 22.6 & 22.5 & 22.3 & 22.4 & 23.0 & 23.0 & 22.8 & 22.6 & 22.6 & 22.9 & 22.9 & 22.8 & 22.8 & 23.0 & 22.6 & 22.5 & 22.7 & 22.8 \\
\hline & $95^{\text {th }}$ ptile & 22.7 & 24.5 & 28.3 & 23.5 & 23.3 & 24.7 & 24.8 & 25.2 & 23.3 & 23.1 & 23.8 & 23.3 & 23.5 & 25.4 & 24.8 & 23.8 & 23.8 & 24.1 & 26.5 & 25.5 & 25.7 & 23.6 & 29.3 & 24.3 & 24.9 & 24.3 & 25.6 \\
\hline
\end{tabular}

Notes: Layouts A, B, C and D refer to the location of the sensors per Figures 25 through 28.

Abbreviations: NA, not available; ptile, percentile; SD, standard deviation. 
Table 4. Monthly relative humidity (\%) at exterior side of stud cavity. Refer to Figures 25 through 28 for sensor location.

\begin{tabular}{|c|c|c|c|c|c|c|c|c|c|c|c|c|c|c|c|c|c|c|c|c|c|c|c|c|c|c|c|c|}
\hline \multirow{3}{*}{ Month } & \multirow{3}{*}{$\begin{array}{l}\text { Descriptive } \\
\text { Statistics }\end{array}$} & \multirow{3}{*}{$\underset{1 \text { st fl }}{\ln }$} & \multirow{3}{*}{$\begin{array}{c}\ln \\
2^{\text {nd ffl }}\end{array}$} & \multirow{3}{*}{ Out } & \multicolumn{5}{|c|}{ North Panels } & \multicolumn{6}{|c|}{ East Panels } & \multicolumn{7}{|c|}{ South Panels } & \multicolumn{6}{|c|}{ West Panels } \\
\hline & & & & & P1-15 & P1-16 & P2-15 & P2-16 & P2-17 & P1-10 & P1-11 & P1-12 & $\mathrm{P} 1-13$ & P1-14 & P2-14 & P1-6 & P1-7 & P1-8 & P1-9 & P2-6 & P2-7 & P2-8 & P1-1 & P1-2 & P1-3 & P1-4 & P1-5 & P2-5 \\
\hline & & & & & $\begin{array}{l}\text { Layout A } \\
\text { RH1A }\end{array}$ & $\begin{array}{l}\text { Layout A } \\
\text { RH1A }\end{array}$ & $\begin{array}{l}\text { Layout A } \\
\text { RH1A }\end{array}$ & $\begin{array}{l}\text { Layout B } \\
\text { RH1B }\end{array}$ & $\begin{array}{l}\text { Layout B } \\
\text { RH1B }\end{array}$ & $\begin{array}{l}\text { Layout C } \\
\text { RH1C }\end{array}$ & $\begin{array}{l}\text { Layout C } \\
\text { RH1C }\end{array}$ & $\begin{array}{l}\text { Layout A } \\
\text { RH1A }\end{array}$ & $\begin{array}{l}\text { Layout A } \\
\text { RH1A }\end{array}$ & $\begin{array}{l}\text { Layout A } \\
\text { RH1A }\end{array}$ & $\begin{array}{l}\text { Layout A } \\
\text { RH1A }\end{array}$ & $\begin{array}{l}\text { Layout A } \\
\text { RH1A }\end{array}$ & $\begin{array}{l}\text { Layout A } \\
\text { RH1A }\end{array}$ & $\begin{array}{l}\text { Layout B } \\
\text { RH1B }\end{array}$ & $\begin{array}{l}\text { Layout B } \\
\text { RH1B }\end{array}$ & $\begin{array}{l}\text { Layout A } \\
\text { RH1A }\end{array}$ & $\begin{array}{l}\text { Layout B } \\
\text { RH1B }\end{array}$ & $\begin{array}{l}\text { Layout A } \\
\text { RH1A }\end{array}$ & $\begin{array}{l}\text { Layout C } \\
\text { RH1C }\end{array}$ & $\begin{array}{l}\text { Layout D } \\
\text { RH2D }\end{array}$ & $\begin{array}{c}\text { Layout B } \\
\text { RH1B }\end{array}$ & $\begin{array}{l}\text { Layout A } \\
\text { RH1A }\end{array}$ & $\begin{array}{l}\text { Layout A } \\
\text { RH1A }\end{array}$ & $\begin{array}{l}\text { Layout B } \\
\text { RH1B }\end{array}$ \\
\hline Sep 09 & an & 51.3 & & NA & 71.4 & 55.8 & NA & 52.1 & 58.0 & 59.9 & 53.9 & 51.6 & 62.0 & 61.0 & 52.5 & 50.4 & 54.3 & 55.5 & 51.1 & 51.6 & 46.9 & 67.0 & 68.3 & 80.8 & 45.5 & 54.1 & 54.1 & 53.3 \\
\hline & & & & $\mathrm{NA}$ & & 3.93 & NA & 3 & 2 & & 4.25 & 1.67 & & 10.7 & 6.90 & 2.27 & 5.38 & 7.6 & 14.0 & & 6.88 & 9.07 & 90 & 66 & 93 & & 14.7 & 6.77 \\
\hline & $5^{\text {th }}$ ptile & 3.3 & & NA & 4 & 49.3 & NA & 50.2 & 56.0 & 9 & 46.1 & 48.5 & 42.1 & 43.5 & 40.6 & 46.6 & 46.2 & 27.9 & 27.4 & 22.4 & 33.8 & 49.8 & 64.9 & 73.9 & 41.3 & 9 & 30.3 & 41.5 \\
\hline & Median & 5 & & NA & .9 & 56.1 & NA & 520 & 581 & & 54.2 & 51.8 & 61.5 & 60.8 & 52.5 & 0.5 & 54.2 & 56.5 & 53.2 & 53.5 & 47.7 & 68.0 & 68.5 & 79.4 & 45.1 & & 52.9 & 53.8 \\
\hline & 95 th $\mathrm{ptil}$ & 54.2 & & NA & 80.7 & 61.3 & NA & 54.4 & 60.1 & 60.7 & 61.5 & 54.1 & 82.3 & 79.3 & 63.9 & 54.3 & 64.7 & 88.6 & 73.9 & 76.7 & 57.2 & 81.1 & 71.0 & 91.7 & 0.4 & .1 & 78.1 & 64.9 \\
\hline Oct 09 & Mean & & & $\mathrm{NA}$ & 7 & 41.0 & 77.2 & 46.1 & 89.7 & 56.6 & 56.9 & 48.1 & 861 & 84.6 & 65.8 & 21.0 & 21.3 & 21.1 & 21.0 & 20.4 & 20.6 & 20.2 & 61.3 & 87.3 & 65.7 & 70.7 & 81.7 & 67.2 \\
\hline & & & & & & & & & & & & & & & & & & 0.66 & & & 0.7 & & 19 & 2.71 & 2.22 & & 9.02 & 4.86 \\
\hline & $5^{\text {th }}$ ptile & 40 & & NA & & 32.9 & 40.2 & 42.4 & 49.0 & & 48.8 & 44.0 & & 74.7 & 58.4 & 20.2 & 20.8 & 20.3 & 20.4 & 19.1 & 19.6 & 18.9 & .0 & 83.4 & 52.9 & & 65.4 & 59.5 \\
\hline & Median & 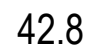 & & & 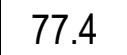 & 41.7 & 59.2 & 45.3 & 52.6 & & 58.5 & 47.7 & & 84.2 & 65.4 & 20.9 & 21. & 20.9 & 20.9 & 20.1 & 20.5 & 20.1 & 0.2 & 87.0 & 5.1 & & 82.4 & 66.9 \\
\hline & & & & & & & 705 & & & & & & & & 74 & & & $2<.0$ & 22 & 23 & 22 & 22.0 & & 92.3 & 0.4 & & 96 & 76.1 \\
\hline Nov 09 & Mean & & & 72 & & ? & 1. & 0 & 47.4 & & 53.4 & 42 & & 87 & 68. & & & 0.4 & 58.6 & 79 & & & 5.8 & 85.0 & 7.5 & & 88.0 & 71.2 \\
\hline & St & & & & & & U & & & & 1 & 1.88 & & & & & & 18.8 & 16 & & & & & 4.19 & 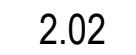 & & 7.54 & 4.97 \\
\hline & $5^{\text {th }}$ & & & & & & & & & & & & & 77 & & & & & & 44 & & & & 0.0 & 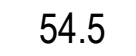 & & 73.2 & 63.0 \\
\hline & Median & & & 7 & & & $\mathrm{~N}$ & 40 & 469 & & & 4 & & 87.6 & & & & 7 & 6 & & & & & 85.5 & & & .1 & 70.6 \\
\hline & & & & 95.0 & & 3 & NA & 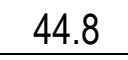 & 51.4 & & 59.5 & 46.7 & 100.6 & 95.4 & 76. & 47.3 & 56.4 & 84.9 & 81.8 & 93.9 & 68.9 & 95.6 & 9.3 & 91.2 & 61.2 & & 98.5 & 79.6 \\
\hline 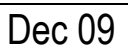 & & & & & & & $\Delta \Lambda$ & & & & & & & & & & & & & & & & & & & & & 80.8 \\
\hline & . & & & & & & TV & & & & & & & 3. & & & & 15.0 & T1. & 13.0 & & & & .36 & 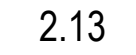 & & 3.92 & 4.21 \\
\hline & $5^{\text {th }}$ & & & & & & NA & & & & 1 & 30 & & 90.0 & 72 & & & 43.4 & 48. & 86.3 & 61.2 & 94 & & 8 & 7 & & 89.0 & 73.1 \\
\hline & Med & & & & & & A & & & & & & & & & & & & & 97 & & & & & & & 0 & 80.9 \\
\hline & 95 & & & & & & $\mathrm{~N}$ & 38 & 44.7 & & 58.0 & 40 & & 100 & 86 & 0.4 & 7 & 93.1 & 88.8 & 10 & 79 & 10 & .6 & 90.3 & 6.8 & & 100 & 87.2 \\
\hline an 10 & & & & & & & & & & & & & & 98.6 & & & & 718 & 781 & 77.2 & & 89.7 & 7 & 23 & .5 & & 95.6 & 81.4 \\
\hline & SL & & & & & & & & & & & & & & & & & & & 13 & 5. & 3. & 37 & .08 & & & 6.74 & 4.37 \\
\hline & $5^{\text {th }}$ & & & & & & $\mathrm{N}$ & & & & & 26 & & 90.5 & 75 & & & 32.2 & 30 & 94 & 63 & 16 & 8 & 70.6 & 8 & & 82.5 & 74.1 \\
\hline & $50^{\text {th }}$ & & & & & & & & & & & & & & & & & & & & & & & & & & & 81.8 \\
\hline & 95 & & & & & & & & & & & & & & & & & 91.7 & & & 81 & & 0.3 & 80.9 & 55.9 & & 00 & 87.8 \\
\hline Feb 10 & & & & & & & $\bar{N}$ & & & & & & & 93 & 71 & & & 70 & & 97 & 7 & 10 & 3.8 & 10 & 58.2 & & 4 & 78.7 \\
\hline & s & & & & & & & & & & & & & & & & & & & & & & & & & & & 7 \\
\hline & & & & & & & & & & & & & & & 73 & & & 47.0 & & 9 & 68 & 10 & 2.6 & 64.3 & 56.5 & & 78.0 & 74.1 \\
\hline & & & & & & & $\mathrm{N}$ & 23 & 83 & & & 26 & & 92 & & & & 71 & 64 & 98 & 73 & 10 & .7 & 66.6 & 7 & & 88 & 78.2 \\
\hline & & & & & & & NA & & & & & & & & & & & & & & & & .1 & & & & & 6 \\
\hline 10 & & & & & & & & & & & & & & & & & & & & & & & & & & & & 67.7 \\
\hline & $\mathrm{Sl}$ & & & & & & TN & & & & & & & & & & & 16.4 & & & & & 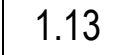 & 5. & 4.49 & & 8 & 5.51 \\
\hline & & & & & & & $\mathrm{N}$ & & & & & & & & & & & & & & & & & & & & & 3 \\
\hline & & & & & & & & & & & & & & & & & & & & & & & & & & & & \\
\hline & & & & & & 29.9 & Tw & 20.0 & 4 & & 40 & 29 & & 0. & 75 & 29.0 & & $v_{1}$ & 0 & $y_{1}$ & 15 & 100 & .1 & 65.8 & 31.0 & & 81.0 & 76.8 \\
\hline Apr 10 & Mea & & & & & & IV & $2 v$ & & & & & & & & & & & & & & $r$ & 5 & 66.2 & & & 48.9 & 54.2 \\
\hline & & & & & & & & 2.8 & & & & & & & & & & & & & & & & & & & & 5. \\
\hline & & 24 & & & & 20 & $\mathrm{~N}$ & 24 & & & & 27 & & & 36 & & & 20 & 20 & 2 & 42 & 6 & 0 & 55.9 & 35.7 & & 27.9 & 45.7 \\
\hline & Med & 28. & 28 & 51 & & 29.9 & NA & 29. & 3 & & J & 29 & & VI. & 45 & $\angle J .1$ & $v_{4}$ & 31.2 & & 49 & & דו & 40.3 & 61.6 & 38.2 & & 48.3 & 54.1 \\
\hline & $95^{\text {th }}$ ptile & 30.0 & 30.9 & 94.9 & 78.3 & 35.0 & NA & 35.1 & 35.5 & 41.8 & 44.5 & 31.8 & 71.7 & 69.0 & 56.1 & 32.4 & 40.0 & 62.9 & 56.1 & 71.9 & 61.5 & 88.8 & 43.6 & 88.0 & 45.0 & 74.2 & 71.5 & 63.0 \\
\hline
\end{tabular}


Table 4. Continuation.

North Panels

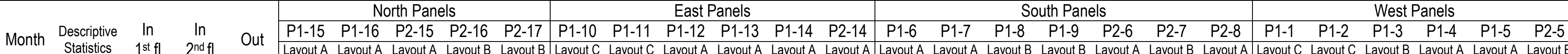

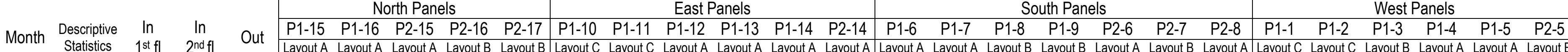

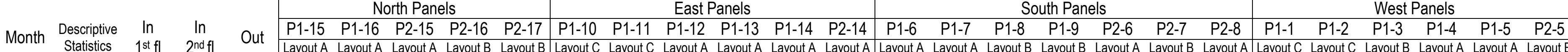
$\begin{array}{llll} & \\ 0\end{array}$

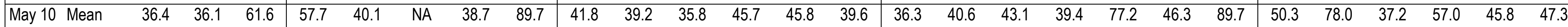
\begin{tabular}{llllllllllllllllllllllllllllllll}
$\mathrm{SD}$ & 4.25 & 3.60 & 17.8 & 10.7 & 6.10 & NA & 3.78 & 3.78 & 1.45 & 3.26 & 2.79 & 11.3 & 10.6 & 5.21 & 2.52 & 3.87 & 12.1 & 9.84 & 13.0 & 4.64 & 3.78 & 4.37 & 10.1 & 1.84 & 9.43 & 15.0 & 5.07 \\
\hline
\end{tabular} \begin{tabular}{lllllllllllllllllllllllllllllll}
$5^{\text {th }}$ ptile & 31.0 & 31.4 & 33.2 & 40.9 & 30.5 & NA & 34.0 & 36.0 & 39.6 & 34.0 & 30.6 & 28.8 & 29.4 & 30.3 & 31.7 & 36.6 & 24.7 & 23.7 & 22.0 & 37.4 & 38.4 & 42.1 & 57.8 & 34.1 & 39.2 & 23.1 & 38.2 \\
\hline Median & 35.1 & 35.2 & 60.7 & 58.3 & 39.6 & NA & 37.4 & 40.3 & 41.5 & 39.1 & 35.3 & 44.2 & 44.7 & 40.0 & 36.1 & 39.6 & 43.0 & 39.6 & 42.7 & 47.1 & 57.2 & 52.3 & 80.1 & 37.2 & 58.2 & 44.6 & 47.6
\end{tabular} \begin{tabular}{lllllllllllllllllllllllllllll} 
Median & 35.1 & 35.2 & 60.7 & 58.3 & 39.6 & NA & 37.4 & 40.3 & 41.5 & 39.1 & 35.3 & 44.2 & 44.7 & 40.0 & 36.1 & 39.6 & 43.0 & 39.6 & 42.7 & 47.1 & 57.2 & 52.3 & 80.1 & 37.2 & 58.2 & 44.6 & 47.6 \\
\hline
\end{tabular} \begin{tabular}{llllllllllllllllllllllllllll}
$95^{\text {th }}$ ptile & 44.3 & 43.0 & 94.2 & 75.8 & 50.8 & NA & 45.4 & 48.1 & 44.4 & 44.7 & 41.2 & 67.7 & 66.8 & 48.0 & 41.3 & 48.4 & 64.3 & 57.7 & 70.3 & 53.4 & 73.4 & 56.0 & 90.8 & 39.8 & 69.2 & 72.1 & 55.7 \\
\hline Mean & 46.8 & 48.9 & 72.7 & 59.3 & 52.9 & NA & 50.7 & 54.2 & 48.7 & 47.4 & 47.7 & 50.3 & 49.2 & 42.1 & 48.4 & 54.9 & 57.3 & 50.2 & 48.6 & 47.0 & 56.8 & 59.2 & 86.0 & 40.1 & 50.0 & 46.9 & 46.4
\end{tabular} \begin{tabular}{llllllllllllllllllllllllllllll}
$\mathrm{SD}$ & 3.80 & 4.78 & 16.1 & 9.02 & 6.08 & $\mathrm{NA}$ & 4.66 & 4.10 & 2.33 & 4.32 & 3.83 & 9.3 & 8.58 & 4.88 & 4.62 & 6.37 & 12.9 & 10.2 & 11.7 & 3.95 & 7.37 & 2.47 & 7.05 & 2.73 & 8.13 & 12.2 & 4.59 \\
\hline
\end{tabular}

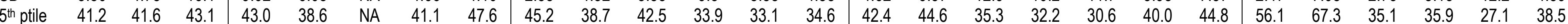

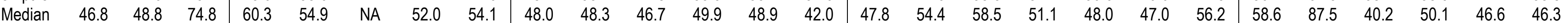
\begin{tabular}{lllllllll|lllllllllllllllllll}
$95^{\text {th }}$ ptile & 52.9 & 57.0 & 95.3 & 73.1 & 59.2 & NA & 56.1 & 60.1 & 53.4 & 53.1 & 54.9 & 64.8 & 62.5 & 50.3 & 56.4 & 66.0 & 76.8 & 65.0 & 70.1 & 53.0 & 69.2 & 63.4 & 93.9 & 45.1 & 62.3 & 66.8 & 53.8 \\
\hline
\end{tabular}

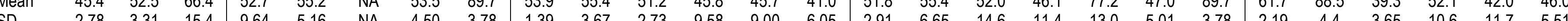
\begin{tabular}{lllllllllllllllllllllllllllllllll} 
SD & 2.78 & 3.31 & 15.4 & 9.64 & 5.16 & NA & 4.50 & 3.78 & 1.39 & 3.67 & 2.73 & 9.58 & 9.00 & 6.05 & 2.91 & 6.65 & 14.6 & 11.4 & 13.0 & 5.01 & 3.78 & 2.19 & 4.4 & 3.65 & 10.6 & 11.7 & 5.51 \\
\hline
\end{tabular} \begin{tabular}{llll|llllllllllllllllllllllllll}
$5^{\text {th }}$ ptile & 40.4 & 46.3 & 41.6 & 36.8 & 44.2 & NA & 45.3 & 52.2 & 51.6 & 50.3 & 46.1 & 30.0 & 30.8 & 30.6 & 46.7 & 44.5 & 30.2 & 28.4 & 24.7 & 38.4 & 37.5 & 58.4 & 79.1 & 34.0 & 33.3 & 21.3 & 36.5
\end{tabular}

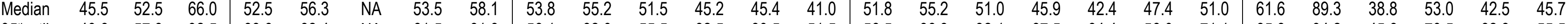

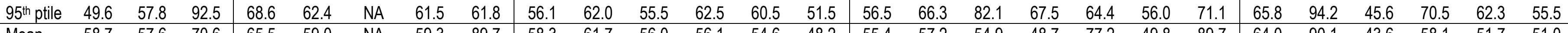

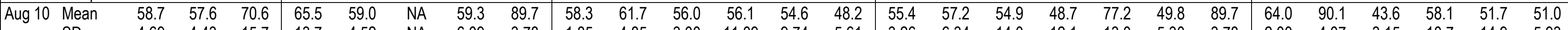
\begin{tabular}{llll|llllllllllllllllllllllllll} 
SD & 4.69 & 4.43 & 15.7 & 13.7 & 4.52 & NA & 6.09 & 3.78 & 1.85 & 4.85 & 3.00 & 11.09 & 9.74 & 5.61 & 3.26 & 6.34 & 14.0 & 12.1 & 13.0 & 5.30 & 3.78 & 2.00 & 4.07 & 3.15 & 10.7 & 14.9 & 5.93
\end{tabular} \begin{tabular}{llllllllllllllllllllllllllll}
$5^{\text {th }}$ ptile & 51.2 & 50.5 & 44.9 & 43.3 & 51.0 & NA & 48.9 & 56.4 & 55.0 & 53.7 & 50.8 & 39.3 & 38.9 & 39.2 & 49.6 & 46.1 & 32.1 & 28.4 & 26.4 & 40.3 & 45.6 & 60.2 & 82.2 & 39.4 & 38.7 & 28.4 & 40.9 \\
\hline
\end{tabular} $\begin{array}{lllllllllllllllllllllllllllll}\text { Median } & 58.2 & 57.8 & 71.0 & 64.7 & 59.3 & \text { NA } & 57.5 & 63.0 & 59.0 & 61.0 & 56.0 & 54.9 & 54.5 & 47.9 & 55.5 & 57.0 & 55.2 & 49.5 & 48.5 & 50.6 & 57.7 & 63.6 & 90.2 & 43.0 & 58.0 & 51.0 & 51.1 \\ 95^{\text {th }} \text { ptile } & 69.1 & 66.3 & 96.0 & 86.9 & 65.4 & \text { NA } & 67.9 & 67.0 & 60.5 & 71.7 & 61.5 & 77.0 & 71.4 & 57.4 & 60.1 & 67.3 & 77.4 & 68.8 & 68.1 & 58.5 & 72.8 & 67.2 & 96.2 & 49.7 & 76.4 & 79.1 & 60.9\end{array}$ Notes: Layouts A, B, C and D refer to the location of the sensors per Figures 25 through 28.

Abbreviations: NA, not available; ptile, percentile; SD, standard deviation. 
Table 5. Monthly water vapor pressure (kPa) at exterior side of stud cavity. Refer to Figures 25 through 28 for sensor location. North Panels East Panels

South Panels

West Panels

\begin{tabular}{|c|c|c|c|c|c|c|c|c|c|c|c|c|c|c|c|c|c|c|c|c|c|c|c|c|c|c|c|c|}
\hline \multirow{3}{*}{ Month } & \multirow{3}{*}{$\begin{array}{c}\text { Descriptive } \\
\text { Statistics }\end{array}$} & \multirow{3}{*}{$\underset{1 \mathrm{st} f \mid}{\ln }$} & \multirow{3}{*}{$\begin{array}{l}\ln \\
2^{\text {nd fl }}\end{array}$} & \multirow{3}{*}{ Out } & & & & \\
\hline & & & & & P1-15 & P1-16 & P2-15 & P2-16 & P2-17 & P1-10 & P1-11 & P1-12 & P1-13 & P1-14 & P2-14 & P1-6 & P1-7 & P1-8 & P1-9 & P2-6 & P2-7 & P2-8 & P1-1 & P1-2 & P1-3 & P1-4 & P1-5 & P2-5 \\
\hline & & & & & Layout A & $\begin{array}{l}\text { Layout A } \\
\end{array}$ & $\begin{array}{l}\text { Layout A } \\
\text { Taut }\end{array}$ & $\begin{array}{l}\text { Layout B } \\
\end{array}$ & $\begin{array}{l}\text { Layout B } \\
\end{array}$ & $\begin{array}{l}\text { Layout C } \\
\text { TAOU }\end{array}$ & $\begin{array}{l}\text { Layout C } \\
\end{array}$ & $\begin{array}{l}\text { Layout A } \\
\text { A }\end{array}$ & $\begin{array}{l}\text { Layout } A \\
\end{array}$ & Layout A & $\begin{array}{l}\text { Layout } A \\
\end{array}$ & Layout A & Layout A & $\begin{array}{l}\text { Layout B } \\
\text { TSOH }\end{array}$ & $\begin{array}{l}\text { Layout B } \\
\end{array}$ & $\begin{array}{l}\text { Layout A } \\
\end{array}$ & Layout $B$ & Layout A & $\begin{array}{l}\text { Layout C } \\
\end{array}$ & $\begin{array}{l}\text { Layout D } \\
\text { ayput }\end{array}$ & Layout B & $\begin{array}{l}\text { Layout A } \\
\end{array}$ & Layout A & $\begin{array}{l}\text { Layout B } \\
\text { TBR/RH1B }\end{array}$ \\
\hline \multirow[t]{5}{*}{ Sep 09} & Mean & 1.32 & 1.33 & NA & 1.34 & 1.37 & NA & 1.30 & 1.48 & 1.52 & 1.35 & 1.30 & 1.44 & 1.51 & 1.15 & 1.33 & 1.48 & 1.48 & 1.35 & 1.18 & 1.13 & 1.41 & 1.74 & 1.96 & 1.15 & 1.35 & 1.32 & $\frac{1.20}{1.20}$ \\
\hline & SD & 0.10 & 0.10 & NA & 0.35 & 0.18 & NA & 0.12 & 0.12 & 0.11 & 0.25 & 0.09 & 0.28 & 0.28 & 0.41 & 0.12 & 0.28 & 0.37 & 0.35 & 0.42 & 0.42 & 0.46 & 0.14 & 0.63 & 0.63 & 0.54 & 0.48 & 0.44 \\
\hline & $5^{\text {th }}$ ptile & 1.16 & 1.17 & NA & 0.82 & 1.04 & NA & 1.11 & 1.29 & 1.35 & 0.98 & 1.15 & 1.07 & 1.10 & 0.71 & 1.16 & 1.06 & 0.93 & 0.86 & 0.71 & 0.67 & 0.84 & 1.53 & 1.18 & 0.60 & 0.78 & 0.73 & 0.71 \\
\hline & Median & 1.32 & 1.32 & NA & 1.30 & 1.38 & NA & 1.28 & 1.46 & 1.51 & 1.32 & 1.29 & 1.38 & 1.48 & 1.02 & 1.31 & 1.43 & 1.46 & 1.28 & 1.05 & 0.98 & 1.29 & 1.74 & 1.82 & 0.94 & 1.18 & 1.23 & 1.08 \\
\hline & $95^{\text {th }}$ ptile & 1.49 & 1.50 & NA & 1.96 & 1.65 & NA & 1.50 & 1.67 & 1.70 & 1.75 & 1.45 & 2.02 & 2.02 & 2.10 & 1.54 & 2.00 & 2.11 & 2.03 & 2.08 & 2.04 & 2.30 & 1.97 & 3.23 & 2.43 & 2.50 & 2.30 & 2.10 \\
\hline \multirow[t]{5}{*}{ Oct 09} & Mean & 1.11 & 1.12 & NA & 0.95 & 0.94 & NA & 1.07 & 1.23 & 1.30 & 1.04 & 1.11 & 1.29 & 1.25 & 0.98 & 1.14 & 1.09 & 1.12 & 1.07 & 1.20 & 0.91 & 1.26 & 1.39 & 1.46 & 0.86 & 1.03 & 1.23 & 0.92 \\
\hline & SD & 0.08 & 0.08 & NA & 0.25 & 0.15 & NA & 0.08 & 0.09 & 0.07 & 0.16 & 0.08 & 0.25 & 0.25 & 0.34 & 0.11 & 0.27 & 0.37 & 0.30 & 0.51 & 0.32 & 0.43 & 0.08 & 0.32 & 0.31 & 0.32 & 0.35 & 0.25 \\
\hline & $5^{\text {th }}$ ptile & 1.01 & 1.03 & NA & 0.56 & 0.71 & NA & 0.97 & 1.12 & 1.18 & 0.75 & 1.02 & 0.9 & 0.90 & 0.60 & 1.01 & 0.73 & 0.63 & 0.69 & 0.72 & 0.56 & 0.73 & 1.27 & 1.06 & 0.53 & 0.64 & 0.80 & 0.59 \\
\hline & Median & 1.08 & 1.09 & NA & 0.94 & 0.95 & NA & 1.04 & 1.20 & 1.28 & 1.03 & 1.08 & 1.25 & 1.2 & 0. & 1.10 & 1.06 & 1.10 & 1.0 & 1.05 & 0.82 & 1.18 & 1.38 & 1.42 & 0.80 & 0.97 & 1.17 & 0.89 \\
\hline & 95th ptile & 1.25 & 1.25 & NA & 1.40 & 1.17 & NA & 1.21 & 1.38 & 1.41 & 1.30 & 1.24 & 1.77 & 1.72 & 1.62 & 1.32 & 1.58 & 1.72 & 1.66 & 2.41 & 1.68 & 2.15 & 1.55 & 2.09 & 1.42 & 1.67 & 1.91 & 1.37 \\
\hline \multirow[t]{5}{*}{ Nov 09} & Mean & 0.96 & 0.97 & 0.71 & 0.81 & 0.79 & NA & 0.92 & 1.07 & 1.16 & 0.89 & 0.96 & 1.12 & 1.10 & 0.86 & 0.98 & 0.89 & 0.93 & 0.90 & 1.06 & 0.83 & 1.10 & 1.22 & 1.25 & 0.74 & 0.93 & 1.10 & 0.80 \\
\hline & SD & 0.04 & .04 & 21 & 0.22 & 0.09 & $\mathrm{~N}$ & 0.05 & 0.05 & 0.06 & 0. & 0.04 & 0.2 & 0.2 & 0.2 & 0.06 & 0.18 & 0.29 & 0.24 & 0.51 & 0. & 0. & 0.07 & 0.29 & 0.26 & 0.31 & 0.36 & 0.25 \\
\hline & $5^{\text {th }}$ ptile & 0.88 & 0.90 & 0.43 & 0.48 & 0.64 & NA & 0.84 & 0.98 & 1.07 & 0.70 & 0.90 & 0.81 & 0.75 & 0.5 & 0.89 & 0.65 & 0.51 & 0.61 & 0.59 & 0.47 & 0.59 & 1.09 & 0.88 & 0.43 & 0.55 & 0.69 & 0.48 \\
\hline & Median & 0.96 & 0.97 & .68 & 0.77 & 0.78 & NA & 0.93 & 1.07 & 1.14 & 0.88 & 0.95 & 1.10 & 1.0 & 0.8 & 0.96 & 0.86 & 0.90 & 0.85 & 0.92 & 0.74 & 1. & 1.22 & 1.21 & 0.69 & 0.88 & 1.03 & 0.78 \\
\hline & $95^{\text {th }}$ ptile & 1.05 & 1.06 & 1.14 & 1.20 & 0.93 & $\mathrm{~N}$ & 1.02 & 1.16 & 1.27 & 1. & 1.05 & 1.5 & 1.5 & 1.5 & 1.10 & 1.21 & 1.47 & 1.44 & 2.32 & 1. & 2.05 & 1.34 & 1. & 1.23 & 1.53 & 1.78 & 1.25 \\
\hline \multirow[t]{5}{*}{ Dec 09} & Mean & 0.71 & 0.72 & 0.40 & - & 0.50 & $\mathrm{~N}$ & 0.69 & 0.80 & 0.93 & 0.63 & 0.72 & 0.7 & 0.74 & 0.4 & 0.73 & 0.62 & 0.65 & 0.6 & 0.75 & 0.54 & 0.68 & 0.90 & 0.85 & 0.49 & 0.60 & 0.73 & 0.52 \\
\hline & SD & 0 & 0 & 0.21 & 0. & 0.15 & $\mathrm{~N}$ & 0.10 & 0.1 & 0 & & 0.10 & 0.2 & 0.2 & & 0.09 & 0. & 0.23 & 0. & 0.43 & 0 & & 0.13 & & 0. & 0.21 & 0.24 & 0.18 \\
\hline & $5^{\text {th }}$ ptile & 0.60 & 0.61 & 15 & 0 & 0.29 & $\mathrm{~N}$ & 0.56 & 0.68 & 0 & 0. & 0.60 & 0.4 & 0. & 0. & 0.60 & 0. & 0. & 0.4 & 0.33 & 0. & 0 & 0.7 & 0.52 & 0.2 & 0. & 0.37 & 0.25 \\
\hline & Median & 0.69 & 0.70 & 0.34 & 0.48 & 0.52 & NA & 0.67 & 0.78 & & 0. & 0.70 & 0.7 & 0. & 0. & 0.7 & 0. & 0. & 0.6 & 0.66 & 0. & & 0.8 & 0.84 & 0.48 & 0.59 & 0.72 & 0.52 \\
\hline & $95^{\text {th }}$ ptile & 0.88 & 0.89 & 75 & 0.85 & 0.76 & $\mathrm{~N} f$ & 0.86 & 0.99 & 1.1 & 0.8 & 0.89 & 1.1 & 1. & 0. & 0.8 & 0.9 & 1.0 & 1.0 & 1.53 & 0. & 1. & 1.1 & 1.8 & 0.77 & 0.99 & 1.14 & 0.83 \\
\hline Jan 10 & Mean & 0.60 & 0.60 & 37 & 0.44 & 0.42 & $\mathrm{~N} /$ & 0.57 & 0.66 & 0 & 0.5 & 0.59 & 0.6 & 0. & 0. & 0.6 & & 0.5 & 0.5 & 0.72 & 0 & 0. & 0.7 & 0. & 0.44 & 0.5 & 0.64 & 0.47 \\
\hline & SD & 0 & 0.05 & 19 & 0 & 0.11 & $\mathrm{~N}$ & 0.06 & 0.05 & 04 & 0.0 & 0.04 & 0.1 & 0.2 & 0.2 & 0.0 & & 0.2 & 0.1 & 0.59 & 0.2 & 0. & 0.08 & 0 & 0. & 0.25 & 0.25 & 0.20 \\
\hline & $5^{\text {th }}$ & 0 & 0.51 & 0 & & 0.23 & $\mathrm{~N}$ & 0.46 & 0.55 & 0.74 & & 0.52 & 0. & 0.3 & & & 0 & 0. & 0. & 0.26 & 0. & & 0.6 & & 0. & 0. & & 0.20 \\
\hline & $50^{\text {th }}$ & 0.60 & 0.61 & .34 & 0 & 0.41 & $\mathrm{~N}$ & 0.58 & 0.67 & 0 & 0.5 & 0.59 & 0.6 & 0. & 0.4 & 0.6 & 0.5 & 0.5 & 0.5 & 0.60 & 0.46 & 0. & 0.7 & 0. & 0. & 0. & 0.62 & 0.45 \\
\hline & $95^{\text {th }}$ & 0.68 & 0.69 & 0.63 & 0 & 0.57 & $\mathrm{~N}$ & 0.66 & 0.74 & 0.87 & 0.71 & 0.66 & 1.0 & 1.0 & 0. & 0.70 & 0.91 & 1. & 0.9 & 1.38 & 0.92 & 1. & 0.8 & 1.0 & 0.76 & 1. & 1.05 & 0.83 \\
\hline Feb 10 & Mean & & & & & & $\cdots$ & & 0.63 & & & 0.55 & 0.6 & & & & & & 0. & 0. & & & 0.6 & & & & & 0.49 \\
\hline & $S$ & 0 & 0 & c & & 0.09 & $\mathrm{~N}$ & 0.04 & U & 0.02 & 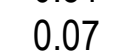 & 0 & 0. & 0 & & & 0. & 0.2 & 0. & 0.40 & 0. & & 0.0 & 0.14 & 0. & 0. & 0.18 & 0.14 \\
\hline & $5^{\text {th }}$ ptile & 0.50 & 0.51 & 0.16 & 0. & 0.28 & $\mathrm{~N}$ & 0.48 & 0.56 & 0.72 & 0. & 0.50 & 0.5 & 0.4 & 0. & 0.51 & 0.40 & 0.3 & 0.4 & 0.38 & 0. & 0. & 0.62 & 0.54 & 0.28 & 0.34 & 0.42 & 0.30 \\
\hline & Medi & 0.57 & 0.58 & 0.36 & & 0. & $\mathrm{~N}$ & 0.54 & 0.63 & & & 0 & 0.6 & & & & & & 0. & 0. & 0. & & 0.6 & & & & & 0.48 \\
\hline & $95^{\text {th }} \mathrm{pt}$ & 0. & 0. & 0.58 & & 0 & 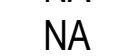 & 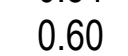 & 0 & 0 & & 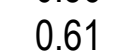 & 0.8 & & & 0.6 & 0 & 0.8 & 0.7 & 1. & 0. & & 0.7 & 0.8 & 0. & 0. & 0.97 & 0.74 \\
\hline Mar 10 & Mean & 0.66 & 0.66 & 0.54 & 0 & 0.54 & $\mathrm{NA}$ & 0.61 & 0.72 & 0.8 & 0.6 & 0.64 & 0.8 & 0. & 0. & 0 & 0.6 & 0.6 & 0.6 & 0.95 & 0.85 & 1. & 0.81 & 0.93 & 0.65 & 0.92 & 0.77 & 0.74 \\
\hline & SD & 0 & 0.06 & 0.19 & & 0.1 & $\mathrm{~N}$ & 0.07 & 0.07 & 0. & 0.1 & 0. & 0.1 & 0.2 & 0. & 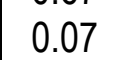 & 0. & 0.2 & 0. & 0.61 & 0 & 0. & 0.0 & 0.2 & 0. & 0. & 0.28 & 0.30 \\
\hline & $5^{\text {th }} \mathrm{p}$ & 0.56 & 0.57 & 0.21 & & 0.3 & $\mathrm{~N}$ & 0.48 & 0.6 & & & 0. & 0.5 & 0.5 & & & 0 & 0.3 & 0. & 0.51 & 0. & & 0.7 & 06 & 0. & 0. & & 0.40 \\
\hline & Median & 0.65 & 0.66 & 0.54 & 0.59 & 0.55 & NA & 0.61 & 0.71 & 0 & 0.6 & 0.63 & 0.7 & 0. & 0. & 0.65 & 0.6 & 0. & 0.6 & 0.71 & 0. & 1. & 0.79 & 0.8 & 0.57 & 0.80 & 0.70 & 0.67 \\
\hline & $95^{\text {th }}$ ptile & 0.78 & 0.78 & 0.90 & 0.8 & 0.69 & NA & 0.74 & 0.86 & 1.06 & 0.8 & 0.76 & 1.21 & 1.2 & 1.7 & 0.8 & 0.92 & 0.9 & 1.0 & 2.34 & 1.8 & 2.44 & 0.99 & 1.59 & 1. & 1. & 1.44 & 1.42 \\
\hline 10 & Mean & 0.77 & 0. & 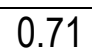 & & 0.7 & $\mathrm{~N}$ & 0.7 & 0.8 & 1. & & 0.7 & 0.6 & 0. & & & & 0. & 0.7 & 0.8 & 0. & & 1.0 & 1. & 0. & 1. & 0.91 & 0.92 \\
\hline & $\mathrm{SD}$ & 0.17 & 0.17 & 0.30 & & 0.22 & NA & 0.19 & 0.20 & & 0. & 0.17 & 0. & 0. & 0. & & 0. & 0. & 0.2 & 0.39 & 0. & 0. & 0.24 & 0.64 & 0.47 & 0. & 0.39 & 0.45 \\
\hline & $5^{\text {th }}$ ptile & 0.58 & 0.59 & 0.38 & 0. & 0.48 & NA & 0.56 & 0.63 & 0.74 & 0.5 & 0.57 & 0.6 & 0.5 & 0.4 & 0.58 & 0.51 & 0.46 & 0.4 & 0.49 & 0.47 & 0.59 & 0.75 & 0.71 & 0.36 & 0.57 & 0.45 & 0.45 \\
\hline & Median & 0.70 & 0.7 & 0.62 & & 0.64 & NA & 0.67 & 0.77 & & 0.7 & 0.69 & 0.8 & 0. & 0. & 0.7 & 0.7 & 0.7 & 0.7 & 0.74 & 0.8 & 1.02 & 0.9 & 1.19 & 0.62 & 1.02 & 0.81 & 0.79 \\
\hline & $95^{\text {th }}$ ptile & 1.12 & 1.13 & 1.34 & 1.73 & 1.19 & NA & 1.14 & 1.23 & 1.43 & 1.30 & 1.09 & 1.59 & 1.59 & 2.03 & 1.12 & 1.25 & 1.32 & 1.25 & 1.71 & 1.91 & 2.47 & 1.48 & 2.65 & 1.69 & 2.50 & 1.69 & 1.81 \\
\hline
\end{tabular}


Table 5. Continuation.

North Panels

East Panels

South Panels

West Panels

\begin{tabular}{|c|c|c|c|c|c|c|c|c|c|c|c|c|c|c|c|c|c|c|c|c|c|c|c|c|c|c|c|c|}
\hline \multirow{3}{*}{ Month } & \multirow{3}{*}{$\begin{array}{l}\text { Descriptive } \\
\text { Statistics }\end{array}$} & \multirow{3}{*}{$\underset{1 \mathrm{stff}}{\ln }$} & \multirow{3}{*}{$\underset{2^{\text {nd } f l}}{\ln }$} & \multirow{3}{*}{ Out } & \multicolumn{5}{|c|}{ North Panels } & \multicolumn{6}{|c|}{ East Panels } & \multicolumn{7}{|c|}{ South Panels } & \multicolumn{6}{|c|}{ West Panels } \\
\hline & & & & & P1-15 & P1-16 & P2-15 & P2-16 & P2-17 & P1-10 & P1-11 & $\mathrm{P} 1-12$ & P1-13 & P1-14 & P2-14 & P1-6 & P1-7 & P1-8 & P1-9 & P2-6 & P2-7 & P2-8 & P1-1 & P1-2 & P1-3 & P1-4 & P1-5 & P2-5 \\
\hline & & & & & Lavout $A$ & Layout A & Layout A & Layout B & Layout B & Layout C & Layout C & Layout A & Layout A & Layout A & Layout A & Layout A & Layout A & Layout B & Layout B & Layout A & Layout B & Layout A & ayout $C$ & Layout C & & Layout A & Layout A & Layout B \\
\hline 10 & Mean & 0.94 & 0.95 & 1.17 & 1.17 & 1.00 & NA & 0.97 & 1.04 & 1.08 & 1.02 & 0.91 & 1.11 & $\frac{1 A R H 1 A}{1.12}$ & $\frac{[1 / A R H 1 A}{1.07}$ & $\frac{1 \text { ANRH1A }}{0.94}$ & $\frac{\text { TIA/RH1A }}{1.07}$ & $\frac{3 B / R H 1 B}{1.10}$ & $\frac{3 B / R H 1 B}{1.00}$ & $\frac{1 \mathrm{ARR} \text { R } 1 \mathrm{~A}}{0.96}$ & $\frac{3 \mathrm{~B} / \mathrm{RH} 1 \mathrm{E}}{110}$ & $\frac{1 \mathrm{ARR} \text { 1A }}{118}$ & $\frac{1 \mathrm{CIRH} 1 \mathrm{C}}{130}$ & 193 & $\frac{3 \mathrm{~B} / \mathrm{RH} 1 \mathrm{~B}}{101}$ & $\begin{array}{l}\text { RH1A } \\
50\end{array}$ & $\frac{1 \mathrm{~A} / \mathrm{RH} 1 \mathrm{~A}}{111}$ & $\frac{\mathrm{RH} 1 \mathrm{E}}{13}$ \\
\hline & SD & 0.17 & 0.18 & 0.43 & 39 & 0.25 & NA & 0.19 & 0.20 & 0.17 & 0.25 & 0.16 & 0.29 & 0.28 & 0.57 & 0.17 & 0.28 & 0.30 & 0.27 & 0.33 & 0.46 & 0.42 & 0.27 & 0.66 & 0 & 0.72 & 0.42 & 0.53 \\
\hline & $5^{\text {th }}$ ptile & 0.63 & 0.64 & 0.52 & 0.58 & 0.55 & NA & 0.65 & 0.6 & 0.76 & 0.6 & 0.63 & 0.68 & 0.66 & 0.4 & 0.6 & 0.63 & 0.63 & 0.59 & 0.57 & 0.47 & 0. & 0.8 & 1. & 0.38 & 0.58 & 0.61 & 0.45 \\
\hline & Median & 0.95 & 0.96 & 1.11 & 1. & 1.02 & NA & 0.98 & 1.0 & 1.08 & 1.0 & 0.9 & 1.06 & 1.0 & 0.8 & 0.9 & 1.0 & 1.10 & 0.98 & 0.84 & 1.01 & 1.0 & 1.3 & 1. & 0.81 & 1.35 & 0.99 & 1.00 \\
\hline & $95^{\text {th }}$ ptile & 1.20 & 1.21 & 1.87 & 1.88 & 1.41 & NA & 1.30 & 1.39 & 1.30 & 1.48 & 1.15 & 1.69 & 1.6 & 2.3 & 1.2 & 1.57 & 1.68 & 1.49 & 1.67 & 2.03 & 2.0 & 1.64 & 3.39 & 2.41 & 3.02 & 2.02 & 2.24 \\
\hline \begin{tabular}{|l|} 
Jun 10 \\
\end{tabular} & Mean & 1.14 & 1.23 & 1.65 & 116 & 1.30 & NA & 1.27 & 1.36 & 123 & 1.28 & 1.17 & 1.37 & 1.36 & 1.2 & 1.23 & 1.45 & 1.58 & 1.38 & 1.26 & 1.21 & 13 & 15 & 2 & 1.22 & 1.50 & 1.35 & 1.26 \\
\hline & SD & 0.15 & 0.22 & 0.43 & $0 .{ }^{2}$ & 0.2 & NA & 0.2 & 0.2 & 0.16 & 0.2 & 0. & 0 & 0.29 & 0. & 0 & 0. & 0. & 0. & 0.36 & 0. & 0.3 & 0 & & 0.58 & 53 & 45 & 0.41 \\
\hline & $5^{\text {th }}$ ptile & 0.91 & 0.91 & 0.93 & 0. & 0.89 & NA & 0.91 & 1.02 & 1.01 & 0.95 & 0.9 & 0.96 & 0.9 & 0.7 & 0. & 1.0 & 1.05 & 0.93 & 0.8 & 0.7 & 0. & 1.2 & 1. & 0. & 0.88 & 0.79 & 0.77 \\
\hline & Median & 1 & 1.22 & 1.64 & & 28 & NA & 1.25 & 1.3 & 1.25 & 1.25 & 1. & 1.3 & 13 & 1. & & 1.4 & 1.49 & & 1. & 1. & & & & & 1.37 & 1.27 & 1.16 \\
\hline & $95^{\text {th }}$ ptile & 1.37 & 1.59 & 2.33 & 2.09 & 1.64 & NA & 1.61 & 1.73 & .50 & 1.67 & 1.49 & 1.96 & 1.89 & 2.3 & 1.58 & 1.94 & 2.25 & 1.92 & 2.00 & 1.83 & 1. & 1.8 & 3.47 & 2.39 & 2.59 & 2.26 & 2.12 \\
\hline 17 & Mean & 1.23 & 1.49 & 1.93 & 1.61 & 1.54 & $\overline{\mathrm{NA}}$ & 1.53 & 1.6 & 1.5 & 1.8 & 1.4 & 1. & 1.6 & 1.5 & 1.5 & 1. & 1.84 & 1.6 & 1.4 & 1.5 & 1. & 1. & 2. & 1.55 & 2.03 & 1.55 & 1.62 \\
\hline & SD & 0.08 & 0.11 & 0.45 & 0. & 0.17 & NA & 0.15 & 0. & 0 & 0 & 0 & 0 & 0. & 0. & 0. & 0. & 0.37 & 0.29 & 0. & 0.4 & 0. & . & 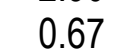 & 0.81 & 0.68 & 15 & 0.53 \\
\hline & $5^{\text {th }} p$ & & & 1. & & 1.14 & $\mathrm{~N}$ & 1 & 1. & & & & & 1.23 & 0 & & 1. & 1 & & & 1.02 & 1 & & & & 7 & 15 & 1.02 \\
\hline & Median & 1.23 & 1.50 & 1.93 & 1.58 & 1.57 & $\mathrm{~N} A$ & 1.55 & 1.6 & 1.57 & 1.83 & 1.43 & 1.56 & 1.60 & 1.5 & 1.5 & 1.7 & 1.77 & 1.61 & 1.30 & 1.4 & 1. & 1. & 2. & 1. & 1.88 & 1.44 & 1.45 \\
\hline & $95^{\text {th }}$ ptile & 1.34 & 1.64 & 2.64 & 2.15 & 1.73 & NA & 1.75 & 1.82 & 1.66 & 2.35 & 1.5 & 2. & 2. & 2. & 1. & 2.1 & 2. & 2.1 & 2.1 & 2.3 & 2. & 1. & 4. & 3.49 & 3.51 & 2.39 & 2.79 \\
\hline 10 & Mean & 1.5 & & 1.82 & & 1.59 & NA & & 1 & & & & & & & & 1. & 1 & & & 1. & 1. & & & & & & 1. \\
\hline & SD & 0.13 & 0.15 & 0.36 & 0. & 0.14 & $\mathrm{~N} /$ & 0.1 & 0. & 0. & 0.3 & 0. & 0 & 0. & 0 & 0. & 0. & 0.32 & 0. & 0. & 0. & 0. & 0. & 0 & 0 & 0.62 & 0.45 & 0.47 \\
\hline & $5^{\text {th }}$ ptile & 1.38 & 1.41 & 1.21 & 1.24 & 1.33 & NA & 1.38 & 1.5 & 1.5 & 1.4 & 1.3 & 1. & 1.3 & 0. & 1. & 1.4 & 1.2 & 1. & 0. & $1 .(\mathrm{C}$ & 1. & 1. & 1. & 0. & 1.31 & 1.04 & 1.03 \\
\hline & Median & 1.5 & 1.58 & 1.81 & 1.64 & 1.60 & NA & 1.63 & 1.72 & 1.67 & 1.8 & 1.5 & 1. & 1.6 & 1. & 1.5 & 1.7 & 1.7 & 1.5 & 1. & 1.4 & 1. & 1. & 2. & 1. & 1.73 & 1.55 & 1.40 \\
\hline & $95^{\text {th }}$ & 1.88 & 1.94 & 2.43 & 2.42 & 1.83 & NA & 1.92 & 1.99 & 1.75 & 2.47 & $1.6 s$ & 2.39 & 2.22 & 2.80 & $1.7 \xi$ & 2.07 & 2.29 & 2.09 & 2.32 & 2.49 & 2.47 & 1.9 & 3.9 & 3.10 & 3.27 & 2.53 & 54 \\
\hline
\end{tabular}

Notes: Layouts A, B, C and D refer to the location of the sensors per Figures 25 through 28.

Abbreviations: NA, not available; ptile, percentile; SD, standard deviation. 
Table 6. Monthly heat flux at interior side of stud cavity; data was obtained by averaging measurements from two transducers. Refer to Figures 25 through 28 for sensor location. North Panels East Panels South Panels

West Panels

\begin{tabular}{|c|c|c|c|c|c|c|c|c|c|c|c|c|c|c|c|c|c|c|c|c|c|c|c|c|c|}
\hline \multirow{3}{*}{ Month } & \multirow{3}{*}{$\begin{array}{l}\text { Descriptive } \\
\text { Statistics }\end{array}$} & \multicolumn{5}{|c|}{ North Panels } & \multicolumn{6}{|c|}{ East Panels } & \multicolumn{7}{|c|}{ South Panels } & \multicolumn{6}{|c|}{ West Panels } \\
\hline & & -15 & P1-16 & P2-15 & P2-16 & P2-17 & $1-10$ & $1-11$ & P1-12 & P1-13 & P1-14 & P2-14 & P1-6 & P1-7 & P1-8 & P1-9 & $\mathrm{P} 2-6$ & P2-7 & P2-8 & P1-1 & P1-2 & P1-3 & P1-4 & P1-5 & P2-5 \\
\hline & & Layout A & Layout A & Layout A & Layout B & Layout B & Layout C & Layout C & Layout A & Layout A & Layout A & Layout A & Layout A & Layout A & Layout B & Layout B & Layout A & Layout B & Layout A & Layout C & Layout D & Layout B & Layout A & Layout A & Layout B \\
\hline \multirow[t]{5}{*}{ ep 09} & Mean & $\mid-1.21$ & -1.25 & -1.17 & -1.56 & -1.56 & -0.50 & -1.24 & -0.62 & -0.32 & -0.34 & -0.52 & 0.61 & 0.25 & 0.24 & 0.17 & -0.18 & -0.37 & -0.85 & -0.13 & -1.6 & -0.39 & -0.29 & -0.26 & -0.70 \\
\hline & & 1.09 & 0.80 & 0.90 & 0.71 & 0.72 & 0.93 & 2.51 & 1.61 & 1.14 & 1.02 & 1.53 & 2.40 & 1.14 & 1.59 & 1.50 & 2.40 & 1.63 & 1.43 & 1.12 & 12.1 & 1.80 & 2.07 & 1.61 & 1.67 \\
\hline & $5^{\text {th }}$ ptile & -2.89 & -2.52 & -2.58 & -2.77 & -2.75 & -2.43 & -5.86 & -3.27 & -2.06 & -1.92 & -2.37 & -2.97 & -1.52 & -2.00 & -1.98 & -2.60 & -2.25 & -2.58 & -2.23 & -18.3 & -2.67 & -2.63 & -2.31 & -2.69 \\
\hline & Median & -1.27 & -1.24 & -1.27 & -1.56 & -1.55 & -0.26 & -1.01 & -0.74 & -0.49 & -0.52 & -0.88 & 0.14 & 0.14 & -0.11 & -0.18 & -1.08 & -0.86 & -1.21 & 0.02 & -4.0 & -0.78 & -0.87 & -0.62 & -1.13 \\
\hline & 95 th ptil & 0.66 & -0.01 & 0.29 & -0.52 & -0.52 & 0.63 & 2.33 & 2.18 & 1.81 & 1.49 & 2.44 & 5.12 & 2.31 & 3.18 & 2.91 & 5.09 & 2.93 & 2.07 & 1.49 & 22.7 & 3.28 & 3.91 & 2.95 & 2.65 \\
\hline \multirow[t]{5}{*}{ Cct 09} & Mean & -2.64 & -3.05 & -2.44 & -3.01 & -3.06 & -2 & -7.67 & -3.29 & -1 & -1.83 & -1.96 & -2.14 & -1 & -1.41 & -1.42 & -1.73 & -1.77 & -2.18 & -2.40 & -16.3 & & 2.23 & -1.95 & -2.34 \\
\hline & $\mathrm{SL}$ & 0.95 & 1.08 & 0.79 & 0.90 & 0.93 & 0.90 & 2.73 & 1.41 & 0.9 & 0.7 & 1.08 & 2.23 & 1.14 & 1.46 & 1.3 & 2.01 & 1.27 & 1.15 & 0.84 & 8.90 & 1.25 & 1.33 & 1.04 & 1.00 \\
\hline & $5^{\text {th }}$ ptile & -4.24 & -4.87 & -3.80 & -4.50 & -4.64 & -4.50 & -12.3 & -5.58 & -3.32 & -3.02 & -3.56 & -4.88 & -2.69 & -3.11 & -3.07 & -3.72 & -3.37 & -3.82 & -3.81 & -29.5 & -4.25 & -3.96 & -3.31 & -3.75 \\
\hline & Median & -2.58 & -2.92 & -2.42 & -2.94 & -2.96 & -2.84 & -7.52 & -3.27 & -2.01 & -1.87 & -2.05 & -2.64 & -1.43 & -1.75 & -1.75 & -2.25 & -1.99 & -2.29 & 38 & -16.9 & -2.49 & -2.43 & -2.10 & -2.41 \\
\hline & $95^{\text {th }}$ ptile & -0.97 & -1.40 & -1.10 & -1.58 & -1.63 & -1.49 & -3.41 & -0.77 & -0.30 & -0.41 & 0.02 & 2.75 & 1.30 & 1.94 & 1.82 & 3.20 & 1.20 & 0.25 & -1.05 & 0.4 & -0.13 & 0.22 & -0.01 & -0.56 \\
\hline \multirow[t]{5}{*}{ Nov 09} & Mean & -3.32 & -3.92 & -3.02 & -3.70 & -3.77 & -3.60 & -9.65 & -4.16 & -2.46 & -2.28 & -2.49 & -2.79 & -1.5 & -1.78 & -1.81 & -2.20 & -2.17 & -2 & -3.12 & -21.9 & 9 & -2.90 & -2.49 & -2.91 \\
\hline & $\mathrm{SL}$ & 1.02 & 1.21 & 0.83 & 0.95 & 0.99 & 0.86 & 2.81 & 1.48 & 0.9 & 0.7 & 1.0 & 2.44 & 1.25 & 1.58 & 1.52 & 2.1 & 1.4 & 1. & 0.81 & 8.85 & & & 0 & 0.99 \\
\hline & ile & -5.02 & -5.83 & -4.43 & -5.22 & -5.33 & -4.86 & -14.03 & -6.46 & -3.74 & -3.33 & -4.00 & -5.80 & -3.11 & -3.64 & -3.57 & -4.31 & -3.83 & -4.39 & -4.78 & -34.7 & -5.09 & -4.69 & -3.76 & -4.28 \\
\hline & Median & -3.19 & -3.81 & -2.91 & -3.66 & -3.73 & -3.66 & -9.54 & -4.17 & -2.5 & -2.3 & -2.54 & -3.23 & -1.82 & -2.14 & -2.12 & -2.73 & -2.44 & -2.77 & -3.11 & -21.7 & -3.15 & -3.02 & -2.61 & -2.92 \\
\hline & 9 & -1.65 & -2.12 & -1.69 & -2.27 & -2.27 & -2.09 & -4.57 & -1.64 & -0.8 & -0.8 & -0.50 & 2.29 & $0 . c$ & 1.53 & 1.41 & 2.91 & 1.23 & -0.04 & 01 & -5.5 & $.9 L$ & -0.48 & -0.67 & -1.16 \\
\hline \multirow[t]{5}{*}{ Dec 09} & Mean & -5.05 & -6.58 & -4.53 & -5.55 & -5.78 & -6.24 & -16.8 & -7.09 & -4.06 & -3.59 & -4.15 & -5.94 & -3.29 & -3.66 & -3.68 & -3.98 & -3.84 & -4 & -5.54 & -37.7 & 32 & 2 & -3.88 & -4.37 \\
\hline & S & 1.26 & 2.36 & 1.03 & 1.35 & 1.44 & 1.27 & 3.52 & 1.62 & & 0.7 & 1. & 1. & 0 & 1.15 & 1.1 & 1.5 & 1.10 & 1. & & 9.69 & & & & 0.89 \\
\hline & $5^{\text {th }}$ ptile & -7.10 & -10.32 & -6.27 & -7.66 & -8.1 & -8.22 & -22.5 & -9.71 & -5.6 & -4.7 & -5.8 & -8.63 & -4.7 & -5.34 & -5.29 & -5.82 & -5.46 & $-6 .(1$ & 7.98 & -54.0 & 7 & .69 & -5.37 & -5.74 \\
\hline & Median & -4.92 & -6.06 & -4.48 & -5.39 & -5.60 & -6.19 & -16.9 & -7.06 & -4.00 & -3.55 & -4.13 & -6.14 & -3.32 & -3.74 & -3.78 & -4.19 & -3.89 & -4.32 & -5.34 & -37.0 & -5.19 & -4.62 & -3.78 & -4.32 \\
\hline & 95 & .10 & -3.61 & -2.90 & -3.54 & -3.64 & -4.00 & -10.8 & -4.22 & -2.5 & -2.3 & -2 & -2.46 & -1 & -1.55 & -1.61 & -1.35 & -1.80 & -2.39 & -3.39 & -22.8 & -3.11 & -2.87 & -2.54 & -3.00 \\
\hline \multirow[t]{5}{*}{ Jan 10} & Mear & -5.44 & -7.37 & -4.87 & -6.00 & -6.2 & -6.85 & -19.1 & -7.67 & -4.2 & -3.7 & -4. & -6 & -3 & -3.84 & -3.5 & -4 & -4 & -4 & 2 & -44 & -5.75 & -5.08 & -4.12 & -4.60 \\
\hline & $\mathrm{SL}$ & 1. & 2.42 & 1.11 & 1.41 & 1.52 & 1.29 & 3.83 & 1.76 & 0.6 & 0.7 & 1. & 2.37 & 1. & 1.46 & 1.4 & 2.1 & 1.3 & 1.2 & 63 & 11.95 & 1.54 & 1. & 1. & 0.96 \\
\hline & $5^{\text {th }}$ & 87 & -11.67 & -6.75 & -8.51 & -9.00 & -8.88 & -25.6 & -10.51 & & & -6 & & & -5.78 & & -6. & -5 & -6.44 & & 3.3 & & & -5.77 & -6.07 \\
\hline & $50^{\text {th }}$ & 34 & -6.91 & -4.85 & -5.94 & -6.1 & 86 & -19.2 & -7.69 & -4 & -3.7 & -4 & -6.68 & -3 & -3.99 & -4.0 & -4.45 & -4 & -4 . & 05 & -43.5 & -5.65 & -5.02 & -4.06 & -4.63 \\
\hline & $95^{\text {th }}$ & -3.33 & -4.37 & -3.17 & -3.97 & -4.09 & -4.90 & -13.4 & -4.88 & -2.5 & -2.4 & -2.5 & -1.49 & -0 & -0.95 & -1.13 & -1.77 & -1.97 & -2 & 3.97 & -24.6 & -3.05 & -2.74 & -2.40 & -2.82 \\
\hline \multirow[t]{5}{*}{ Feb 10} & & -5.07 & -7.05 & -4 & -5.77 & -6 & & $-1 \varepsilon$ & -7 & -3 & & & & & -3 & -3 . & & & & & & & & & -4.52 \\
\hline & & 0. & 1.54 & 0. & 0.84 & 0. & 0.83 & 2. & 1.27 & 0.7 & 0.6 & 0. & 1.71 & $0 . \varepsilon$ & 1.05 & 1. & 1. & 0. & 0. & 1.01 & 8.98 & 1.0 & 1.04 & 0. & 0.67 \\
\hline & $5^{\text {th }}$ ptile & -6.63 & -9.80 & -5.84 & -7.01 & -7.40 & -7.87 & -22.0 & -9.10 & -5.0 & -4.48 & -5.48 & -8.23 & -4.4 & -4.94 & -4.9 & -5.57 & -5.16 & -5.68 & 7.69 & -54.8 & -6.97 & -6.30 & -4.99 & -5.41 \\
\hline & & & -6.99 & -4.66 & -5.79 & -6. & & -18.1 & -7.31 & -4 & -3 & -4 & & & -3 & -3. & -4 & -4 & -4 & -6.21 & -42.6 & & & -4.08 & -4.62 \\
\hline & 9 & -3.40 & -448 & -3.3 & -4.32 & -4.5 & -5.02 & -14.2 & -484 & -2 & 2 & -2 & -2.79 & -1.8 & -1.69 & -1. & -1. & -2 & -3.0 & -4.13 & -26 & 0 & -2 & -264 & -3.29 \\
\hline \multirow[t]{5}{*}{ Mar 10} & Mean & -3.74 & -5.14 & -3.48 & -4.50 & -4.64 & -4.16 & $\begin{array}{l}-11.5 \\
\end{array}$ & -4.56 & -2.4 & -2. & -2.74 & -3.01 & -1 & -1.86 & -1.89 & -2.37 & -2.45 & -3.03 & -3.72 & -26.8 & -3.52 & -3.10 & -2.65 & \begin{tabular}{|c|}
-3.26 \\
\end{tabular} \\
\hline & & & 1.69 & 0.8 & 0.8 & 1.0 & & 2.9 & 1.71 & 1. & 0. & 1. & 2.6 & 1. & 1.66 & 1.6 & 2.3 & 1. & 1. & & 12.1 & & & & 1.11 \\
\hline & $5 t$ & 5 & -8.29 & -4.94 & -6.54 & -6.7 & -5 & 16 & -7.35 & & & -4 . & $6>0$ & & -384 & & -4 & -4 . & -4 & & -45 & & -5 & & -4.80 \\
\hline & Median & -3.68 & -4.73 & -3.45 & -4.29 & -4.45 & -4.10 & -11.6 & -4.71 & -2.5 & -2.4 & -2.94 & -3.79 & -2.20 & -2.44 & -2.47 & -3.19 & -2.76 & -3.24 & -3.64 & -28.2 & -3.68 & -3.32 & -2.79 & -3.45 \\
\hline & $95^{\text {th }} \mathrm{ptil}$ & -2.21 & -3.19 & -2.36 & -3.22 & -3.34 & $-2 . \varepsilon$ & -6.5 & -1.22 & -0.3 & -0. & 0.4 & 2.49 & 0. & 1.8 & 1.6 & 3.37 & 1. & -0.15 & -2.12 & -0.7 & -0. & 0.33 & -0.15 & -0.89 \\
\hline \multirow[t]{5}{*}{ Apr 10} & & & -3.21 & -2.35 & -3.0 & -3. & -2 . & -6.08 & $-2.4 \varepsilon$ & -1.2 & 1. & -1 & & & -1 & -1 & -1. & -1. & -2 & 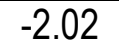 & -1 & & -1. & & -2. \\
\hline & S & 1.24 & 1.36 & 1.02 & 0.96 & 1.00 & 1.34 & 3.13 & 1.88 & 1.1 & 1.0 & 1. & 2.23 & 1. & 1.5 & 1. & 2. & 1. & 1. & 1. & 15.0 & 1. & 2.02 & & 1.56 \\
\hline & $5^{\text {th }}$ ptile & -4.52 & -5.80 & -4.12 & -4.82 & -4.89 & -4.51 & -11.61 & -5.43 & -2.9 & -2.8 & -3.8 & -4.72 & -2.56 & -3.0 & -3.0 & -3.92 & -3.59 & -4 . & -4.77 & -35.2 & -4.60 & -4.12 & -3.48 & -4.22 \\
\hline & & -2.54 & -2.99 & -2.44 & -2.88 & -2.91 & -2.09 & -5.74 & -2.58 & -1.6 & -1.6 & -1.9 & -1.99 & -1. & -1.28 & -1.32 & -2.20 & -1.91 & -2 & -1.97 & -16.4 & -2.34 & -2.24 & -1.91 & -2.34 \\
\hline & $95^{\text {th }}$ ptile & -0.24 & -1.27 & -0.55 & -1.66 & -1.74 & -0.21 & -1.26 & 0.97 & 0.87 & 0.71 & 2.10 & 2.63 & 1.22 & 1.99 & 1.85 & 2.92 & 1.47 & 0.60 & 0.19 & 16.7 & 1.98 & 2.53 & 1.77 & 1.33 \\
\hline
\end{tabular}


Table 6. Continuation.

\begin{tabular}{|c|c|c|c|c|c|c|c|c|c|c|c|c|c|c|c|c|c|c|c|c|c|c|c|c|c|}
\hline \multirow{3}{*}{ Month } & \multirow{3}{*}{$\begin{array}{l}\text { Descriptive } \\
\text { Statistics }\end{array}$} & \multicolumn{5}{|c|}{ North Panels } & \multicolumn{6}{|c|}{ East Panels } & \multicolumn{7}{|c|}{ South Panels } & \multicolumn{6}{|c|}{ West Panels } \\
\hline & & $51-15$ & $\mathrm{P} 1-16$ & P2-15 & P2-16 & P2-17 & $\mathrm{P} 1-10$ & P1-11 & P1-12 & P1-13 & P1-14 & P2-14 & $\mathrm{P} 1-6$ & P1-7 & P1-8 & P1-9 & P2-6 & P2-7 & P2-8 & P1-1 & $\mathrm{P} 1-2$ & P1-3 & P1-4 & P1-5 & P2-5 \\
\hline & & Layout A & Layout A & Layout A & Layout B & Layout B & Layout C & Layout C & Layout A & Layout A & Layout A & Layout A & Layout A & Layout A & Layout B & Layout B & Layout $\mathrm{A}$ & Layout B & Layout A & Layout C & Layout C & Layout B & Layout A & Layout A & Layout B \\
\hline \multirow[t]{5}{*}{ May 10} & Mean & -0.85 & -0.98 & -0.88 & -1.28 & -1.28 & -0.38 & -0.6 & -0.24 & -0.11 & -0.06 & -0.10 & 0.21 & 0.04 & 0.07 & 0.04 & -0.35 & -0.48 & -0.86 & -0.11 & -0.7 & -0.25 & -0.17 & -0.16 & -0.55 \\
\hline & SD & 1.55 & 1.62 & 1.32 & 1.27 & 1.25 & 1.59 & 4.47 & 2.39 & 1.48 & 1.42 & 1.97 & 2.44 & 1.20 & 1.53 & 1.51 & 2.03 & 1.51 & 1.41 & 1.72 & 18.2 & 2.24 & 2.62 & 1.92 & 1.94 \\
\hline & $5^{\text {th }}$ ptile & -3.23 & -3.77 & -2.82 & -3.32 & -3.39 & -2.71 & -7.6 & -3.66 & -2.36 & -2.14 & -2.53 & -3.27 & -1.85 & -2.26 & -2.30 & -2.84 & -2.50 & -2.88 & -2.94 & -26.4 & -3.35 & -2.97 & -2.68 & -3.04 \\
\hline & Median & -1.08 & -1.01 & -1.05 & -1.45 & -1.43 & -0.55 & -1.1 & -0.59 & -0.30 & -0.26 & -0.64 & -0.11 & -0.04 & -0.14 & -0.15 & -0.97 & -0.78 & -1.10 & -0.27 & -3.9 & -0.70 & -0.97 & -0.57 & -1.02 \\
\hline & $95^{\text {th }}$ ptile & 1.85 & 1.53 & 1.57 & 0.85 & 0.73 & 2.23 & 7.3 & 4.55 & 2.73 & 2.70 & 3.80 & 4.65 & 2.12 & 2.81 & 2.68 & 3.64 & 2.46 & 1.88 & 2.65 & 36.1 & 4.31 & 5.90 & 3.70 & 3.35 \\
\hline \multirow[t]{5}{*}{ Jun 10} & Mean & 0.08 & 0.24 & -0.08 & -0.26 & -0.24 & 0.83 & 2.05 & 0.81 & 0.49 & 0.54 & 0.4 & 0.9 & 0.44 & 0.50 & 0.50 & 0.22 & 0.6 & -0.19 & 1.28 & 7.0 & 0.75 & 6 & 58 & 0.24 \\
\hline & & 1.25 & 1.20 & 1.05 & 1.06 & 1.06 & 1.24 & 3.42 & 1.95 & 1.19 & 1.15 & 1.53 & 1.90 & 0.90 & 1.15 & 1.13 & 1.51 & 1.08 & 1.08 & 1.35 & 15.4 & 1.90 & 2.36 & 1.65 & 1.61 \\
\hline & $5^{\text {th }}$ ptile & -2.07 & -2.15 & -1.91 & -2.25 & -2.24 & -1.33 & -3.81 & -2.39 & -1.33 & -1.25 & -1.58 & -2.12 & -1.01 & -1.34 & -1.33 & -1.97 & -1.71 & -2.01 & -1.11 & -14.1 & -1.97 & -1.95 & -1.71 & -2.04 \\
\hline & Median & -0.01 & 0.27 & -0.09 & -0.20 & -0.18 & 0.89 & 1.91 & 0.67 & 0.38 & 0.43 & 0.25 & 0.82 & 0.39 & 0.40 & 0.44 & -0.04 & -0.08 & -0.26 & 1.35 & 3.9 & 0.43 & -0.02 & 0.25 & -0.02 \\
\hline & $95^{\text {th }}$ ptile & 2.12 & 2.12 & 1.69 & 1.45 & 1.55 & 3.12 & 8.24 & 4.56 & 2.78 & 2.74 & 3.21 & 4.37 & 1.98 & 2.54 & 2.48 & 3.14 & 2.10 & 1.76 & 3.46 & 36.3 & 4.30 & 5.41 & 3.69 & 3.16 \\
\hline \multirow[t]{5}{*}{ Jul 10} & Mean & 0.57 & 0.77 & 0.37 & 0.29 & 0.25 & 1.65 & 1.7 & 1.82 & 1.11 & 1.15 & 1.09 & 2.13 & 1.00 & 1.15 & 1.16 & 0.82 & 0.57 & 0.29 & 1.87 & 13.1 & 1.46 & 40 & 1.25 & 0.86 \\
\hline & & 1 & 1.12 & 1.08 & 1.01 & 0.97 & 1.0 & 1.03 & 2.01 & 1.2 & 1.20 & 1.72 & 2.0 & 0.9 & 1.26 & 1.2 & 1.78 & 1.24 & 1.17 & 1.14 & 17.3 & 2.10 & 3.37 & 1.86 & 1.83 \\
\hline & $5^{\text {th }}$ ptile & -1.53 & -1.22 & -1.41 & -1.68 & -1.67 & -0.26 & -0.3 & -1.30 & -0.82 & -0.67 & -1.17 & -1.12 & -0.55 & -0.86 & -0.80 & -1.69 & -1.36 & -1.65 & -0.46 & -9.09 & -1.27 & -2.12 & -1.10 & -1.54 \\
\hline & Median & 0.49 & 0.76 & 0.24 & 0.31 & 0.25 & 1.74 & 1.7 & 1.62 & 0.96 & 1.02 & 0.75 & 1.93 & 0.97 & 1.01 & 1.05 & 0.39 & 0.34 & 0.15 & 1.95 & 8.41 & 0.99 & 0.33 & 0.75 & 0.42 \\
\hline & $95^{\text {th }}$ ptile & 2.59 & 2.55 & 2.25 & 2.01 & 1.79 & 3.54 & 3.5 & 5.32 & 3.29 & 3.15 & 4.37 & 5.68 & 2.54 & 3.31 & 3.18 & 4.19 & 2.72 & 2.31 & 3.61 & 47.1 & 5.77 & 8.97 & 4.96 & 4.68 \\
\hline \multirow[t]{5}{*}{ Aug 10} & Mean & -0.03 & -0.01 & -0.16 & -0.32 & -0.31 & 1.01 & 1.0 & 1.05 & 0.59 & 0.56 & 0.46 & 1.72 & 0.76 & 0.81 & 0.88 & 0.57 & 0.33 & 0.00 & 1.01 & 7.0 & 0.76 & 0.76 & 0.62 & 0.25 \\
\hline & SD & 1.18 & 0.98 & 0.94 & 0.83 & 0.85 & 0.96 & 0.96 & 1.86 & 1.12 & 1.03 & 1.46 & 2.27 & 0.98 & 1.26 & 1.30 & 1.95 & 1.34 & 1.20 & 0.95 & 15.1 & 1.87 & 2.80 & 1.61 & 1.57 \\
\hline & $5^{\text {th }}$ ptile & -1.94 & -1.76 & -1.67 & -1.84 & -1.80 & -0.77 & -0.8 & -1.96 & -1.24 & -1.08 & -1.52 & -1.55 & -0.71 & -1.04 & -0.96 & -1.81 & -1.38 & -1.77 & -0.74 & -12.1 & -1.63 & -2.12 & -1.36 & -1.73 \\
\hline & Median & -0.08 & 0.07 & -0.19 & -0.24 & -0.24 & 1.09 & 1.1 & 0.89 & 0.47 & 0.46 & 0.14 & 1.40 & 0.65 & 0.60 & 0.63 & 0.00 & 0.03 & -0.16 & 1.05 & 2.92 & 0.35 & -0.06 & 0.21 & -0.05 \\
\hline & $95^{\text {th }}$ ptile & 1.89 & 1.52 & 1.53 & 0.97 & 1.00 & 2.49 & 2.5 & 4.38 & 2.62 & 2.42 & 3.42 & 5.94 & 2.46 & 3.17 & 3.20 & 4.47 & 2.86 & 2.21 & 2.46 & 38.7 & 4.69 & 7.04 & 3.89 & 3.47 \\
\hline
\end{tabular}

Notes: Layouts A, B, C and D refer to the location of the sensors per Figures 25 through 28 .

Abbreviations: NA, not available; ptile, percentile; SD, standard deviation. 


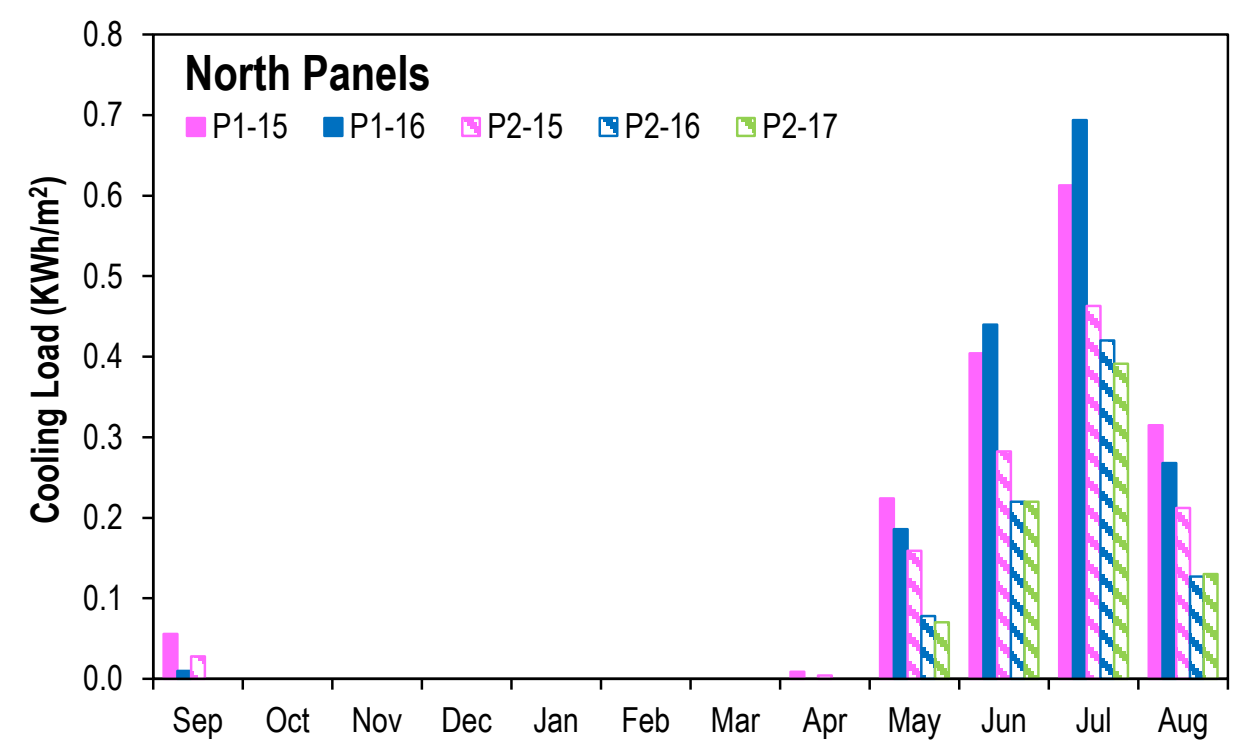

Figure 29. Monthly cooling loads from panels facing north.

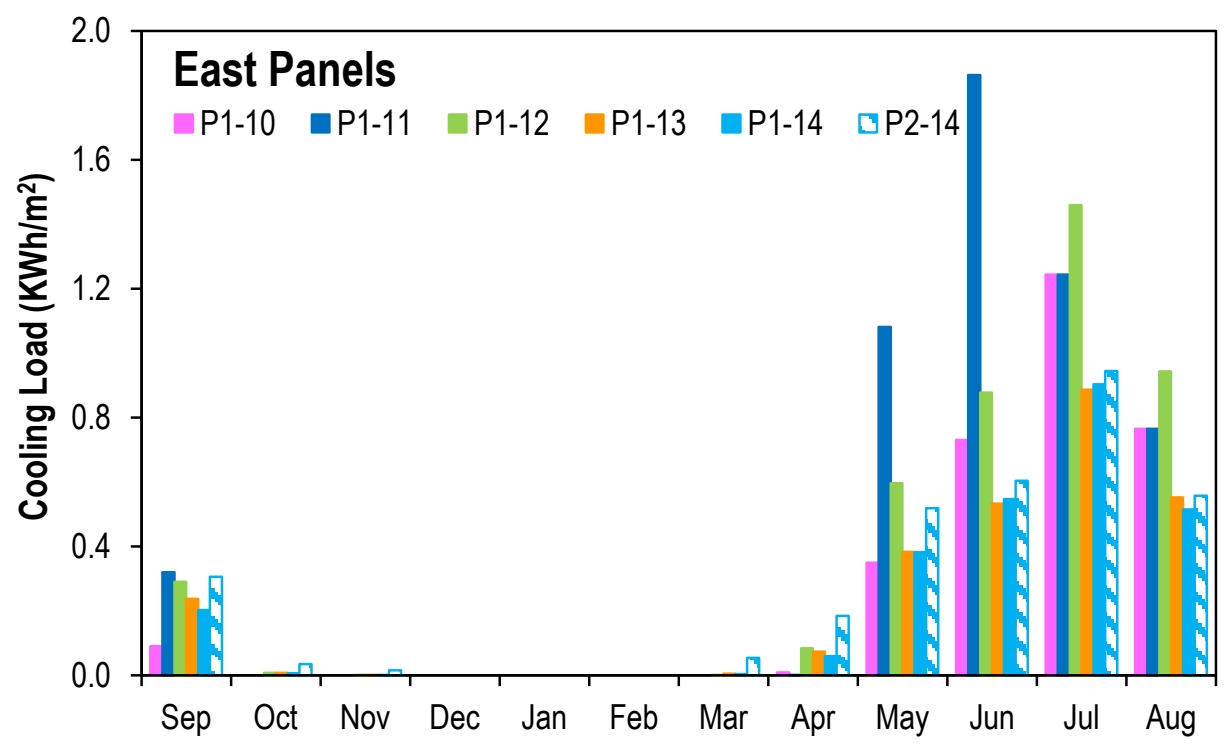

Figure 30. Monthly cooling loads from panels facing east. 


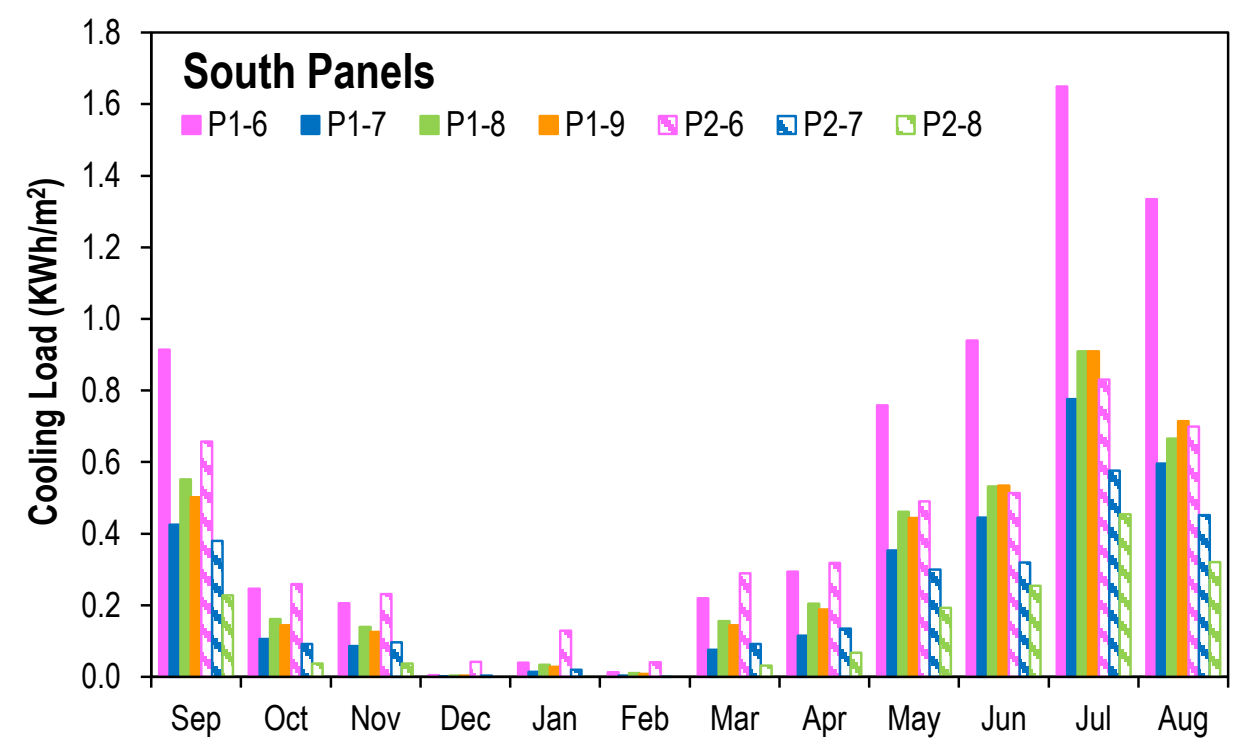

Figure 31. Monthly cooling loads from panels facing south.

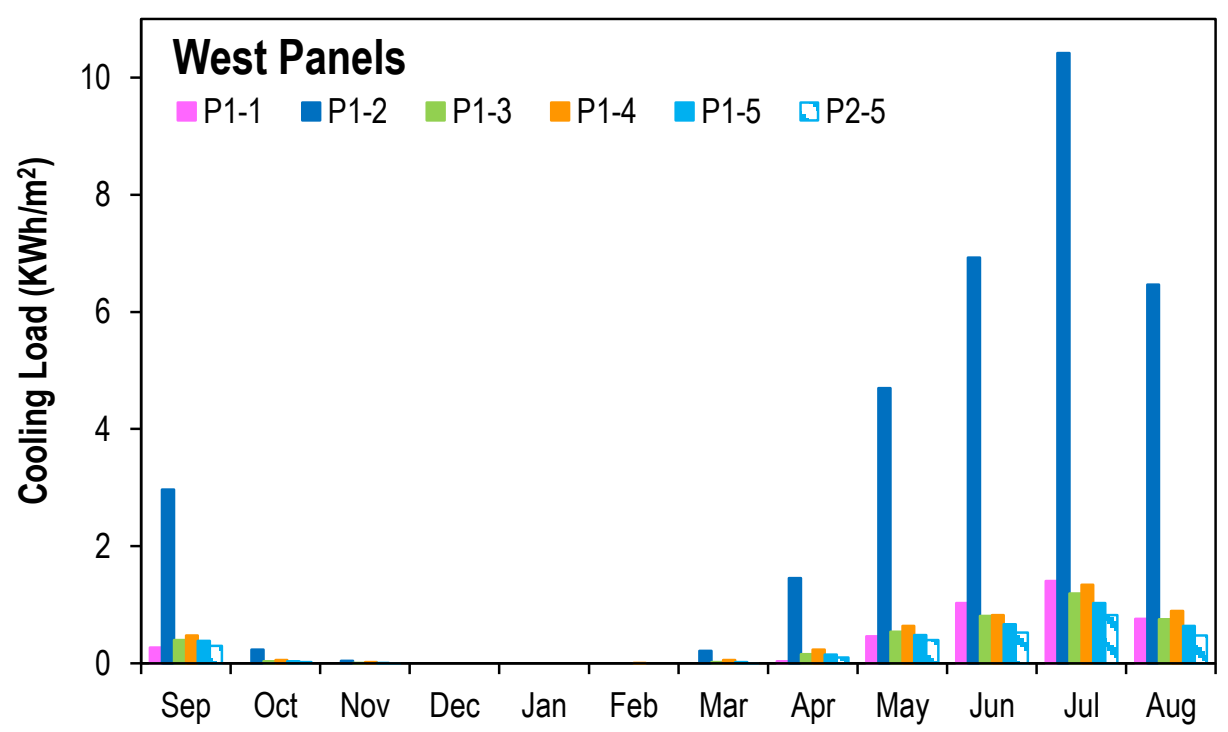

Figure 32. Monthly cooling loads from panels facing west. 


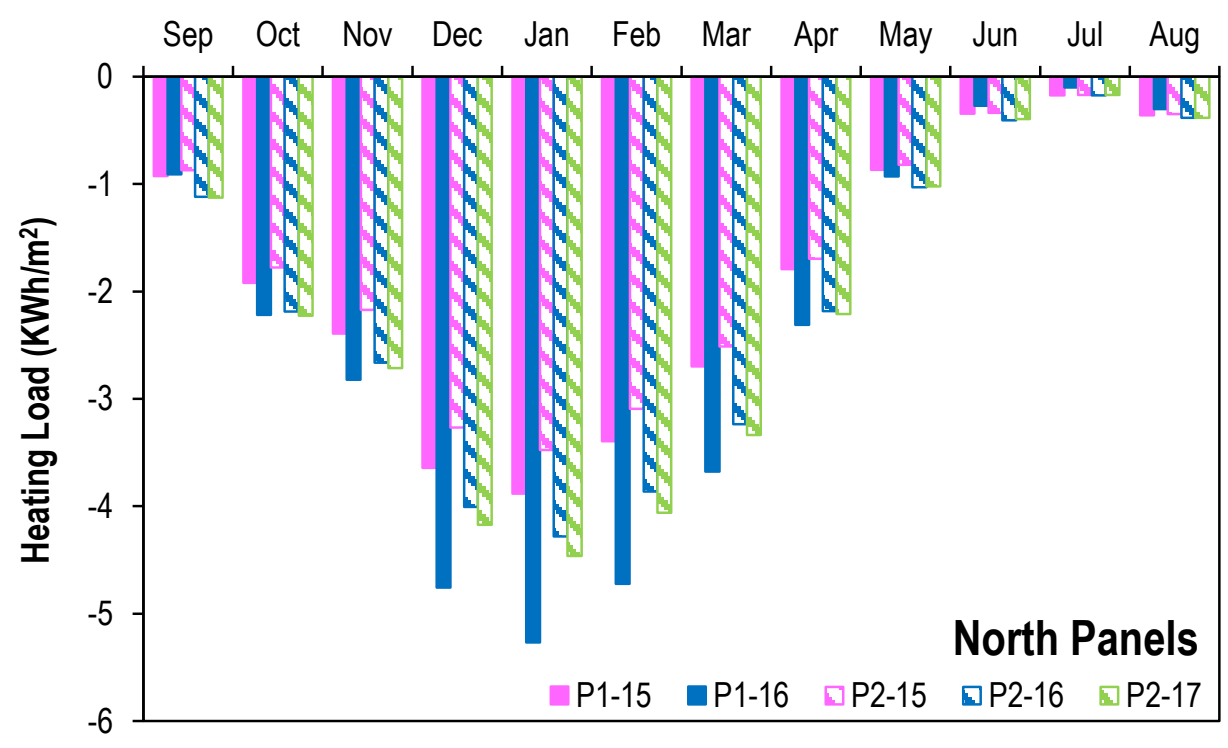

Figure 33. Monthly heating loads from panels facing north.

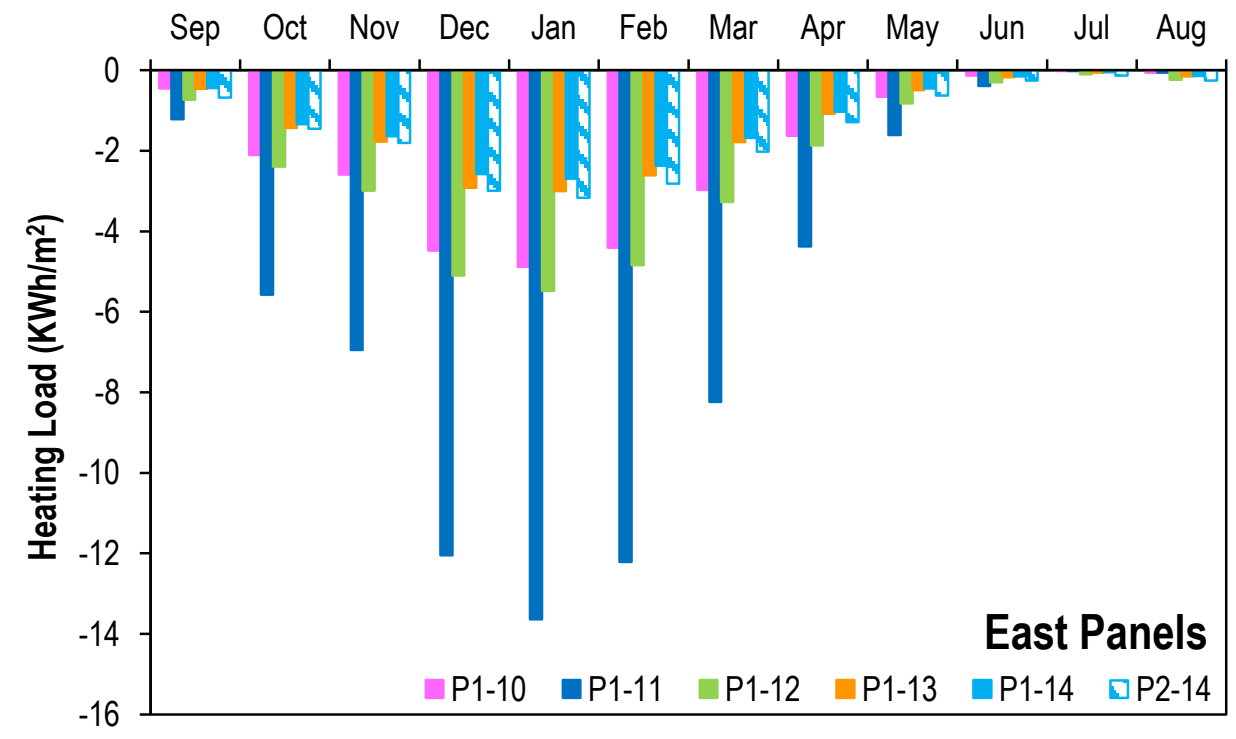

Figure 34. Monthly heating loads from panels facing east. 


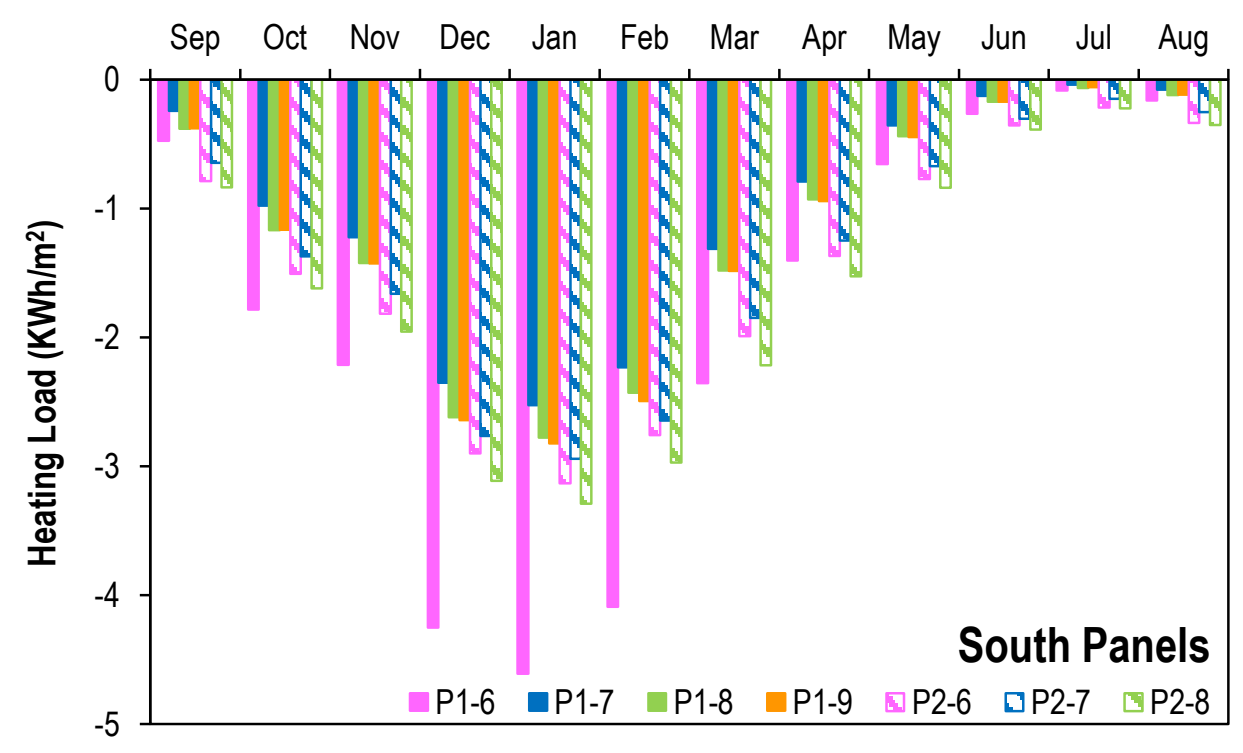

Figure 35. Monthly heating loads from panels facing south.

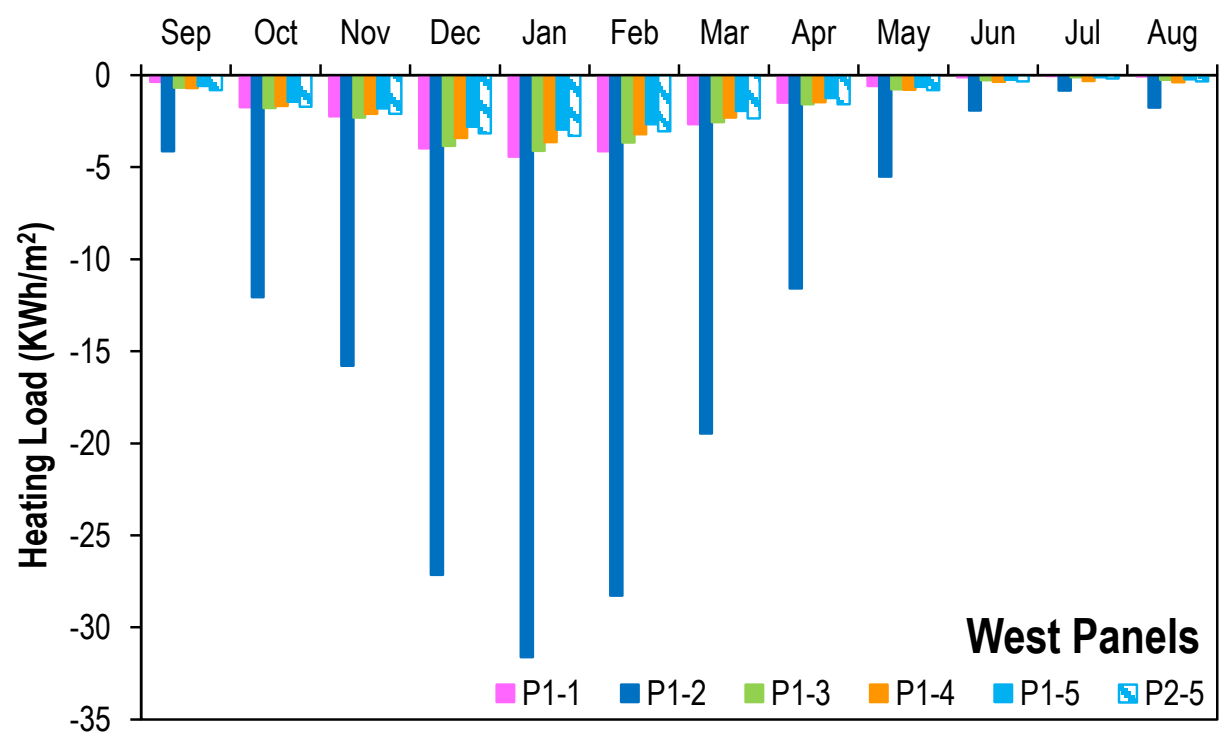

Figure 36. Monthly heating loads from panels facing west. 

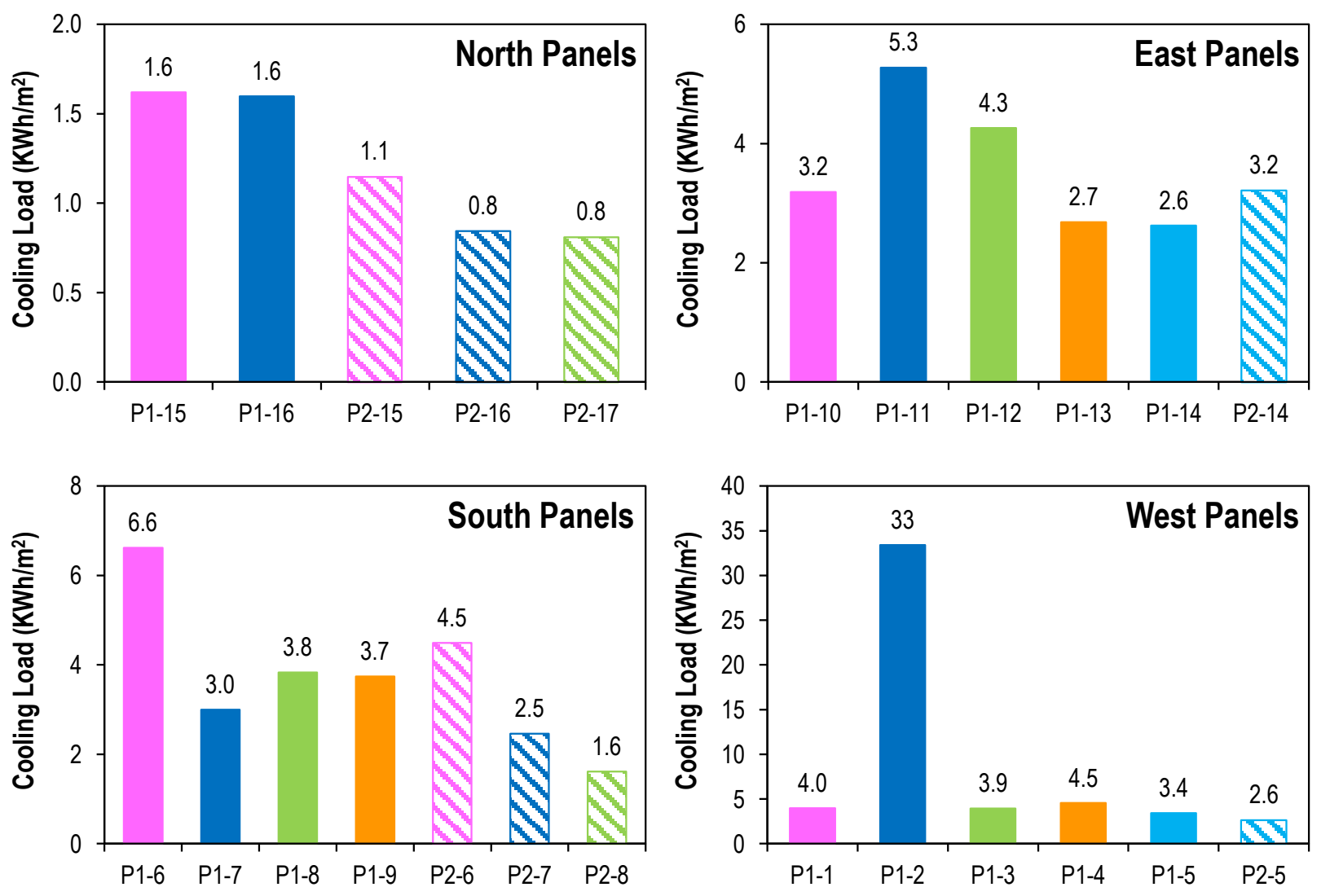

Figure 37. Annual cooling loads. 

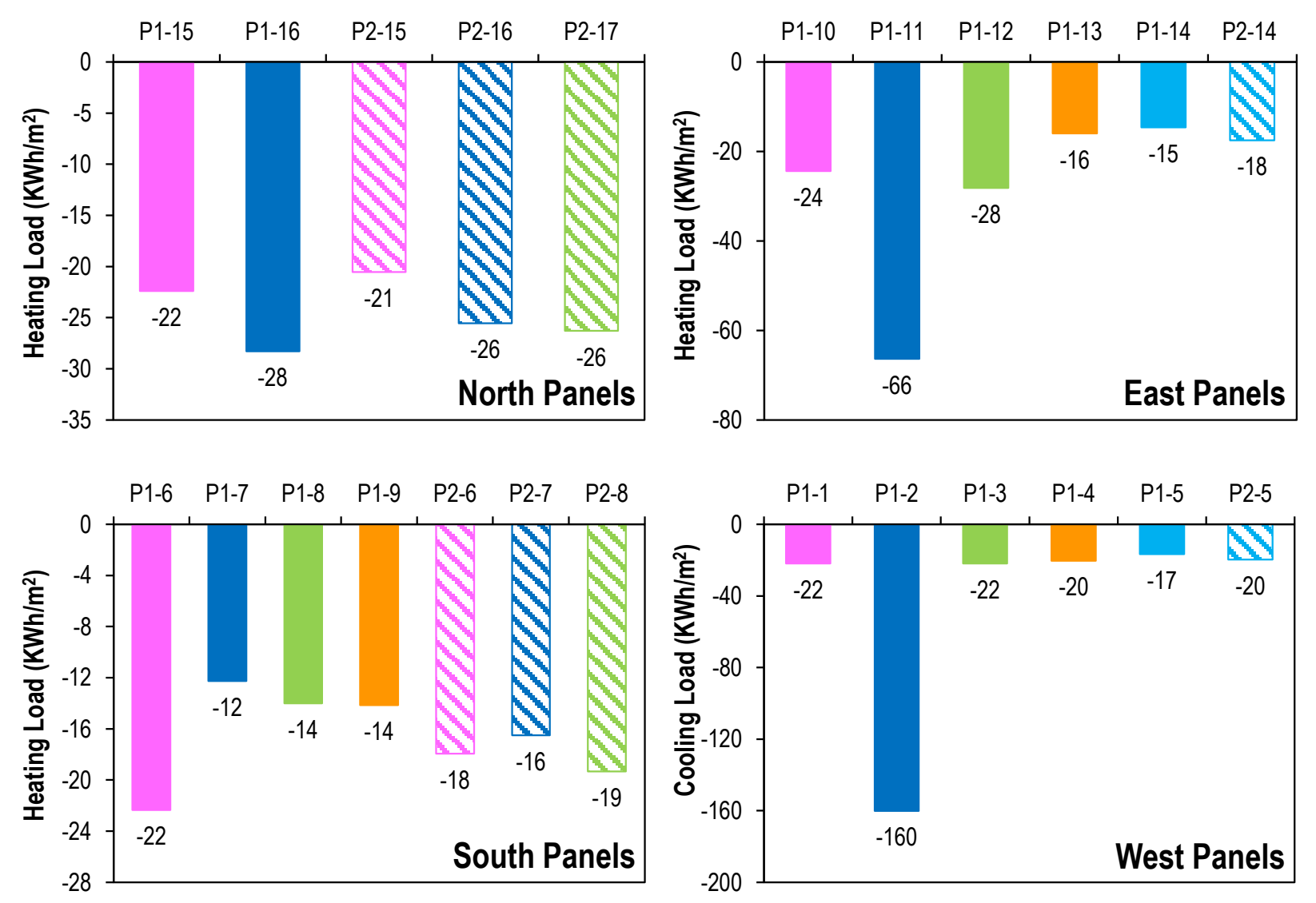

Figure 38. Annual heating loads.

Table 7. Monthly total solar insolation $\left(\mathrm{kW} / \mathrm{m}^{2}\right)$ on each side of the BEST lab.

\begin{tabular}{|c|cccc|}
\hline Month & East & North & South & West \\
\hline Sep 09 & 34 & 13 & 56 & 37 \\
Oct 09 & 28 & 13 & 62 & 30 \\
Nov 09 & 33 & 12 & 81 & 30 \\
Dec 09 & 23 & 13 & 60 & 24 \\
Jan 10 & 29 & 20 & 64 & 32 \\
Feb 10 & 31 & 27 & 56 & 37 \\
Mar 10 & 65 & 27 & 116 & 67 \\
Apr 10 & 73 & 29 & 104 & 84 \\
May 10 & 82 & 46 & 94 & 99 \\
Jun 10 & 67 & 46 & 72 & 94 \\
Jul 10 & 87 & 49 & 97 & 112 \\
Aug 10 & 73 & 34 & 97 & 83 \\
\hline
\end{tabular}


Table 8. Monthly average wind speeds (m/s).

\begin{tabular}{|c|c|c|c|c|c|}
\hline Month & Descriptive Statistics & North & East & South & West \\
\hline \multirow[t]{5}{*}{ Sep 09} & Mean & 0.42 & 0.77 & 1.59 & 1.49 \\
\hline & SD & 0.77 & 0.42 & 1.31 & 1.06 \\
\hline & $5^{\text {th }}$ ptile & 0.00 & 0.14 & 0.10 & 0.12 \\
\hline & Median & 0.00 & 0.73 & 1.19 & 1.26 \\
\hline & 95th ptile & 2.22 & 1.66 & 4.14 & 3.56 \\
\hline \multirow[t]{5}{*}{ Oct 09} & Mean & 0.82 & 0.93 & 1.82 & 1.80 \\
\hline & SD & 1.05 & 0.52 & 1.94 & 1.33 \\
\hline & $5^{\text {th }}$ ptile & 0.00 & 0.11 & 0.07 & 0.23 \\
\hline & Median & 0.37 & 0.86 & 1.15 & 1.56 \\
\hline & 95th ptile & 3.20 & 1.81 & 6.38 & 4.37 \\
\hline \multirow[t]{5}{*}{ Nov 09} & Mean & 1.49 & 0.97 & 1.54 & 2.14 \\
\hline & SD & 1.28 & 0.52 & 1.37 & 1.42 \\
\hline & $5^{\text {th }}$ ptile & 0.16 & 0.13 & 0.11 & 0.25 \\
\hline & Median & 1.16 & 0.91 & 1.04 & 1.87 \\
\hline & $95^{\text {th }}$ ptile & 3.77 & 1.97 & 4.58 & 5.00 \\
\hline \multirow[t]{5}{*}{$\operatorname{Dec} 09$} & Mean & 2.38 & 1.39 & 2.28 & 3.17 \\
\hline & SD & 6.07 & 2.02 & 8.52 & 3.62 \\
\hline & $5^{\text {th }}$ ptile & 0.15 & 0.13 & 0.13 & 0.43 \\
\hline & Median & 1.33 & 1.03 & 1.41 & 2.52 \\
\hline & $95^{\text {th }}$ ptile & 6.43 & 3.13 & 4.89 & 6.74 \\
\hline \multirow[t]{5}{*}{\begin{tabular}{|l|}
$\operatorname{Jan} 10$ \\
\end{tabular}} & Mean & 2.02 & 1.15 & 1.74 & 2.80 \\
\hline & SD & 3.09 & 0.81 & 1.63 & 2.29 \\
\hline & $5^{\text {th }}$ & 0.19 & 0.14 & 0.12 & 0.38 \\
\hline & $50^{\text {th }}$ & 1.31 & 1.02 & 1.11 & 2.45 \\
\hline & $95^{\text {th }}$ & 5.19 & 3.07 & 5.26 & 5.94 \\
\hline \multirow[t]{5}{*}{ Feb 10} & Mean & 2.99 & 1.19 & 5.12 & 8.21 \\
\hline & SD & 10.4 & 0.63 & 20.5 & 39.6 \\
\hline & $5^{\text {th }}$ ptile & 0.27 & 0.41 & 0.05 & 0.59 \\
\hline & Median & 1.44 & 1.14 & 0.87 & 3.34 \\
\hline & $95^{\text {th }}$ ptile & 5.00 & 2.43 & 20.9 & 5.94 \\
\hline \multirow[t]{5}{*}{ Mar 10} & Mean & 2.16 & 1.82 & 1.21 & 2.09 \\
\hline & SD & 1.46 & 1.17 & 1.23 & 1.16 \\
\hline & $5^{\text {th }}$ ptile & 0.14 & 0.39 & 0.07 & 0.37 \\
\hline & Median & 2.09 & 1.50 & 0.85 & 1.98 \\
\hline & $95^{\text {th }}$ ptile & 4.61 & 4.27 & 3.77 & 3.95 \\
\hline \multirow[t]{5}{*}{ Apr 10} & Mean & 2.22 & 0.90 & 1.49 & 2.59 \\
\hline & SD & 1.58 & 0.46 & 1.47 & 1.50 \\
\hline & $5^{\text {th }}$ ptile & 0.21 & 0.14 & 0.09 & 0.35 \\
\hline & Median & 2.05 & 0.91 & 1.00 & 2.41 \\
\hline & $95^{\text {th }}$ ptile & 5.28 & 1.67 & 4.95 & 5.21 \\
\hline
\end{tabular}


Table 8. Continuation.

\begin{tabular}{|ll|cccc|}
\hline Month & Descriptive Statistics & North & East & South & West \\
\hline May 10 & Mean & 1.99 & 0.80 & 1.31 & 2.25 \\
& SD & 1.41 & 0.55 & 1.05 & 1.59 \\
& $5^{\text {th }}$ ptile & 0.19 & 0.10 & 0.12 & 0.22 \\
& Median & 1.65 & 0.69 & 1.02 & 1.95 \\
& $95^{\text {th }}$ ptile & 4.64 & 1.90 & 3.61 & 5.37 \\
\hline Jun 10 & Mean & 1.78 & 0.73 & 1.55 & 2.07 \\
& SD & 1.30 & 0.46 & 1.21 & 1.21 \\
& $5^{\text {th }}$ ptile & 0.14 & 0.12 & 0.13 & 0.31 \\
& Median & 1.52 & 0.63 & 1.21 & 1.97 \\
& 95th ptile & 4.13 & 1.65 & 3.81 & 4.24 \\
\hline Jul 10 & Mean & 1.63 & 0.56 & 1.53 & 1.86 \\
& SD & 1.23 & 0.38 & 1.16 & 0.96 \\
& $5^{\text {th }}$ ptile & 0.10 & 0.11 & 0.15 & 0.37 \\
& Median & 1.35 & 0.44 & 1.15 & 1.81 \\
& $95^{\text {th }}$ ptile & 4.11 & 1.29 & 3.85 & 3.55 \\
\hline Aug 10 & Mean & 1.33 & 0.79 & 1.50 & 1.57 \\
& SD & 1.12 & 0.51 & 1.19 & 1.04 \\
& $5^{\text {th }}$ ptile & 0.10 & 0.09 & 0.12 & 0.21 \\
& Median & 1.04 & 0.75 & 1.15 & 1.31 \\
& $95^{\text {th }}$ ptile & 3.71 & 1.83 & 3.95 & 3.66 \\
\hline
\end{tabular}

Abbreviations: ptile, percentile; SD, standard deviation.

Table 9. Monthly rain accumulation (mm).

\begin{tabular}{|c|c|}
\hline Month & Rain Accumulation (mm) \\
\hline Sep 09 & 42 \\
Oct 09 & 92 \\
Nov 09 & 39 \\
Dec 09 & 45 \\
Jan 10 & 24 \\
Feb 10 & 21 \\
Mar 10 & 57 \\
Apr 10 & 29 \\
May 10 & 53 \\
Jun 10 & 151 \\
Jul 10 & 96 \\
Aug 10 & 150 \\
\hline
\end{tabular}


Table 10. Weekly and monthly pressure differentials $(\mathrm{Pa})$ across building sides $\mathrm{s}^{\mathrm{a}, \mathrm{b}}$.

\begin{tabular}{|c|c|c|c|c|c|c|c|c|c|c|c|c|c|c|c|c|c|c|c|c|c|}
\hline \multirow{3}{*}{ Month } & \multirow{3}{*}{$\begin{array}{c}\text { Descriptive } \\
\text { Statistics }\end{array}$} & \multicolumn{5}{|c|}{ North } & \multicolumn{5}{|c|}{ East } & \multicolumn{5}{|c|}{ South } & \multicolumn{5}{|c|}{ West } \\
\hline & & \multicolumn{4}{|c|}{ Weekly } & \multirow[b]{2}{*}{ Monthly } & \multicolumn{4}{|c|}{ Weekly } & \multirow[b]{2}{*}{ Monthly } & \multicolumn{4}{|c|}{ Weekly } & \multirow[b]{2}{*}{ Monthly } & \multicolumn{4}{|c|}{ Weekly } & \multirow[b]{2}{*}{ Monthl } \\
\hline & & 1 st & $2^{\text {nd }}$ & $3^{\text {rd }}$ & $4^{\text {th }}$ & & 1 st & $2^{\text {nd }}$ & $3^{\text {rd }}$ & $4^{\text {th }}$ & & 1 st & $2^{\text {nd }}$ & $3^{\text {rd }}$ & $4^{\text {th }}$ & & 1st & $2^{\text {nd }}$ & $3^{\text {rd }}$ & $4^{\text {th }}$ & \\
\hline \multirow[t]{9}{*}{ Sep 09} & Mean & - & - & 0.19 & -0.01 & - & - & - & -1.14 & -2.16 & - & - & - & 0.84 & 3.06 & - & - & - & 0.78 & 0.56 & - \\
\hline & SD & . & - & 1.50 & 1.49 & - & - & - & 2.67 & 3.60 & - & - & - & 3.20 & 5.14 & - & - & - & 1.54 & 3.67 & - \\
\hline & & - & - & -5.56 & -9.83 & - & - & - & -23.7 & -28.5 & - & - & - & -22.6 & -11.4 & - & - & - & -12.3 & -22.4 & - \\
\hline & $5^{\text {th }}$ ptile & - & - & -1.08 & -2.07 & - & - & - & -6.25 & -9.29 & - & - & - & -3.54 & -2.50 & - & - & - & -1.31 & -5.83 & - \\
\hline & $25^{\text {th }}$ ptile & - & - & -0.41 & -0.54 & - & - & - & -1.87 & -3.56 & - & - & - & -0.35 & -0.11 & - & - & - & 0.13 & -1.07 & - \\
\hline & Median & - & - & -0.22 & -0.13 & - & - & - & -0.15 & -1.07 & - & - & - & 0.59 & 1.33 & - & - & - & 0.71 & 0.67 & - \\
\hline & $75^{\text {th }}$ ptile & - & - & 0.19 & 0.38 & - & - & - & 0.45 & 0.25 & - & - & - & 1.26 & 5.21 & - & - & - & 1.08 & 2.43 & - \\
\hline & $95^{\text {th }}$ ptile & - & - & 3.30 & 2.46 & - & - & - & 0.94 & 1.21 & - & - & - & 5.89 & 13.3 & - & - & - & 3.69 & 6.46 & - \\
\hline & Max. & & - & 14.4 & 13.4 & - & - & - & 5.11 & 36.5 & - & & - & 26.2 & 39.3 & - & - & - & 10.6 & 20.3 & - \\
\hline \multirow{9}{*}{ Oct 09} & Mean & -0.08 & 0.60 & -0.12 & -0.13 & 0.05 & -1.03 & -0.88 & 0.38 & -1.93 & -0.97 & 1.08 & 0.50 & 1.16 & 3.82 & 1.85 & 2.19 & 2.53 & 1.56 & -0.02 & 1.41 \\
\hline & SD & 2.13 & 2.33 & 1.43 & 1.89 & 1.99 & 3.11 & 2.55 & 1.76 & 5.48 & 3.84 & 3.86 & 2.76 & 1.83 & 8.24 & 5.44 & 4.20 & 2.88 & 1.63 & 5.32 & 4.08 \\
\hline & Min. & -12.2 & -11.0 & -6.44 & -14.1 & -14.1 & -38.6 & -19.1 & -14.3 & -51.7 & -51.7 & -27.0 & -13.7 & -13.7 & -13.3 & -27.0 & -21.0 & -8.05 & -9.96 & -45.8 & -45.8 \\
\hline & $5^{\text {th }}$ ptile & -2.95 & $\begin{array}{l}-2.22 \\
\end{array}$ & -3.07 & -3.18 & -2.94 & $\begin{array}{l}-7.21 \\
\end{array}$ & -5.92 & -3.23 & $\begin{array}{l}-13.0 \\
-1\end{array}$ & -7.43 & -4.50 & -4.35 & -2.28 & -3.15 & -3.51 & -2.66 & -1.37 & -1.40 & -10.8 & -3.81 \\
\hline & $25^{\text {th }}$ ptile & -0.66 & -0.38 & -0.39 & -0.63 & -0.50 & -1.67 & -1.89 & 0.00 & -2.61 & -1.58 & -0.17 & -0.74 & 0.49 & 0.34 & 0.09 & 0.43 & 0.96 & 0.90 & -0.68 & 0.53 \\
\hline & Median & $\begin{array}{l}-0.25 \\
\end{array}$ & -0.01 & -0.22 & -0.21 & -0.20 & 0.01 & -0.08 & 0.82 & 0.21 & 0.29 & 0.95 & 0.68 & 1.21 & 1.15 & 1.04 & 1.36 & 1.94 & 1.55 & 0.92 & 1.39 \\
\hline & $75^{\text {th }}$ ptile & 0.23 & 1.11 & 0.09 & 0.24 & 0.36 & 0.75 & 0.72 & 1.31 & 0.78 & 0.91 & 1.99 & 1.82 & 1.91 & 3.86 & 2.17 & 3.07 & 3.65 & 2.07 & 1.83 & 2.56 \\
\hline & $95^{\text {th }}$ ptile & 2.85 & 5.13 & 2.05 & 2.85 & 3.29 & 1.57 & 1.67 & 2.29 & 1.85 & 1.87 & 6.96 & 4.84 & 3.99 & 22.0 & 9.55 & 10.4 & 8.22 & 4.27 & 6.64 & 7.22 \\
\hline & Max. & 36.9 & 20.5 & 13.9 & 16.2 & 36.9 & 6.11 & 6.29 & 5.38 & 10.4 & 10.4 & 33.2 & 15.0 & 12.4 & 69.9 & 69.9 & 37.1 & 21.3 & 13.9 & 23.4 & 37.1 \\
\hline \multirow[t]{9}{*}{ Nov 09} & Mean & 0.18 & -0.03 & 0.01 & 0.38 & 0.15 & -1.28 & -0.45 & -0.04 & -1.17 & -0.77 & 2.10 & 1.57 & 0.56 & 1.07 & 1.31 & 2.16 & 1.07 & 2.24 & 2.77 & 2.11 \\
\hline & SD & 2.36 & 1.30 & 1.85 & 3.46 & 2.47 & 3.99 & 2.99 & 2.12 & 4.46 & 3.61 & 5.02 & 3.54 & 2.24 & 4.29 & 3.98 & 3.85 & 2.16 & 2.50 & 4.85 & 3.66 \\
\hline & Min. & -9.30 & -6.56 & -8.36 & -10.3 & -10.3 & -34.2 & -21.7 & -18.0 & -39.2 & -39.2 & -18.7 & -11.5 & -15.1 & -27.9 & -27.9 & -21.0 & -17.6 & -9.19 & -16.4 & -21.0 \\
\hline & $5^{\text {th }}$ ptile & -3.37 & -1.89 & -3.08 & -3.39 & -3.10 & -9.42 & -6.82 & -4.26 & -10.2 & -7.66 & -4.17 & -2.07 & -3.14 & -6.13 & -3.65 & -3.83 & -2.87 & -1.50 & -2.76 & -2.72 \\
\hline & $25^{\text {th }}$ ptile & -0.61 & -0.39 & -0.60 & -0.56 & -0.52 & -2.56 & -1.01 & -0.81 & -2.06 & -1.64 & -0.06 & 0.19 & -0.35 & -0.13 & -0.08 & 0.82 & 0.65 & 0.97 & 0.76 & 0.81 \\
\hline & Median & -0.24 & -0.19 & -0.24 & -0.23 & -0.22 & 0.00 & 0.64 & 0.59 & 0.56 & 0.52 & 1.42 & 0.92 & 0.80 & 1.23 & 1.07 & 1.88 & 1.26 & 1.77 & 1.48 & 1.57 \\
\hline & $75^{\text {th }}$ ptile & 0.68 & 0.31 & 0.54 & 0.30 & 0.44 & 1.24 & 1.10 & 1.20 & 1.16 & 1.17 & 2.96 & 1.69 & 1.57 & 2.43 & 2.03 & 3.76 & 1.88 & 3.30 & 3.83 & 3.07 \\
\hline & $95^{\text {th }}$ ptile & 3.88 & 2.12 & 3.34 & 5.79 & 3.55 & 2.32 & 1.92 & 2.24 & 2.35 & 2.21 & 11.6 & 9.36 & 3.44 & 7.45 & 7.92 & 8.48 & 4.01 & 6.83 & 12.2 & 8.15 \\
\hline & Max. & 22.1 & 14.2 & 13.9 & 38.9 & 38.9 & 6.22 & 6.41 & 6.47 & 6.44 & 6.47 & 48.1 & 28.3 & 57.3 & 30.4 & 57.3 & 21.0 & 16.0 & 25.6 & 46.1 & 46.1 \\
\hline \multirow[t]{9}{*}{ Dec 09} & Mean & -0.59 & -0.85 & 1.04 & 0.24 & -0.01 & -0.89 & -3.09 & -0.98 & -0.84 & -1.39 & 3.13 & 3.15 & 0.28 & 1.33 & 1.91 & 2.04 & 3.90 & 4.71 & 3.91 & 3.67 \\
\hline & SD & 1.98 & 2.63 & 3.59 & 3.59 & 3.16 & 3.56 & 8.98 & 4.38 & 5.25 & 5.93 & 4.71 & 6.79 & 3.60 & 4.88 & 5.24 & 4.34 & 6.70 & 4.03 & 5.59 & 5.39 \\
\hline & Min. & -16.5 & -15.9 & -12.0 & -11.6 & -16.5 & -28.3 & -67.3 & -29.3 & -46.5 & -67.3 & -18.9 & -21.9 & -22.3 & -33.7 & -33.7 & -25.9 & -27.7 & -10.3 & -14.3 & -27.7 \\
\hline & $5^{\text {th }}$ ptile & -4.29 & -5.52 & -3.79 & -3.99 & -4.30 & -7.98 & -14.7 & -9.78 & -11.6 & -11.0 & -2.94 & -5.00 & -6.46 & -7.31 & -5.59 & -4.26 & -5.42 & -0.85 & -2.27 & -2.91 \\
\hline & $25^{\text {th }}$ ptile & -1.02 & -1.75 & -0.55 & -0.83 & -0.95 & -2.22 & -4.18 & -2.54 & -1.91 & -2.63 & 0.85 & -0.07 & -1.46 & -0.15 & -0.22 & 0.37 & 0.54 & 2.53 & 1.40 & 1.21 \\
\hline & Median & -0.37 & -0.49 & 0.22 & -0.31 & -0.30 & 0.10 & -0.75 & 0.23 & 1.14 & 0.31 & 2.33 & 2.02 & 1.09 & 1.93 & 1.88 & 1.81 & 2.64 & 3.65 & 2.36 & 2.61 \\
\hline & $75^{\text {th }}$ ptile & 0.05 & 0.31 & 2.06 & 0.43 & 0.60 & 1.34 & 1.18 & 1.99 & 1.91 & 1.66 & 4.81 & 4.71 & 2.46 & 3.04 & 3.36 & 3.57 & 6.98 & 6.21 & 5.14 & 5.37 \\
\hline & $95^{\text {th }}$ ptile & 2.24 & 2.87 & 8.29 & 6.46 & 4.81 & 2.75 & 2.84 & 3.41 & 3.53 & 3.19 & 11.6 & 15.3 & 4.80 & 7.68 & 10.3 & 9.35 & 16.5 & 12.8 & 14.7 & 13.7 \\
\hline & Max. & 13.4 & 22.9 & 30.8 & 38.1 & 38.1 & 17.5 & 46.8 & 7.19 & 9.43 & 46.8 & 42.4 & 108 & 27.1 & 31.1 & 108 & 29.7 & 41.3 & 31.0 & 54.4 & 54.4 \\
\hline
\end{tabular}


Table 10. Continuation.

\begin{tabular}{|c|c|c|c|c|c|c|c|c|c|c|c|c|c|c|c|c|c|c|c|c|c|}
\hline \multirow{3}{*}{ Month } & \multirow{3}{*}{$\begin{array}{c}\text { Descriptive } \\
\text { Statistics }\end{array}$} & \multicolumn{5}{|c|}{ North } & \multicolumn{5}{|c|}{ East } & \multicolumn{5}{|c|}{ South } & \multicolumn{5}{|c|}{ West } \\
\hline & & \multicolumn{4}{|c|}{ Weekly } & \multirow{2}{*}{ Monthly } & \multicolumn{4}{|c|}{ Weekly } & \multirow{2}{*}{ Monthly } & \multicolumn{4}{|c|}{ Weekly } & \multirow{2}{*}{ Monthly } & \multicolumn{4}{|c|}{ Weekly } & \multirow{2}{*}{ Monthly } \\
\hline & & 1 st & $2^{\text {nd }}$ & $3^{\text {rd }}$ & $4^{\text {th }}$ & & 1 st & $2^{\text {nd }}$ & $3^{\text {rd }}$ & $4^{\text {th }}$ & & $1^{\text {st }}$ & $2^{\text {nd }}$ & $3^{\text {rdd }}$ & $4^{\text {th }}$ & & 1 st & $2^{\text {nd }}$ & $3^{\text {rd }}$ & $4^{\text {th }}$ & \\
\hline \multirow[t]{9}{*}{ Jan 10} & Mean & -0.15 & 0.48 & -0.17 & 0.07 & 0.06 & -1.04 & -0.69 & 0.51 & -1.03 & -0.61 & -0.06 & 2.37 & 1.65 & 2.59 & 1.73 & 6.45 & 3.27 & 2.52 & 3.31 & 3.83 \\
\hline & SD & 2.94 & 2.46 & 1.33 & 3.39 & 2.73 & 3.42 & 3.59 & 2.11 & 5.51 & 4.09 & 3.08 & 4.15 & 2.35 & 6.27 & 4.58 & 3.94 & 3.24 & 2.05 & 6.46 & 4.73 \\
\hline & Min. & -12.9 & -7.80 & -8.83 & -20.8 & -20.8 & -33.1 & -21.7 & -18.8 & -53.7 & -53.7 & -25.9 & -16.3 & -10.5 & -33.1 & -33.1 & -5.94 & -17.1 & -11.4 & -32.7 & -32.7 \\
\hline & $5^{\text {th }}$ ptile & -4.39 & -3.34 & -2.88 & -4.14 & -3.77 & -7.33 & -8.00 & -3.56 & -11.0 & -8.11 & -5.75 & -3.46 & -1.82 & -6.4 & -4.59 & 0.98 & -1.80 & -0.79 & -7.7 & -1.97 \\
\hline & $25^{\text {th }}$ ptile & -1.63 & -0.62 & -0.52 & -1.08 & -0.85 & -2.35 & -2.10 & -0.06 & -2.81 & -1.91 & -1.55 & 0.40 & 0.80 & -0.16 & -0.16 & 3.87 & 1.96 & 1.63 & 1.30 & 1.82 \\
\hline & Median & -0.21 & -0.07 & -0.25 & -0.37 & -0.25 & -0.14 & 0.37 & 1.21 & 0.25 & 0.50 & 0.49 & 2.18 & 1.68 & 2.09 & 1.64 & 5.86 & 3.17 & 2.21 & 2.88 & 3.24 \\
\hline & $75^{\text {th }}$ ptile & 1.04 & 1.25 & 0.27 & 0.62 & 0.74 & 1.12 & 1.77 & 1.66 & 1.87 & 1.63 & 1.87 & 3.78 & 2.27 & 4.24 & 2.98 & 8.52 & 4.86 & 3.34 & 5.52 & 5.63 \\
\hline & 95th ptile & 4.13 & 5.44 & 1.95 & 6.54 & 4.55 & 2.48 & 3.09 & 2.73 & 3.78 & 3.12 & 3.97 & 9.23 & 4.57 & 14.8 & 9.01 & 13.6 & 8.56 & 5.92 & 13.8 & 11.6 \\
\hline & Max. & 38.1 & 18.7 & 9.50 & 41.1 & 41.1 & 6.44 & 5.24 & 4.86 & 43.0 & 43.0 & 11.9 & 30.3 & 26.5 & 50.9 & 50.9 & 37.7 & 23.9 & 22.6 & 47.1 & 47.1 \\
\hline \multirow[t]{9}{*}{ Feb 10} & Mean & -0.28 & - & - & - & - & 0.19 & - & - & - & - & 1.41 & - & - & - & - & 3.97 & - & - & - & - \\
\hline & SD & 1.84 & - & - & - & - & 2.38 & - & - & - & - & 2.42 & - & - & - & - & 3.27 & - & - & - & - \\
\hline & Min. & -11.8 & - & - & - & - & -15.7 & - & - & - & - & -9.51 & - & - & - & - & -3.53 & - & - & - & - \\
\hline & $5^{\text {th }}$ ptile & -3.68 & - & - & - & - & -4.51 & - & - & - & - & -3.20 & - & - & - & - & -0.16 & - & - & - & - \\
\hline & $25^{\text {th }}$ ptile & -0.75 & - & - & - & - & -0.96 & - & - & - & - & 0.08 & - & - & - & - & 2.00 & - & - & - & - \\
\hline & Median & -0.36 & - & - & - & - & 0.84 & - & - & - & - & 1.99 & - & - & - & - & 2.98 & - & - & - & - \\
\hline & $75^{\text {th }}$ ptile & 0.53 & - & - & - & - & 1.89 & - & - & - & - & 2.73 & - & - & - & - & 5.57 & - & - & - & - \\
\hline & $95^{\text {th }}$ ptile & 2.72 & - & - & - & - & 2.86 & - & - & - & - & 4.77 & - & - & - & - & 10.4 & - & - & - & - \\
\hline & Max. & 9.90 & - & - & - & - & 4.48 & - & - & - & - & 11.7 & - & - & - & - & 22.5 & - & - & - & - \\
\hline \multirow[t]{9}{*}{ May 10} & Mean & - & - & - & - & - & - & - & - & -1.24 & - & - & - & - & -0.34 & - & - & - & - & 1.09 & - \\
\hline & SD & - & - & - & - & - & - & - & - & 2.29 & - & - & - & - & 2.39 & - & - & - & - & 2.17 & - \\
\hline & Min. & - & - & - & - & - & - & - & - & -15.6 & - & - & - & - & -14.8 & - & - & - & - & -9.23 & - \\
\hline & $5^{\text {th }}$ ptile & - & - & - & - & - & - & - & - & -6.06 & - & - & - & - & -3.99 & - & - & - & - & -1.15 & - \\
\hline & $25^{\text {th }}$ ptile & - & - & - & - & - & - & - & - & -2.15 & - & - & - & - & -1.09 & - & - & - & - & -0.04 & - \\
\hline & Median & - & - & - & - & - & - & - & - & -0.39 & - & - & - & - & -0.43 & - & - & - & - & 0.49 & - \\
\hline & $75^{\text {th }}$ ptile & - & - & - & - & - & - & - & - & 0.30 & - & - & - & - & 0.14 & - & - & - & - & 1.73 & - \\
\hline & 95th ptile & - & - & - & - & - & - & - & - & 0.90 & - & - & - & - & 3.98 & - & - & - & - & 5.58 & - \\
\hline & Max. & - & - & - & - & - & - & - & - & 2.79 & - & - & - & - & 15.3 & - & - & - & - & 15.8 & - \\
\hline \multirow{9}{*}{ Jun 10} & Mean & & - & - & - & - & -1.63 & - & - & - & - & 1.73 & - & - & - & - & 0.67 & - & - & - & - \\
\hline & SD & - & - & - & - & - & 2.16 & - & - & - & - & 3.83 & - & - & - & - & 2.58 & - & - & - & - \\
\hline & Min. & - & - & - & - & - & -13.1 & - & - & - & - & -7.11 & - & - & - & - & -10.6 & - & - & - & - \\
\hline & $5^{\text {th }}$ ptile & - & - & - & - & - & -5.83 & - & - & - & - & -3.57 & - & - & - & - & -3.14 & - & - & - & - \\
\hline & $25^{\text {th }}$ ptile & - & - & - & - & - & -2.66 & - & - & - & - & -0.48 & - & - & - & - & -0.61 & - & - & - & - \\
\hline & Median & - & - & - & - & - & -1.08 & - & - & - & - & 1.07 & - & - & - & - & 0.34 & - & - & - & - \\
\hline & $75^{\text {th }}$ ptile & - & - & - & - & - & -0.16 & - & - & - & - & 3.67 & - & - & - & - & 1.80 & - & - & - & - \\
\hline & 95th ptile & - & - & - & - & - & 0.85 & - & - & - & - & 8.83 & - & - & - & - & 5.40 & - & - & - & - \\
\hline & Max. & - & - & - & - & - & 4.18 & - & - & - & - & 22.2 & - & - & - & - & 15.7 & - & - & - & - \\
\hline
\end{tabular}


Table 10. Continuation.

\begin{tabular}{|c|c|c|c|c|c|c|c|c|c|c|c|c|c|c|c|c|c|c|c|c|c|}
\hline \multirow{3}{*}{ Month } & \multirow{3}{*}{$\begin{array}{c}\text { Descriptive } \\
\text { Statistics }\end{array}$} & \multicolumn{5}{|c|}{ North } & \multicolumn{5}{|c|}{ East } & \multicolumn{5}{|c|}{ South } & \multicolumn{5}{|c|}{ West } \\
\hline & & \multicolumn{4}{|c|}{ Weekly } & \multirow{2}{*}{ Monthly } & \multicolumn{4}{|c|}{ Weekly } & \multirow{2}{*}{ Monthly } & \multicolumn{4}{|c|}{ Weekly } & \multirow{2}{*}{ Monthly } & \multicolumn{4}{|c|}{ Weekly } & \multirow[b]{2}{*}{ Monthly } \\
\hline & & $1^{\text {st }}$ & $2^{\text {nd }}$ & $3^{\text {rd }}$ & $4^{\text {th }}$ & & $1^{\text {st }}$ & $2^{\text {nd }}$ & $3^{\text {rd }}$ & $4^{\text {th }}$ & & 1st & $2^{\text {nd }}$ & $3^{\text {rd }}$ & $4^{\text {th }}$ & & $1^{\text {st }}$ & $2^{\text {nd }}$ & $3^{\text {rdd }}$ & $4^{\text {th }}$ & \\
\hline \multirow[t]{9}{*}{ Jul $10^{c}$} & Mean & 0.34 & 0.42 & 0.07 & 0.45 & 0.33 & -1.47 & -1.68 & -1.92 & -1.83 & -1.79 & -1.21 & 0.81 & -0.15 & -0.23 & 0.04 & - & 0.18 & 0.55 & 1.42 & - \\
\hline & SD & 0.93 & 1.45 & 1.31 & 2.02 & 1.63 & 1.42 & 2.62 & 2.33 & 8.53 & 5.43 & 1.44 & 3.75 & 2.96 & 3.55 & 3.41 & - & 6.24 & 1.63 & 5.65 & - \\
\hline & Min. & -2.13 & -5.34 & -12.9 & -12.5 & -12.9 & -8.10 & -26.7 & -21.4 & -39.1 & -39.1 & -7.45 & -14.7 & -14.8 & -23.2 & -23.2 & - & -38.1 & -8.59 & -34.9 & - \\
\hline & $5^{\text {th }}$ ptile & -0.80 & -0.99 & -1.77 & -1.43 & -1.35 & -4.33 & -6.94 & -6.51 & -18.0 & -8.54 & -3.94 & -3.56 & -4.81 & -5.52 & -4.66 & - & -5.05 & -1.51 & -3.33 & - \\
\hline & $25^{\text {th }}$ ptile & -0.21 & -0.29 & -0.34 & -0.52 & -0.37 & -2.17 & -2.52 & -3.03 & -3.20 & -2.85 & -1.98 & -1.03 & -1.53 & -1.57 & -1.39 & - & -1.13 & -0.29 & -0.10 & - \\
\hline & Median & 0.09 & -0.02 & -0.05 & -0.06 & -0.03 & -1.17 & -0.86 & -1.26 & -0.66 & -0.92 & -0.98 & -0.06 & -0.21 & -0.36 & -0.27 & - & -0.03 & 0.20 & 0.97 & - \\
\hline & $75^{\text {th }}$ ptile & 0.76 & 0.77 & 0.50 & 0.80 & 0.68 & -0.49 & -0.09 & -0.24 & 0.15 & -0.04 & -0.25 & 1.60 & 0.86 & 0.80 & 0.90 & - & 1.16 & 1.19 & 2.60 & - \\
\hline & $95^{\text {th }}$ ptile & 2.21 & 3.22 & 2.04 & 4.66 & 3.31 & 0.26 & 0.95 & 0.52 & 9.40 & 1.44 & 0.78 & 8.38 & 5.29 & 5.50 & 6.13 & - & 6.18 & 3.65 & 8.67 & - \\
\hline & Max. & 4.61 & 14.9 & 14.8 & 16.2 & 16.2 & 1.59 & 7.59 & 3.51 & 57.5 & 57.5 & 3.12 & 37.0 & 19.9 & 37.8 & 37.8 & - & 34.0 & 12.4 & 48.6 & - \\
\hline
\end{tabular}

Positive values denote that pressure was higher outdoos than indoors.

b. Data missing (March, April, August and various weeks) due to equipment malfunction.

c. Measurements obtained while Syracuse University pressurized the test hut to characterize the air leakage of wall panels are not included.

Abbreviations: NA, not available; ptile, percentile; SD, standard deviation. 


\section{References}

ASHRAE. 2005. 2005 ASHRAE Handbook - Fundamentals. Atlanta: American Society of Heating, Refrigeration and Air-Conditioning Engineers, Inc.

Pradhan, Denis; Zhang, Jensen; Thorsell, Thomas; Mocarski, Robbin. 2011. Air Leakage Measurement of 25 Wall Assemblies with Different Types of Air Barriers. Report issued by Syracuse University to the Air Barrier Association of America. 


\section{Appendix A: North Panels}
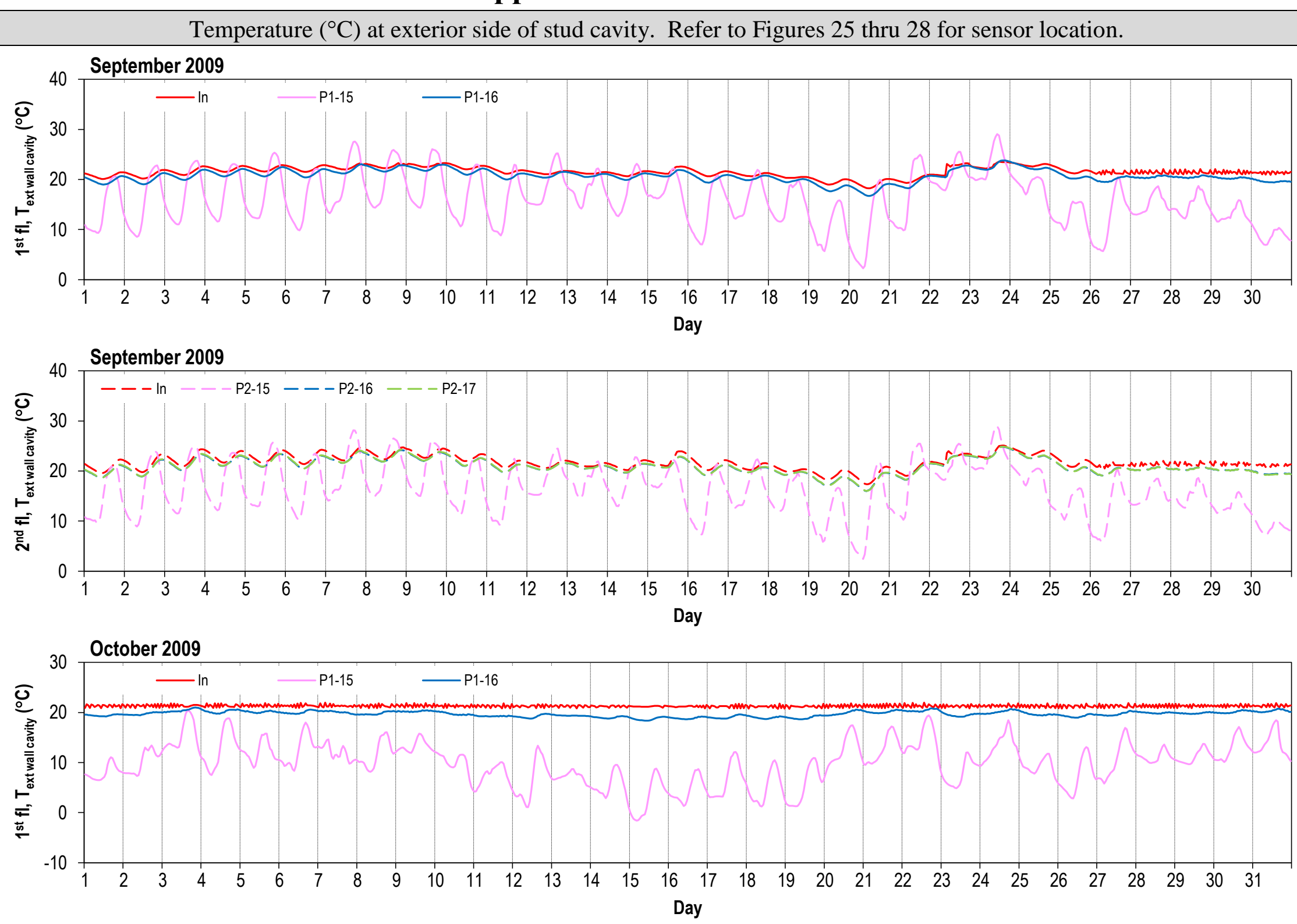

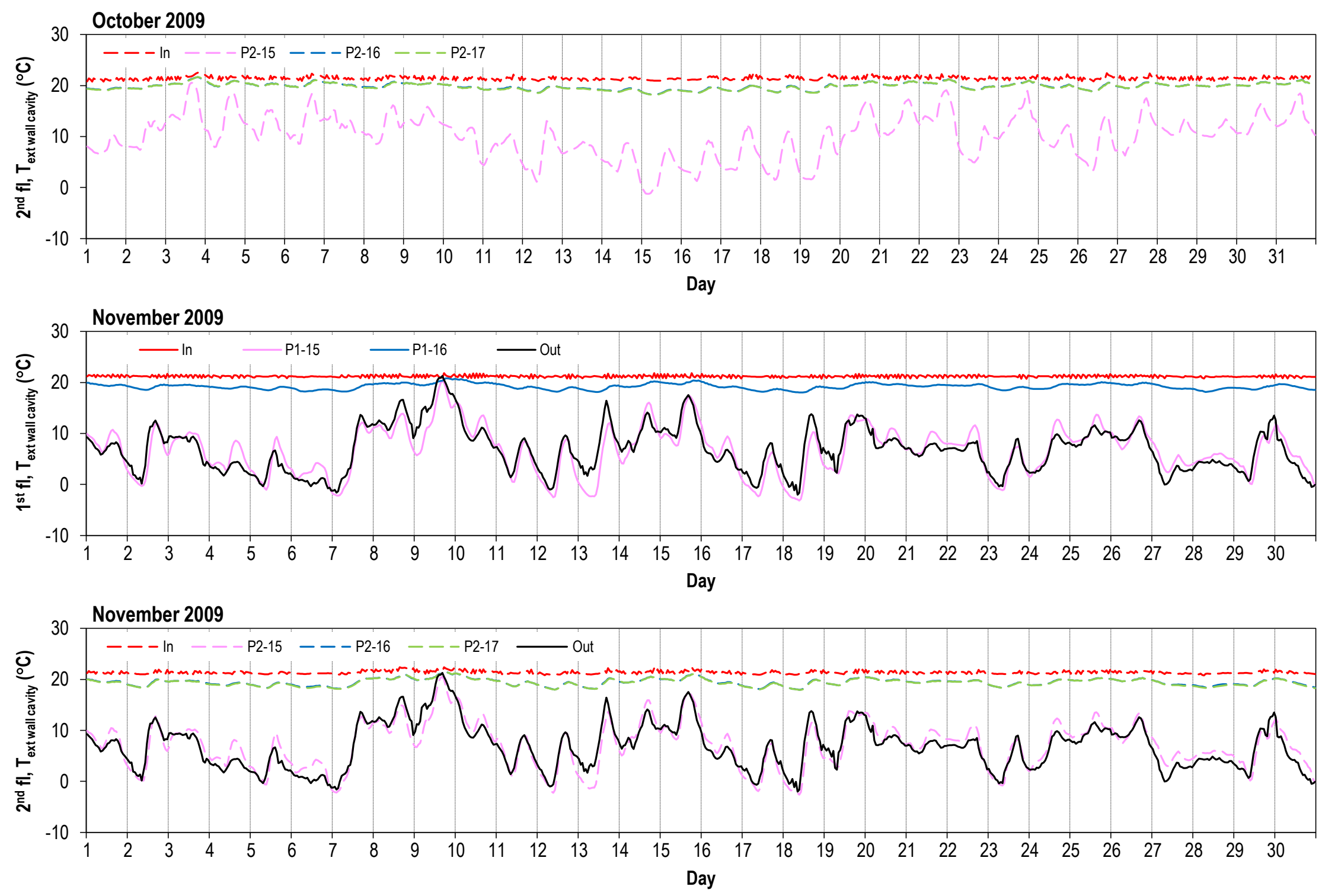

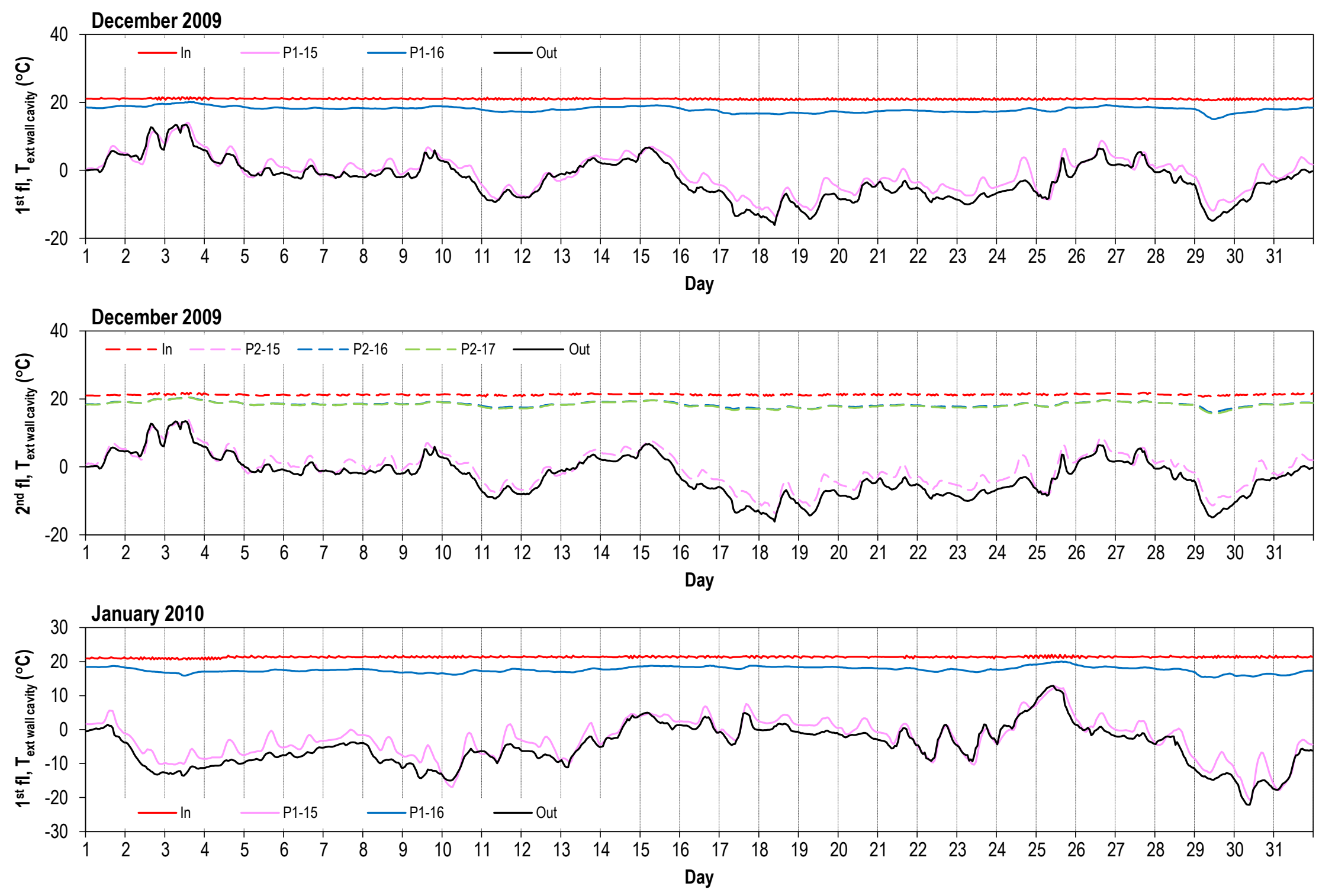

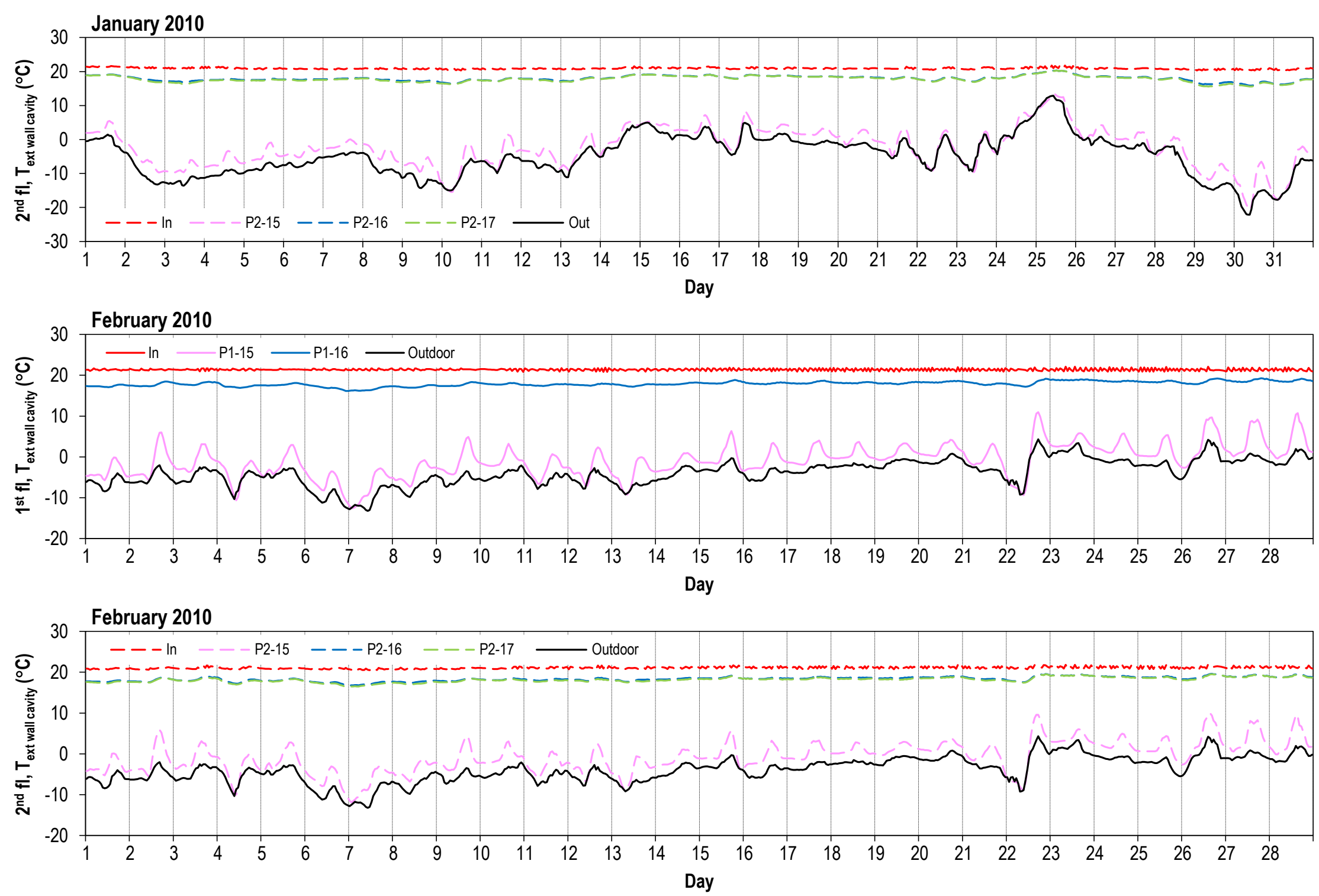

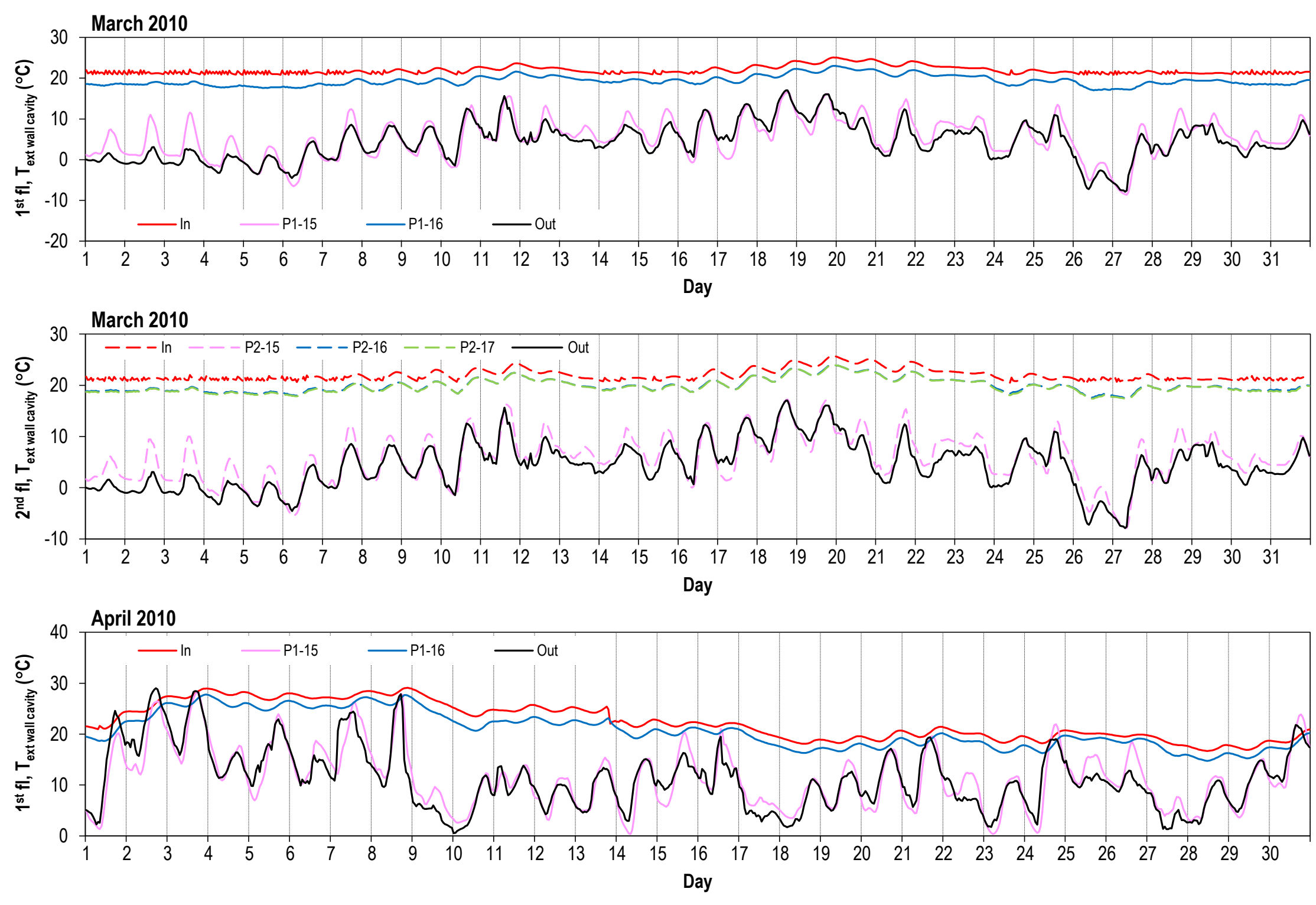

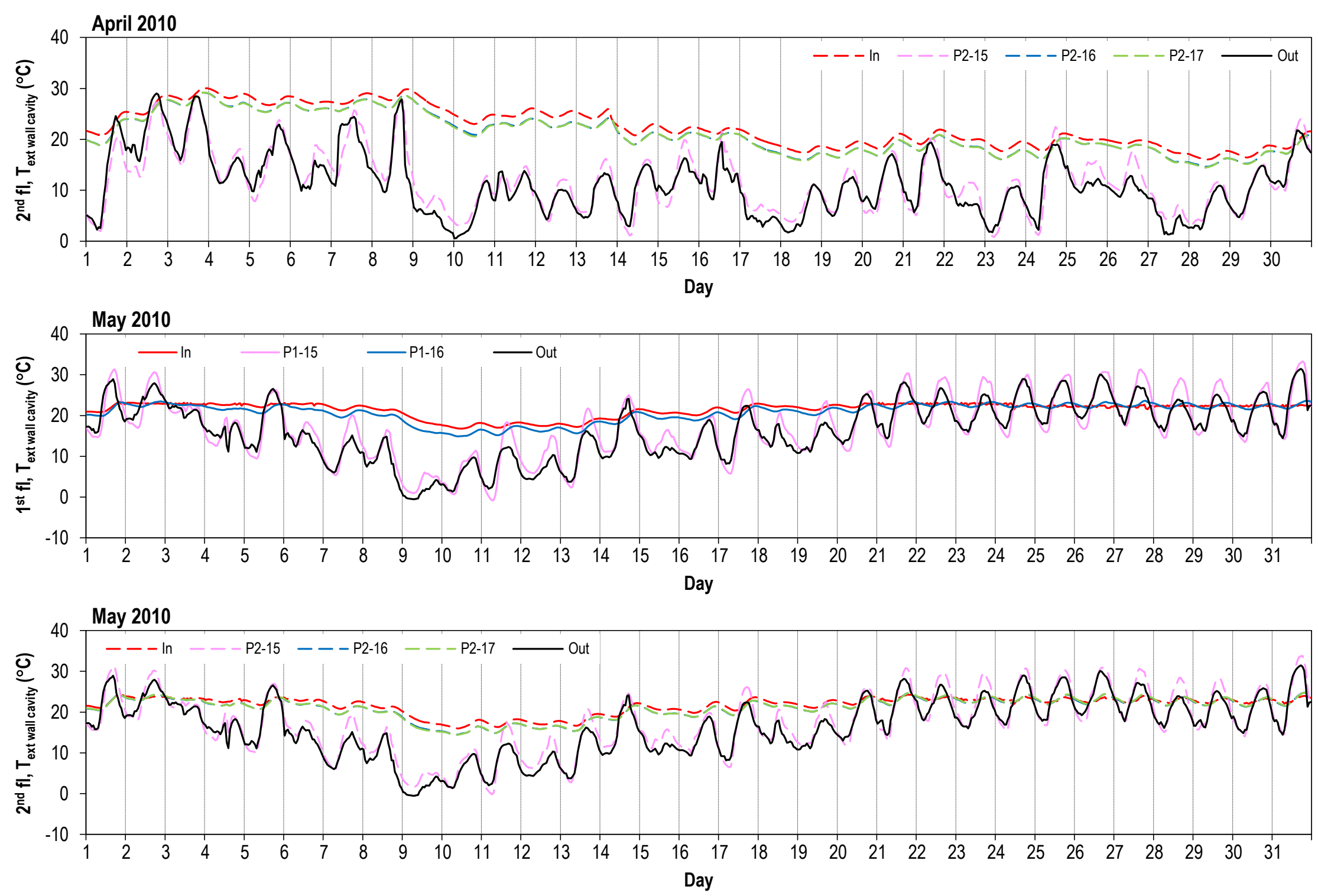

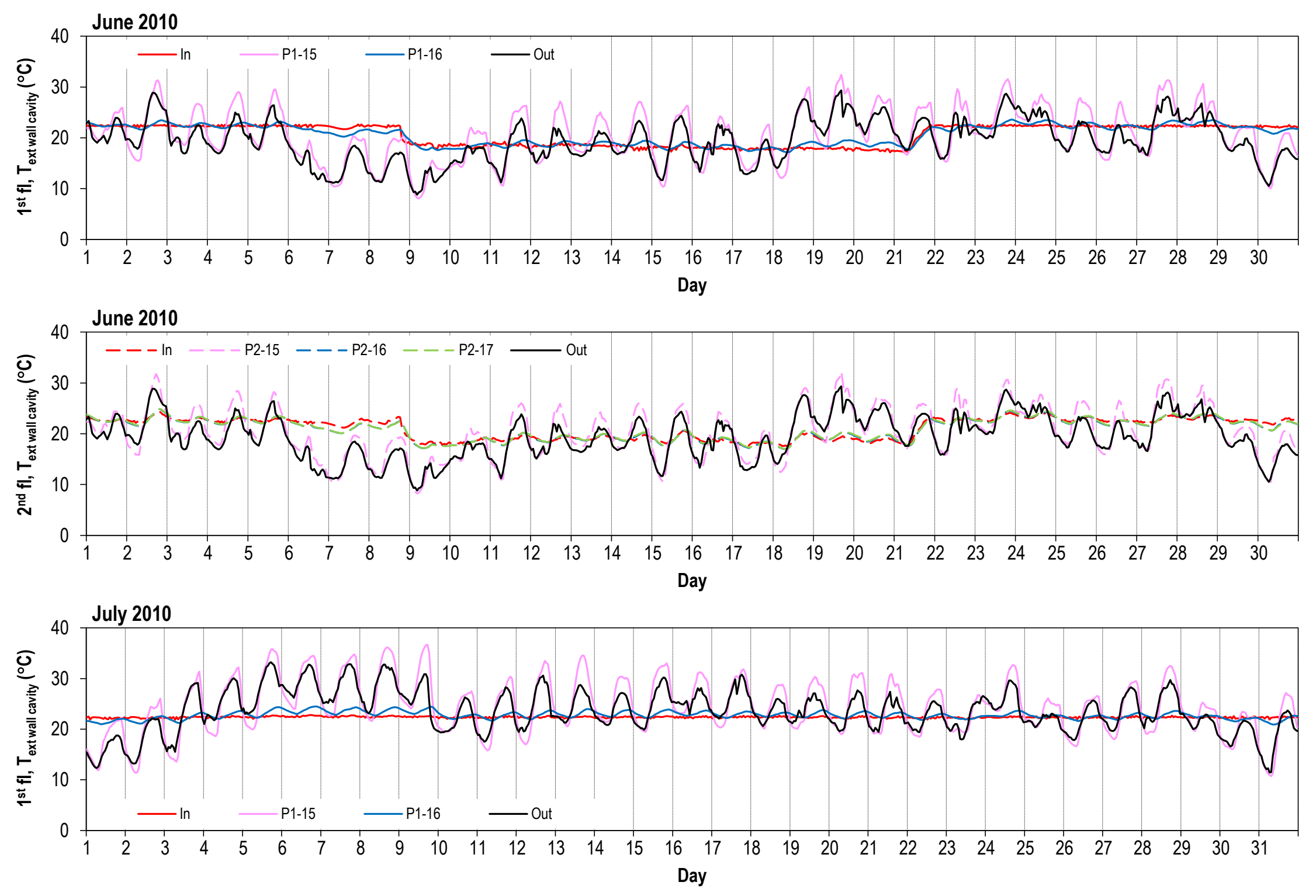

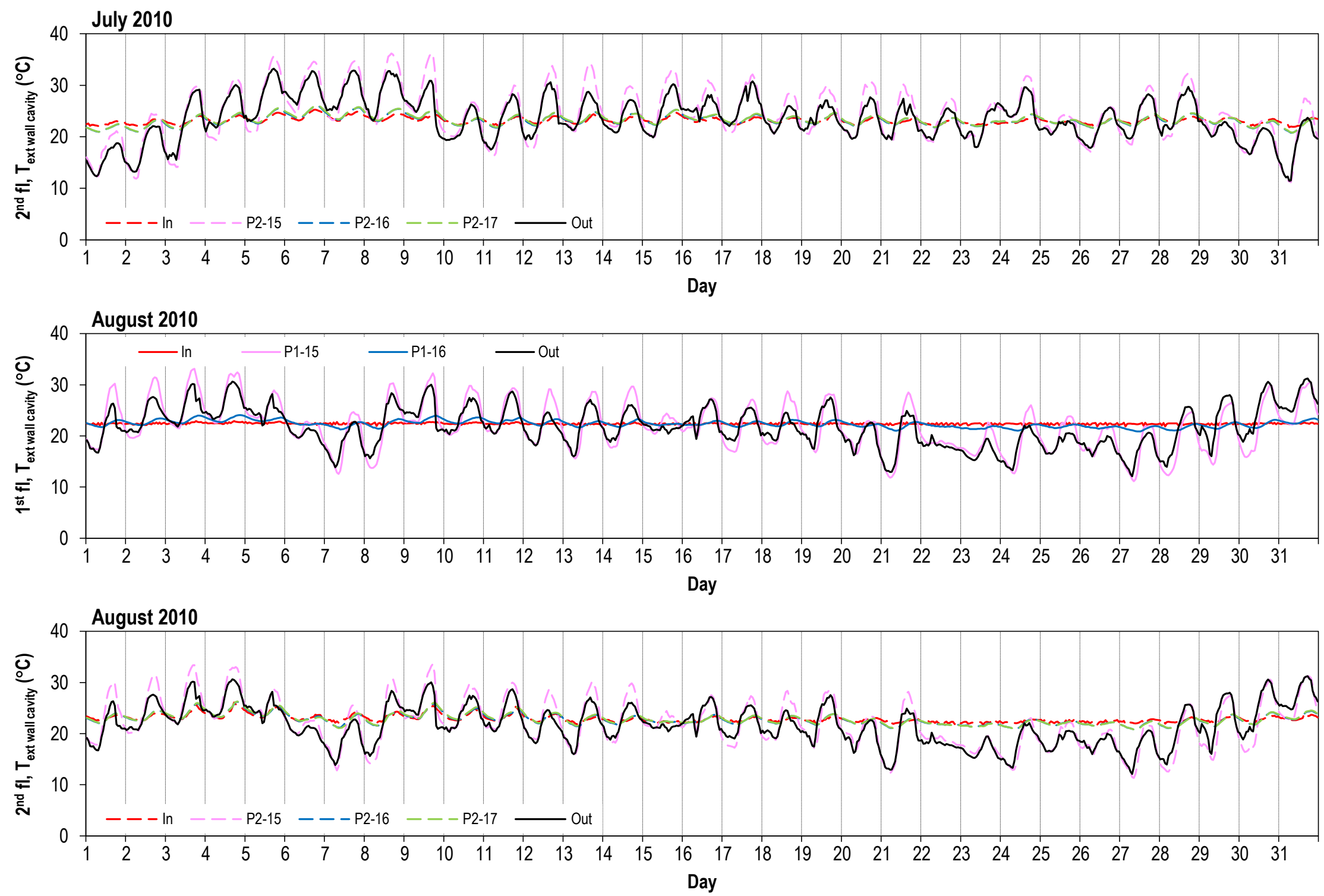
Temperature $\left({ }^{\circ} \mathrm{C}\right)$ at interior side of stud cavity. Refer to Figures 25 thru 28 for sensor location.
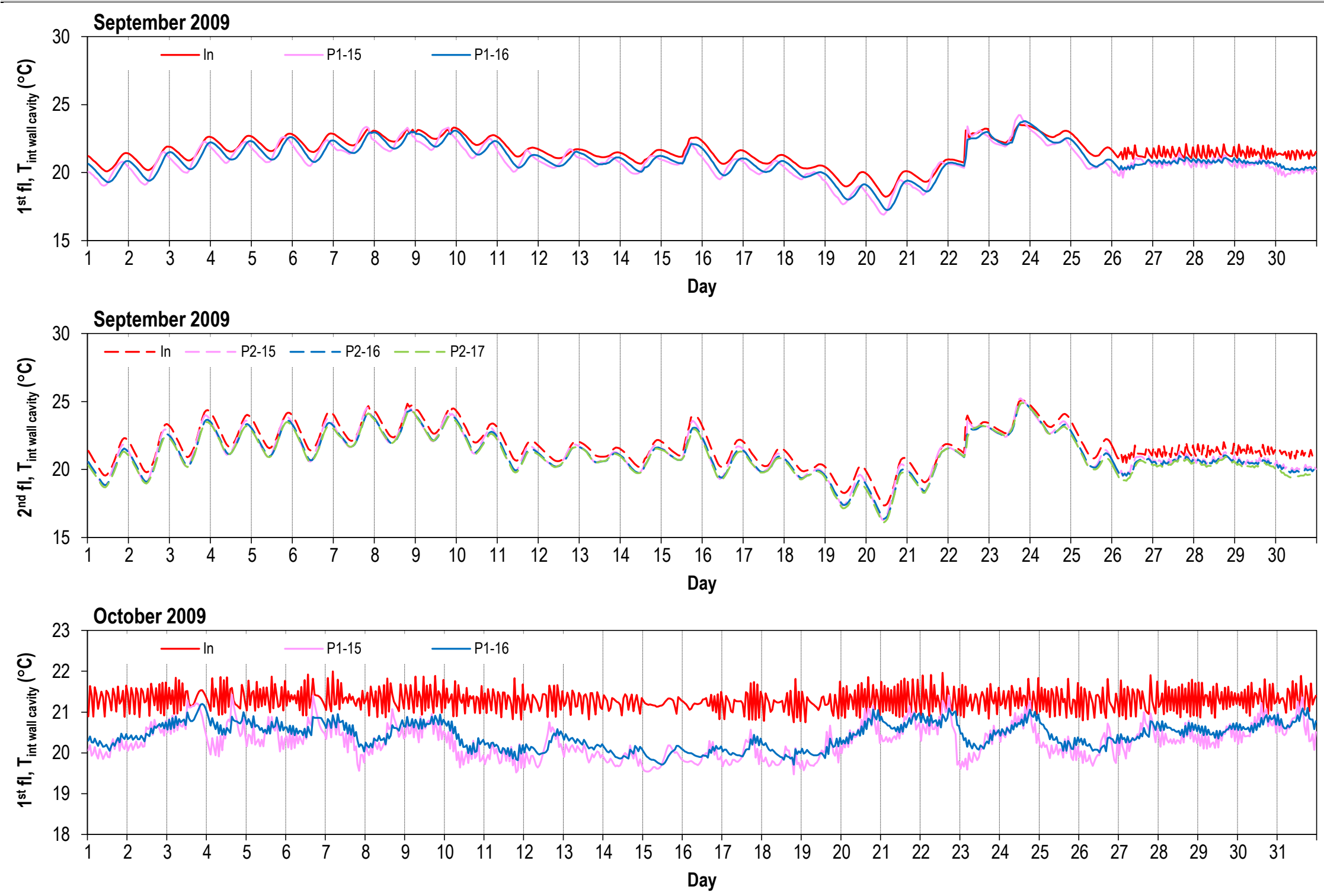

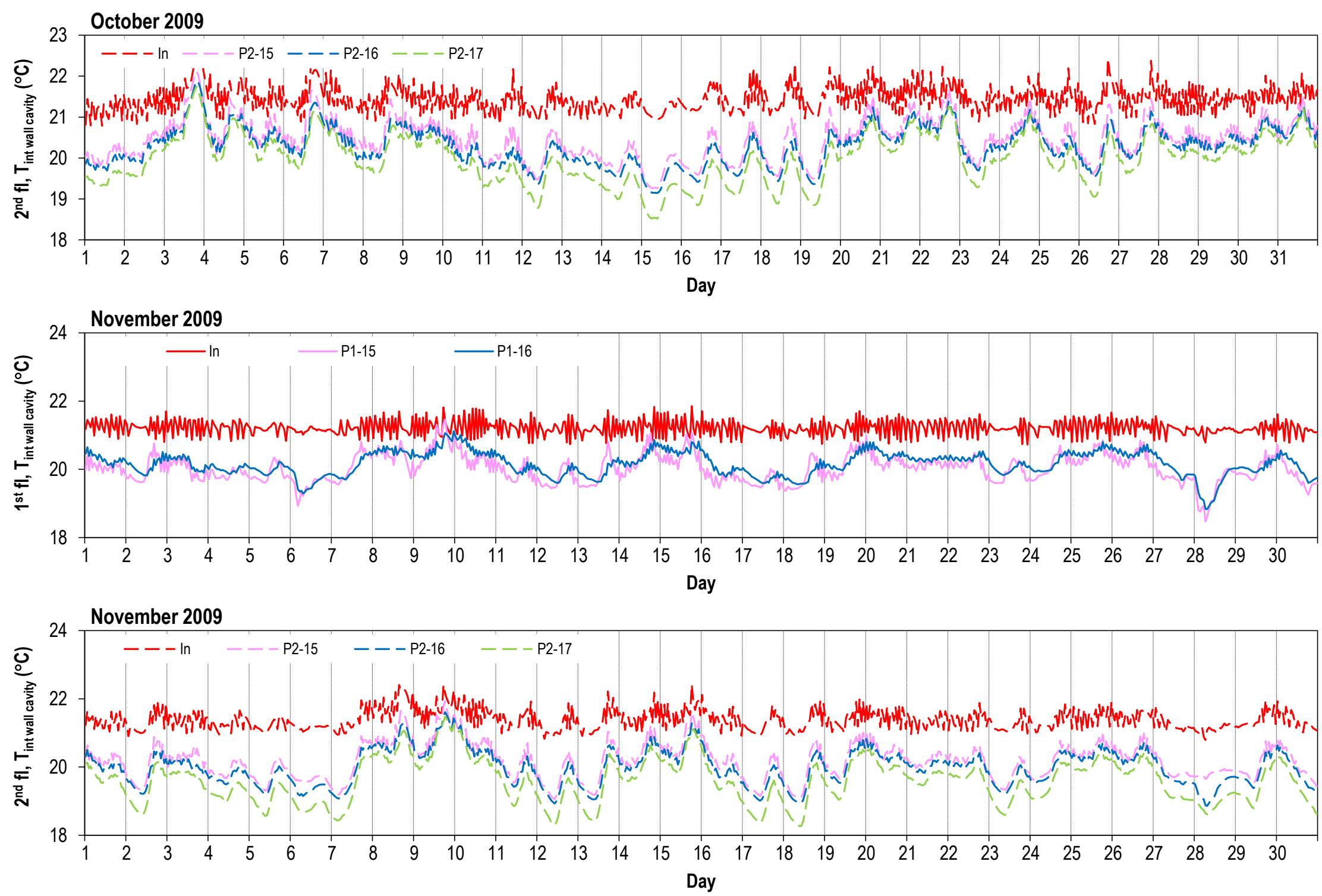

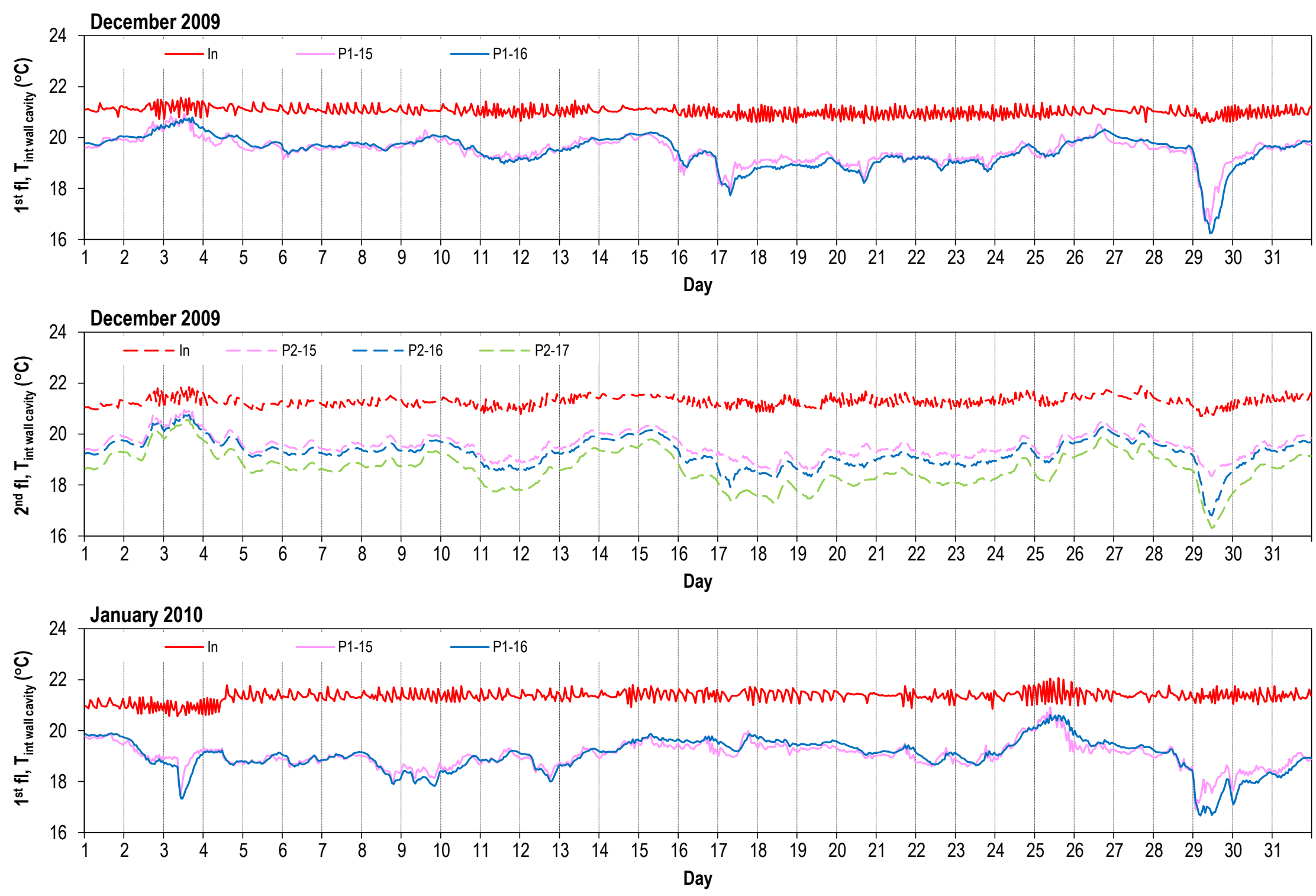

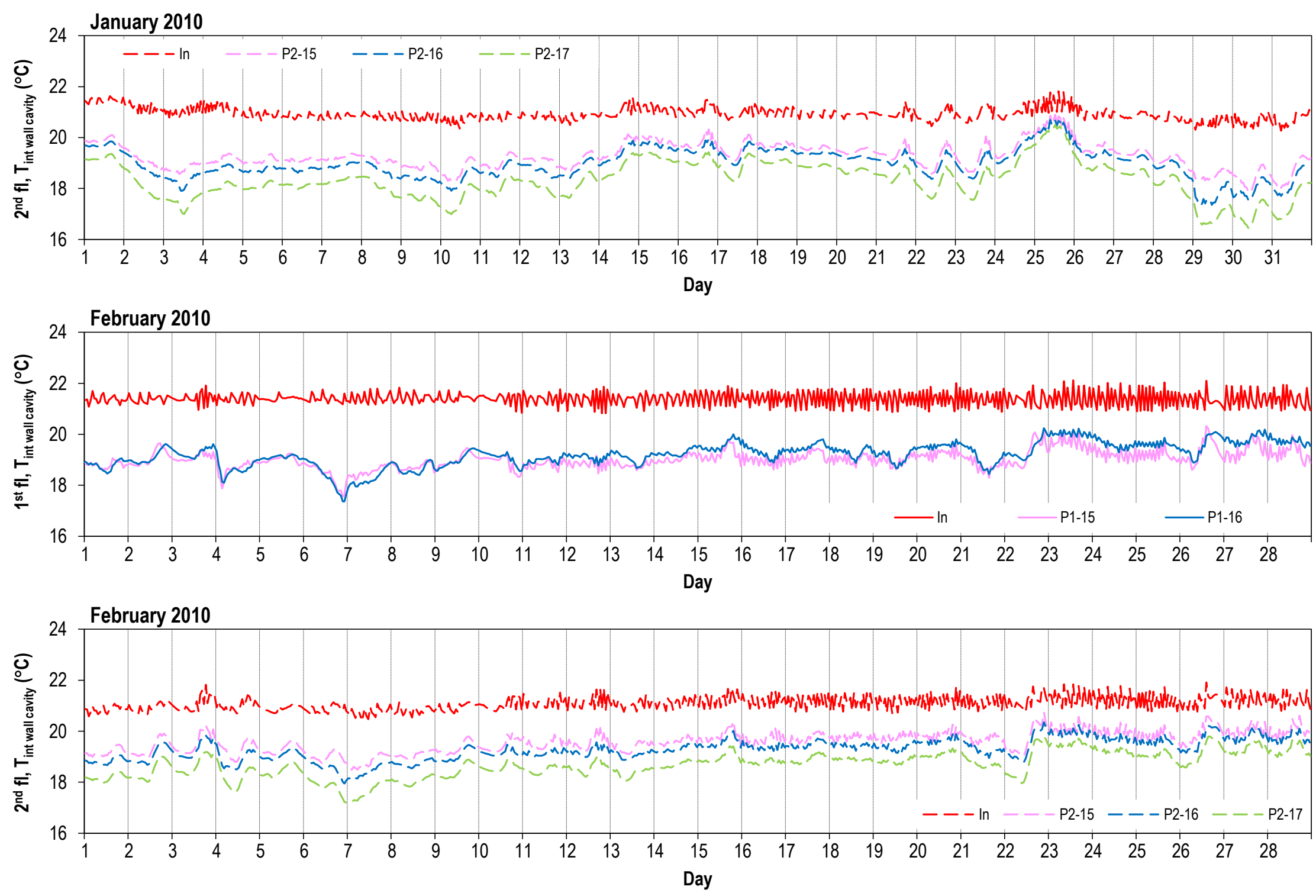

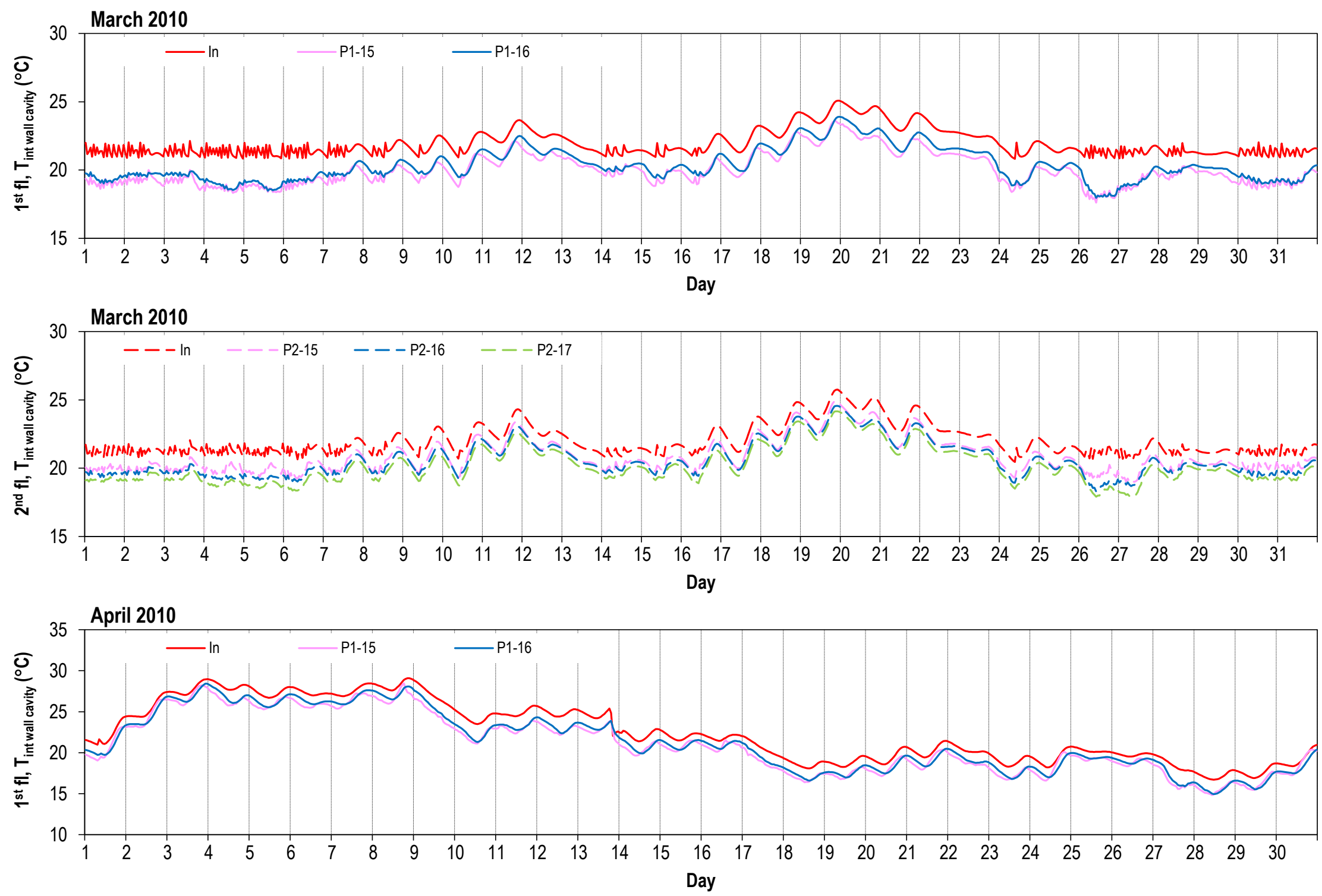

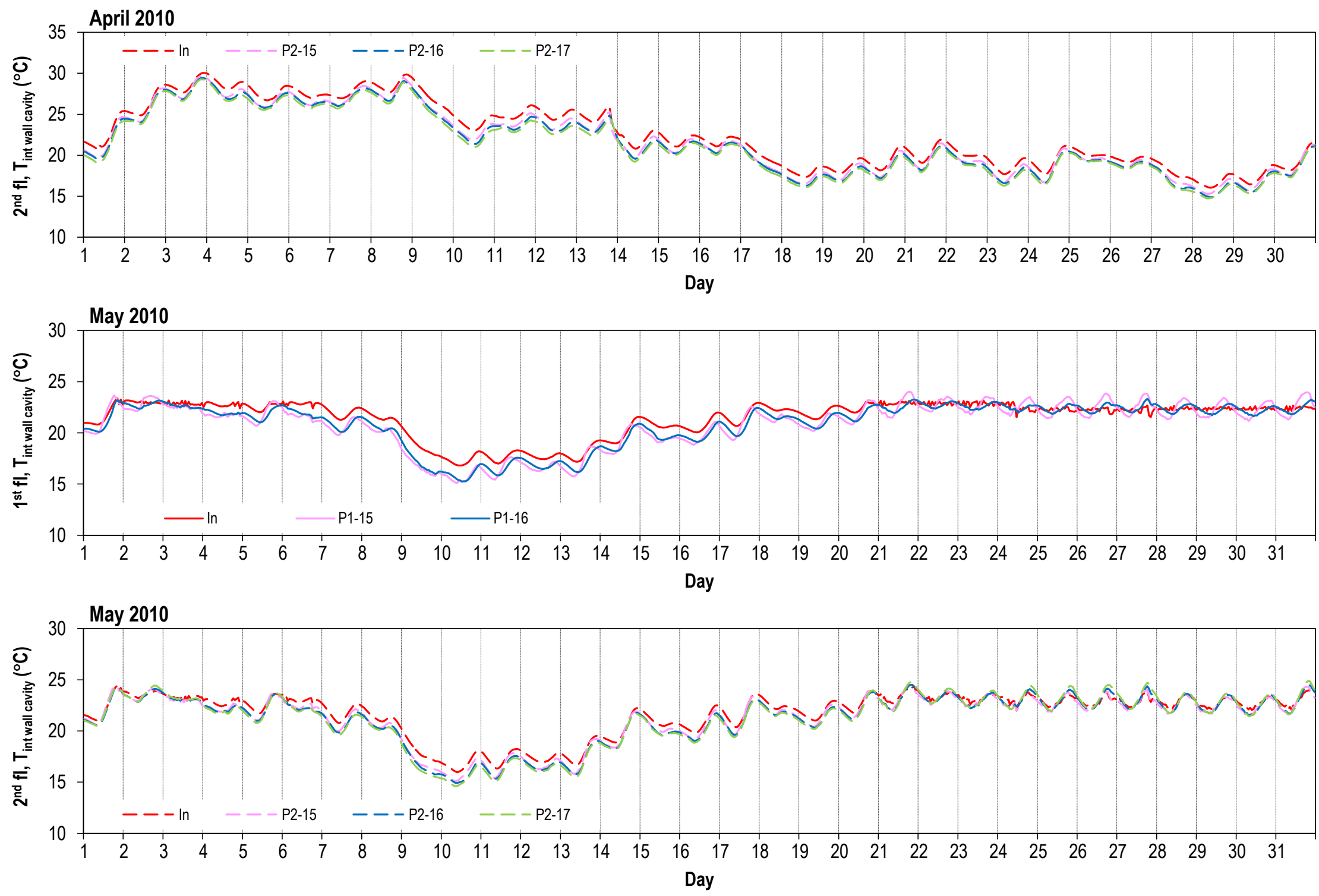

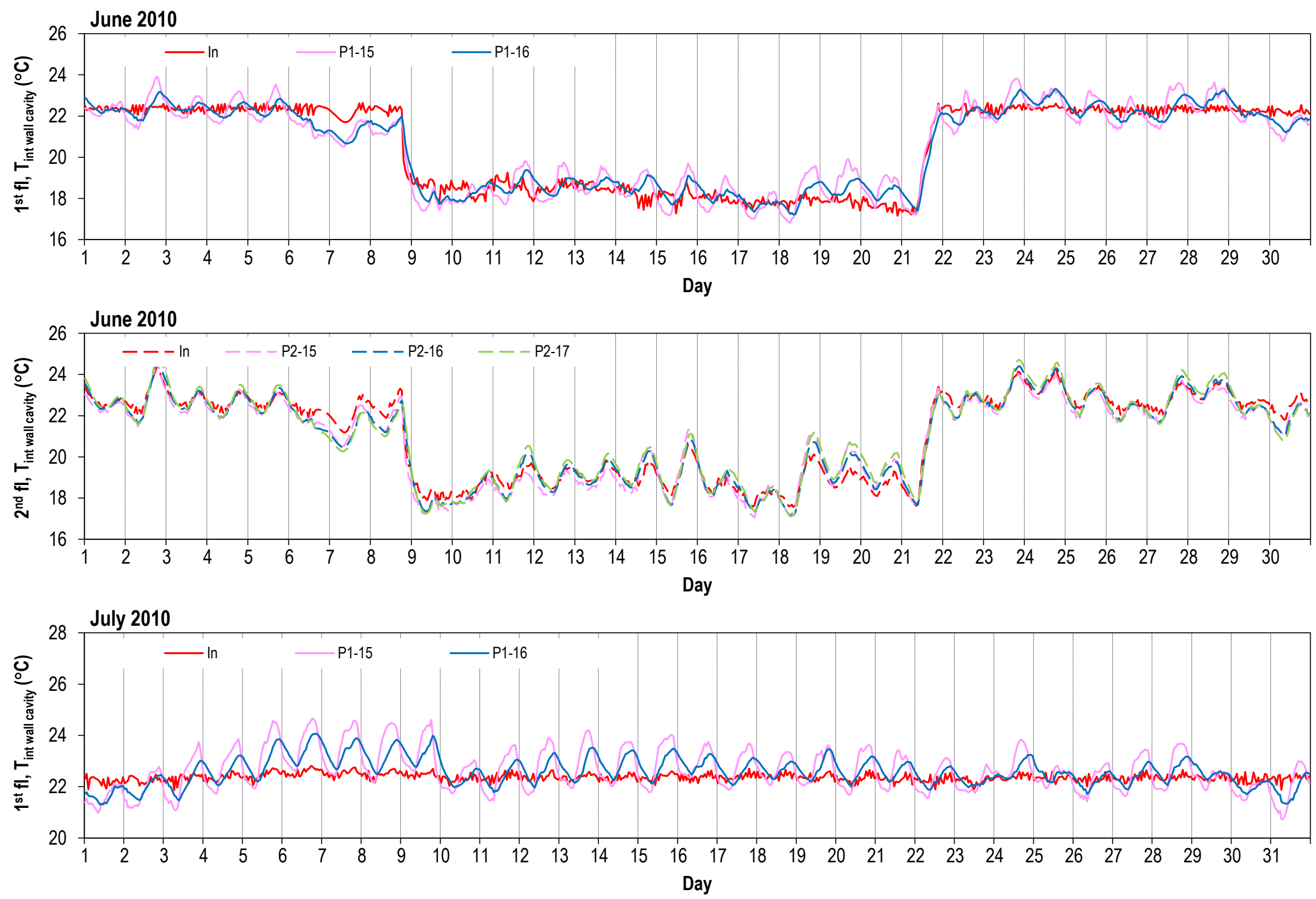

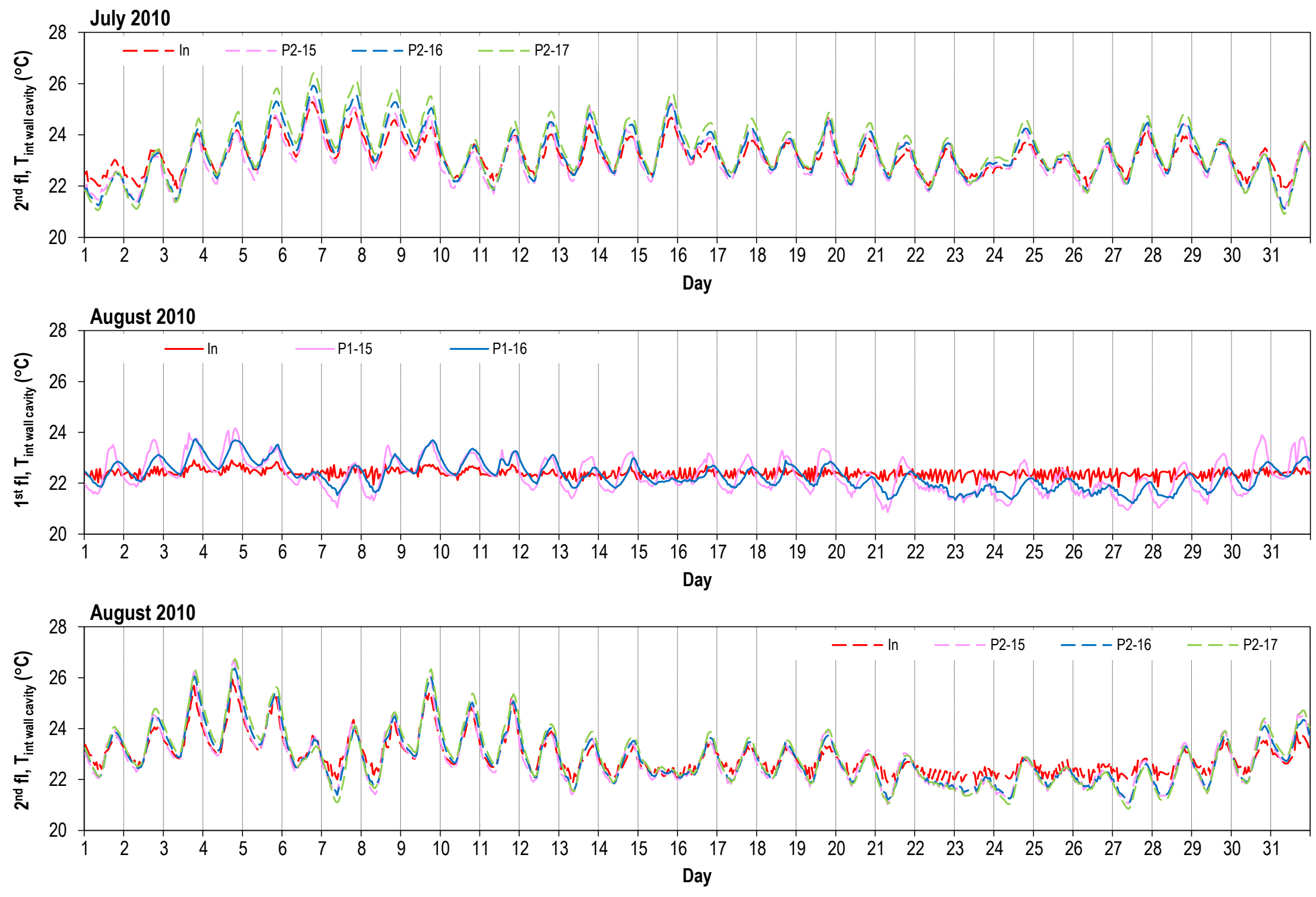
Relative humidity (\%) at exterior side of stud cavity. Refer to Figures 25 thru 28 for sensor location.

Data from panel P2-15 is not shown because of equipment malfunction.
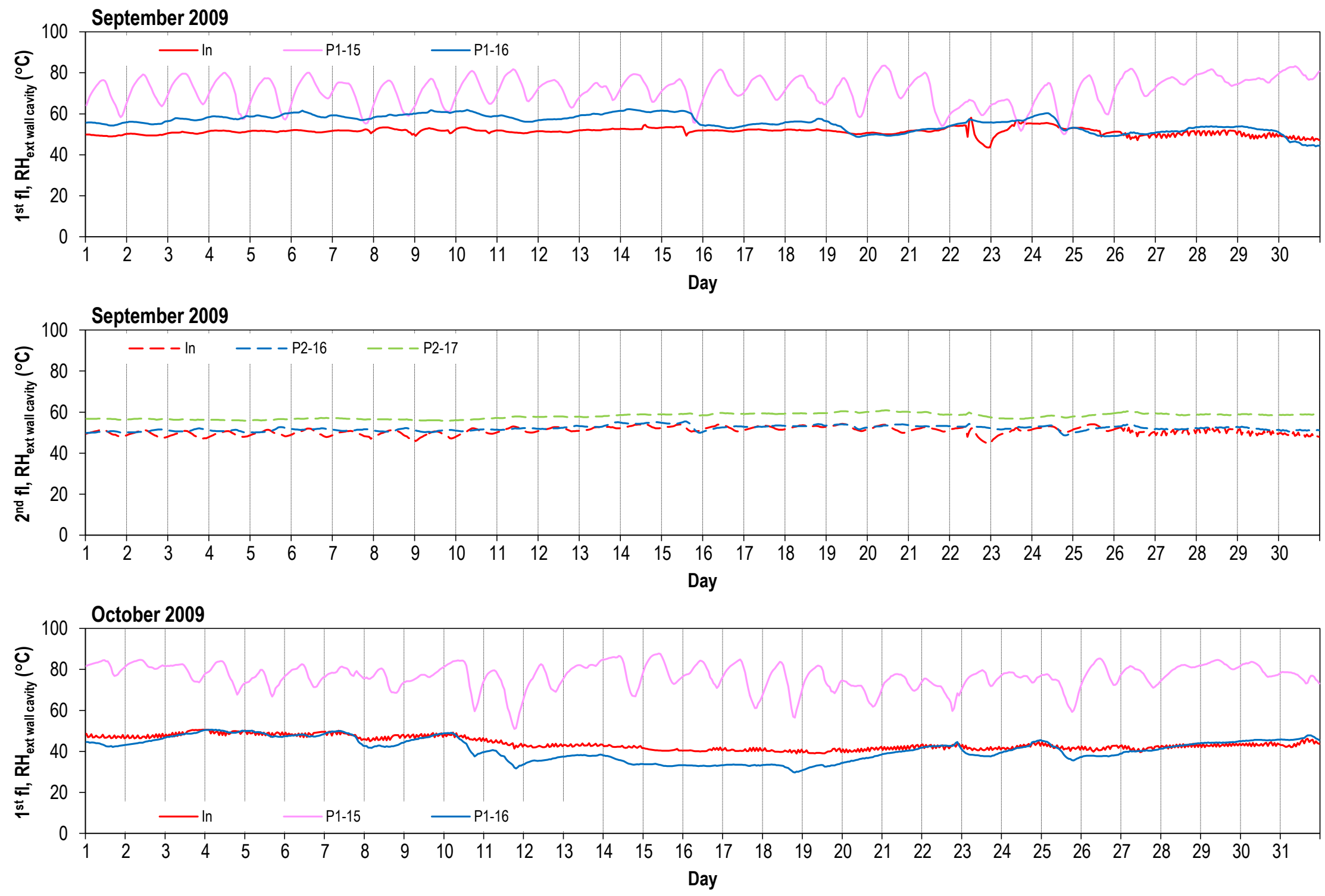

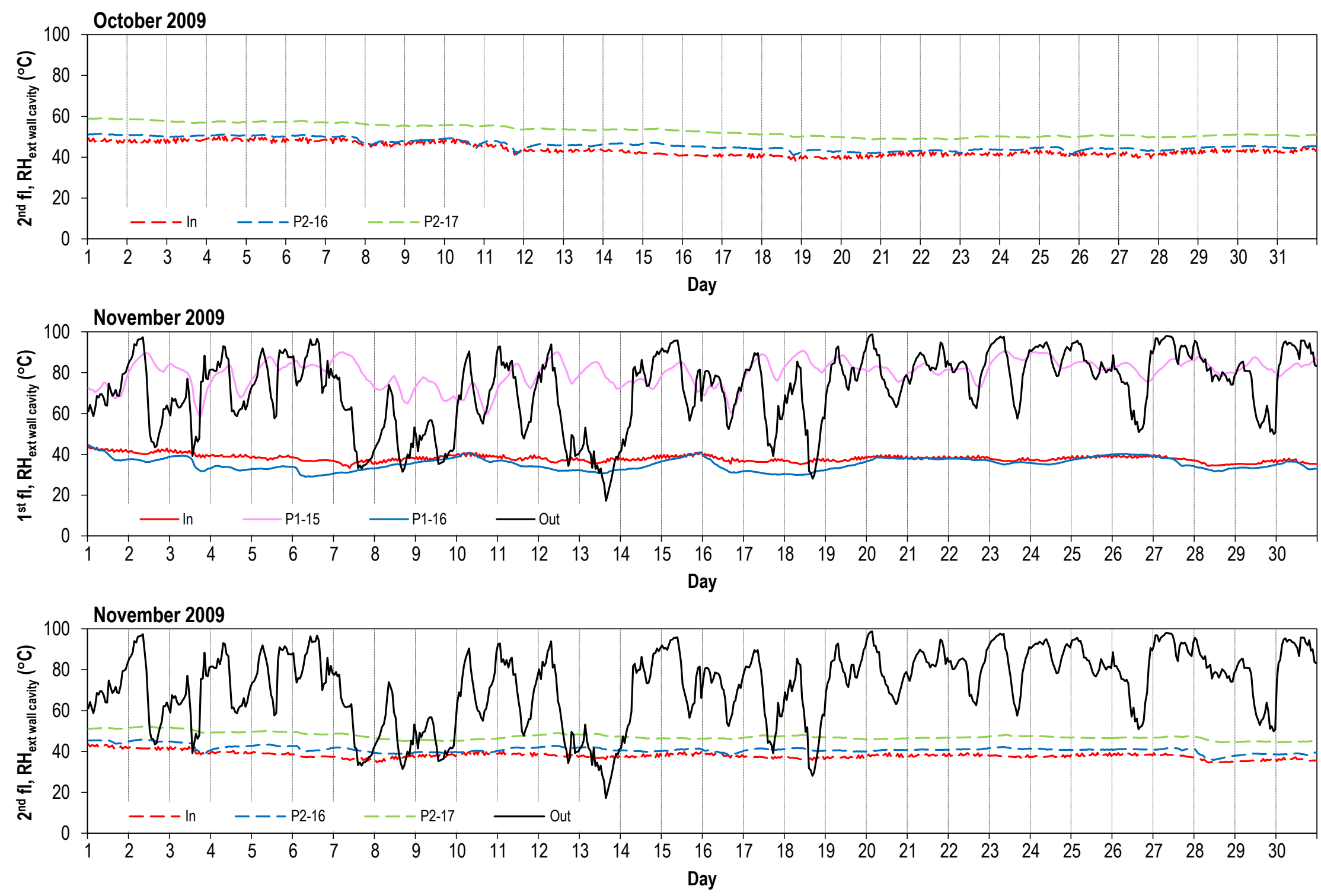

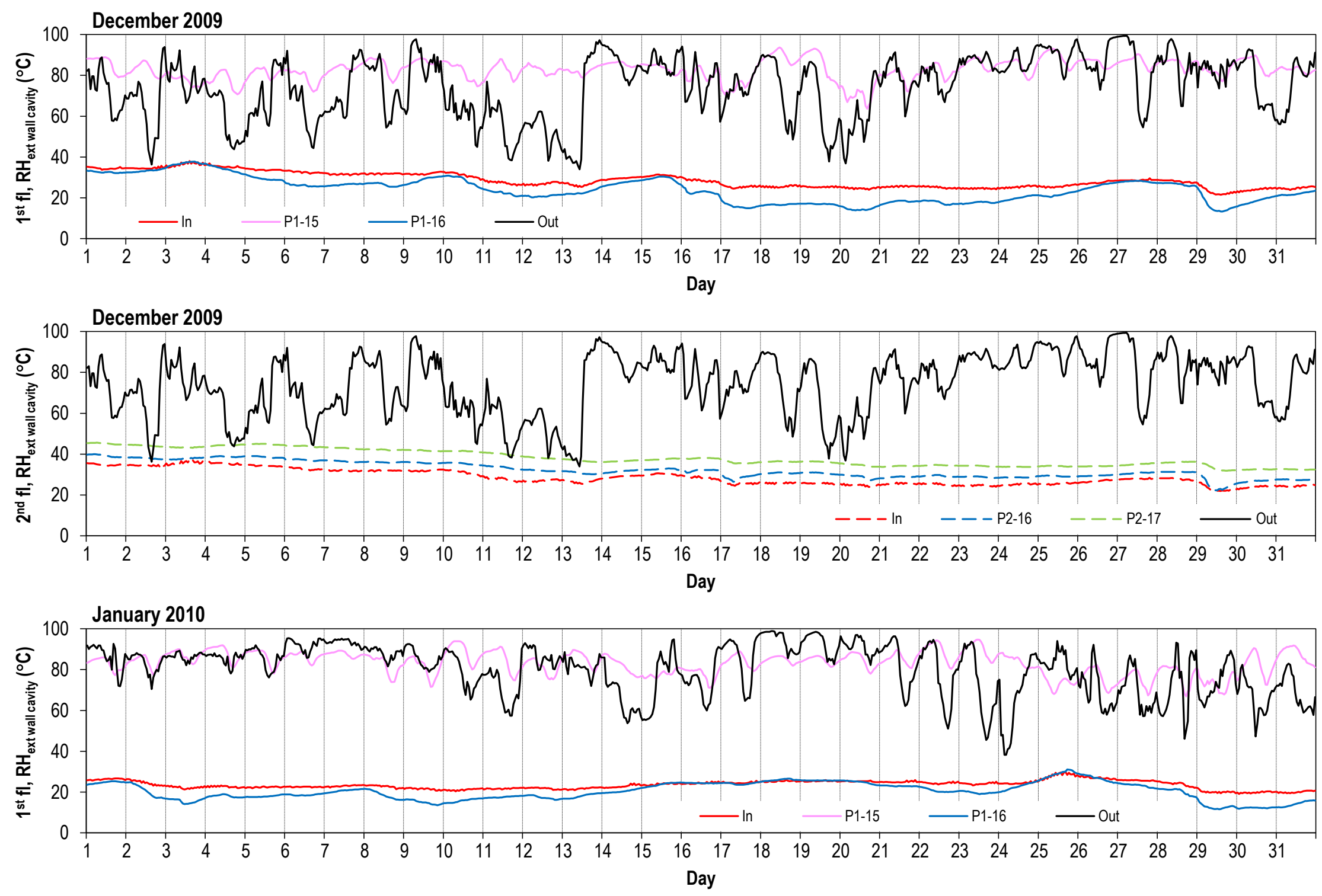

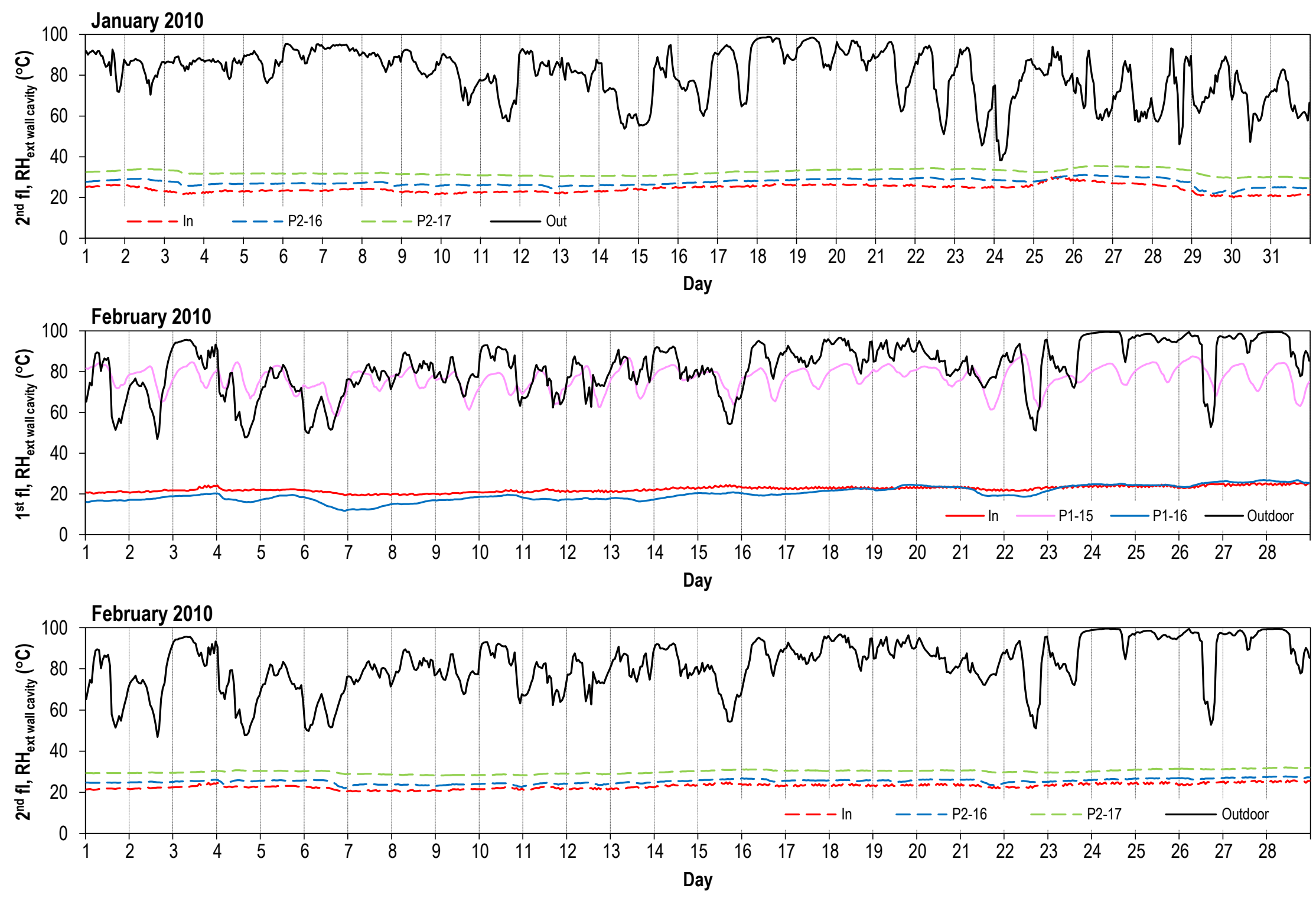

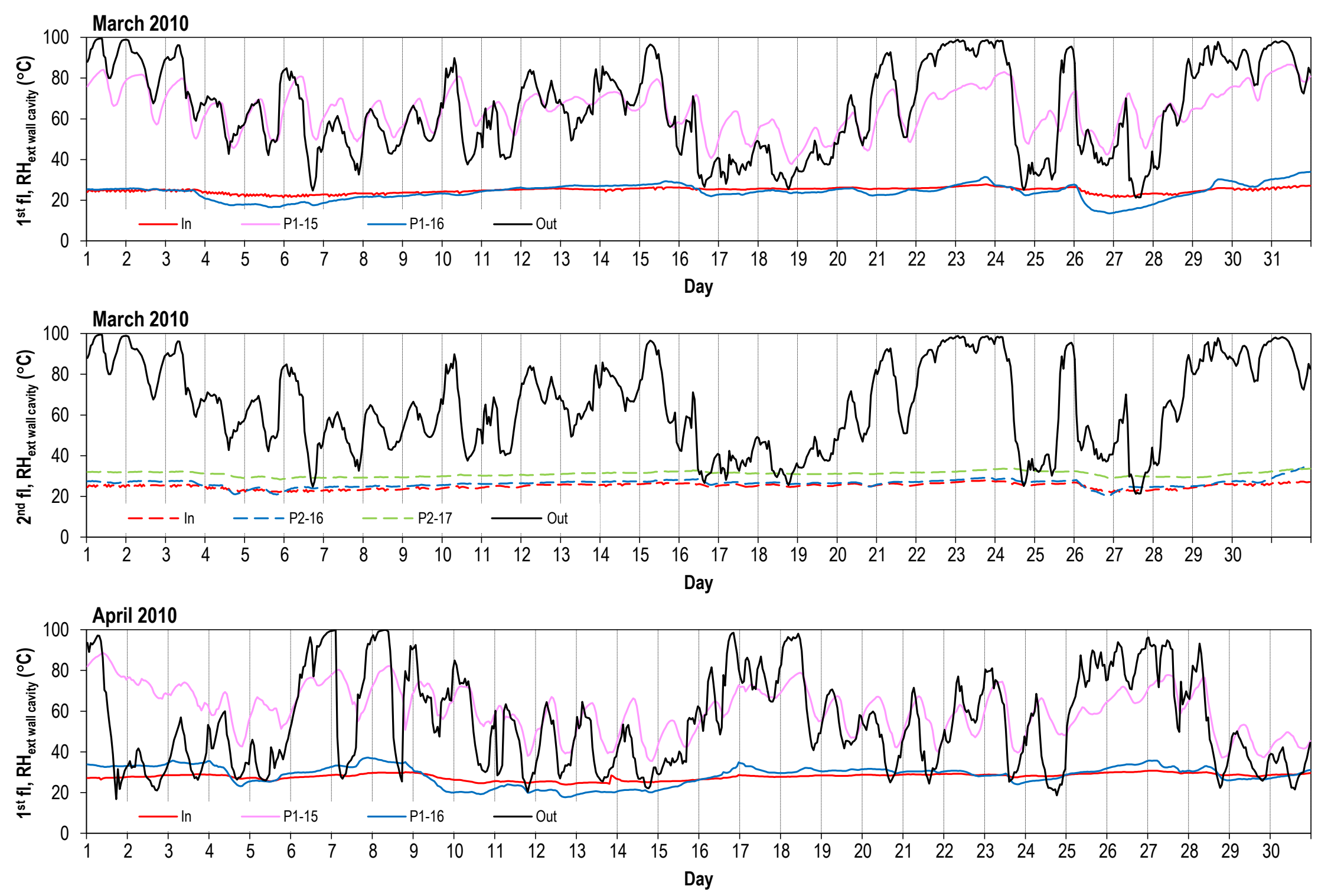

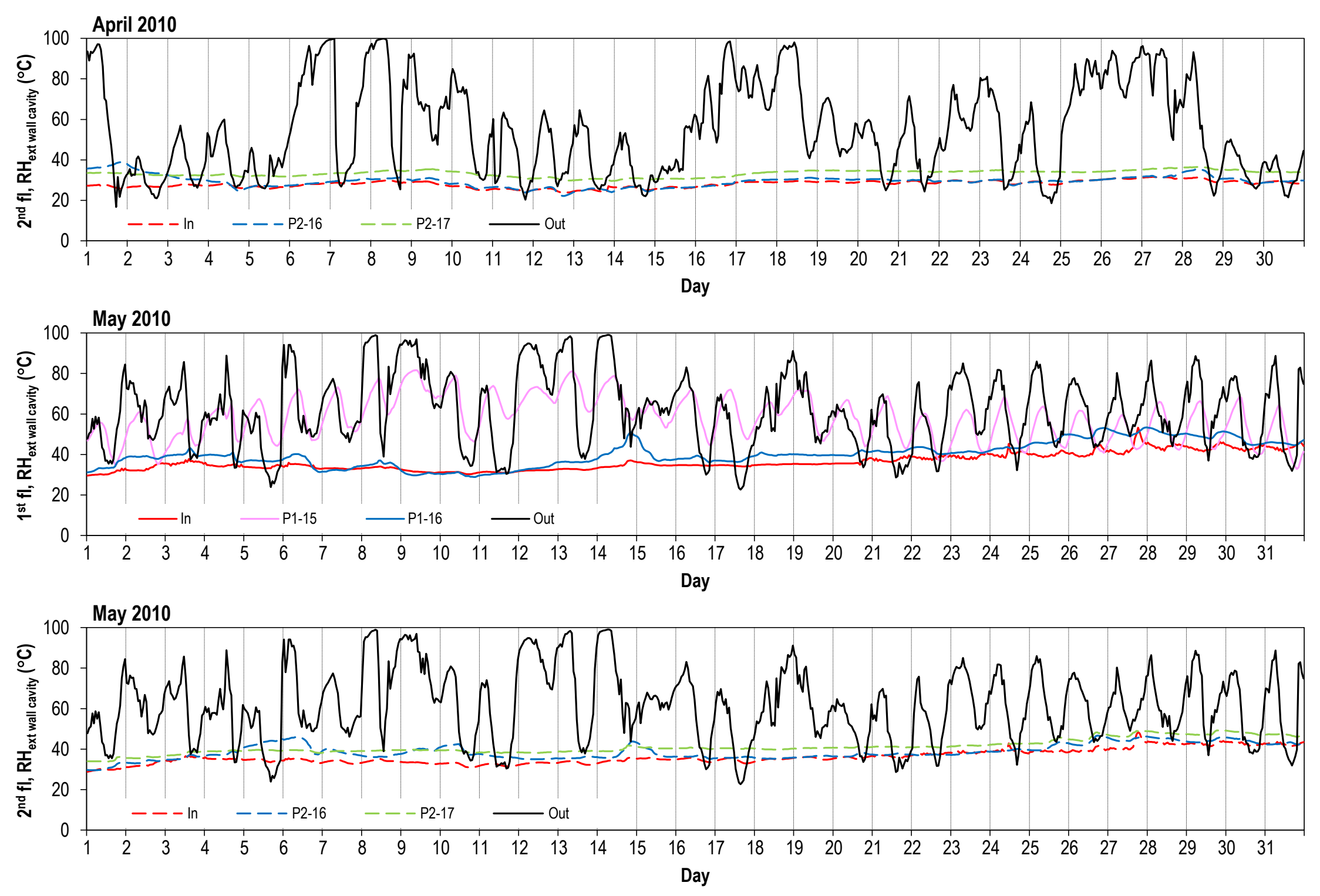

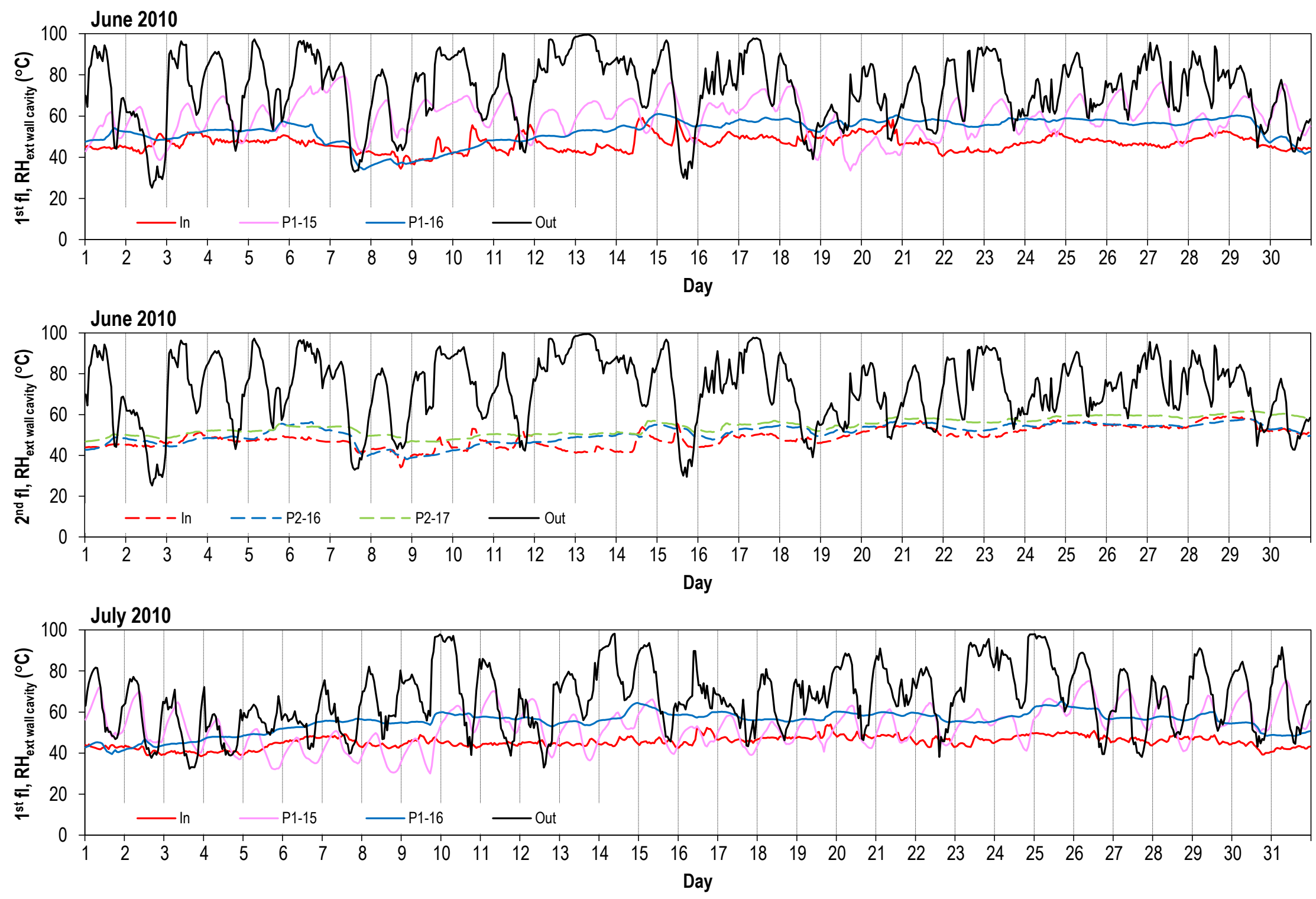

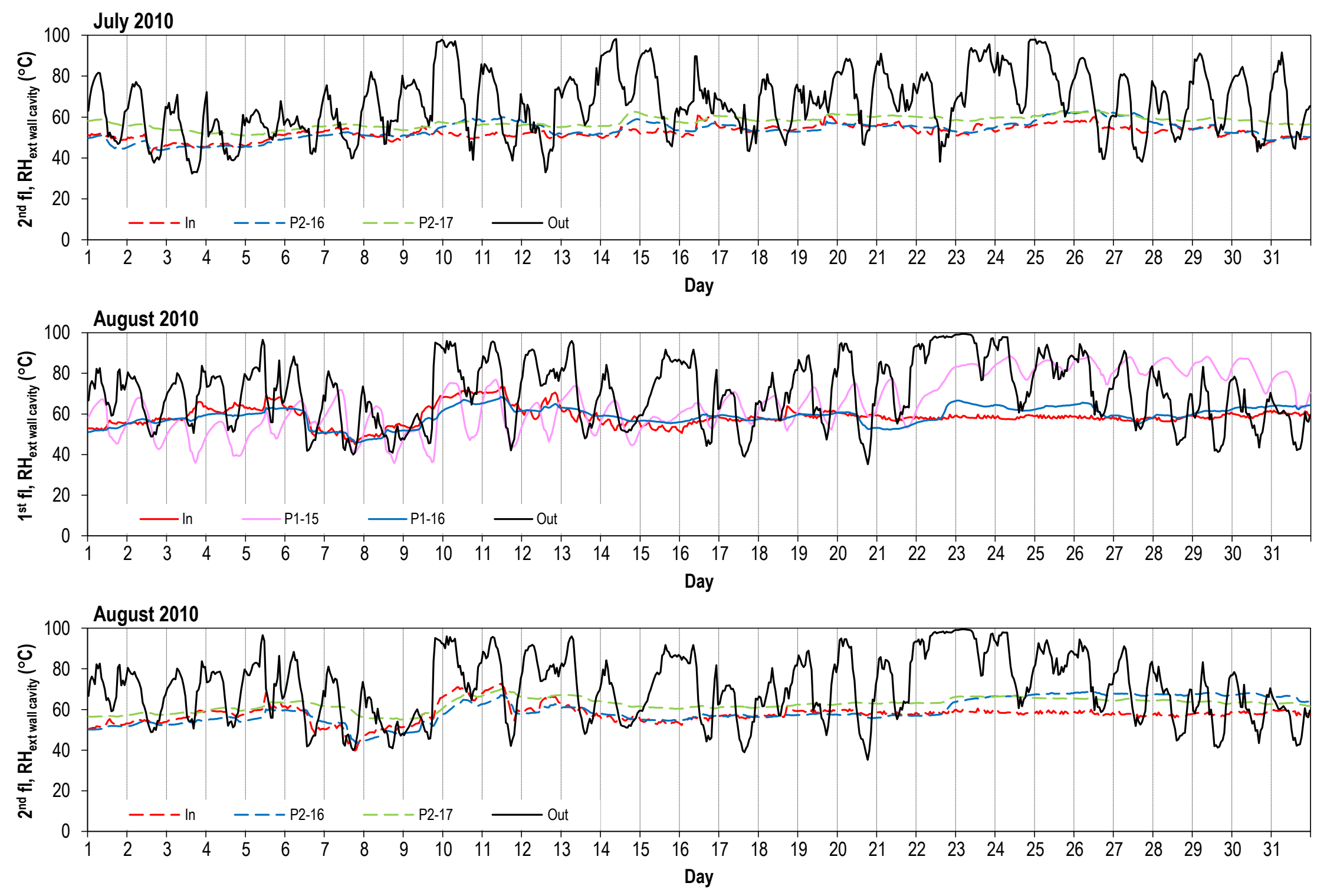

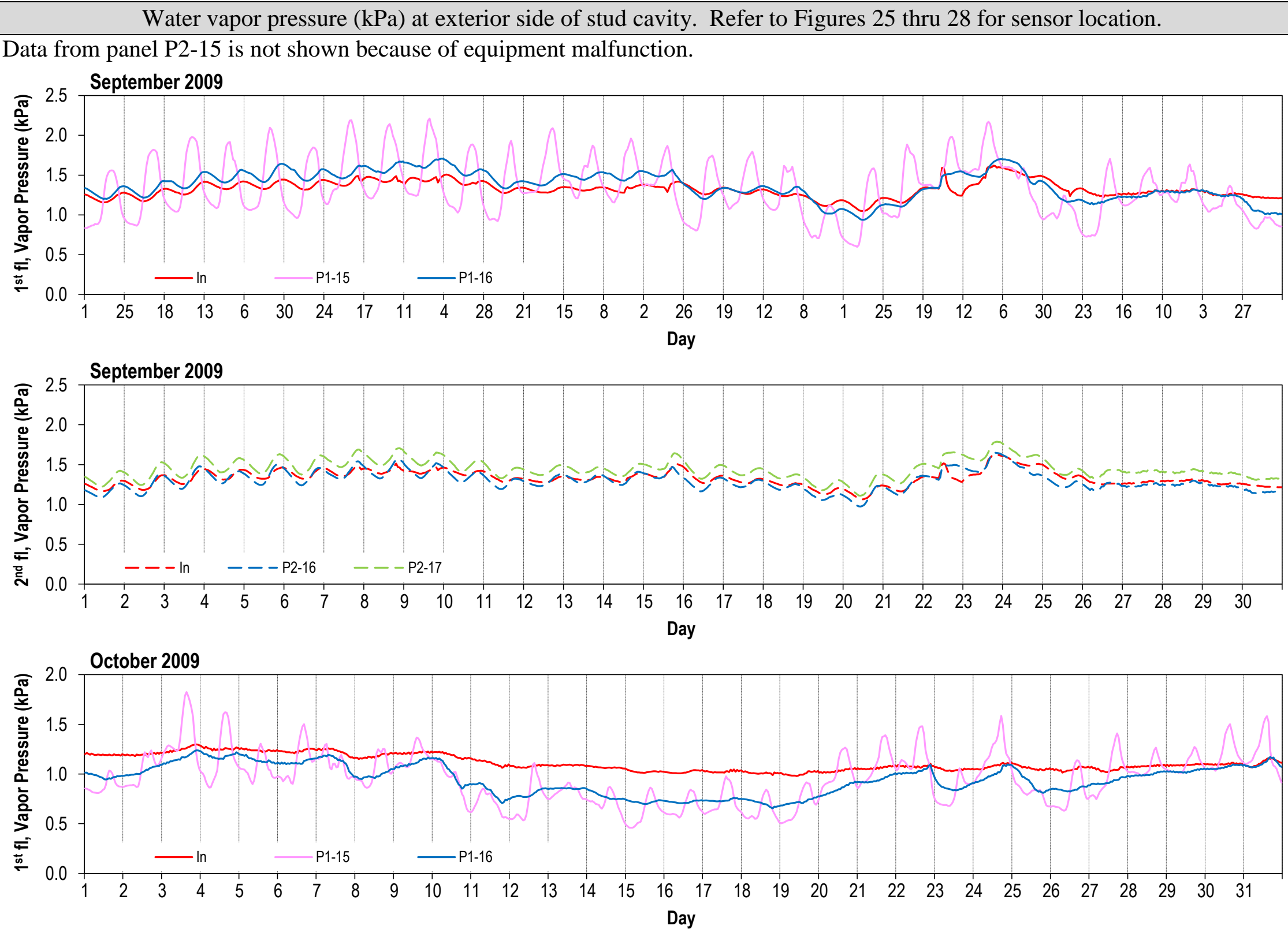

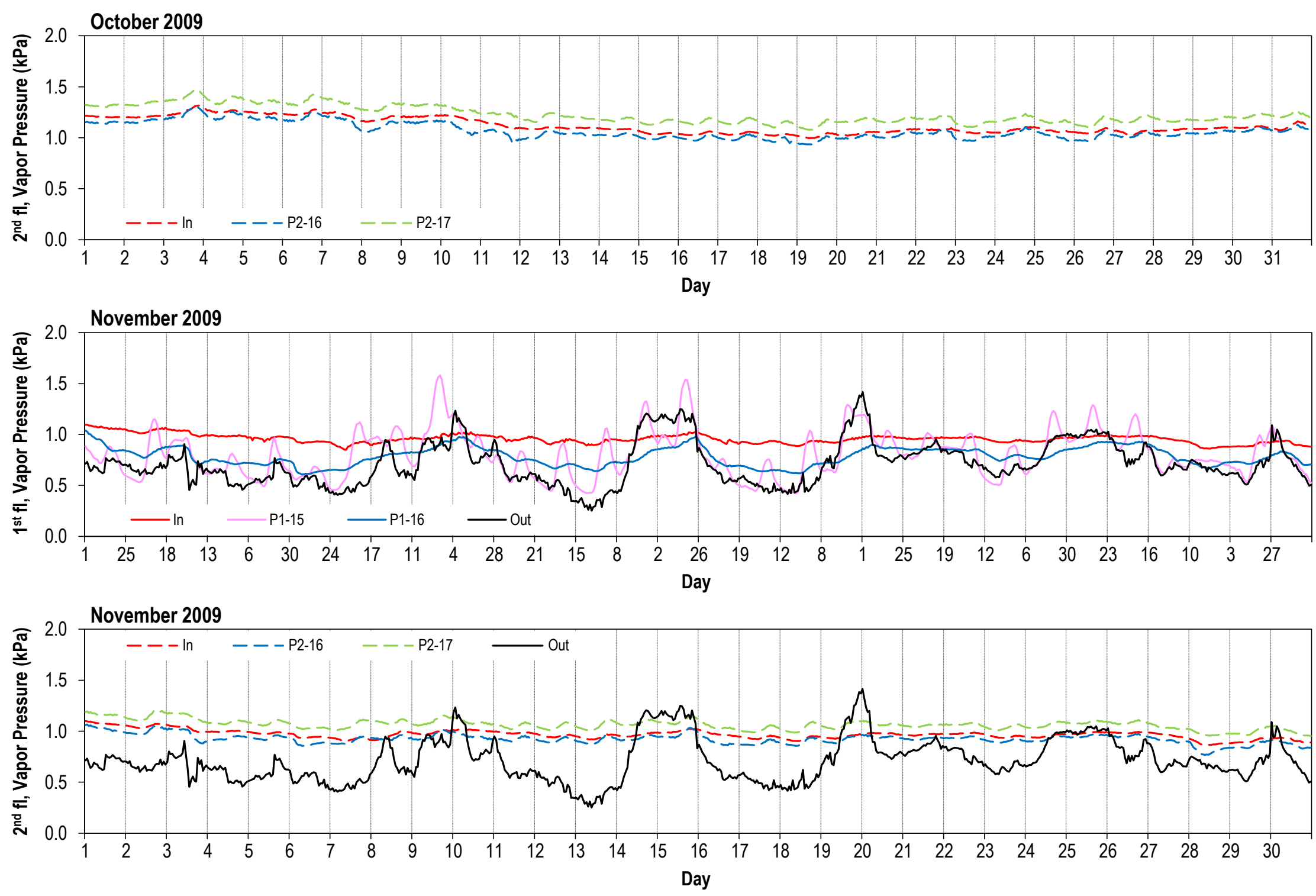

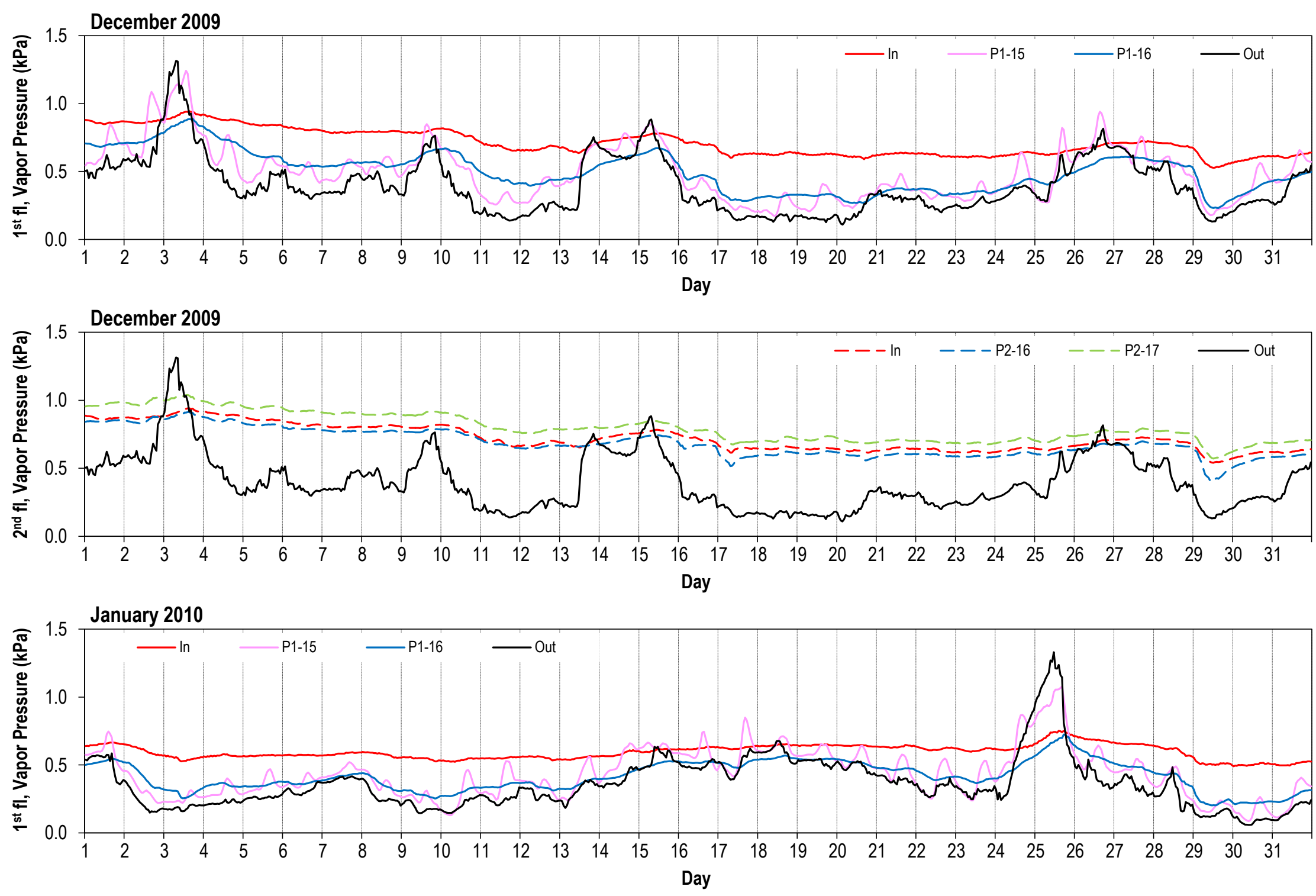

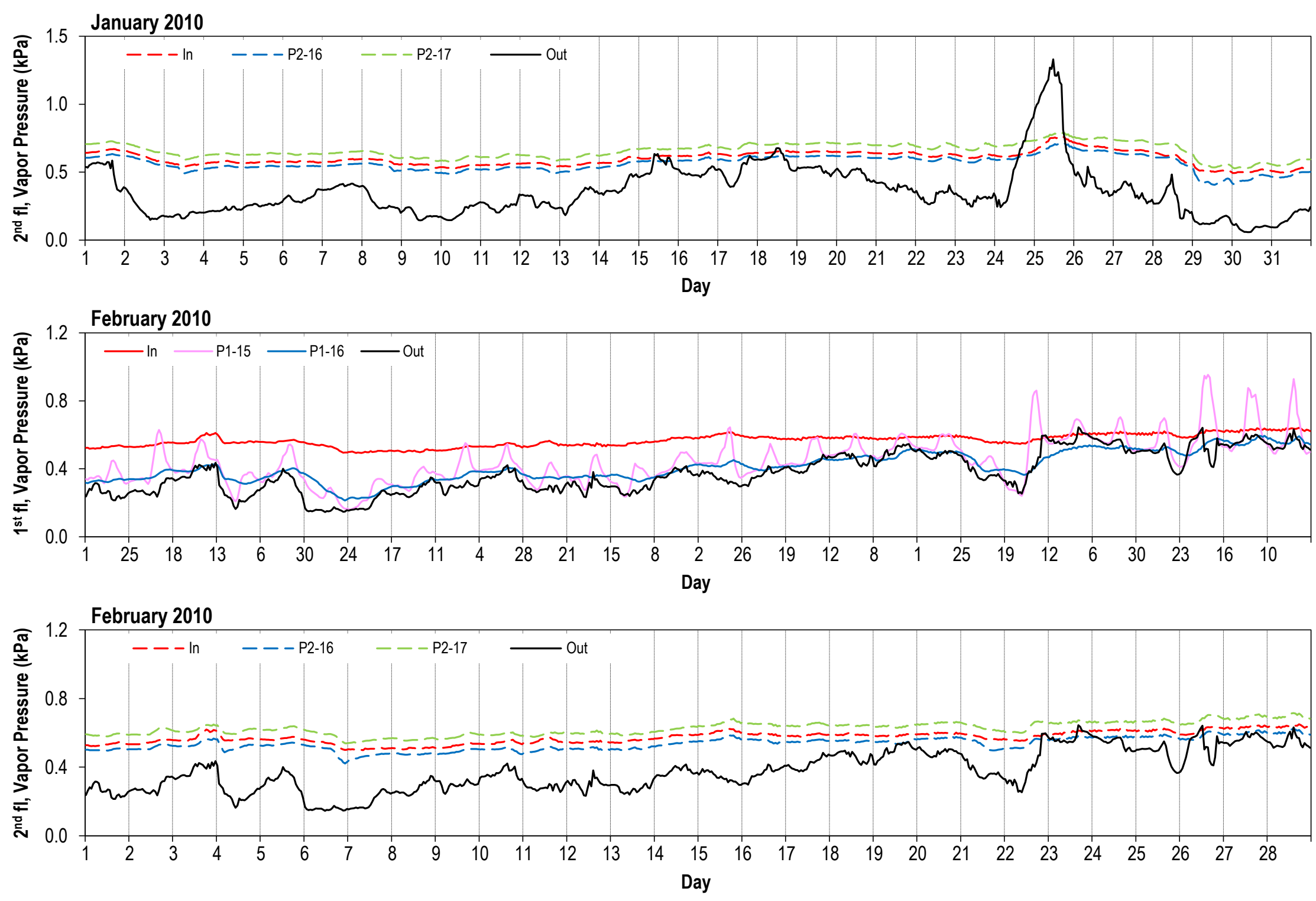

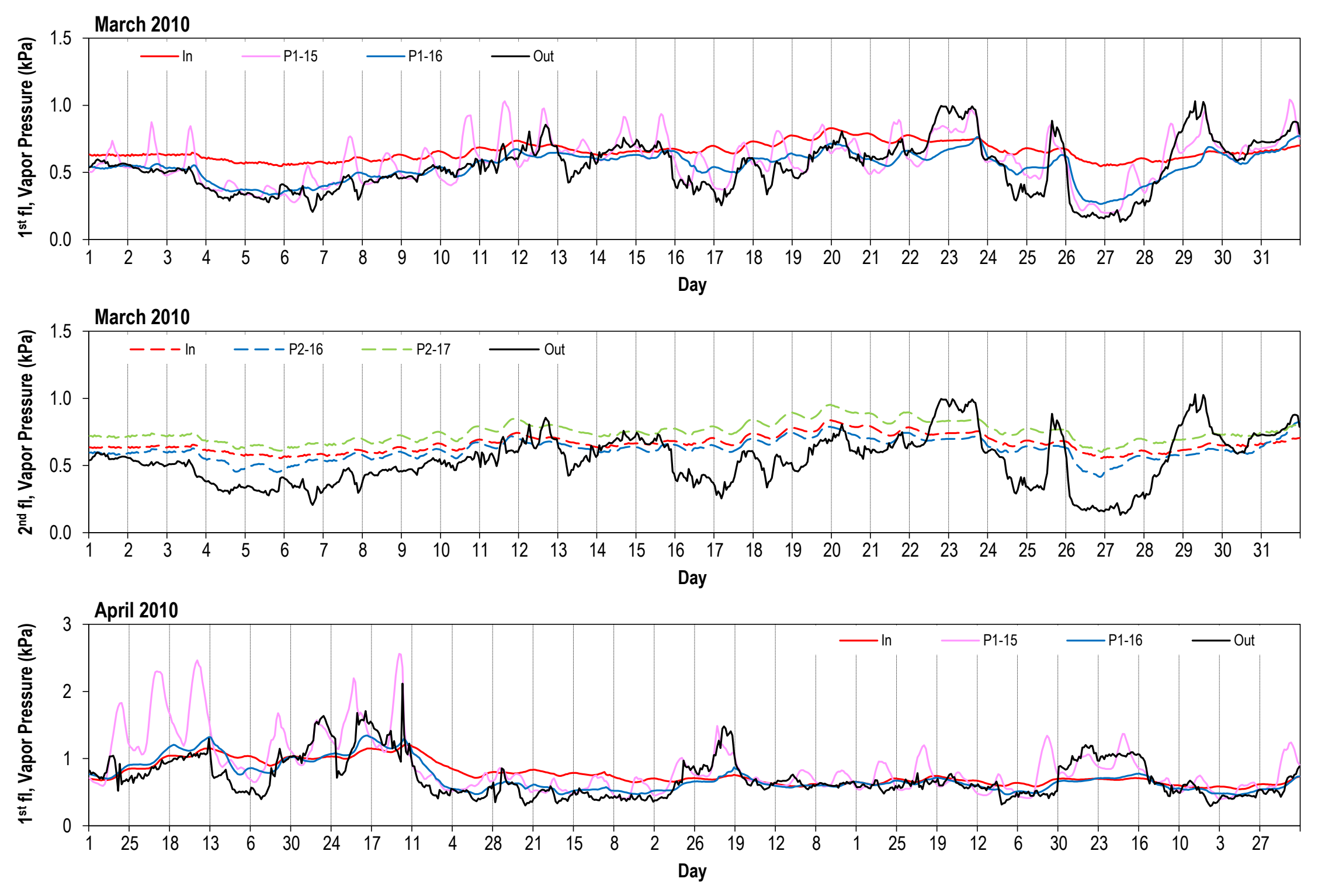

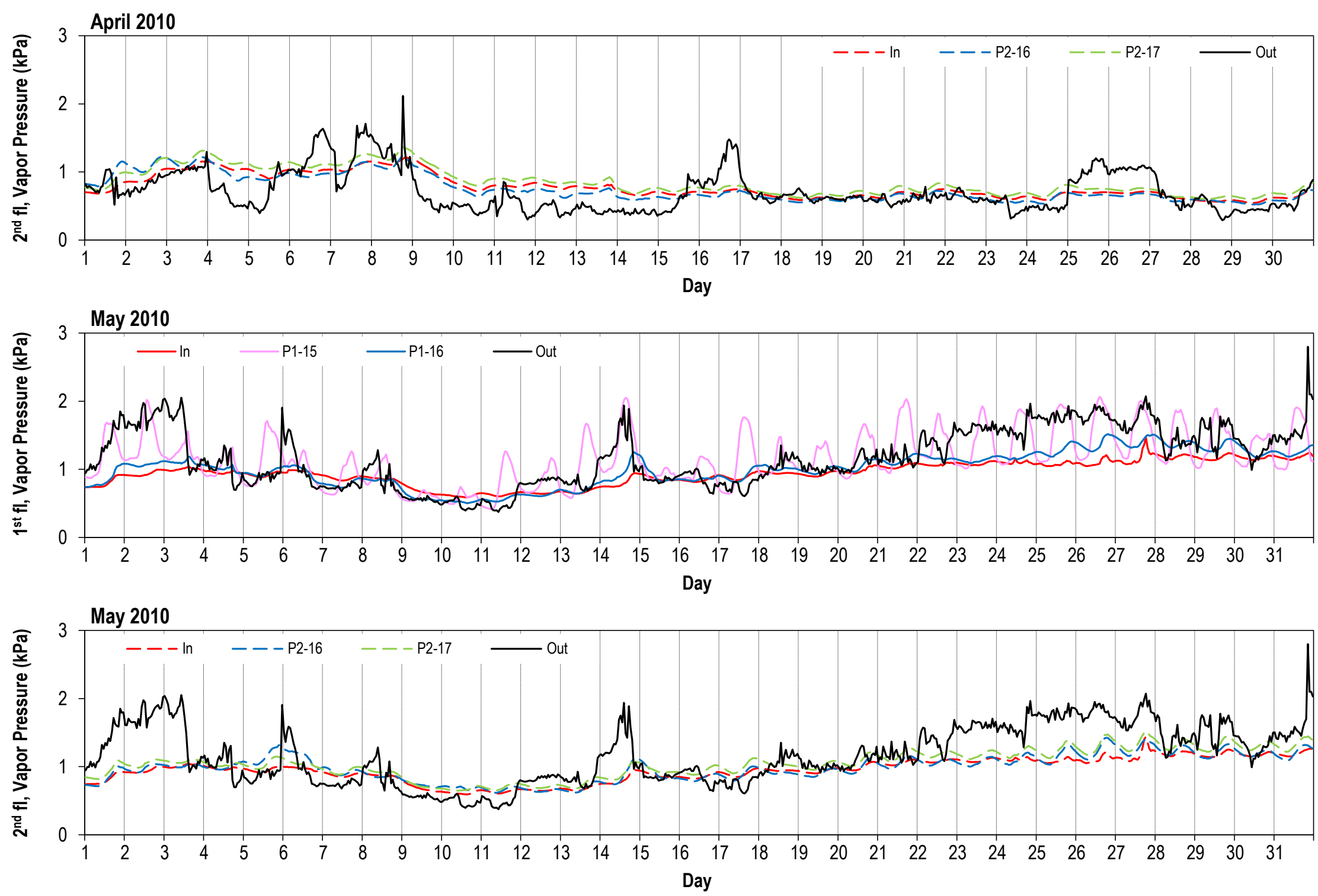

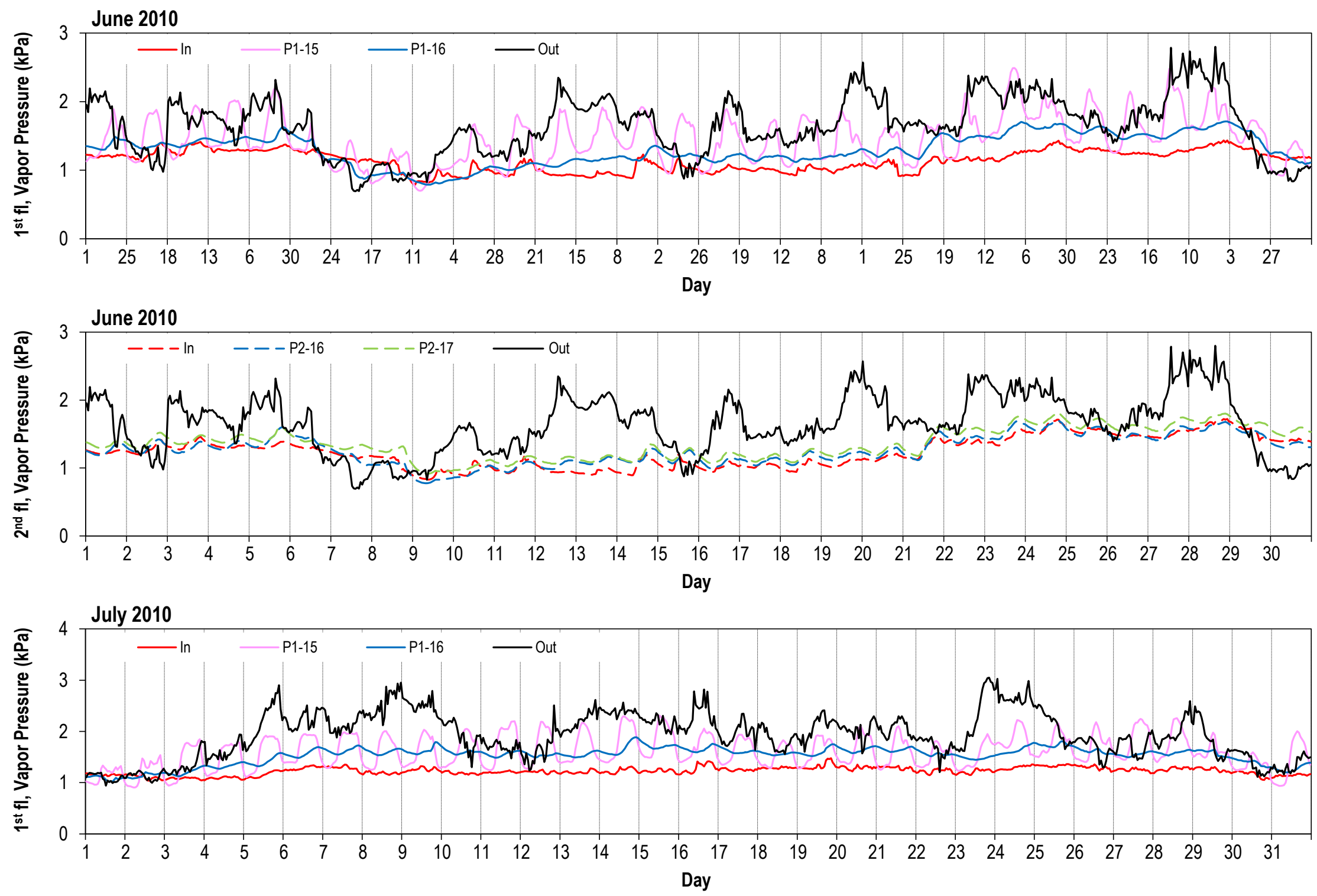

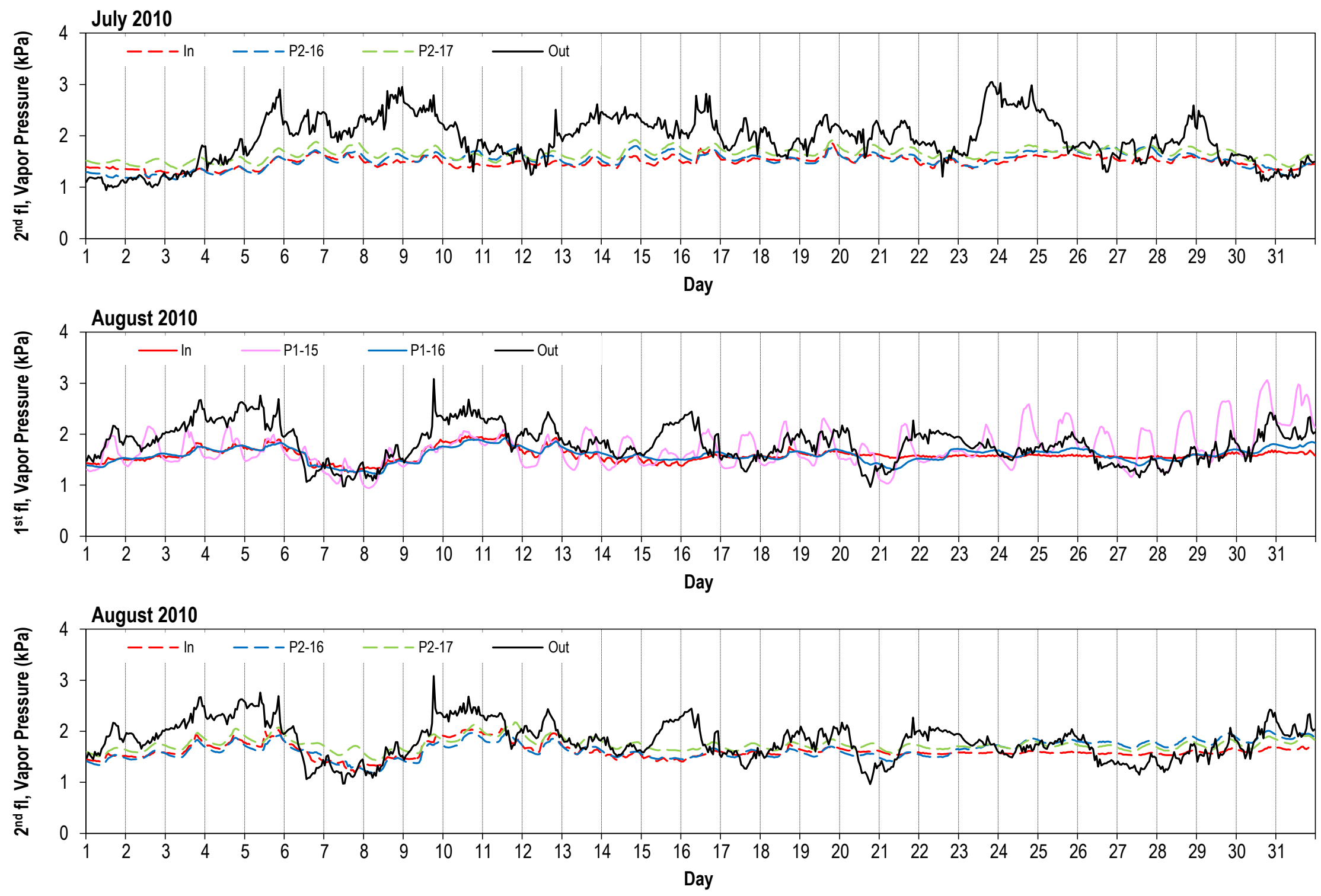
Heat flux $\left(\mathrm{W} / \mathrm{m}^{2}\right)$ thru interior side of stud cavity. Refer to Figures 25 thru 28 for sensor location.
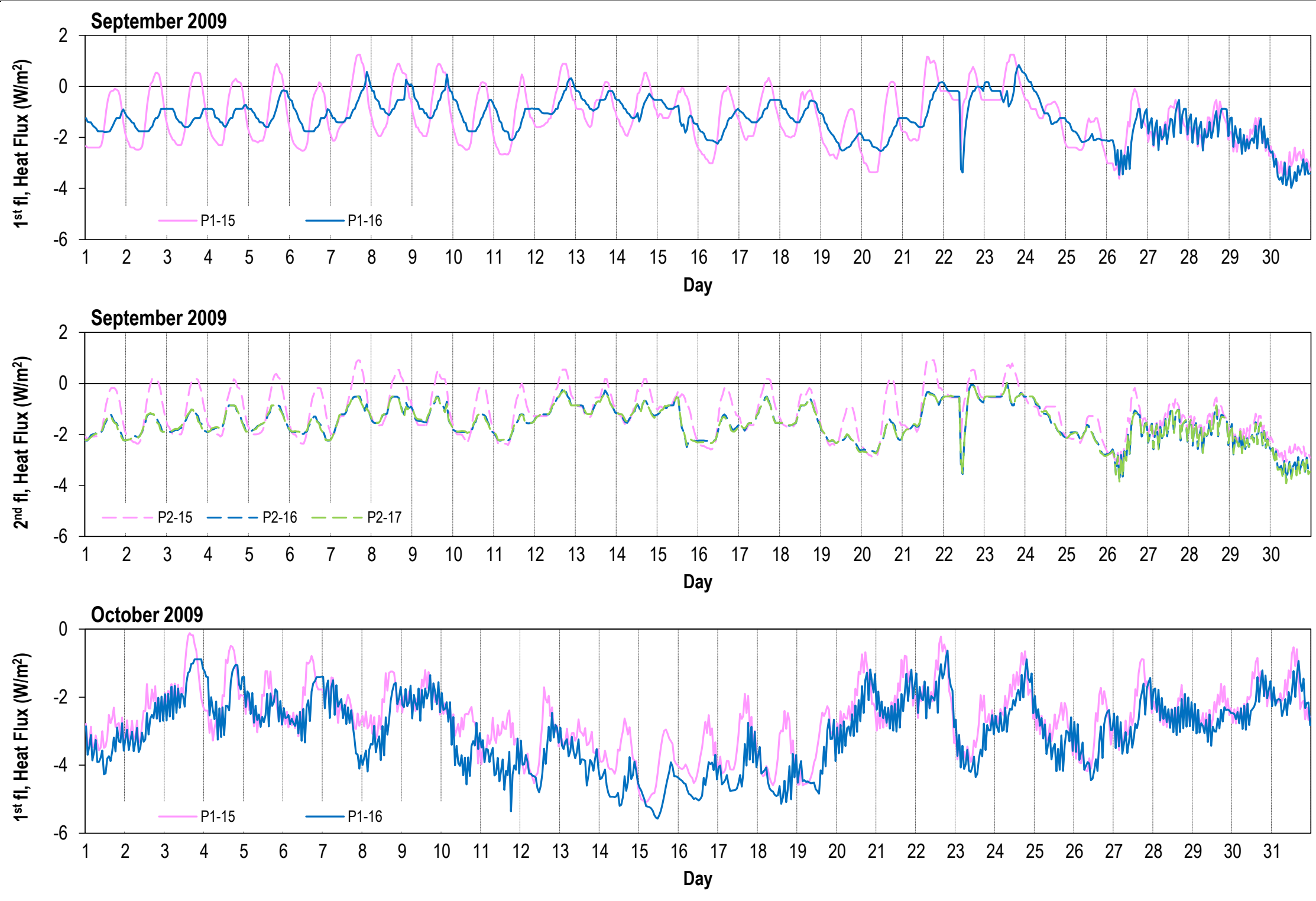

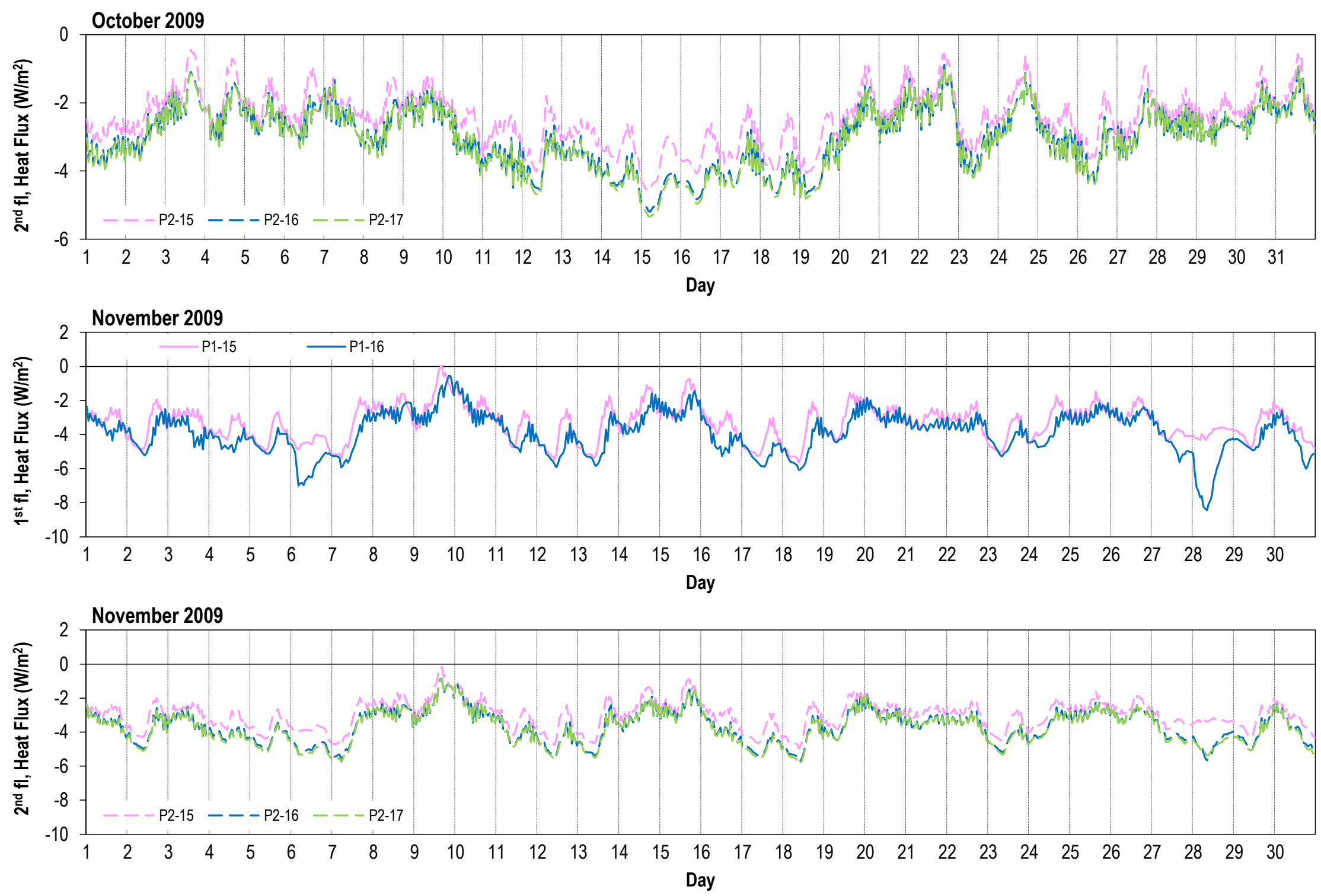

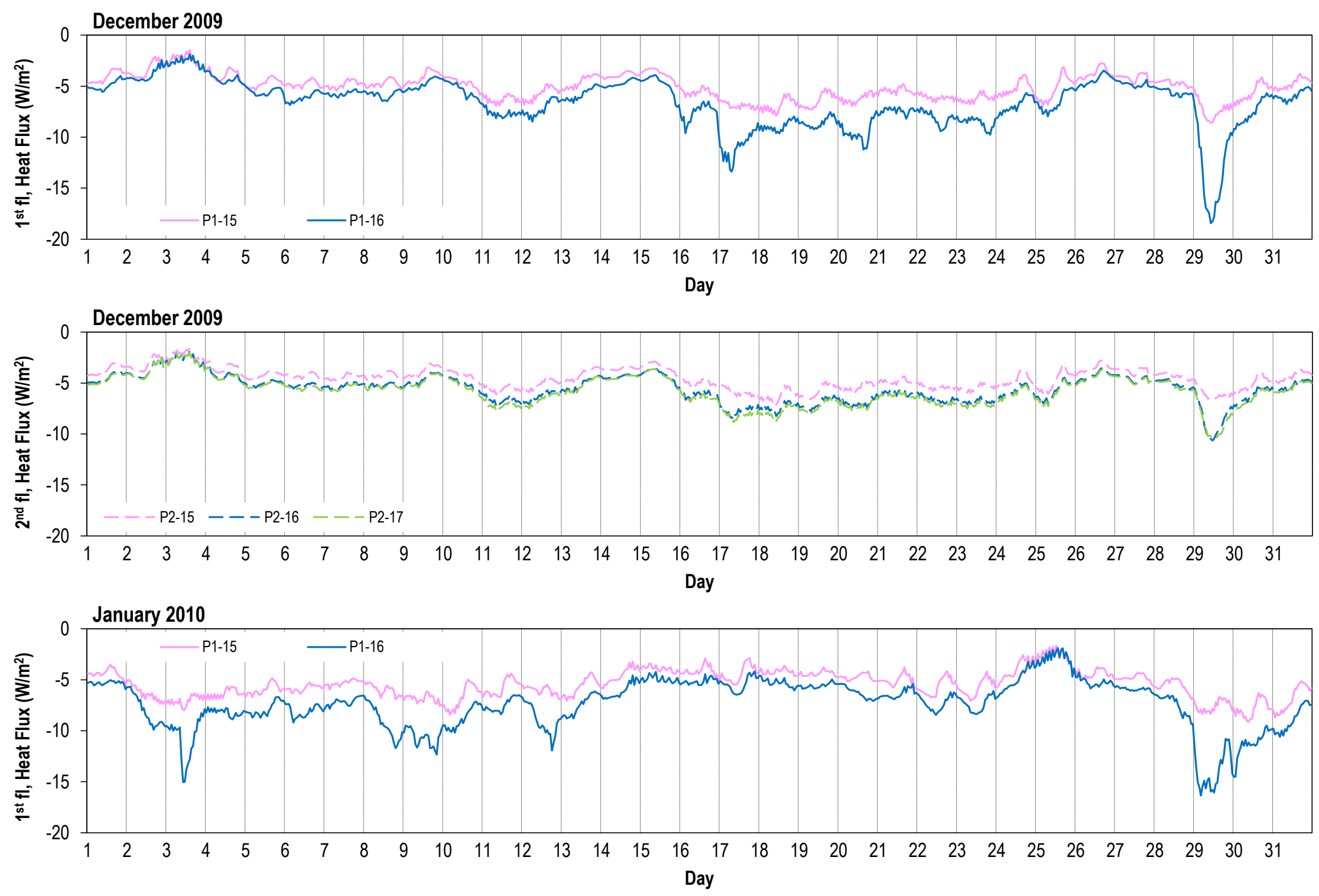

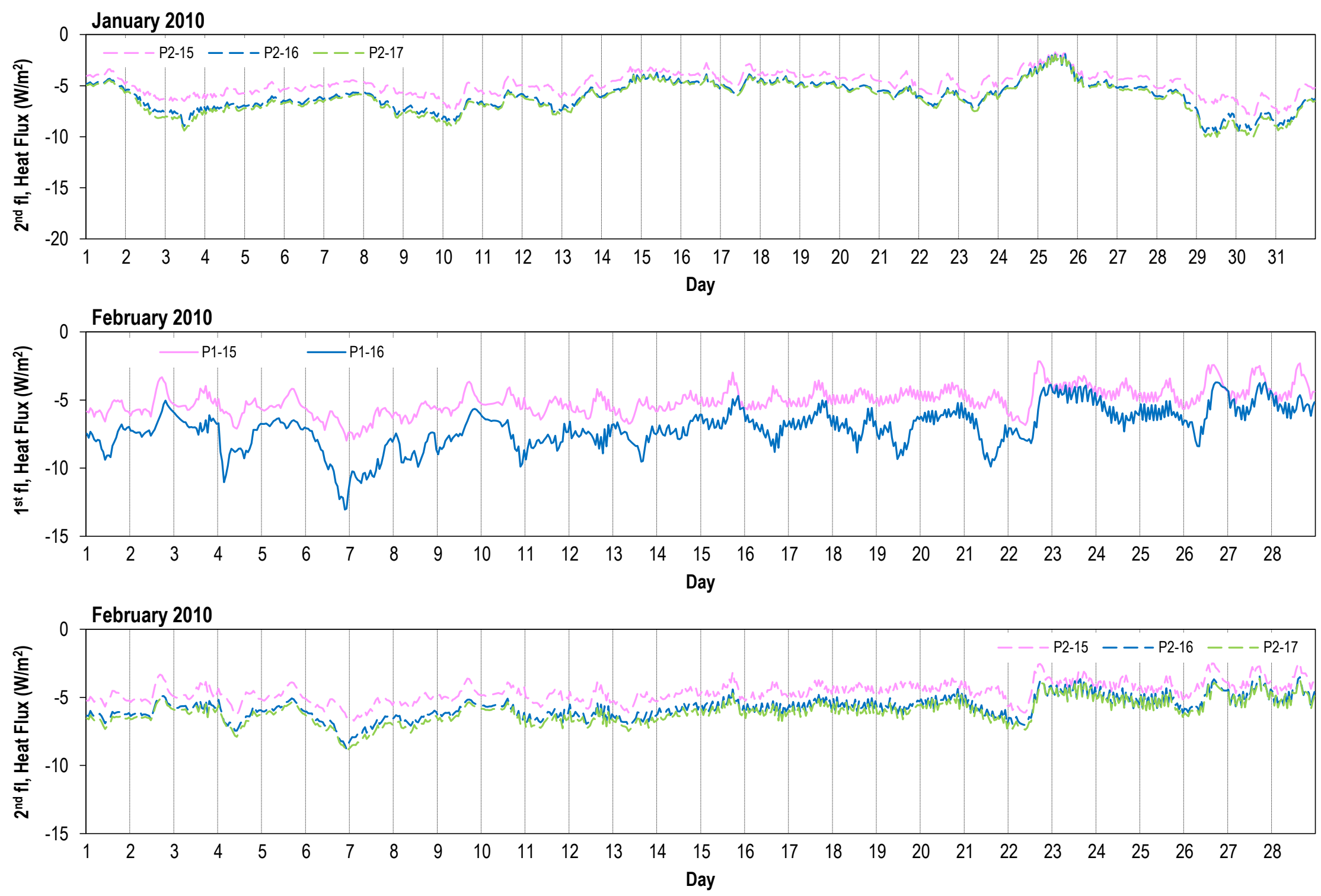

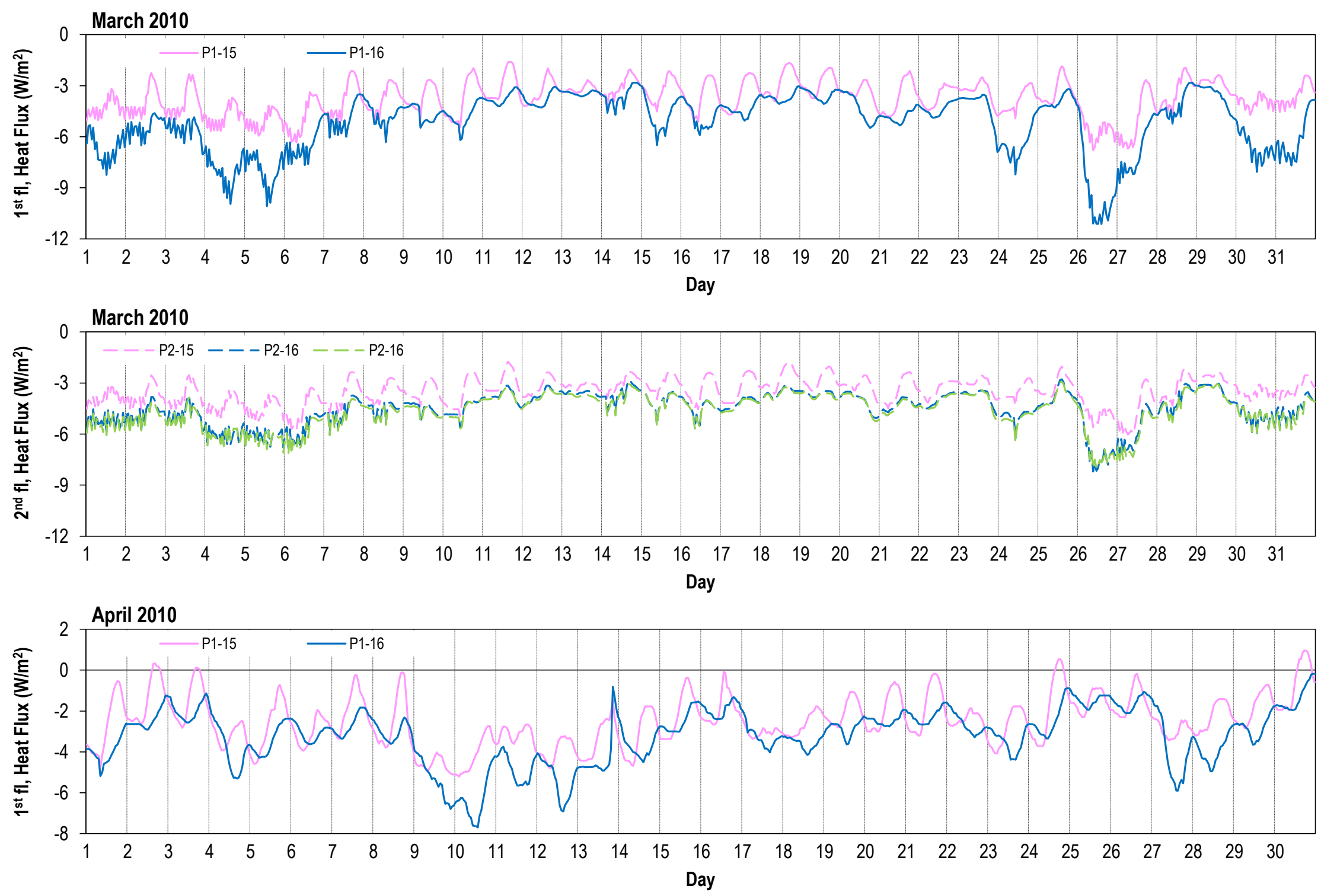

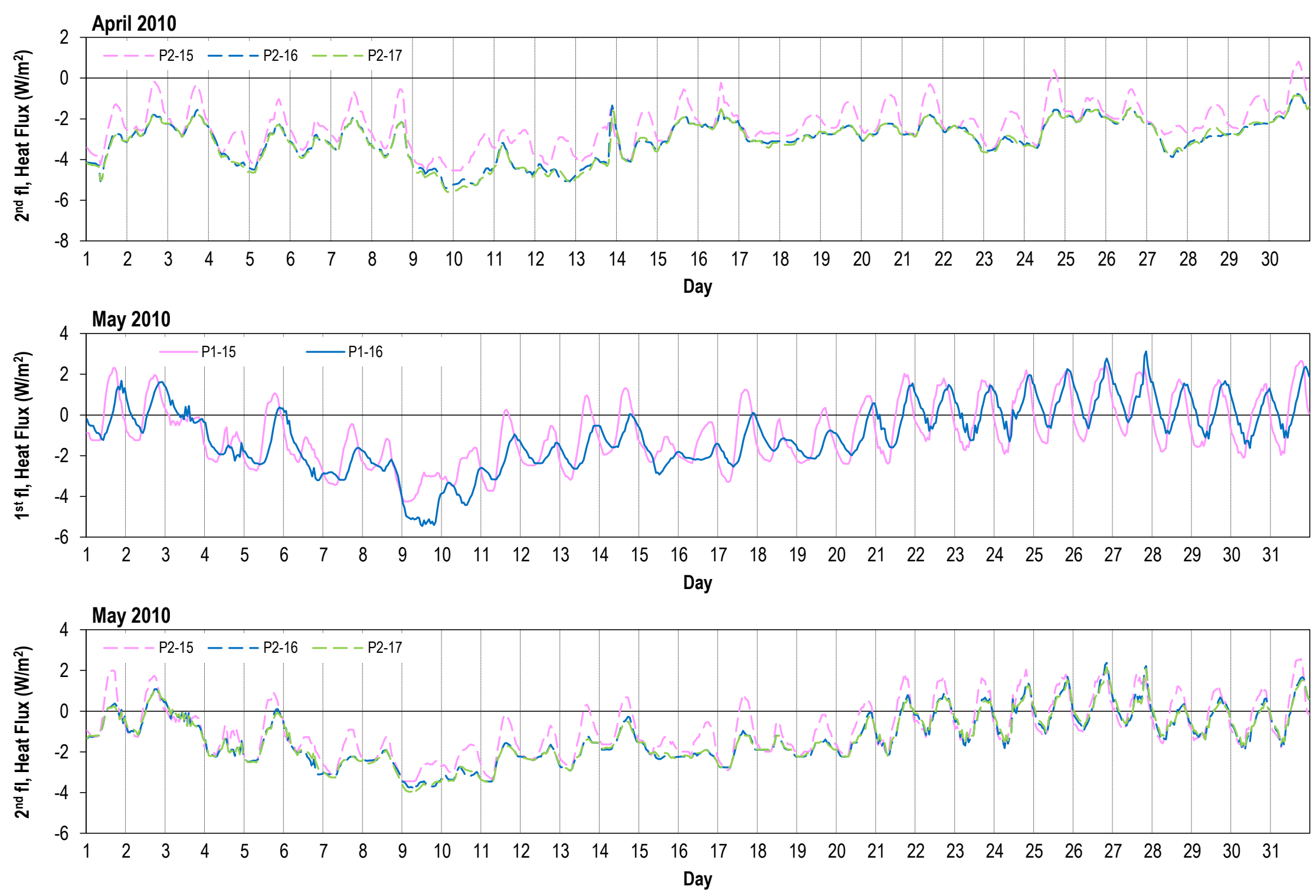

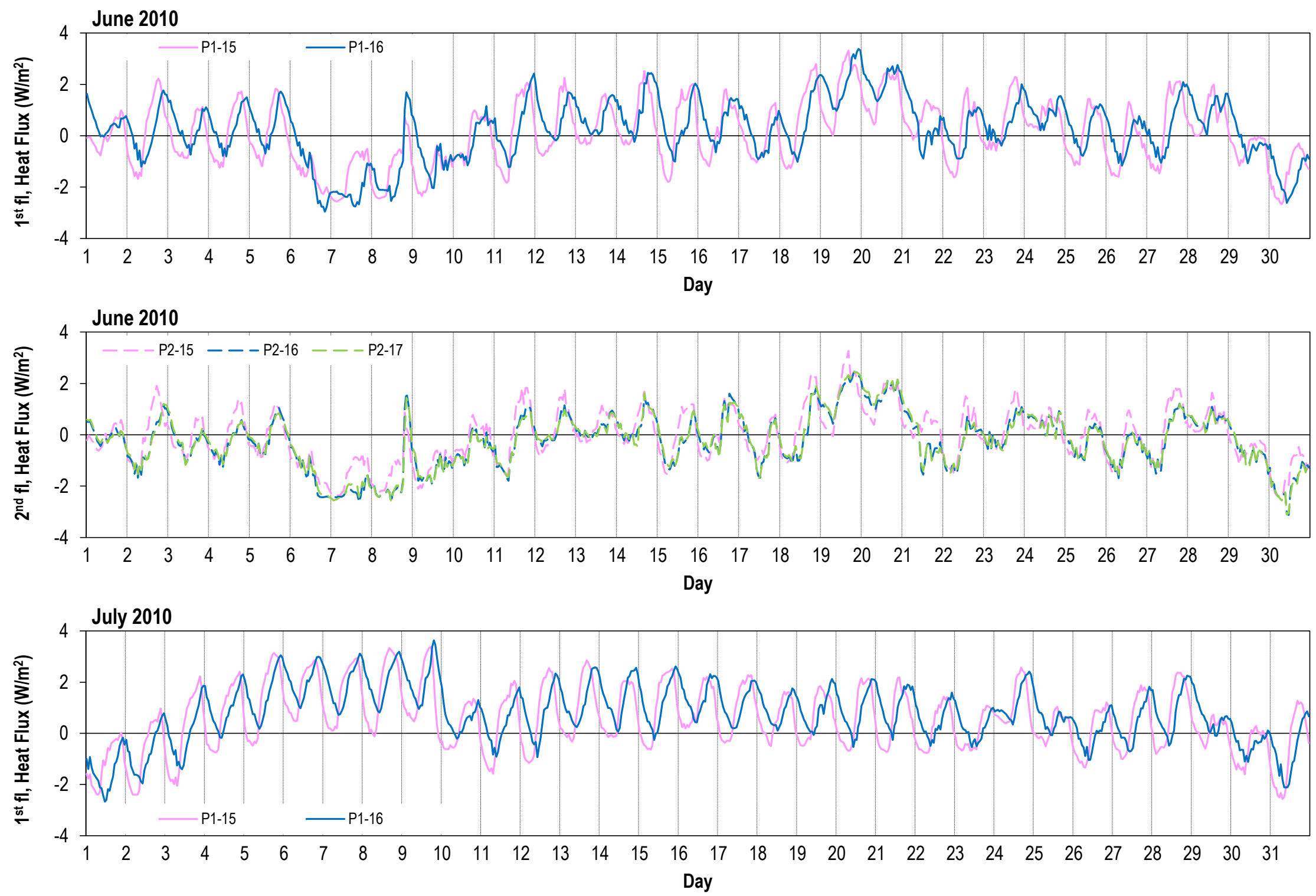

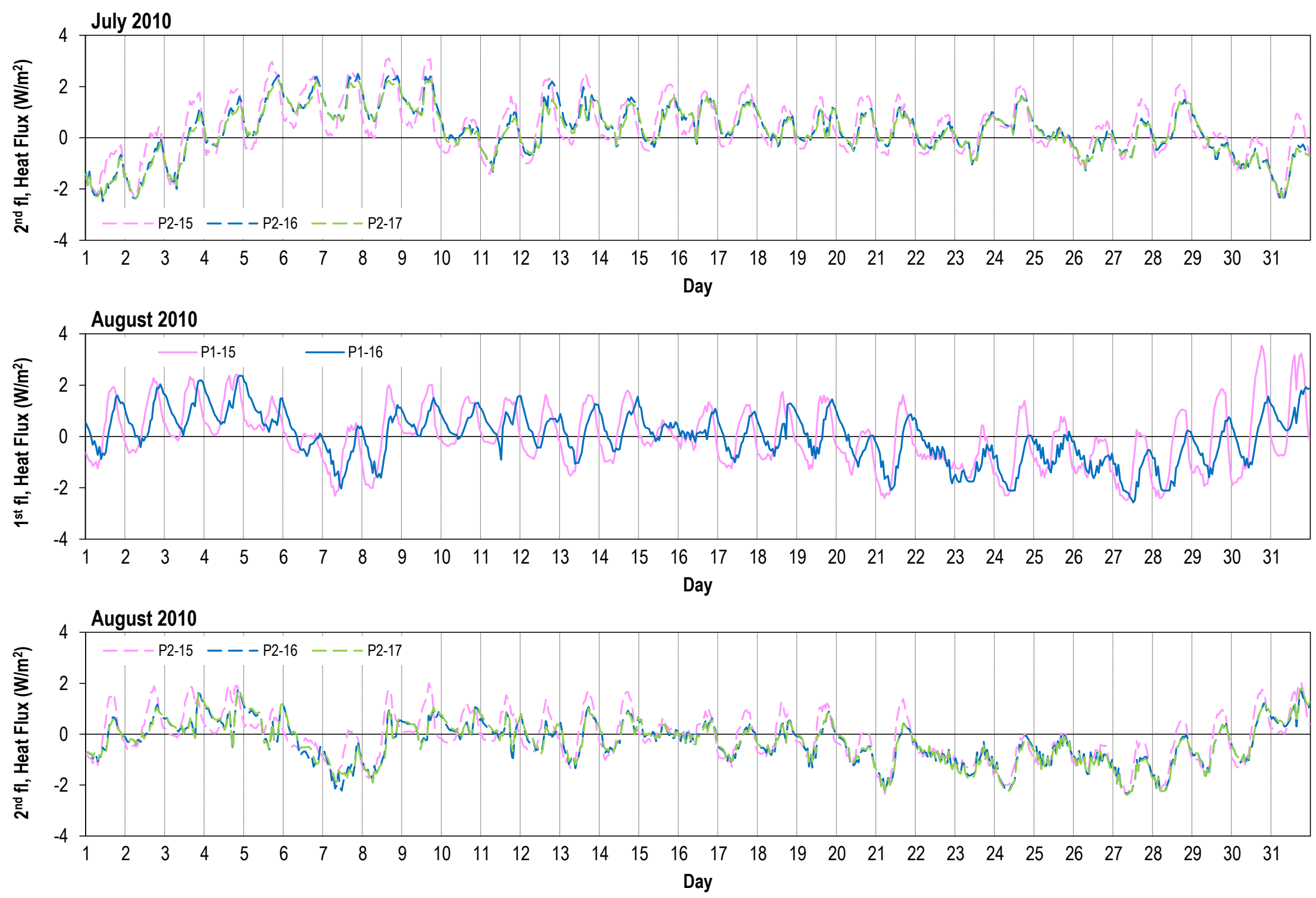

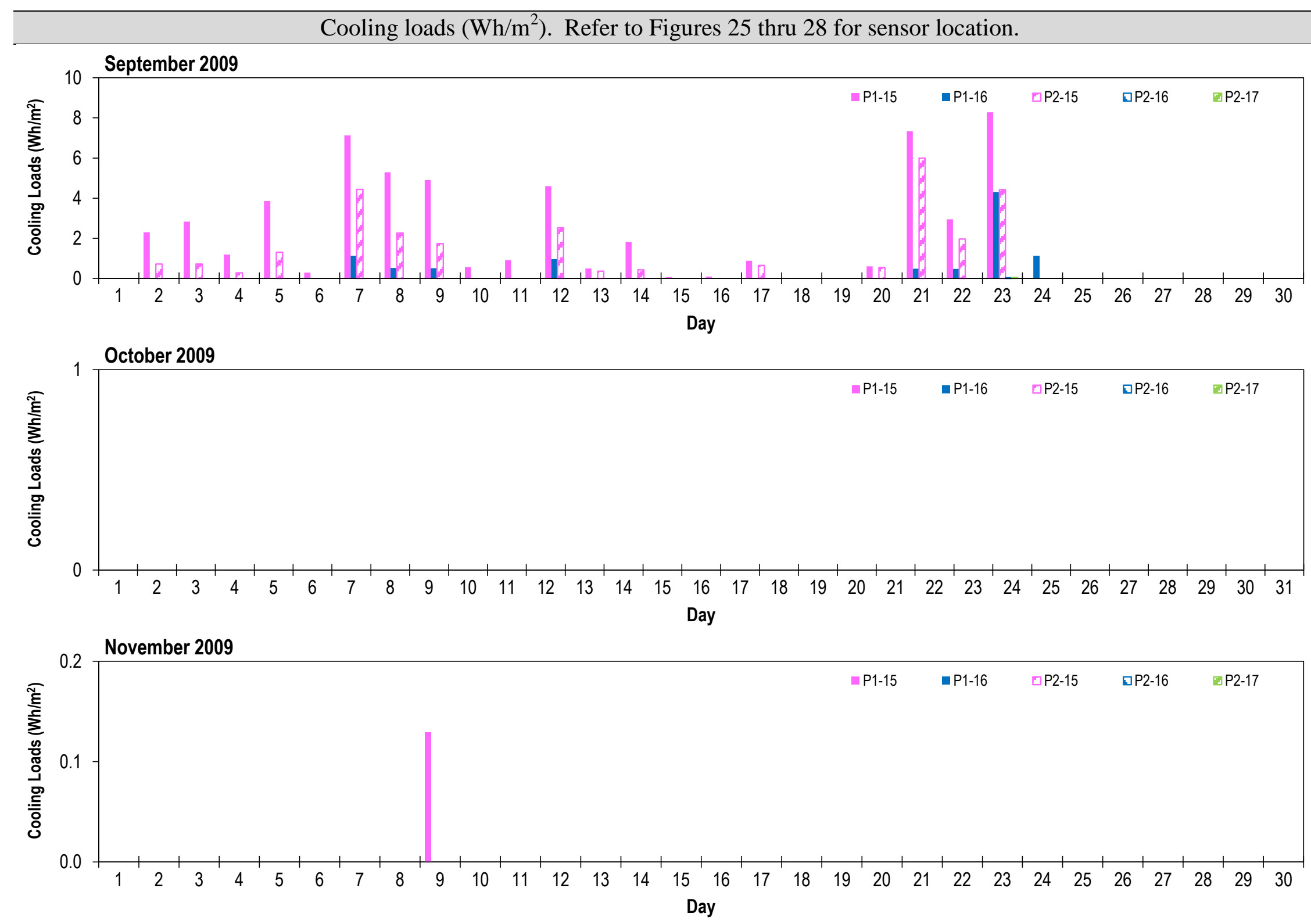

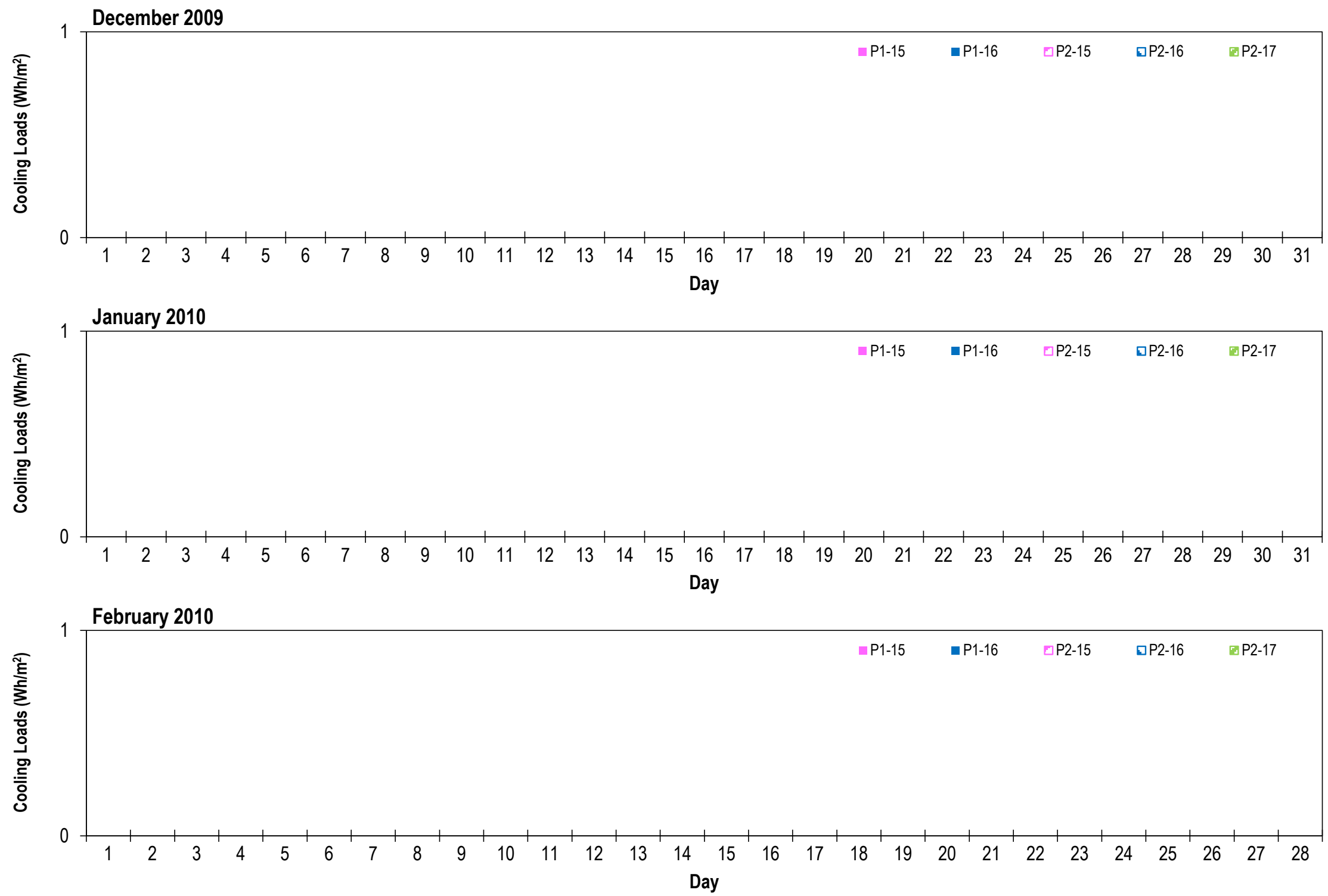

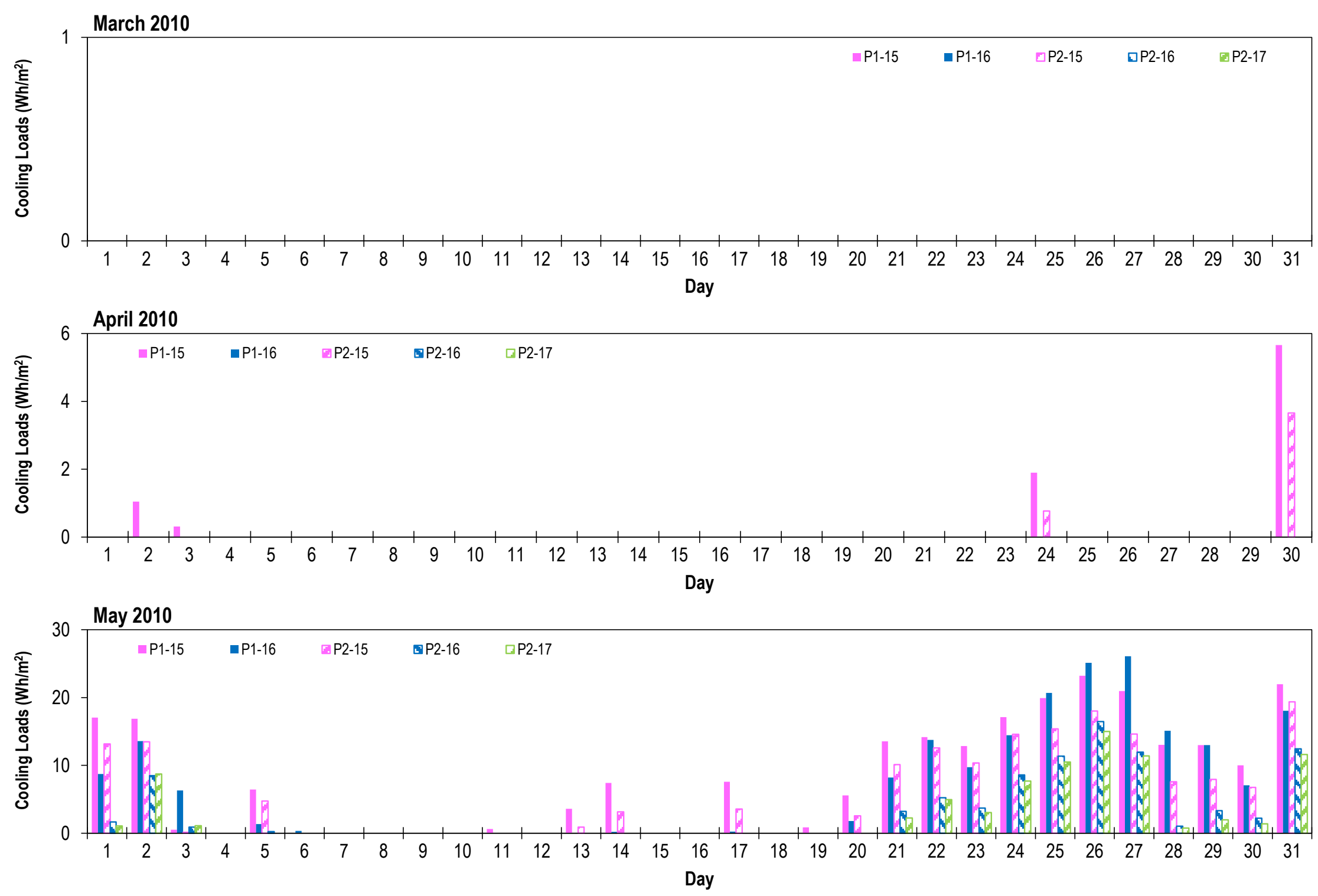

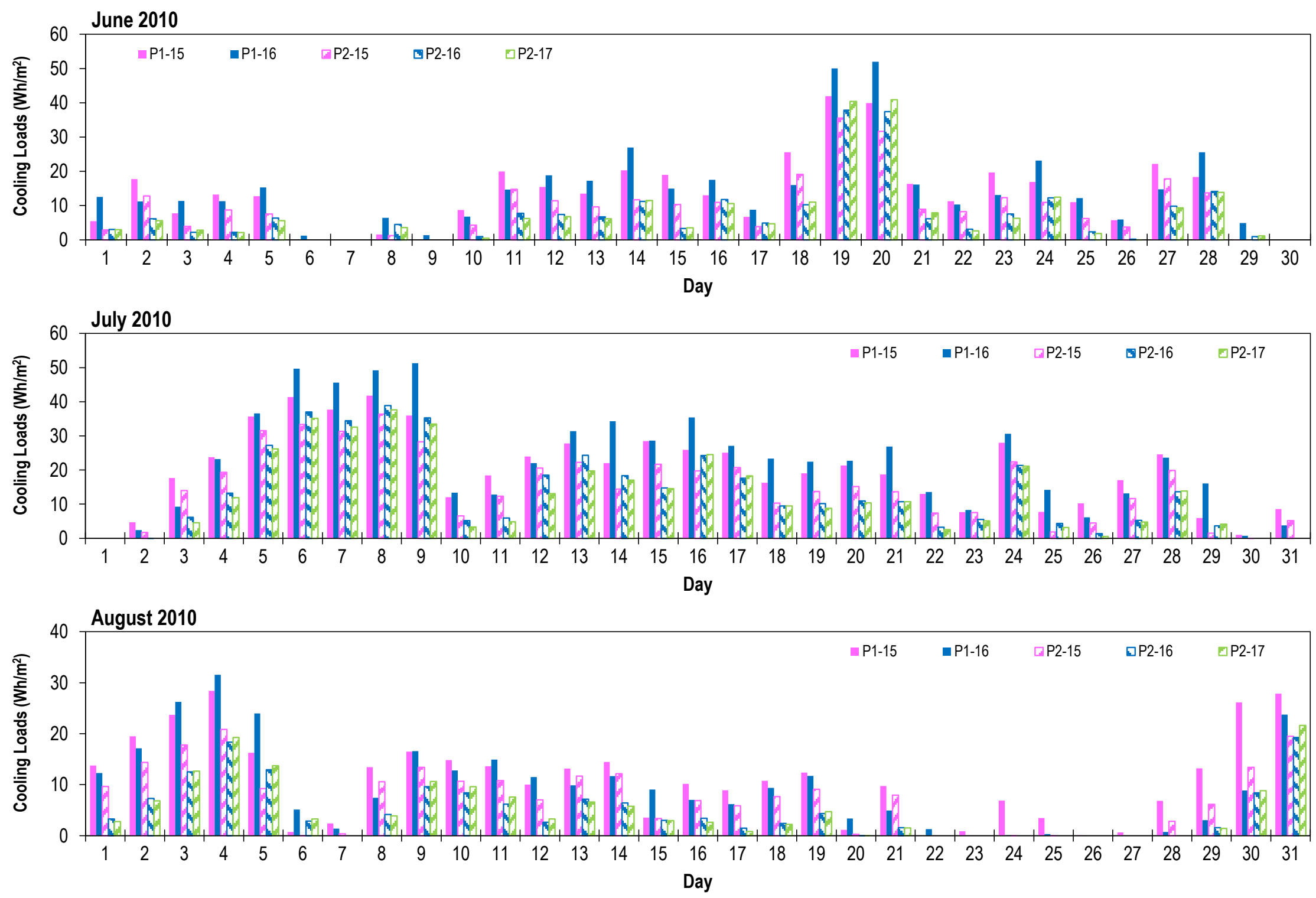
Heating loads $\left(\mathrm{Wh} / \mathrm{m}^{2}\right)$. Refer to Figures 25 thru 28 for sensor location.

\section{September 2009}

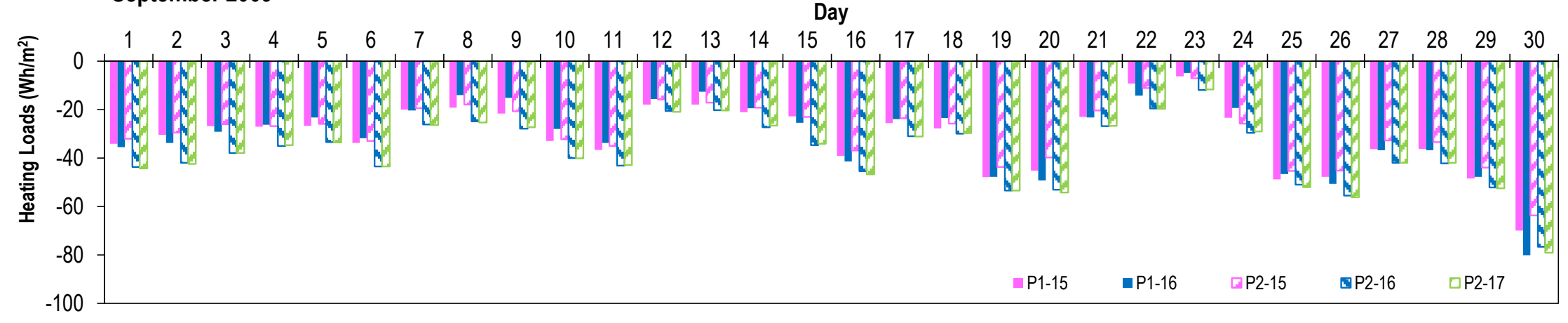

\section{October 2009}

Day

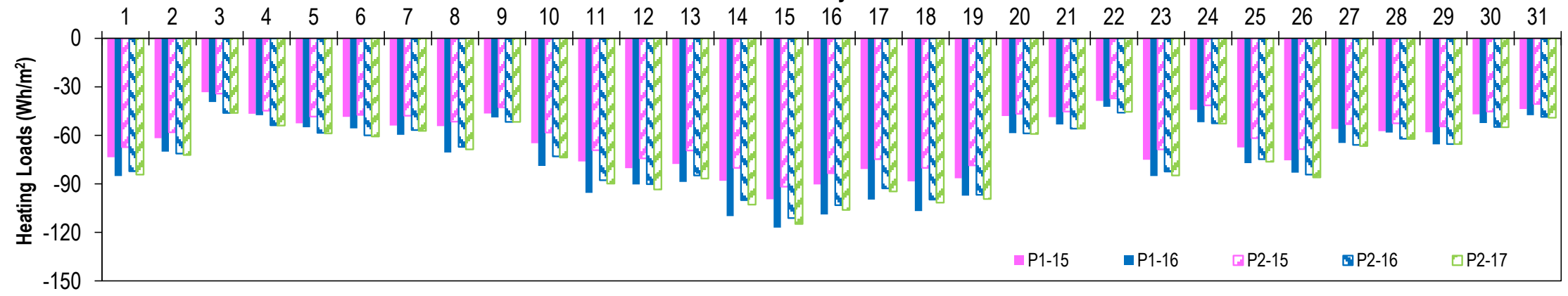

November 2009

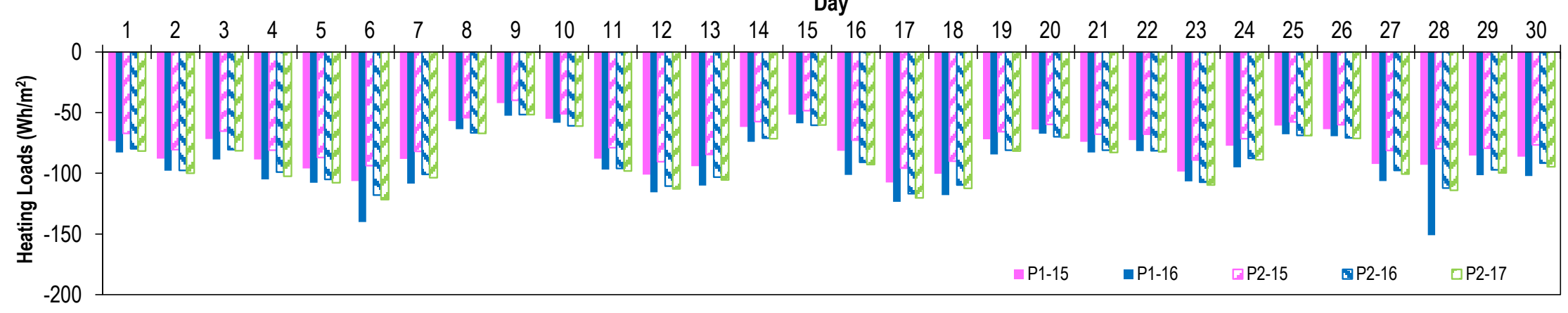




\section{December 2009}
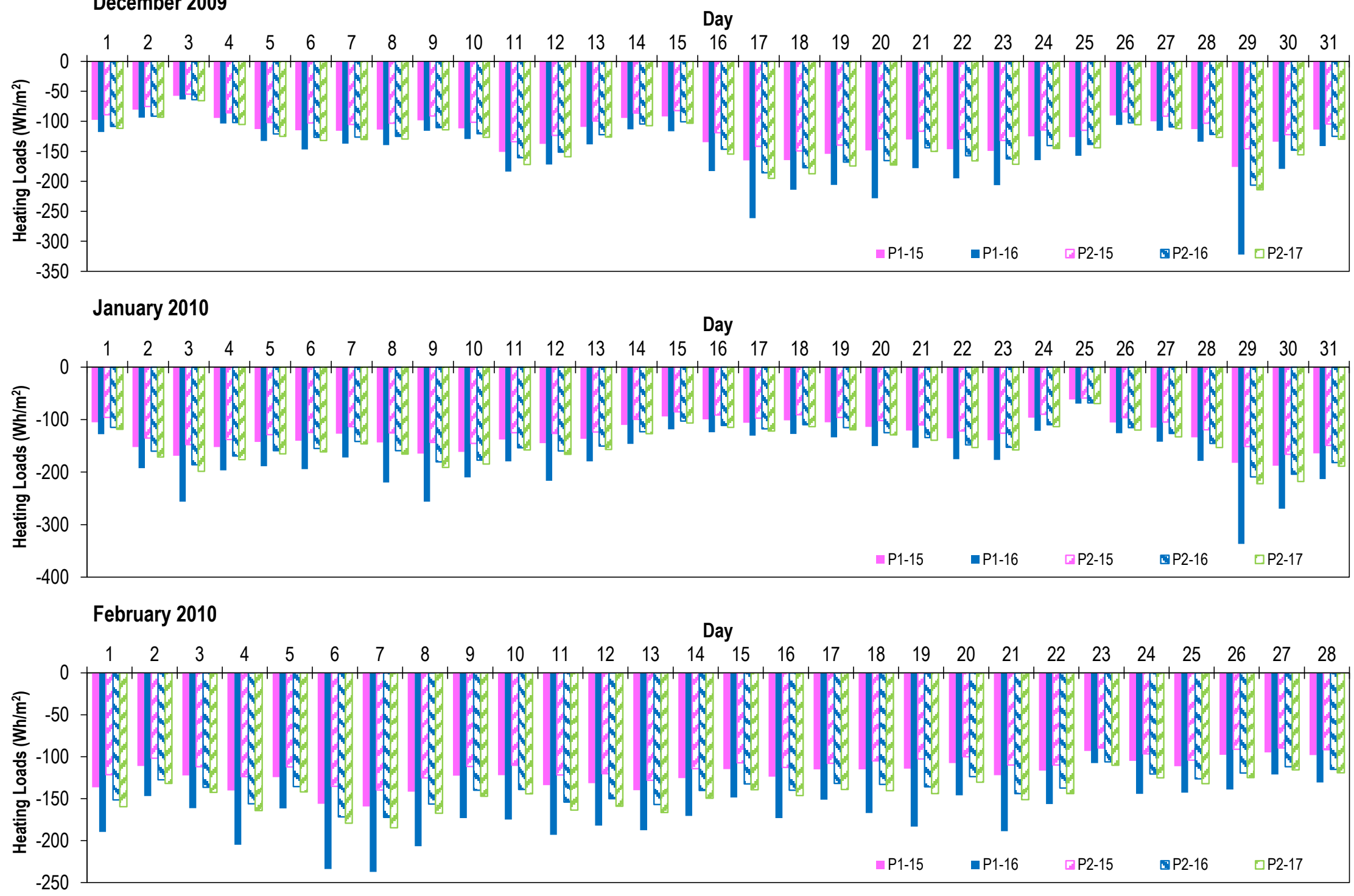


\section{March 2010}
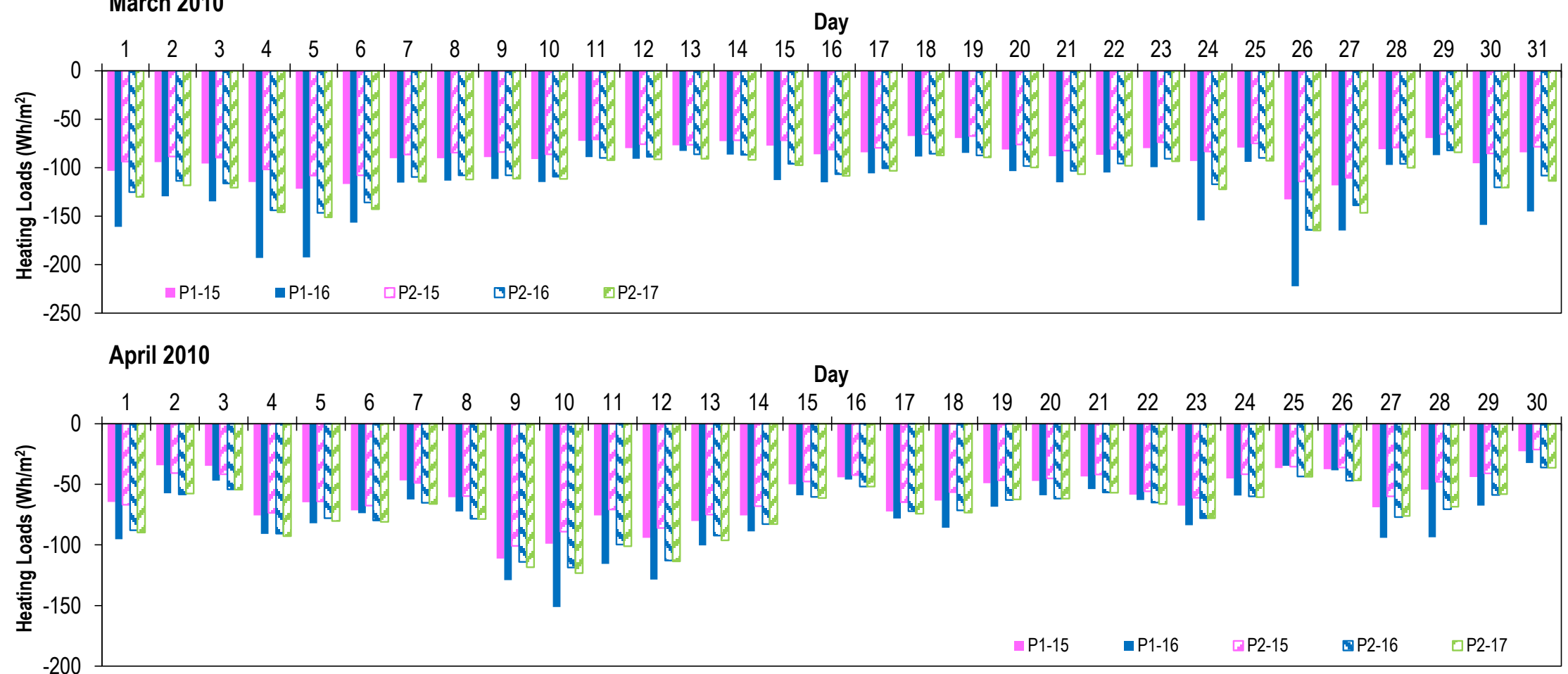

May 2010

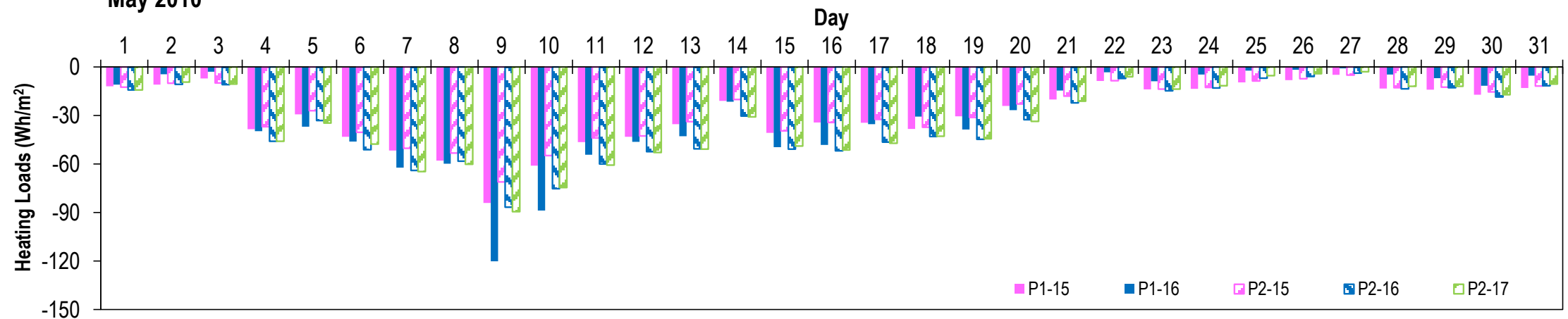


June 2010

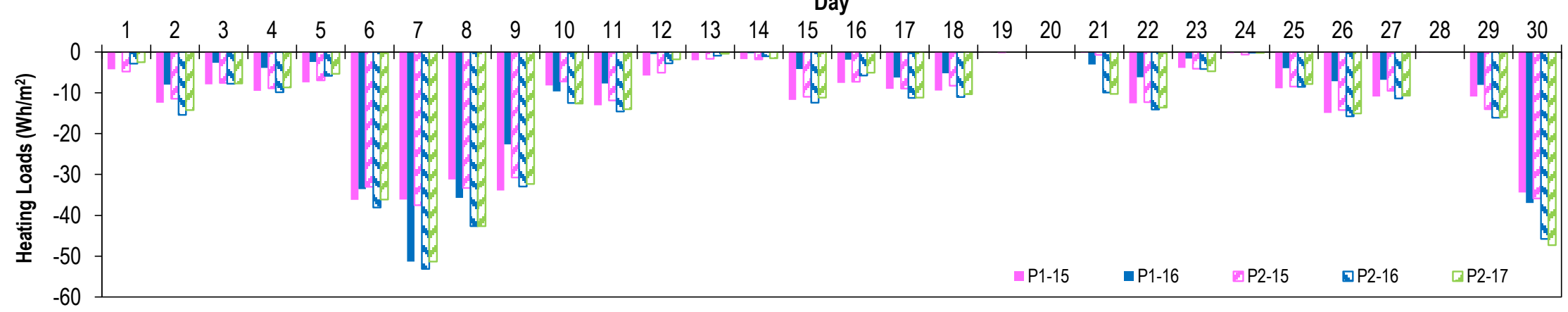

July 2010

Day

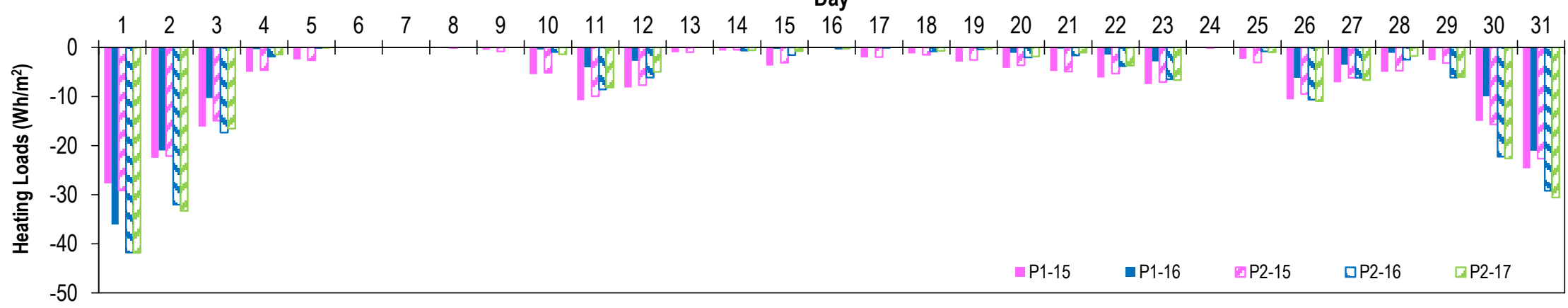

August 2010

Day

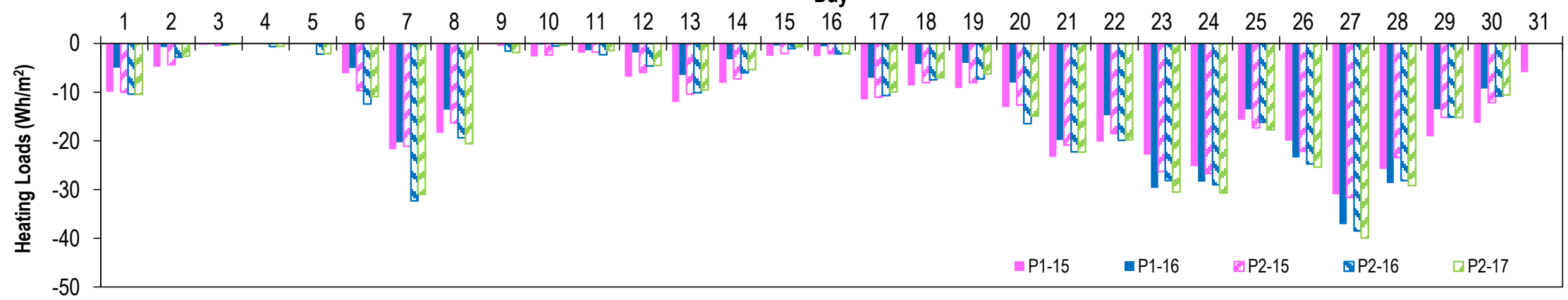




\section{Appendix B: East Panels}

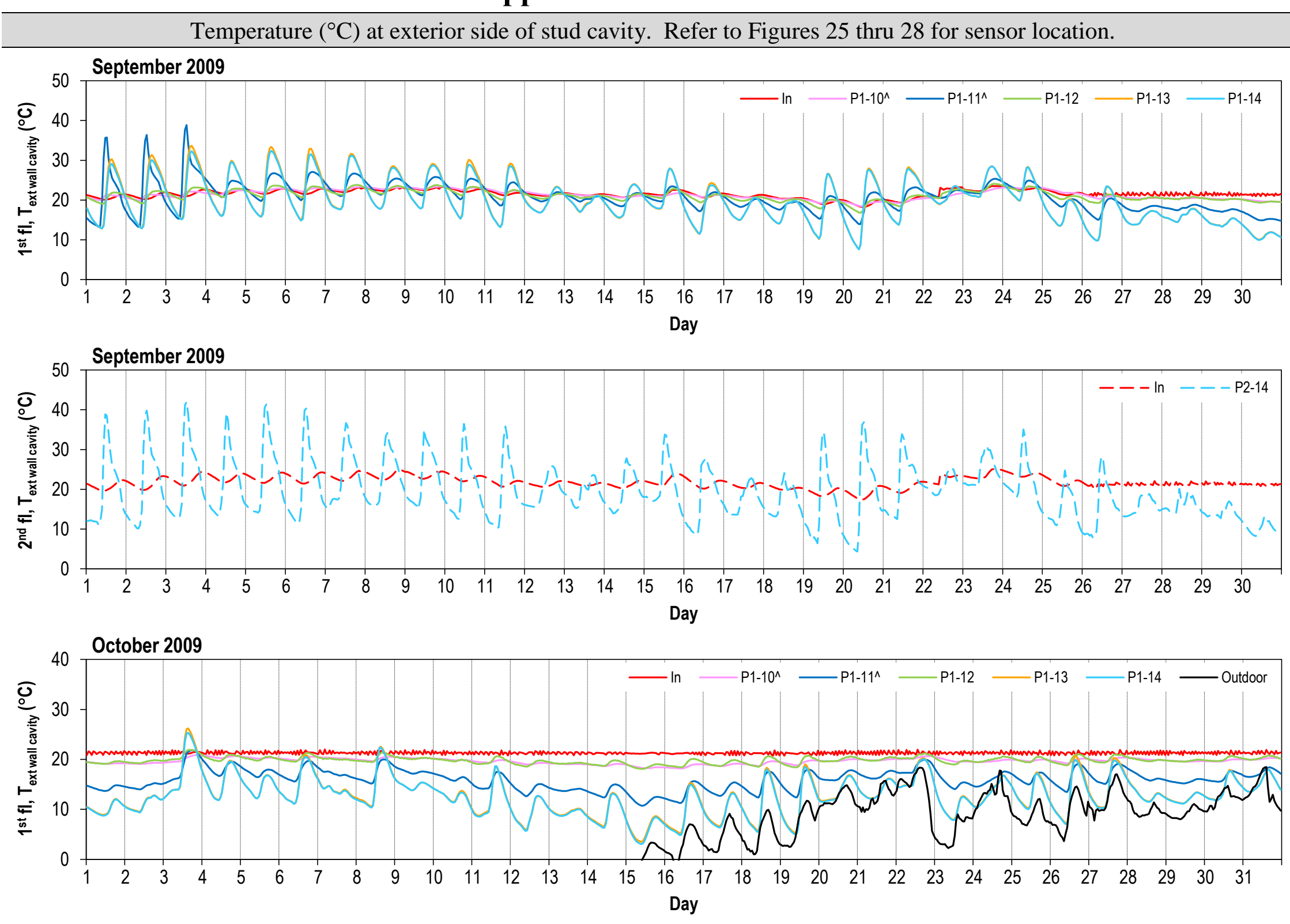



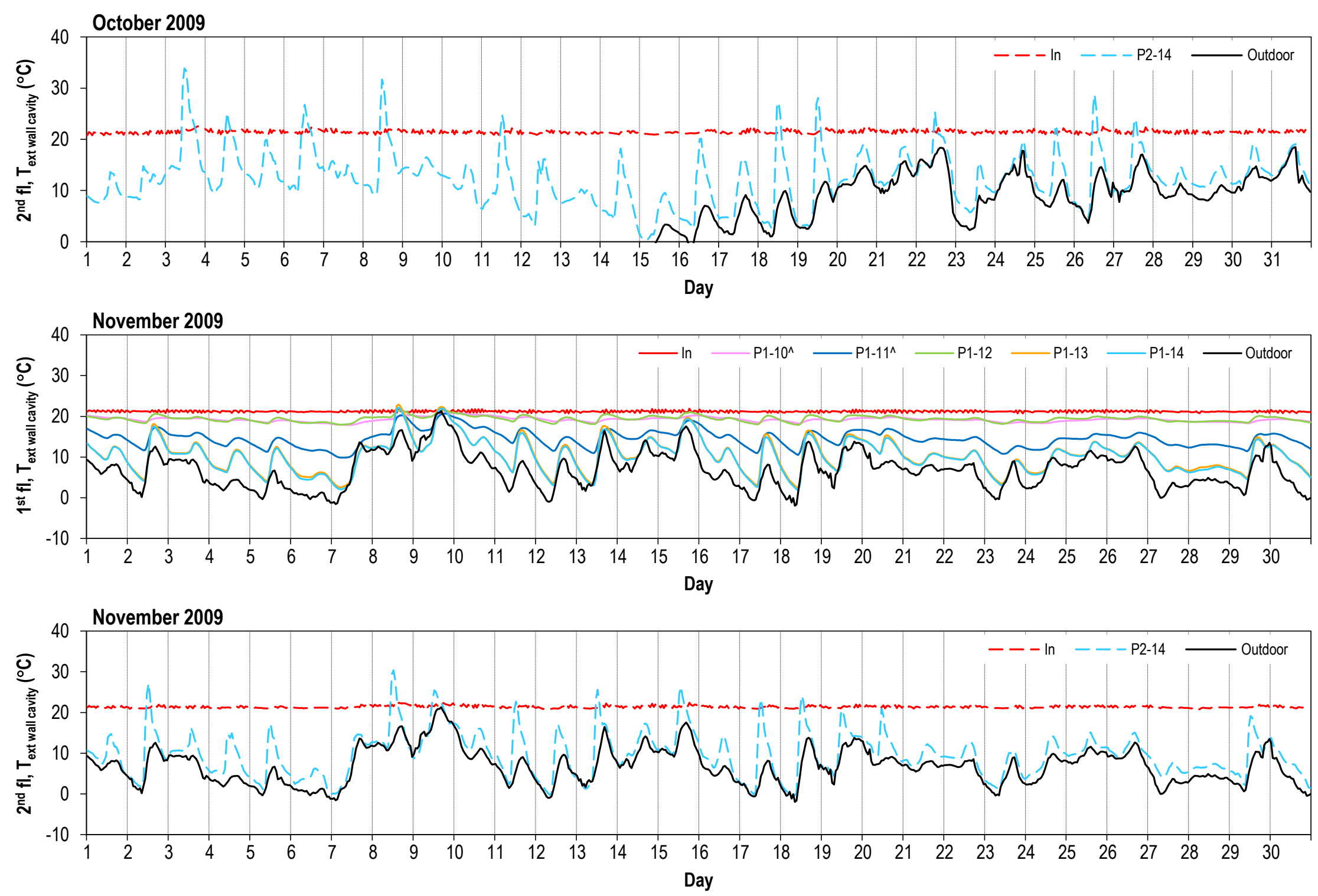

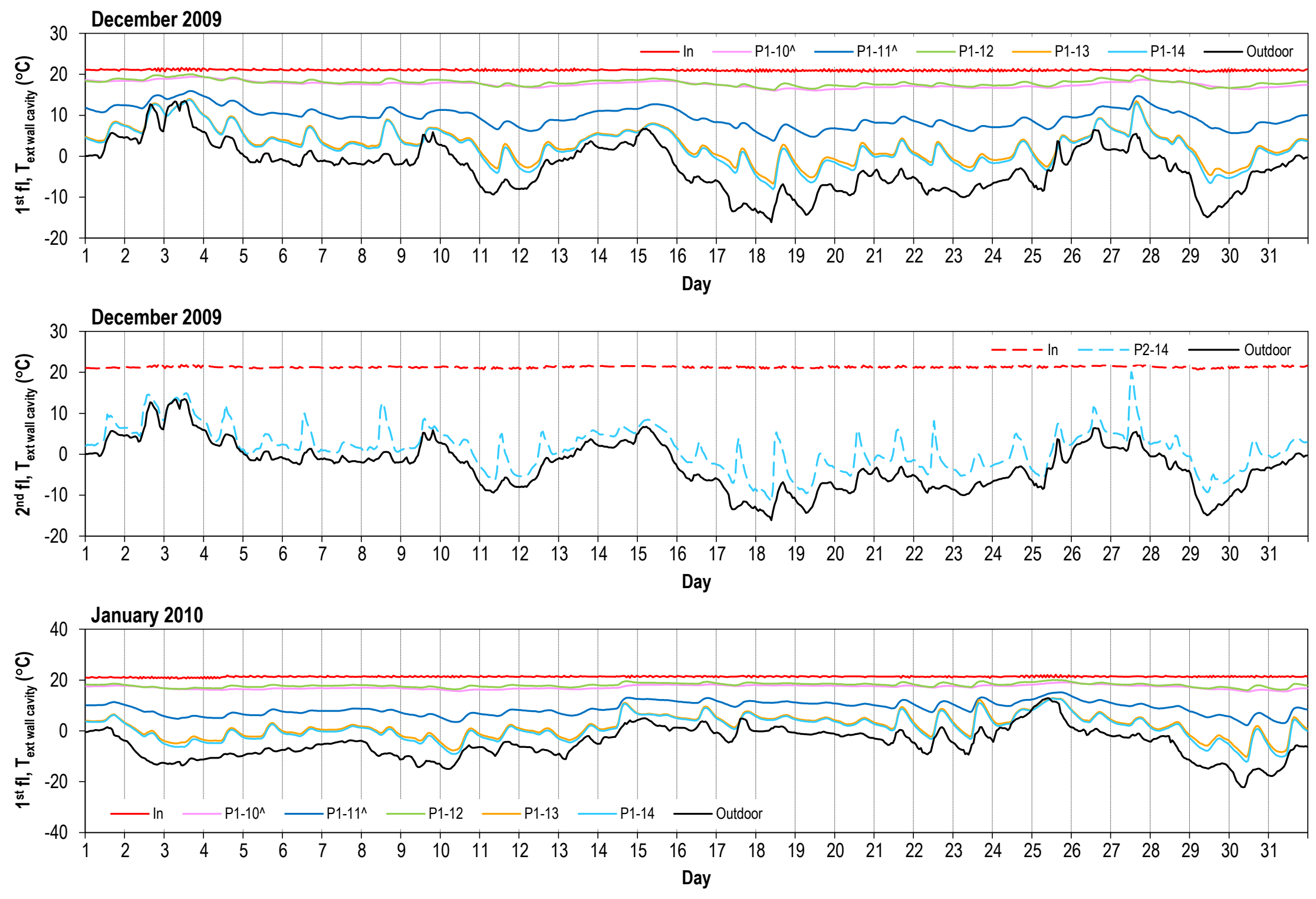

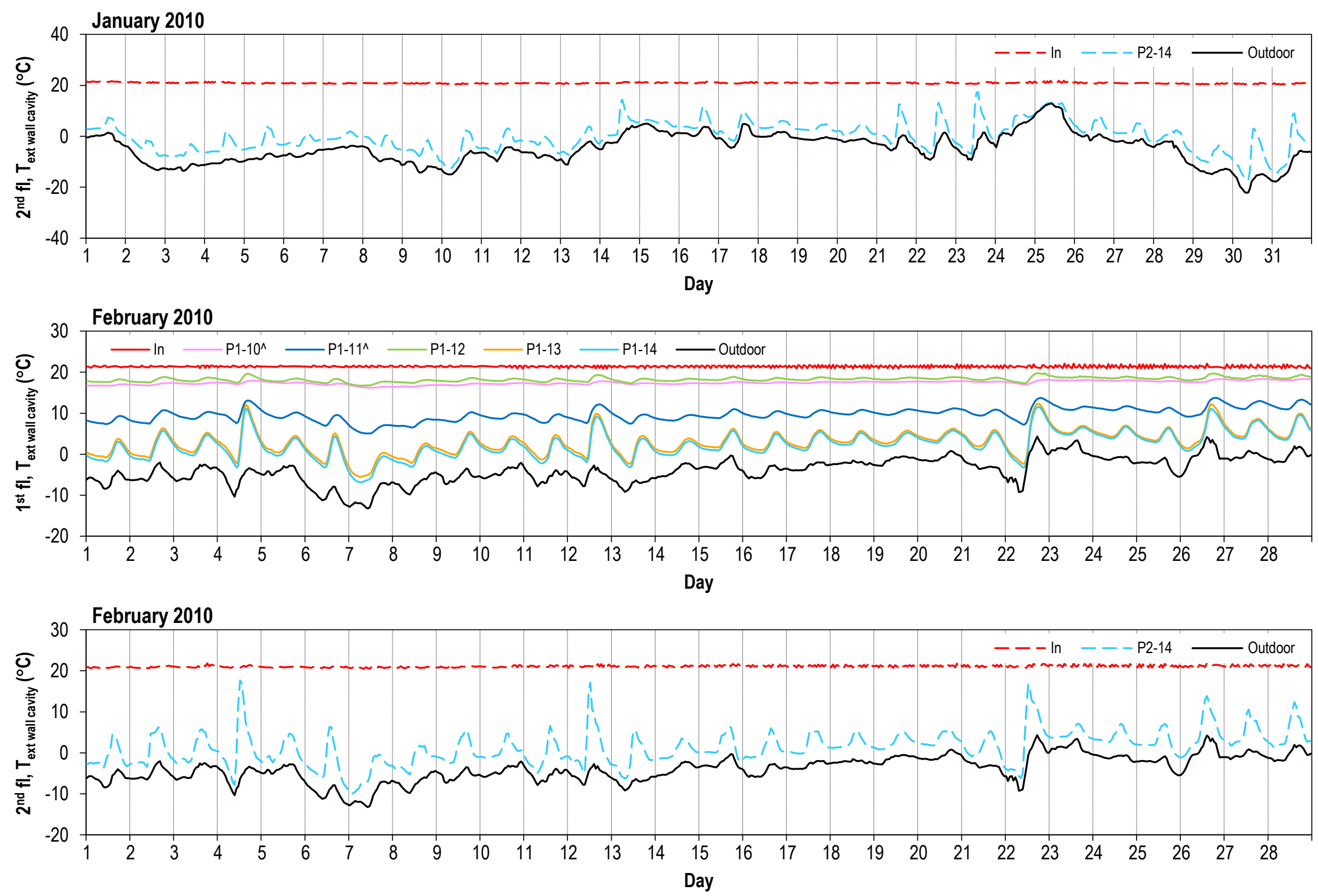

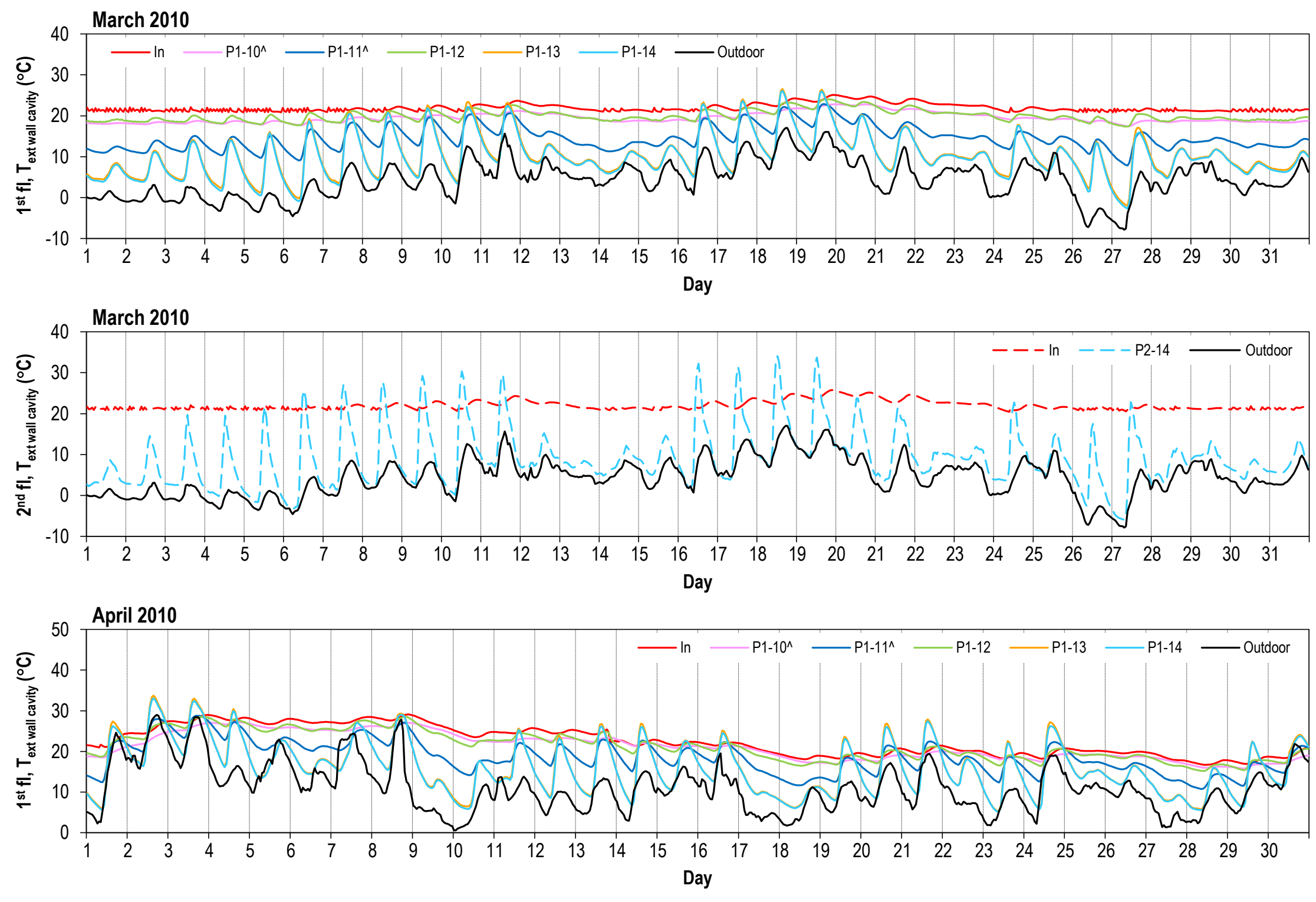

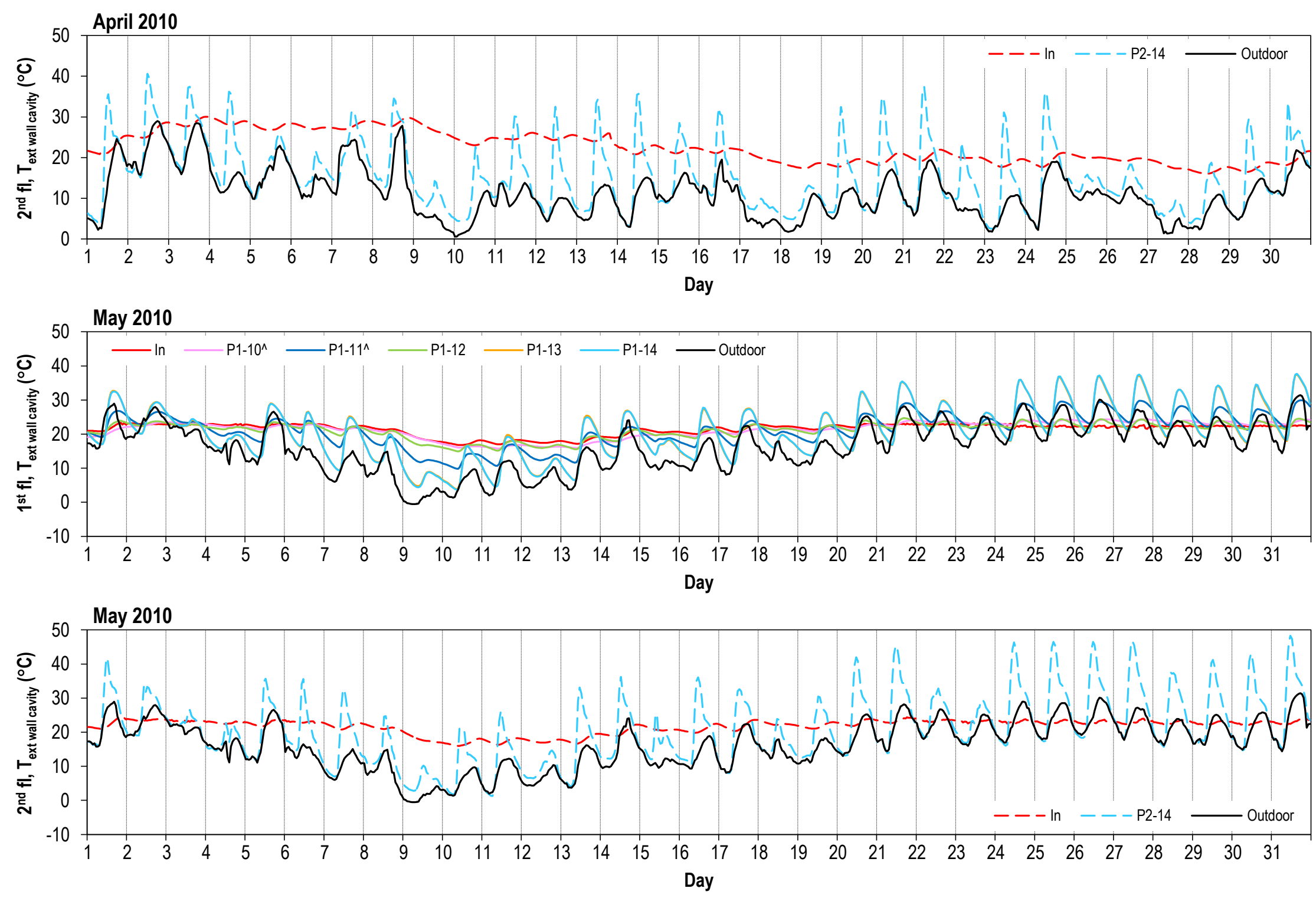

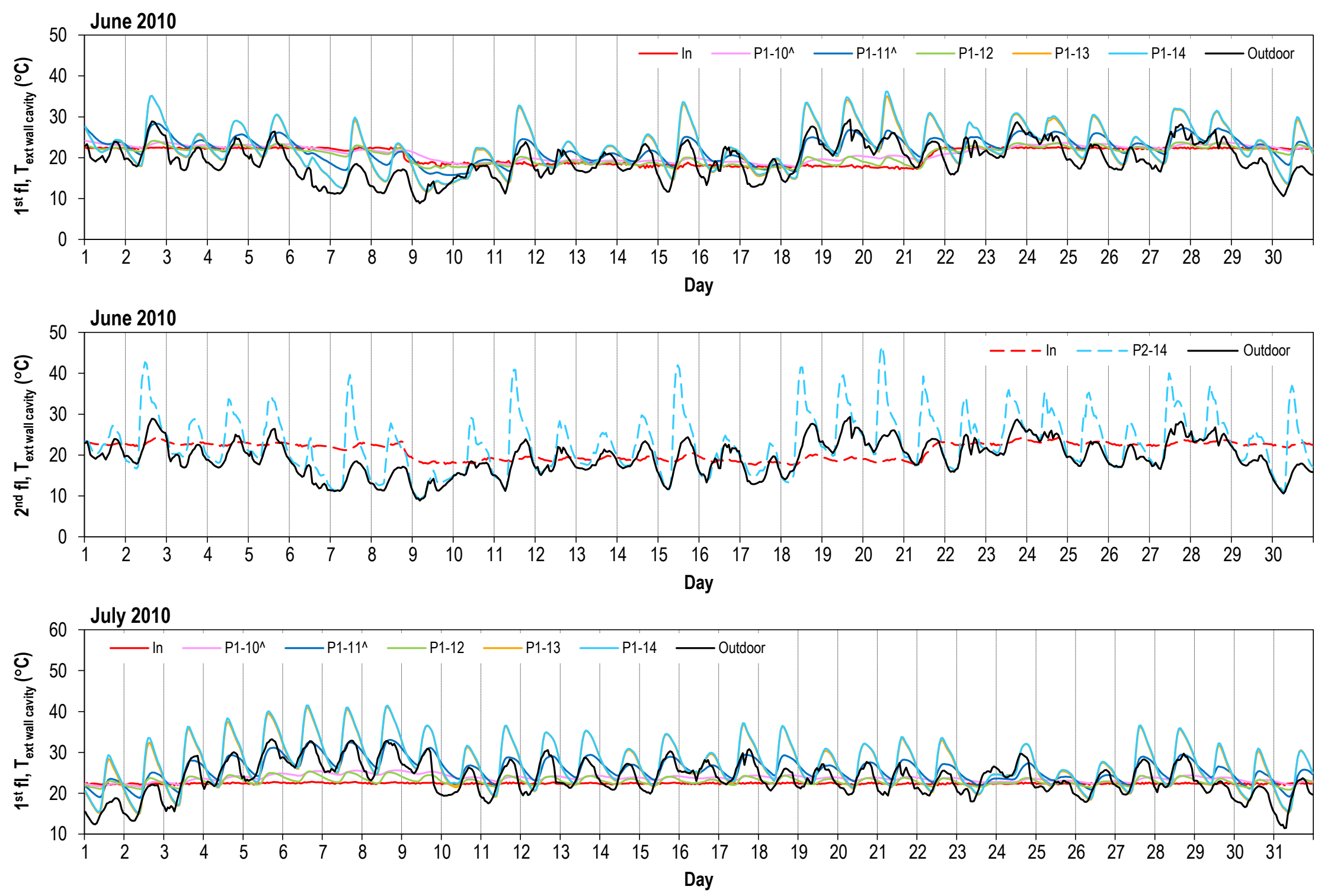

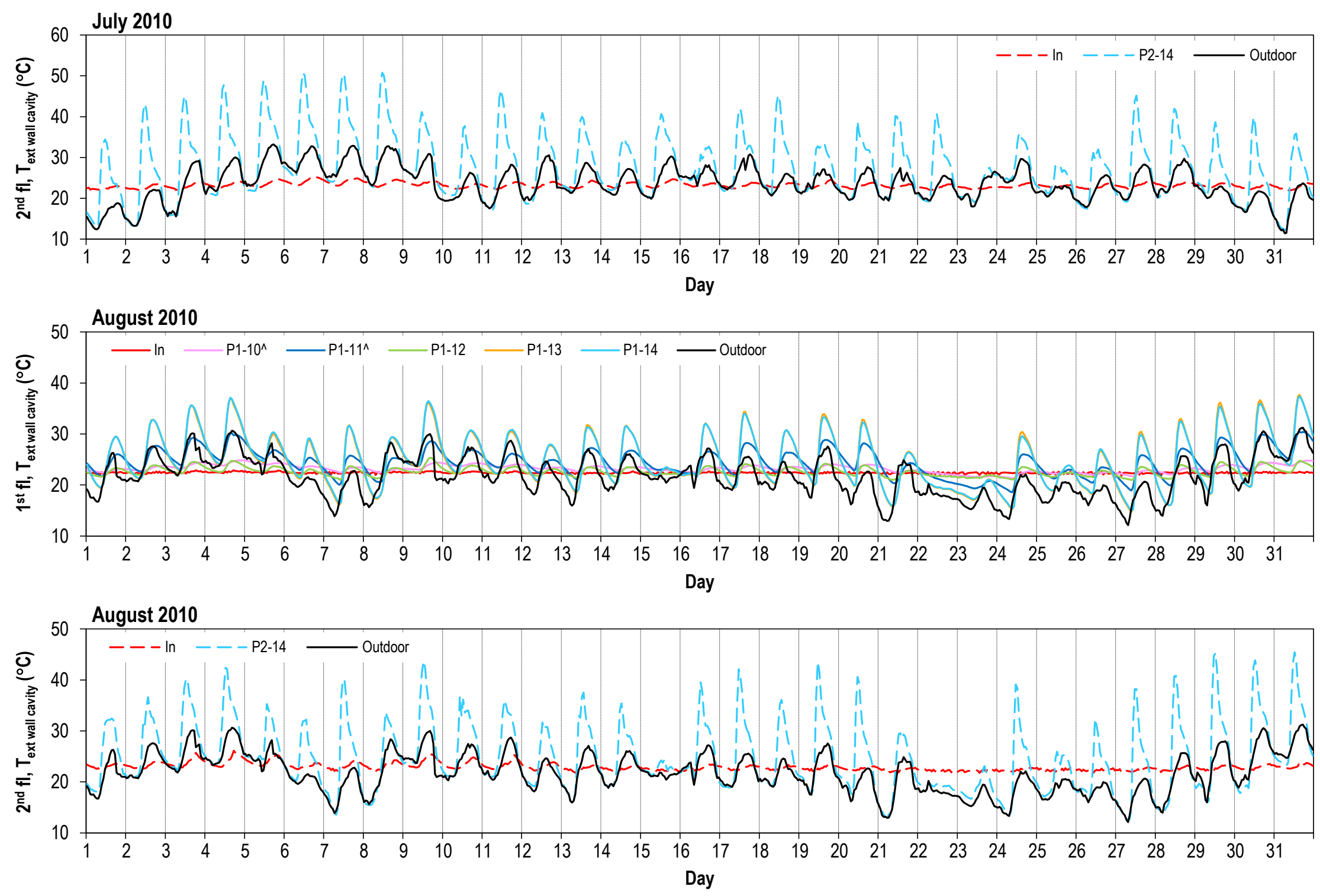
Temperature $\left({ }^{\circ} \mathrm{C}\right)$ at interior side of stud cavity. Refer to Figures 25 thru 28 for sensor location.
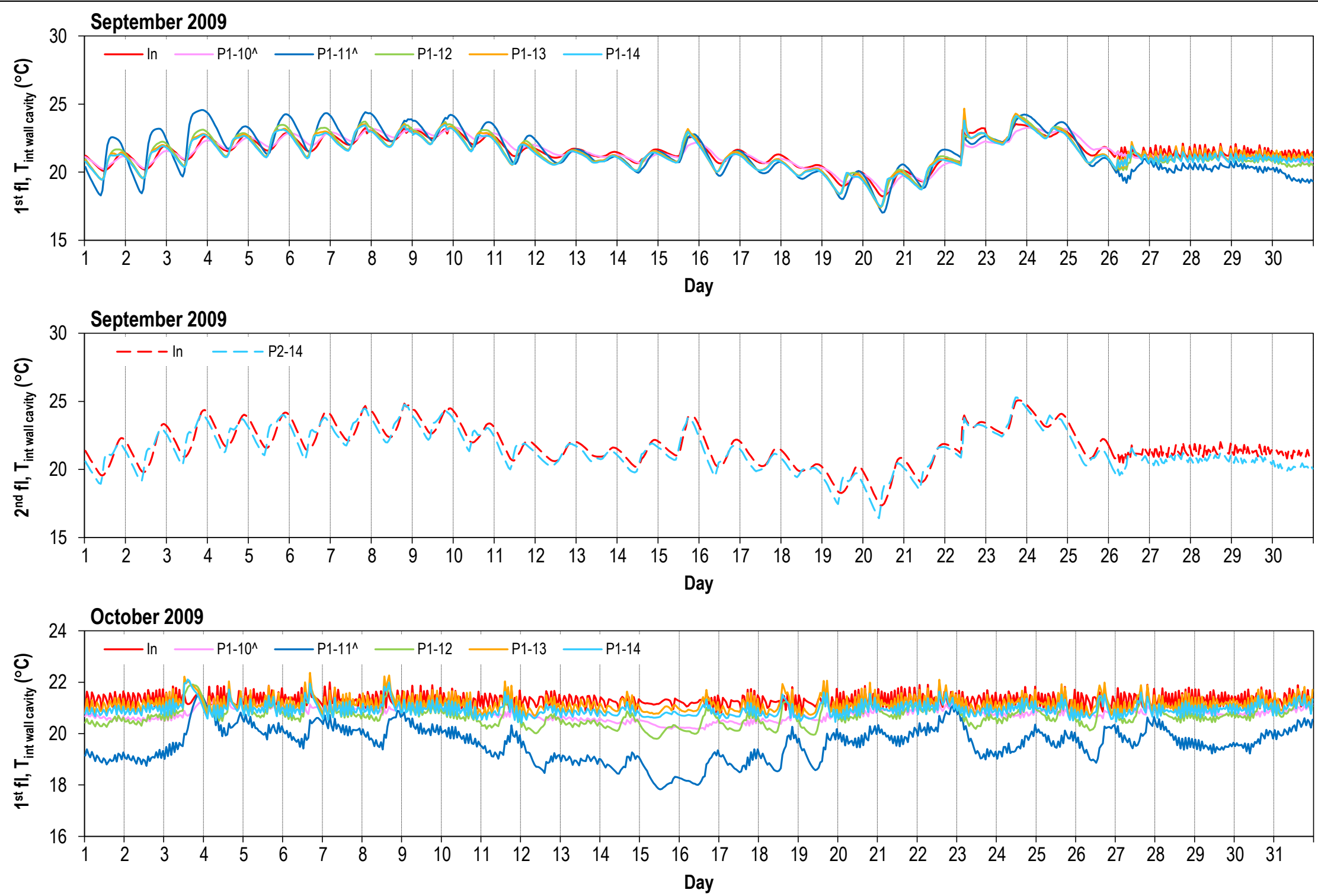

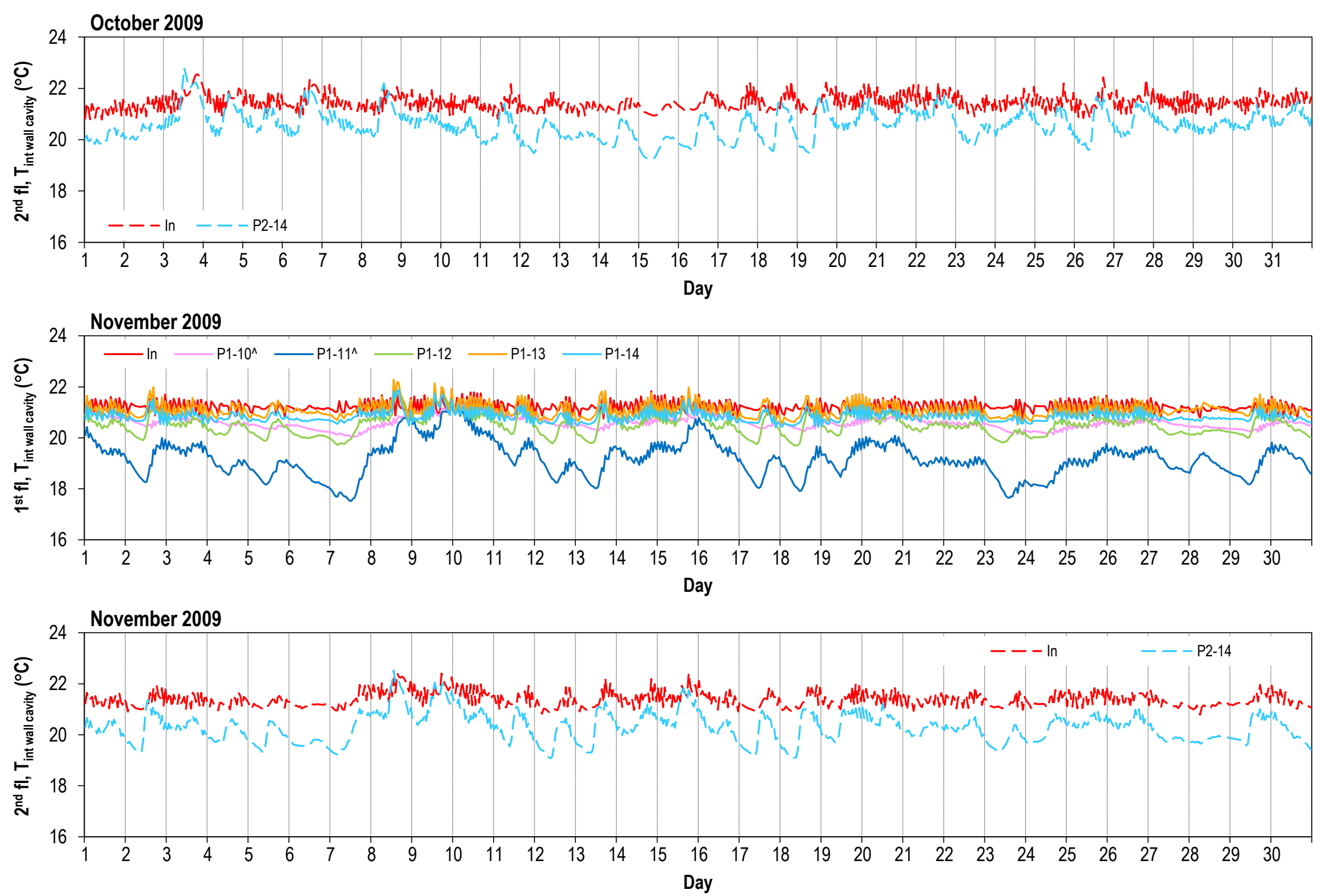

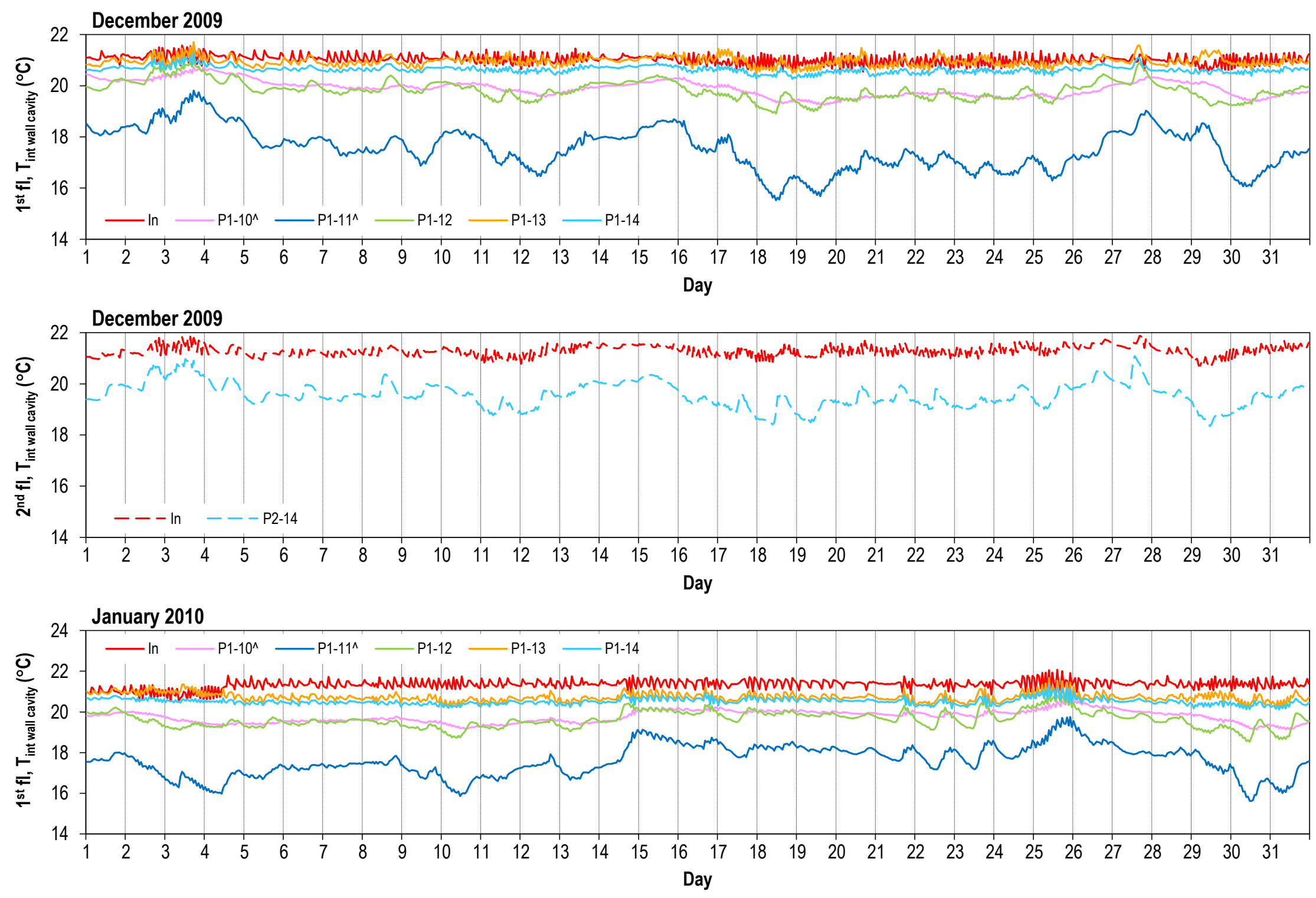

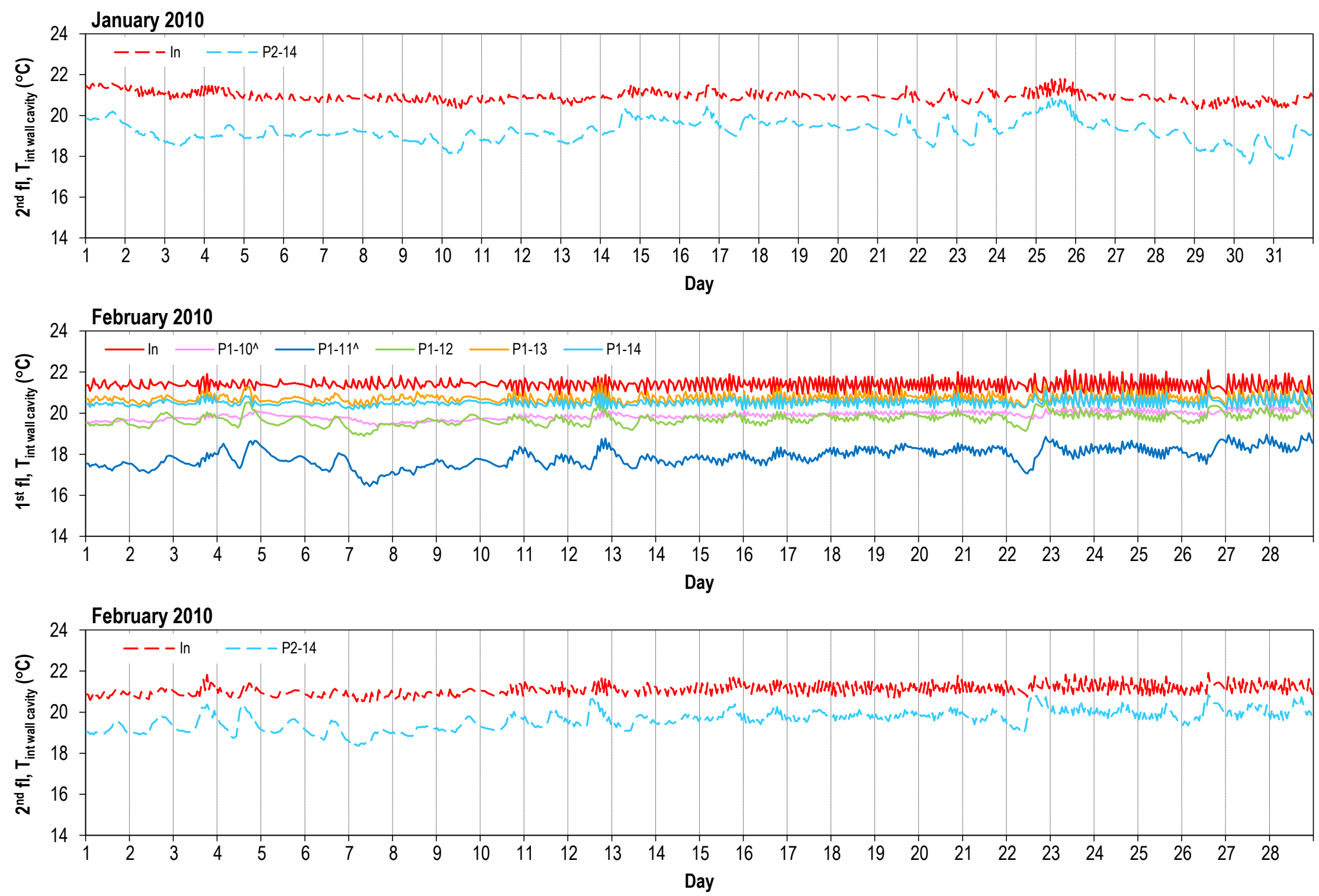

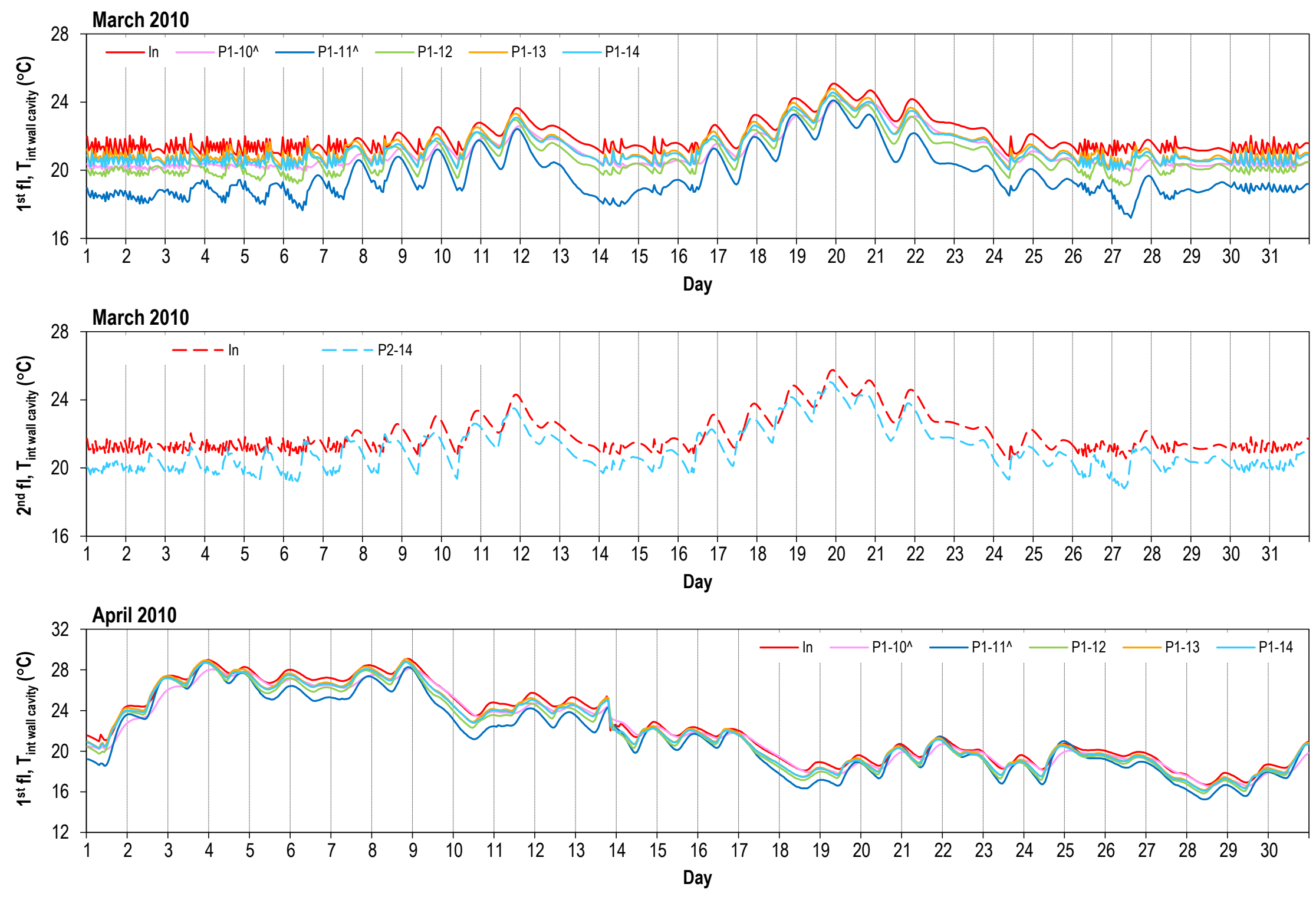

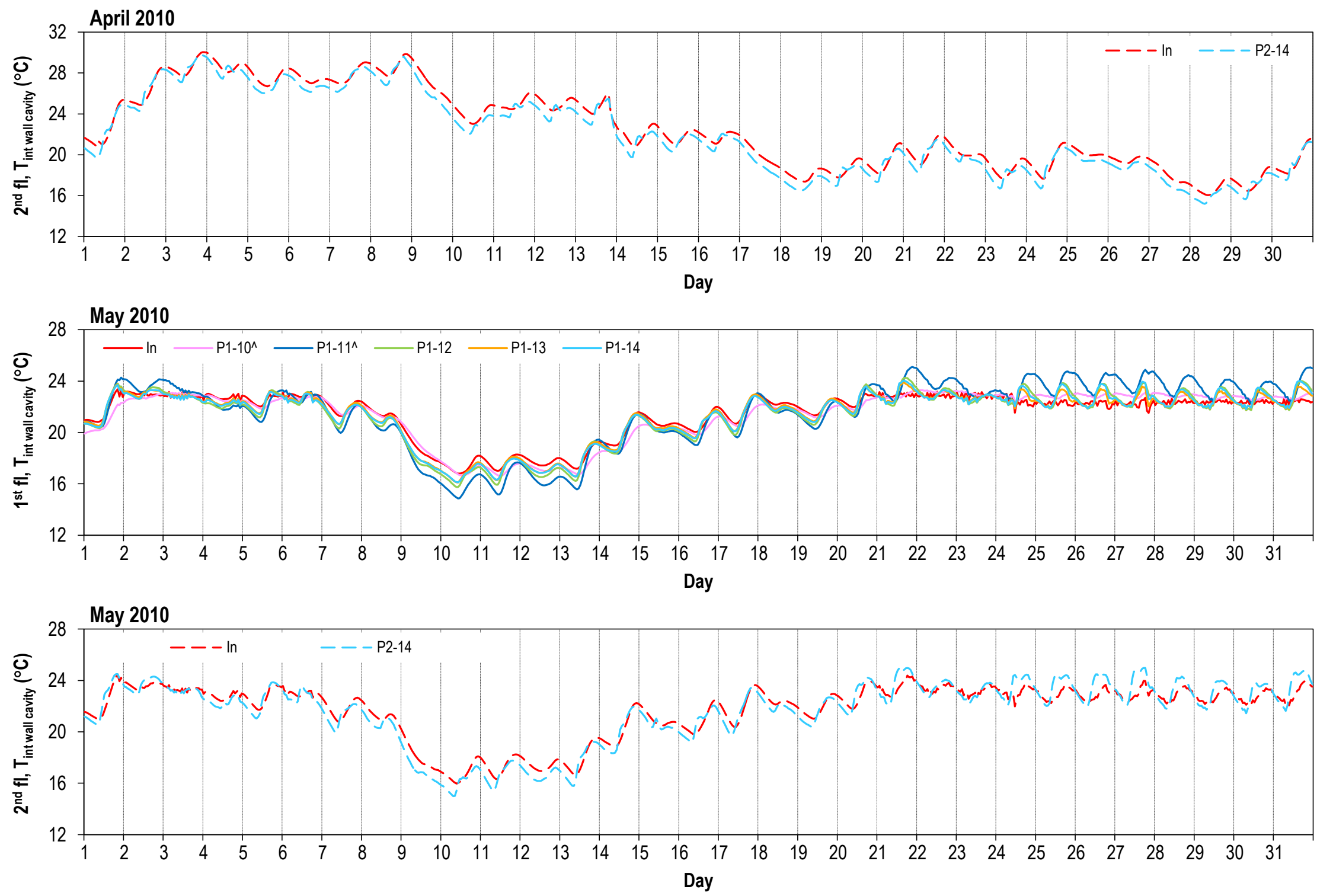

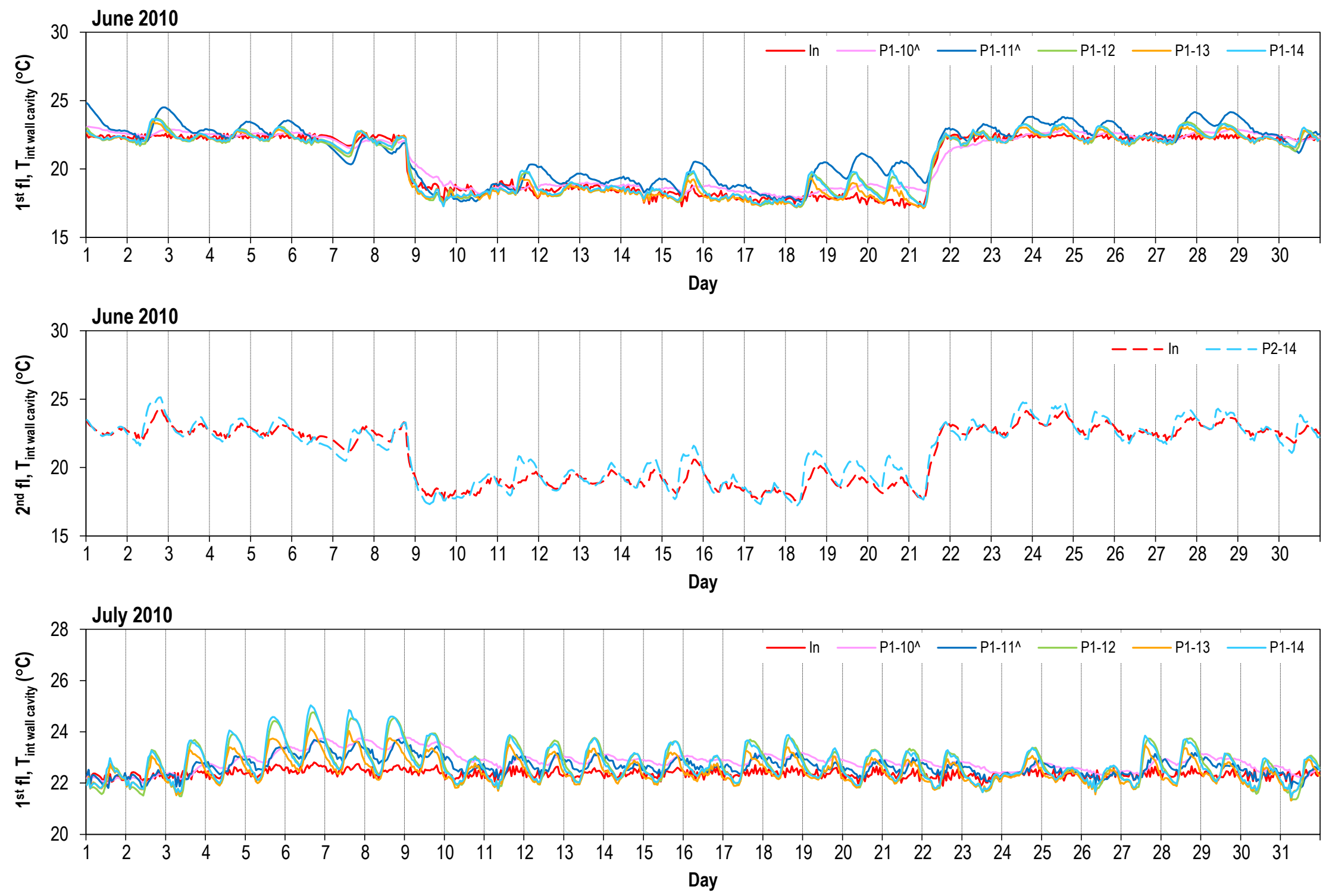

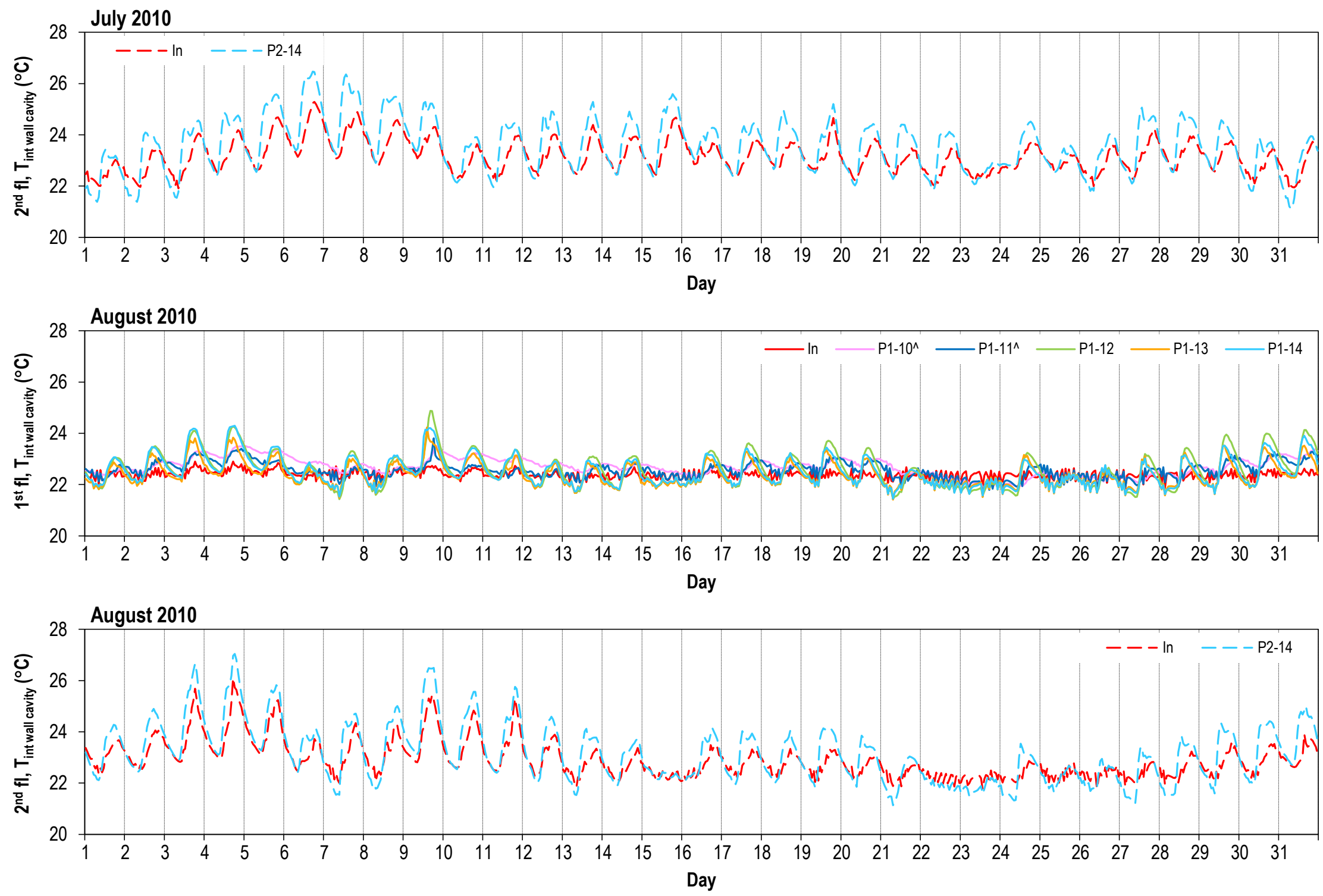

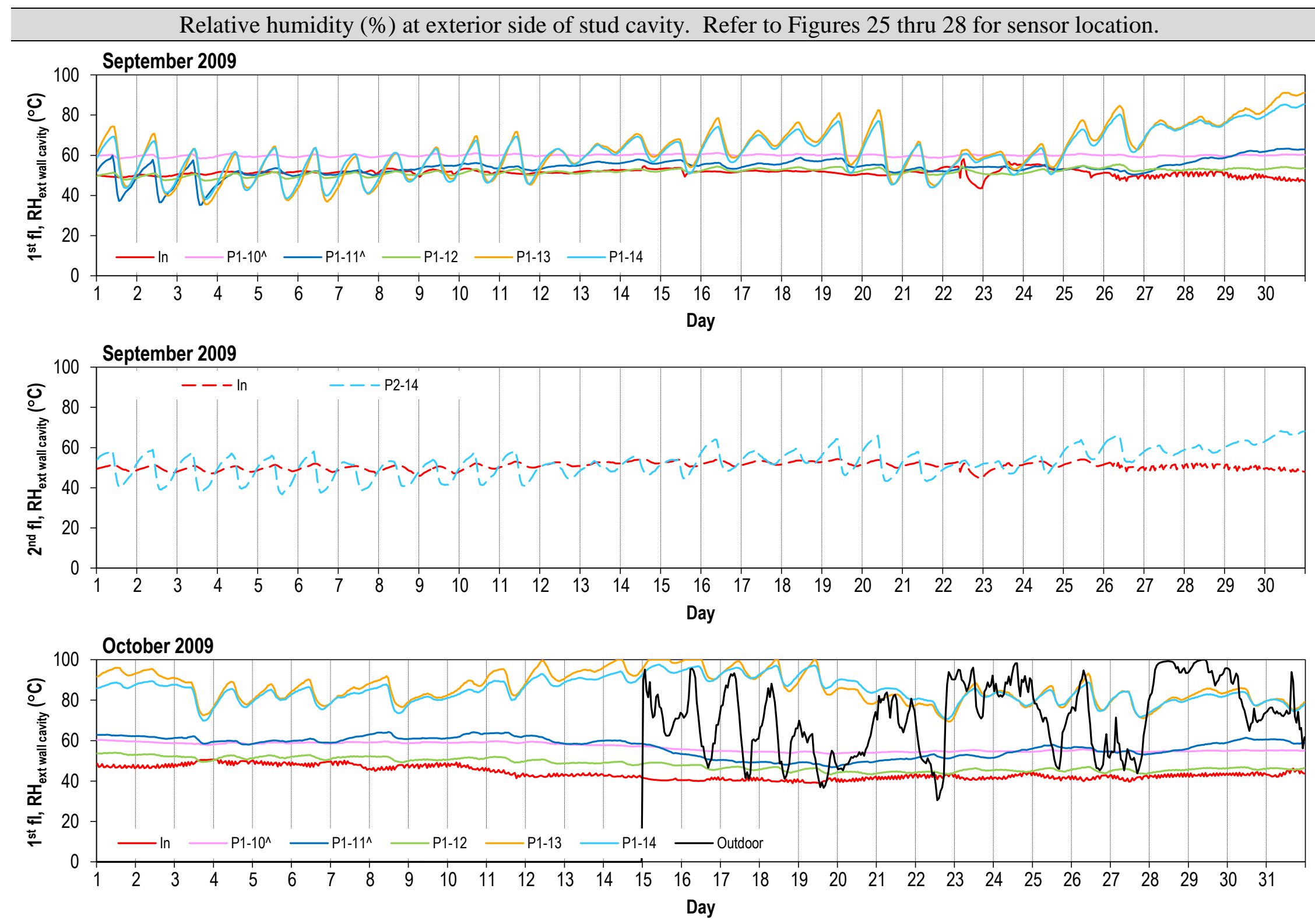

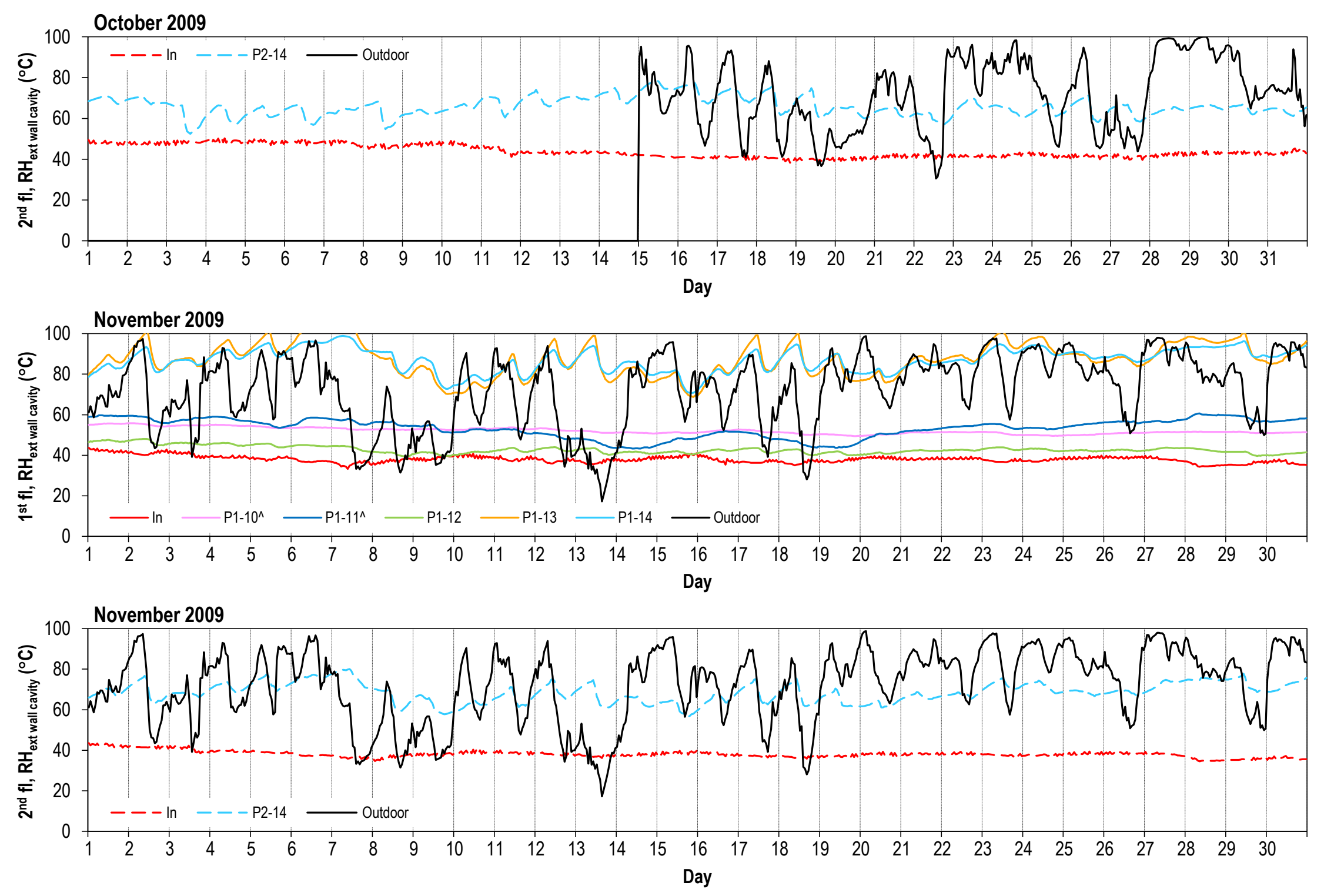

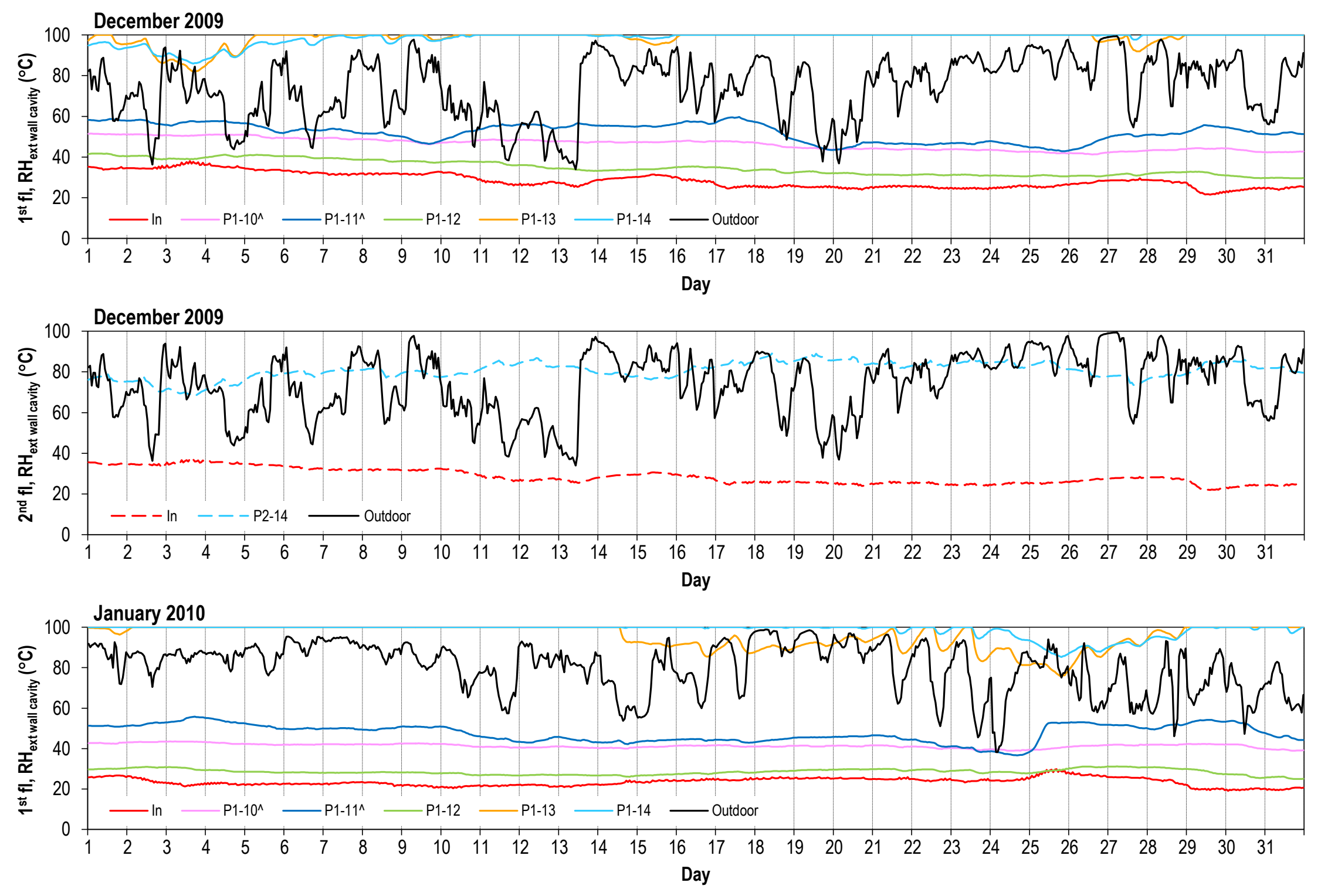

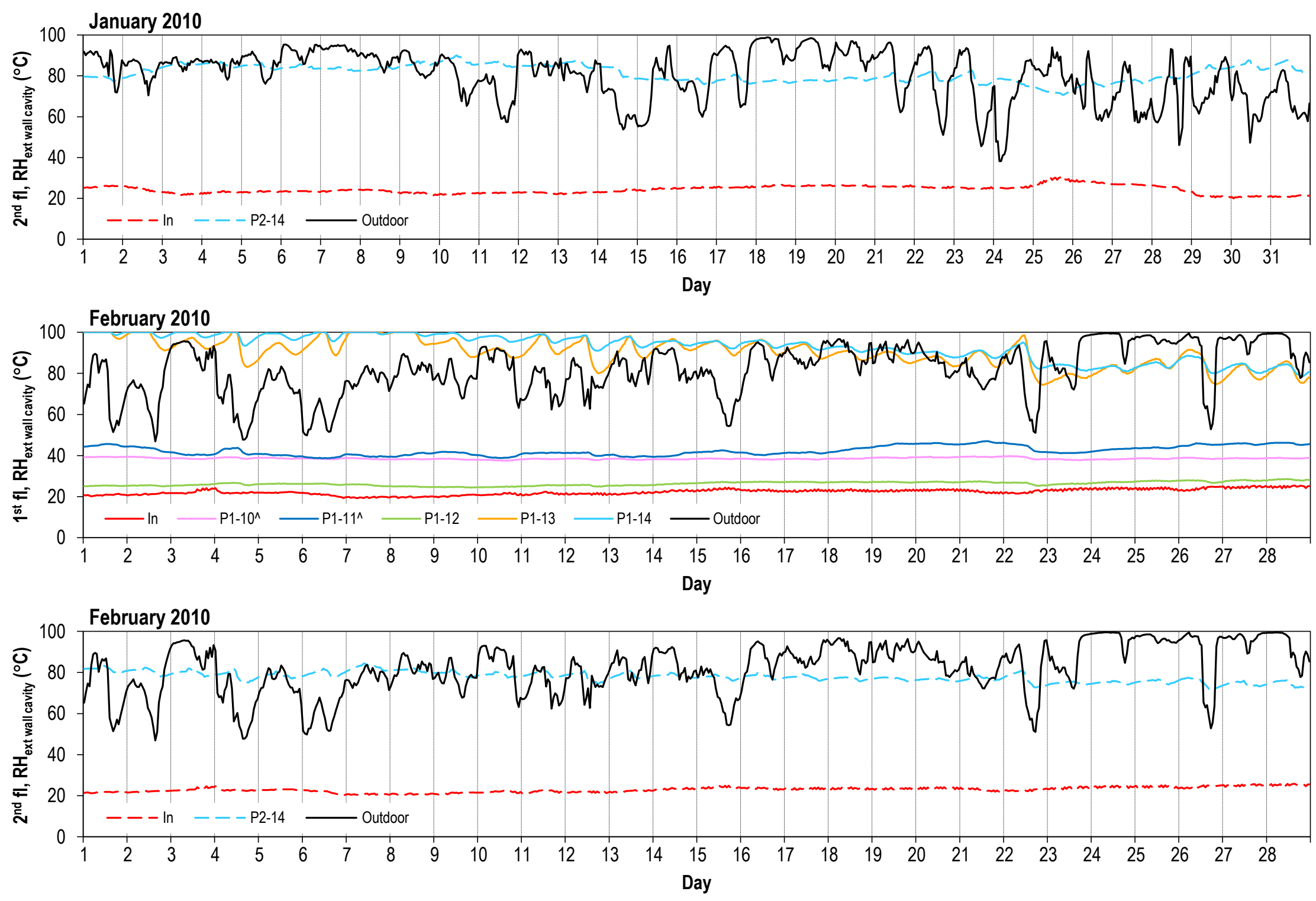
111
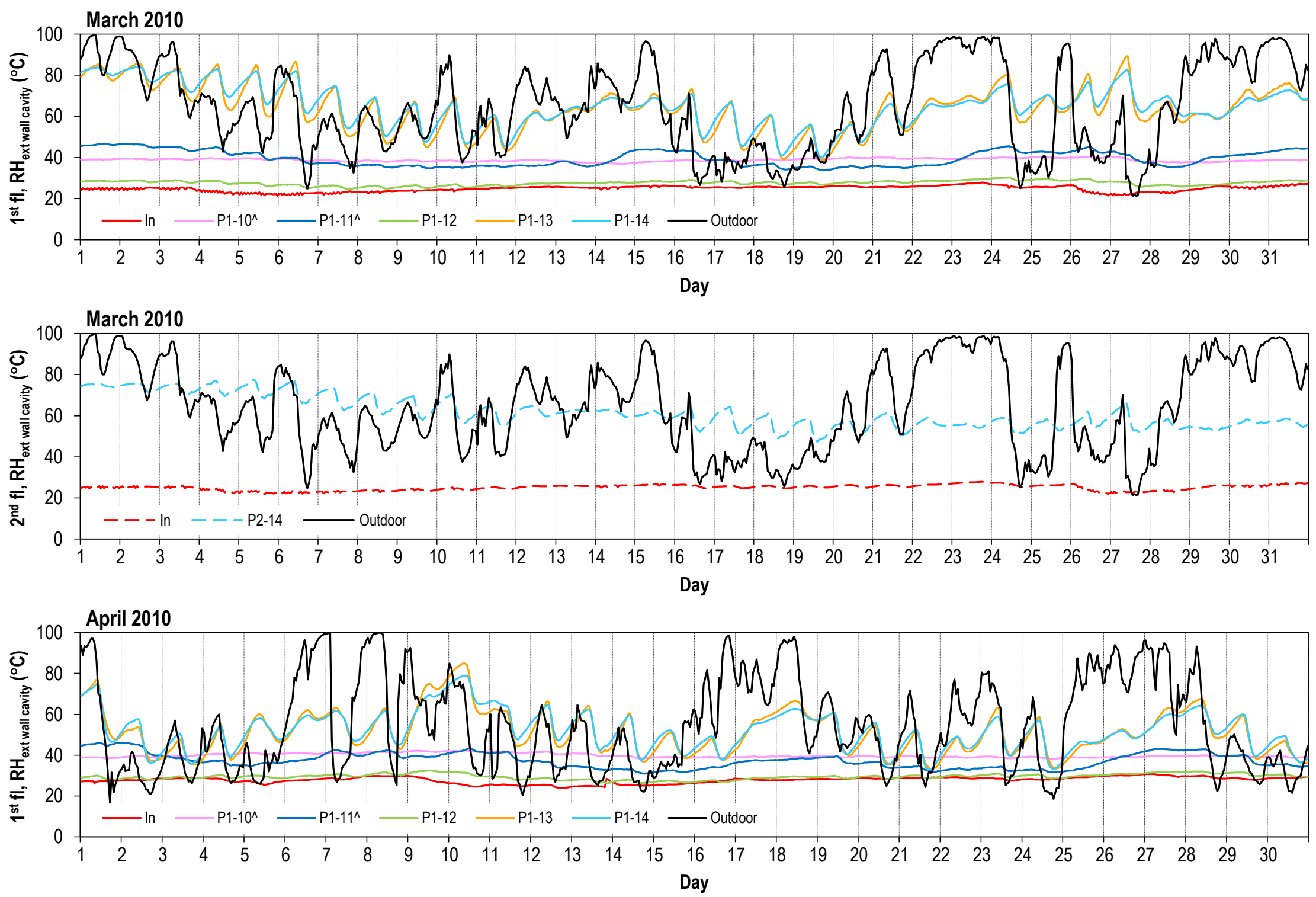

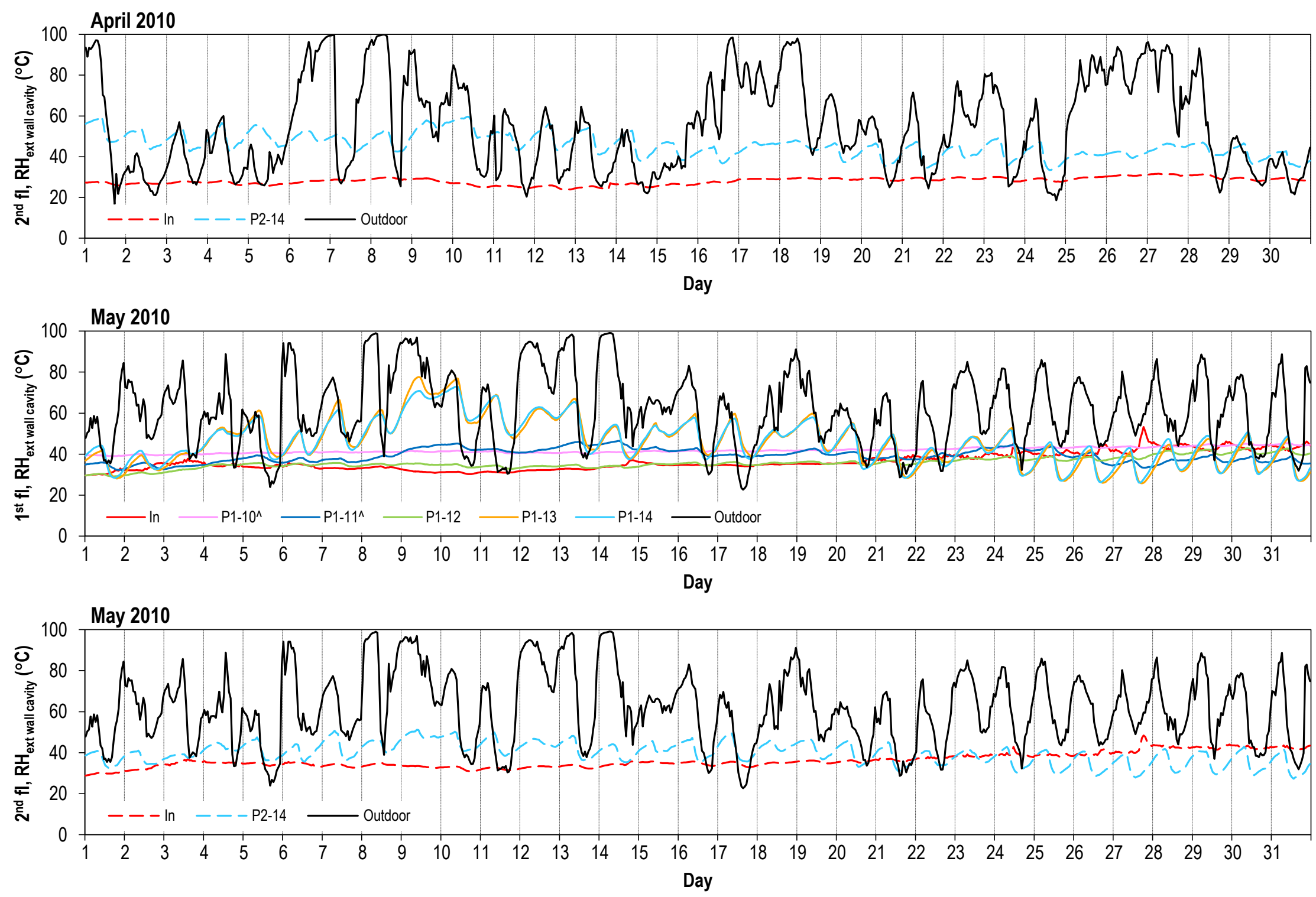

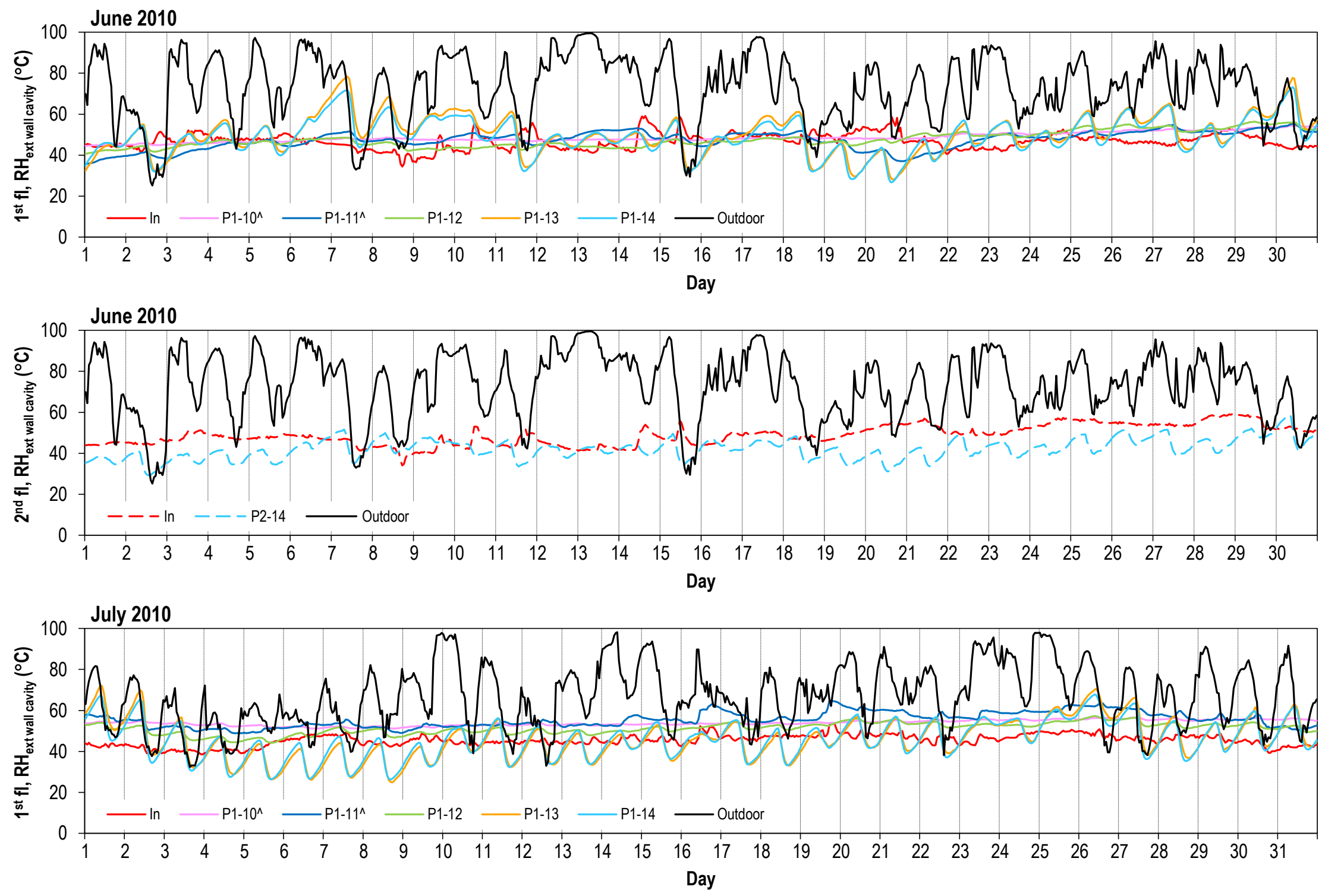

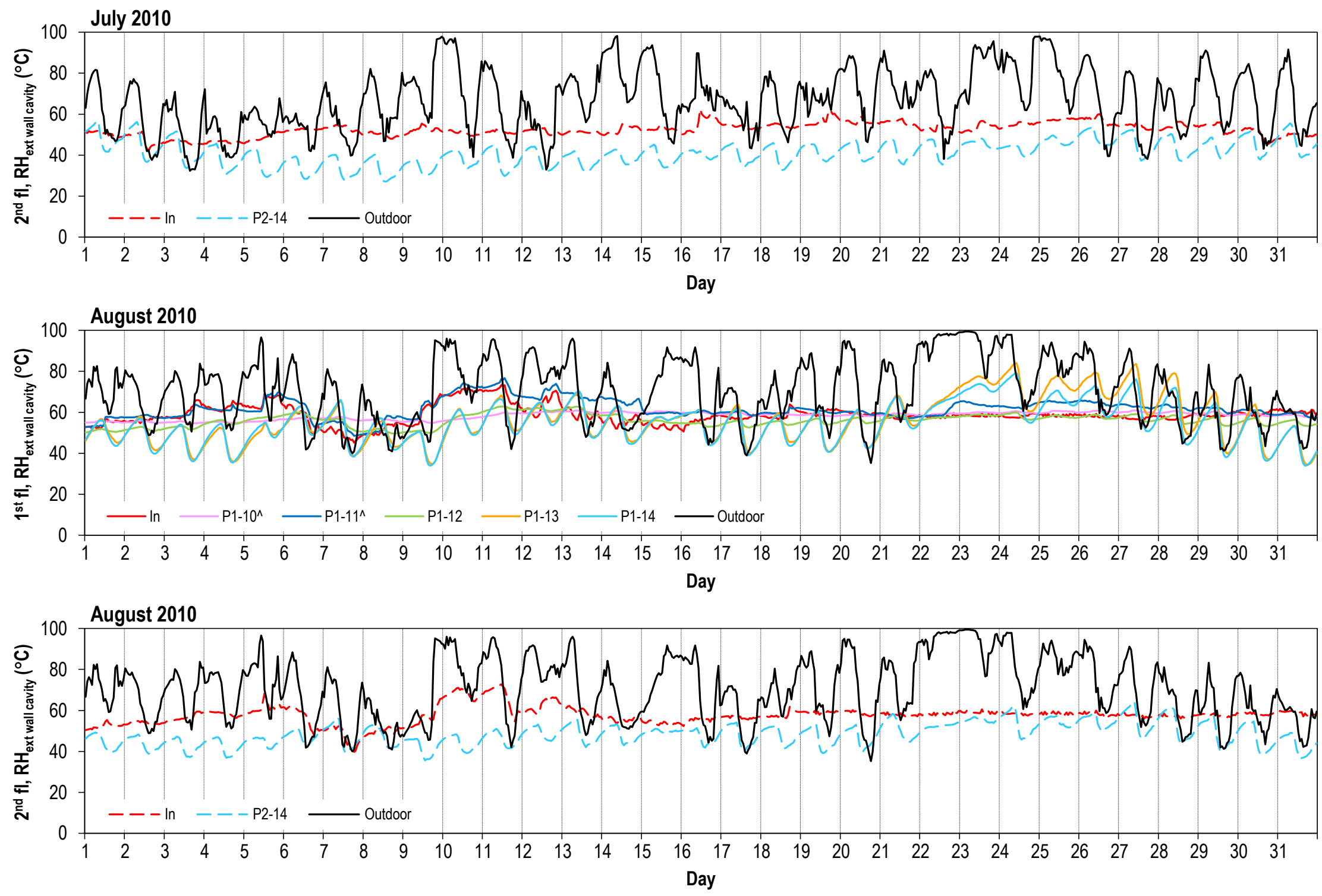

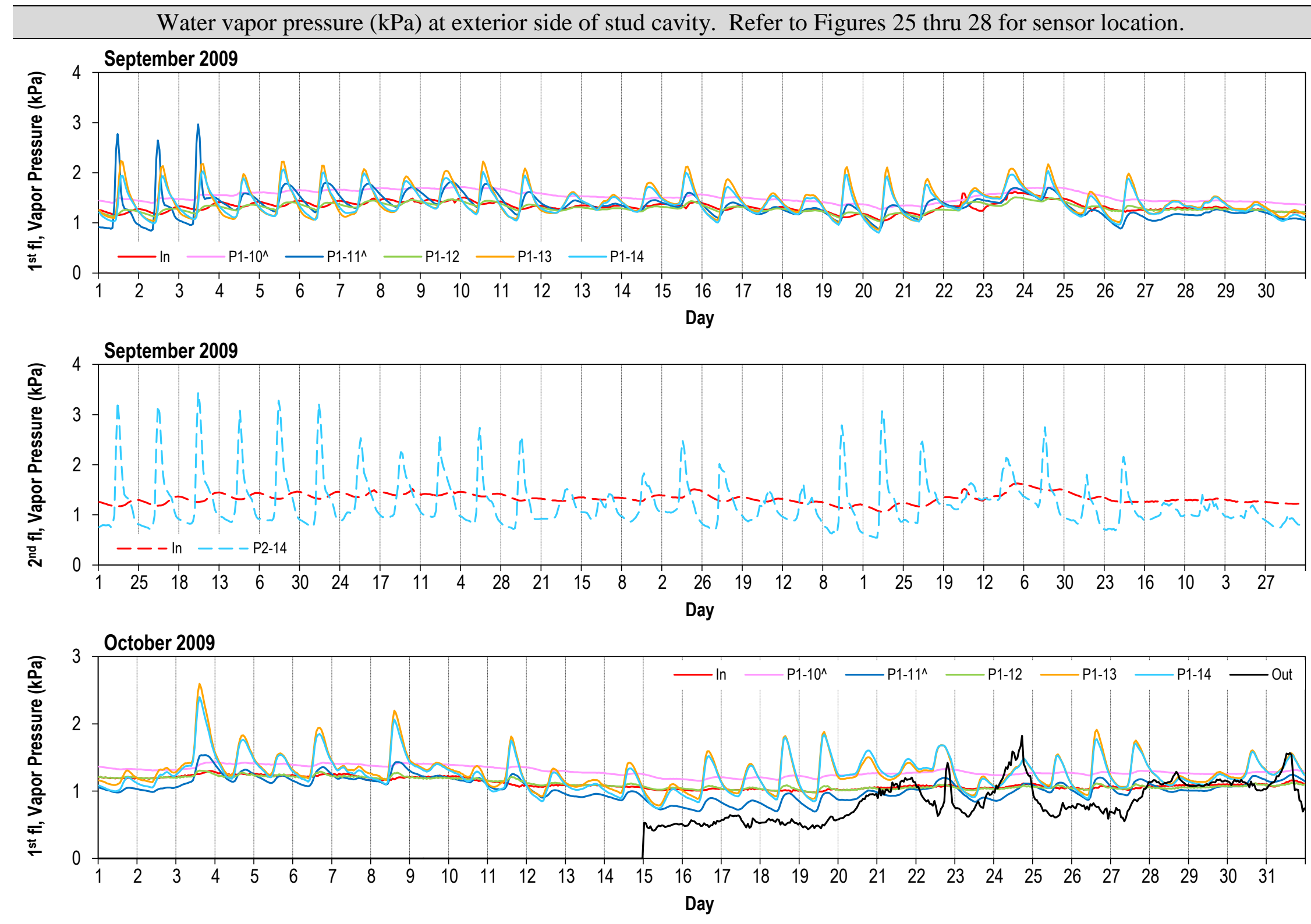

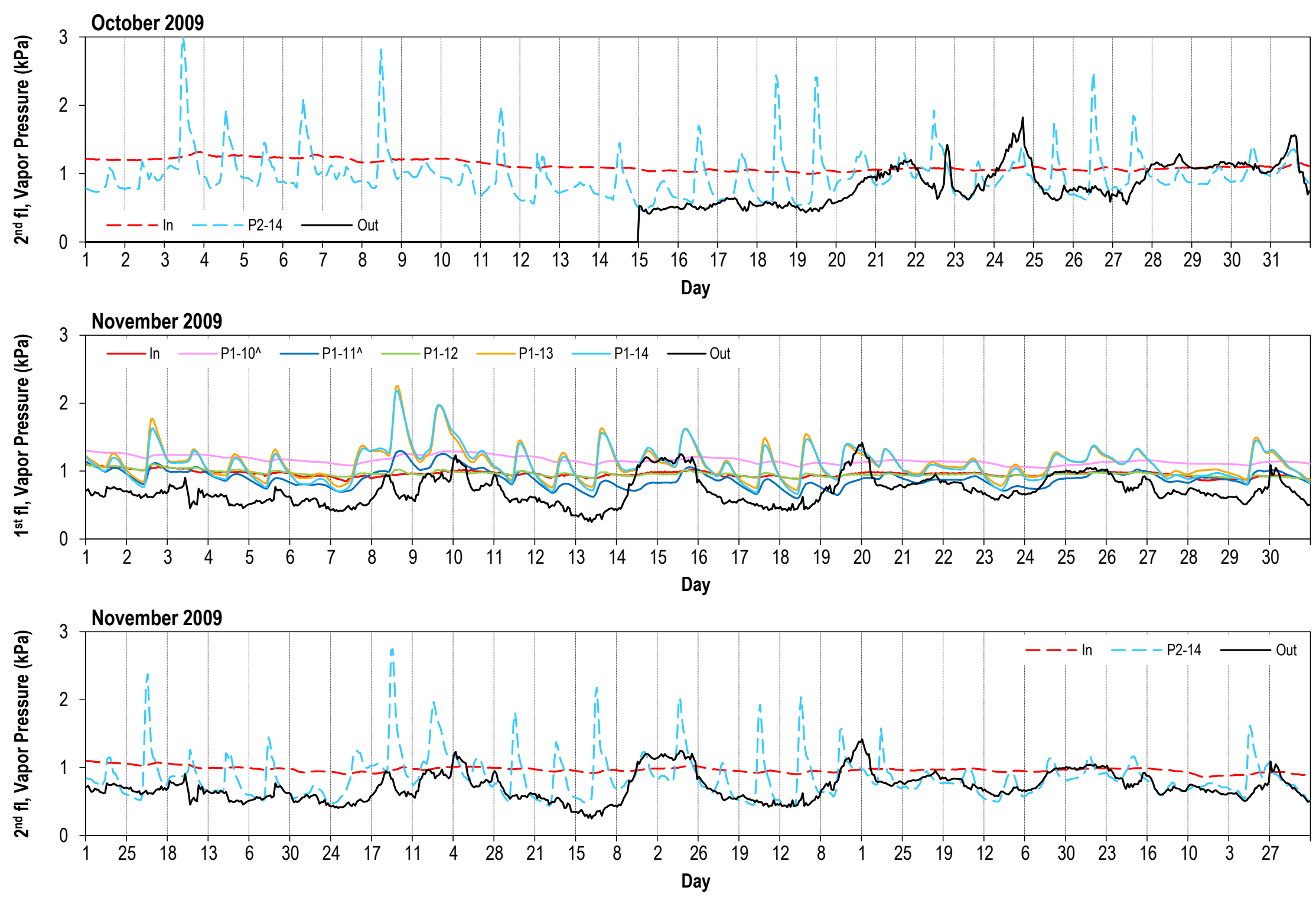

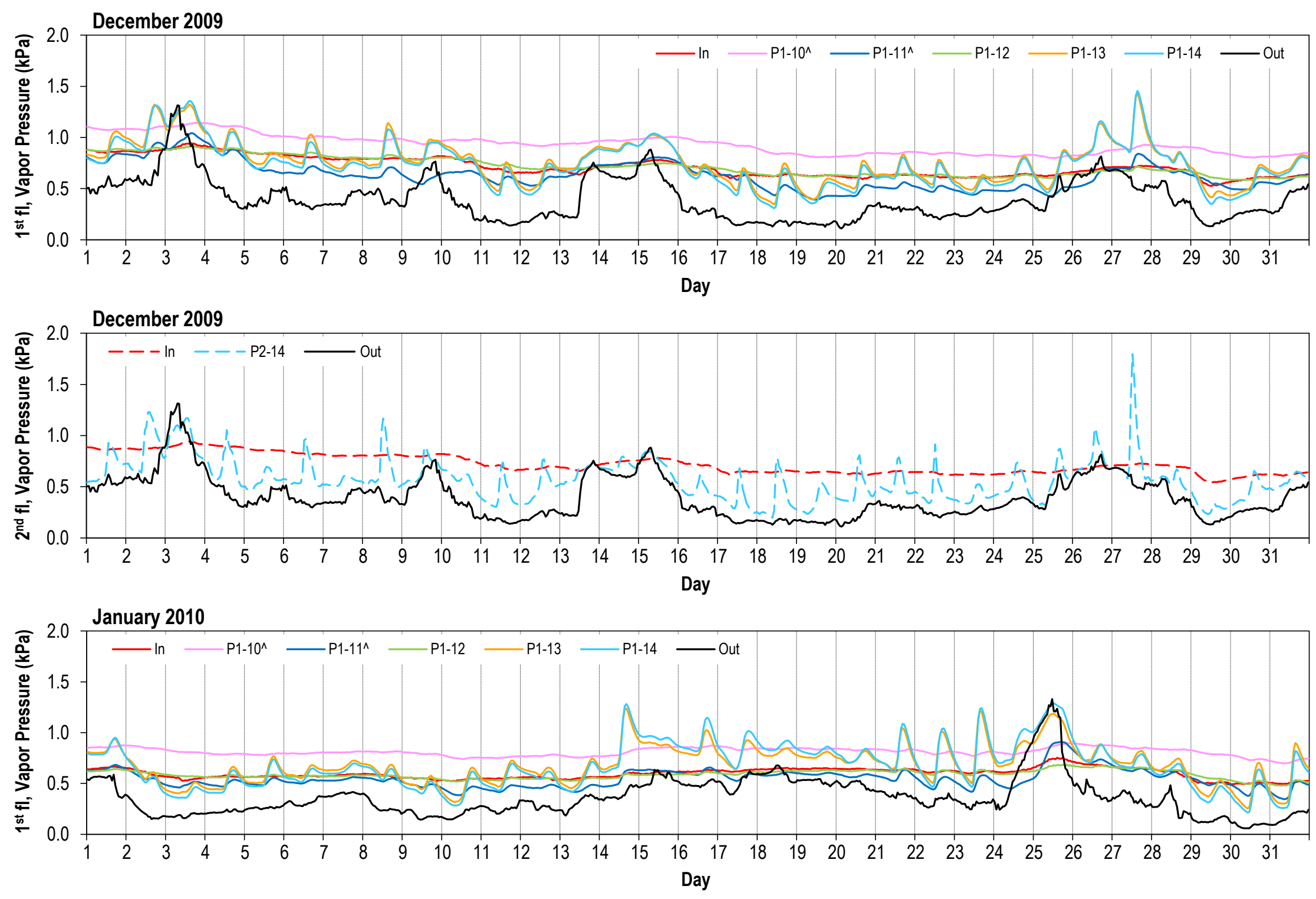

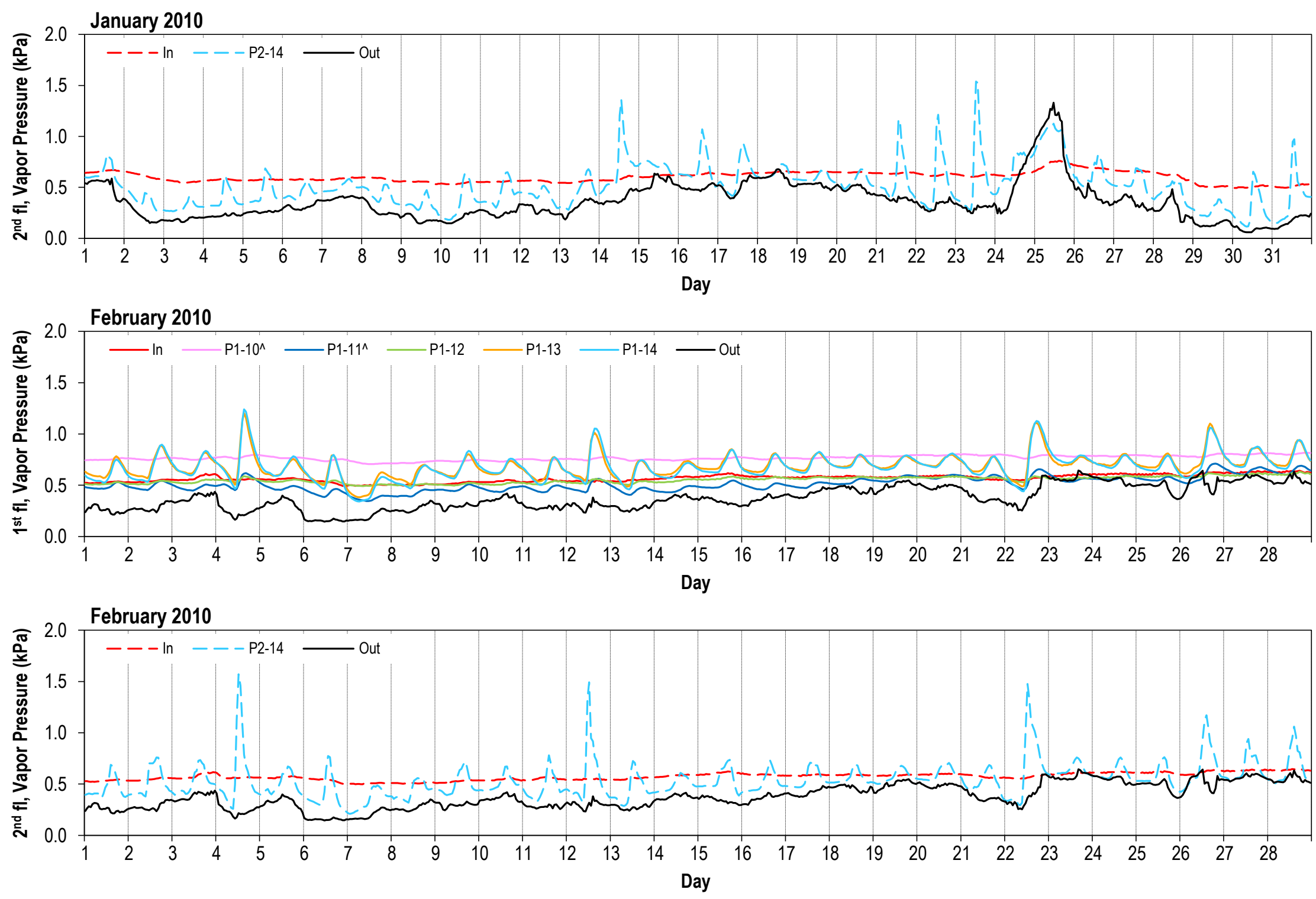

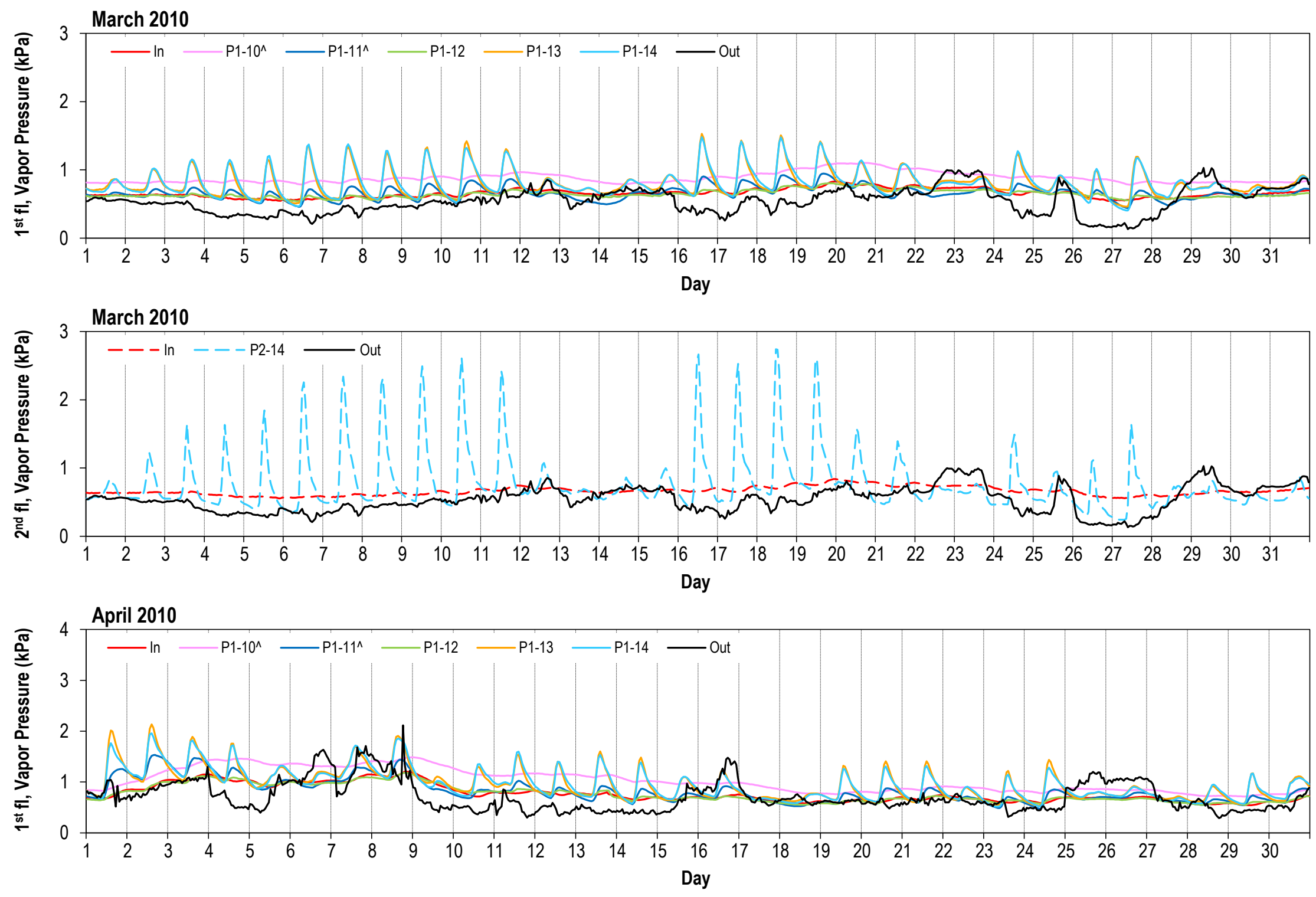

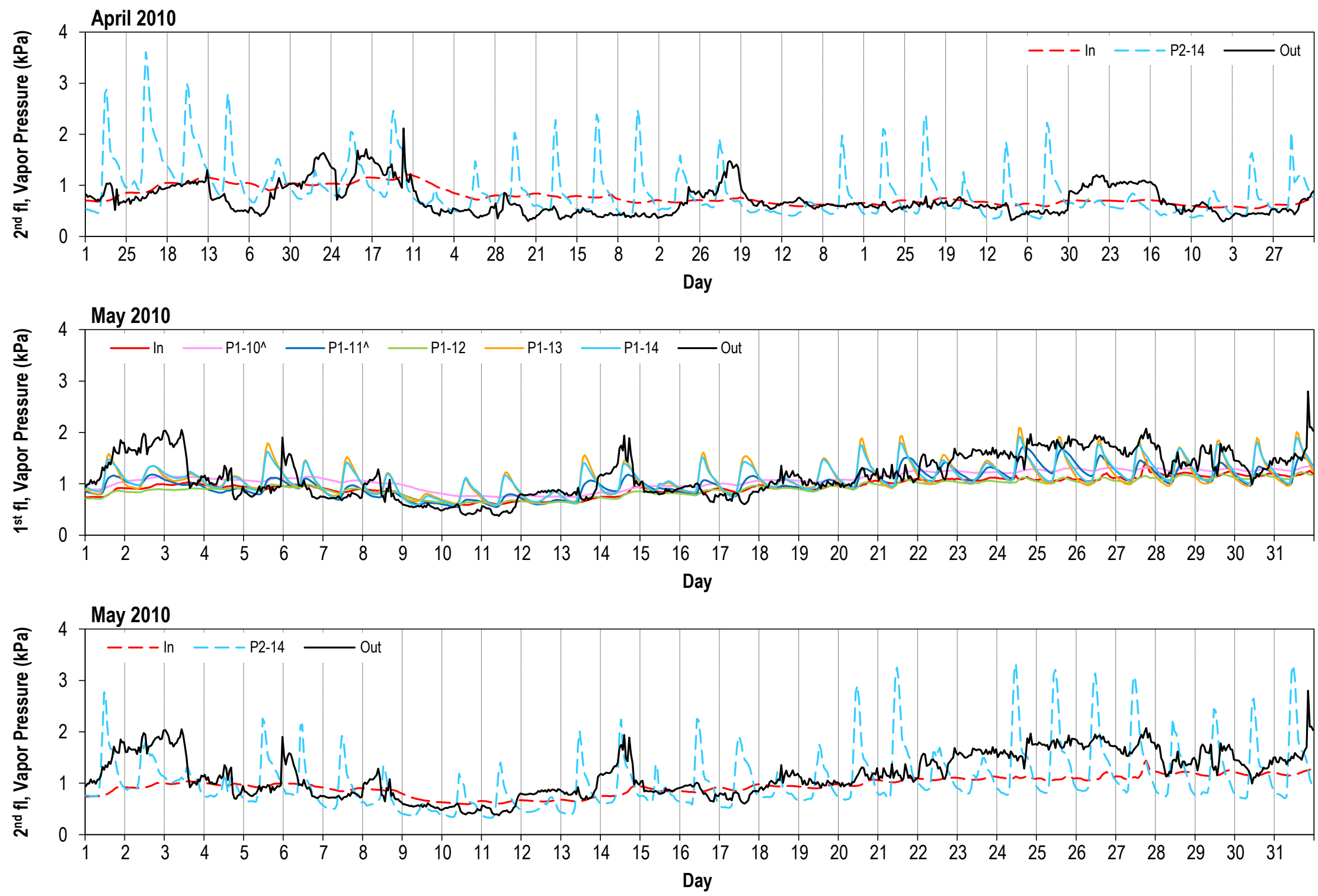
121
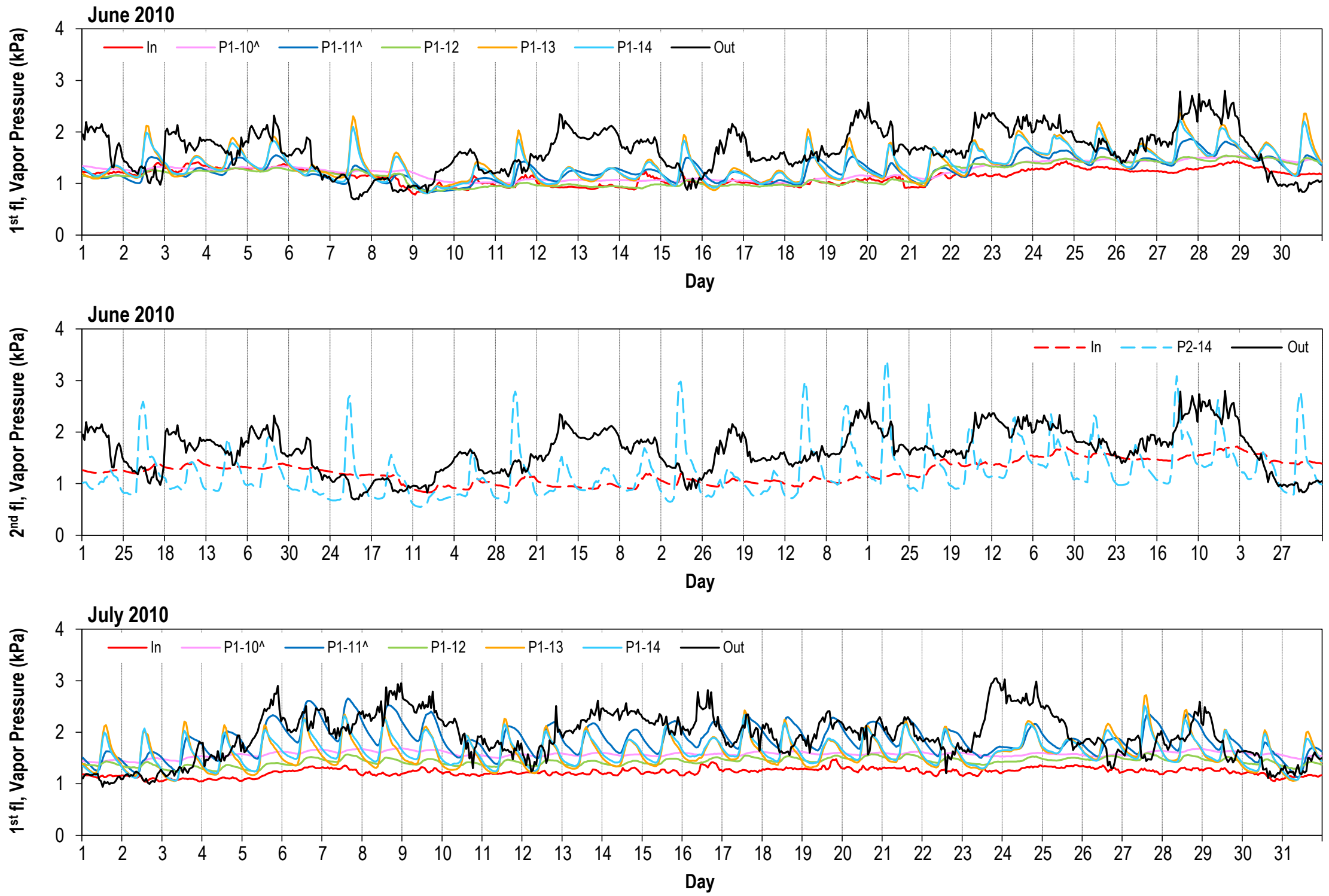

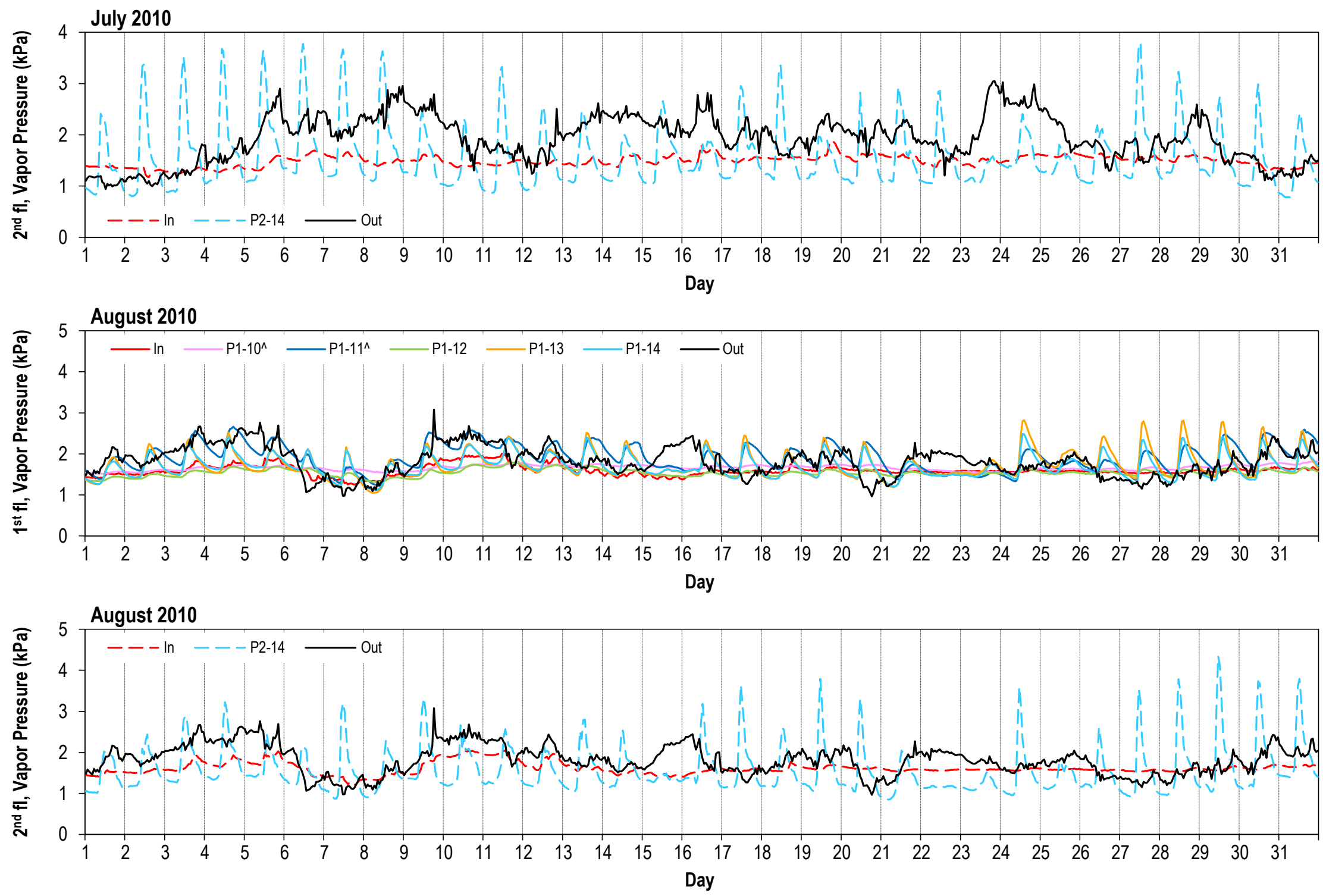
Heat flux $\left(\mathrm{W} / \mathrm{m}^{2}\right)$ thru interior side of stud cavity. Refer to Figures 25 thru 28 for sensor location.
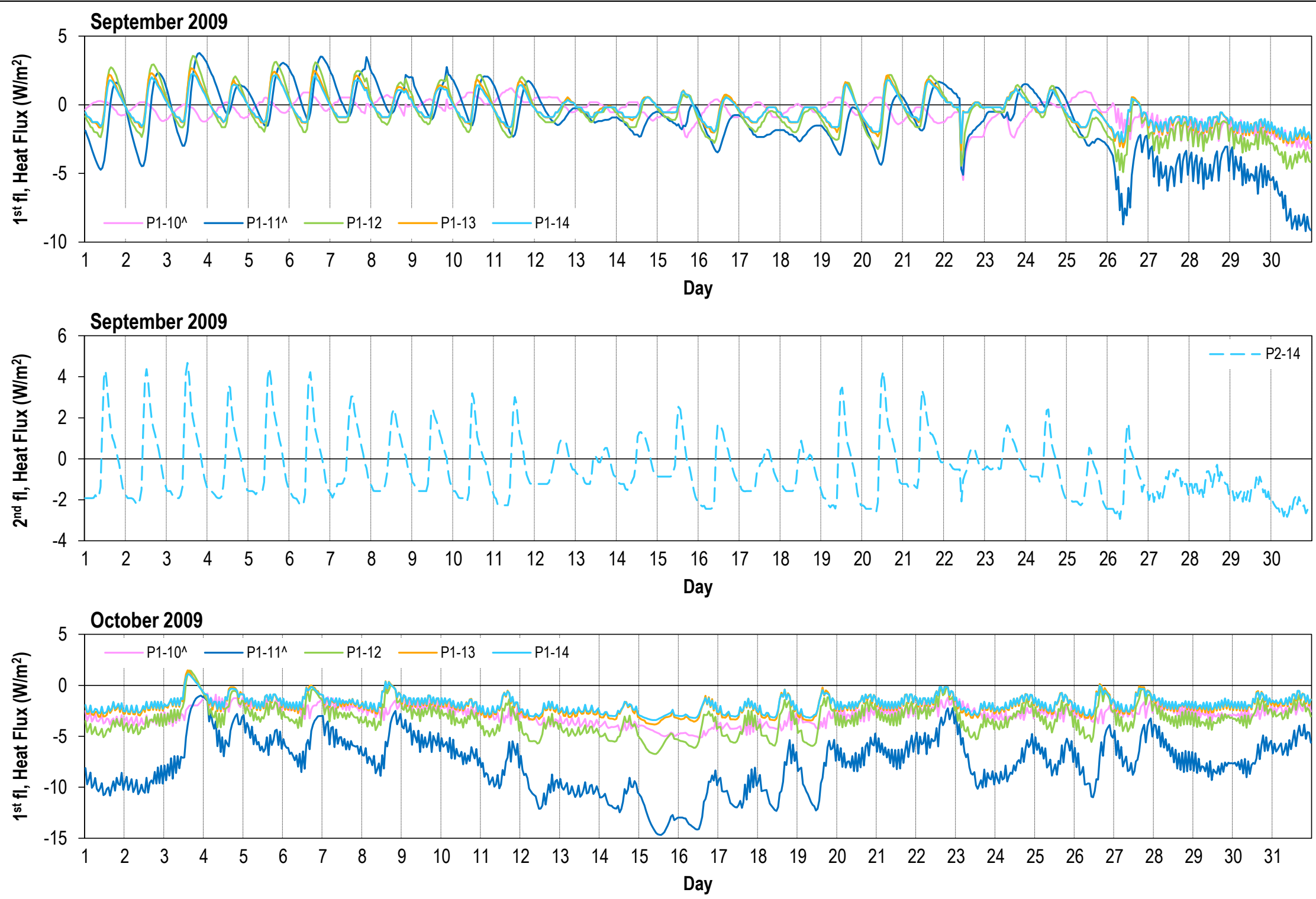

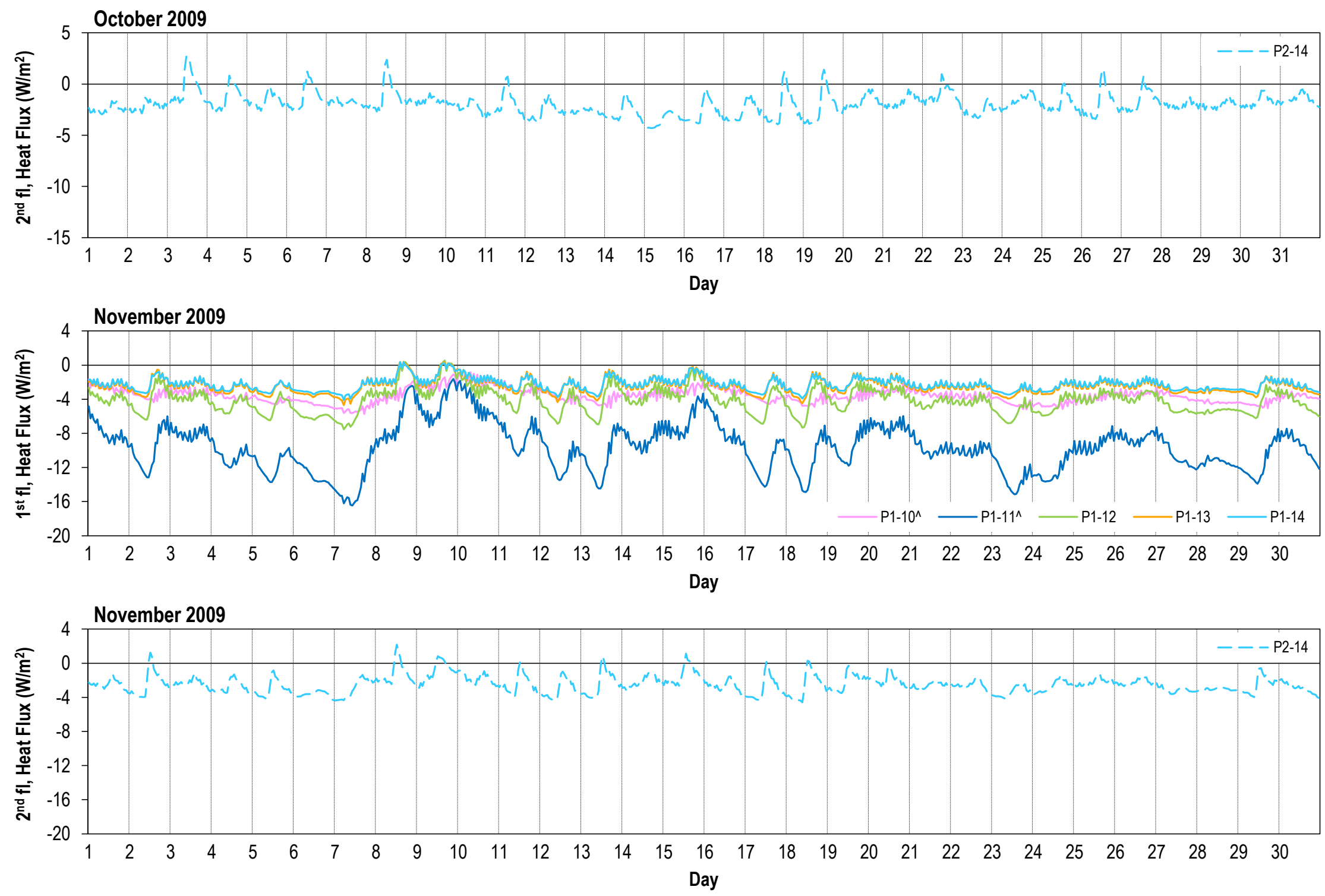

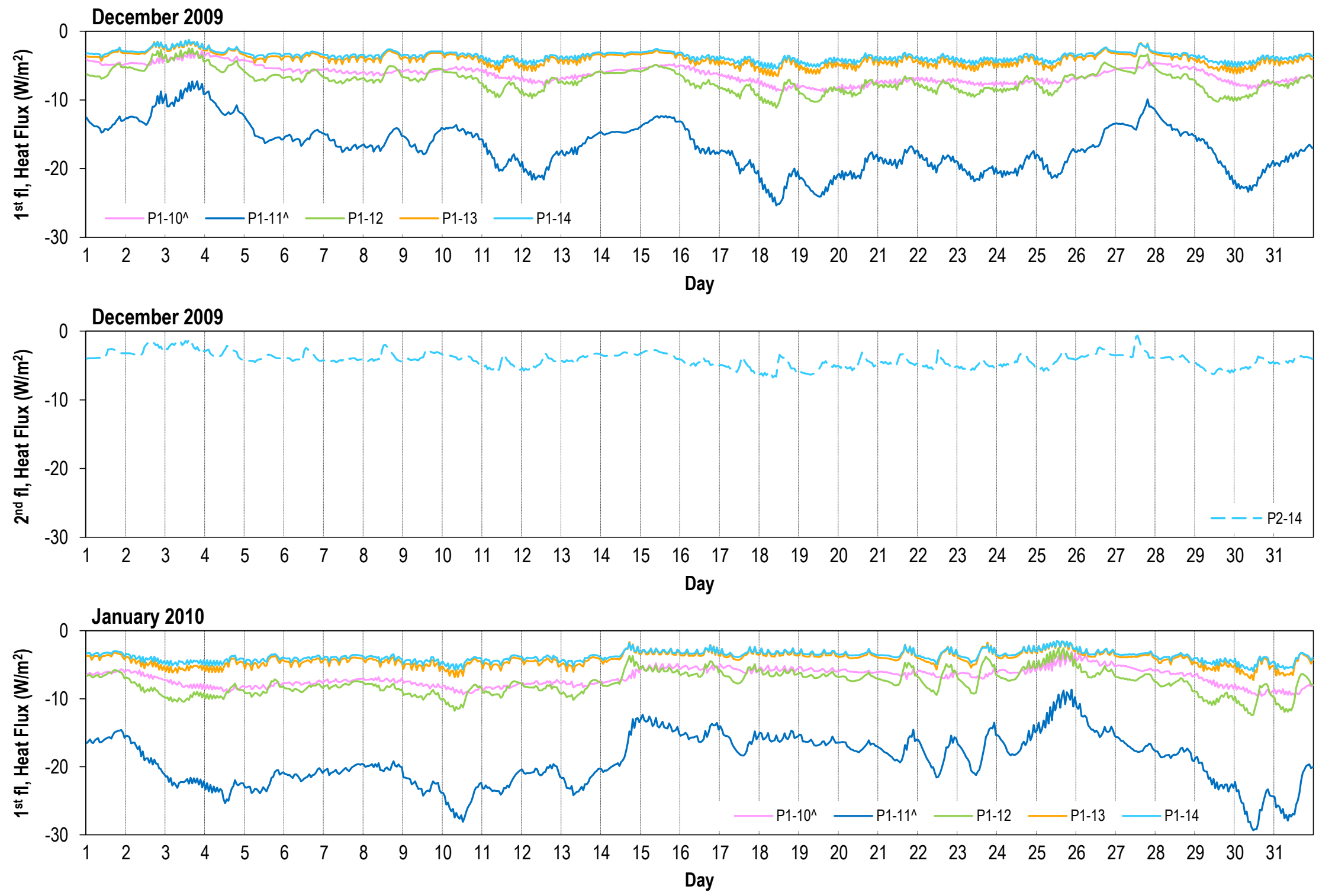

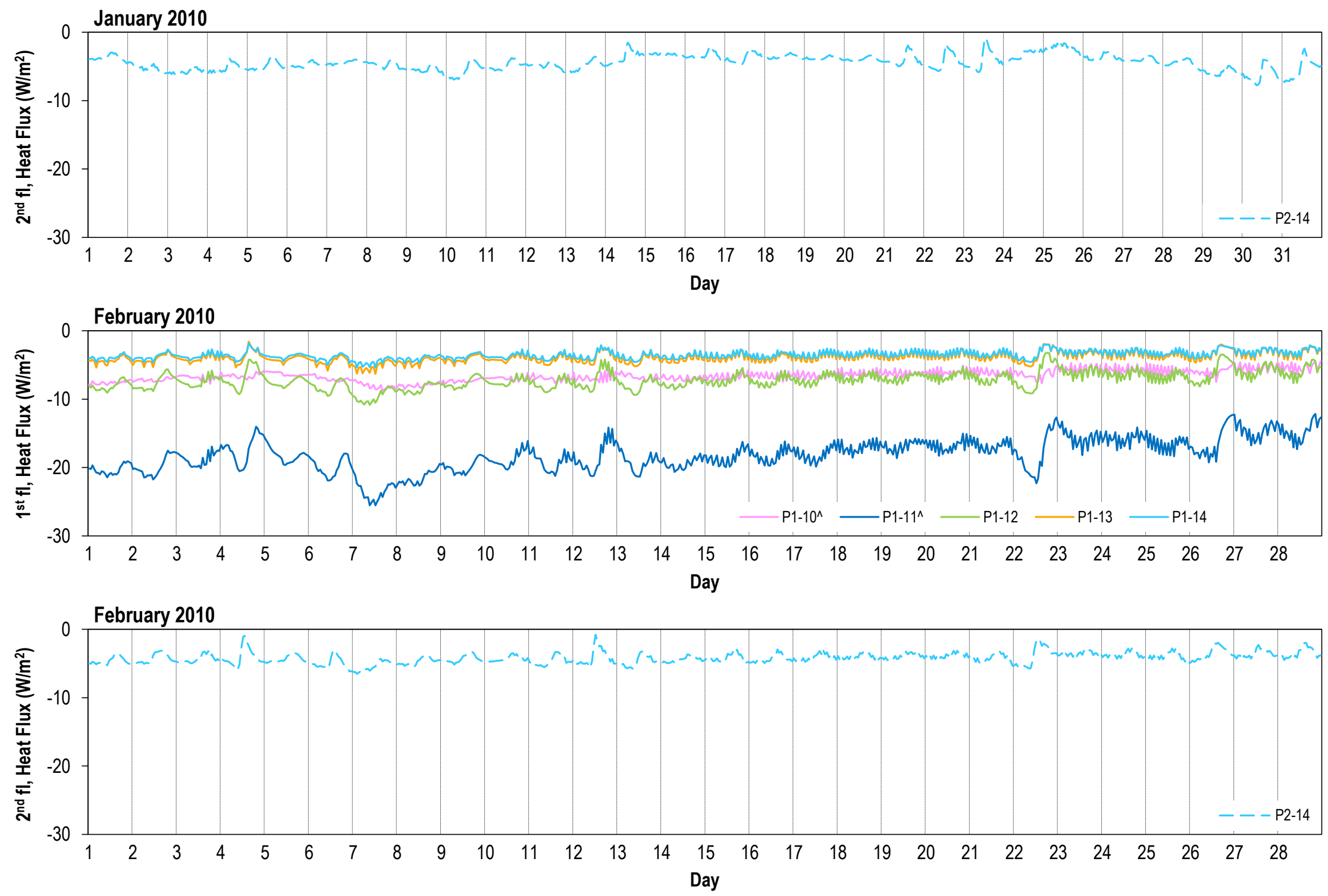

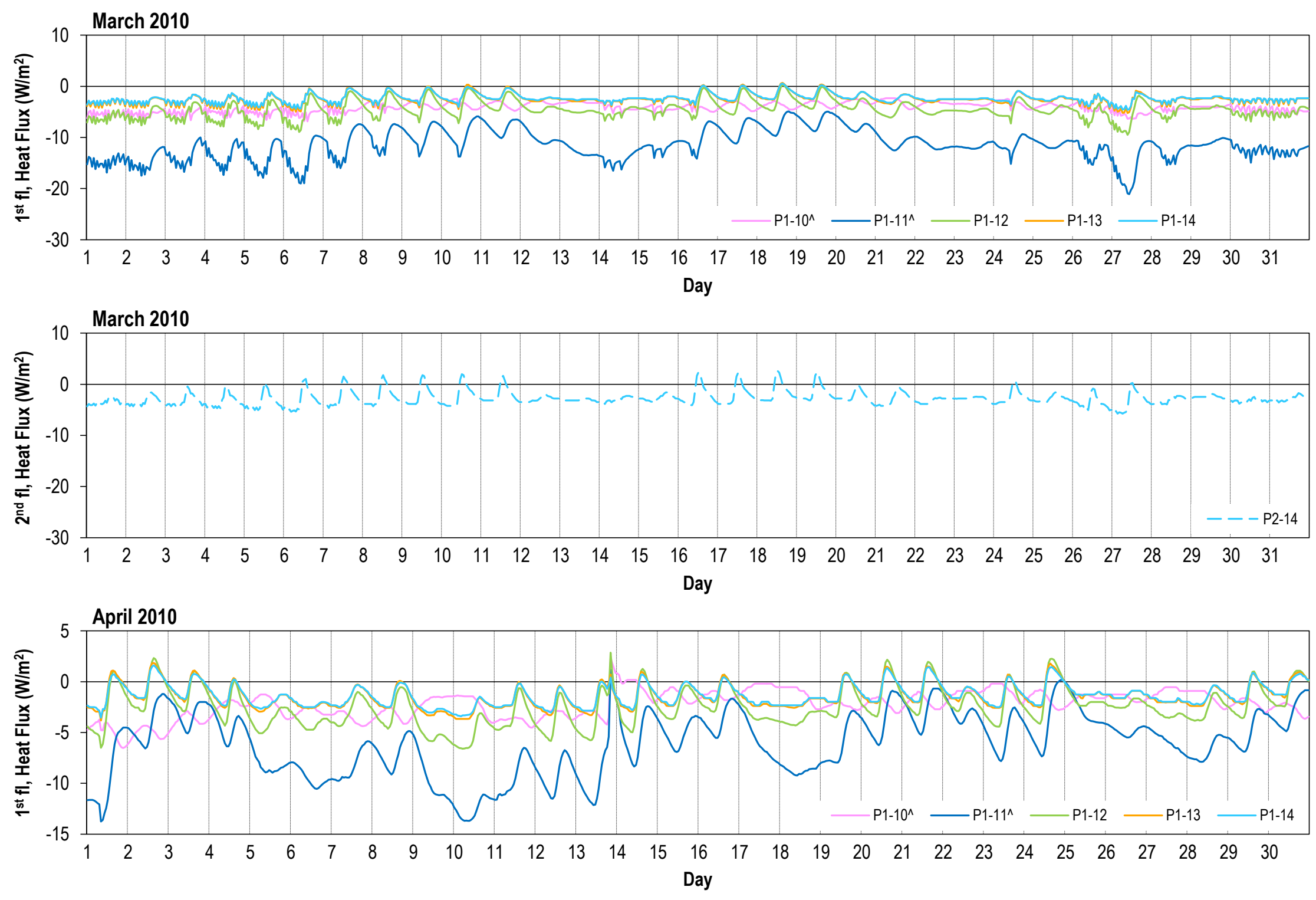

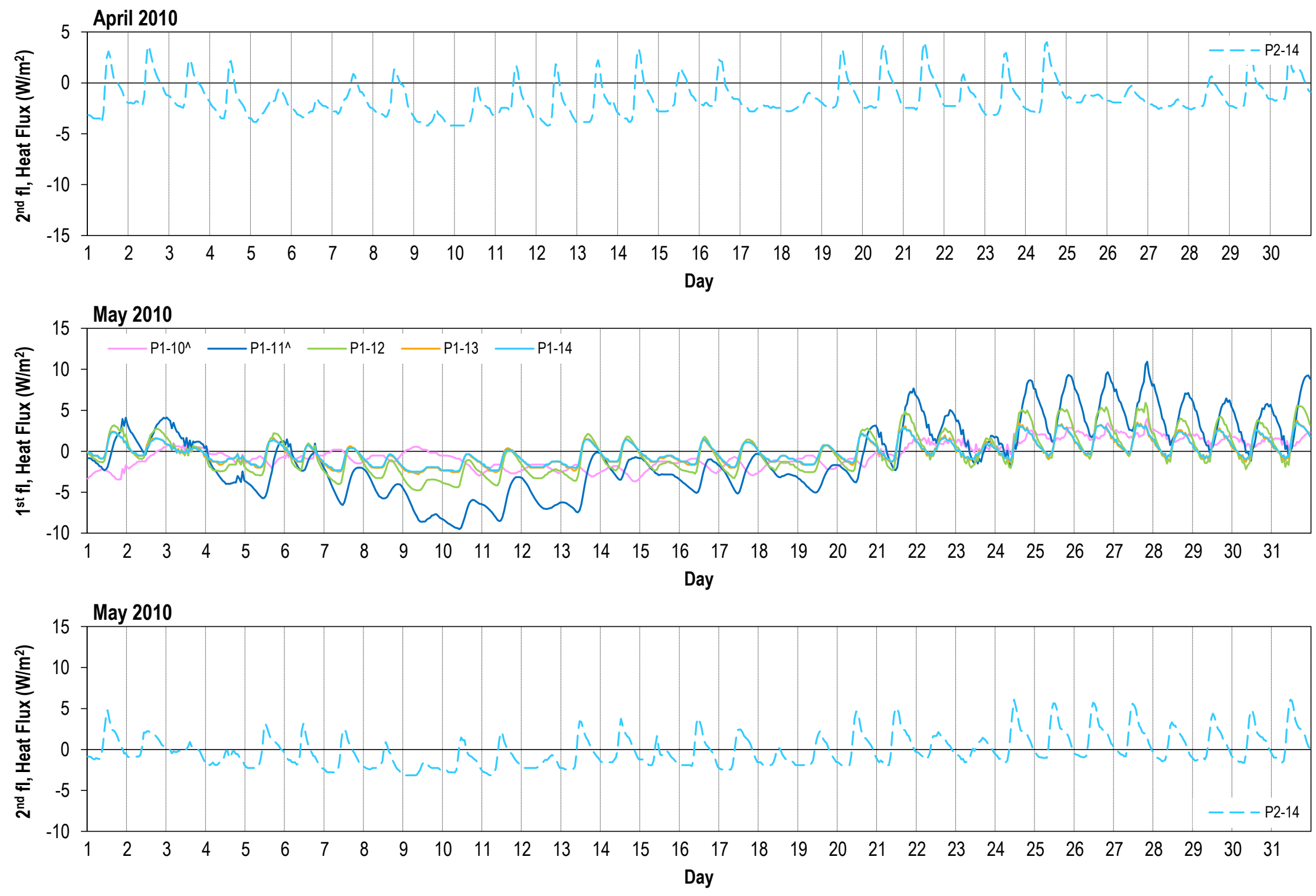

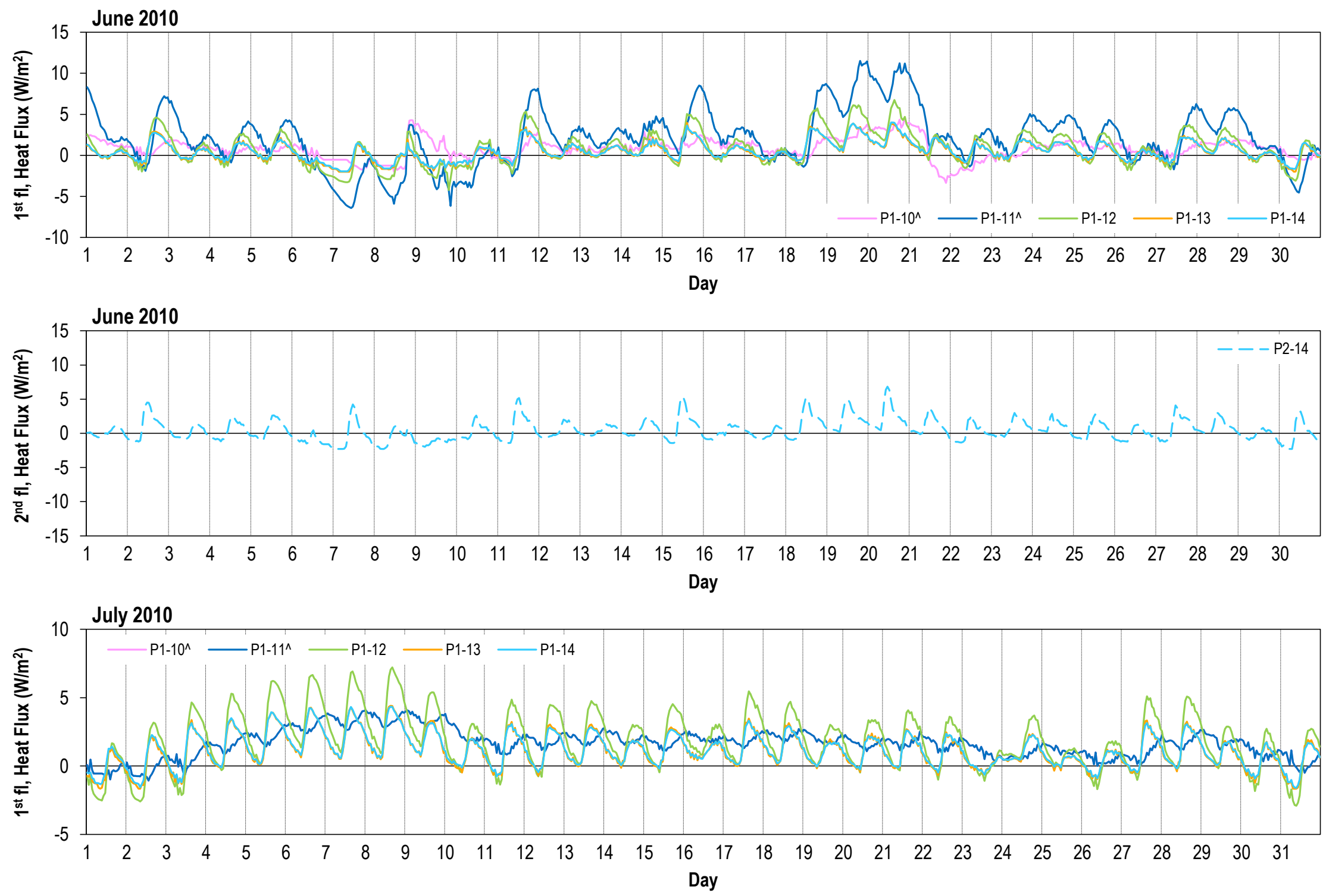

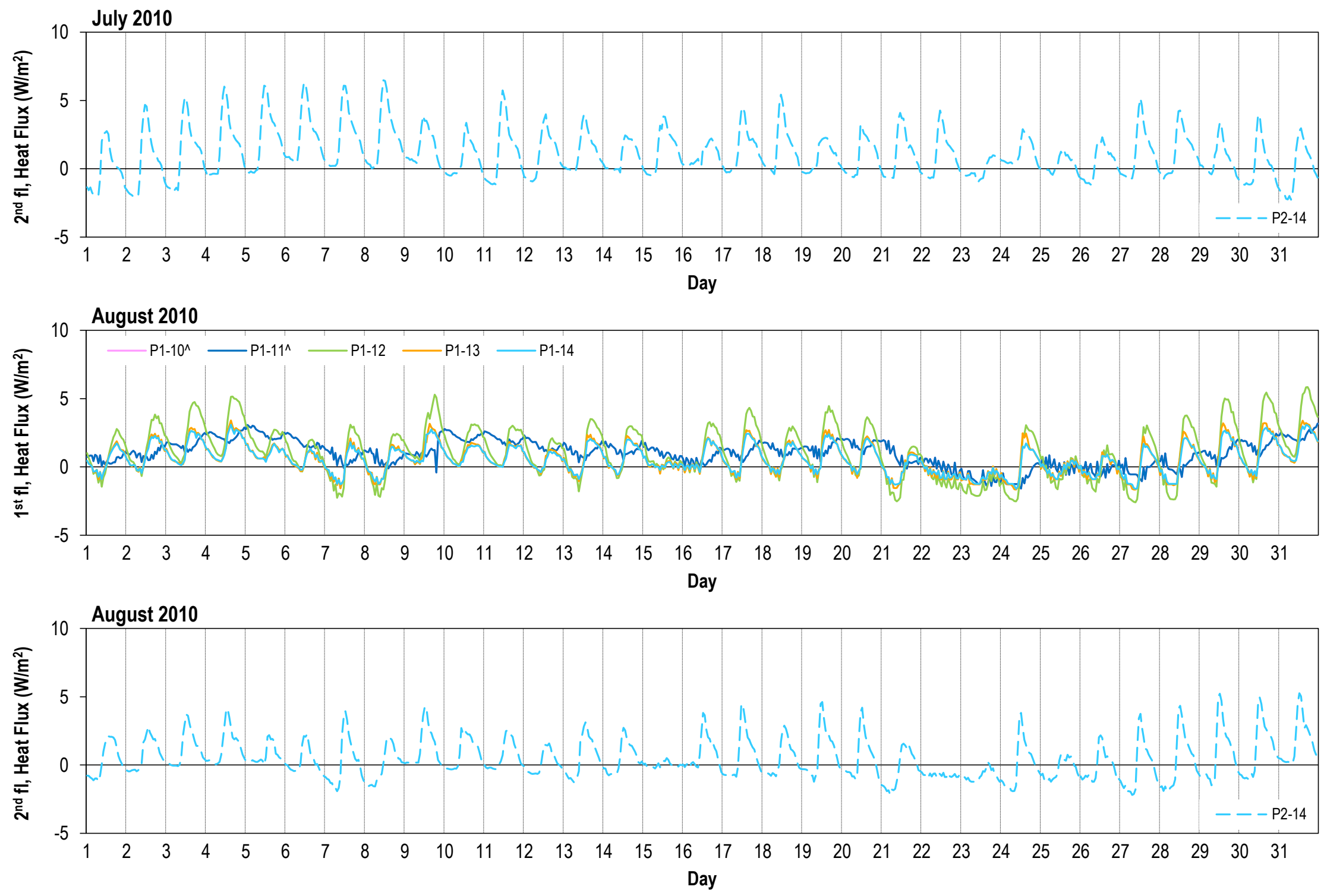
Cooling loads $\left(\mathrm{Wh} / \mathrm{m}^{2}\right)$. Refer to Figures 25 thru 28 for sensor location.
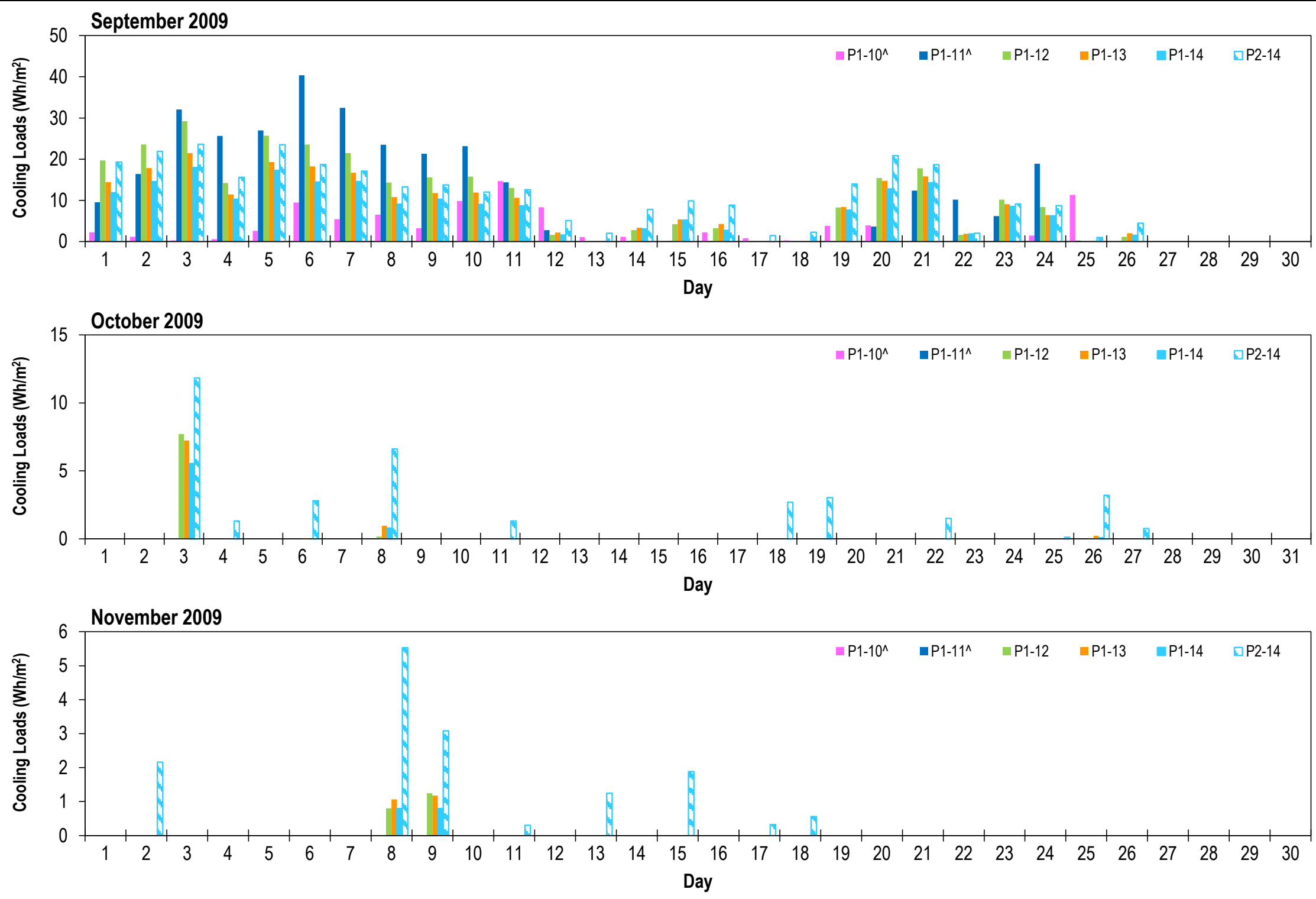

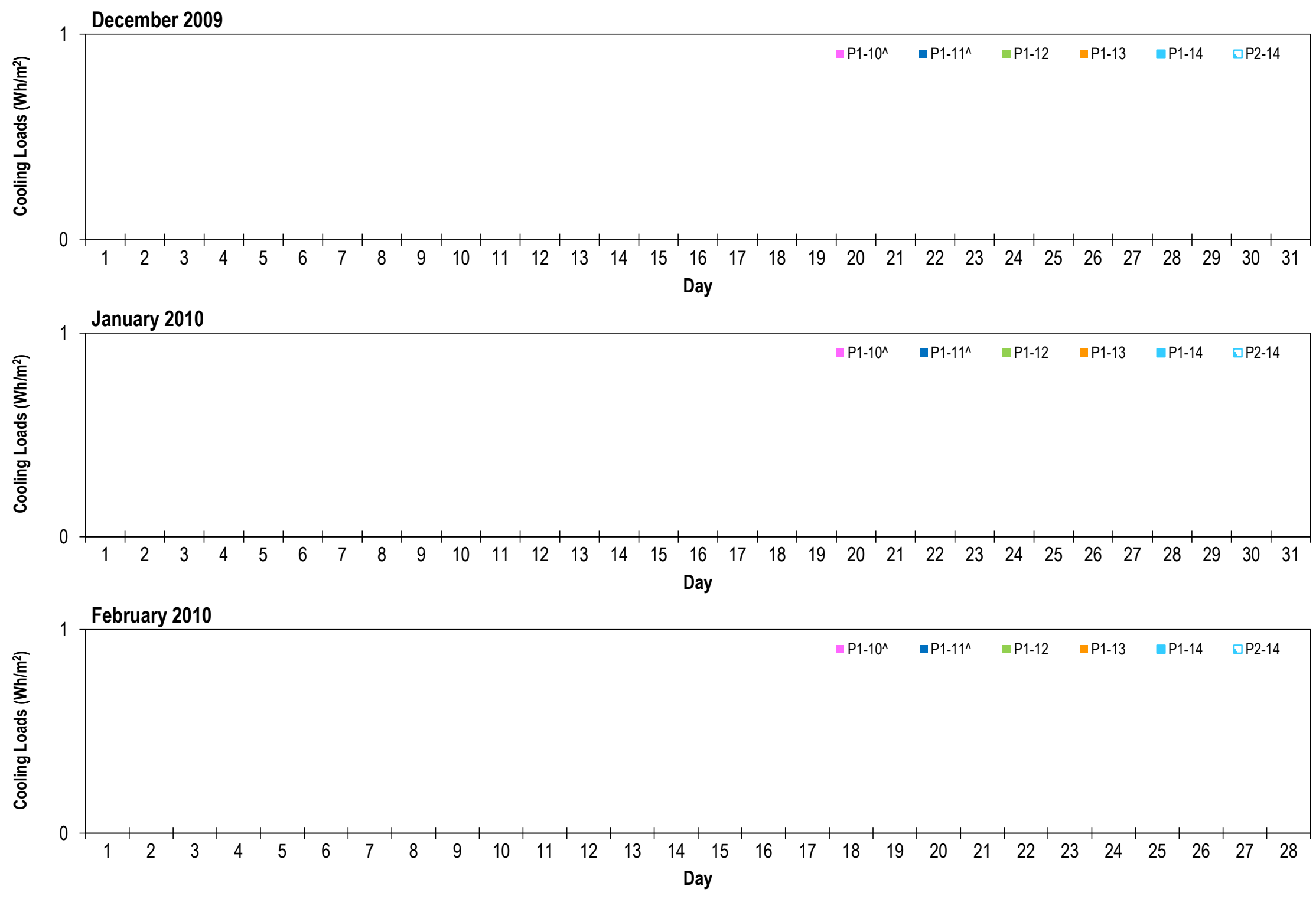

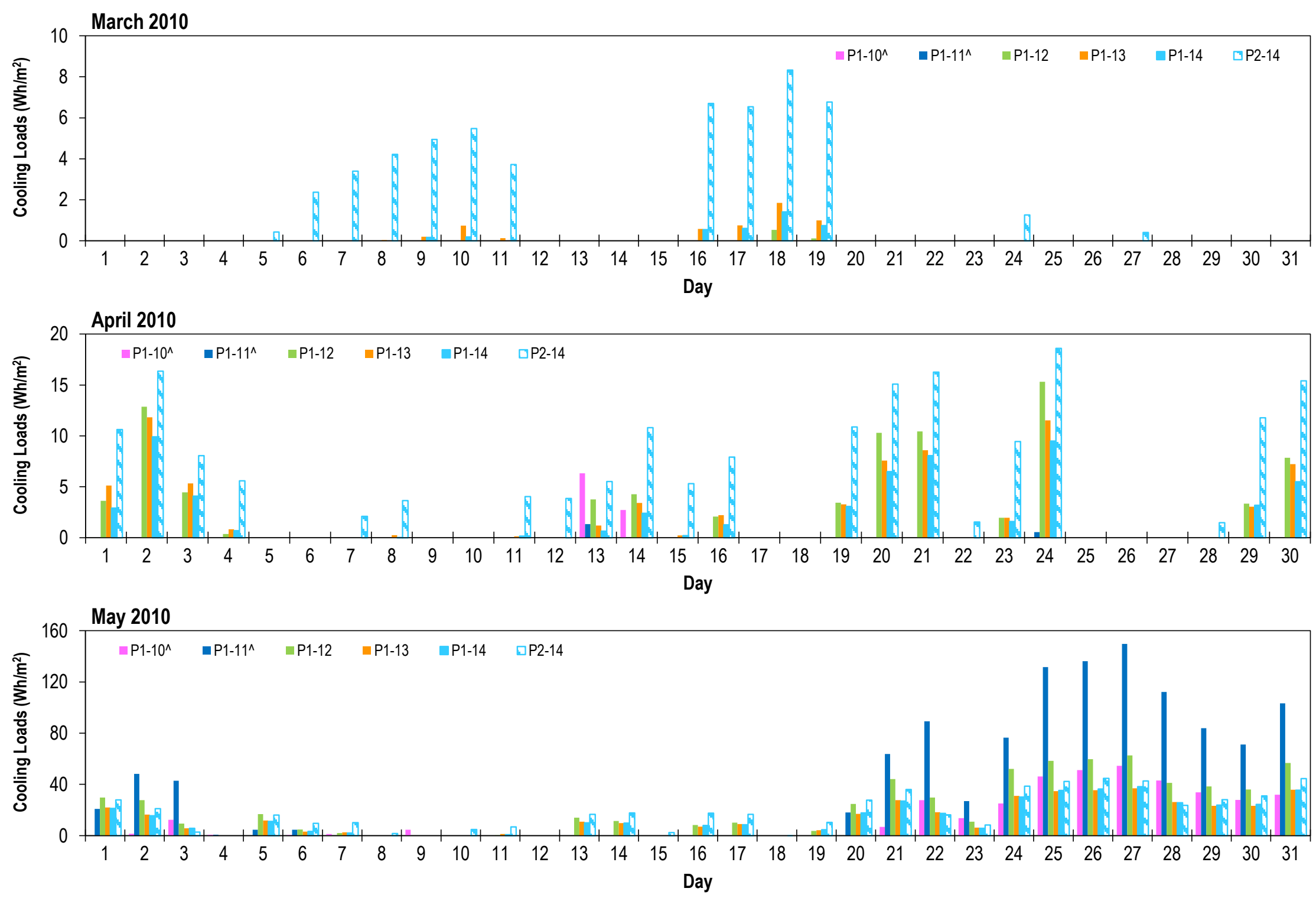

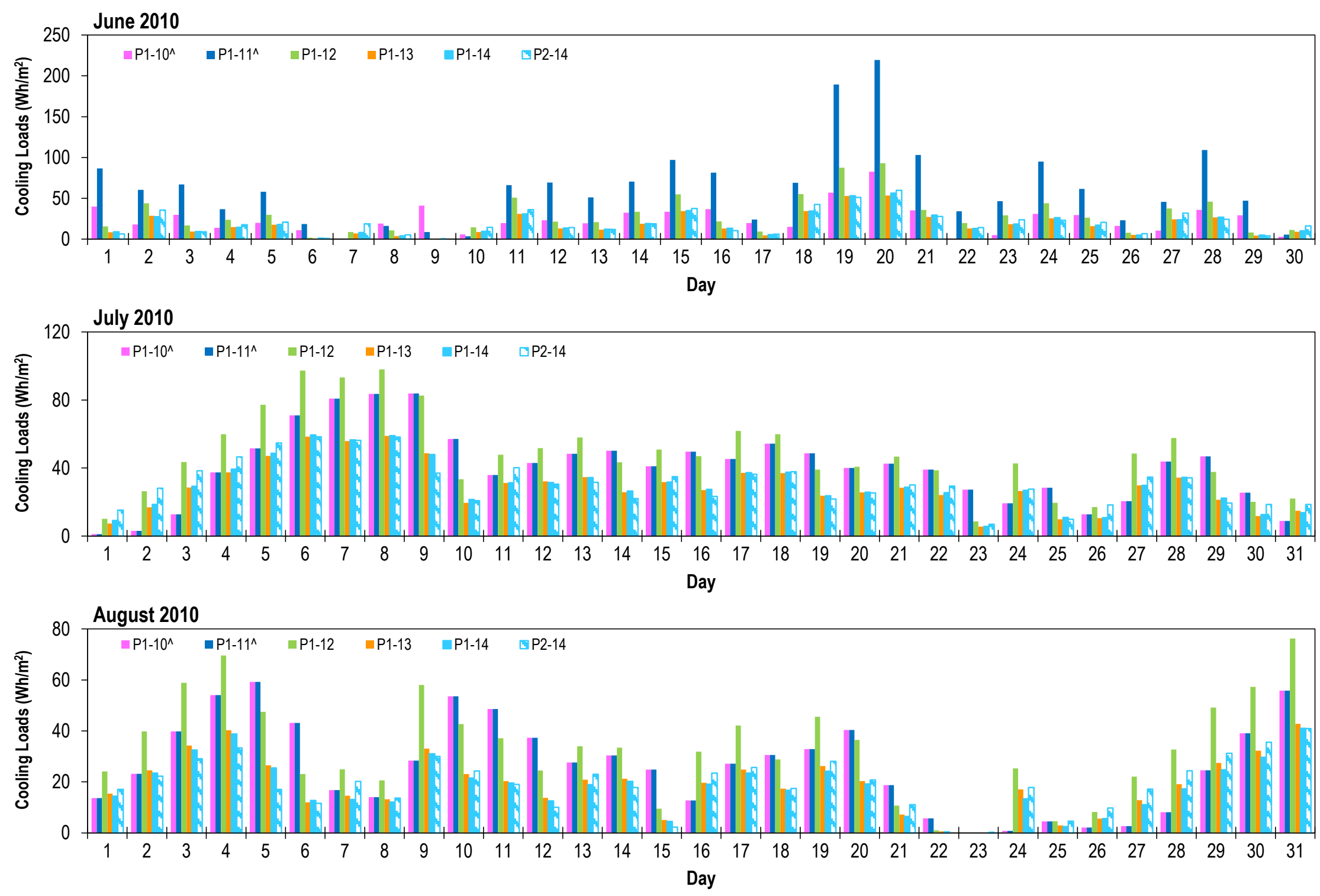
Heating loads $\left(\mathrm{Wh} / \mathrm{m}^{2}\right)$. Refer to Figures 25 thru 28 for sensor location.

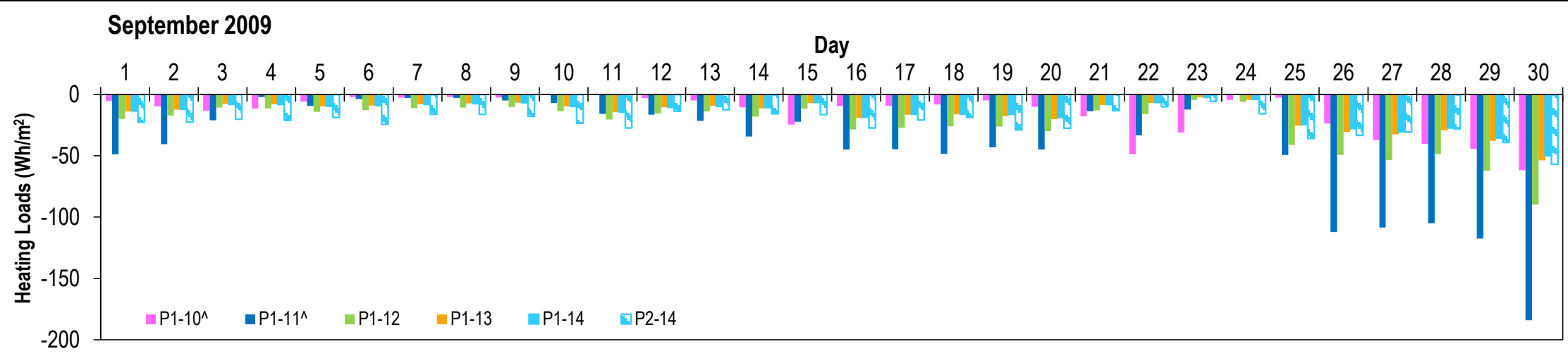

October 2009

Day

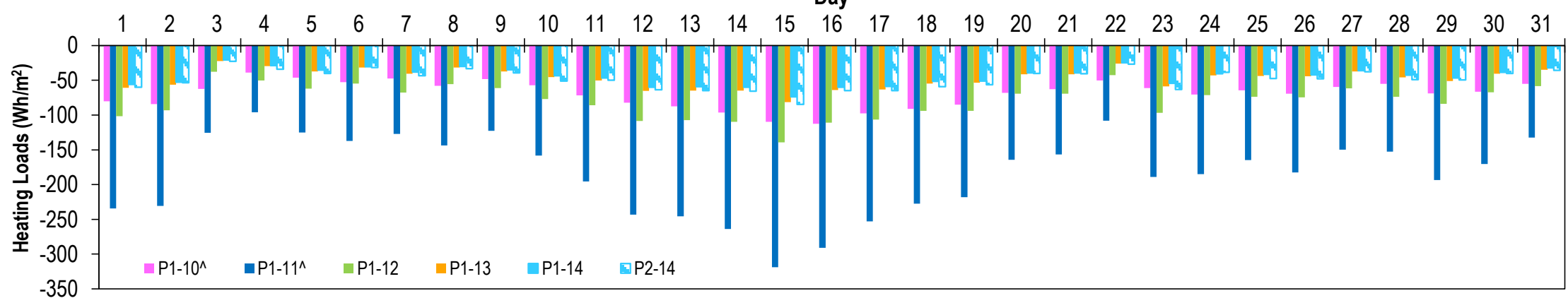

November 2009

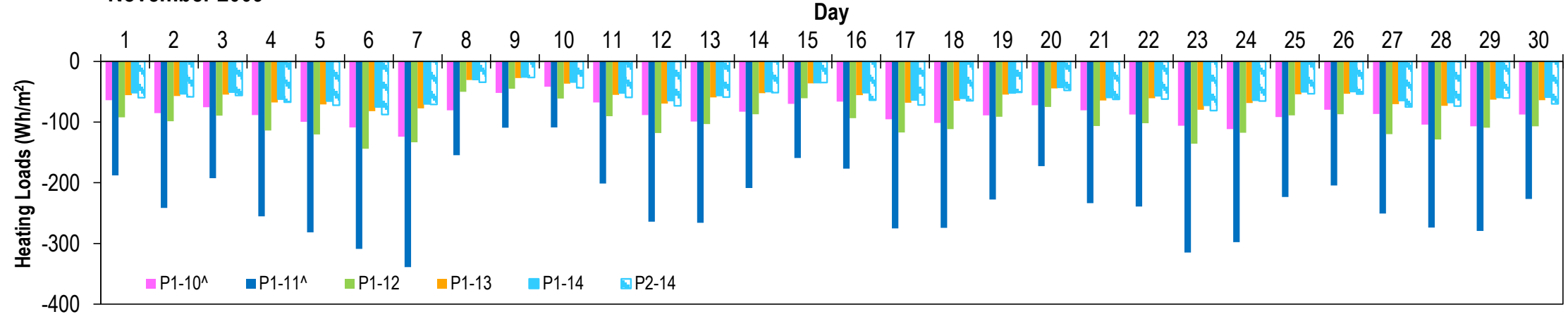



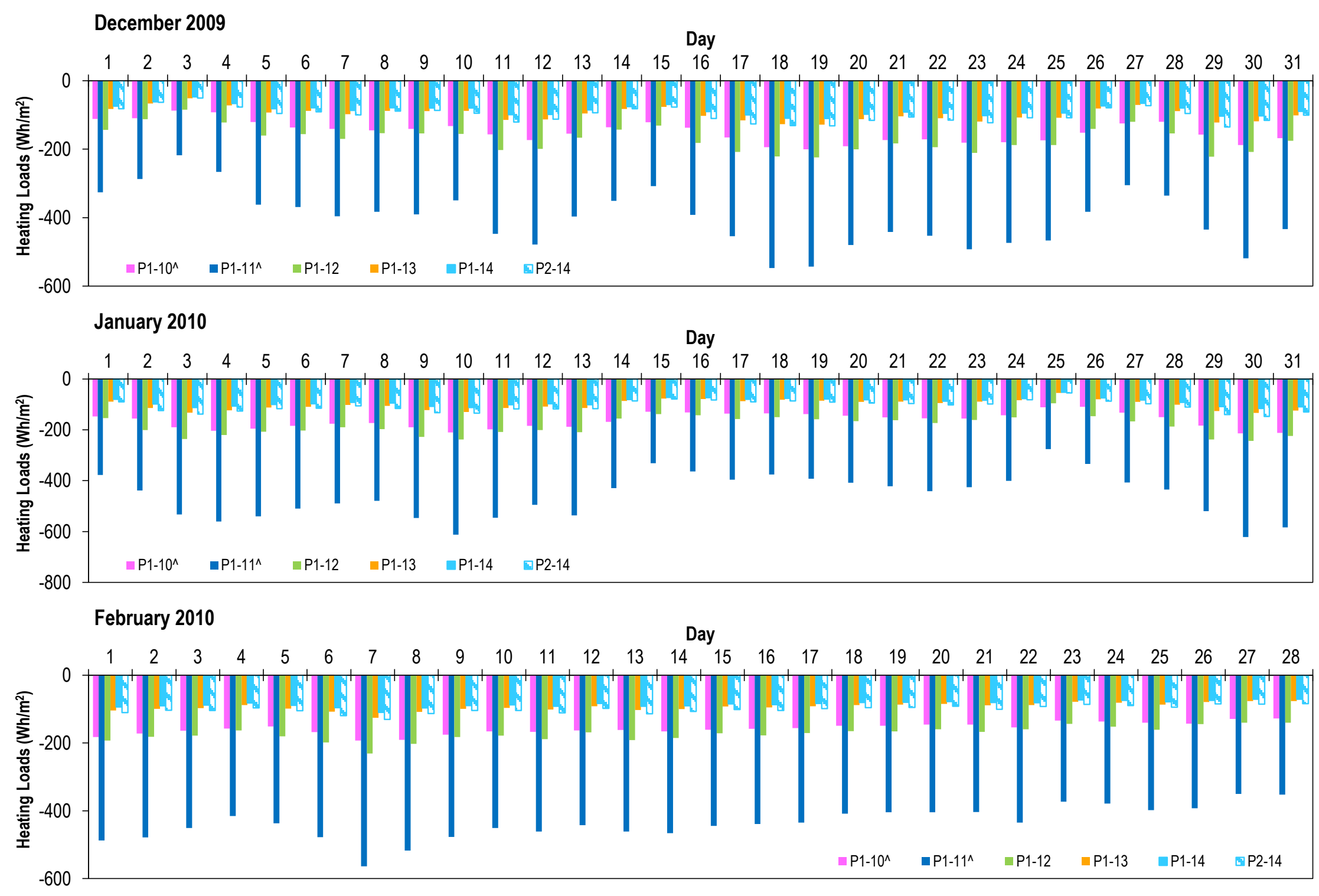
March 2010

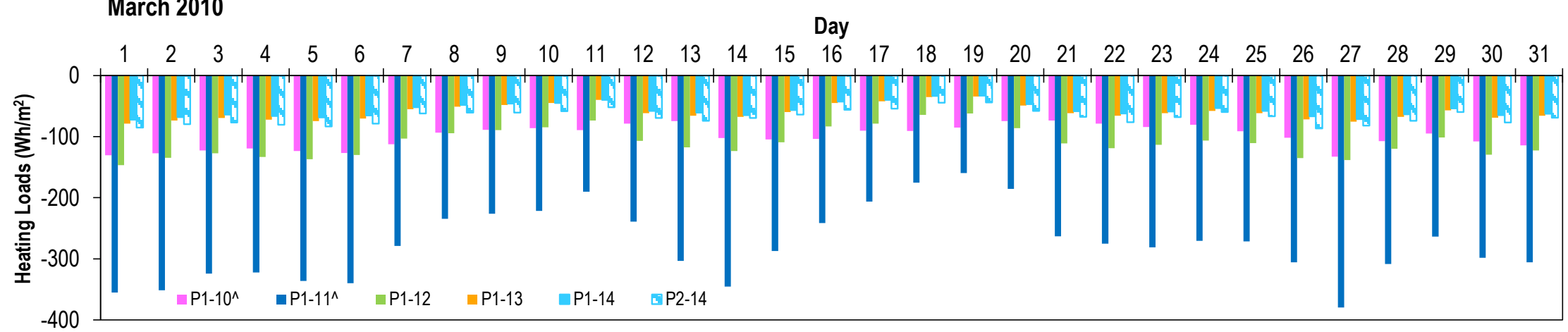

April 2010

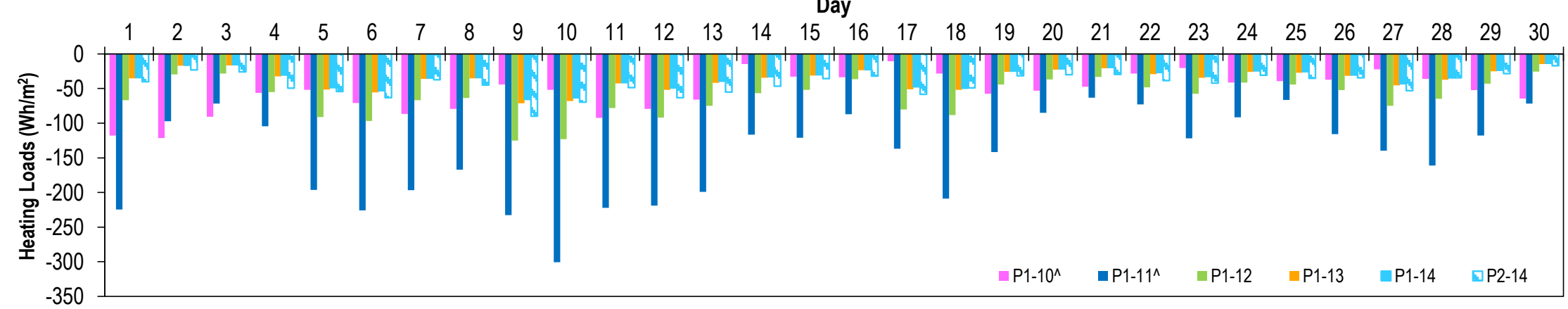

\section{May 2010}

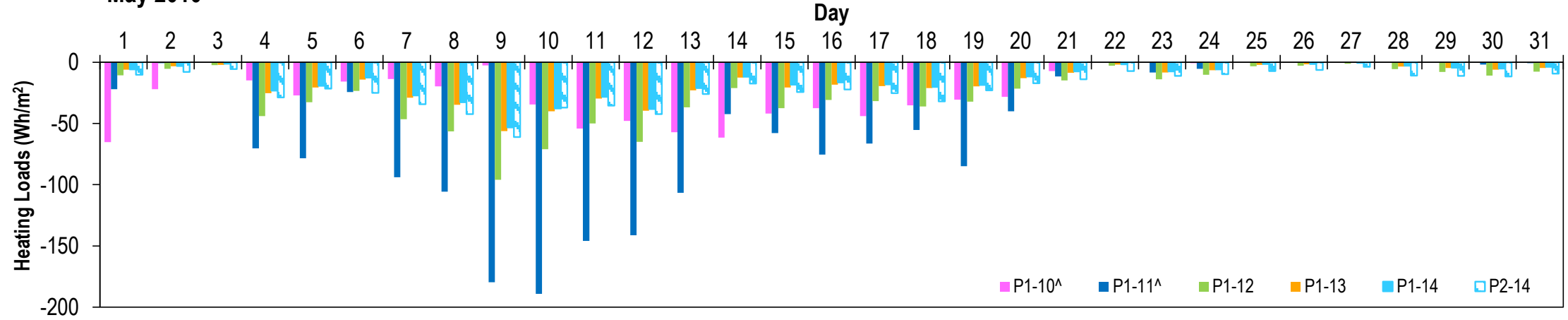


June 2010

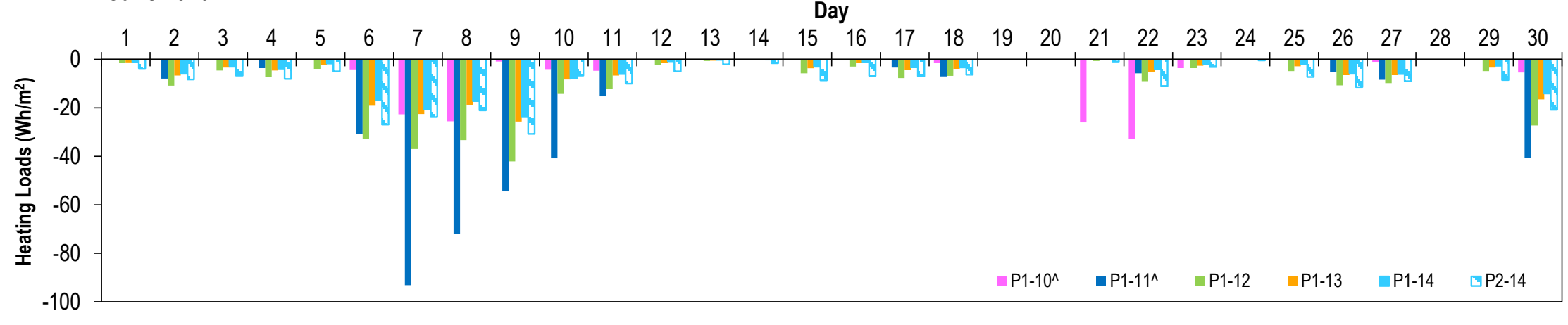

July 2010

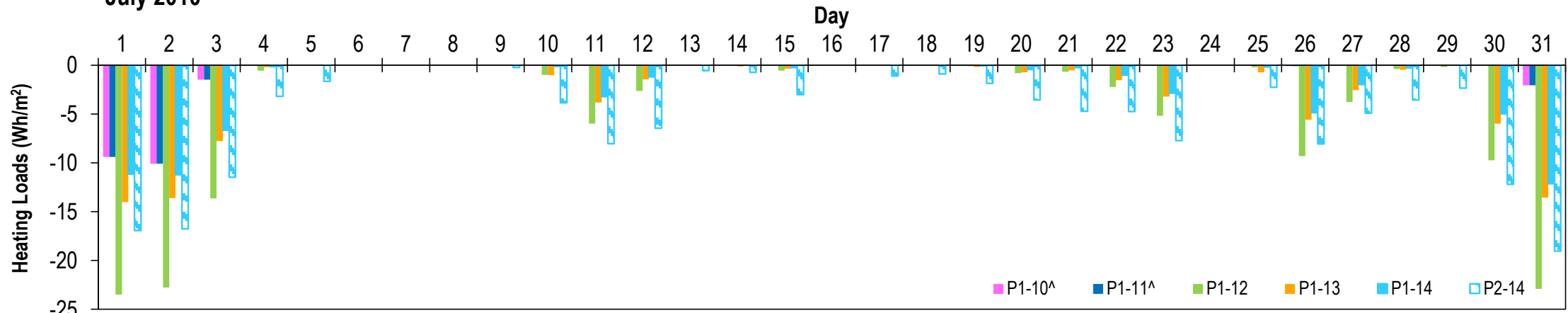

August 2010

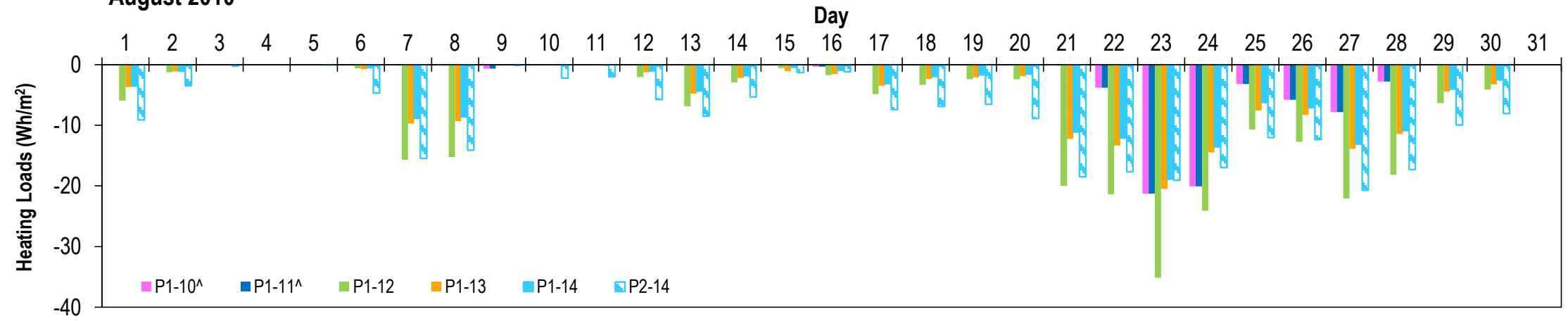




\section{Appendix C: South Panels}

Temperature $\left({ }^{\circ} \mathrm{C}\right)$ at exterior side of stud cavity. Refer to Figures 25 thru 28 for sensor location.
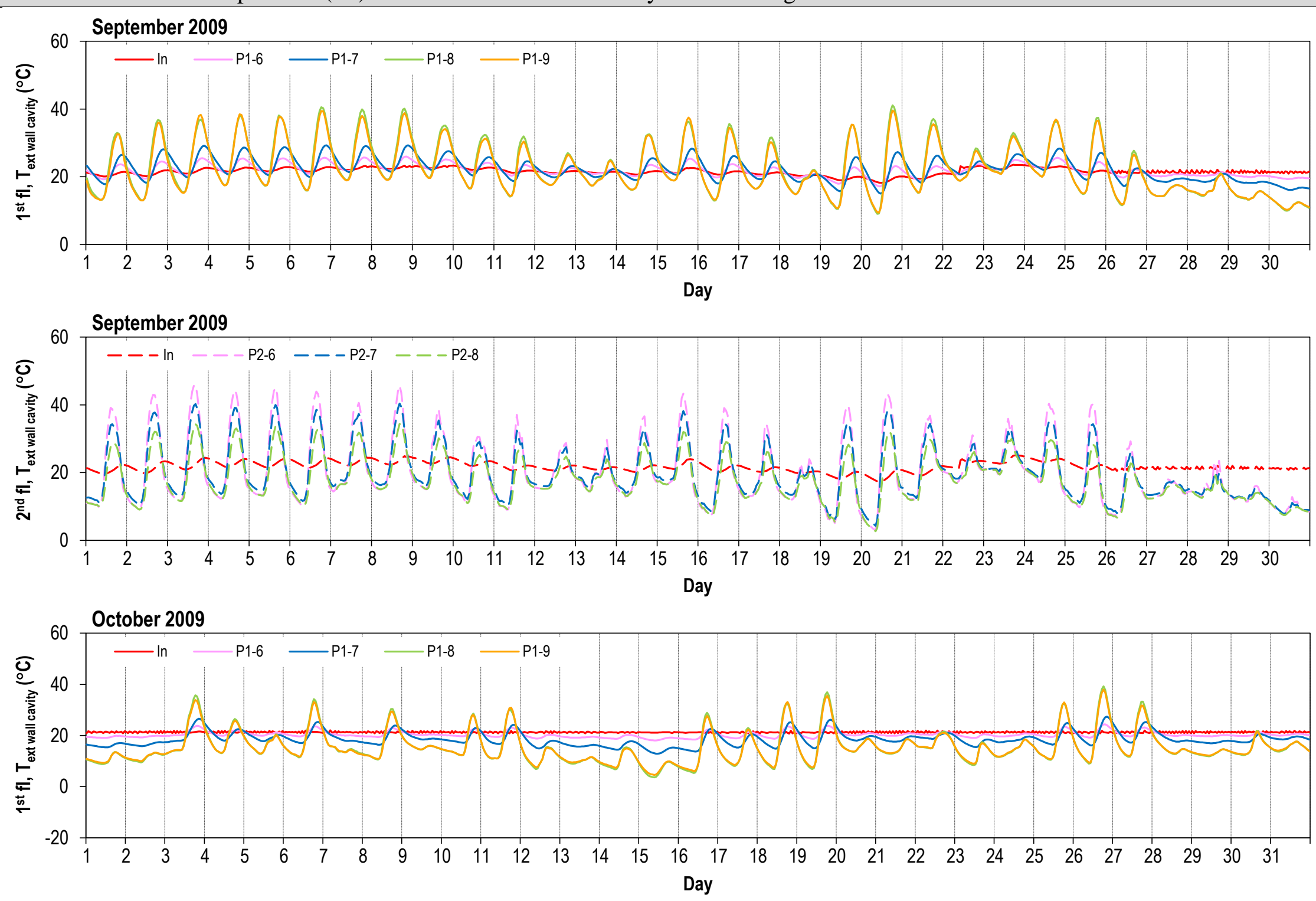

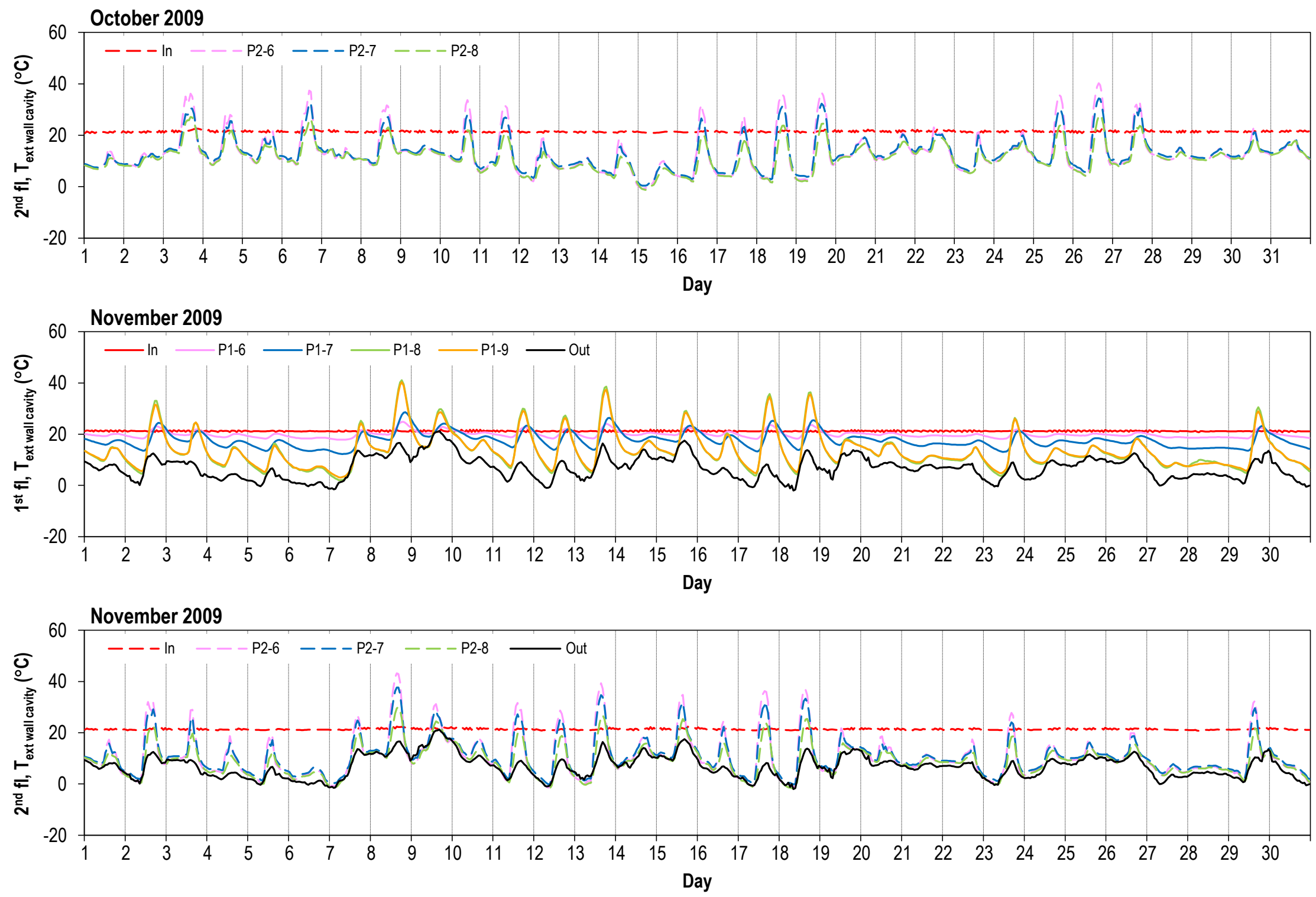

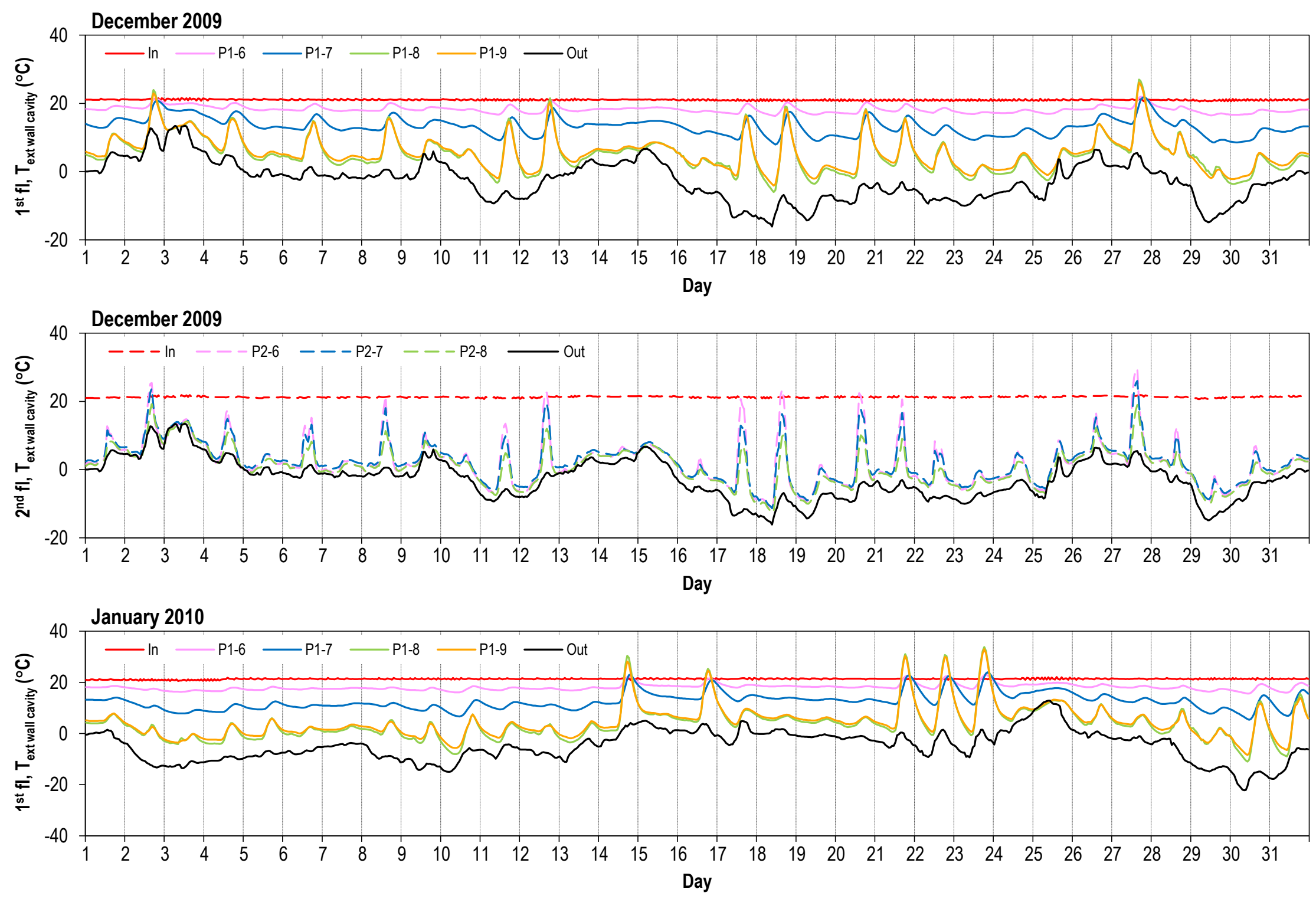

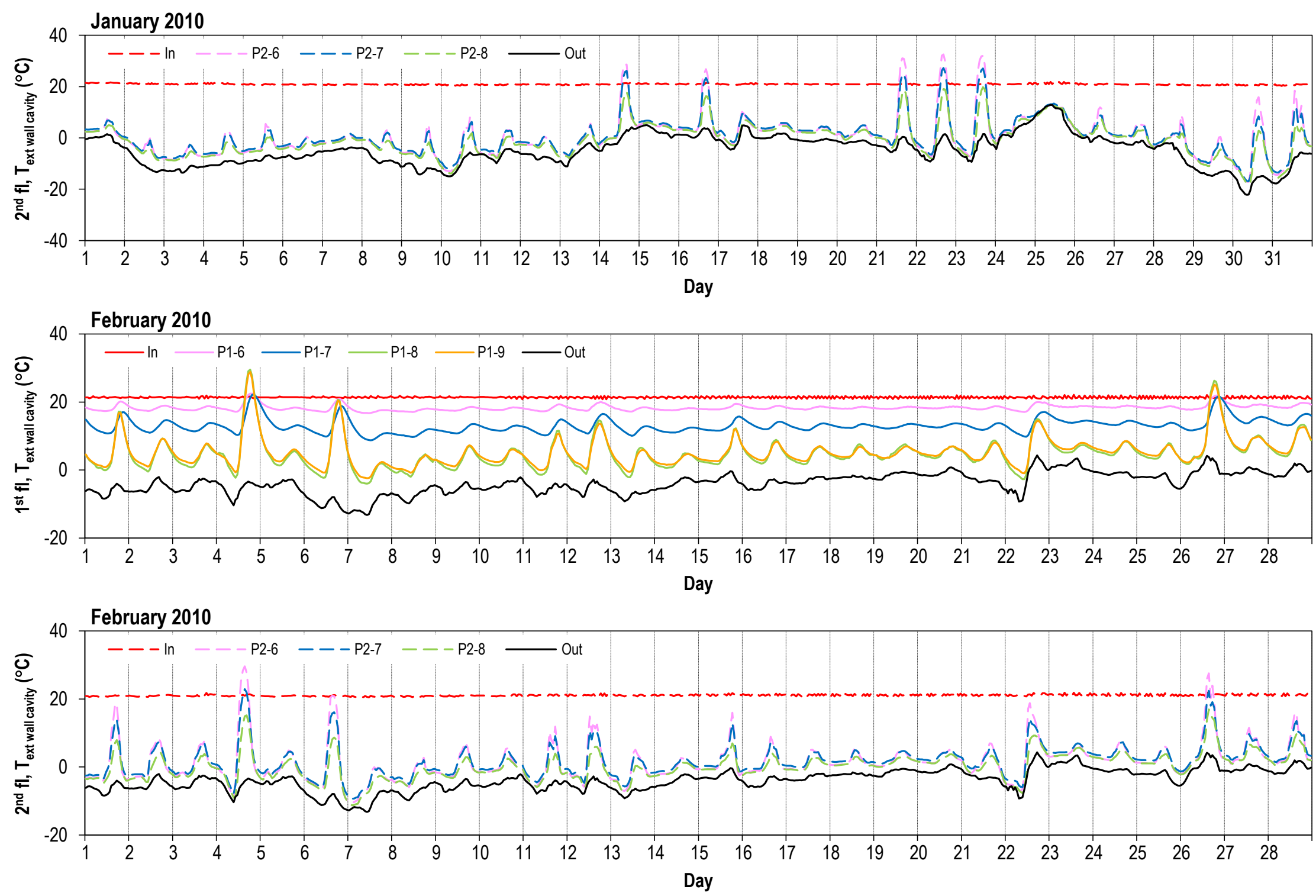

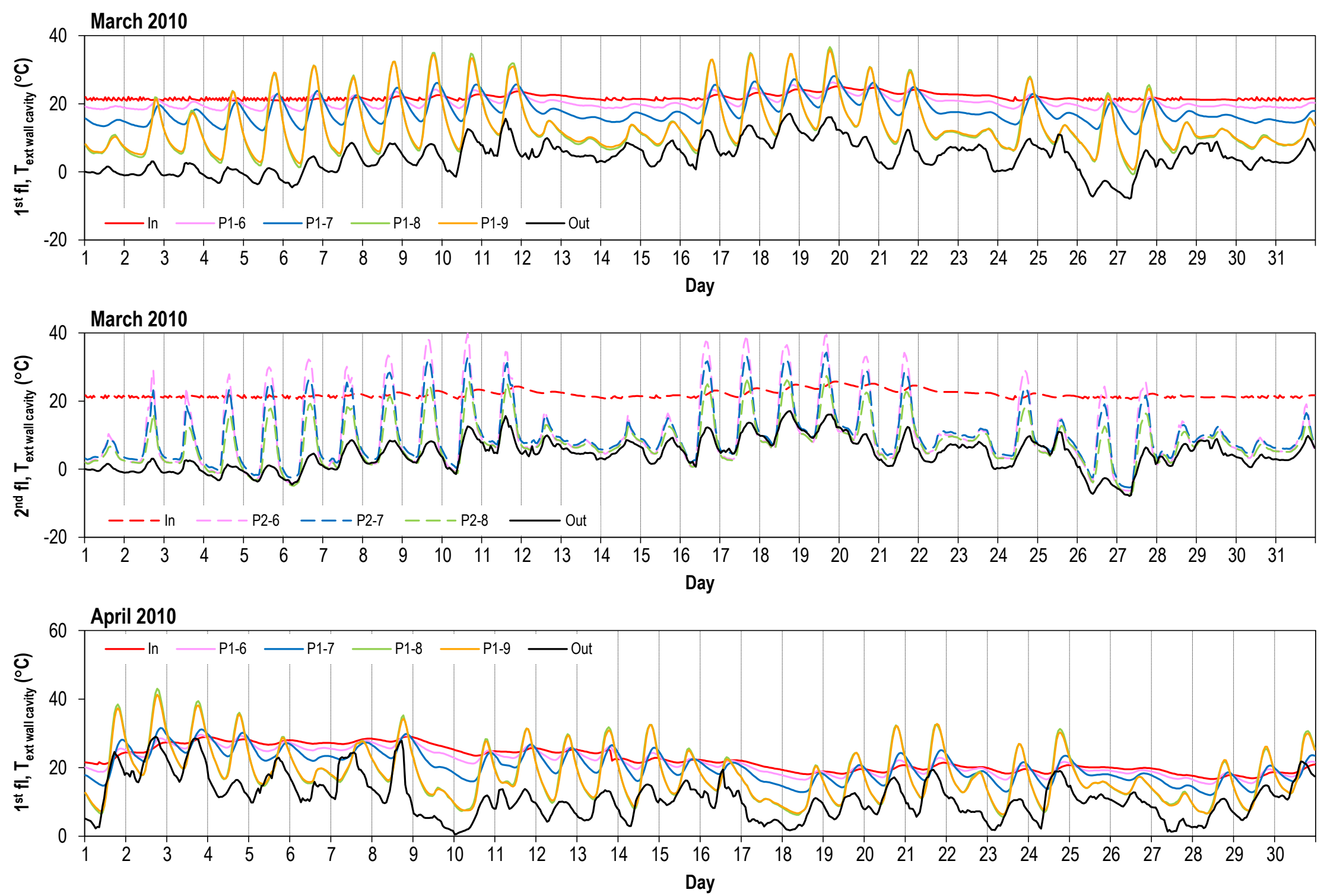

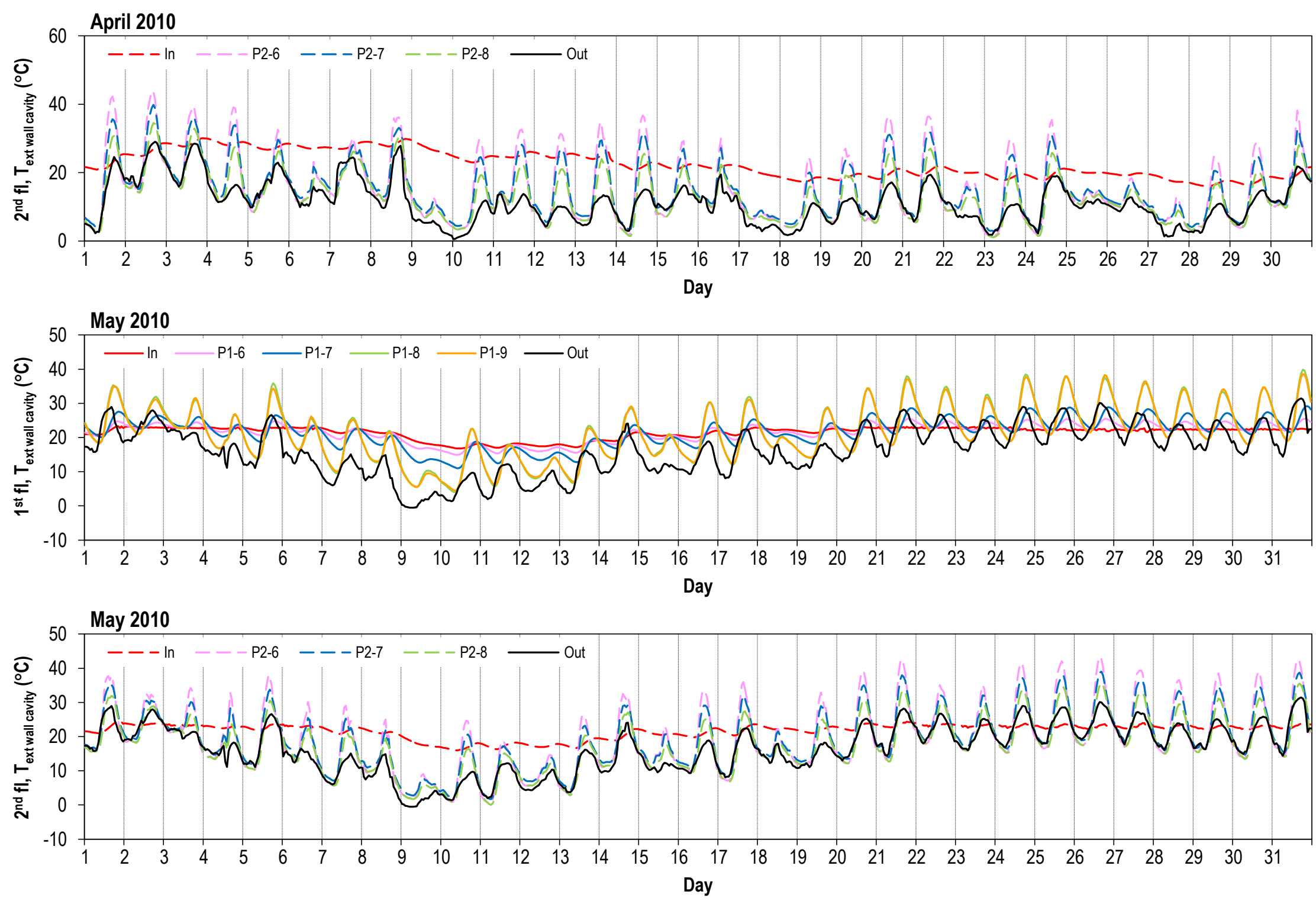

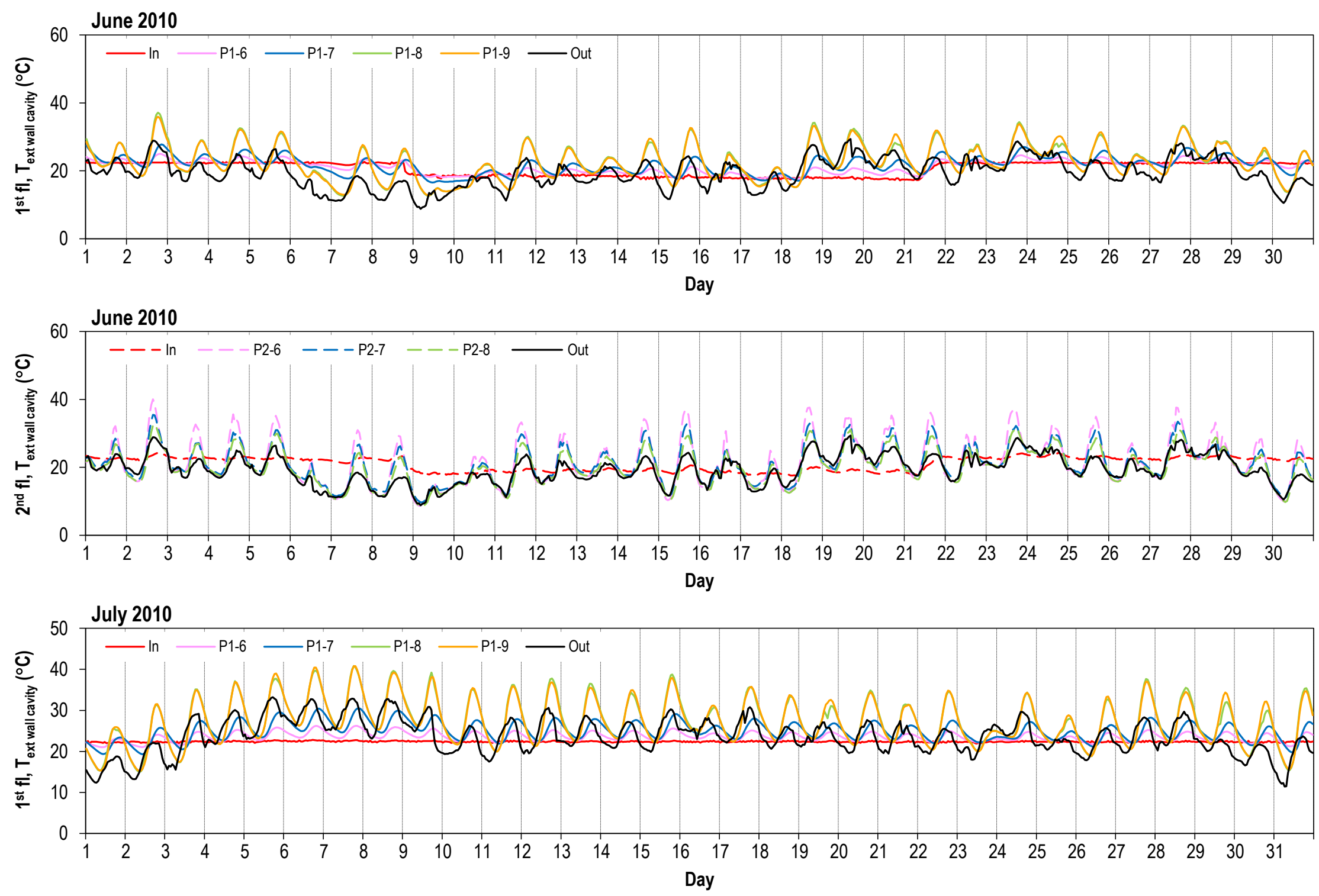

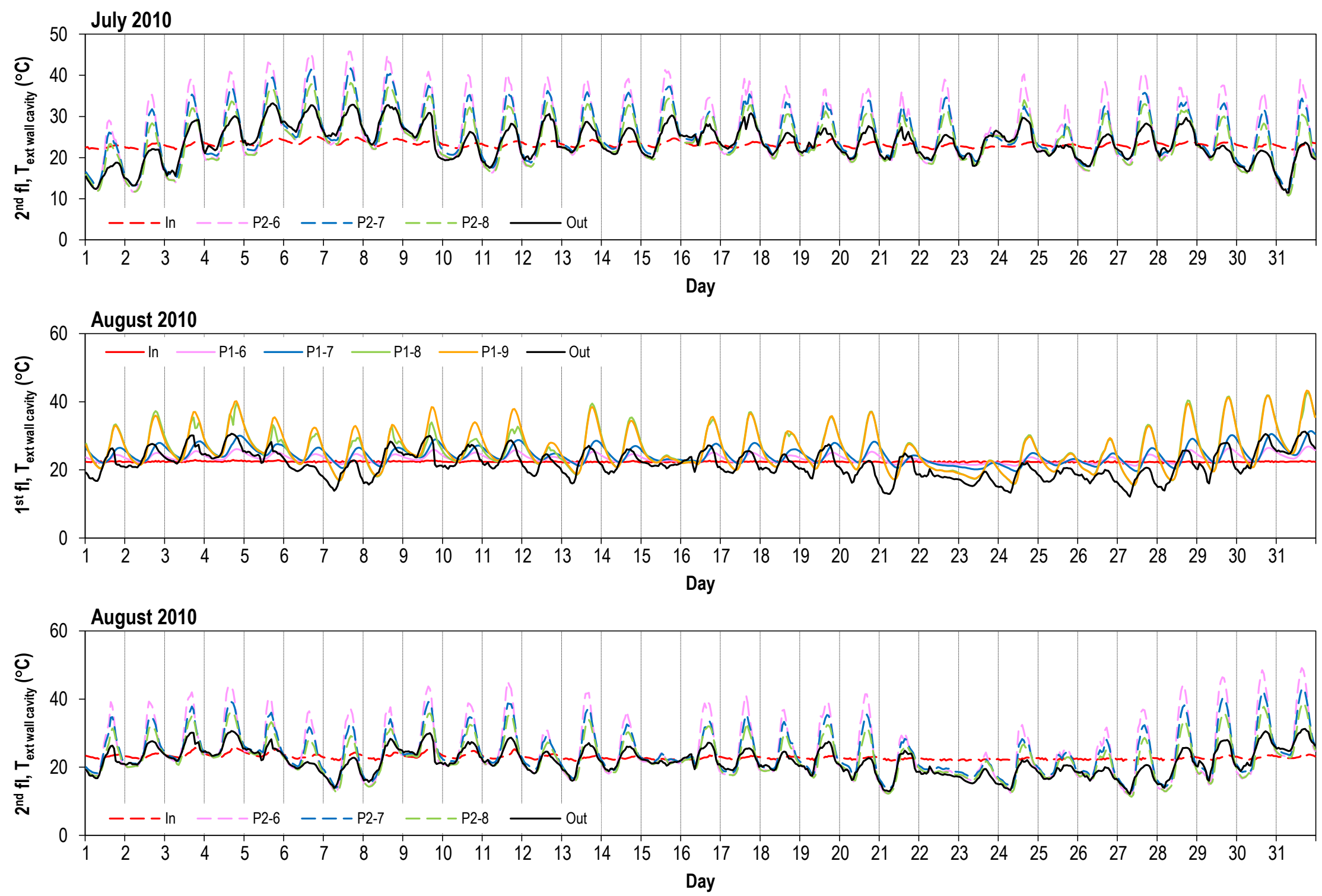
Temperature $\left({ }^{\circ} \mathrm{C}\right)$ at interior side of stud cavity. Refer to Figures 25 thru 28 for sensor location.
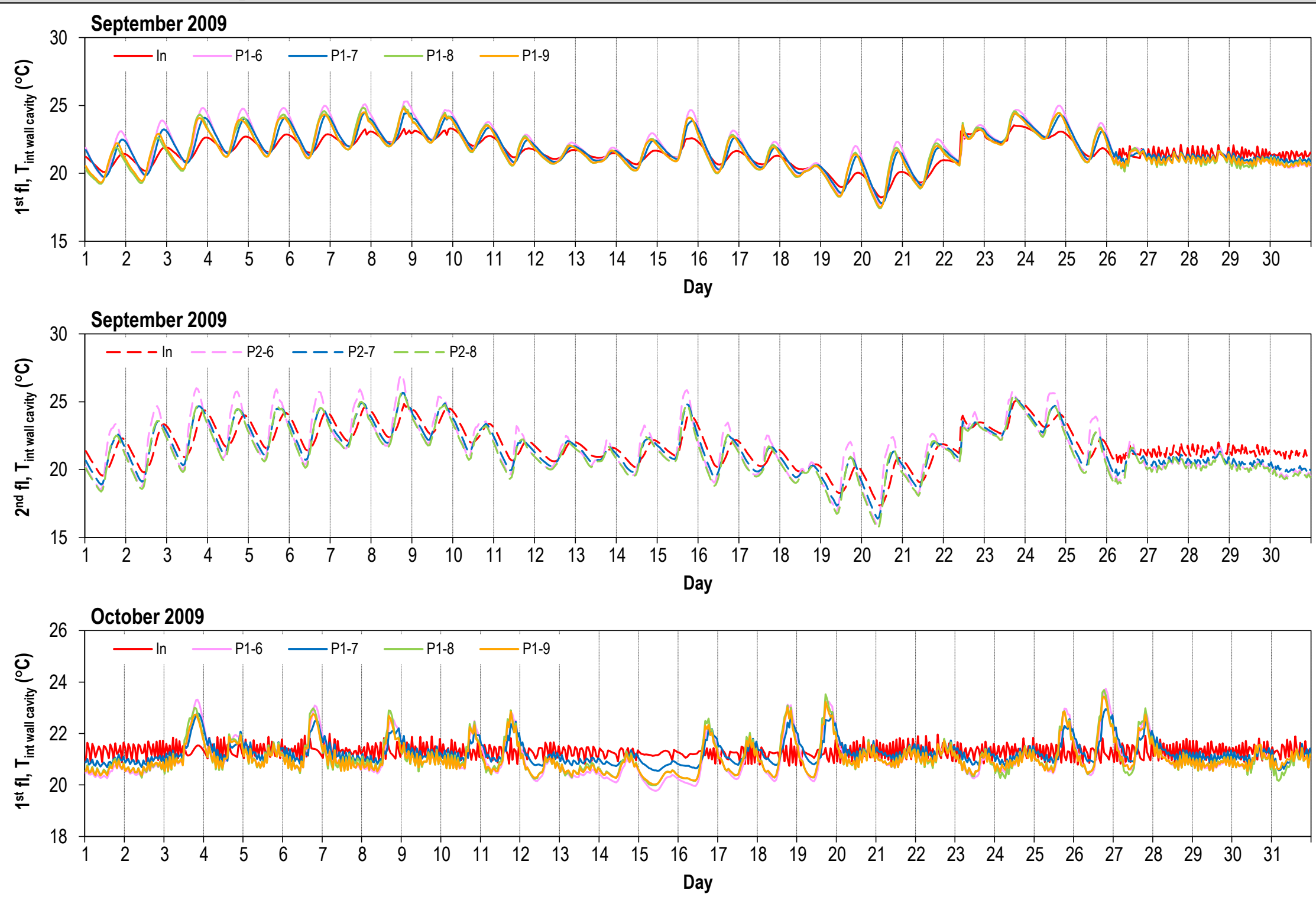

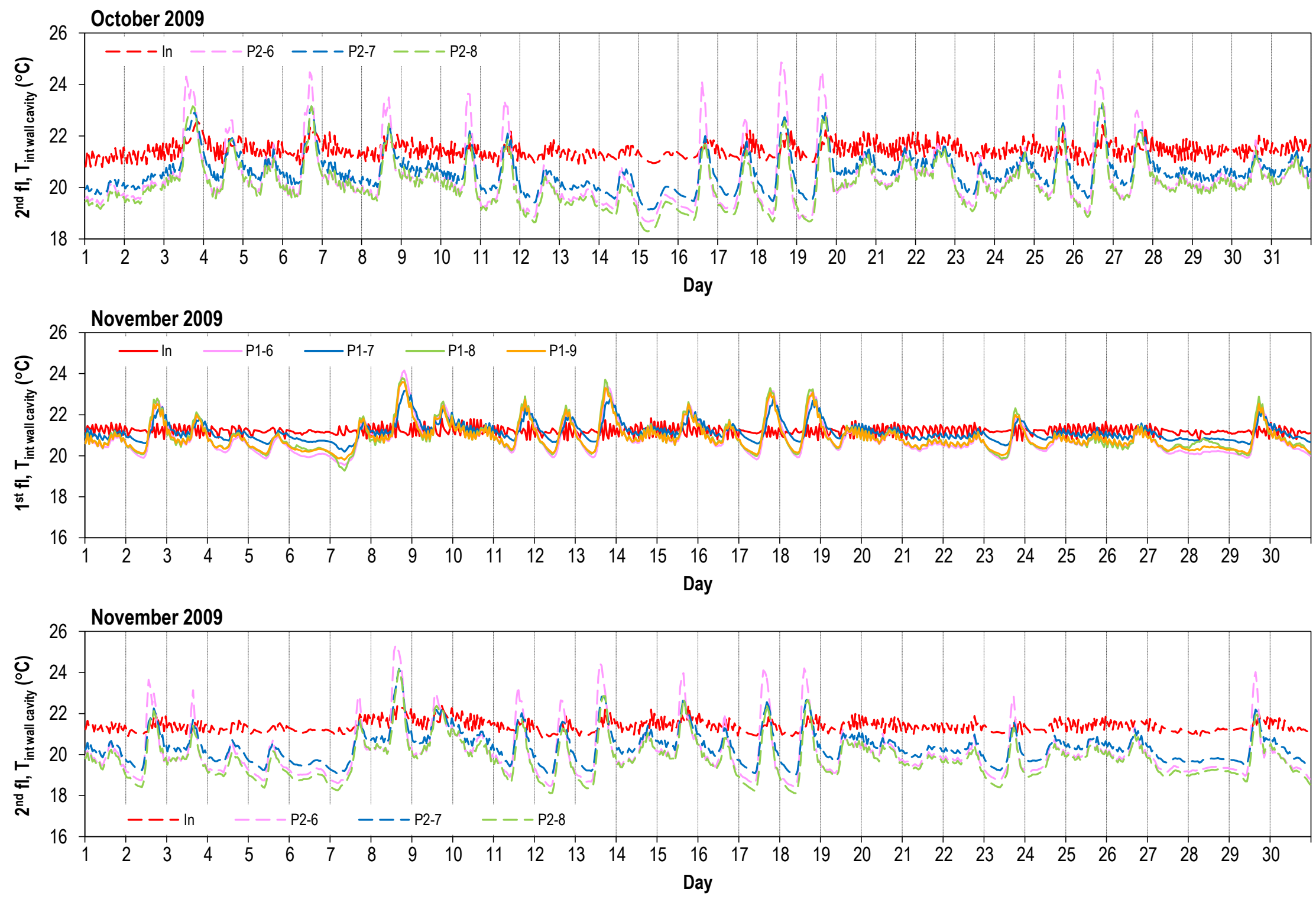

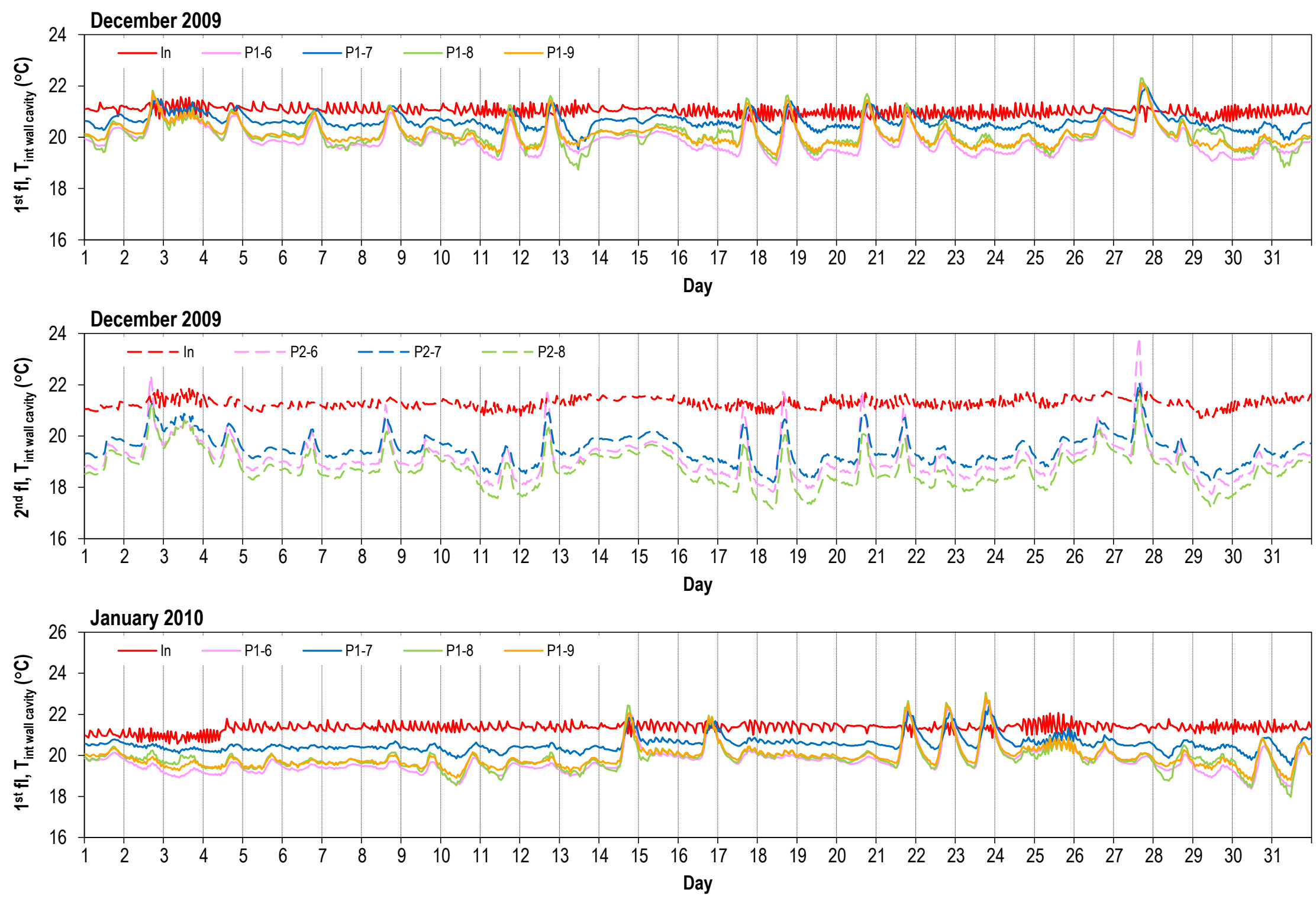

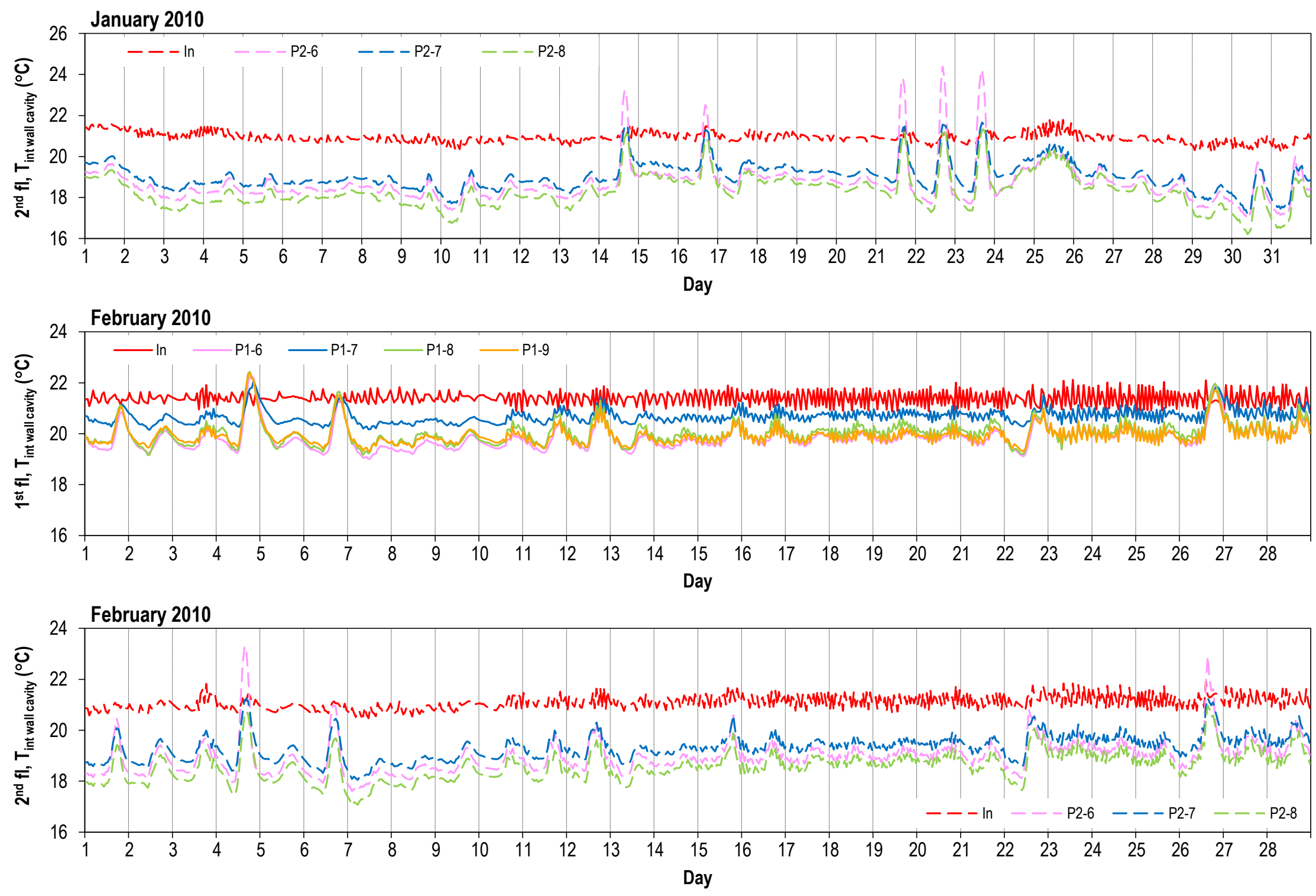

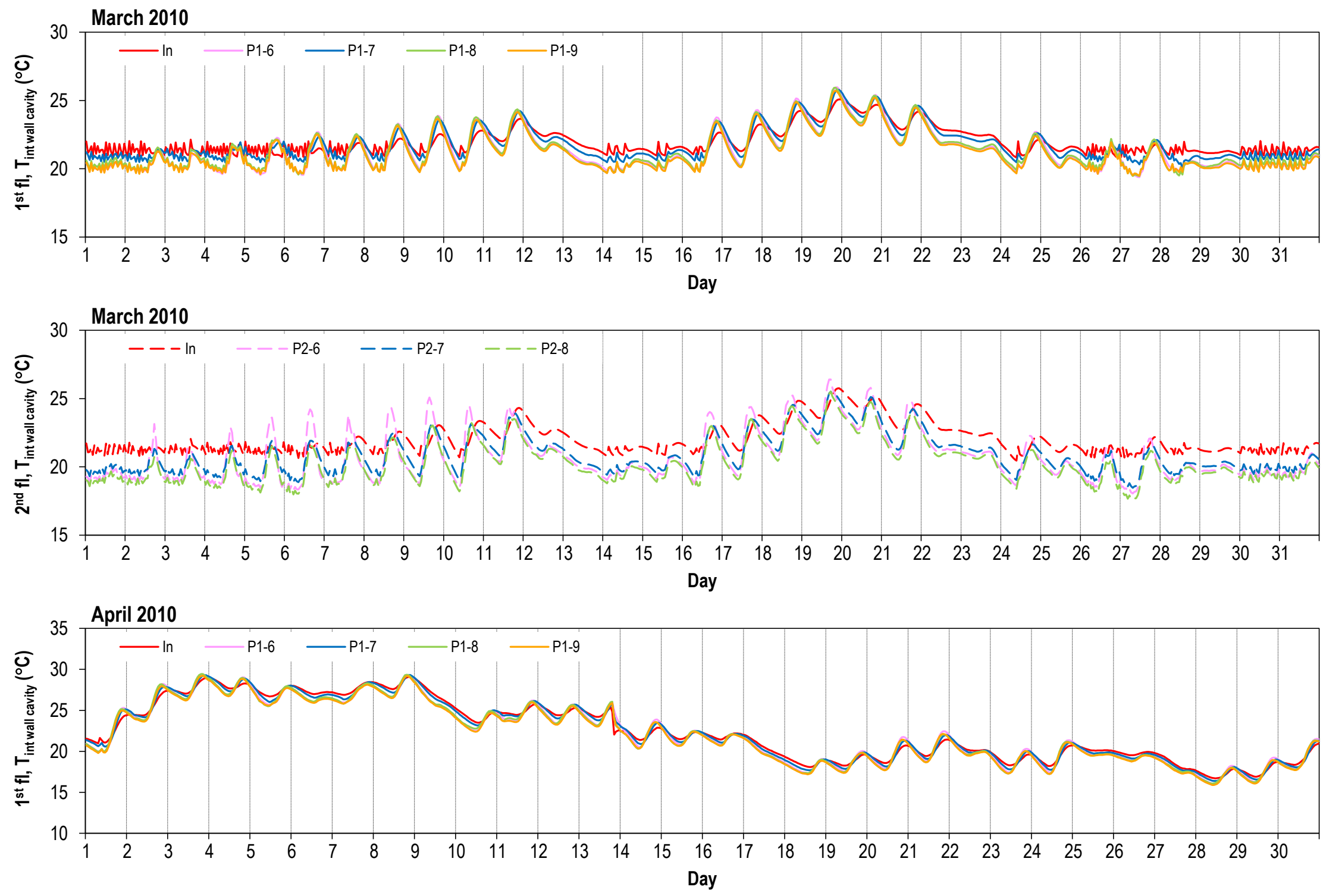

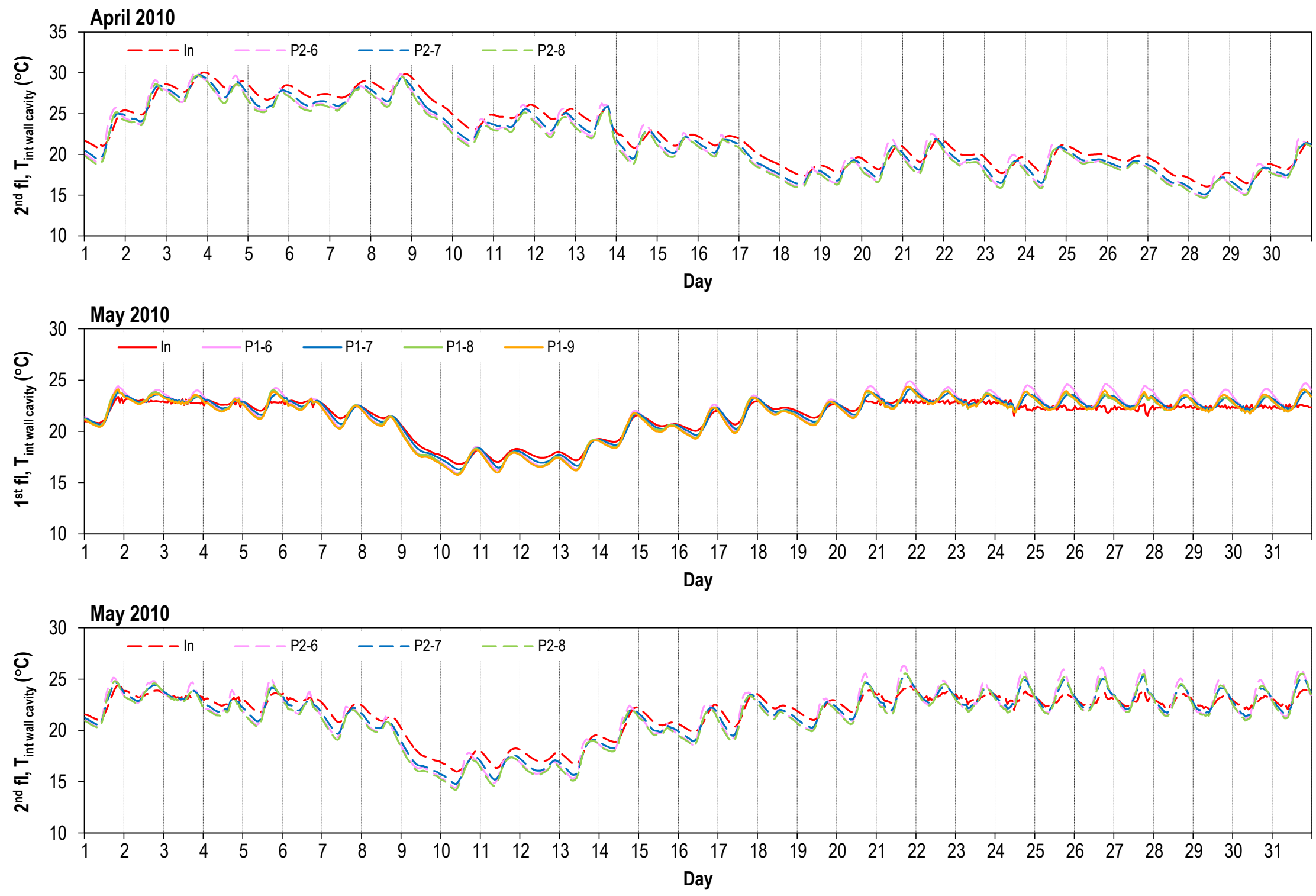

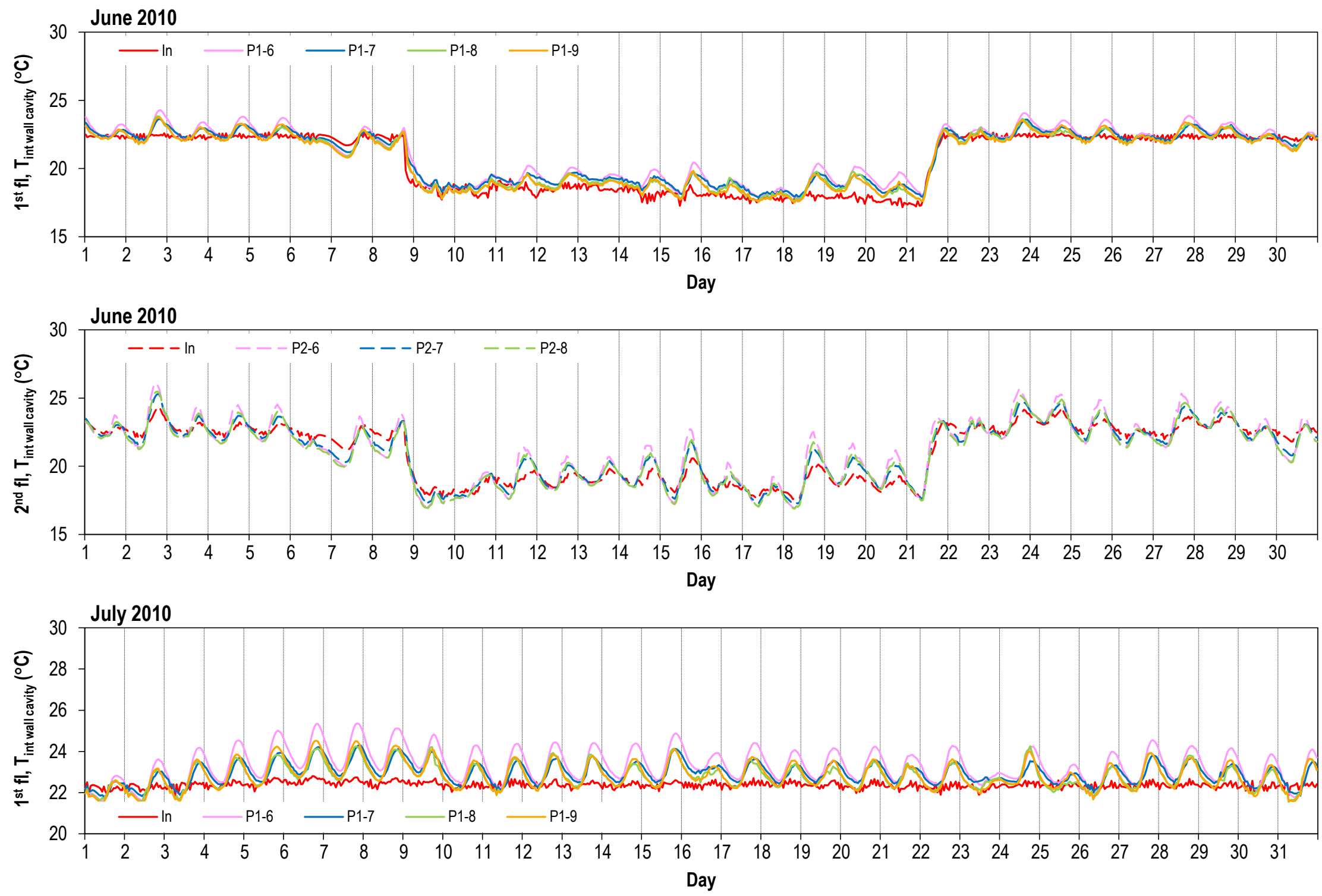

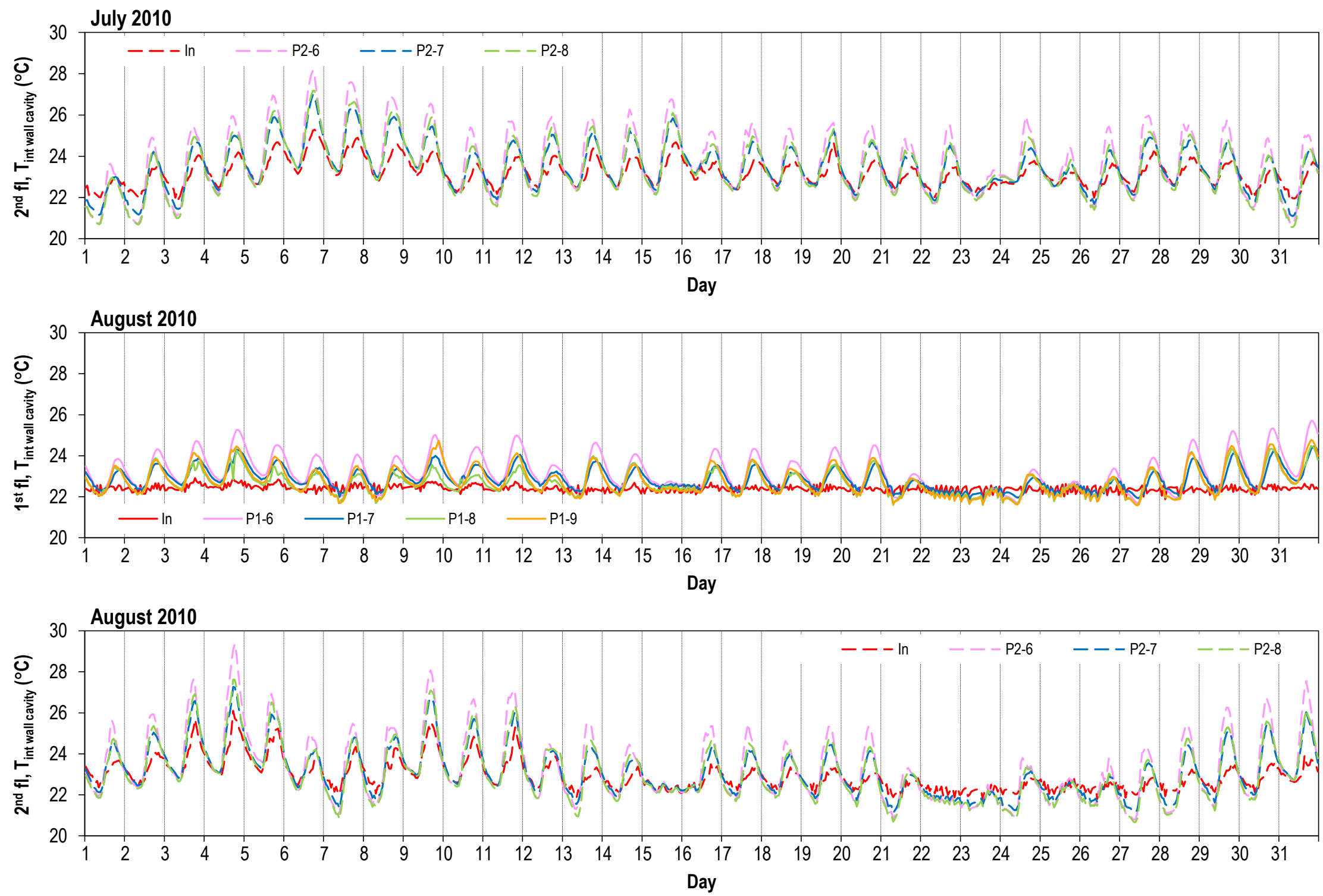
Relative humidity (\%) at exterior side of stud cavity. Refer to Figures 25 thru 28 for sensor location.
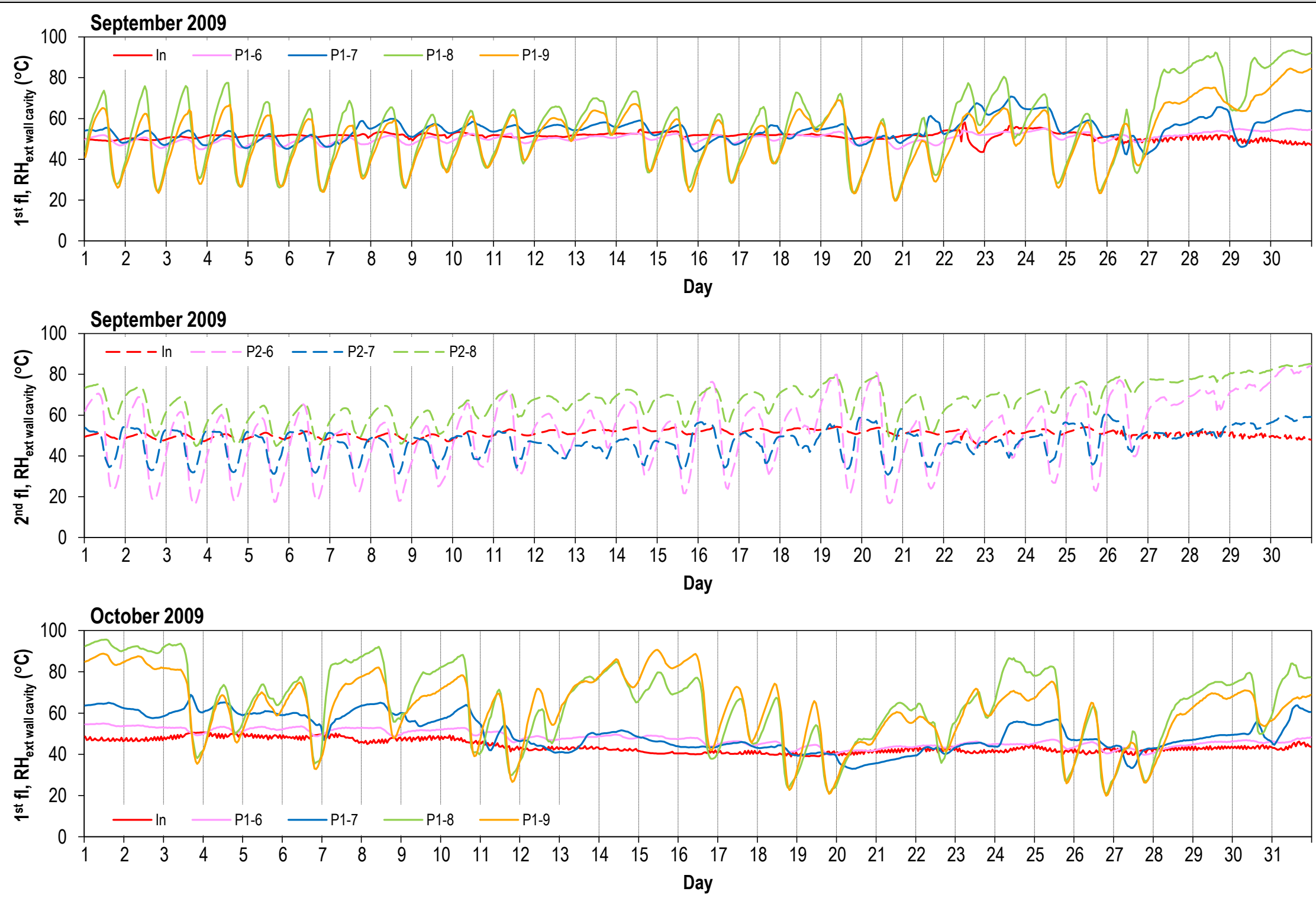
156
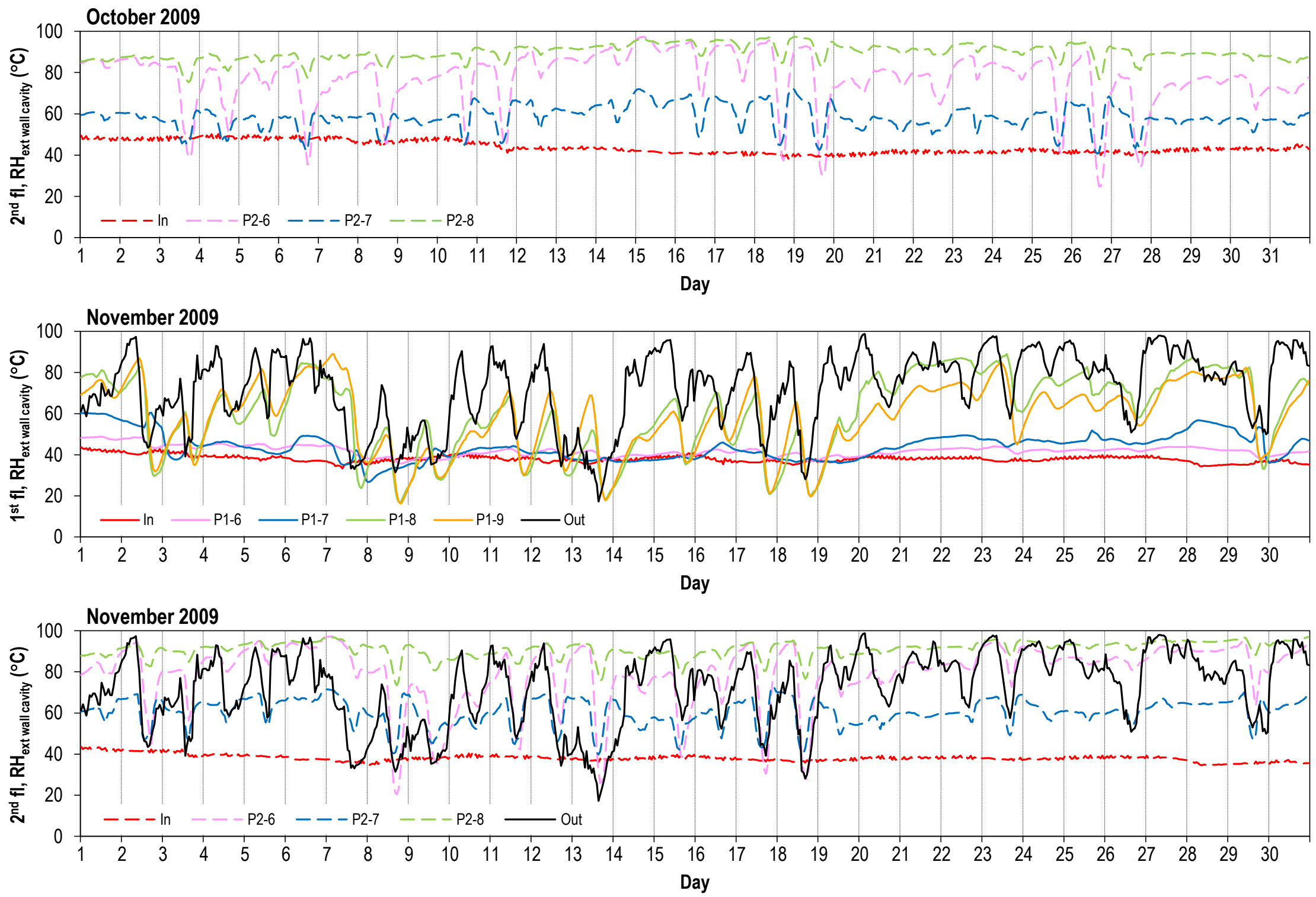

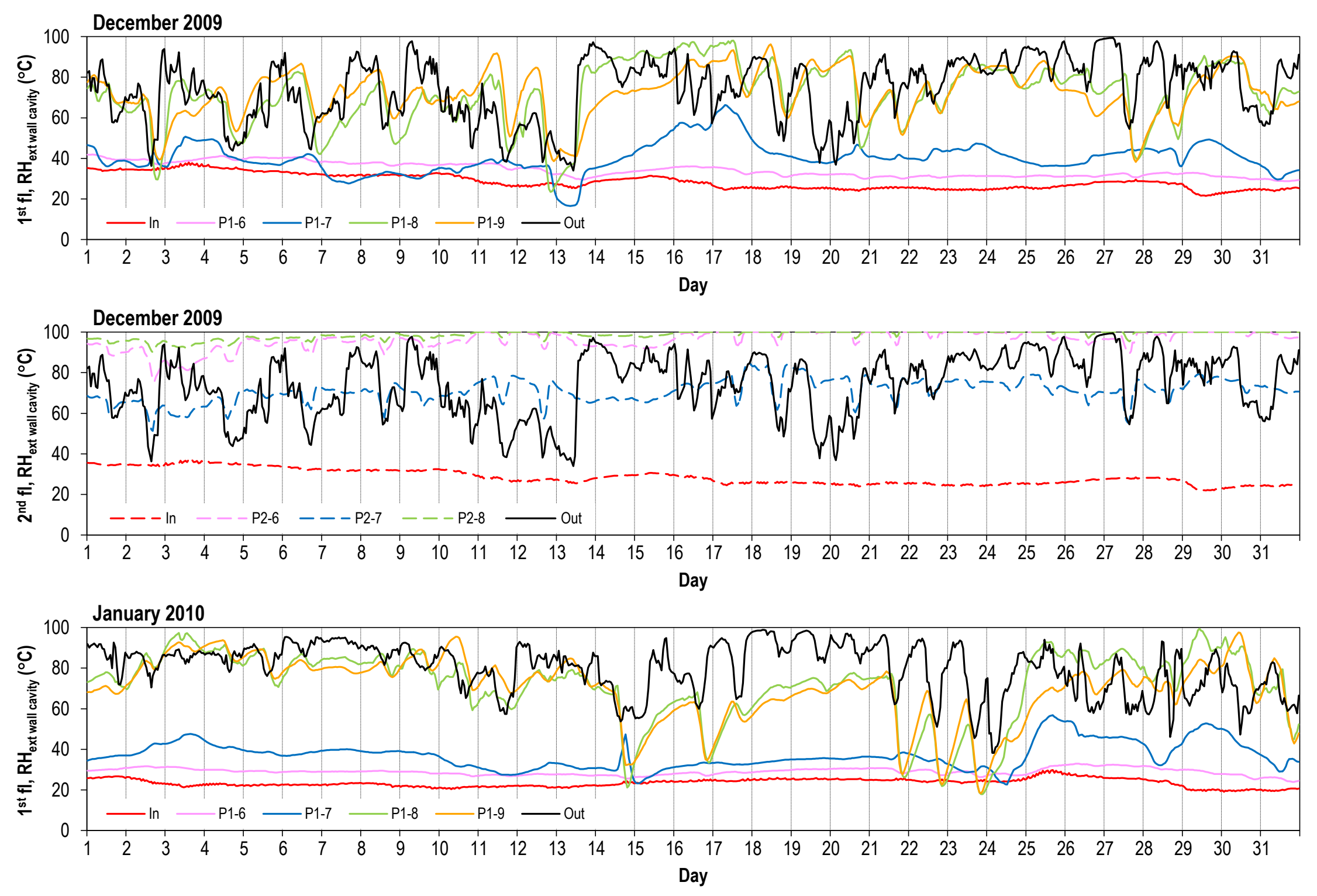

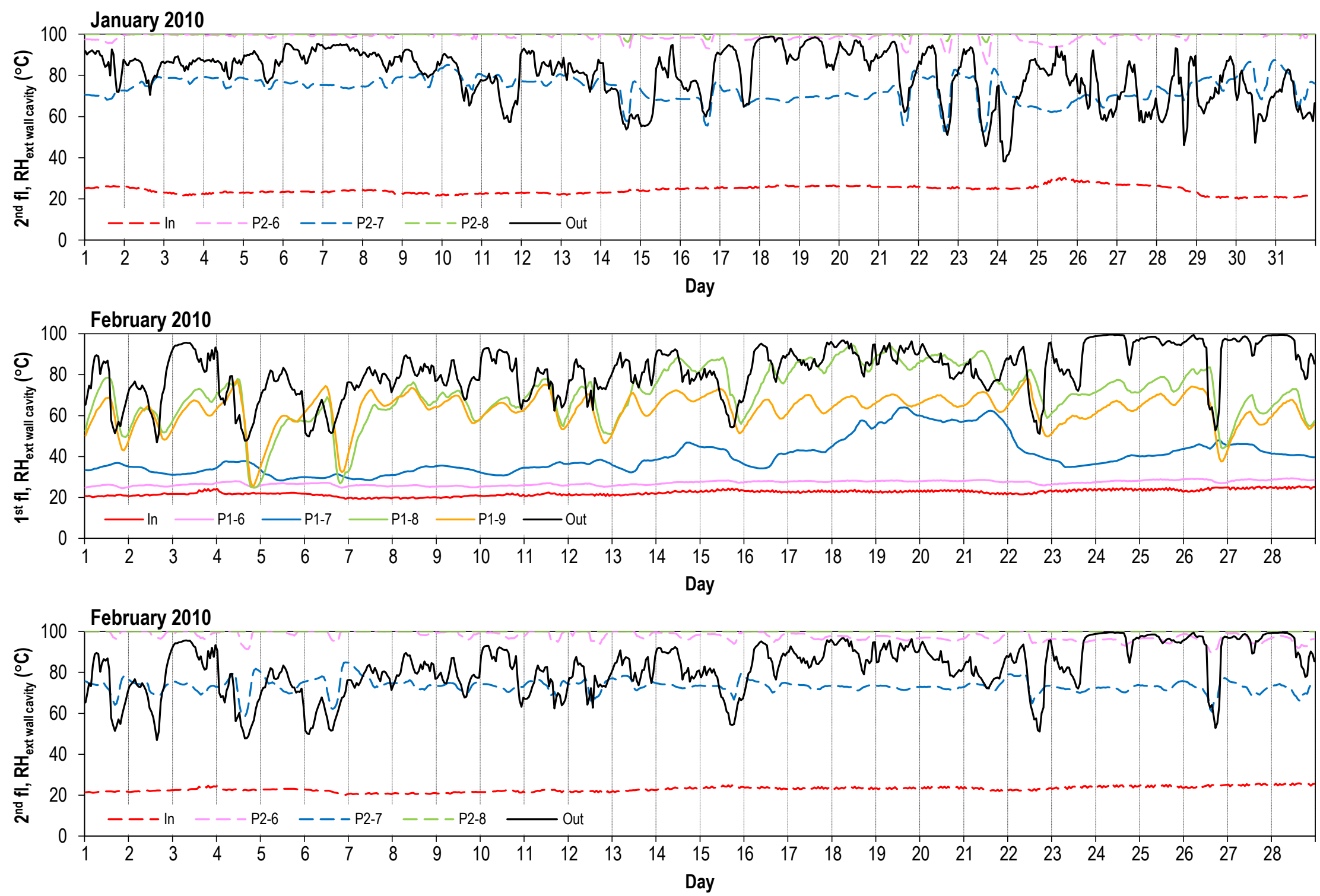
159
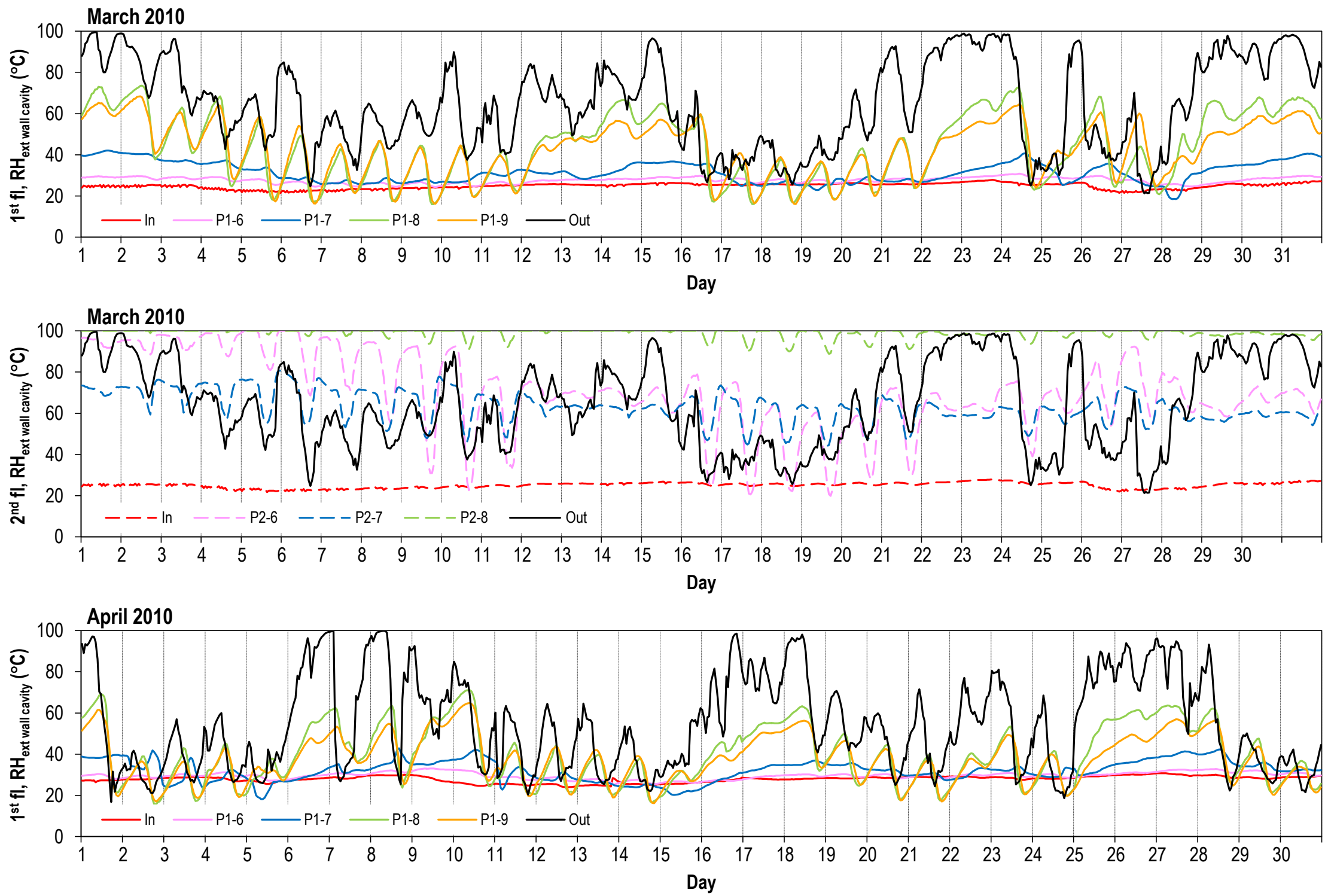

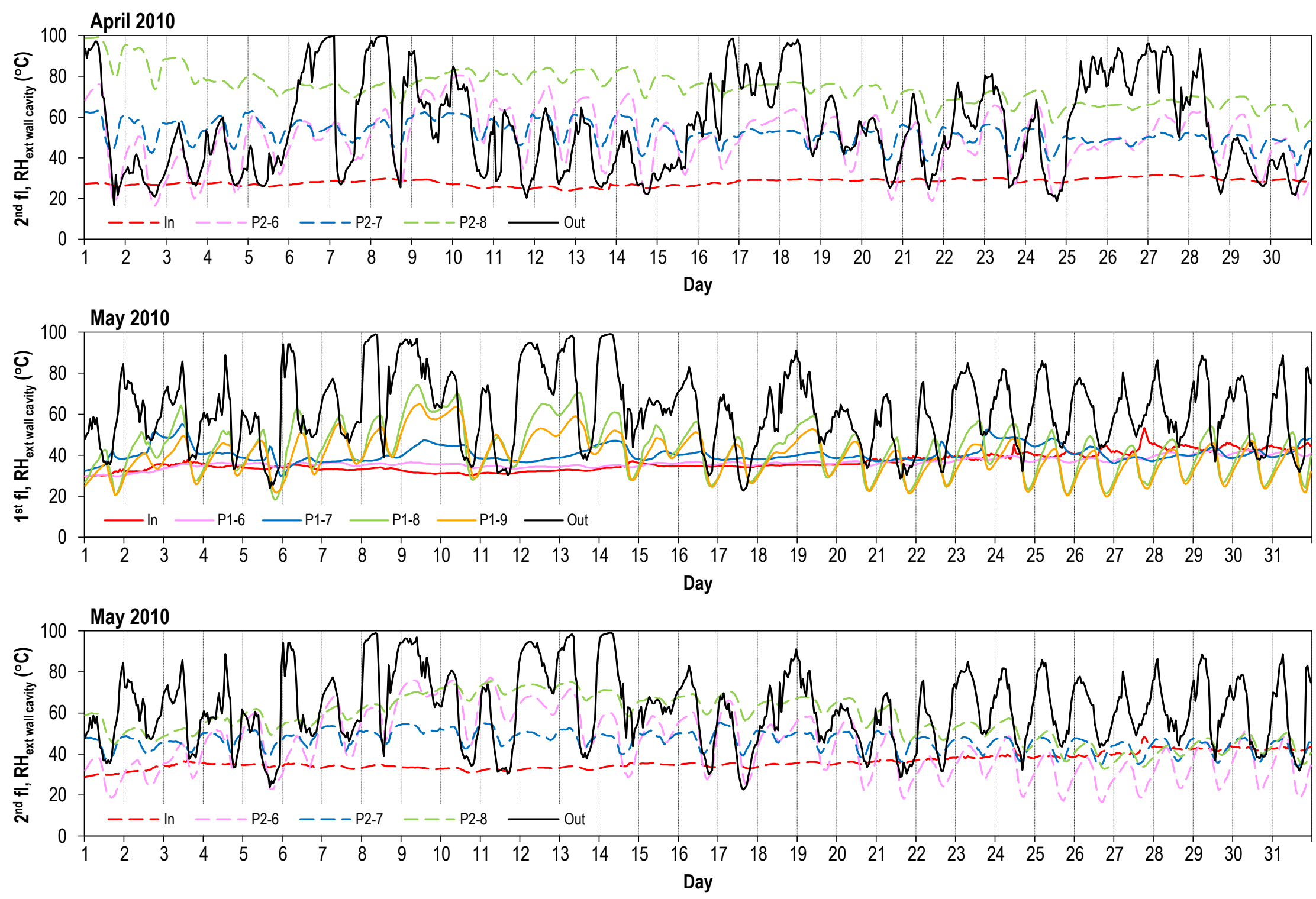
161
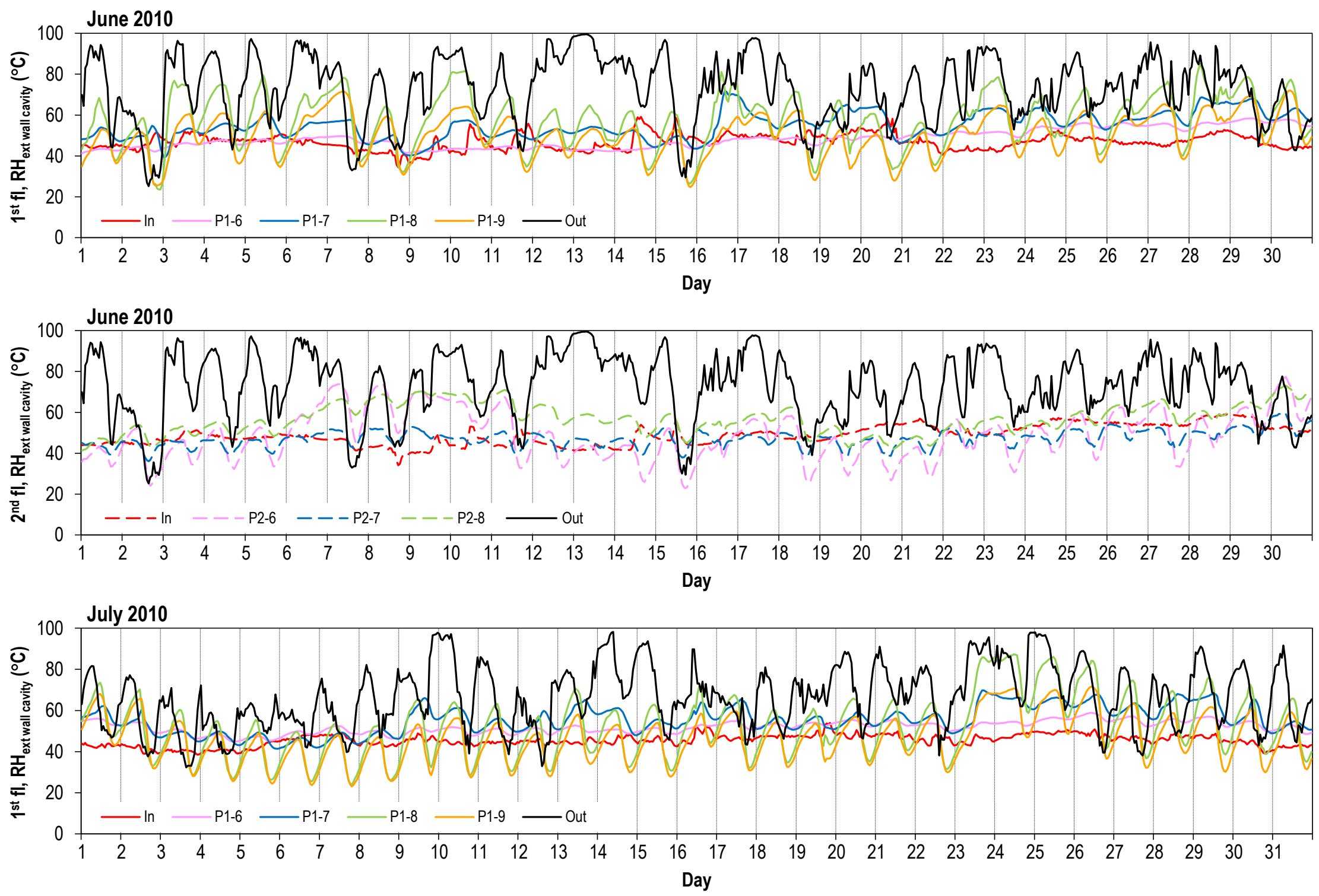

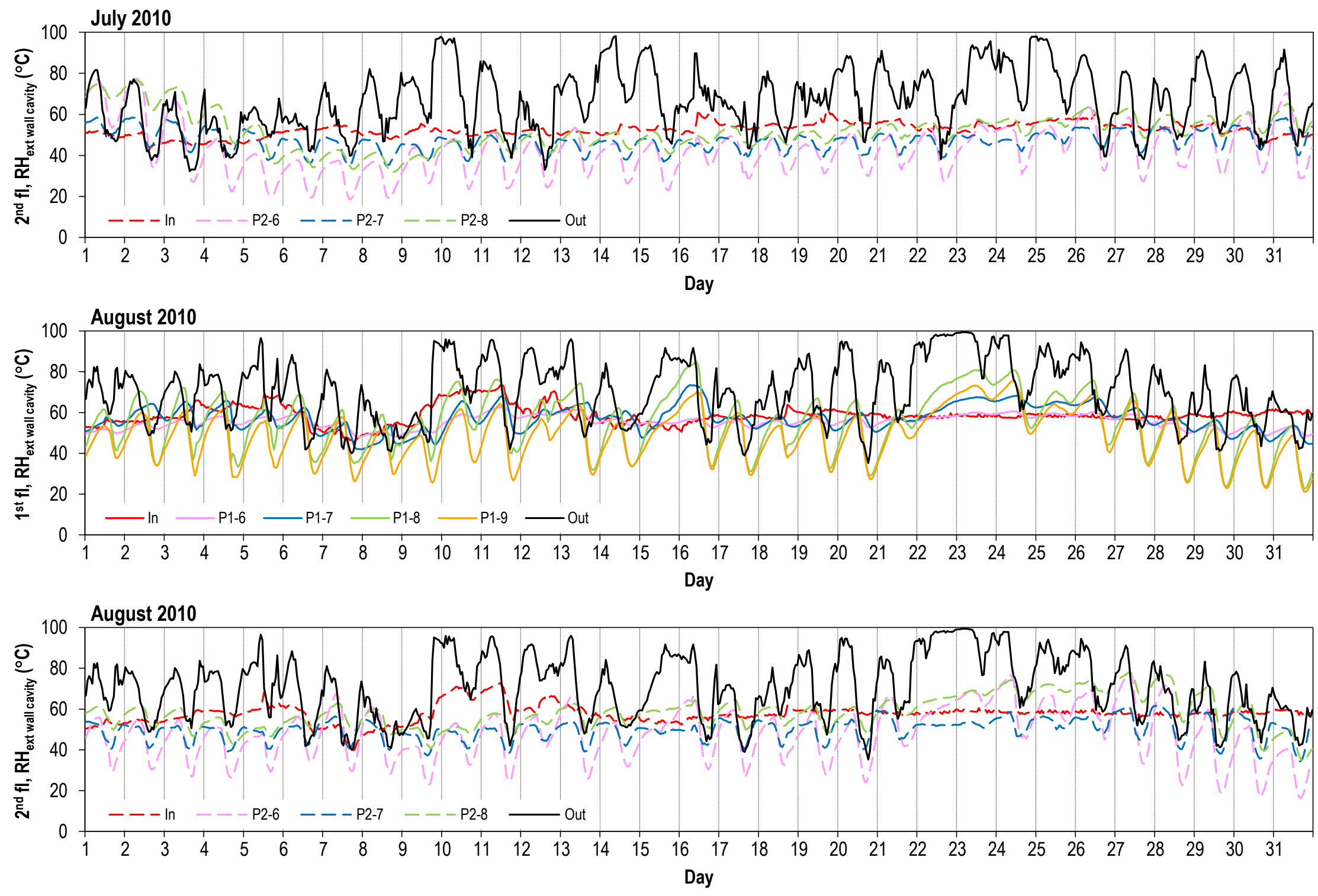

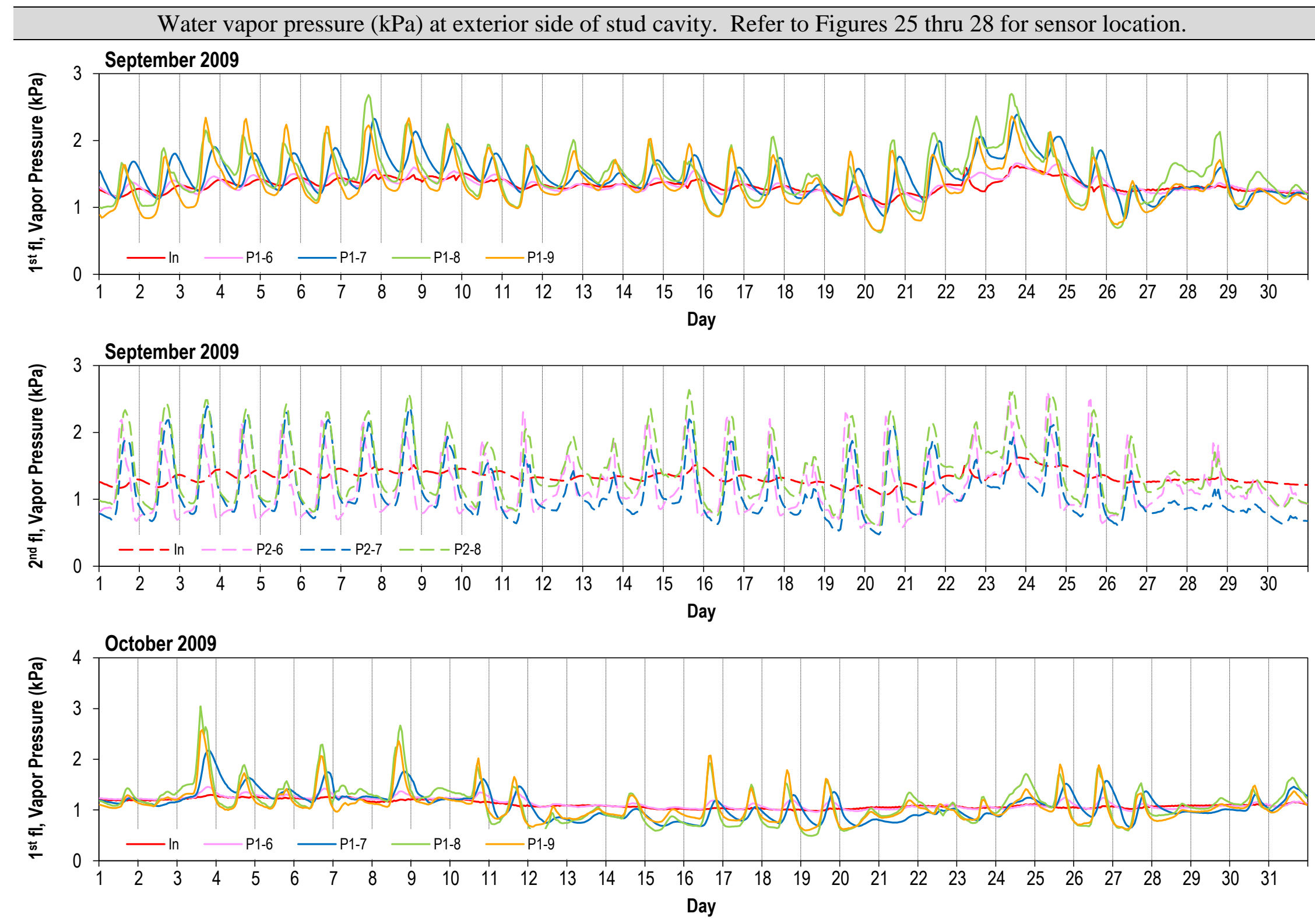

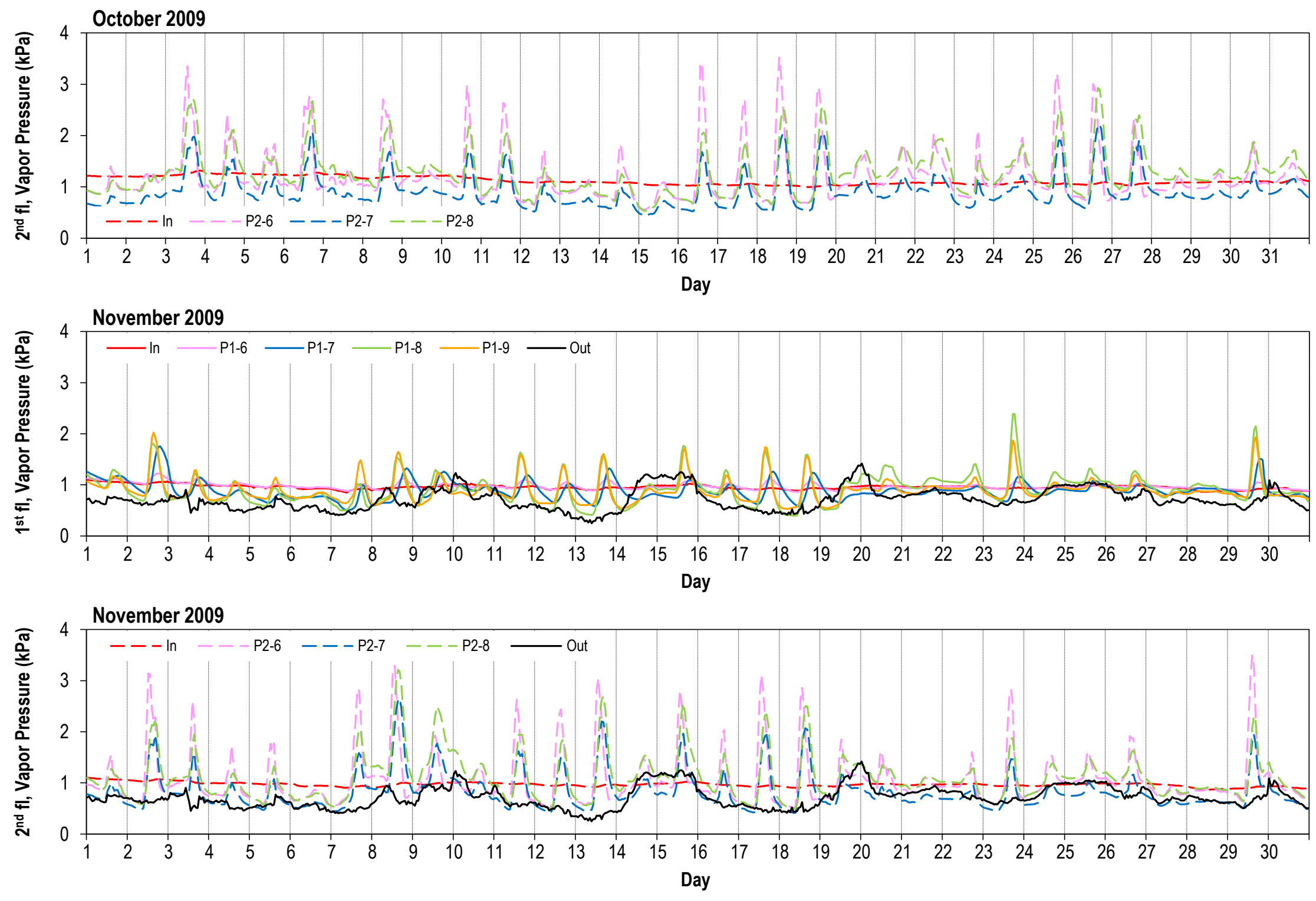

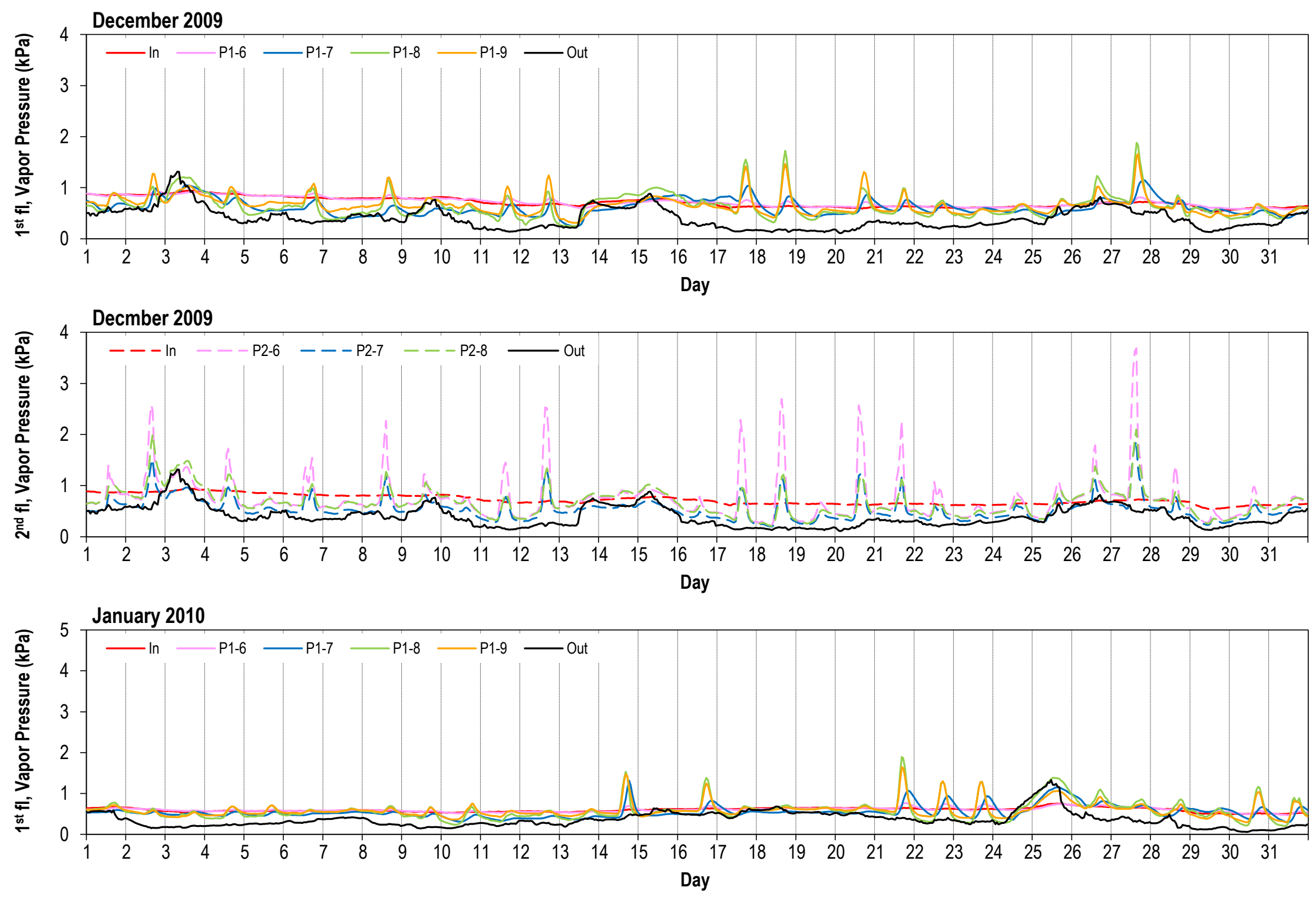

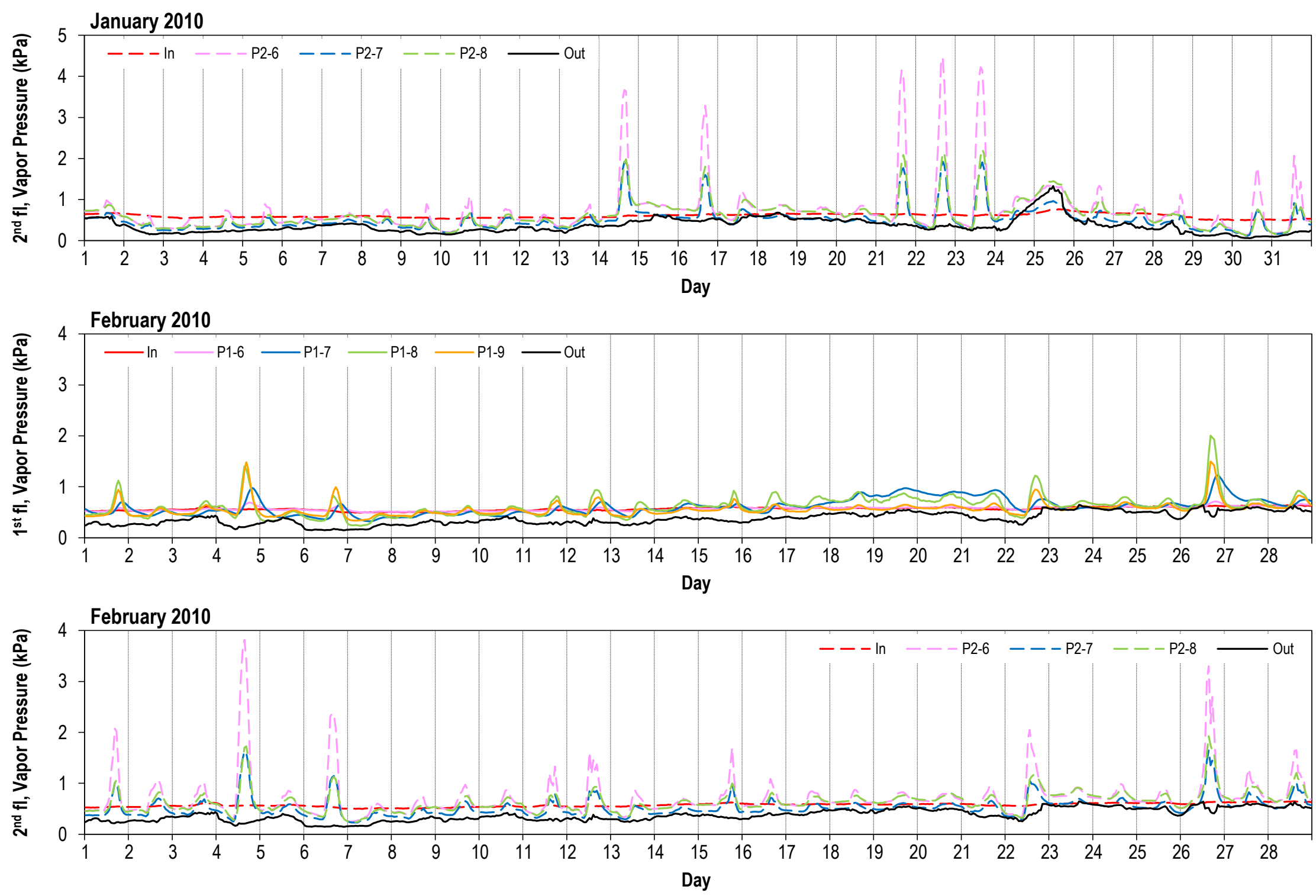

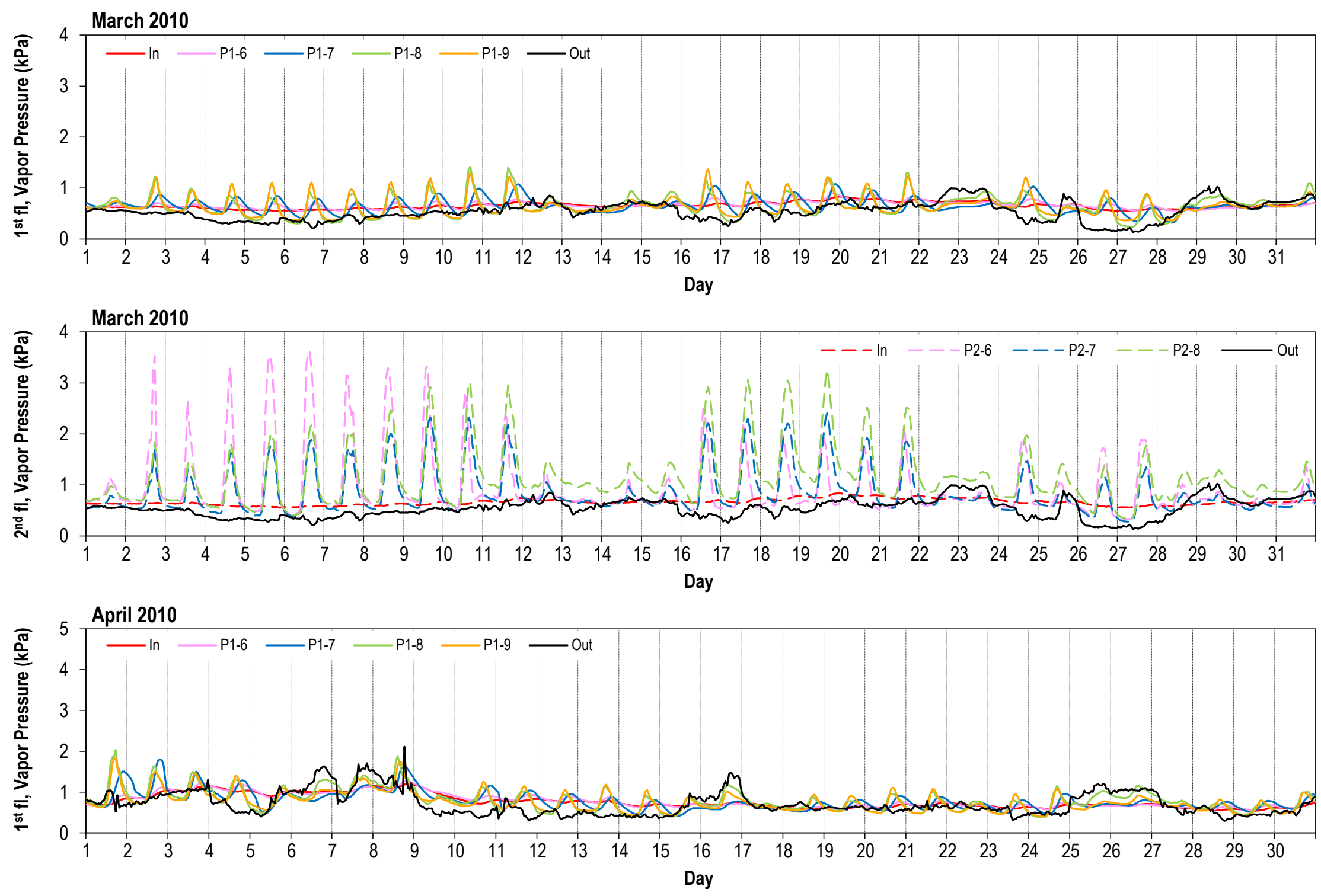

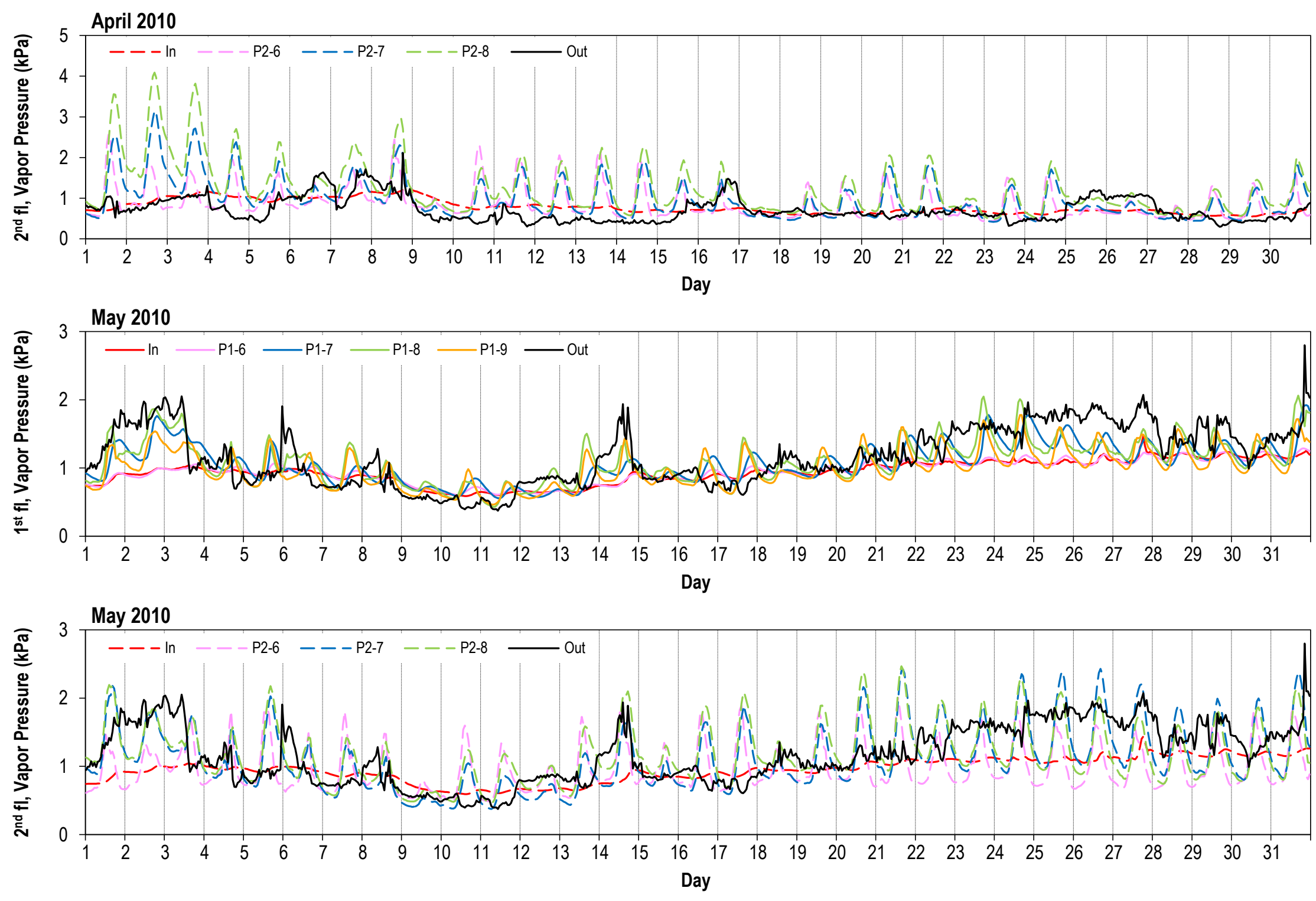

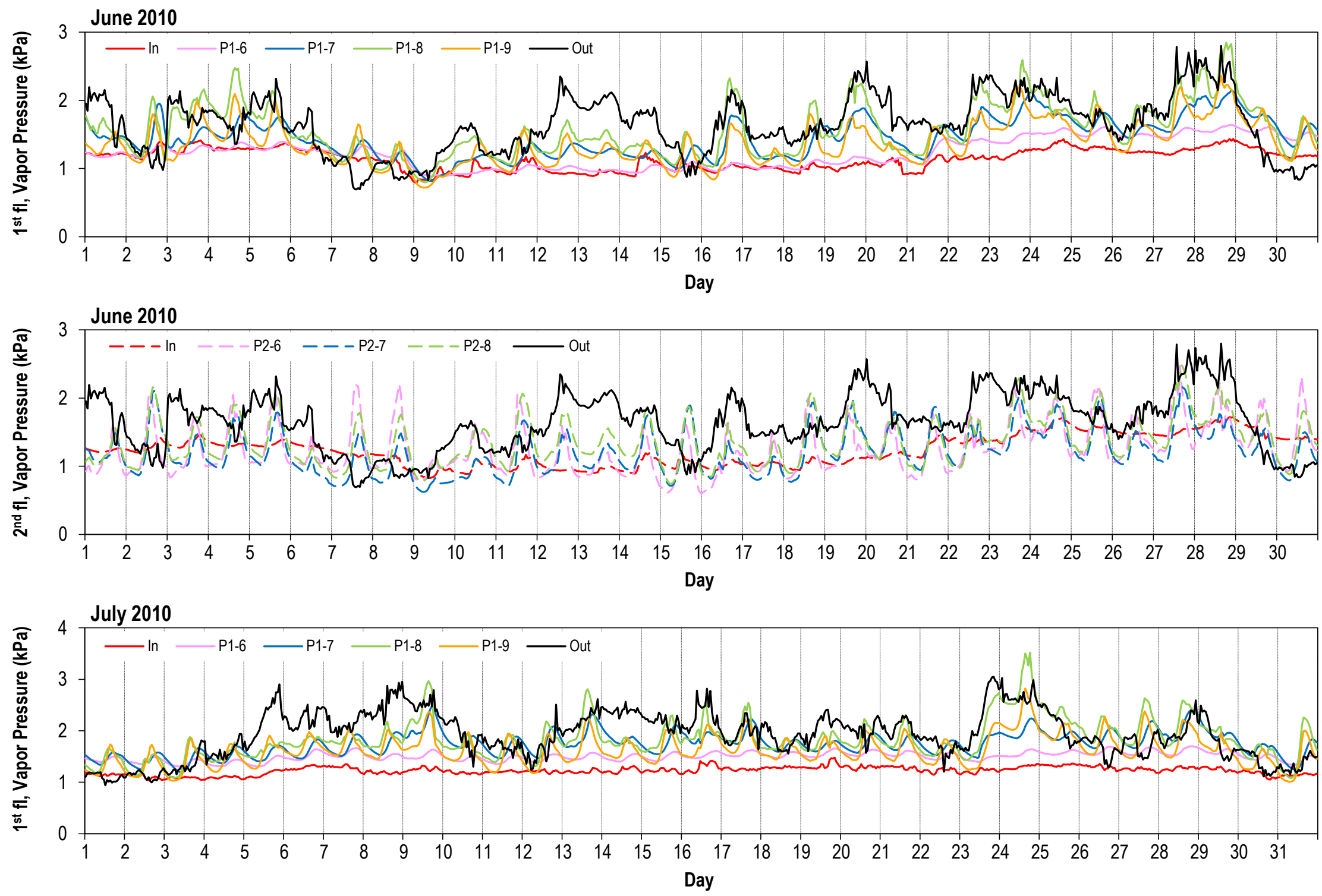

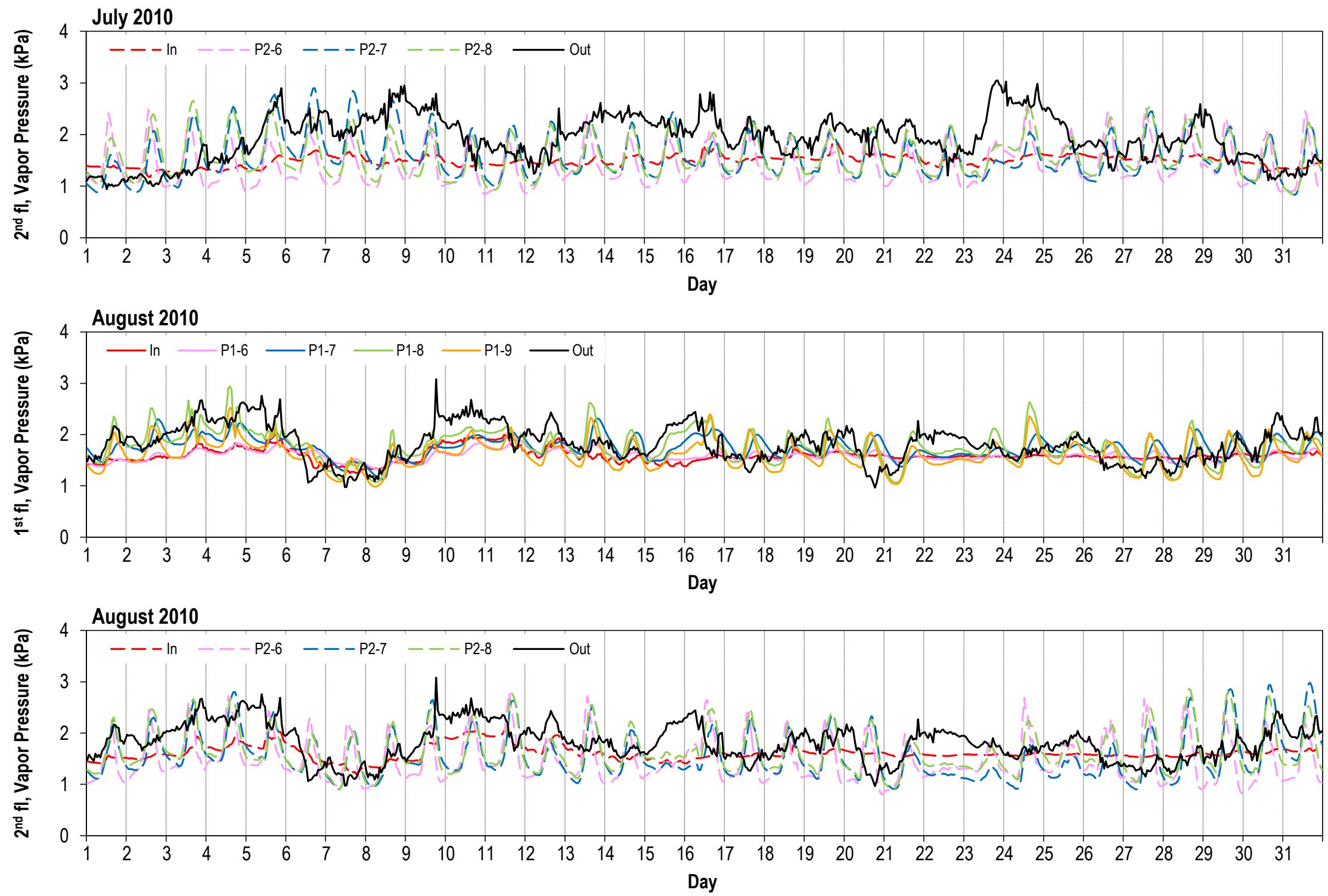

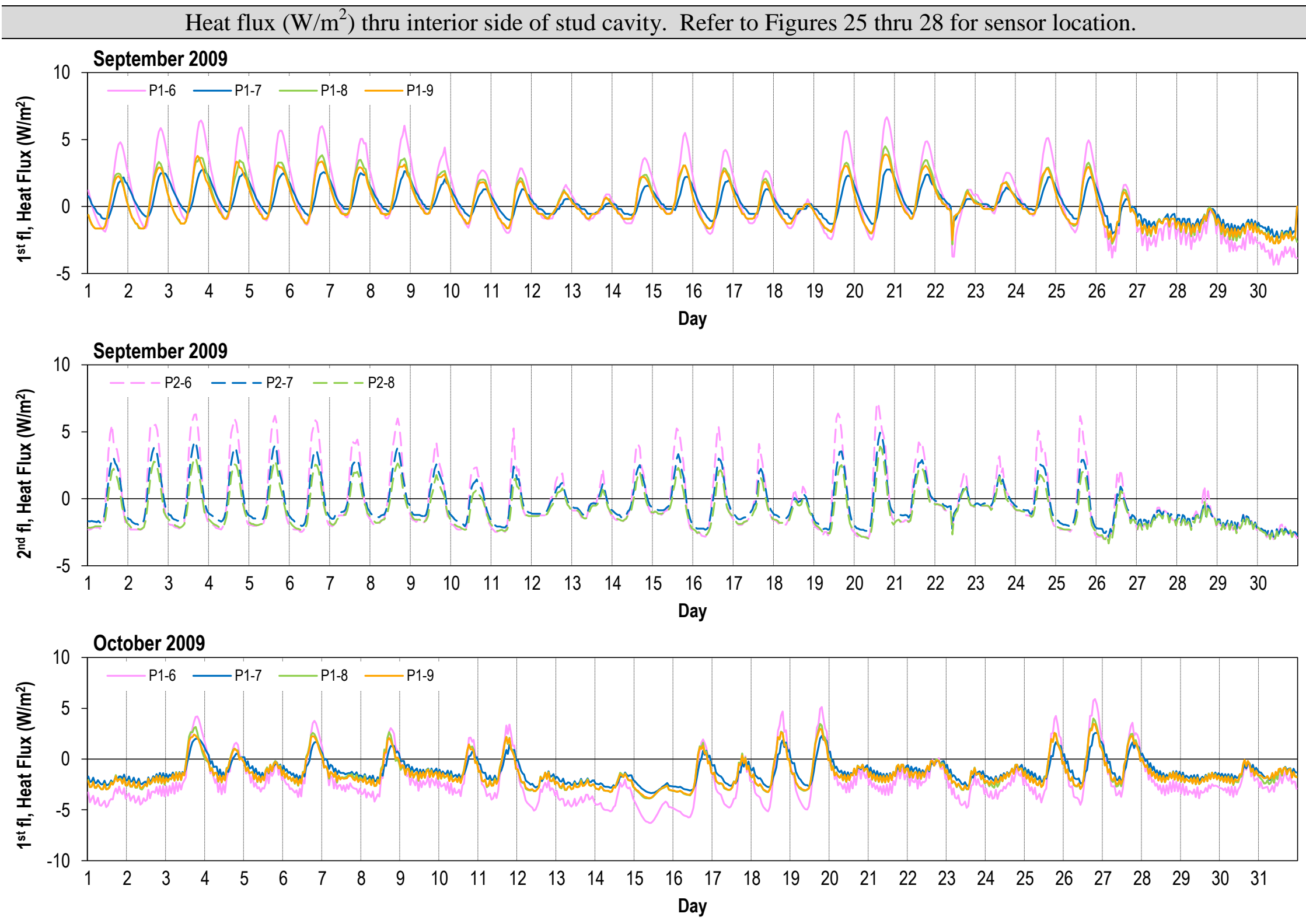

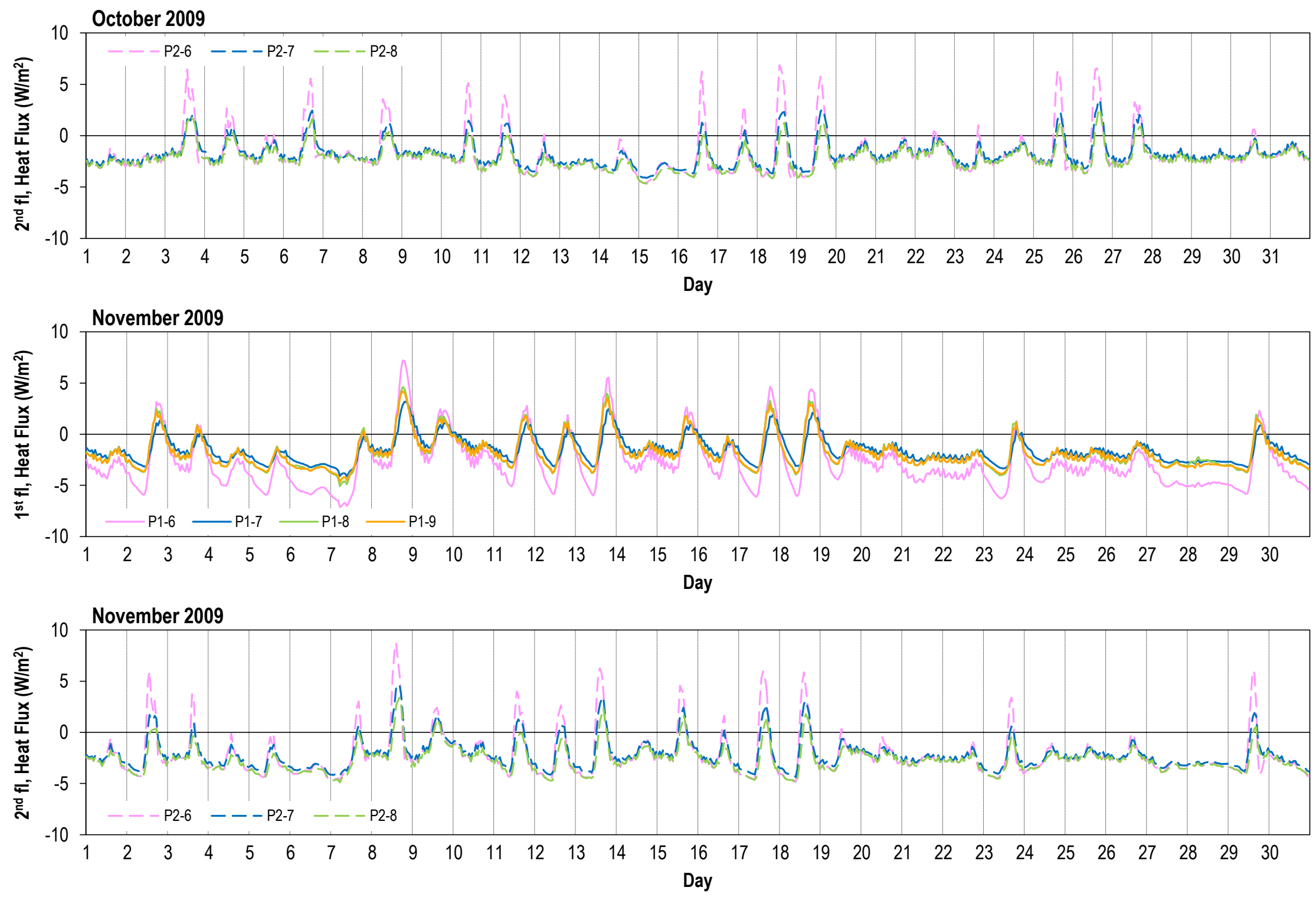

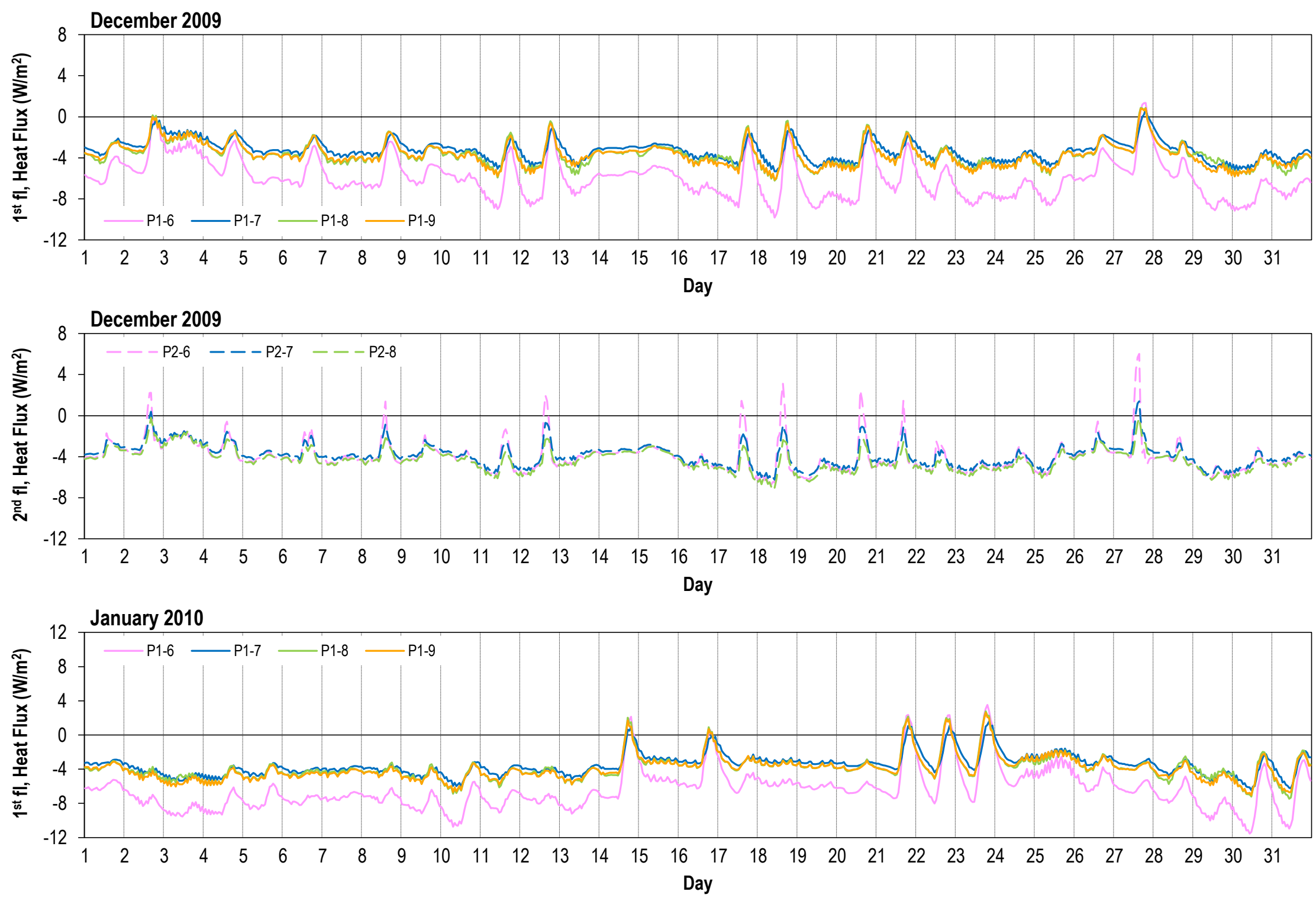

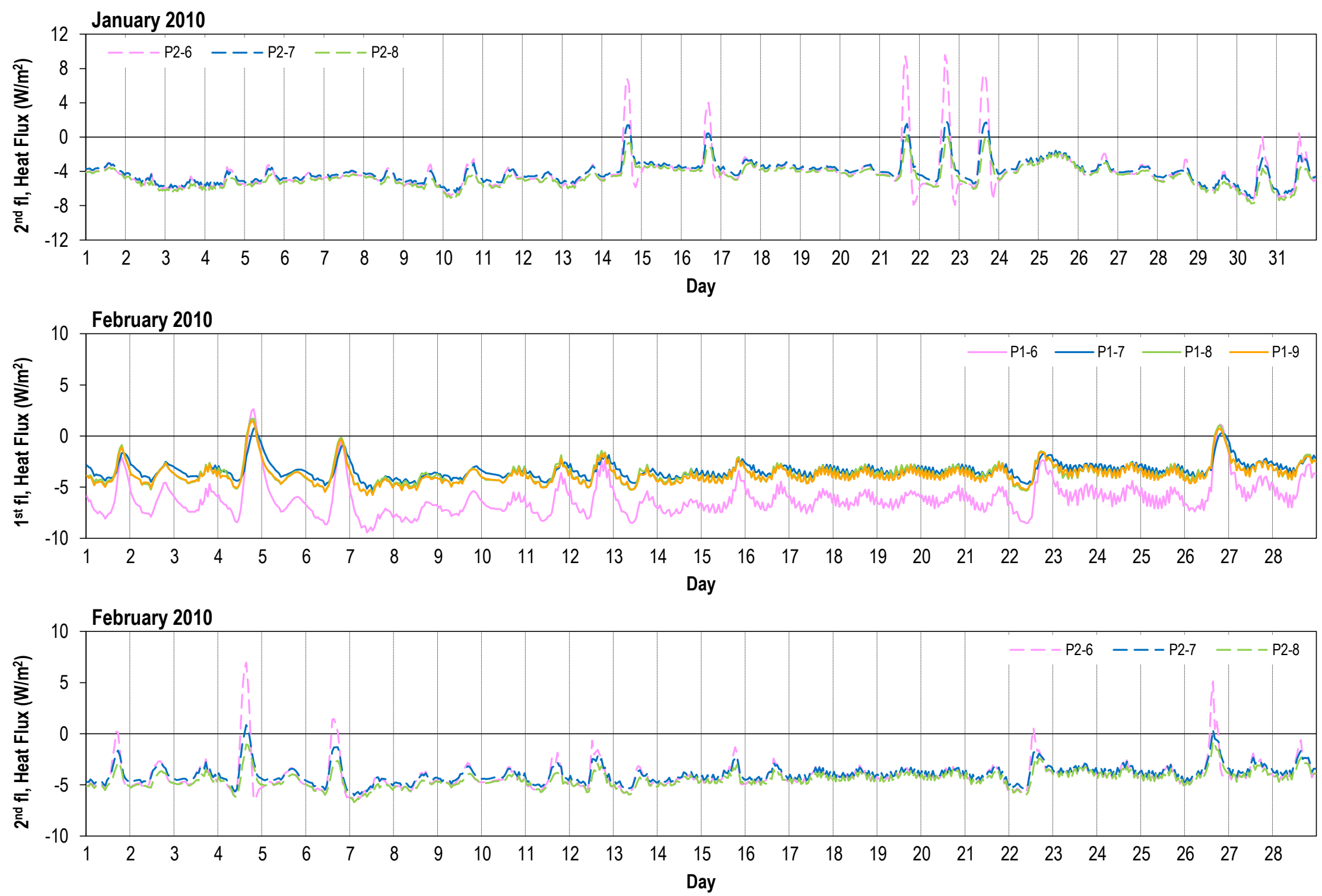

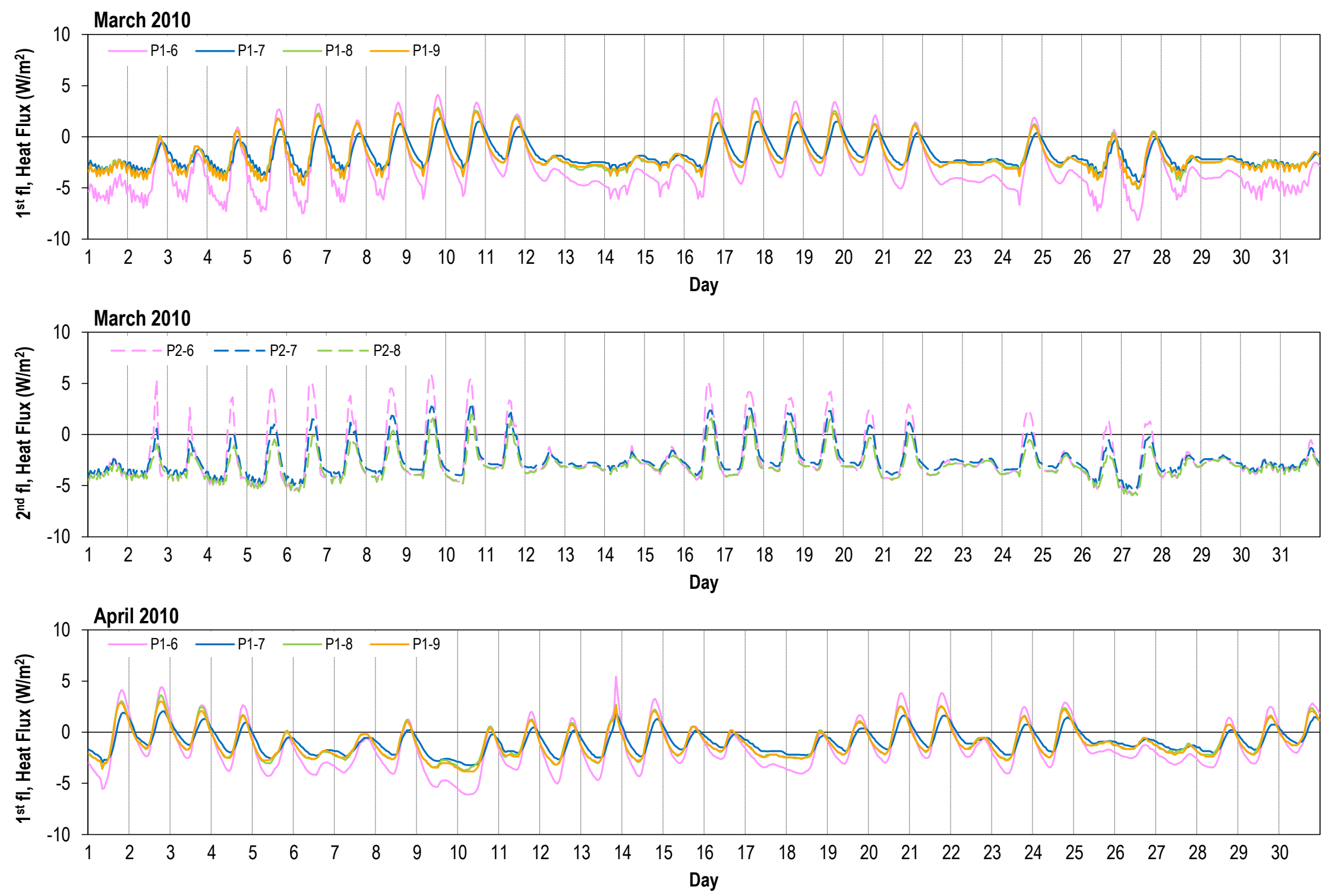

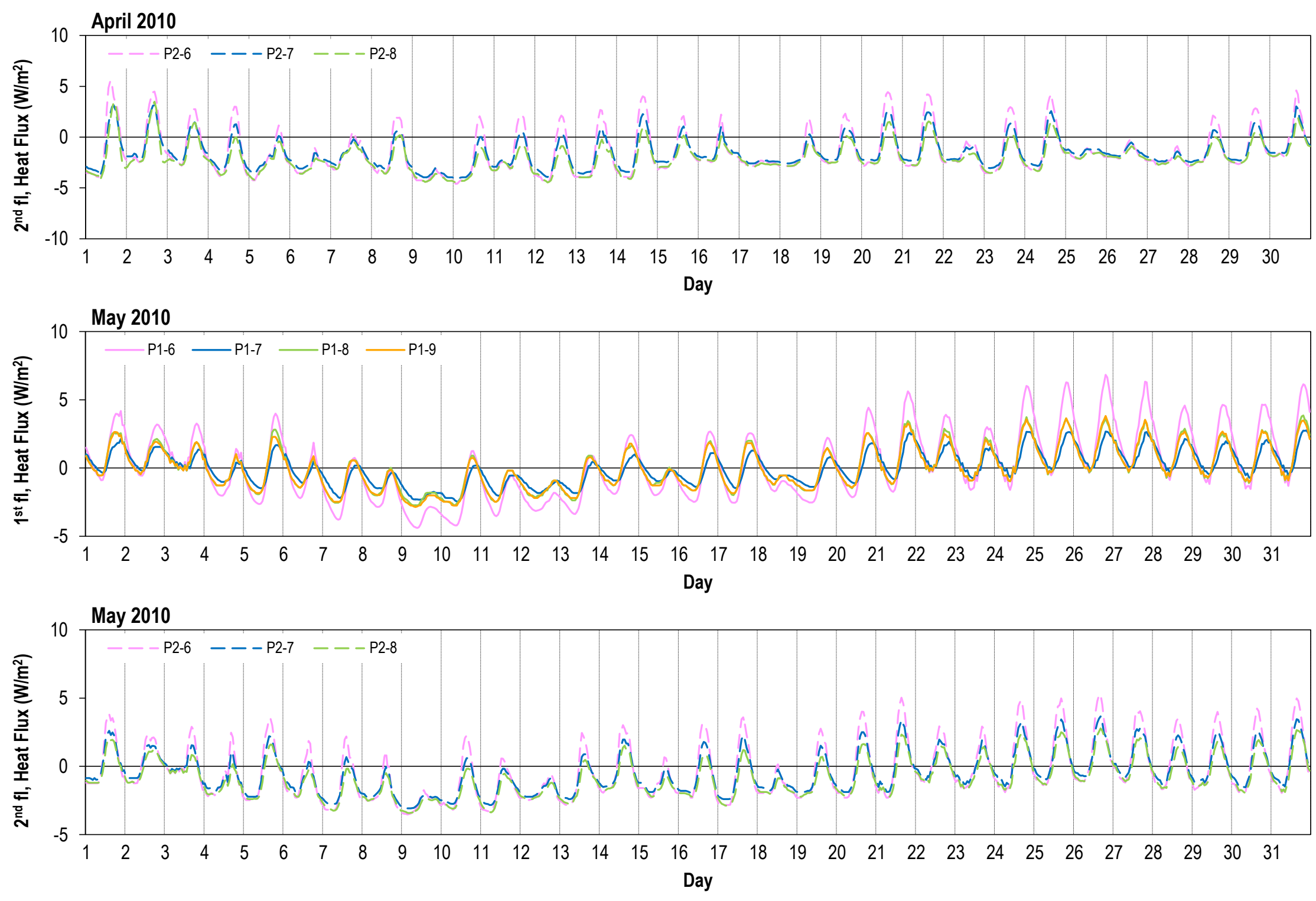

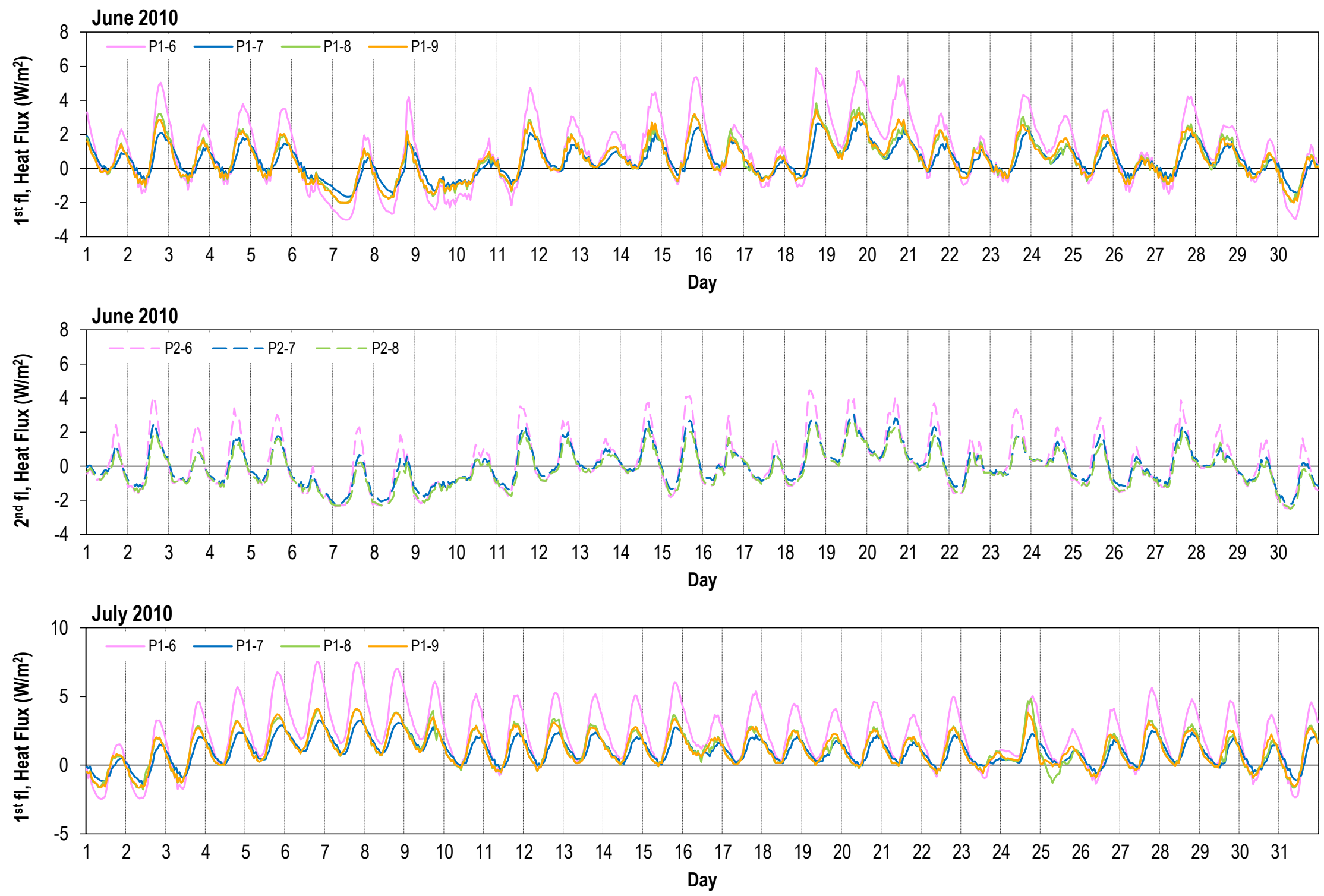

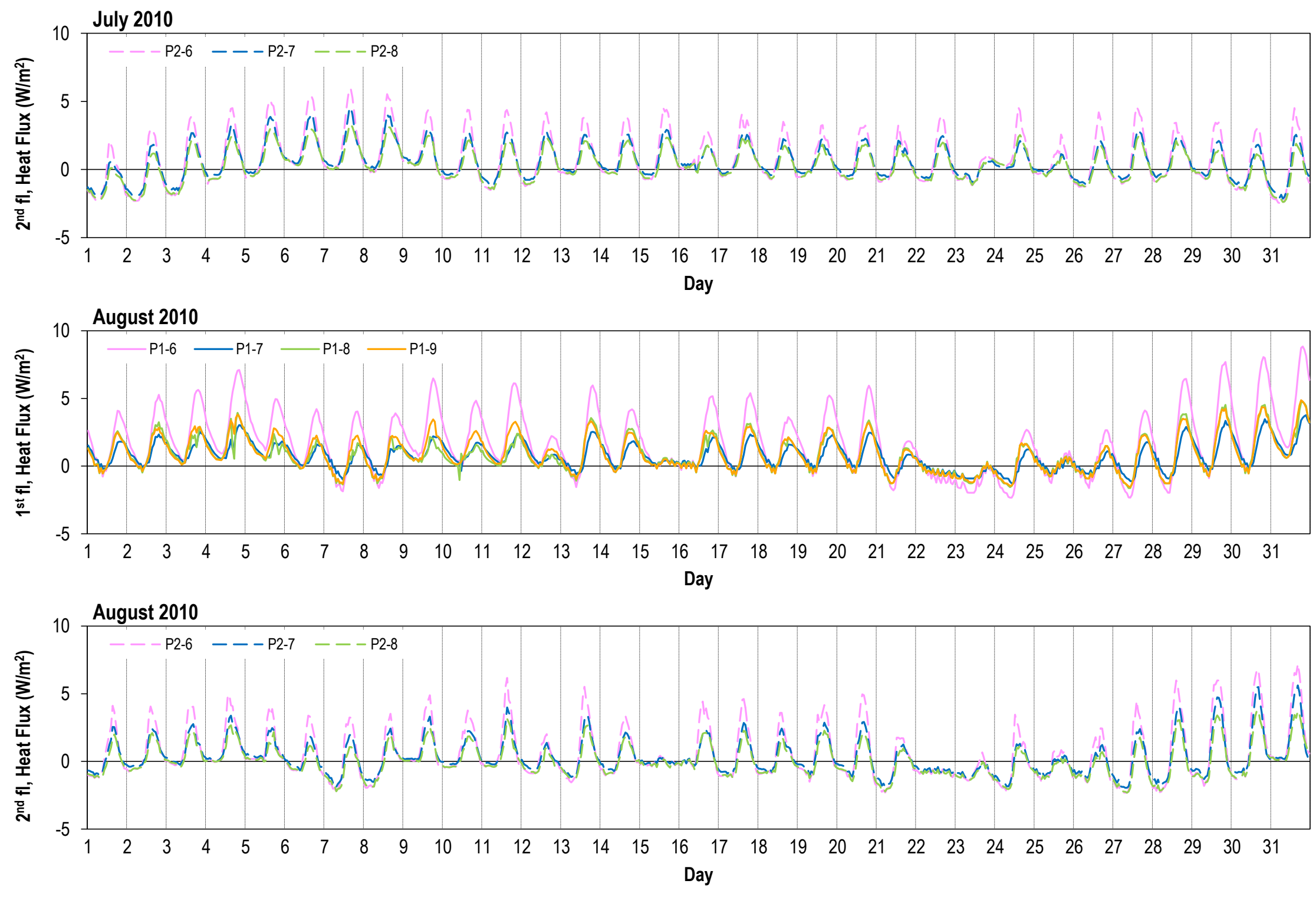
Cooling loads $\left(\mathrm{Wh} / \mathrm{m}^{2}\right)$. Refer to Figures 25 thru 28 for sensor location.
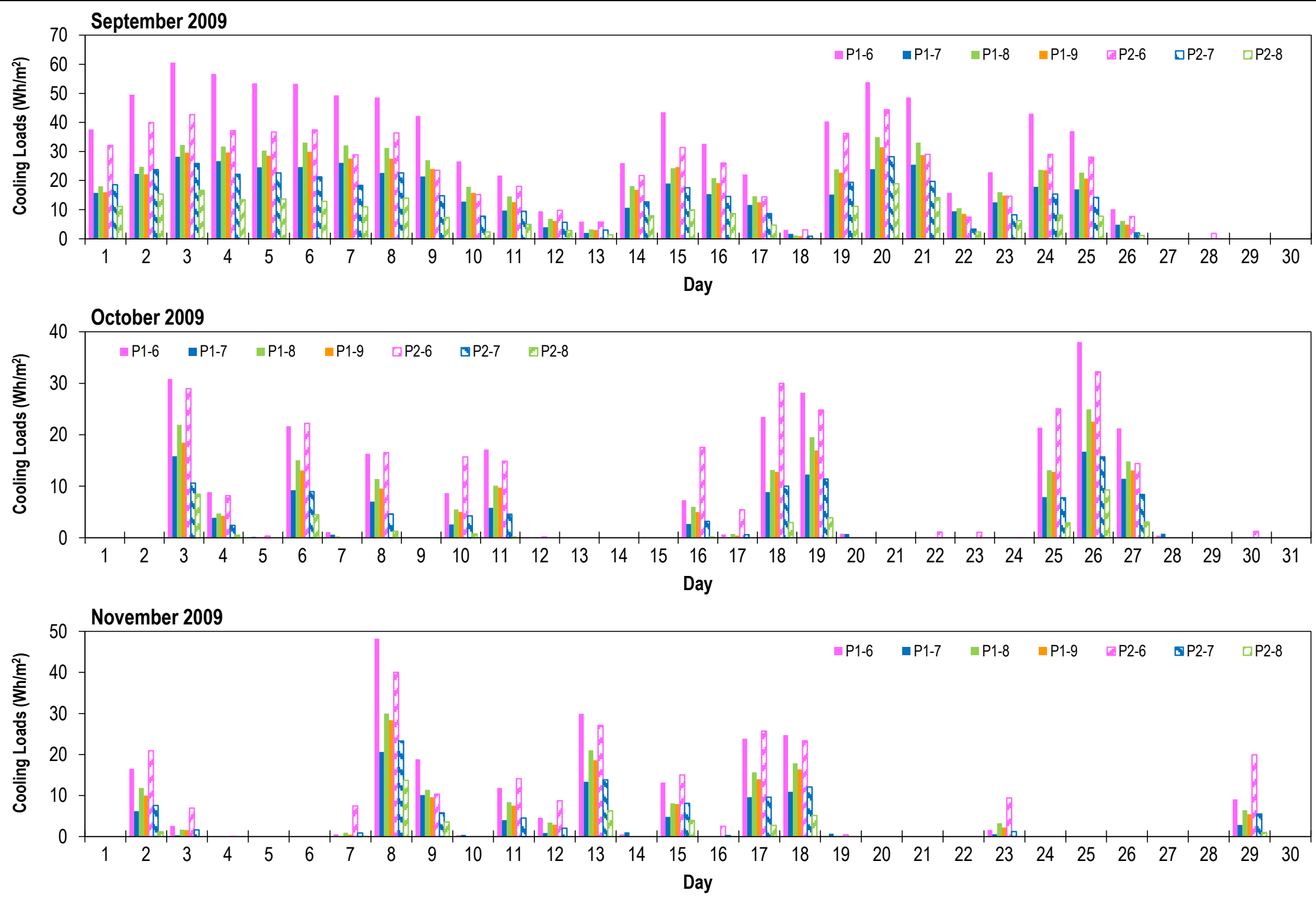

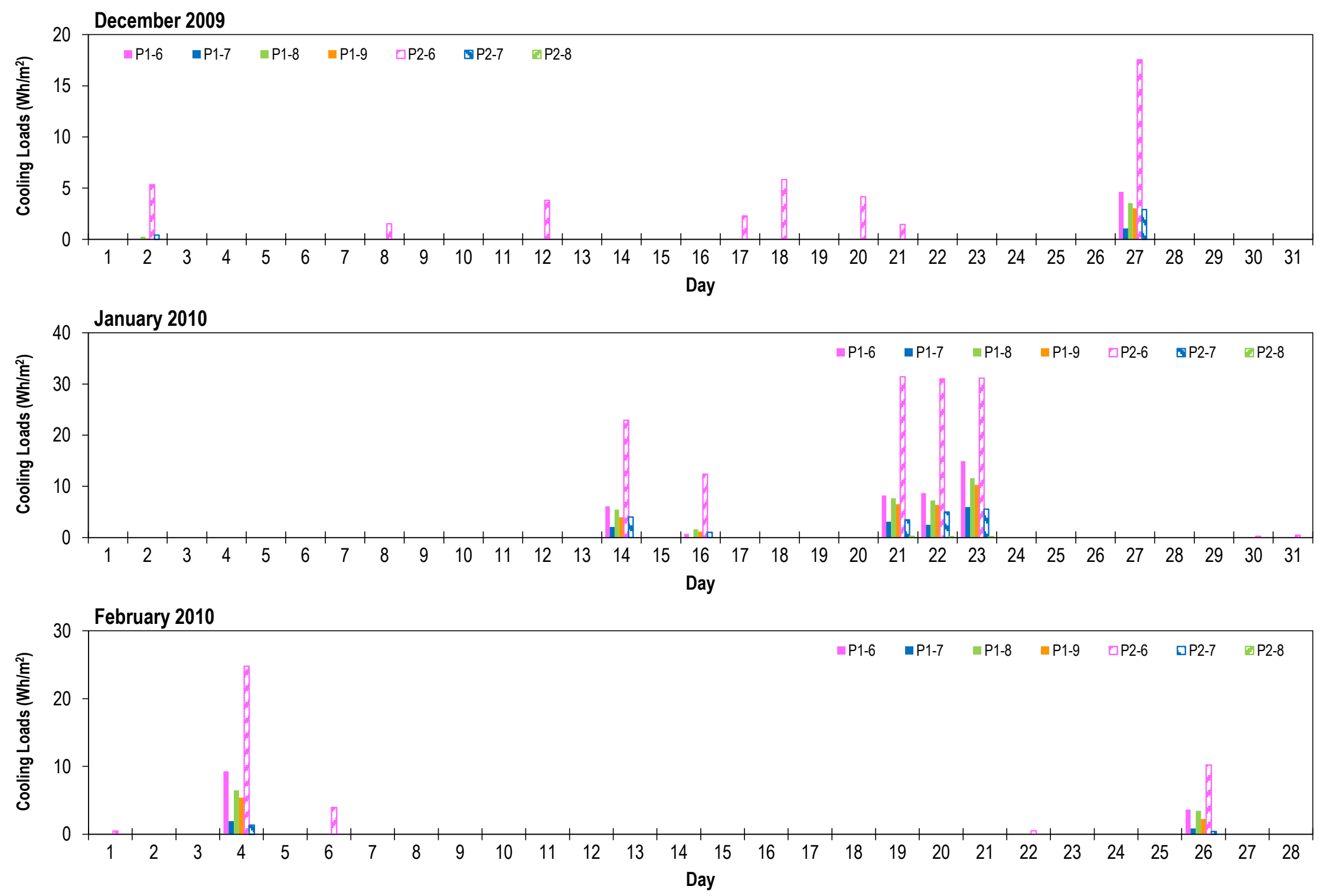

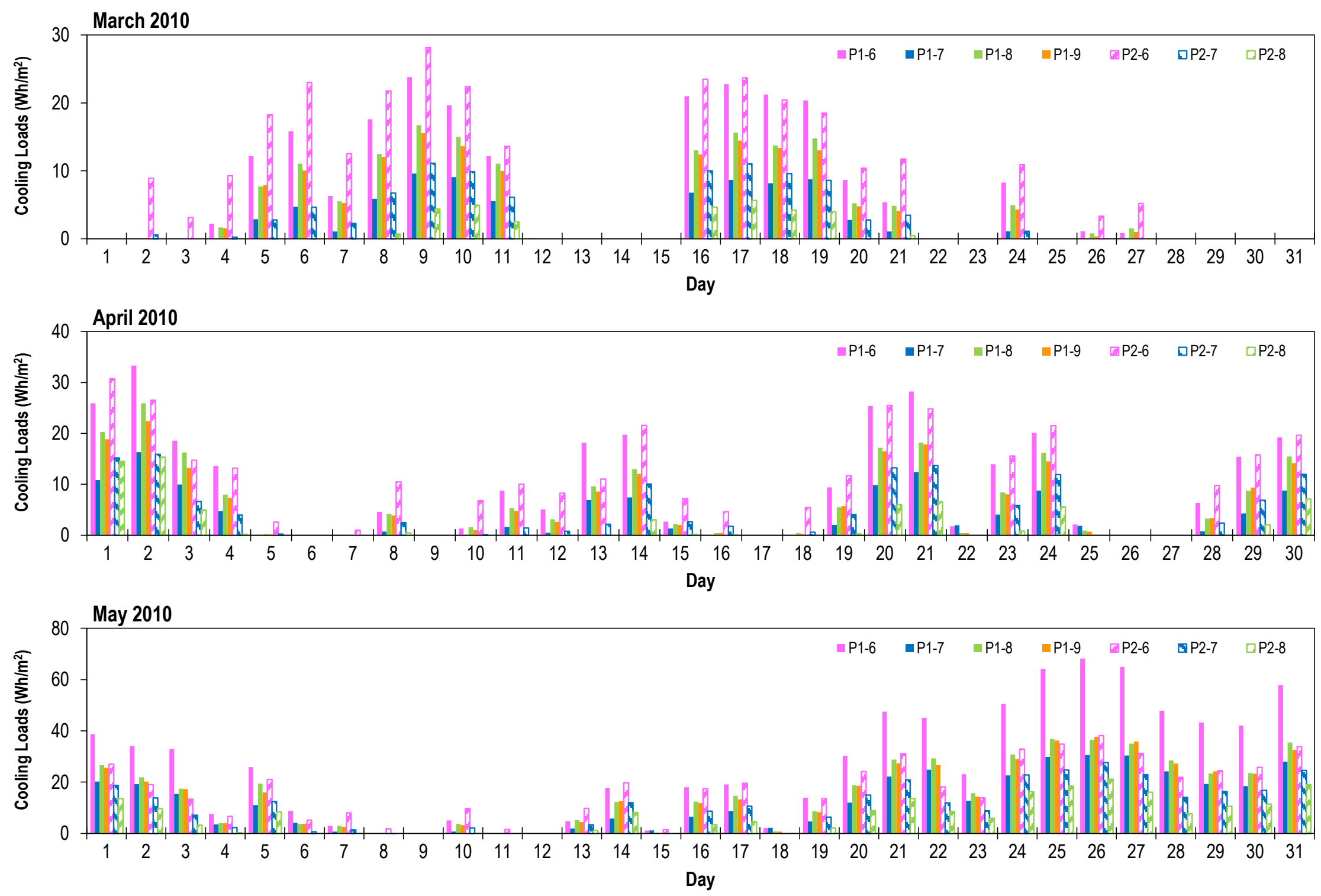

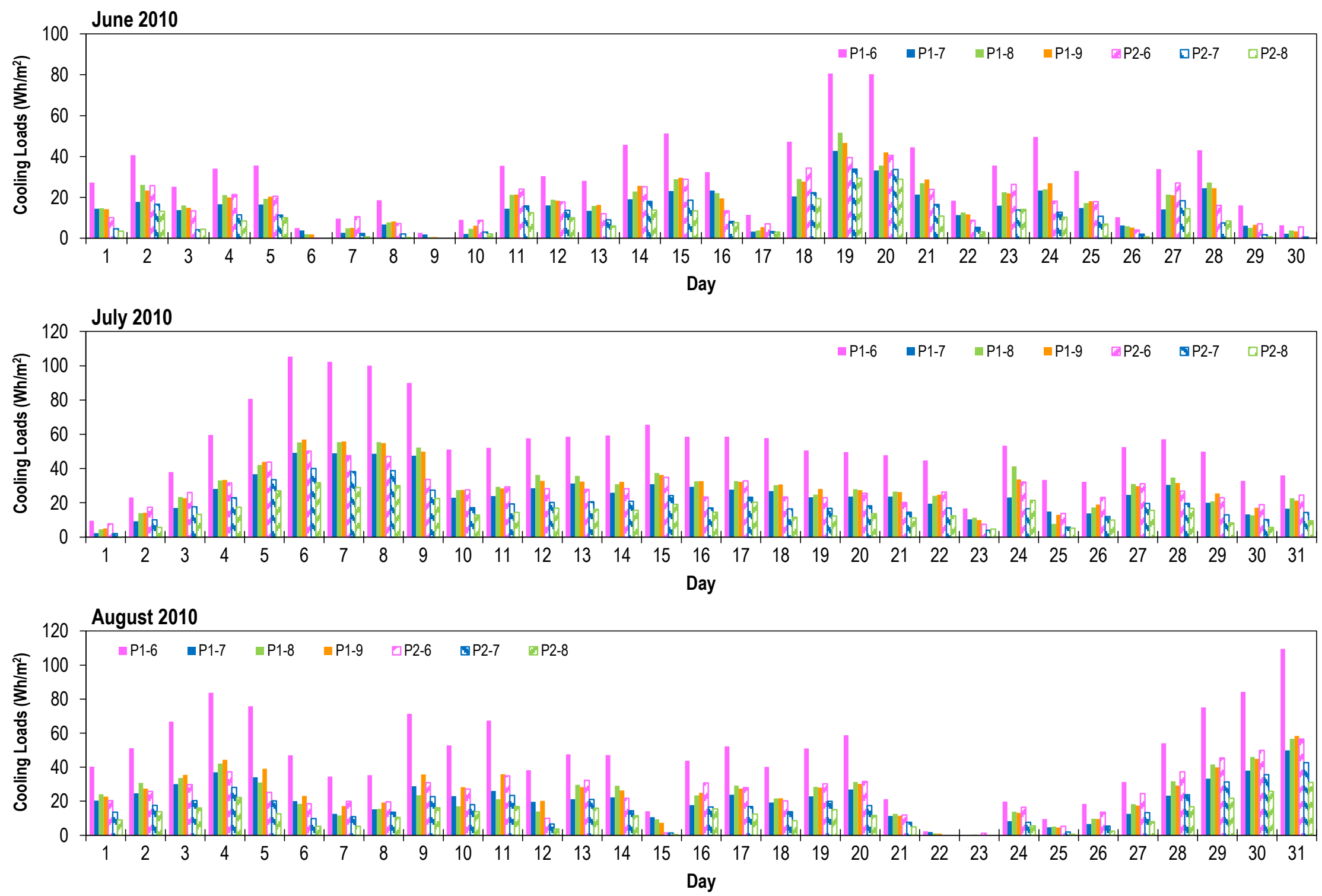
Heating loads $\left(\mathrm{Wh} / \mathrm{m}^{2}\right)$. Refer to Figures 25 thru 28 for sensor location.

\section{September 2009}

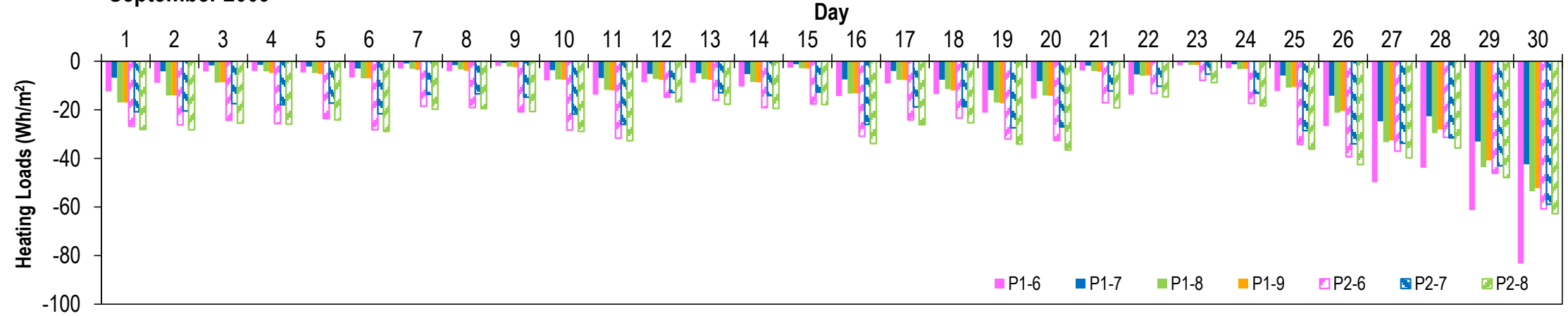

October 2009

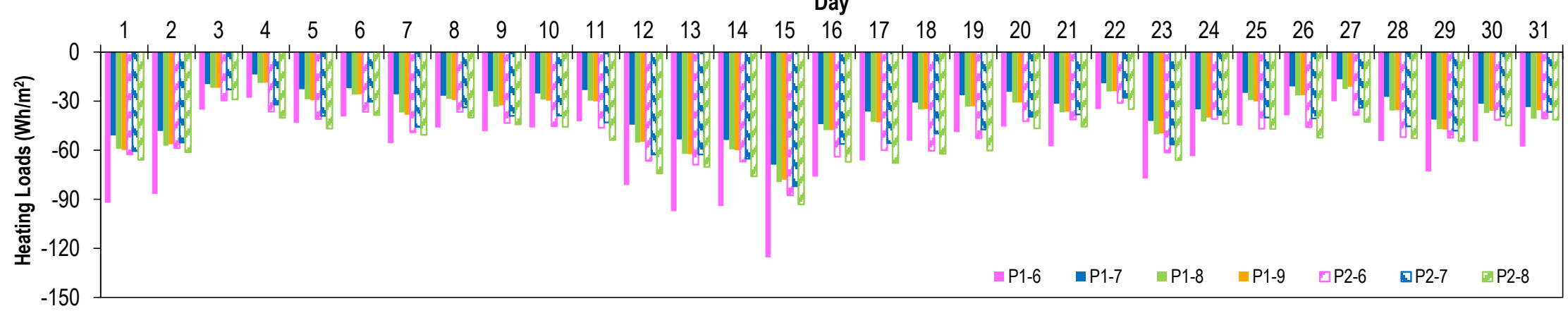

November 2009

Day

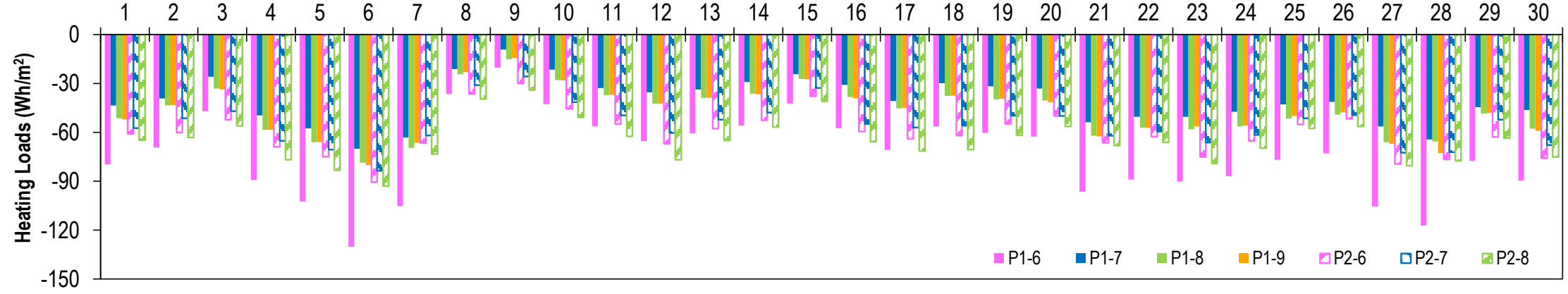




\section{December 2009}

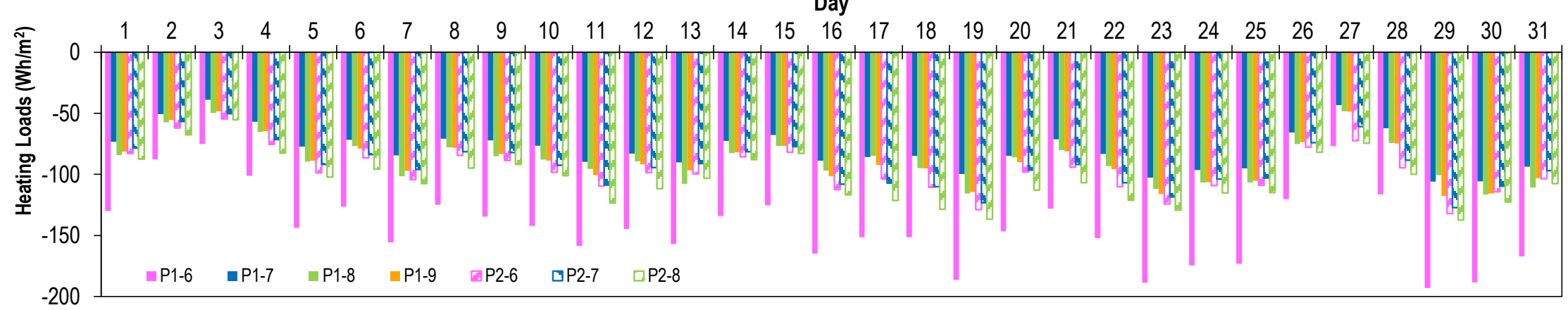

January 2010

Day

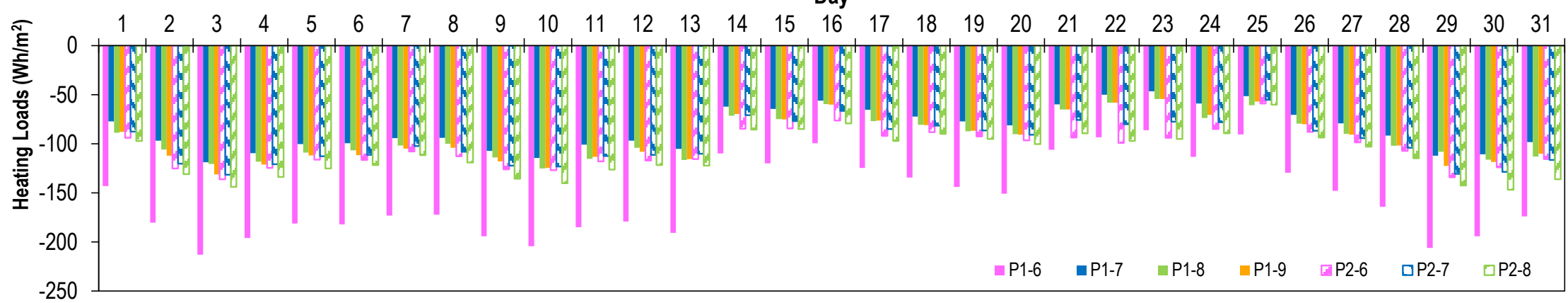

\section{February 2010}

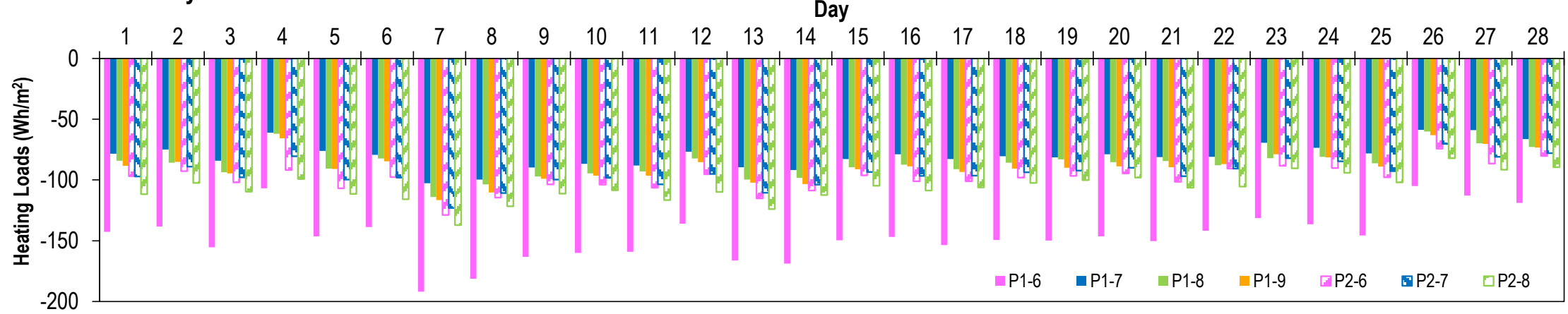




\section{March 2010}

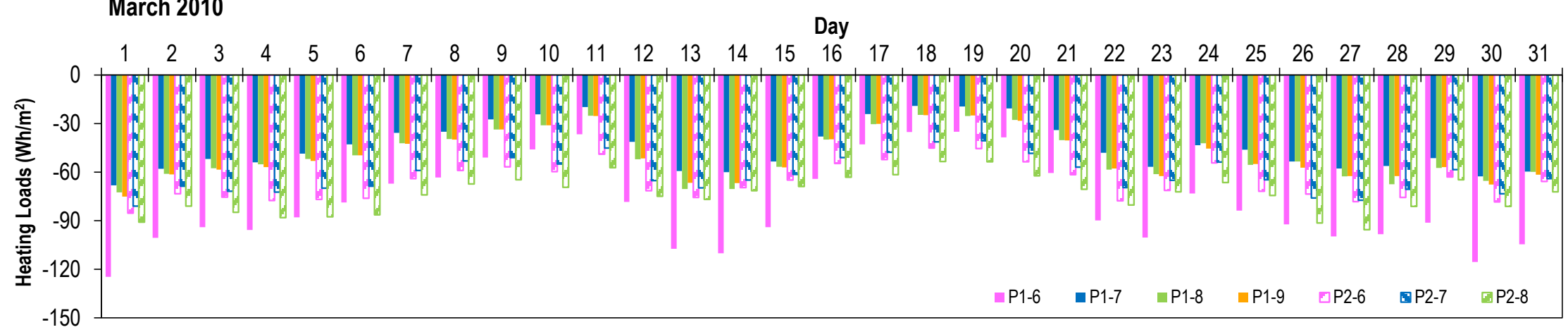

\section{April 2010}

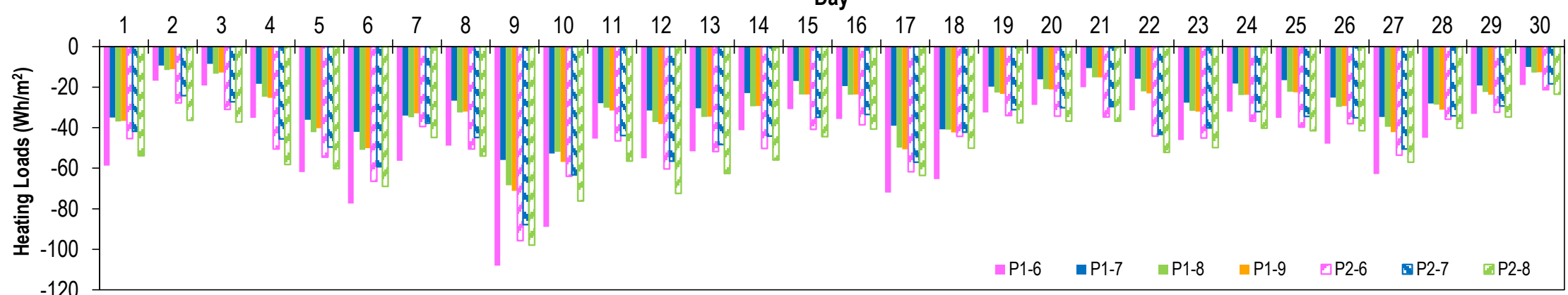

\section{May 2010}

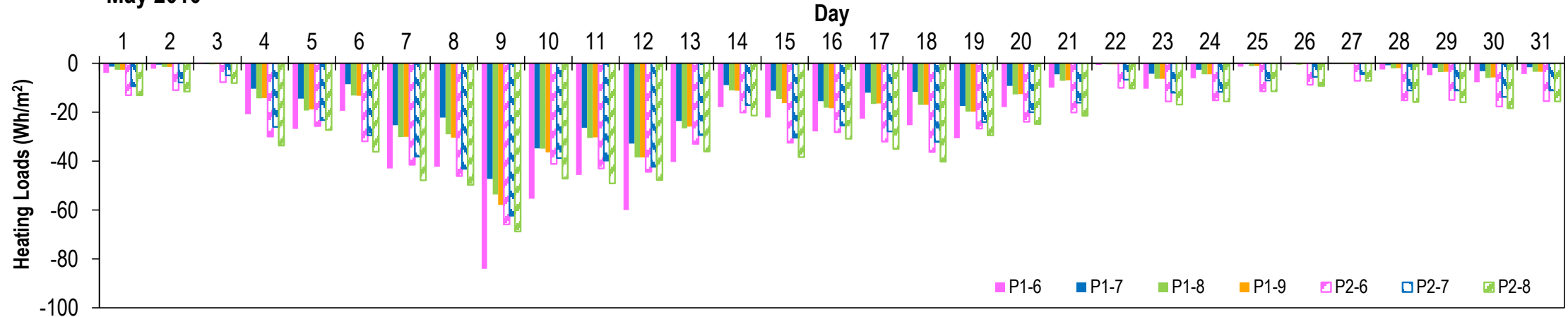


June 2010

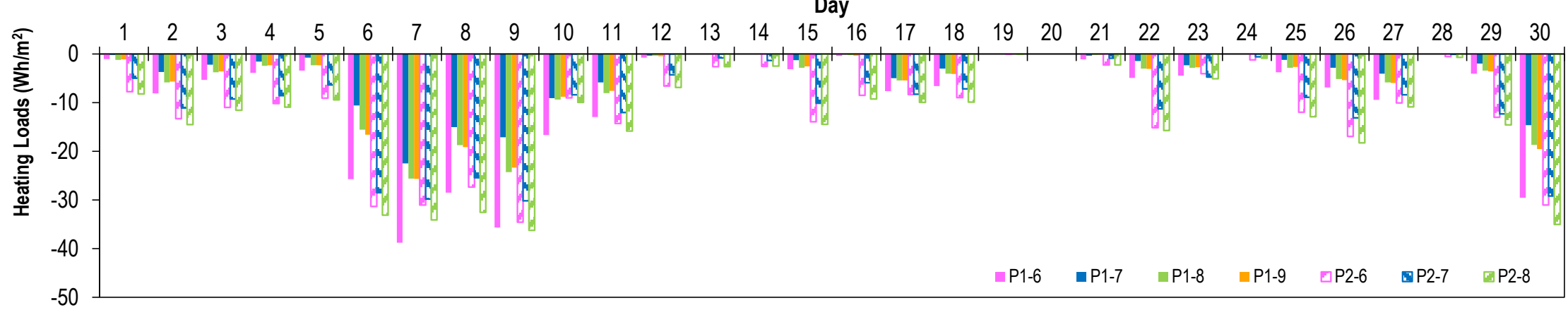

July 2010

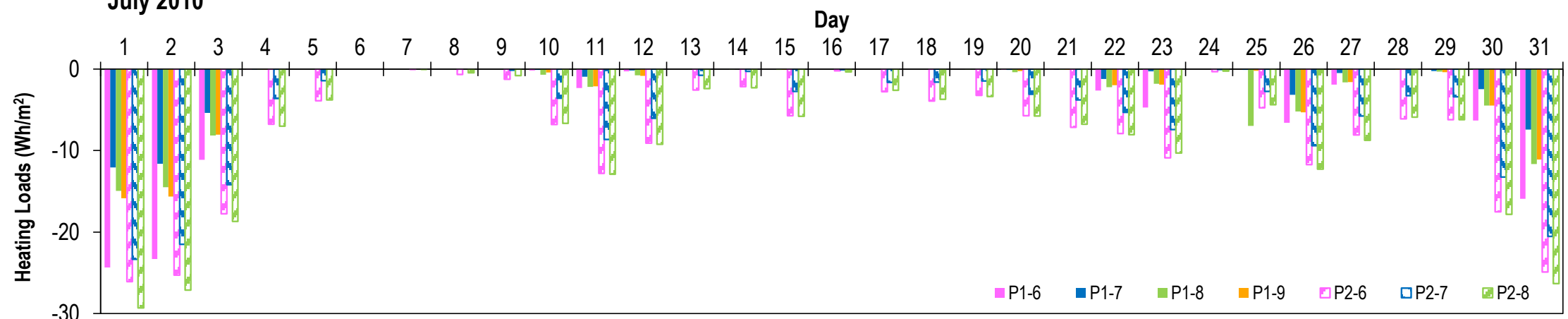

August 2010

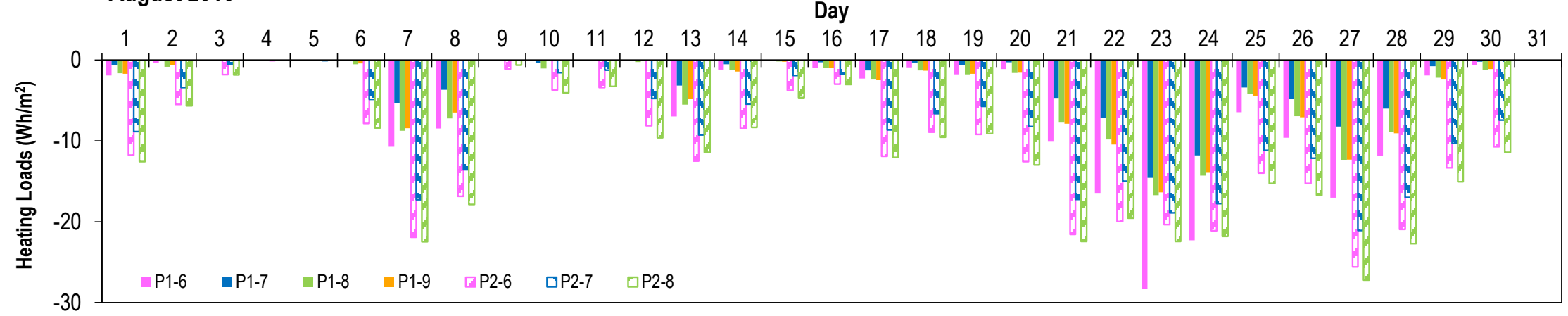




\section{Appendix D: West Panels}

Temperature $\left({ }^{\circ} \mathrm{C}\right)$ at exterior side of stud cavity. Refer to Figures 25 thru 28 for sensor location.
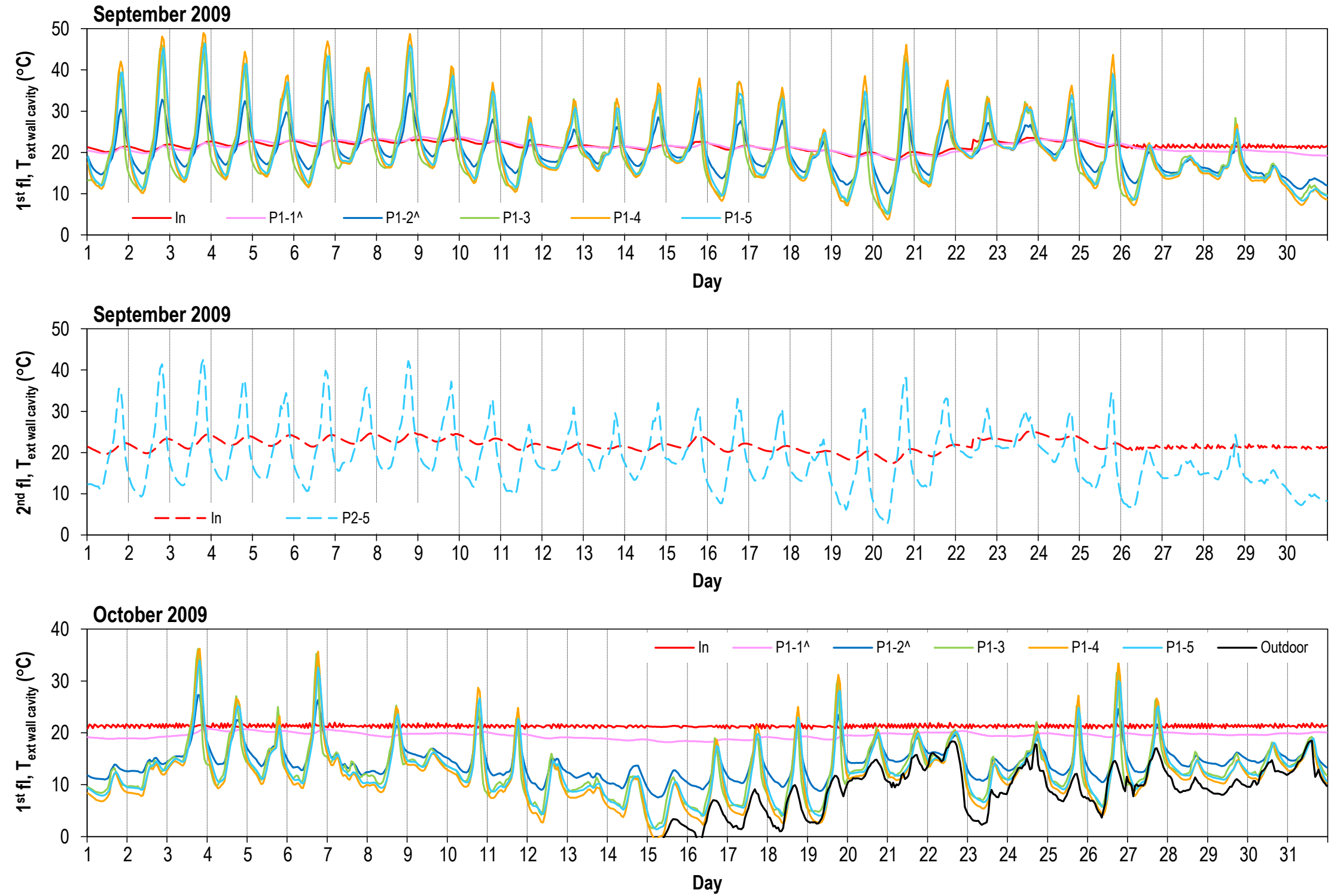

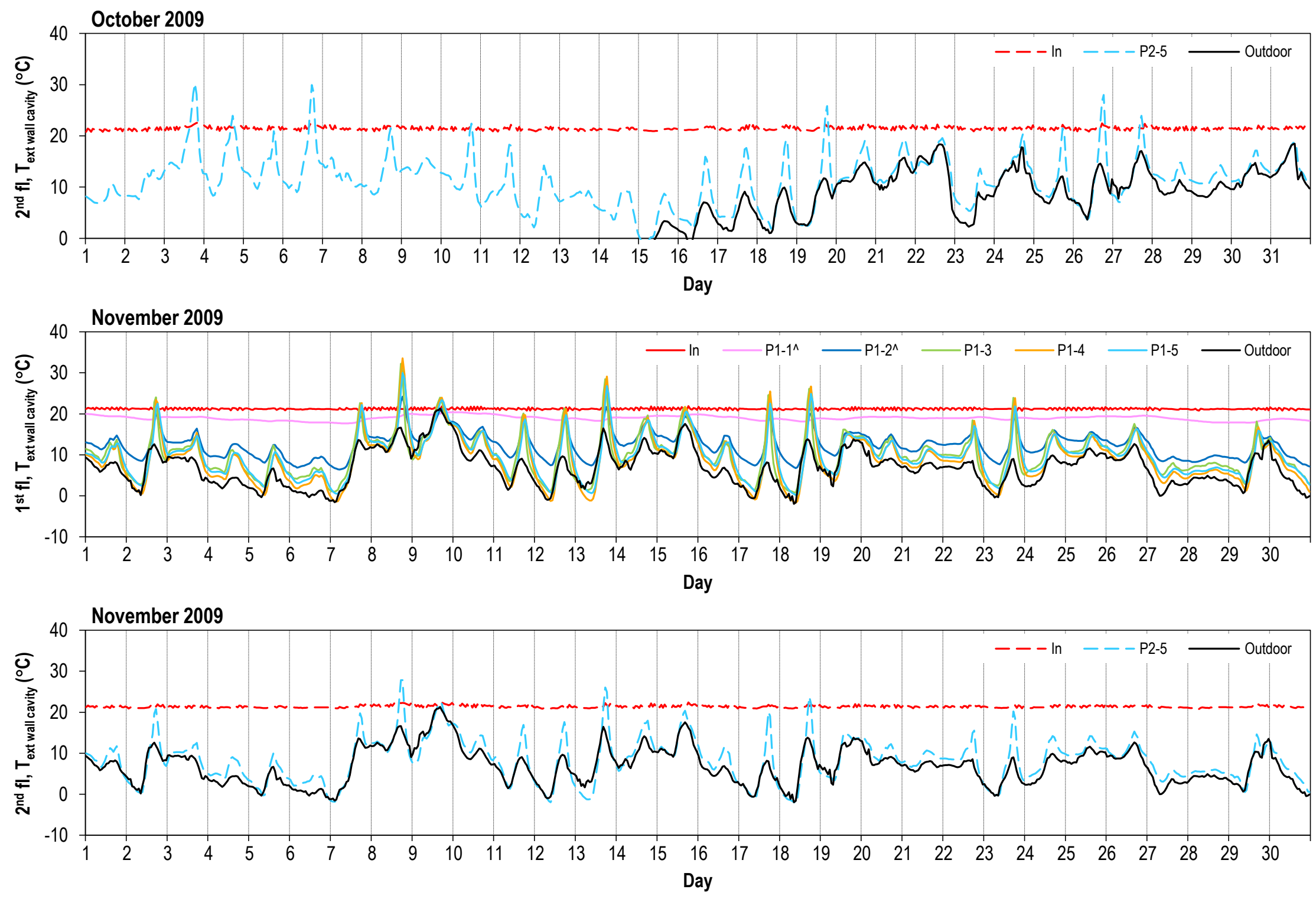

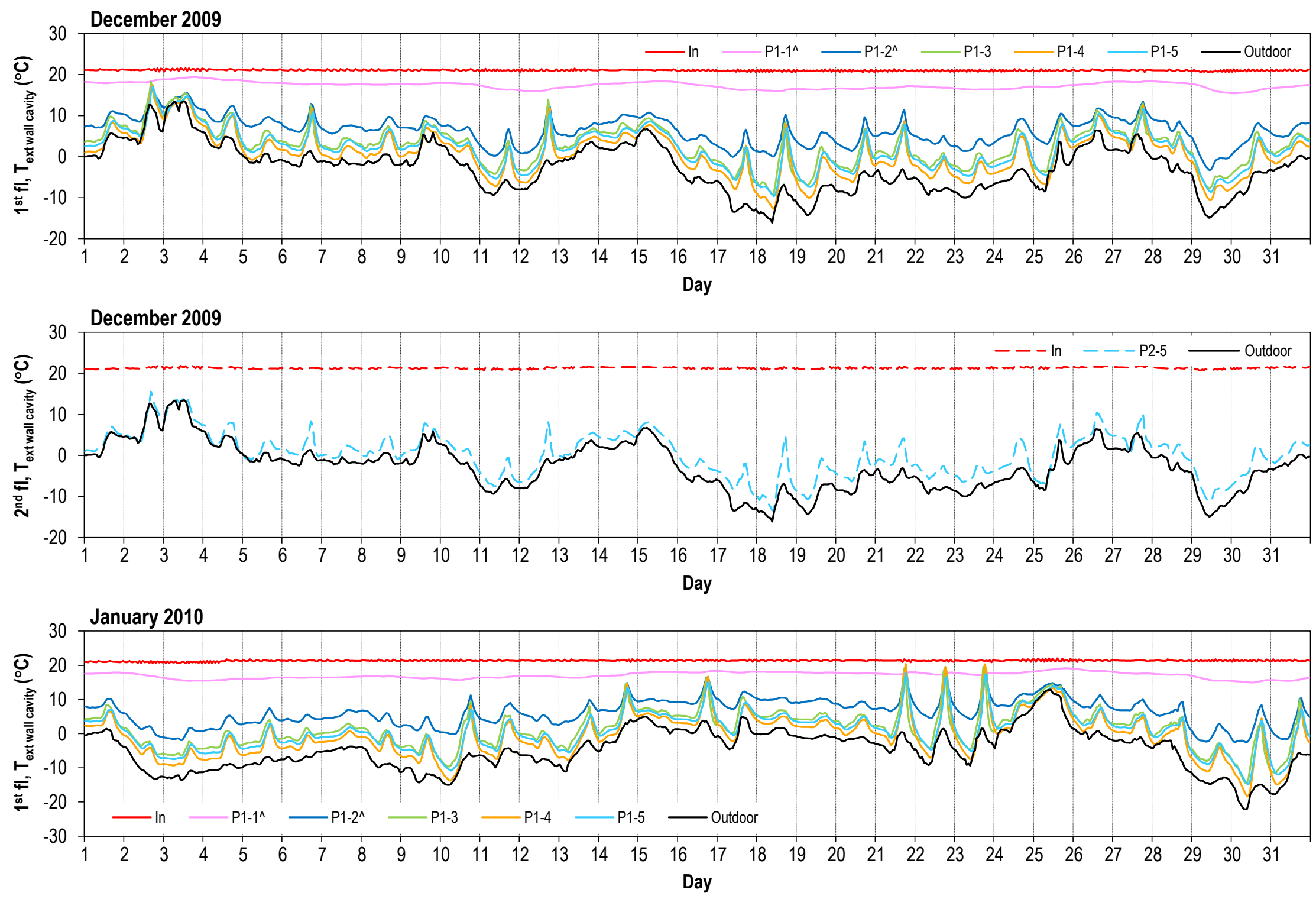

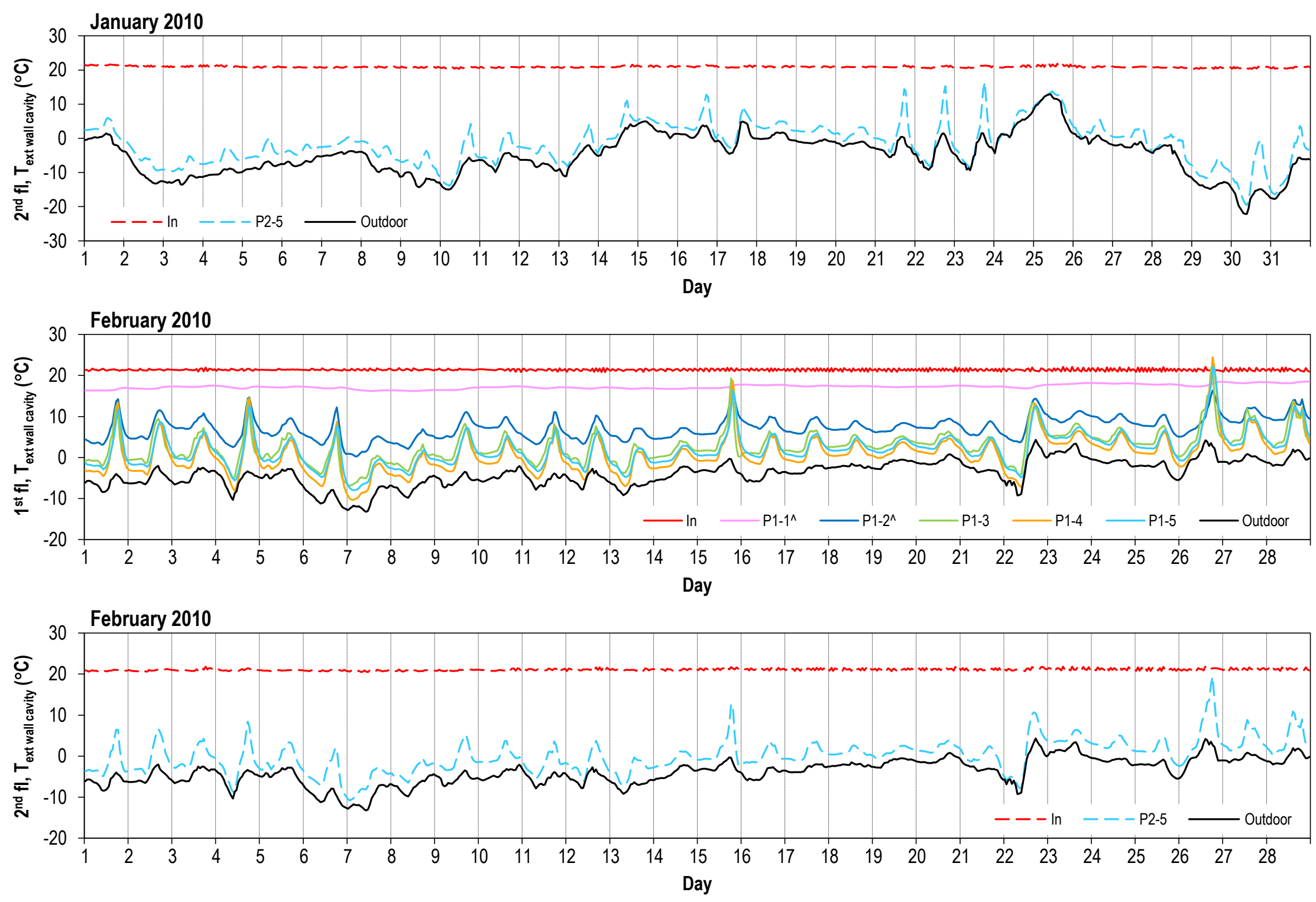

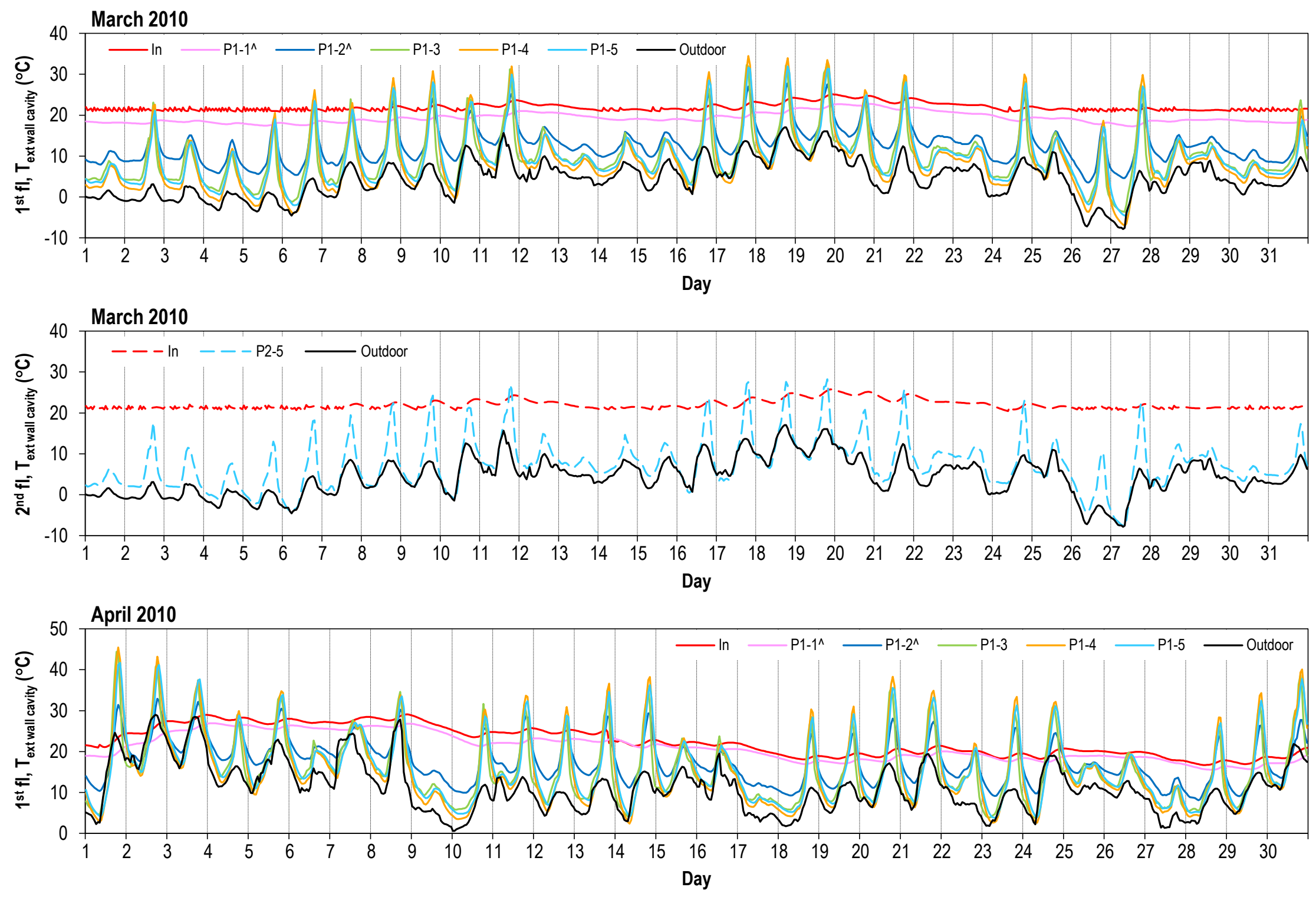

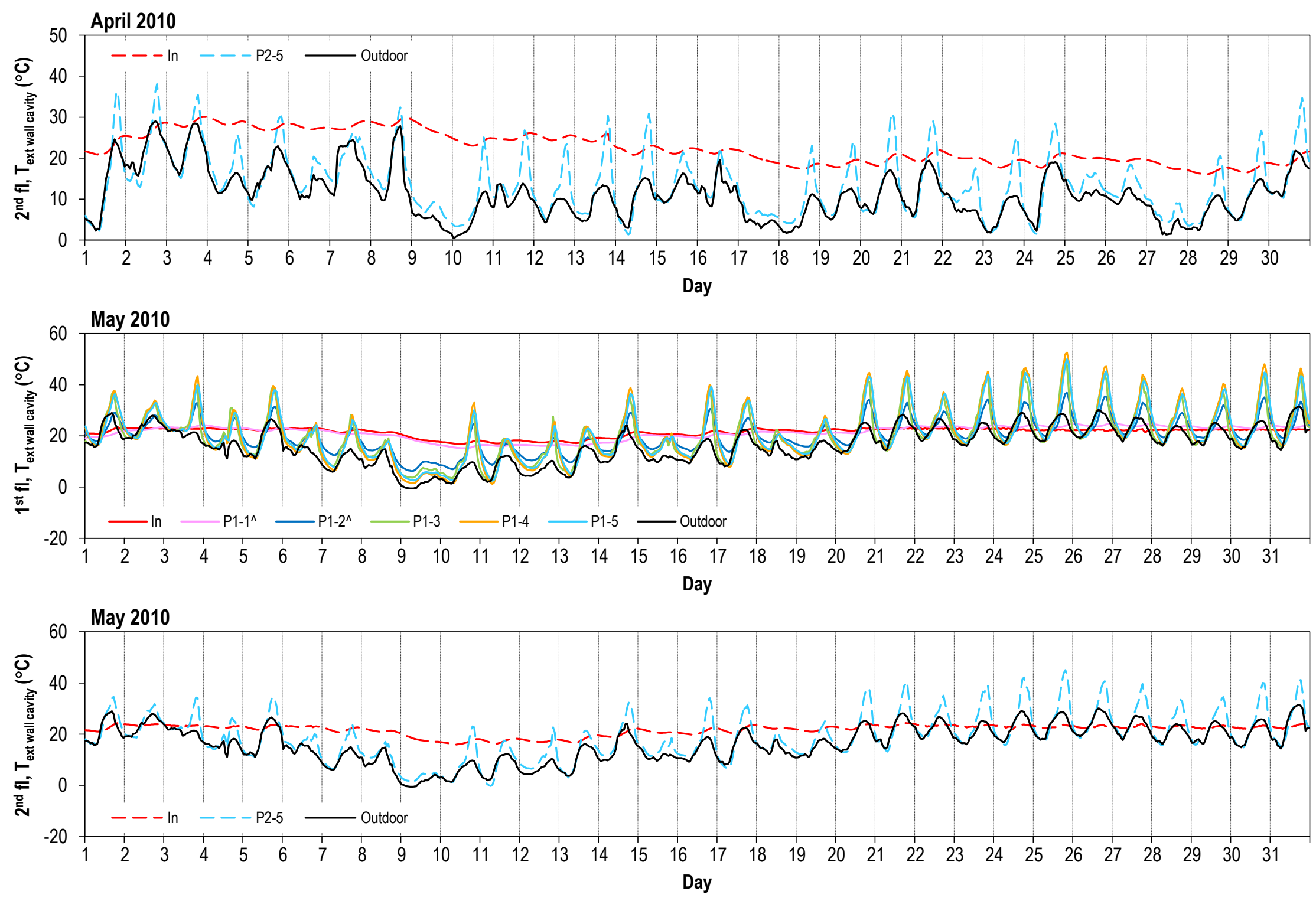

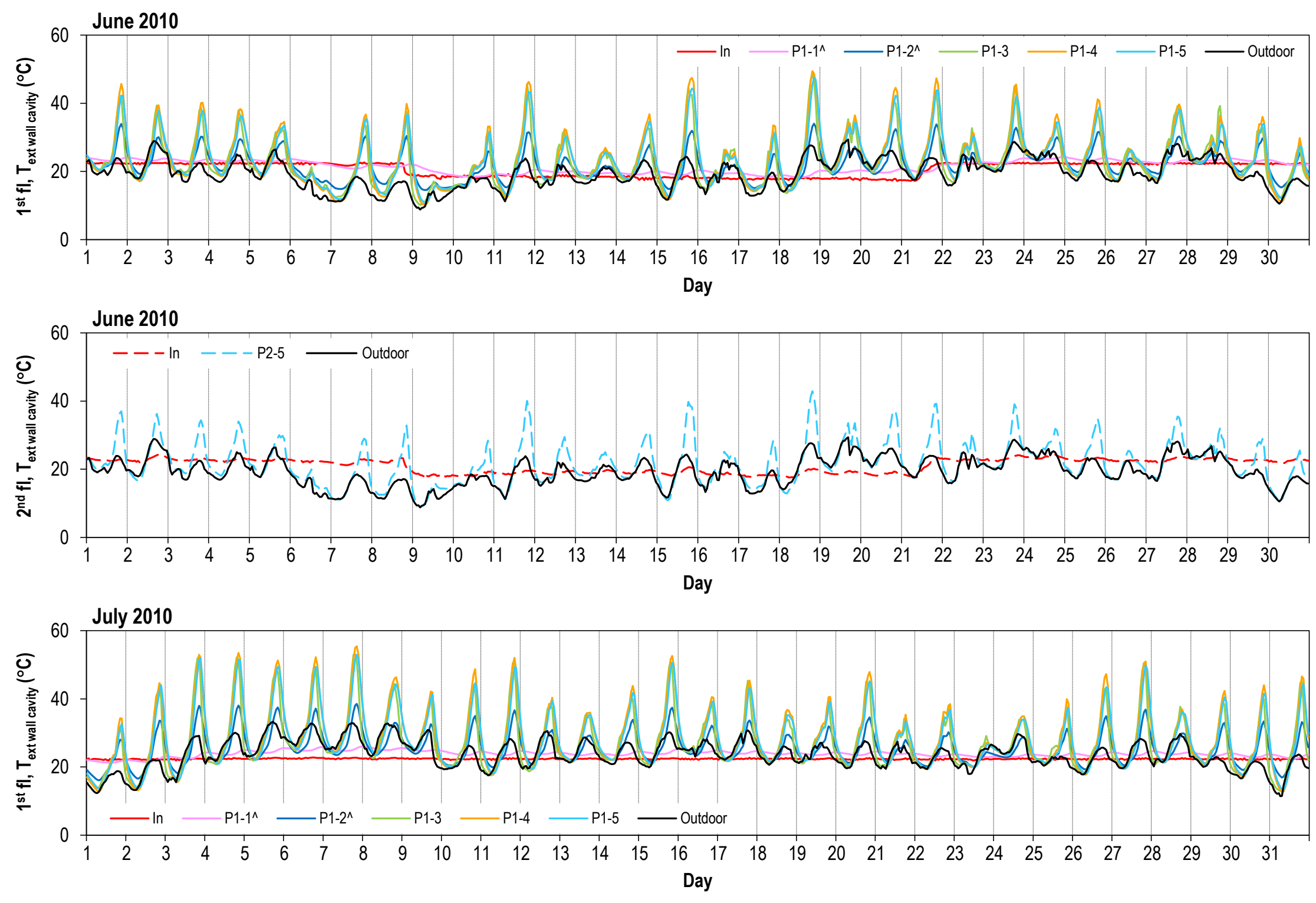

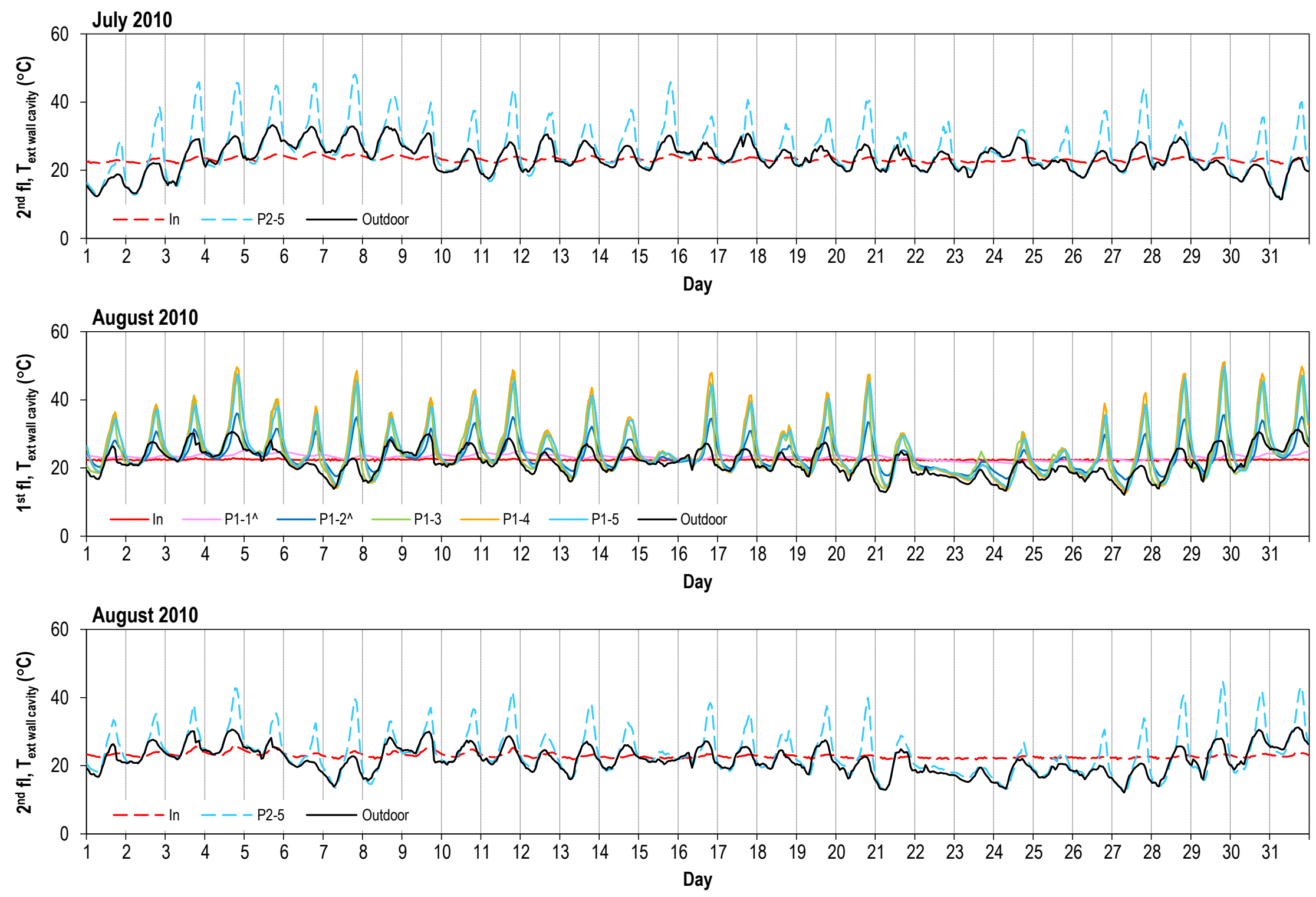
Temperature $\left({ }^{\circ} \mathrm{C}\right)$ at interior side of stud cavity. Refer to Figures 25 thru 28 for sensor location.
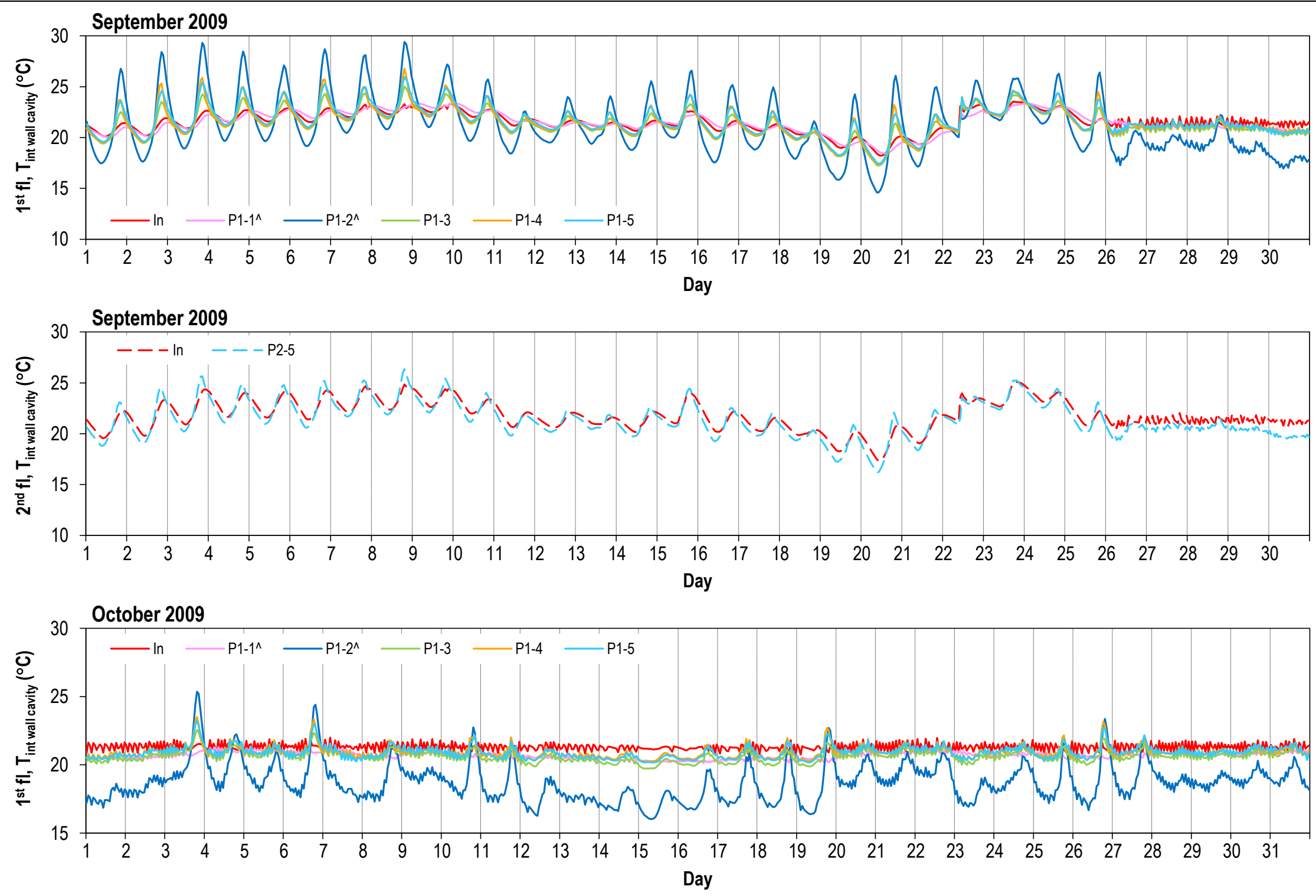

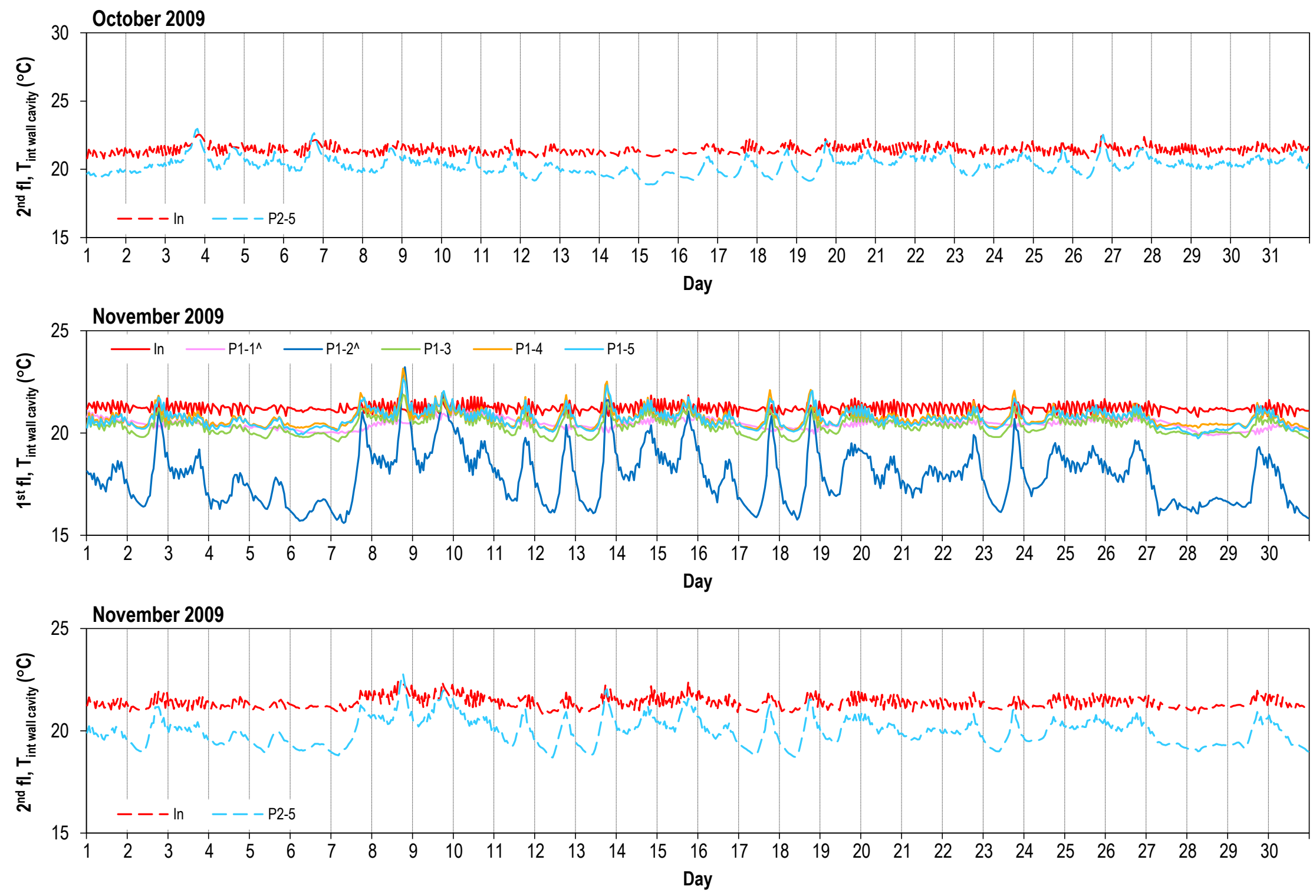

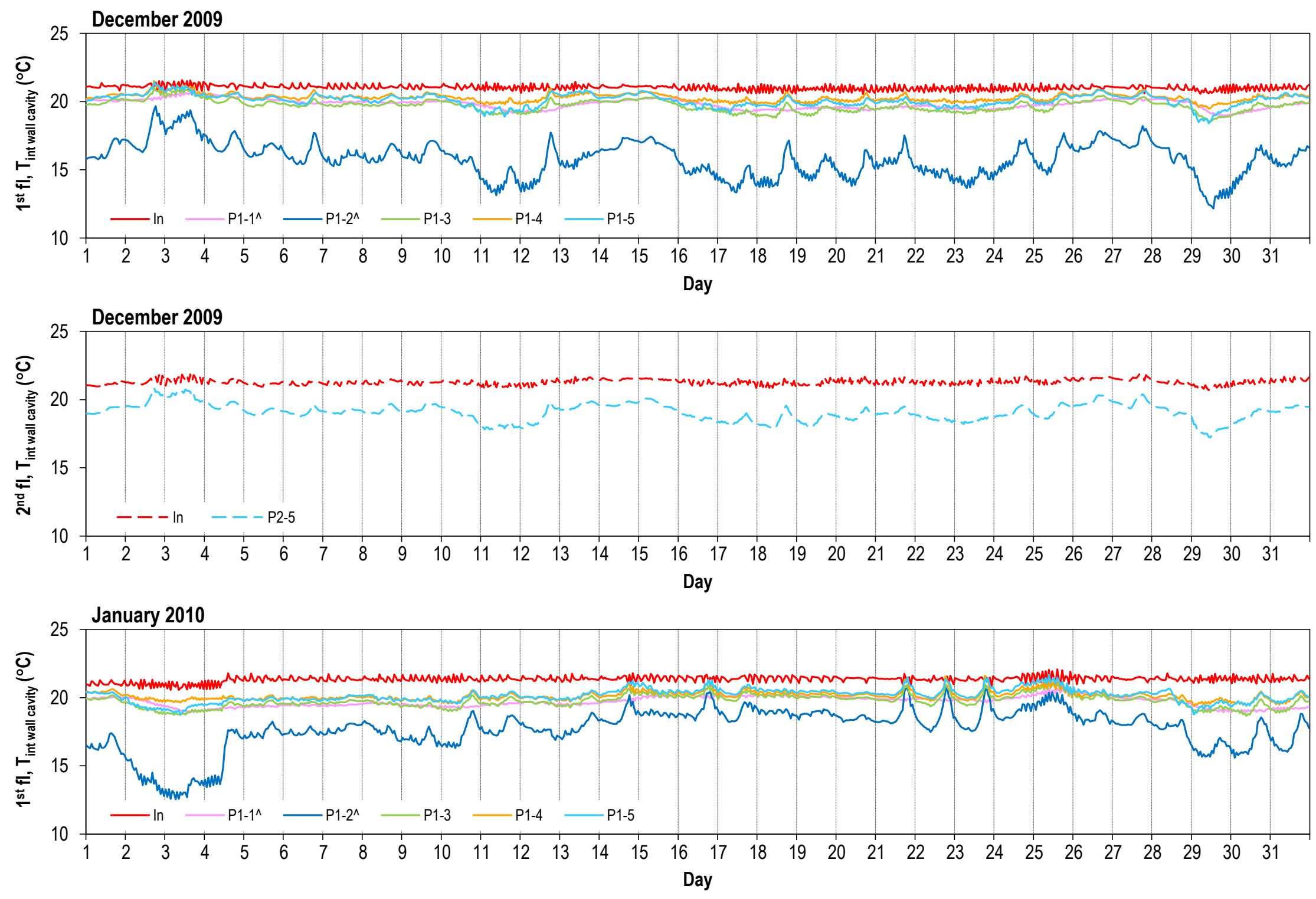

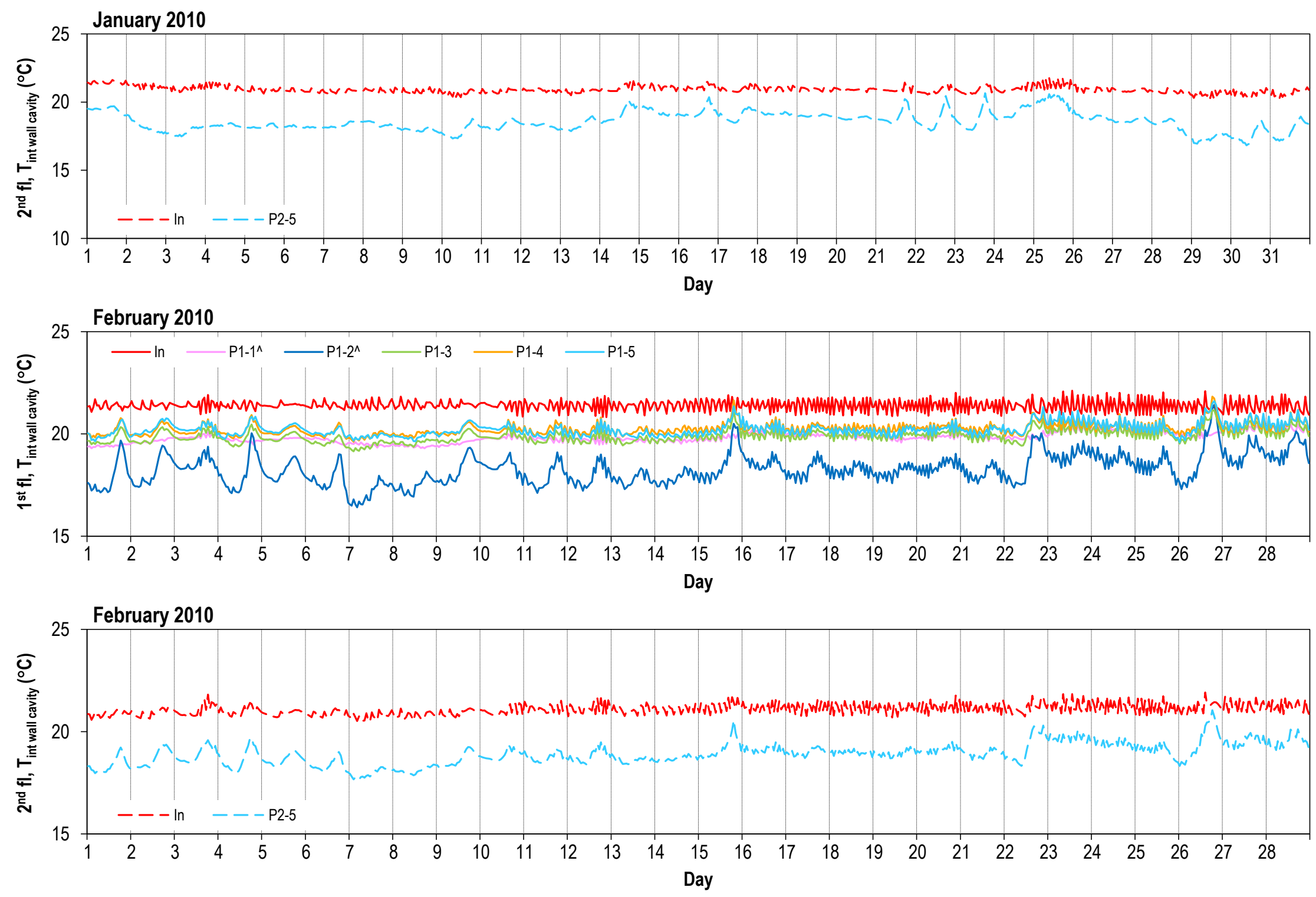

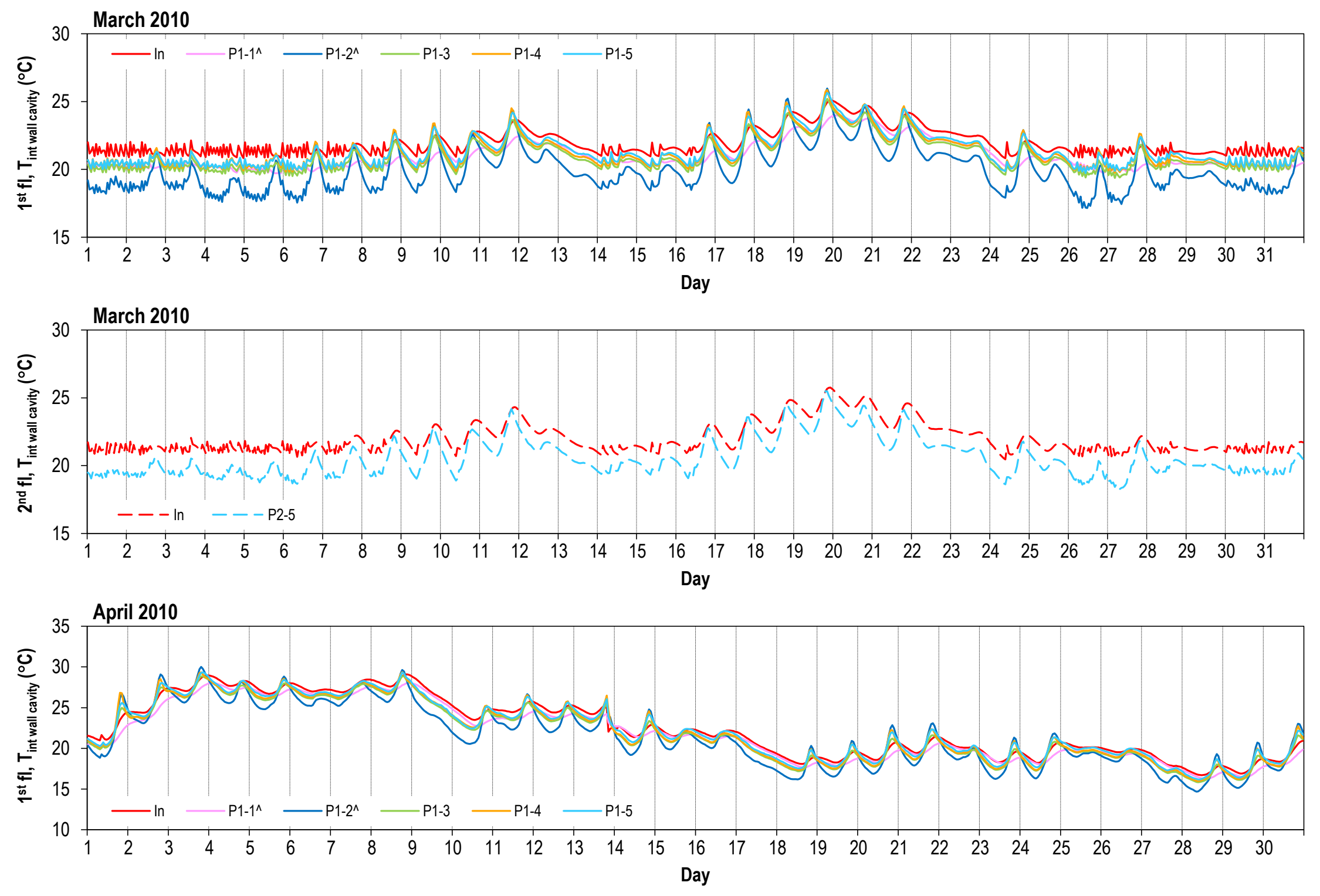

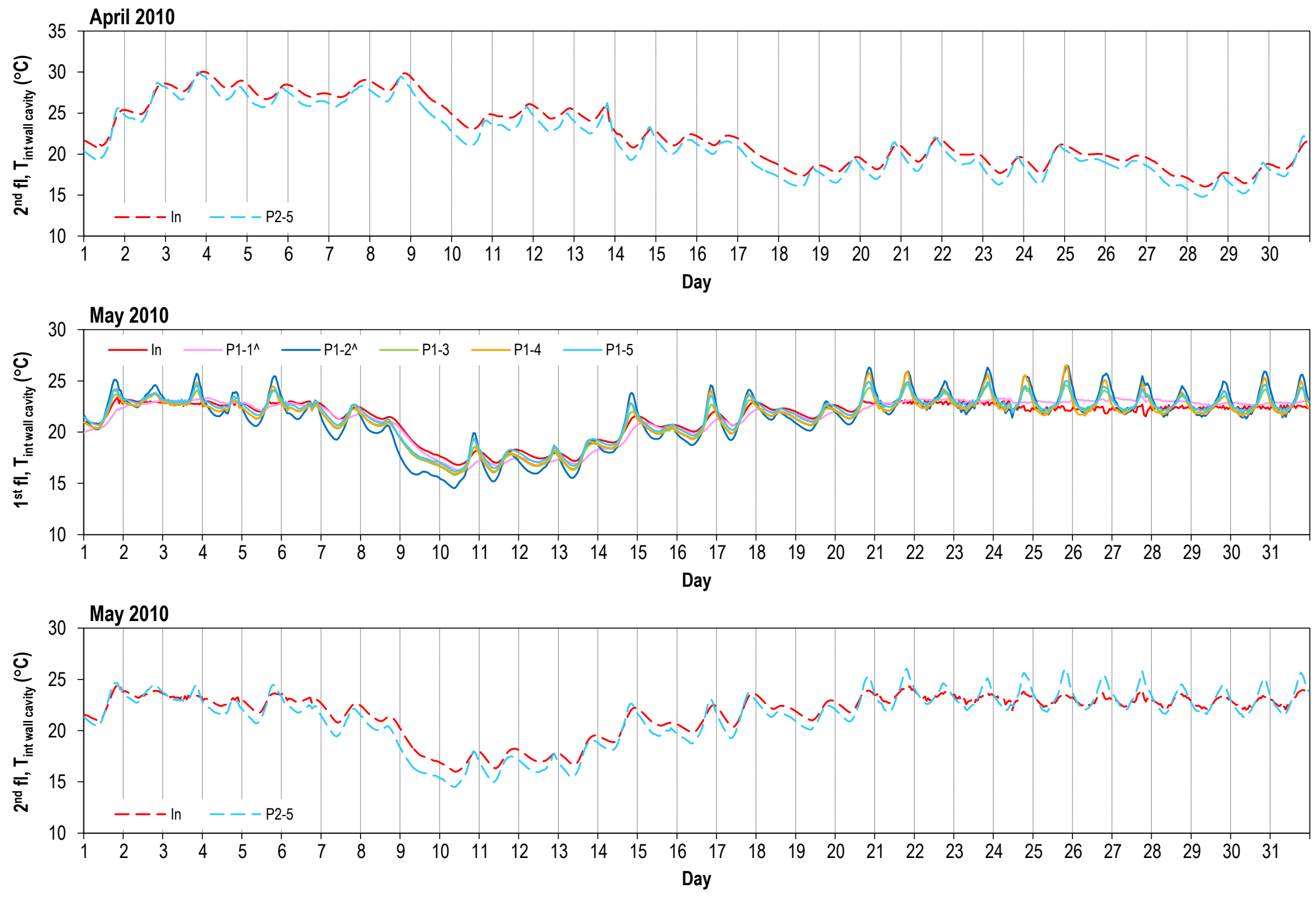

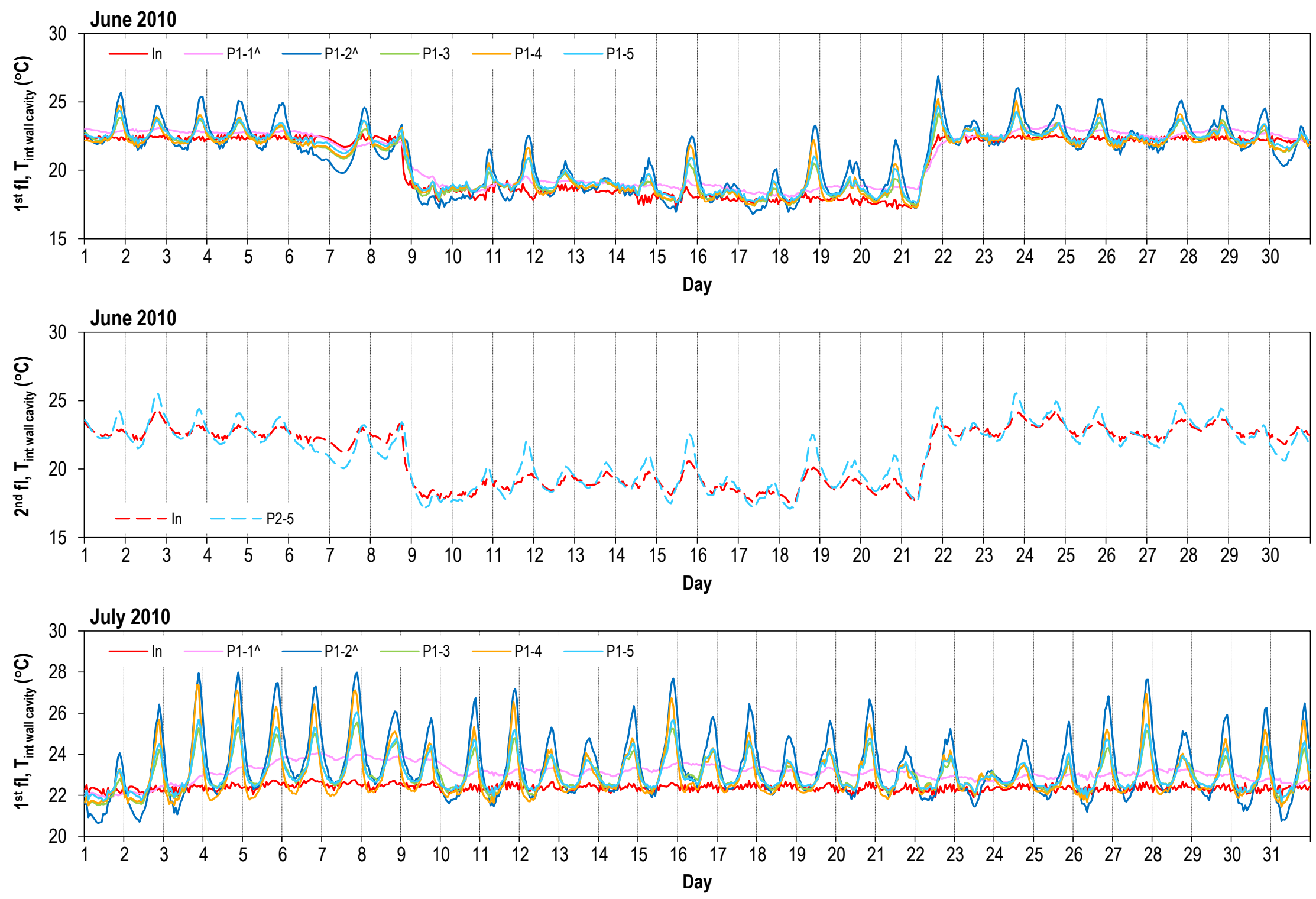

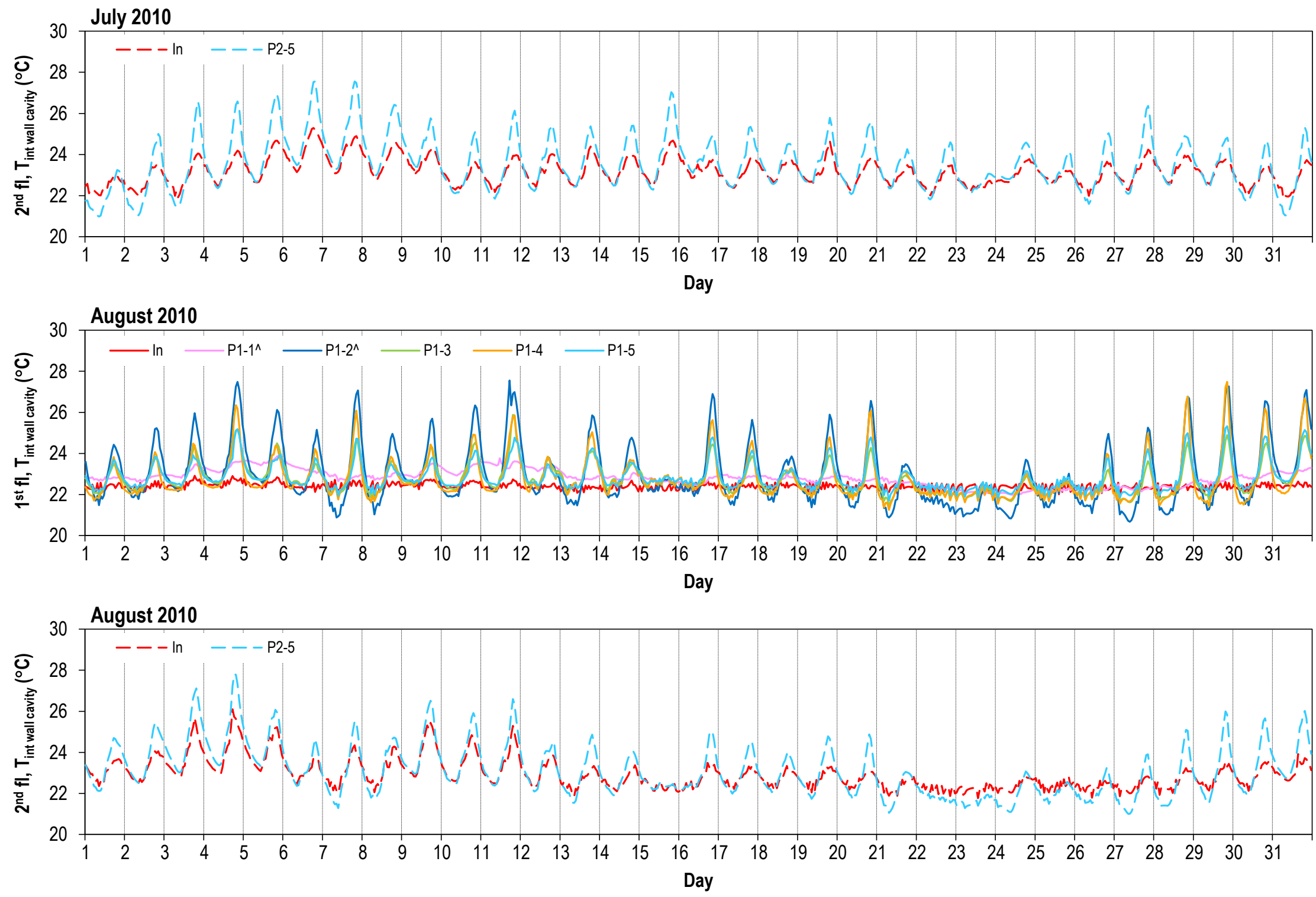

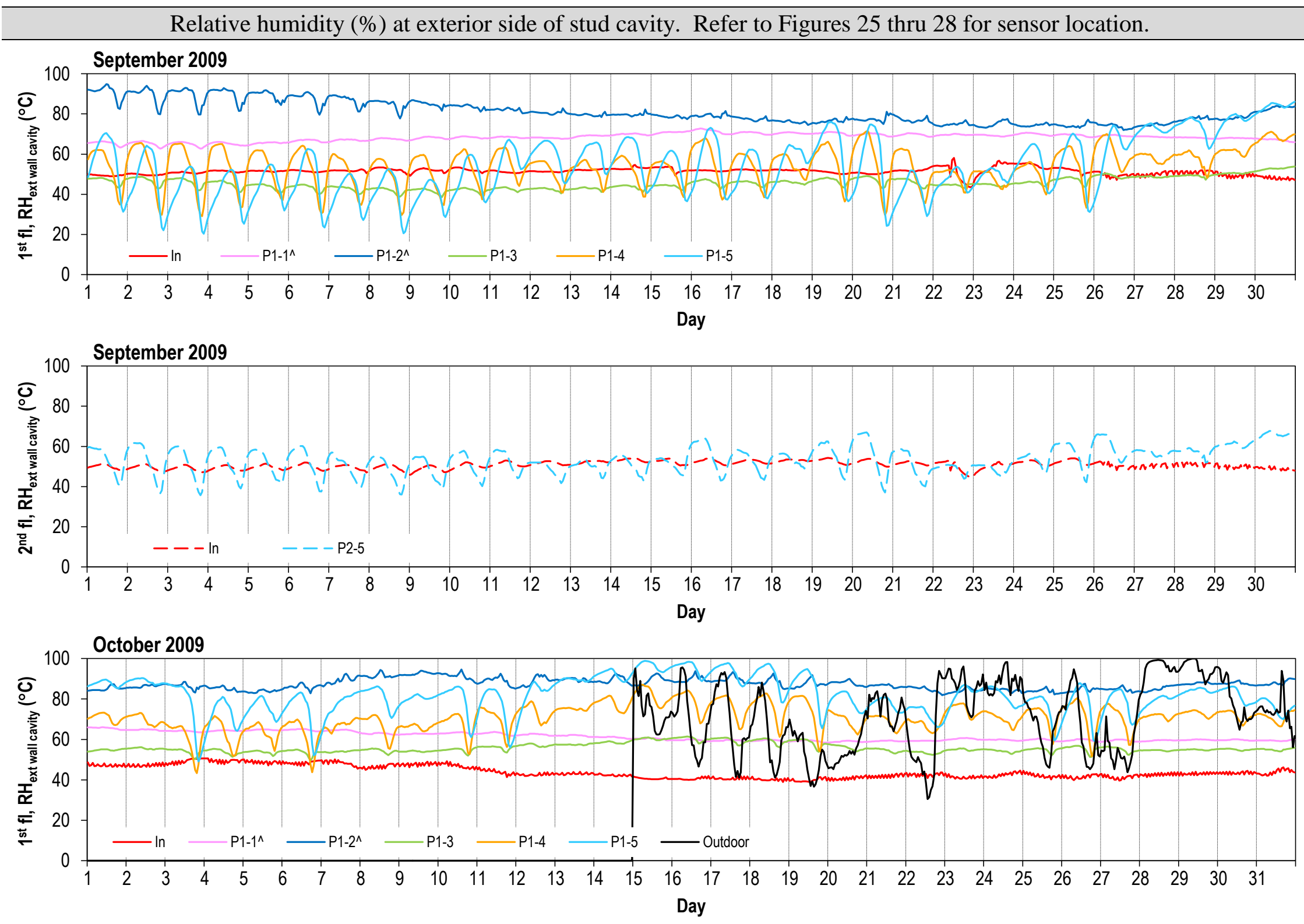

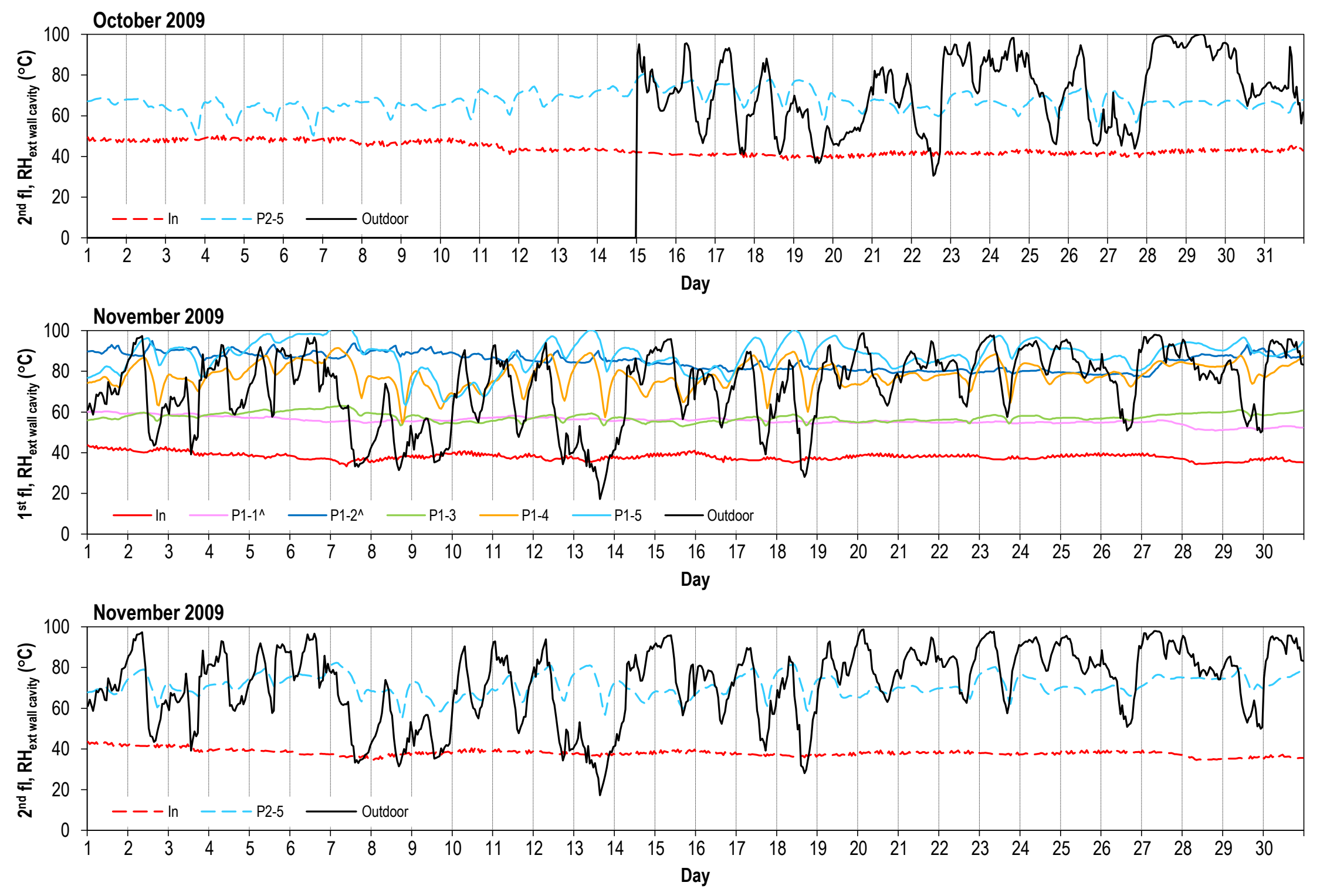

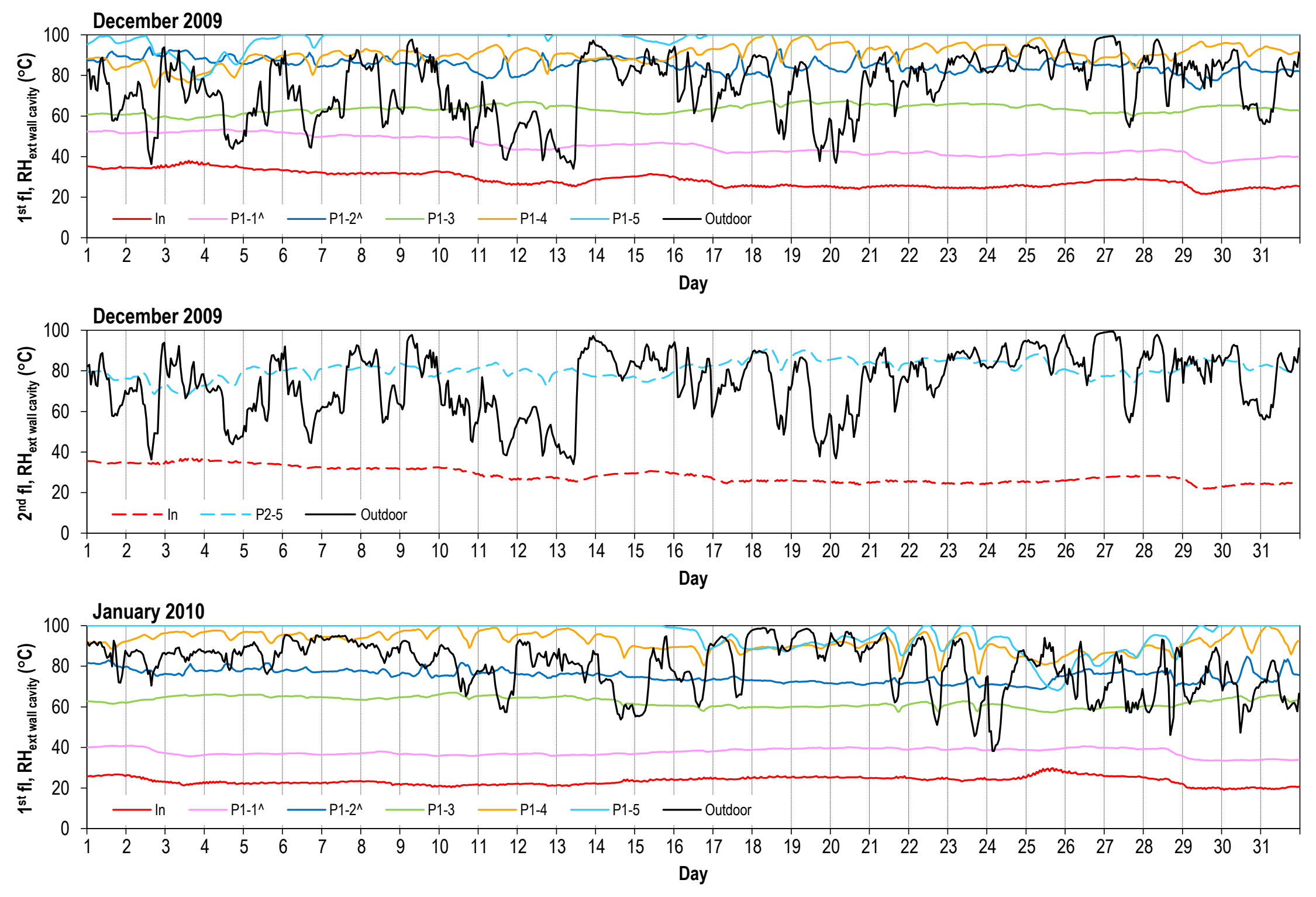

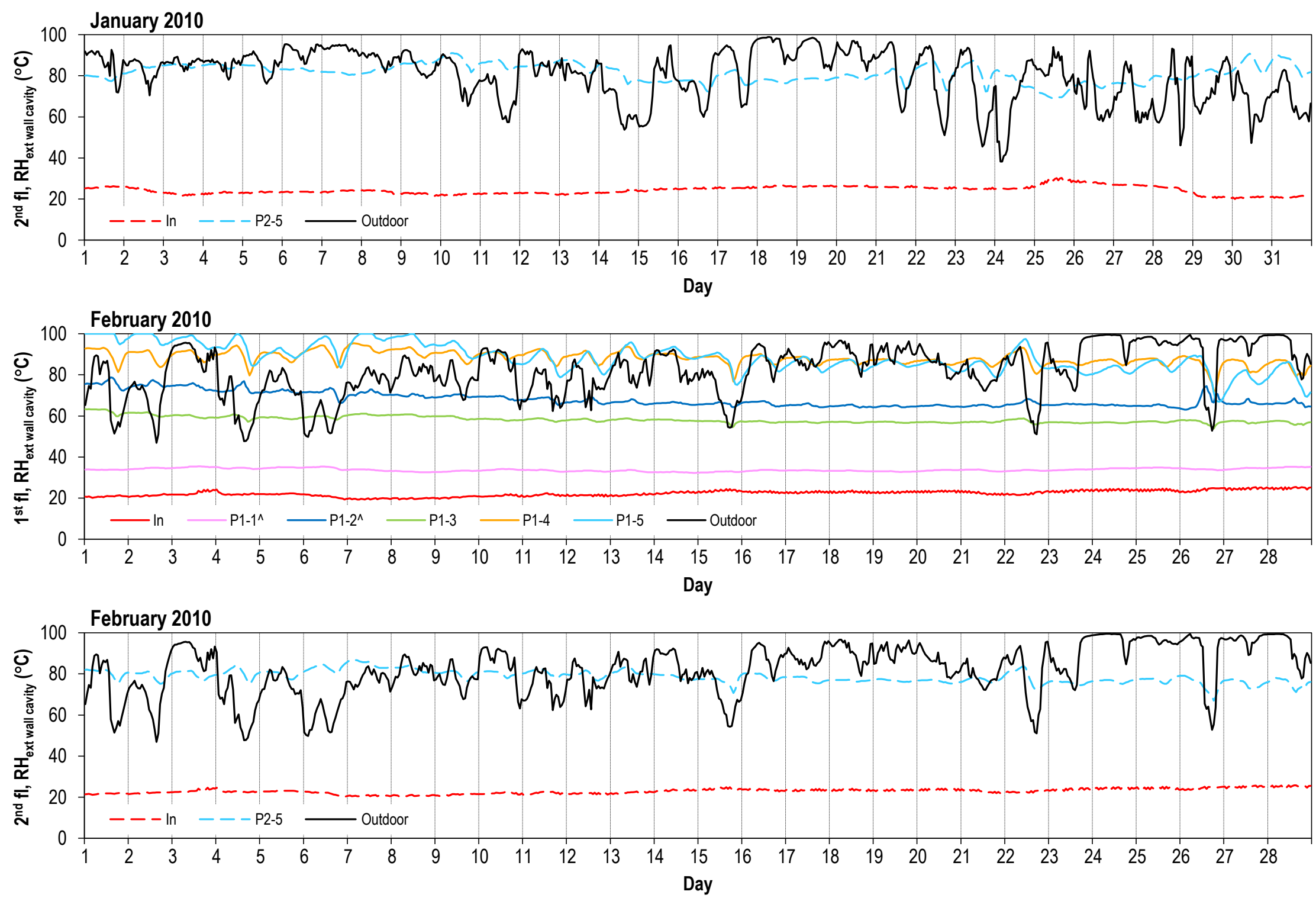
207

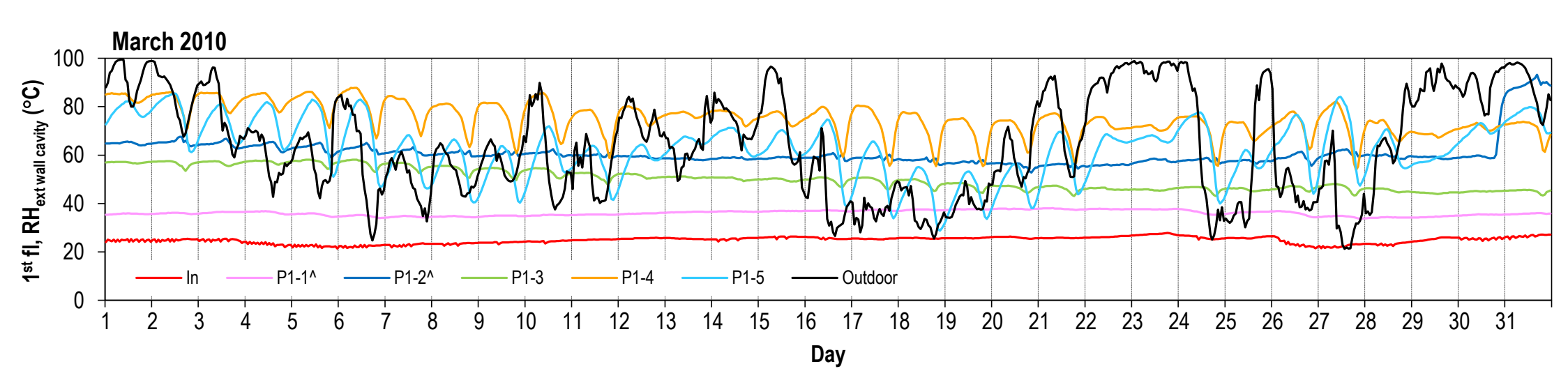

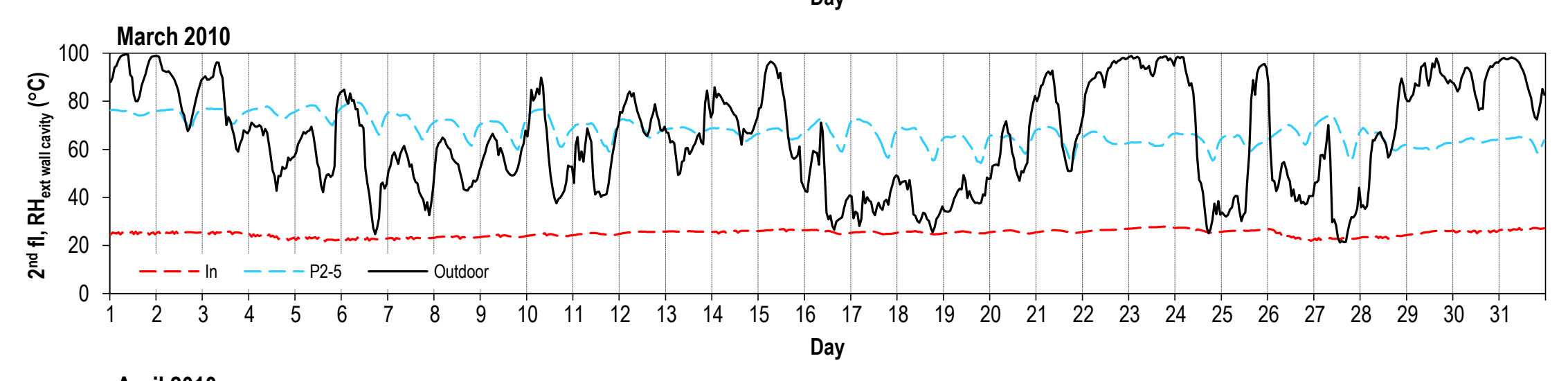

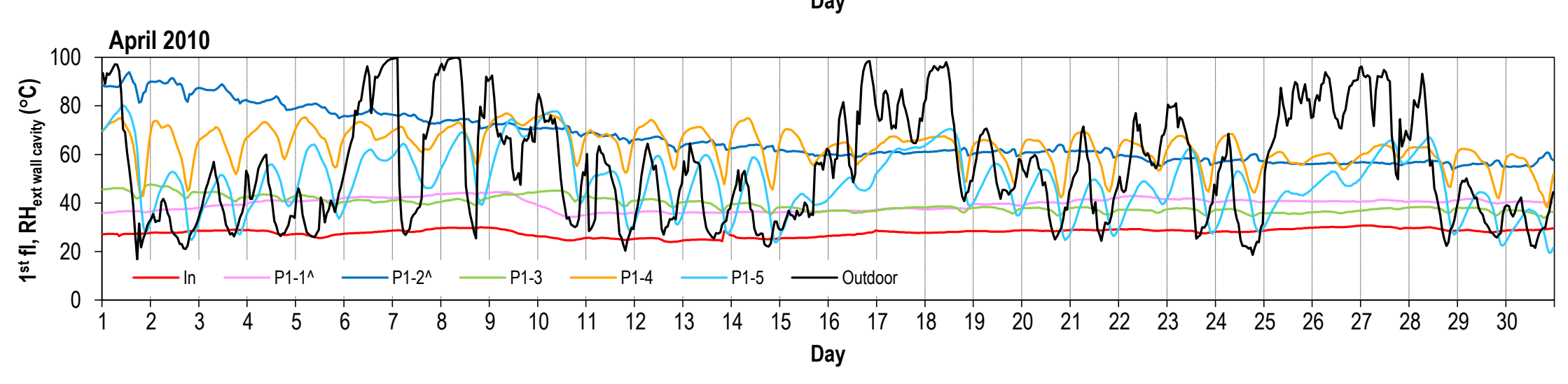



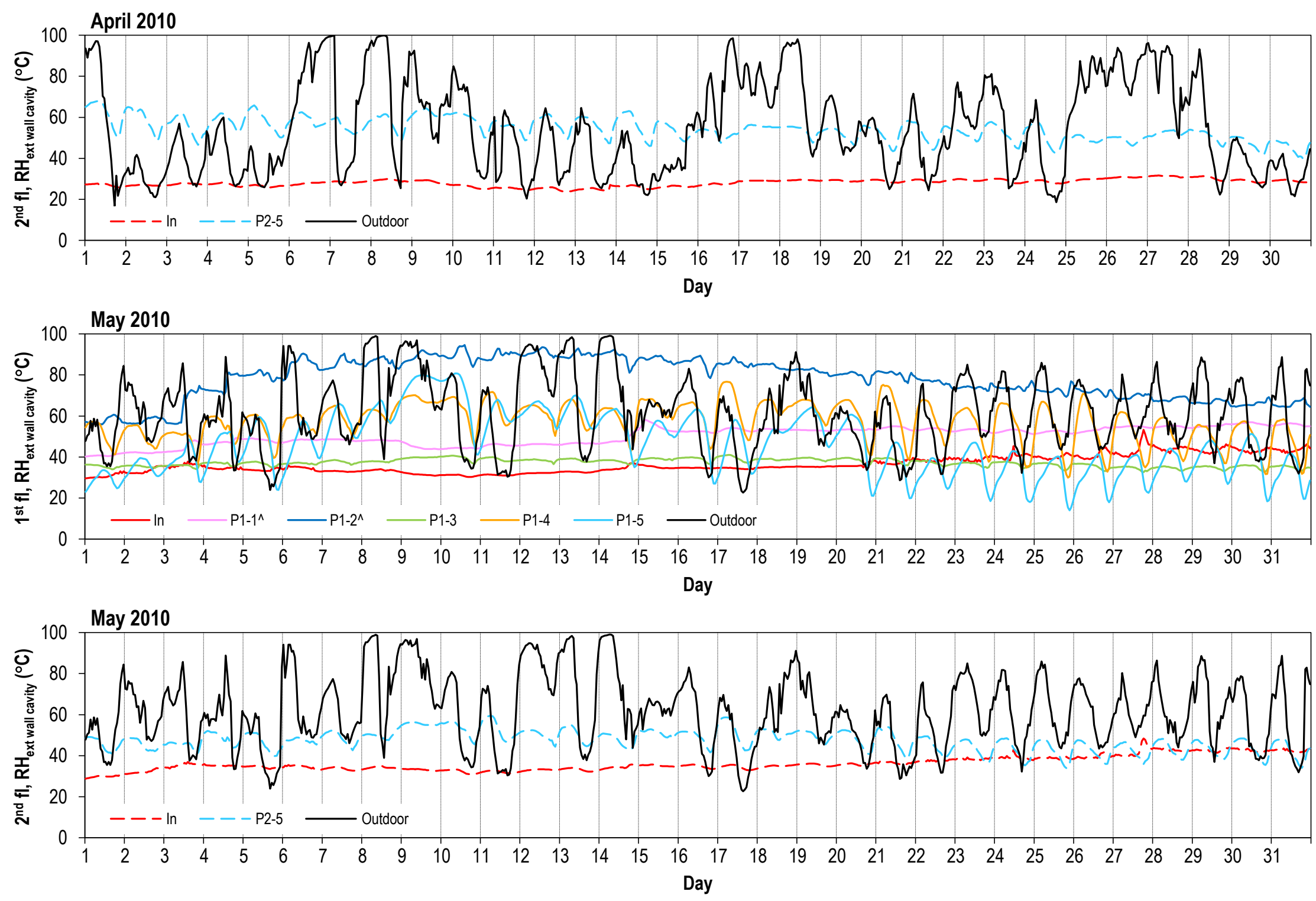

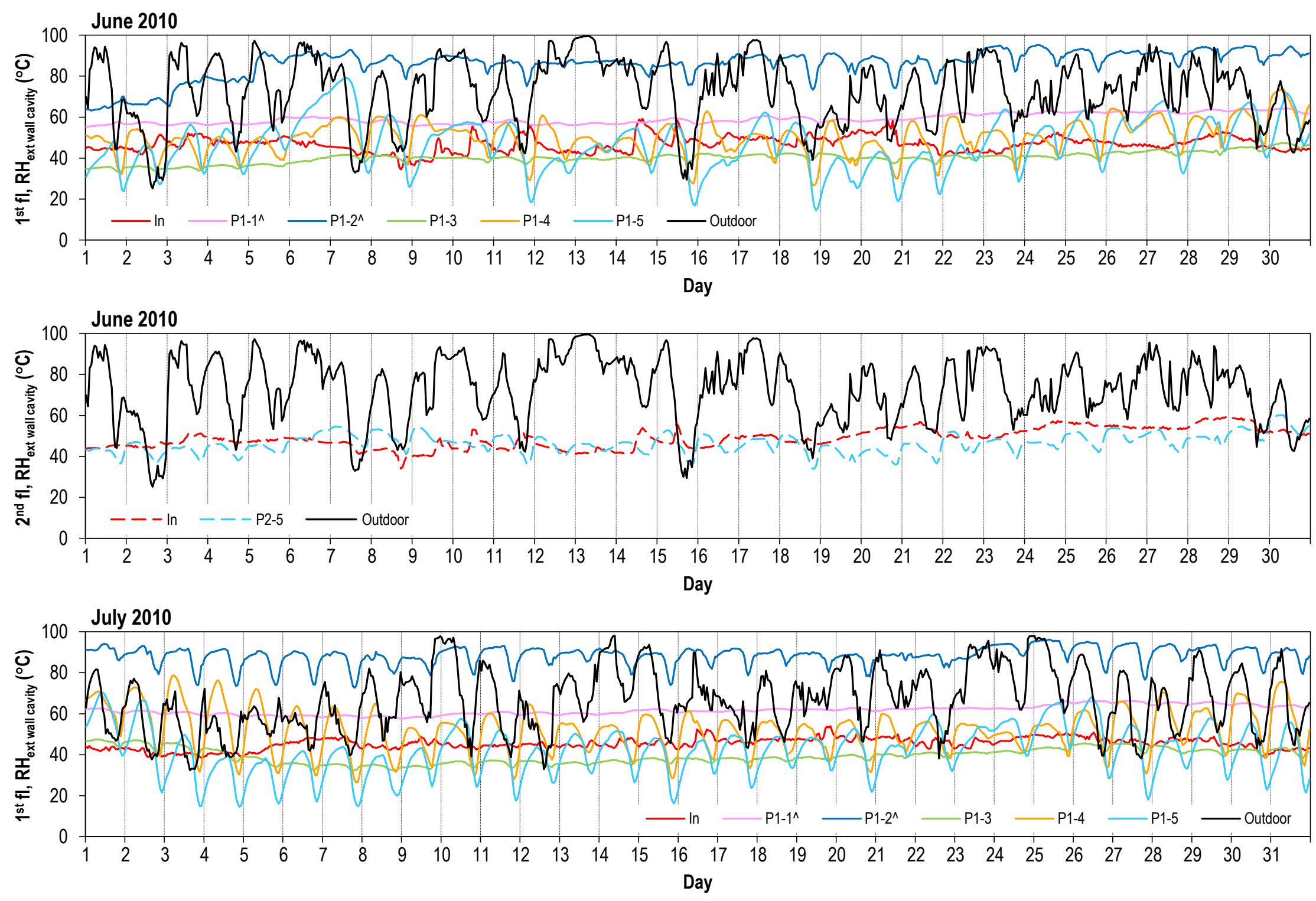

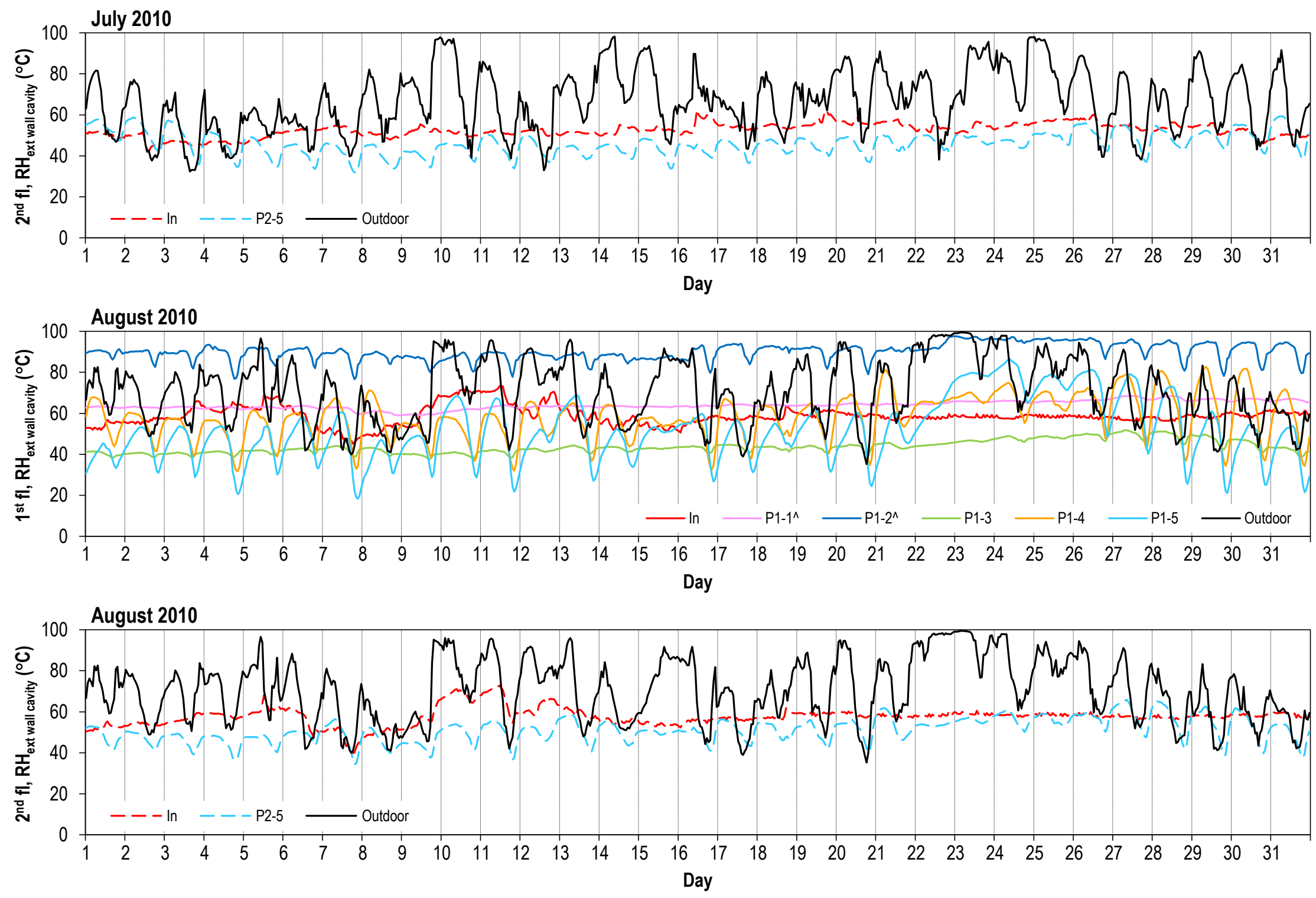


\section{Water vapor pressure ( $\mathrm{kPa}$ ) at exterior side of stud cavity. Refer to Figures 25 thru 28 for sensor location.}
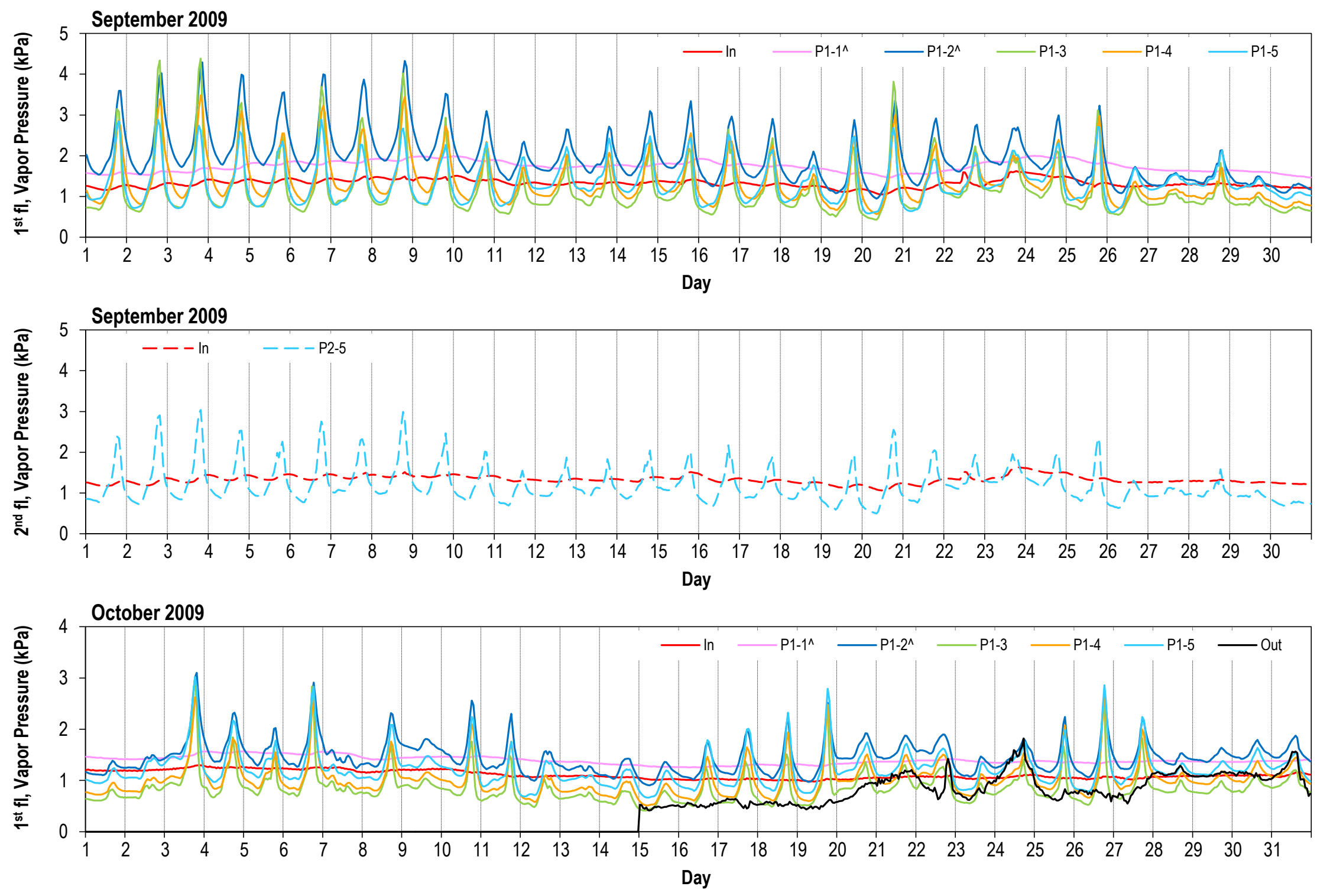

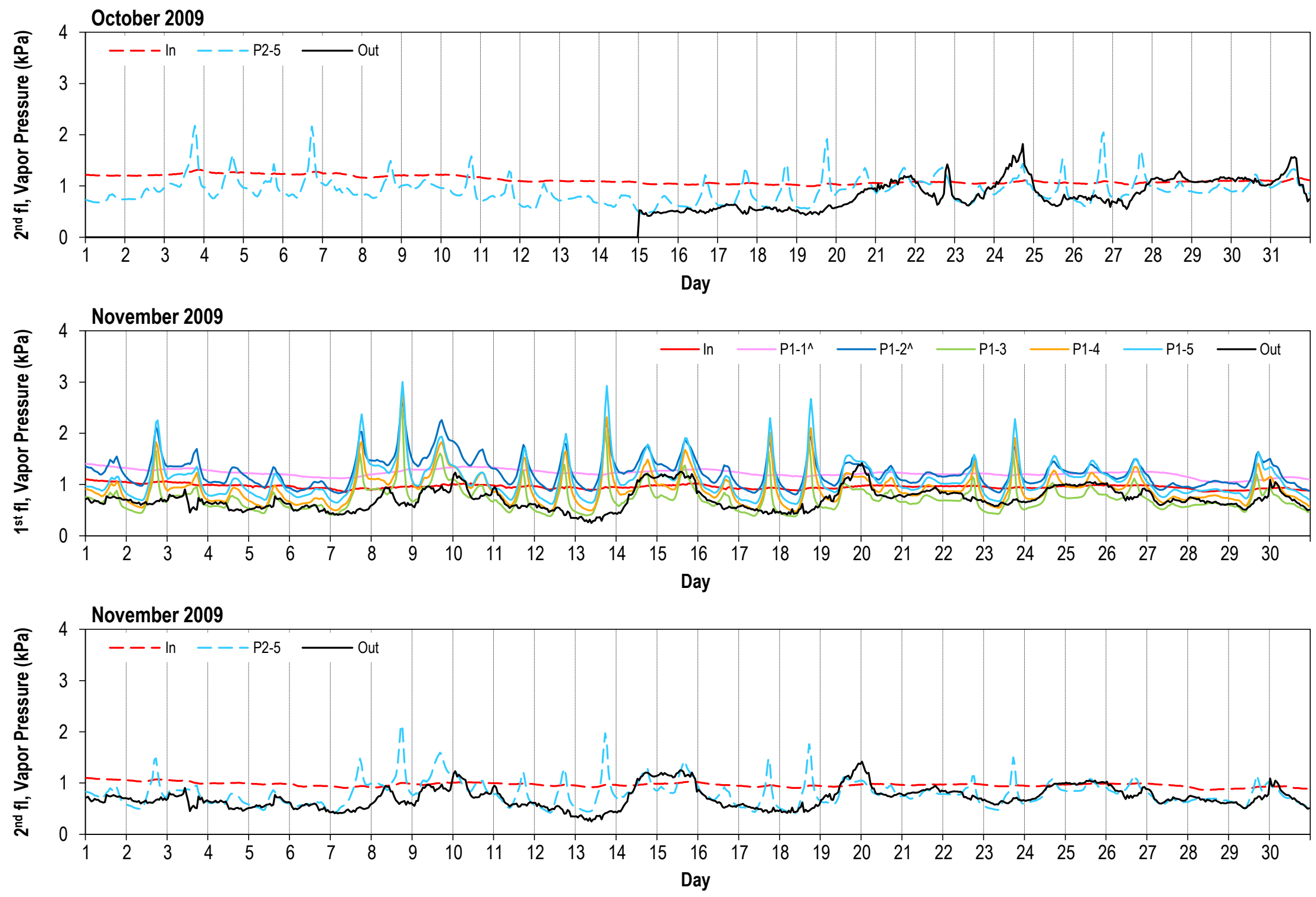

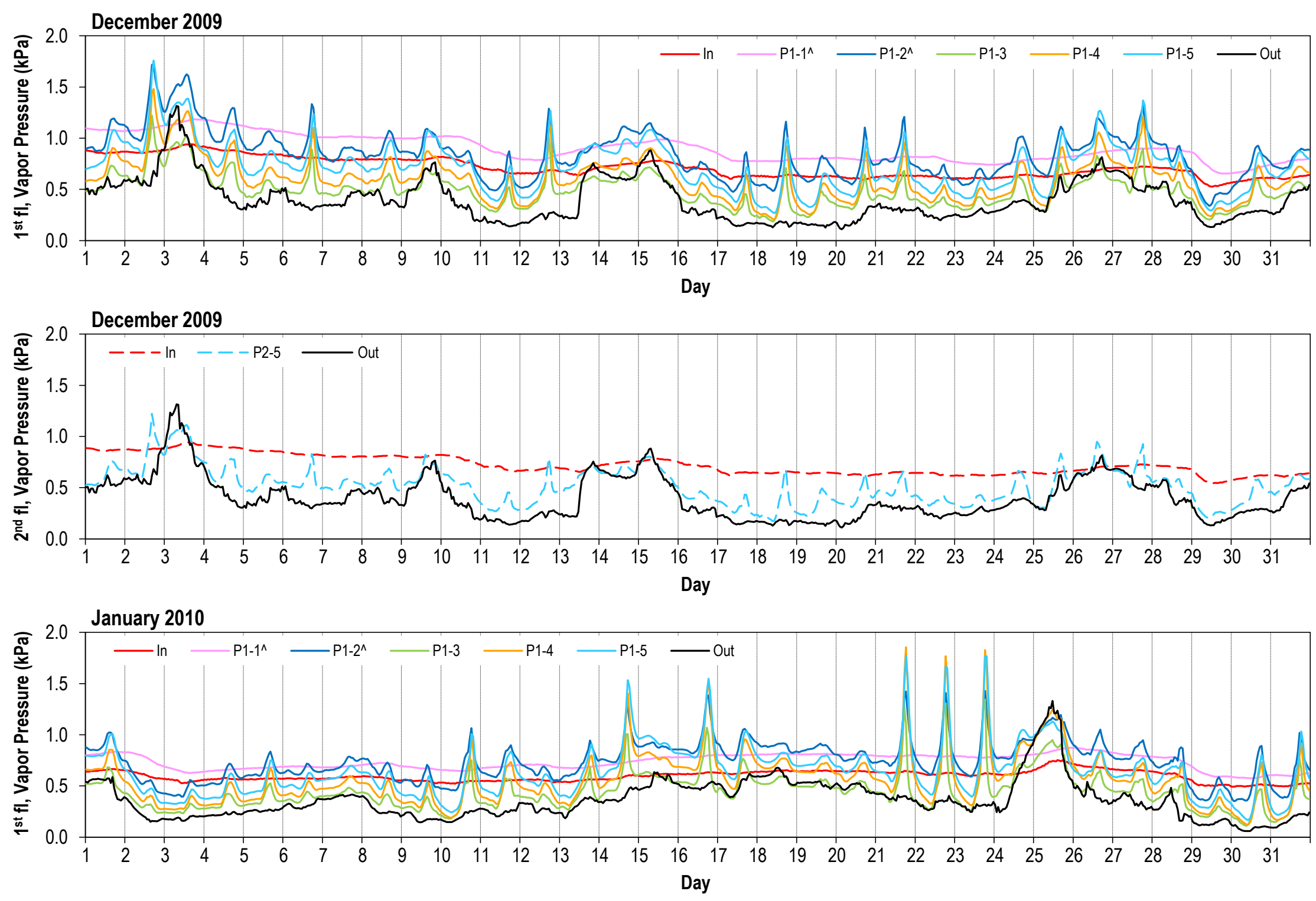

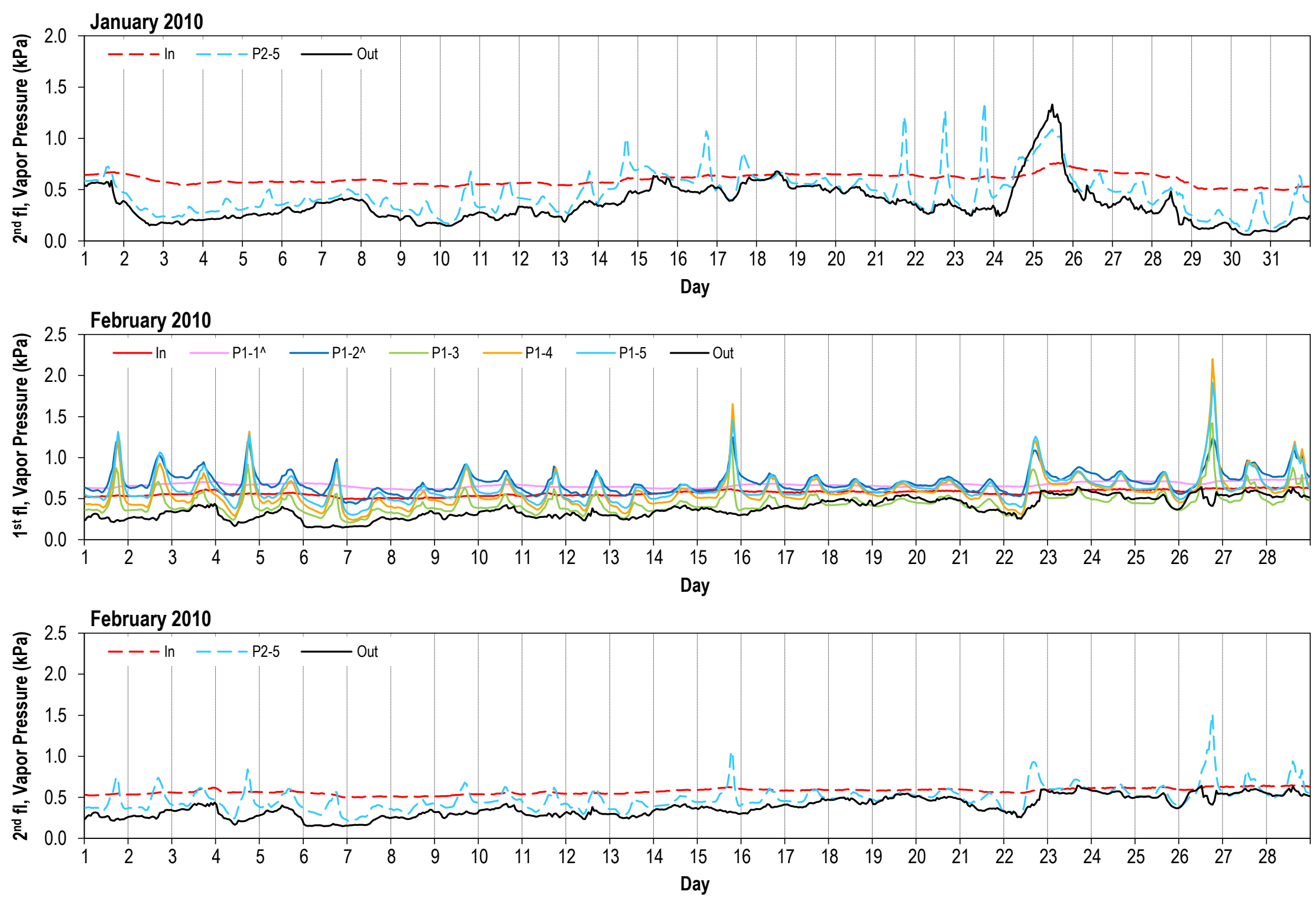

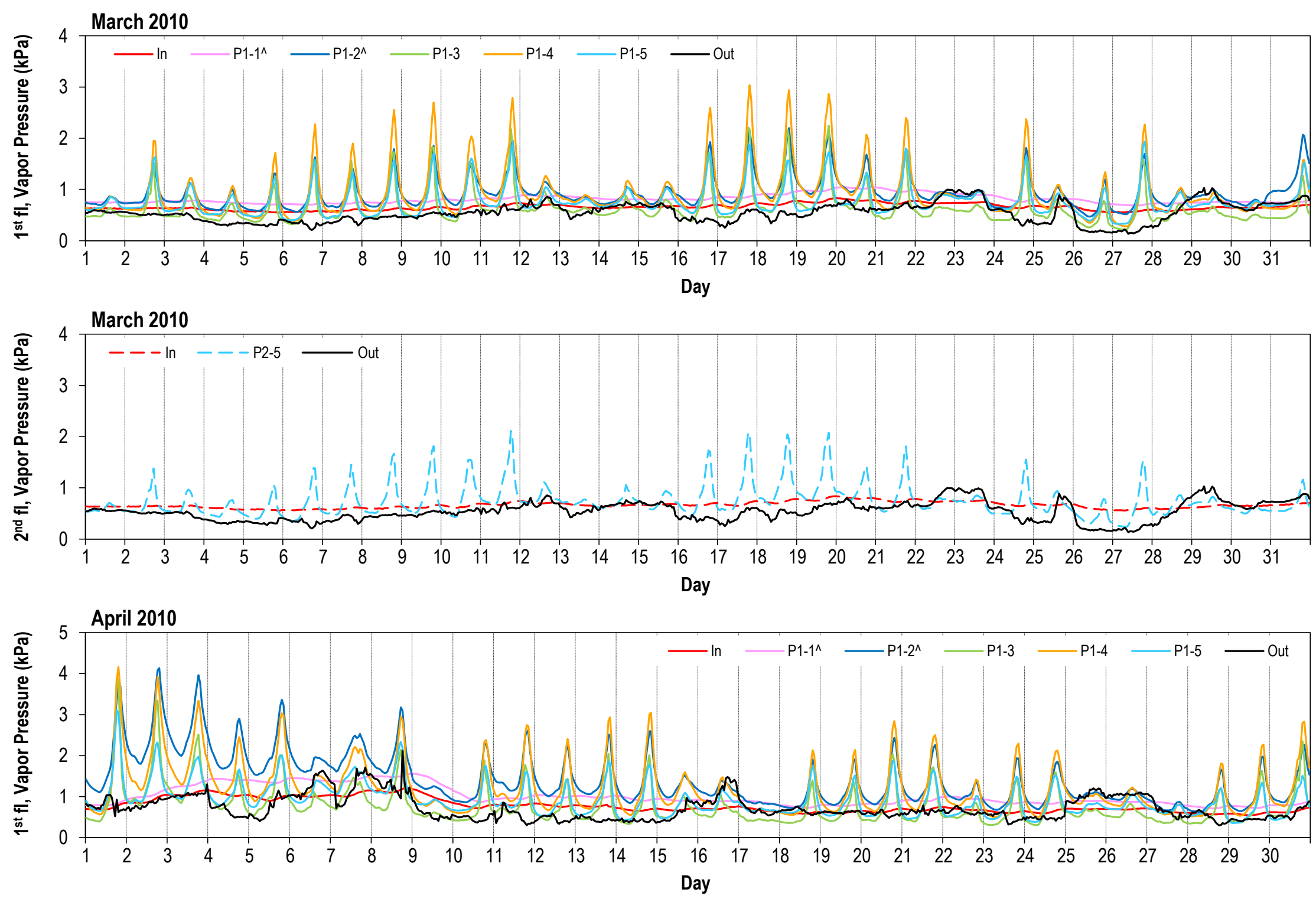

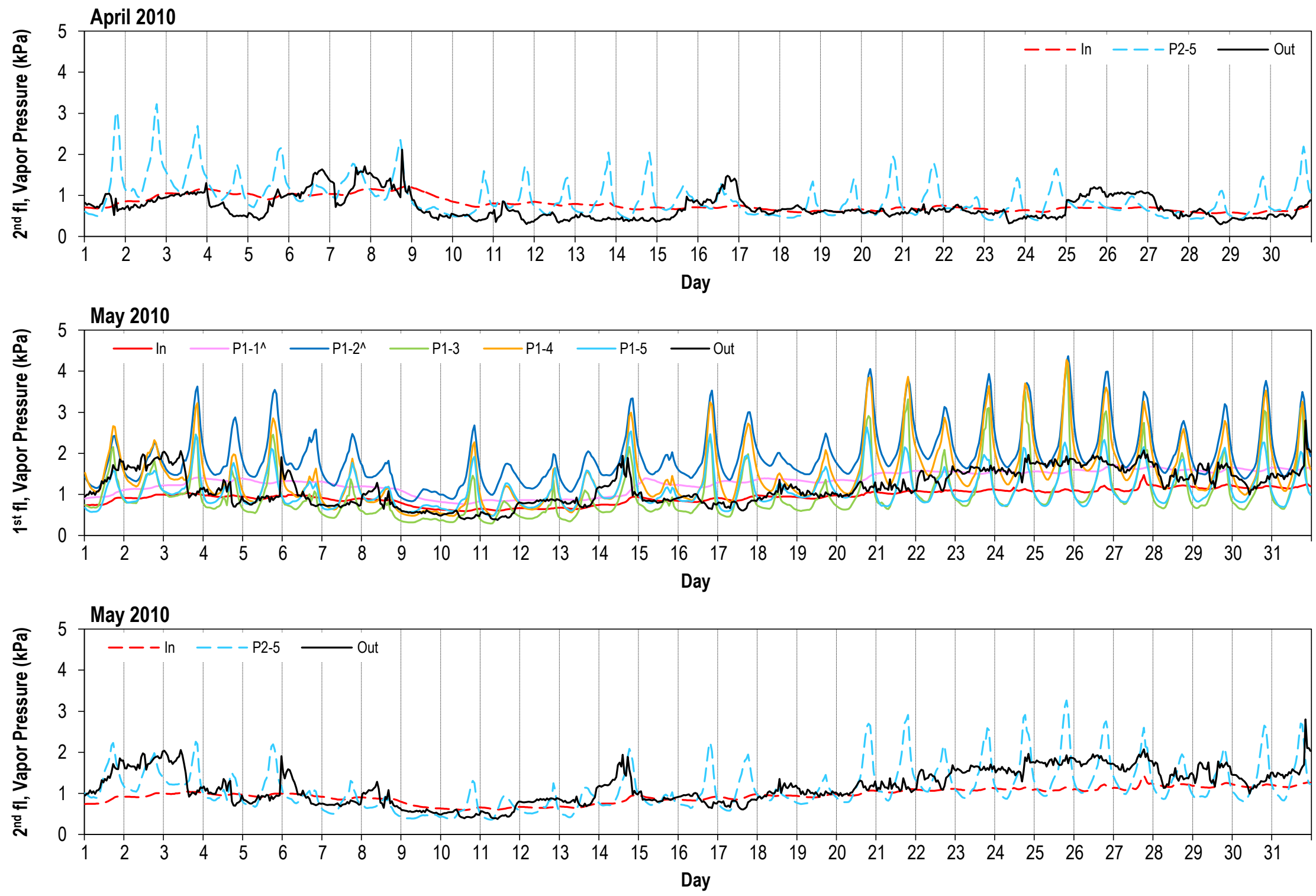

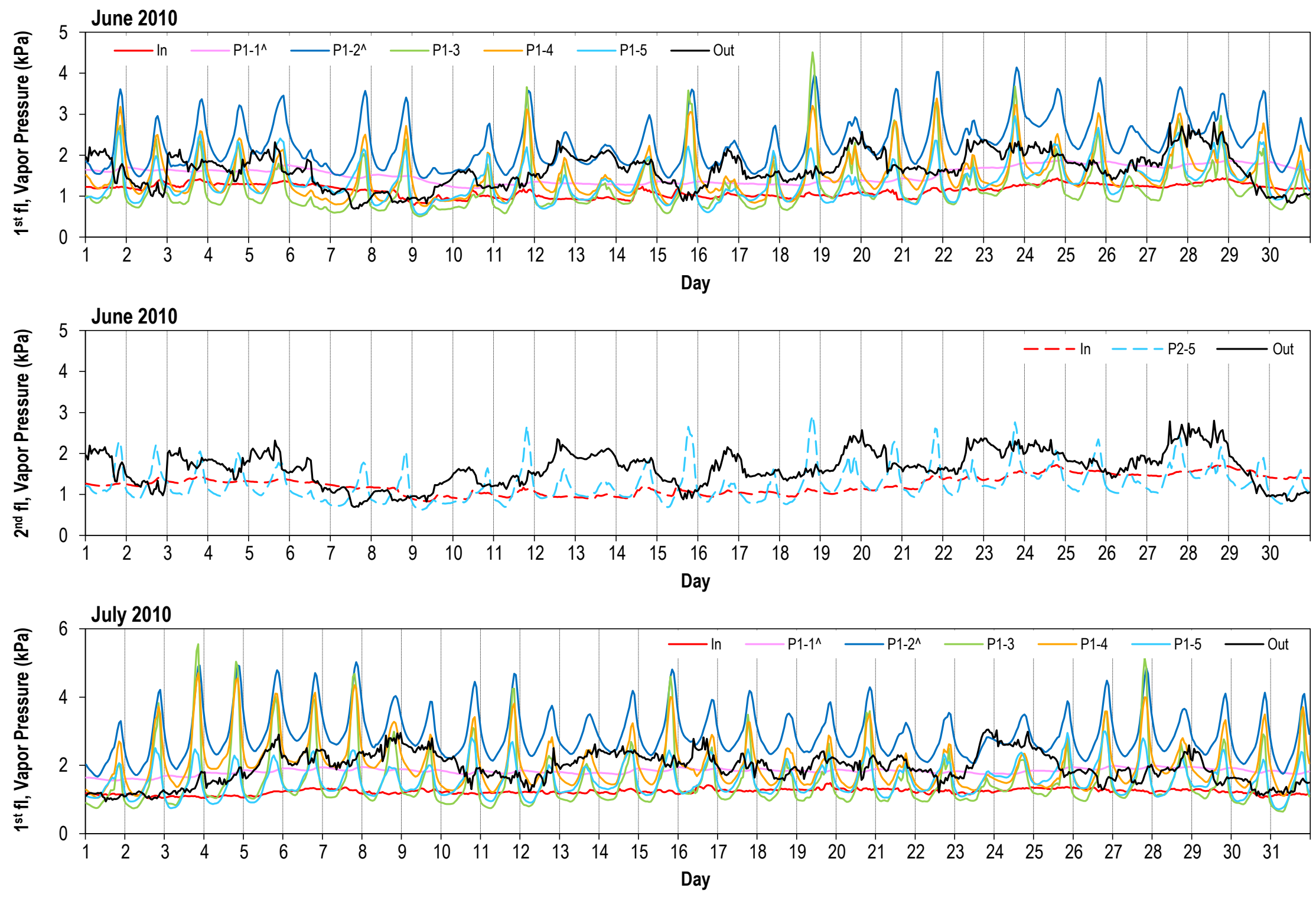

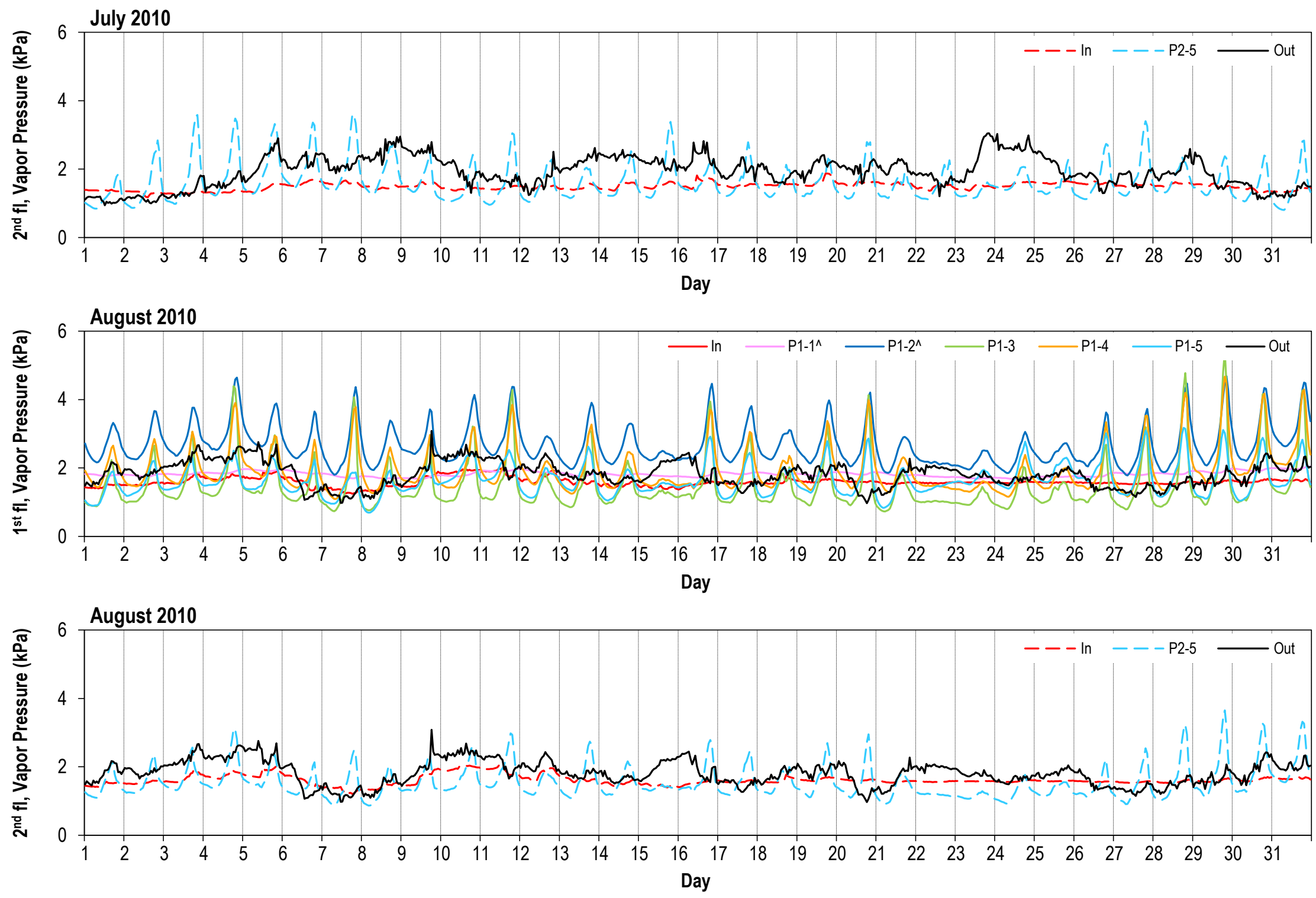
Heat flux $\left(\mathrm{W} / \mathrm{m}^{2}\right)$ thru interior side of stud cavity. Refer to Figures 25 thru 28 for sensor location.
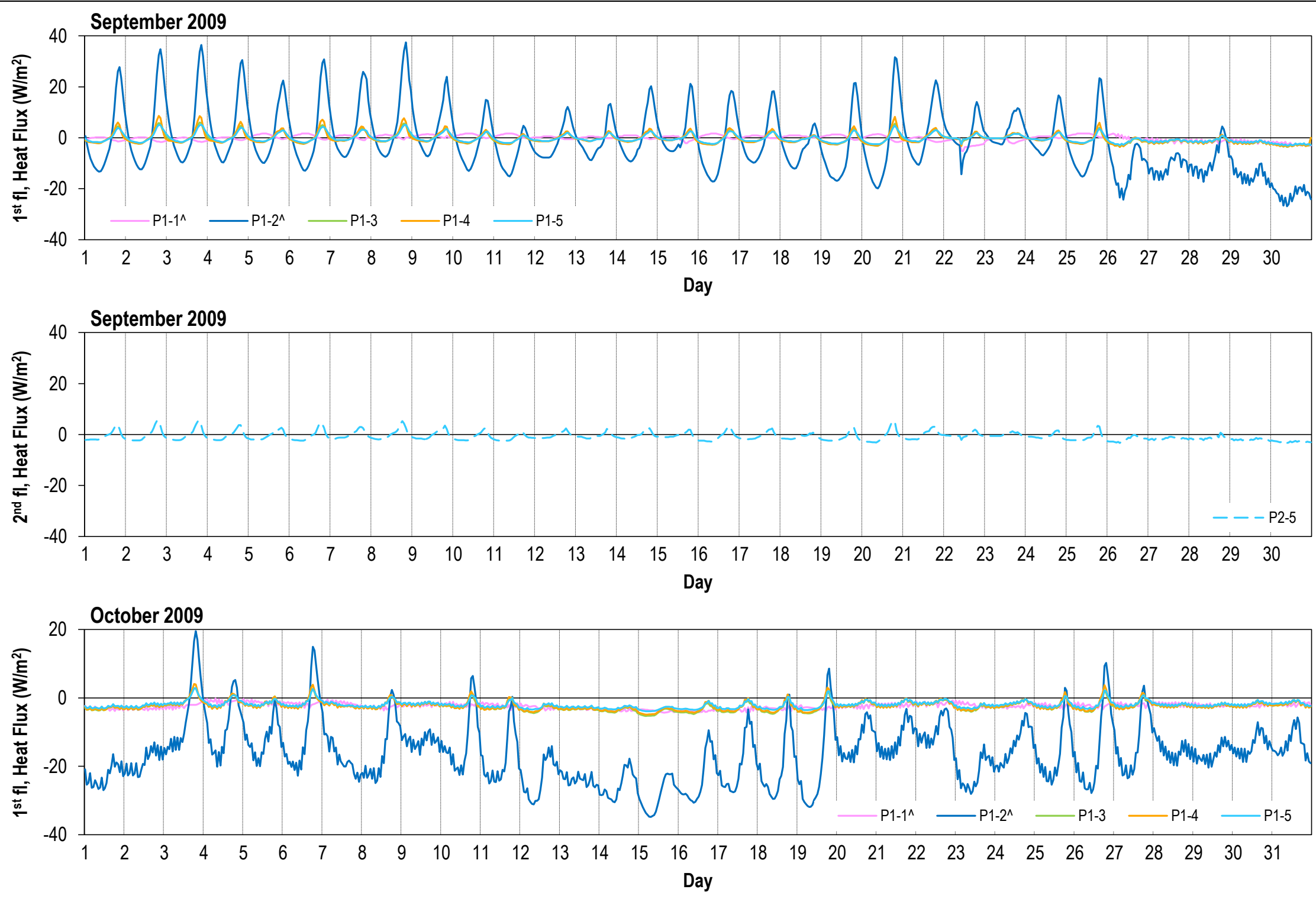

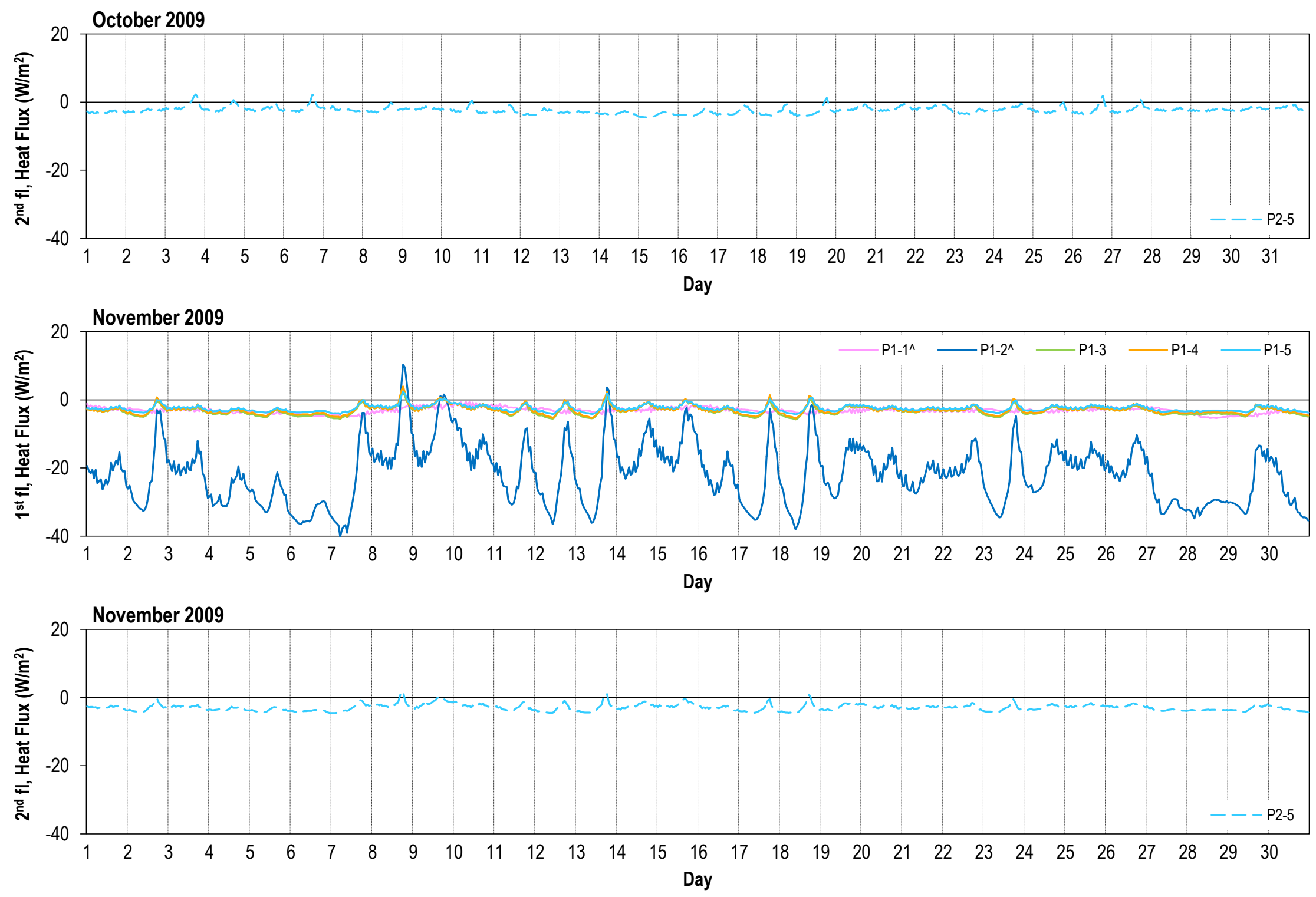

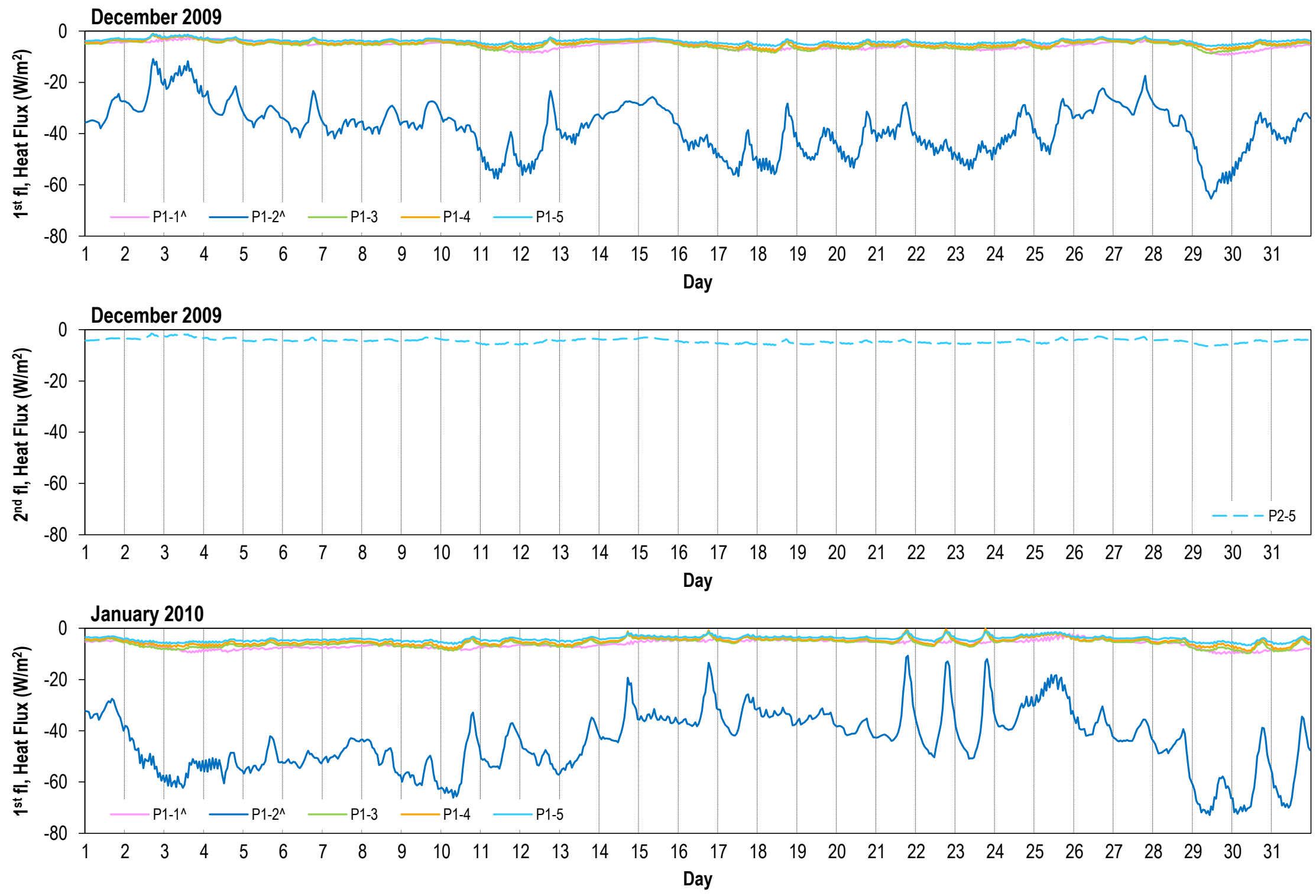

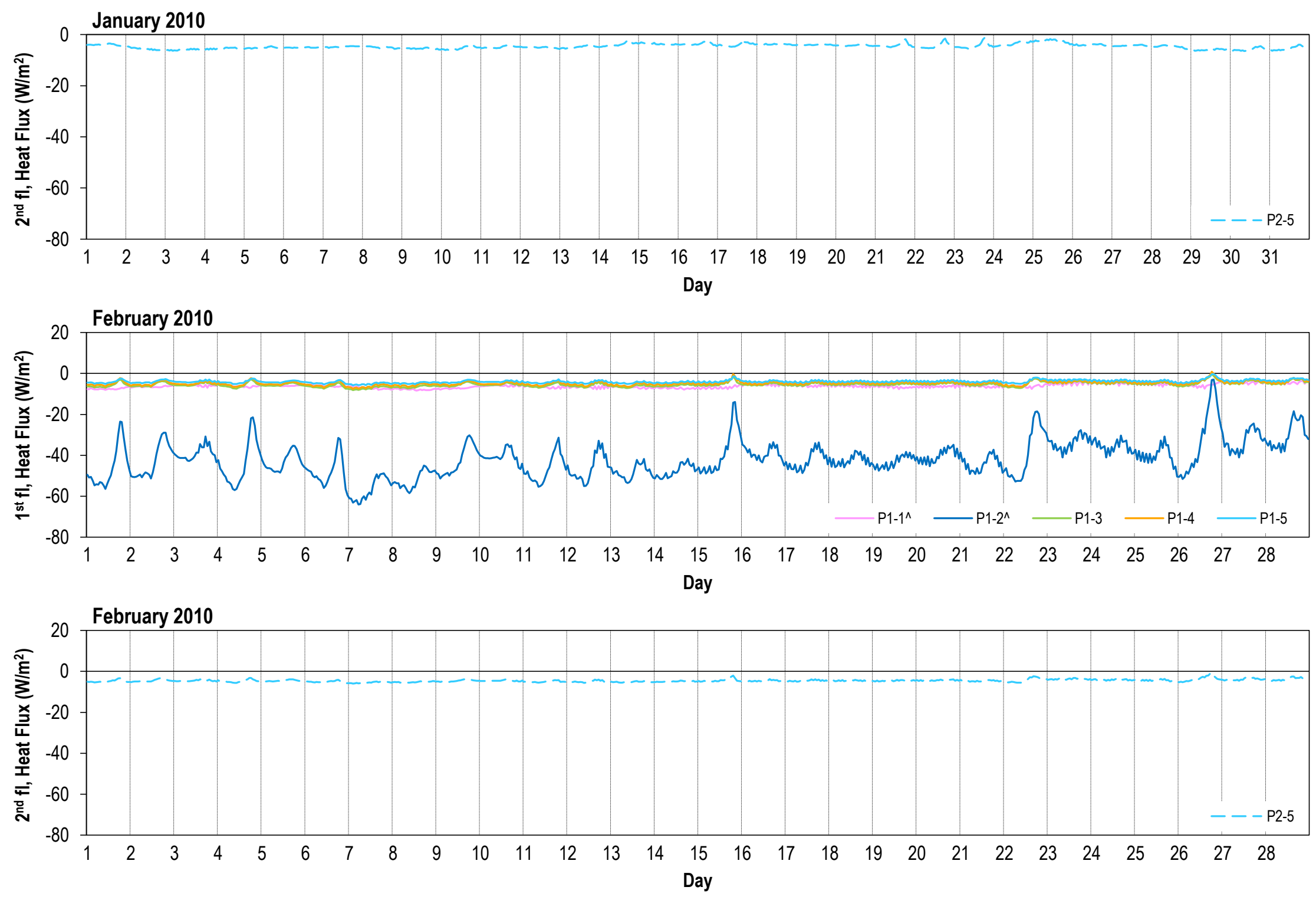

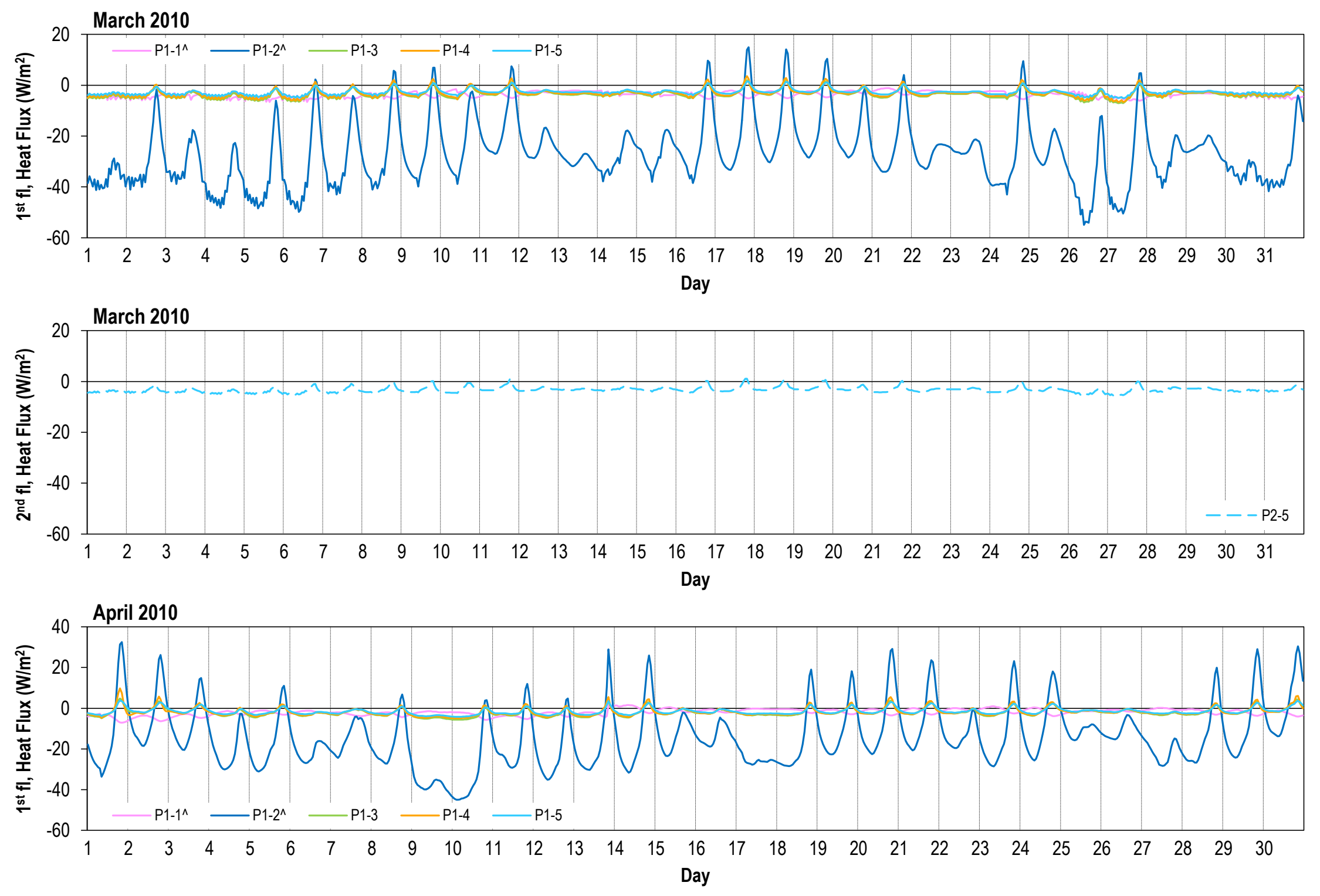

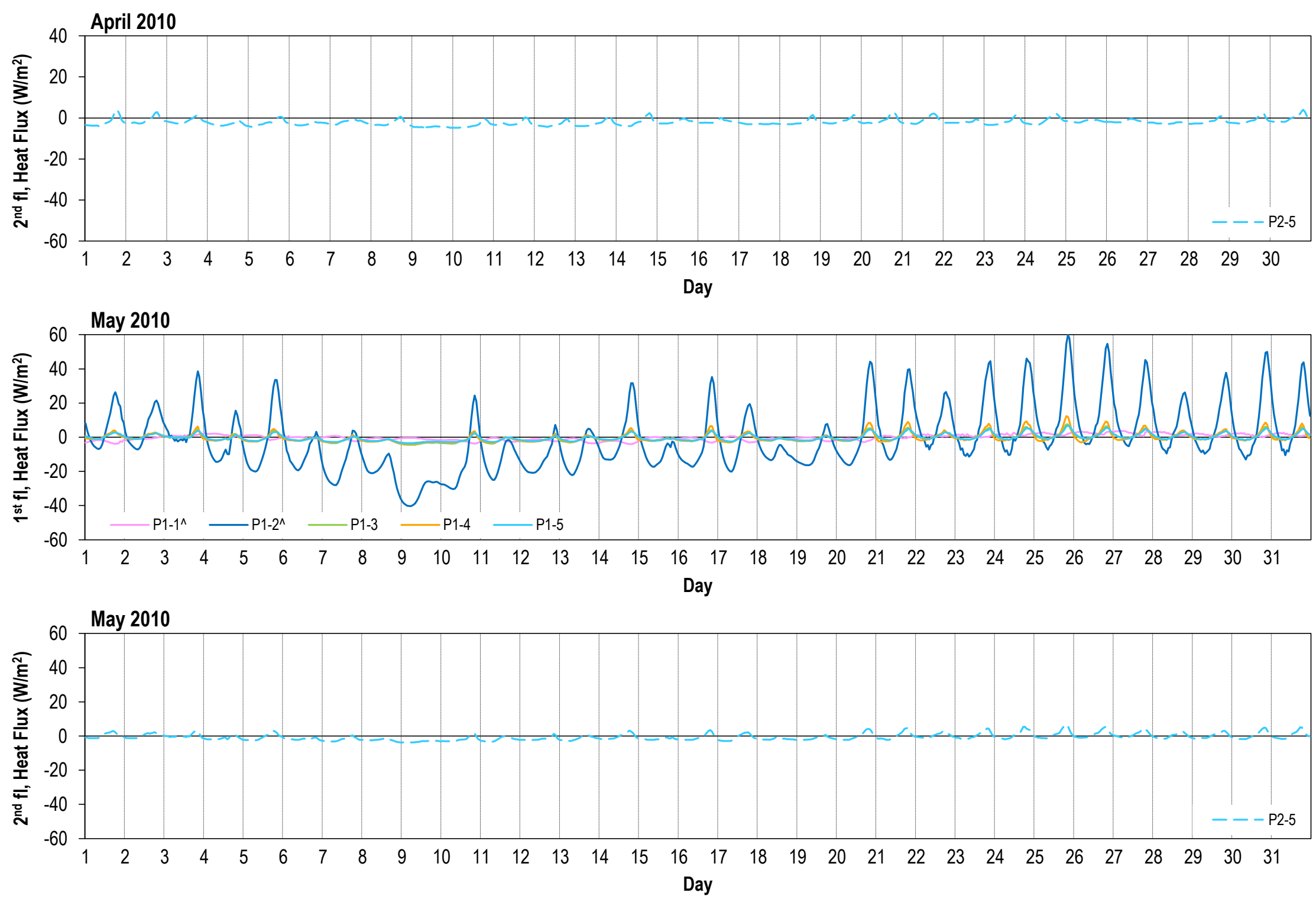

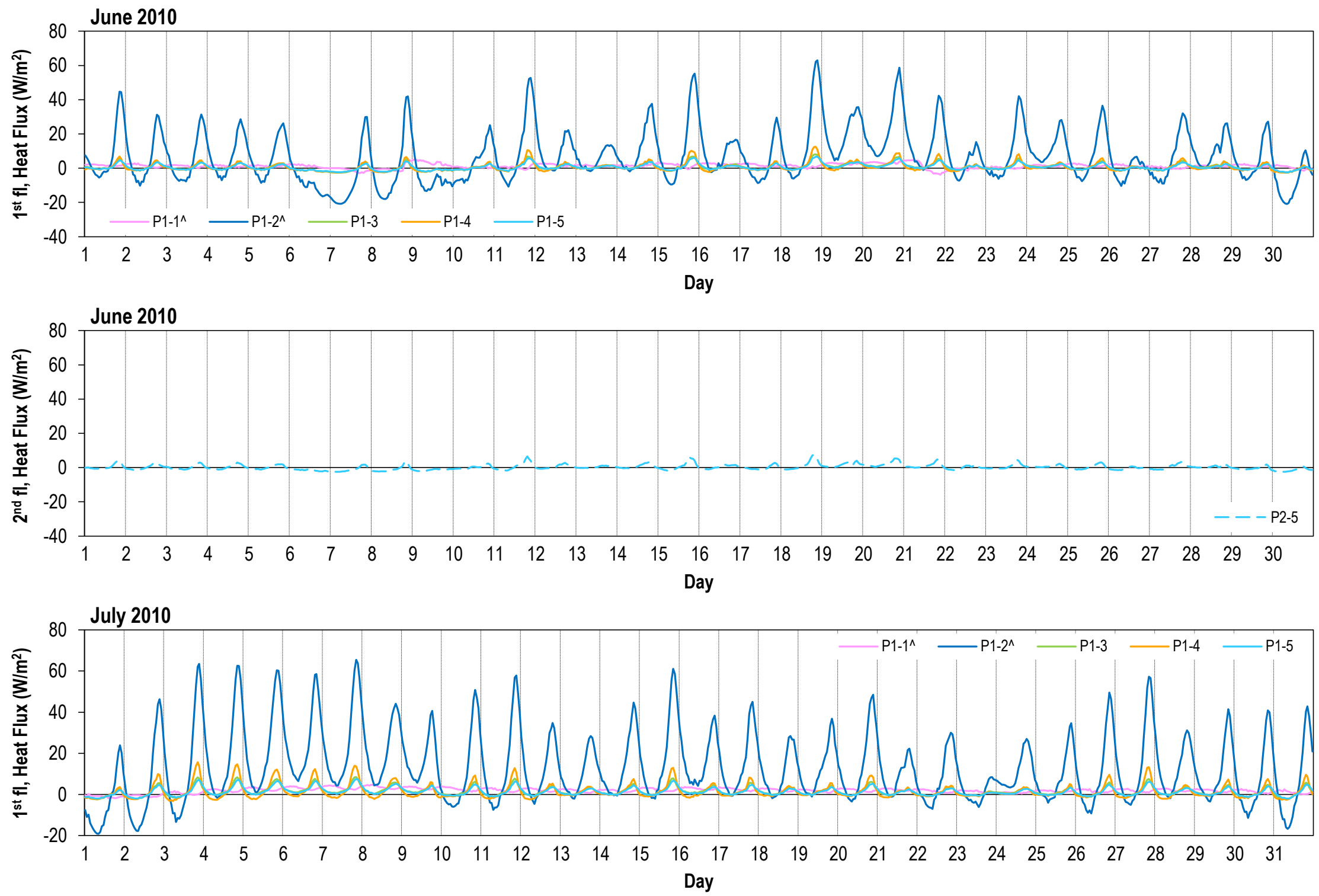

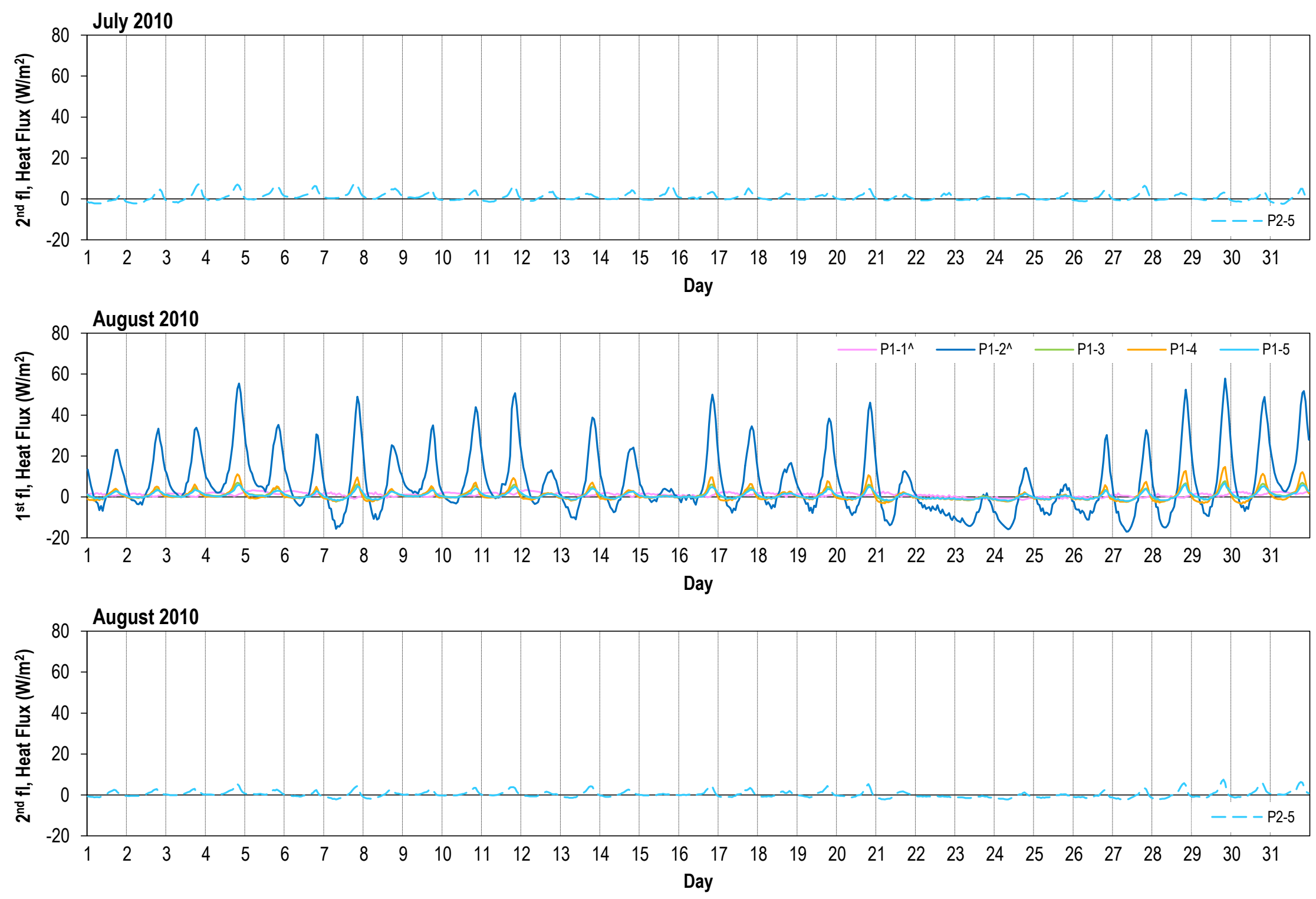

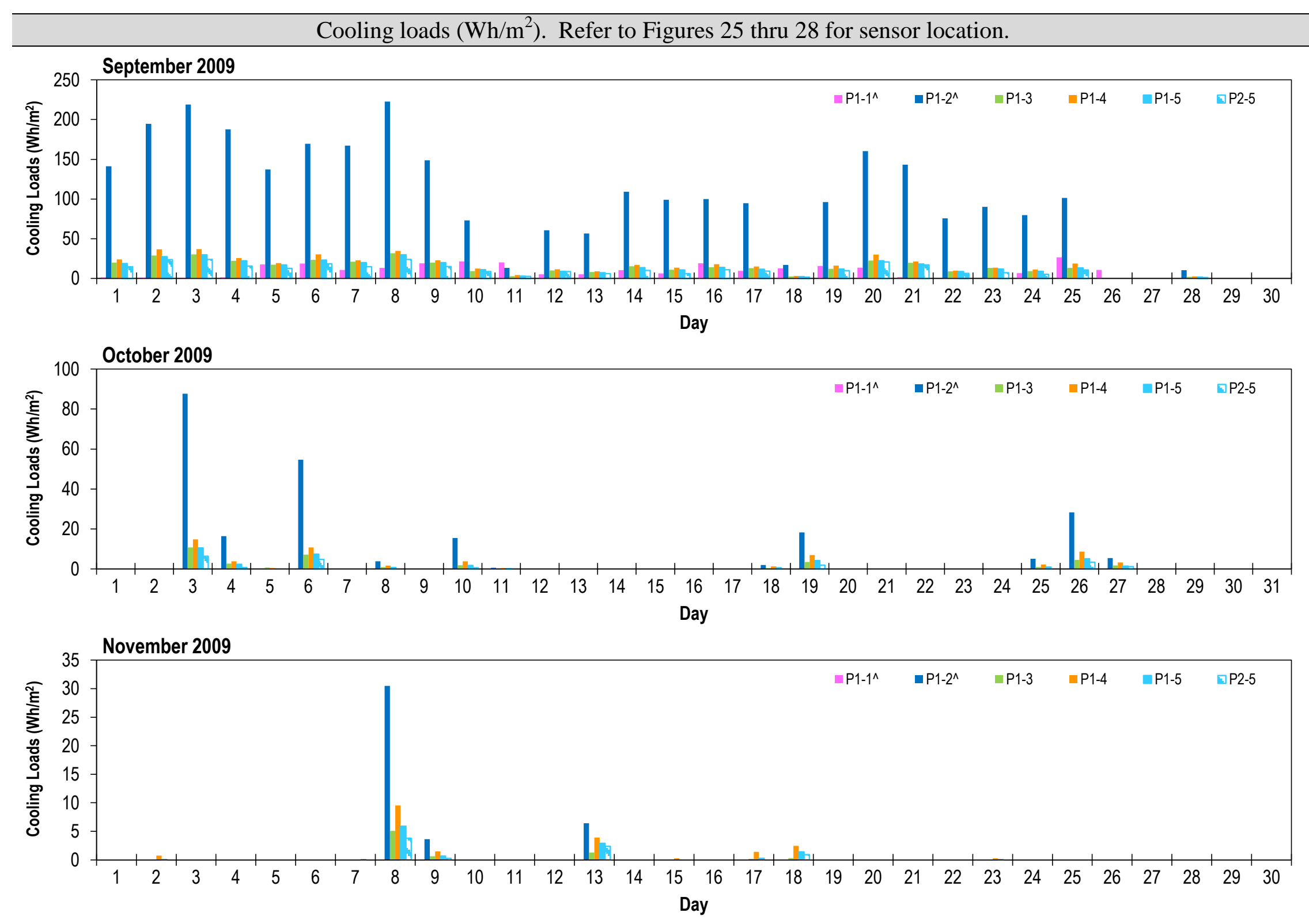

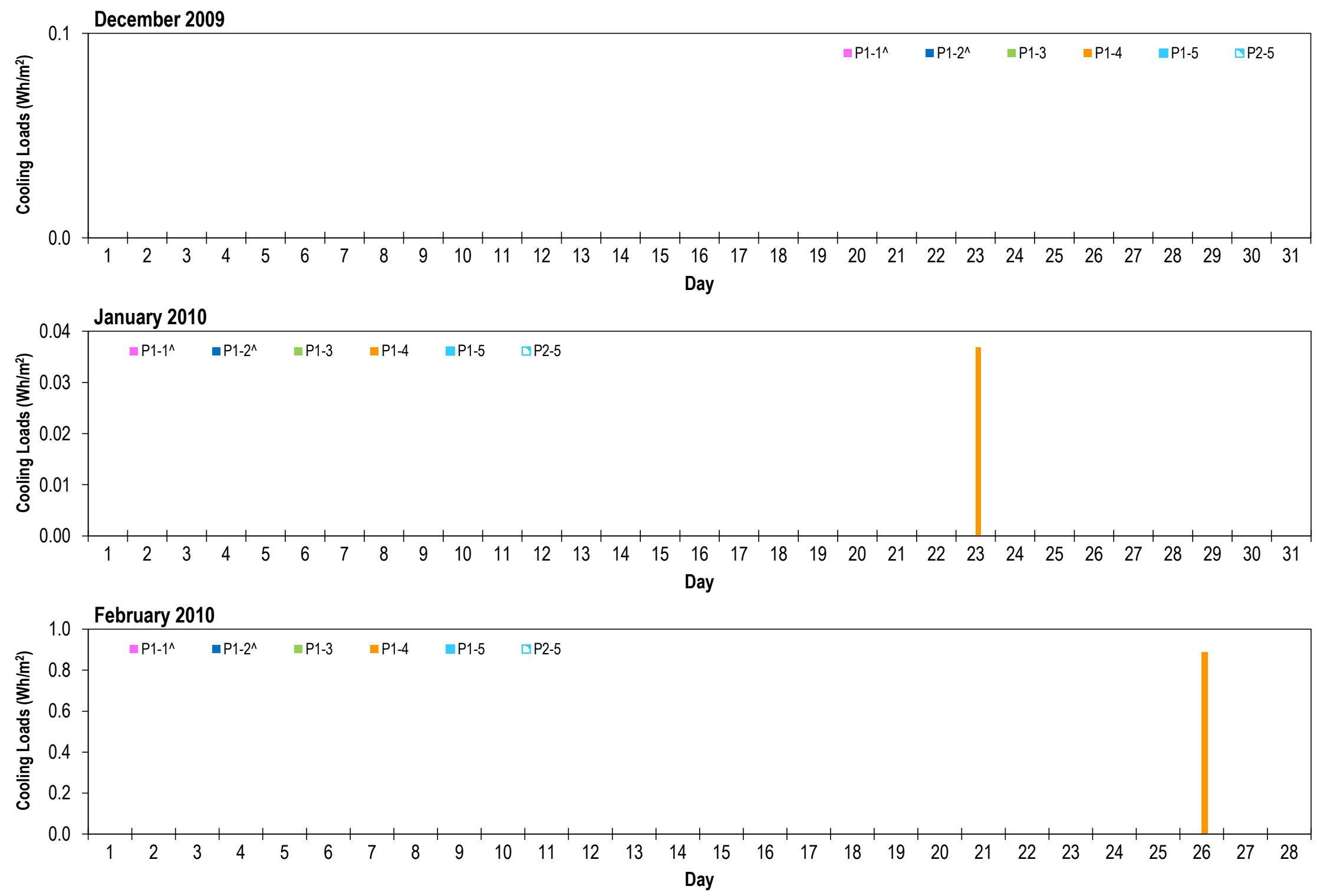

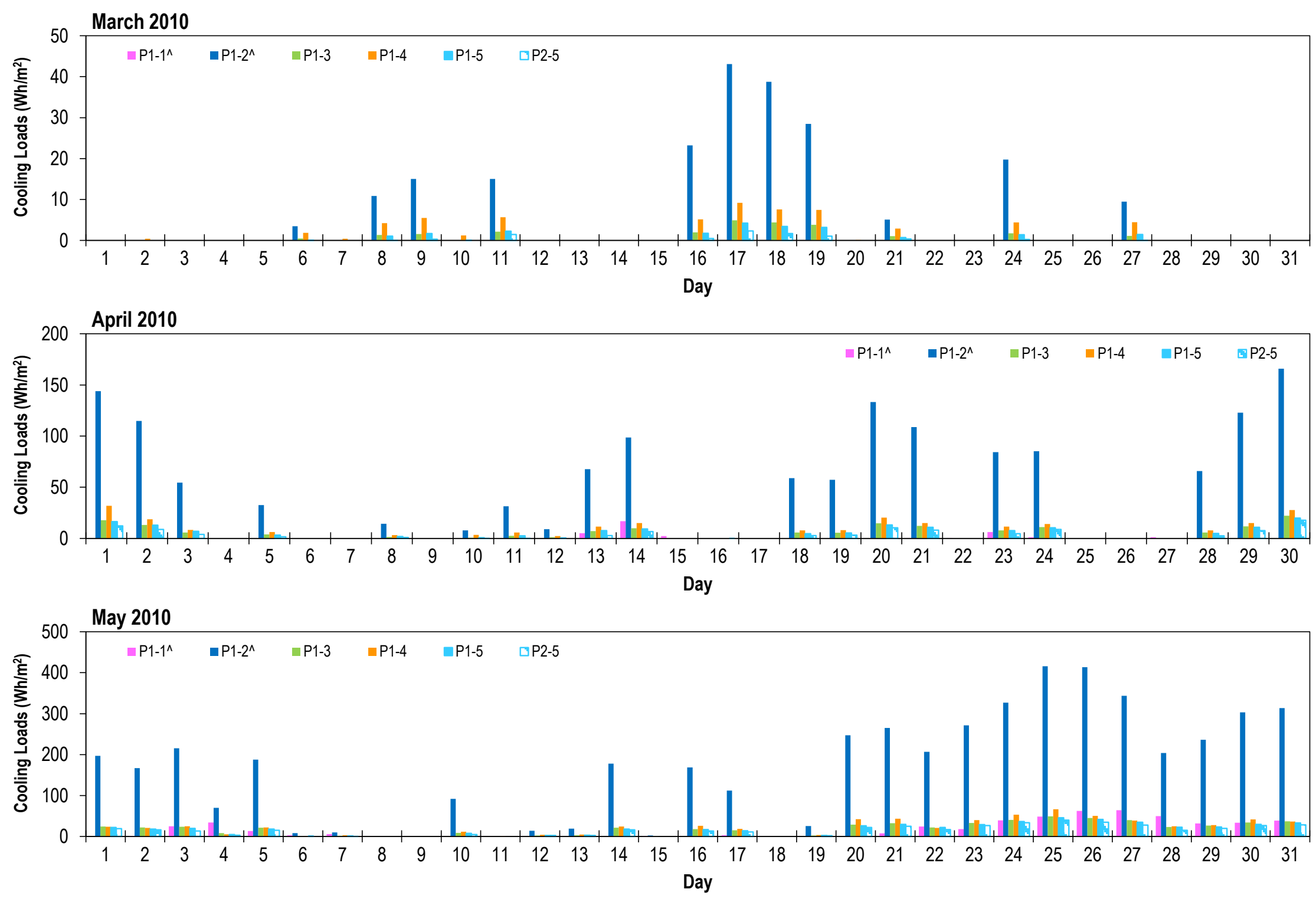

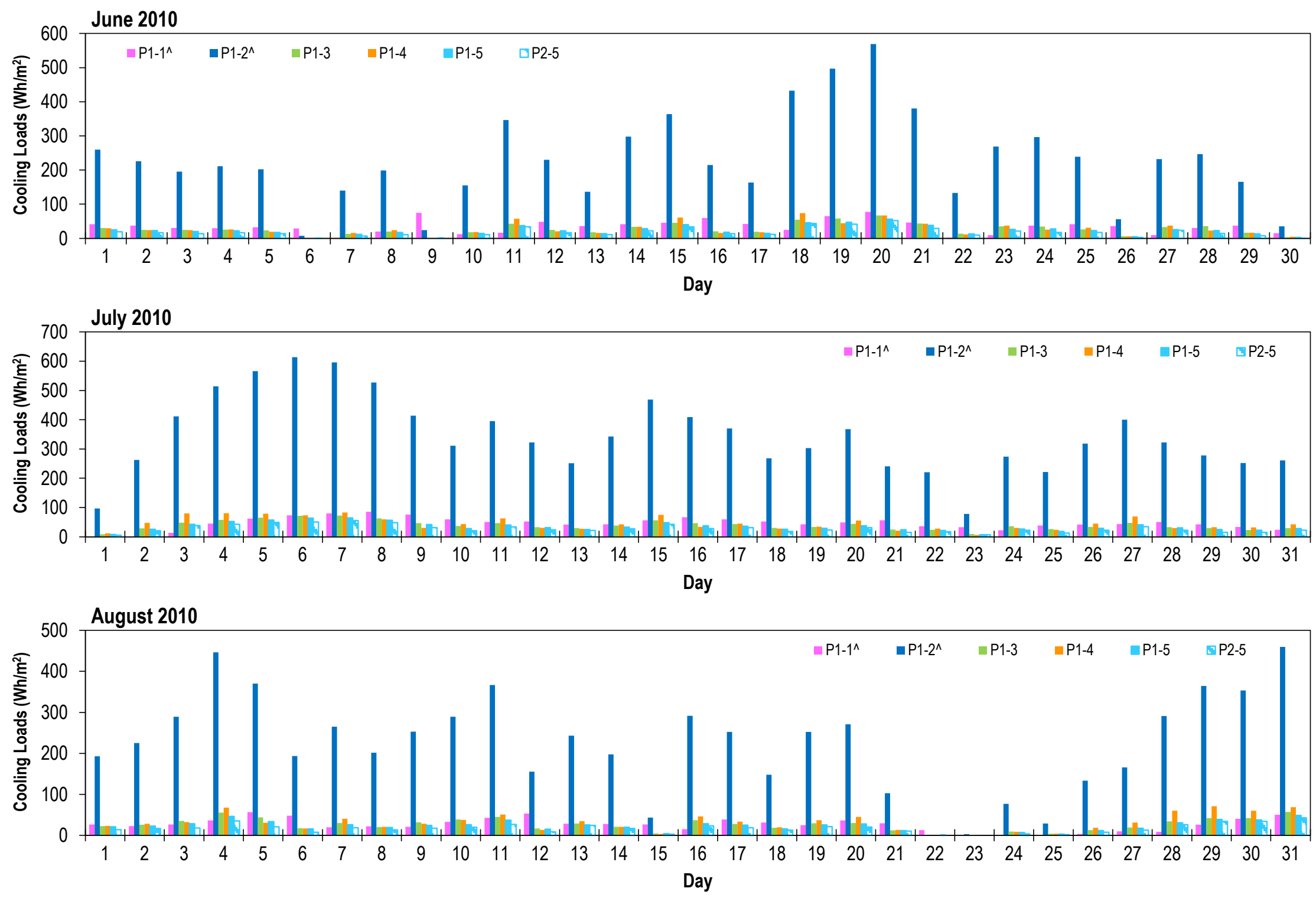
Heating loads $\left(\mathrm{Wh} / \mathrm{m}^{2}\right)$. Refer to Figures 25 thru 28 for sensor location.

September 2009

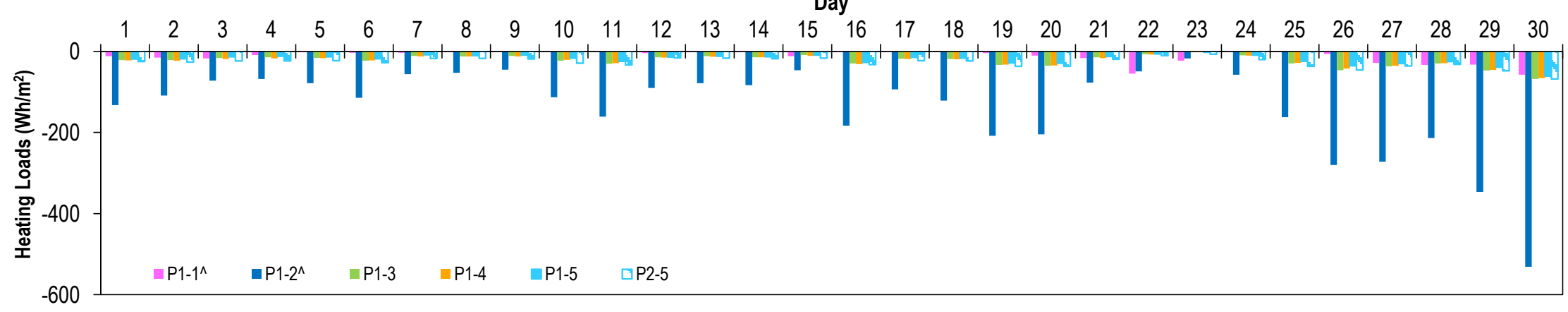

\section{October 2009}

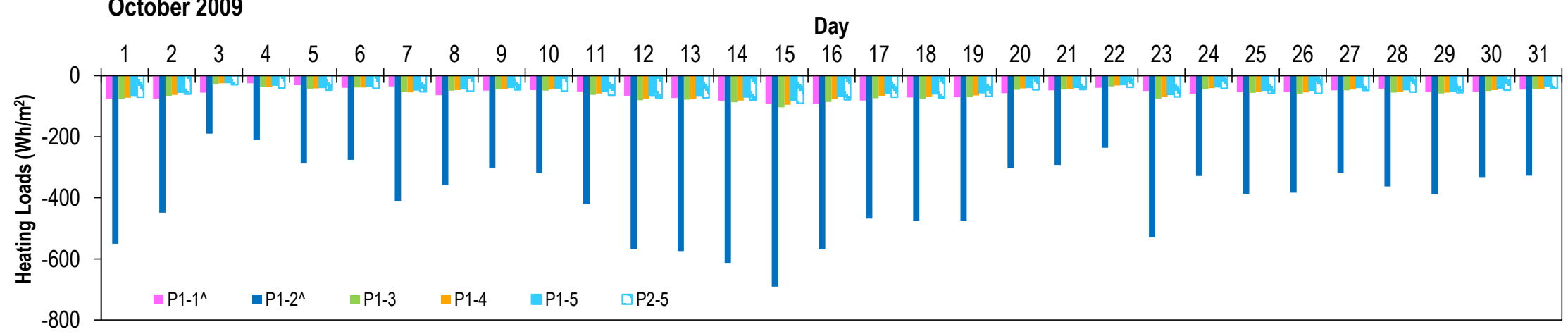

November 2009

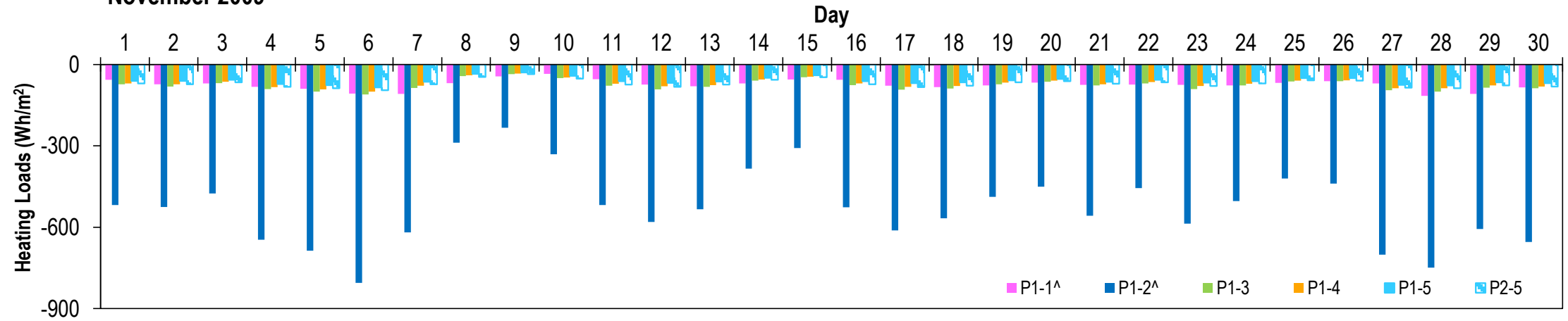



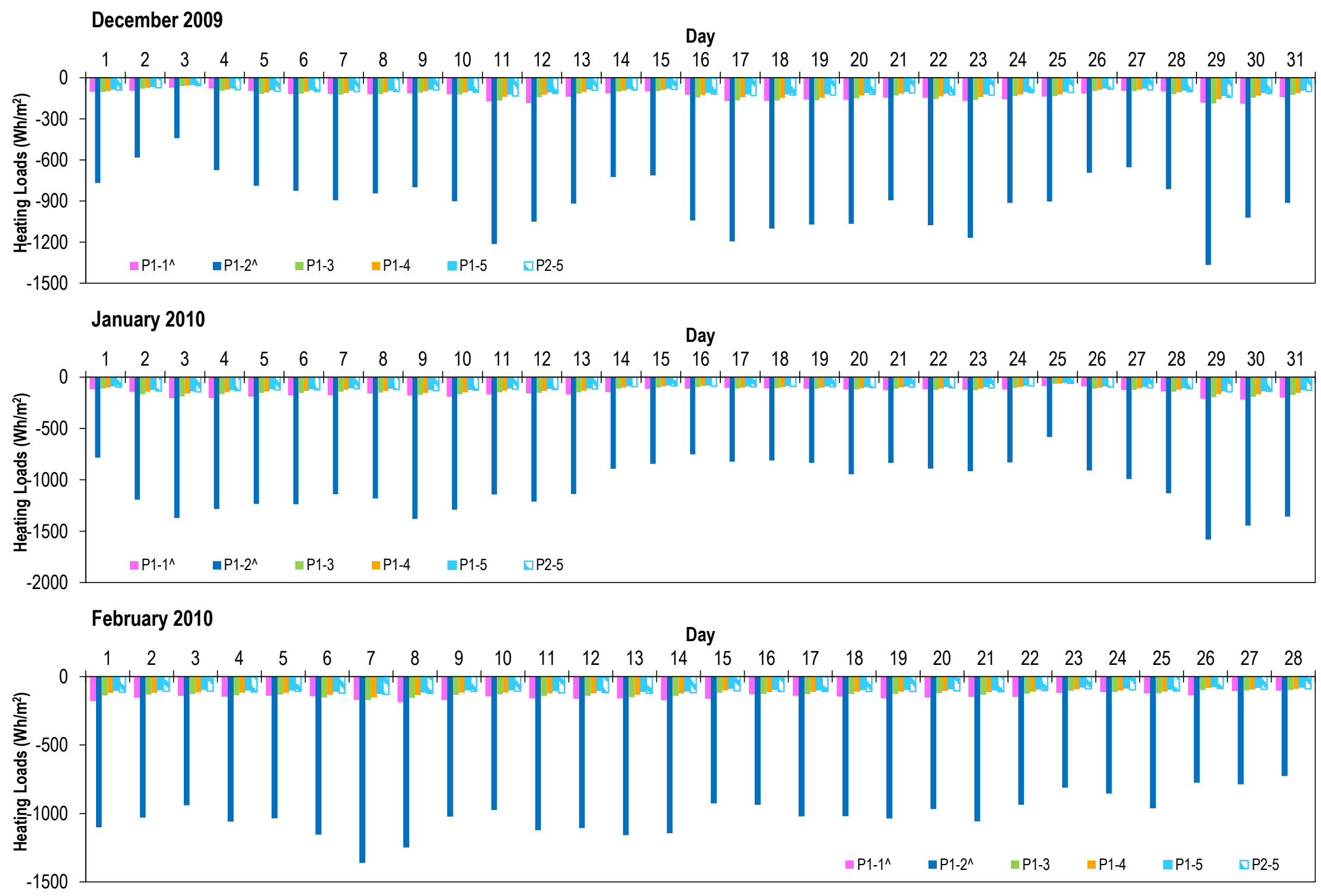
March 2010

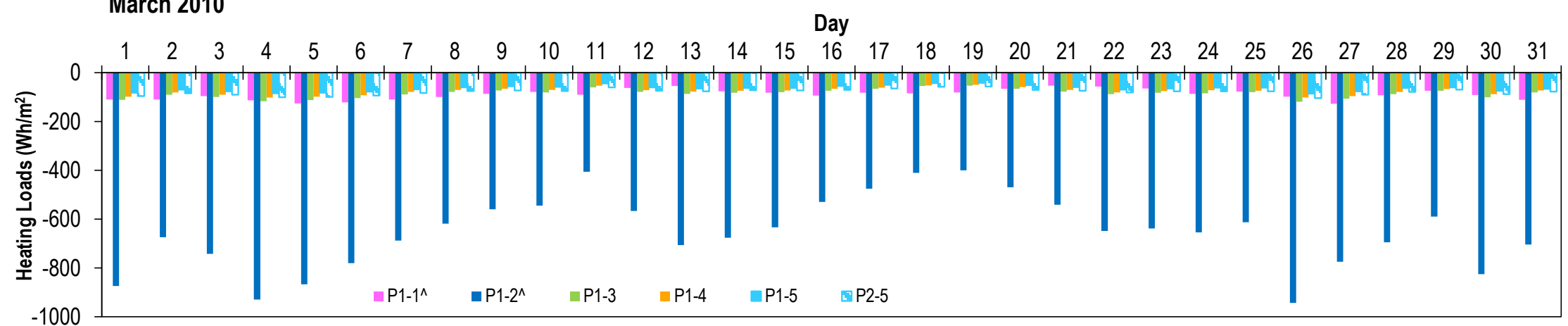

$-1000$

\section{April 2010}

\section{Day}

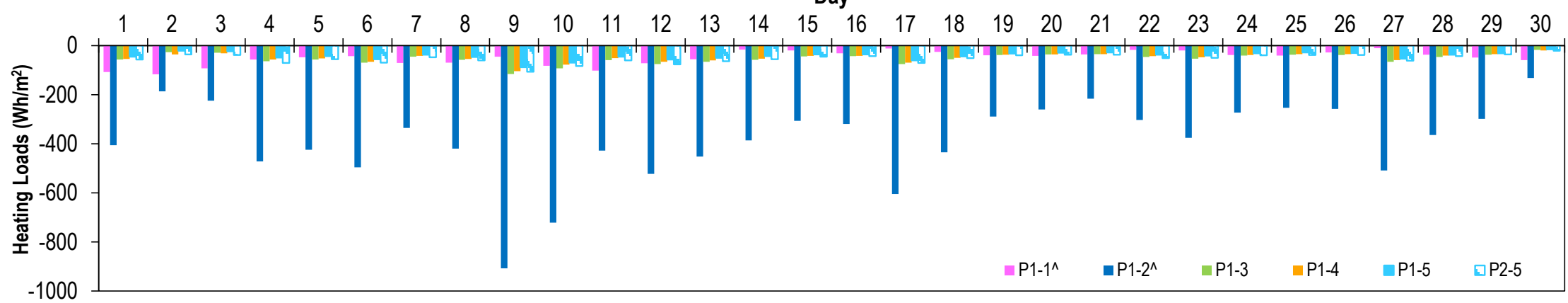

May 2010

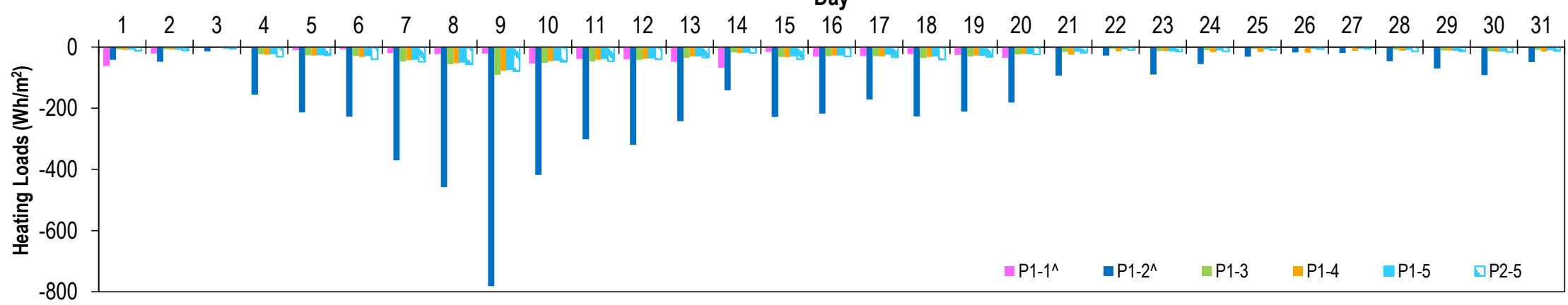


June 2010

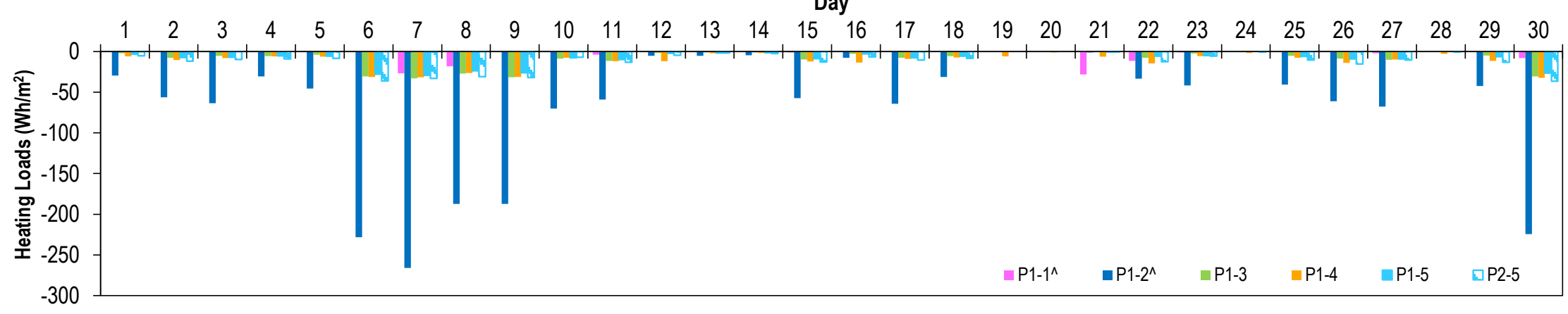

July 2010

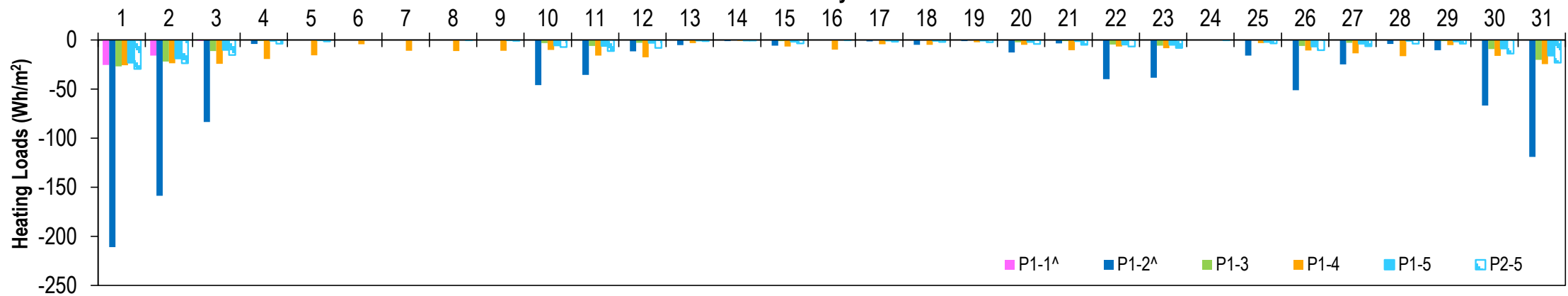

\section{August 2010}

Day

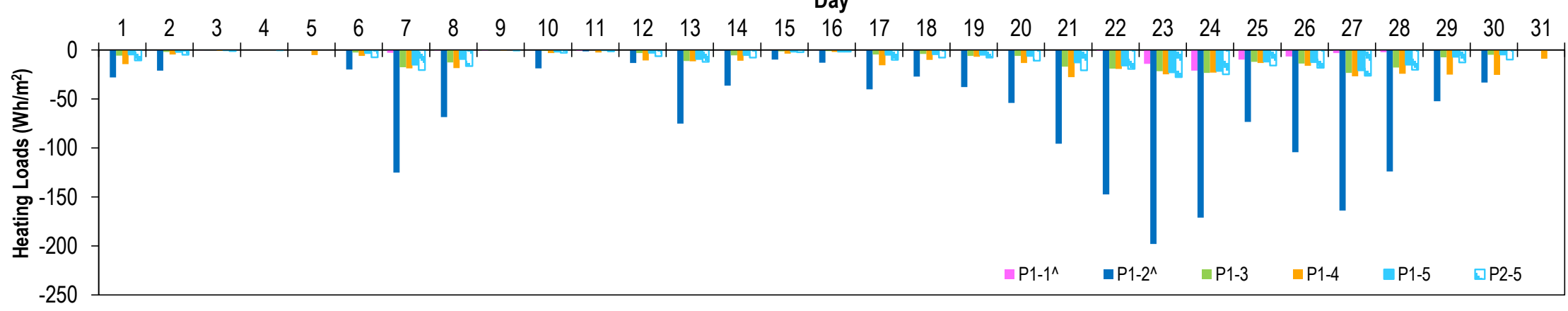


Appendix E: Solar Radiation
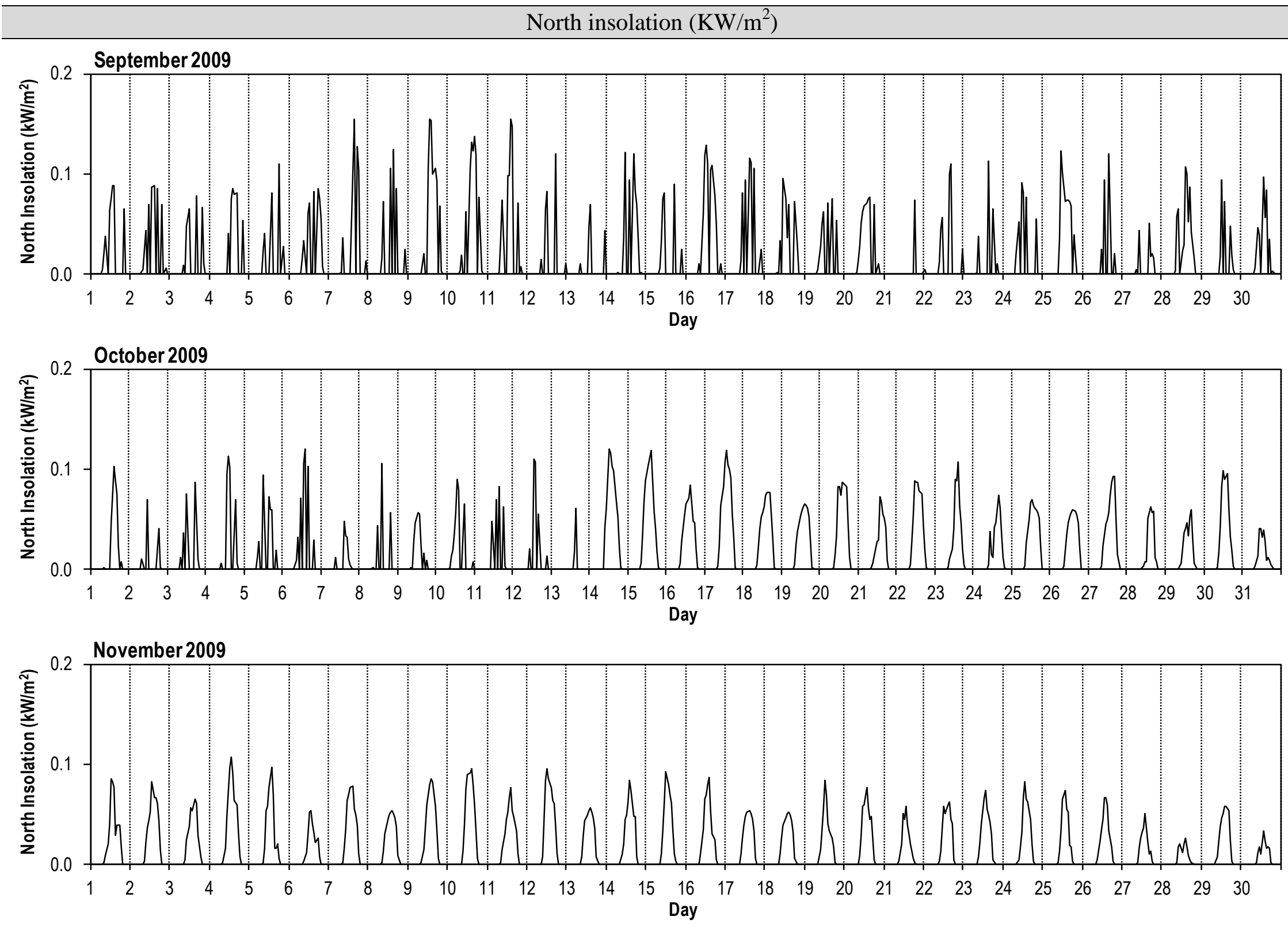

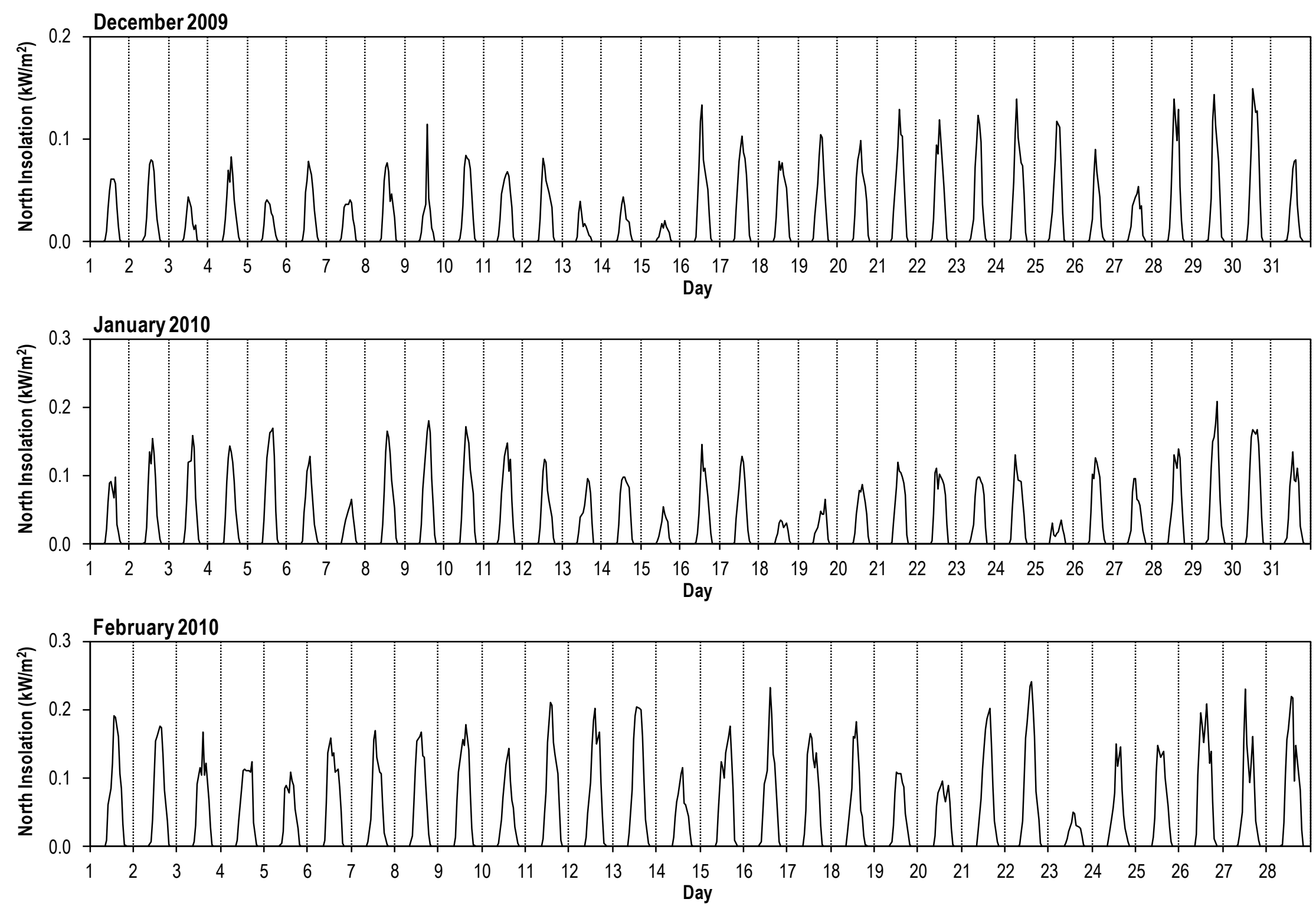
237
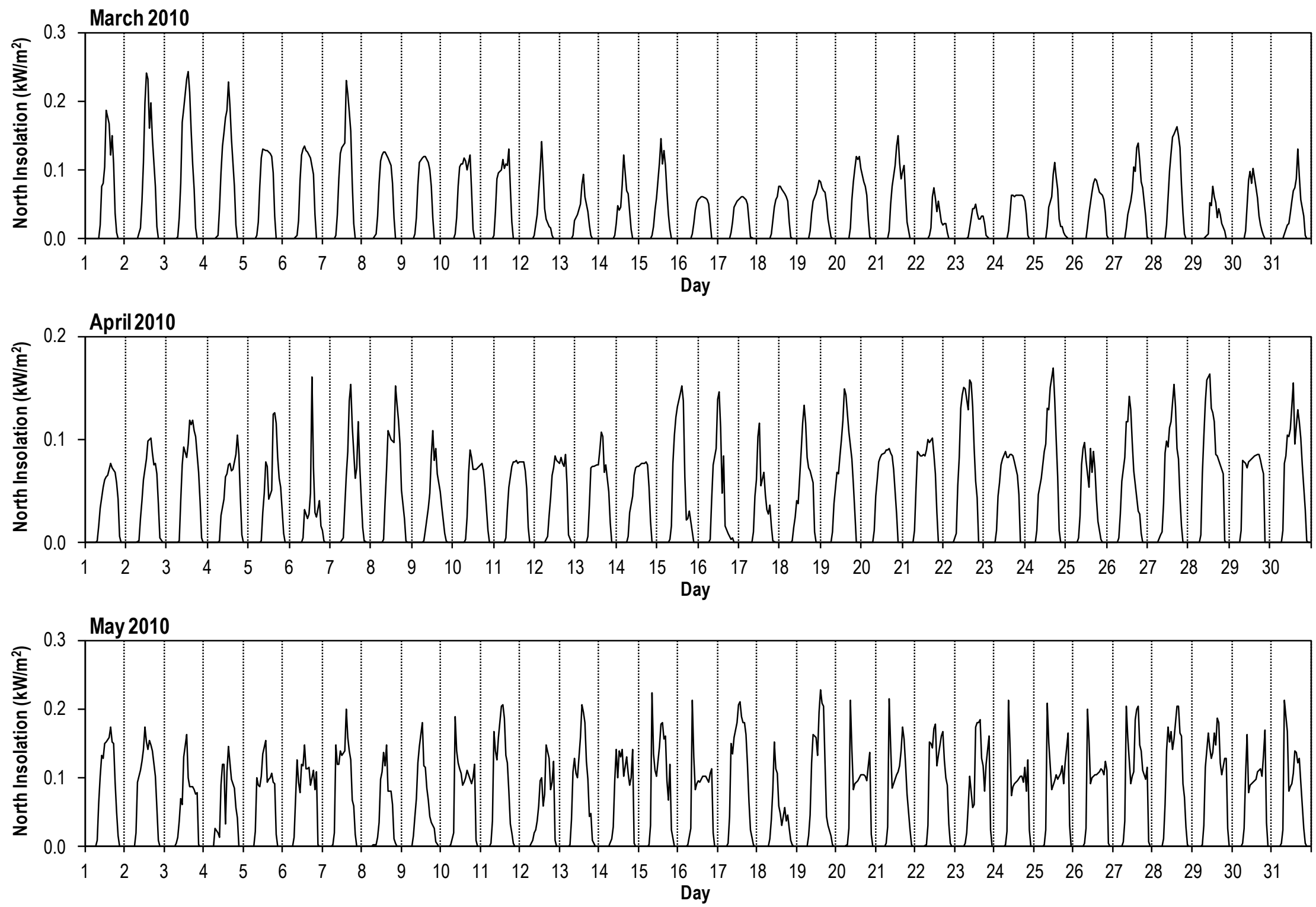

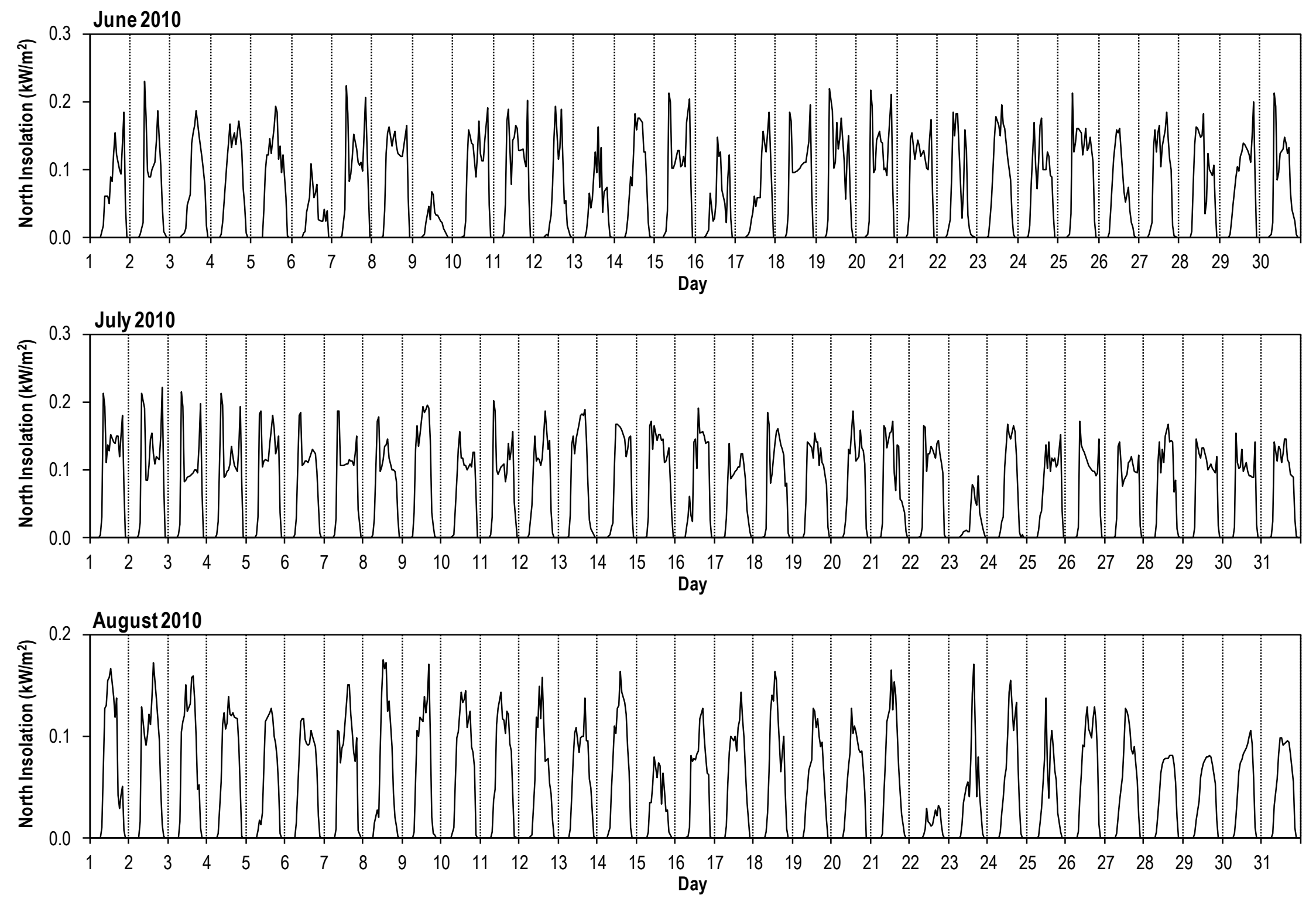
East insolation $\left(\mathrm{KW} / \mathrm{m}^{2}\right)$
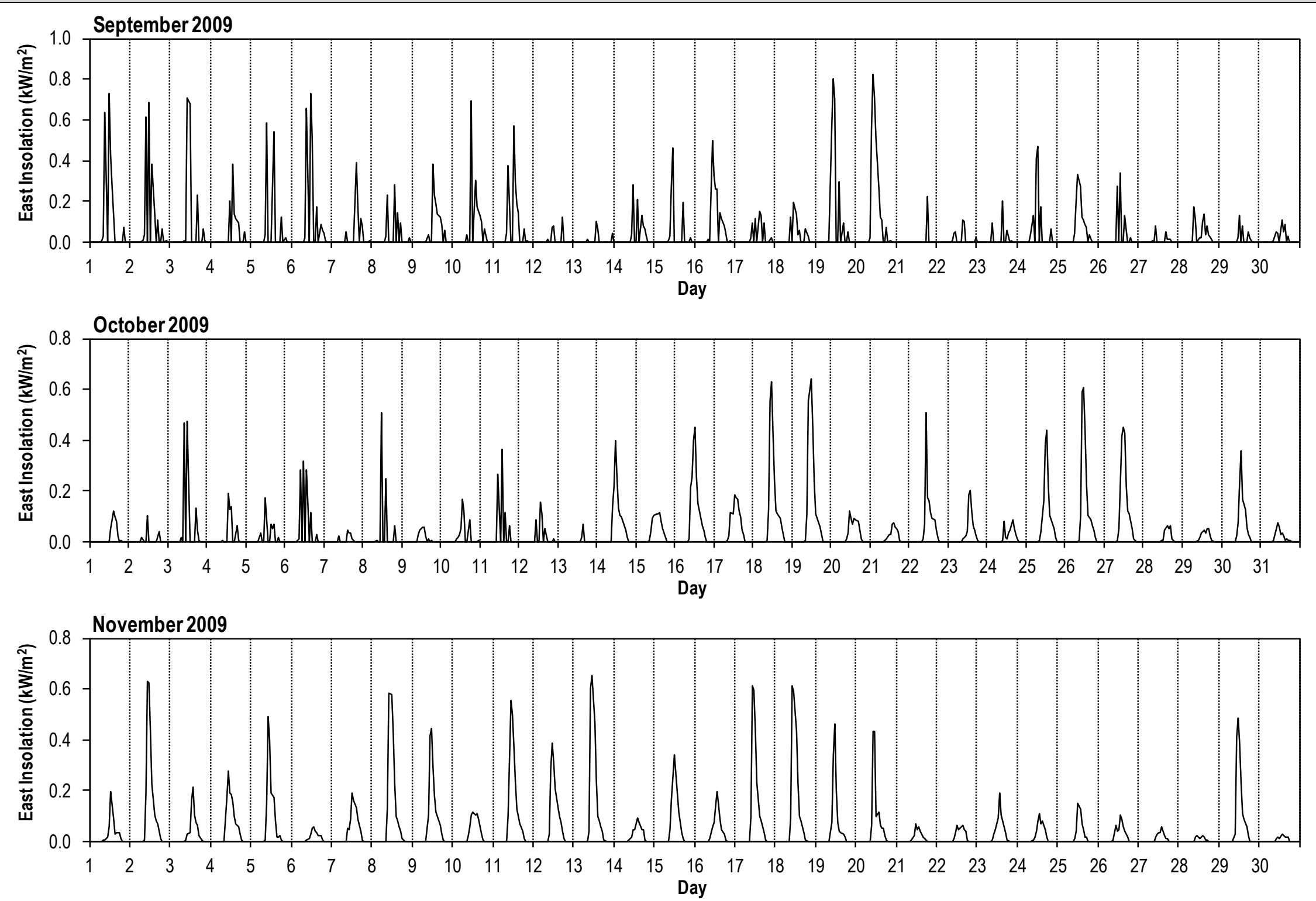

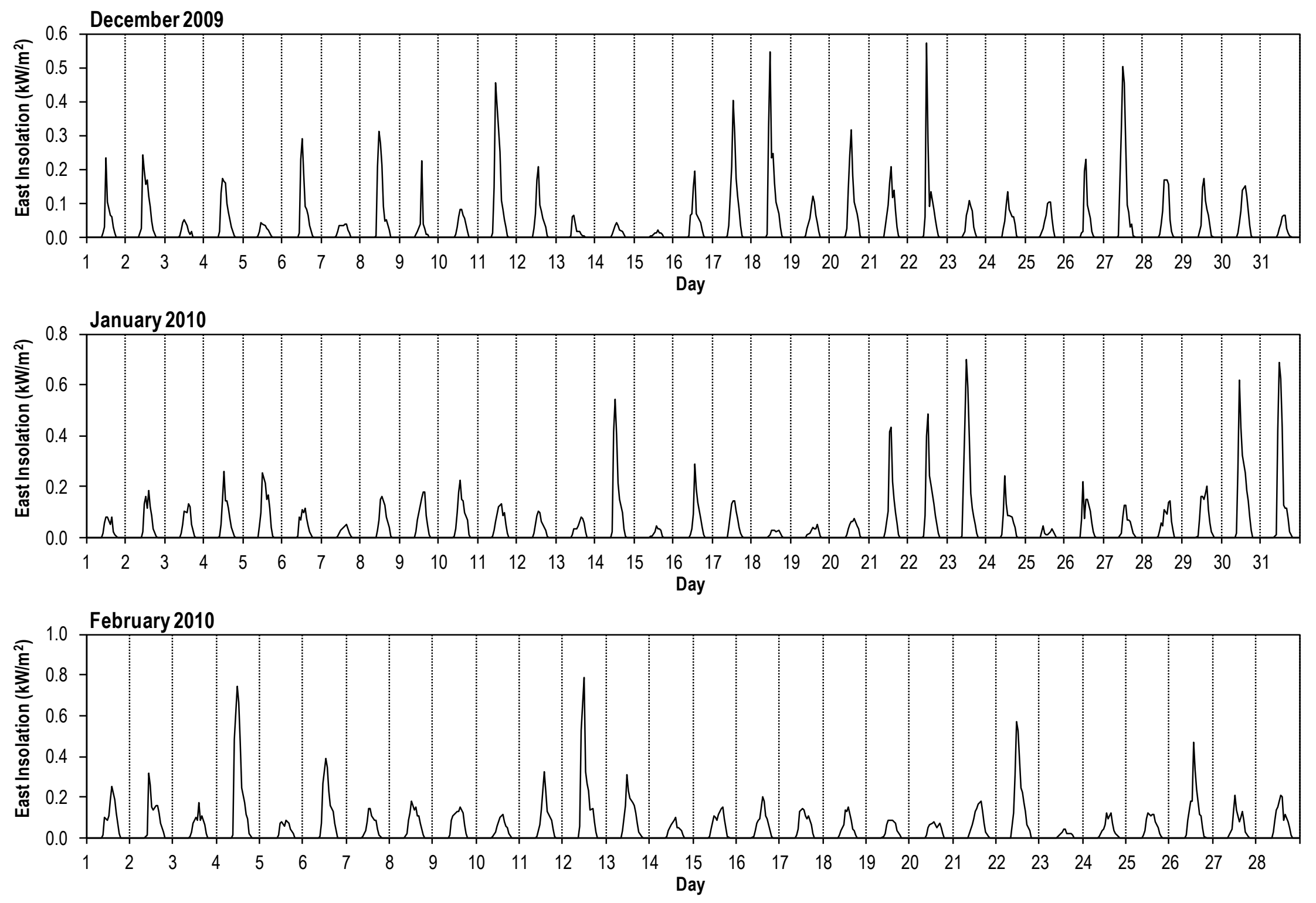

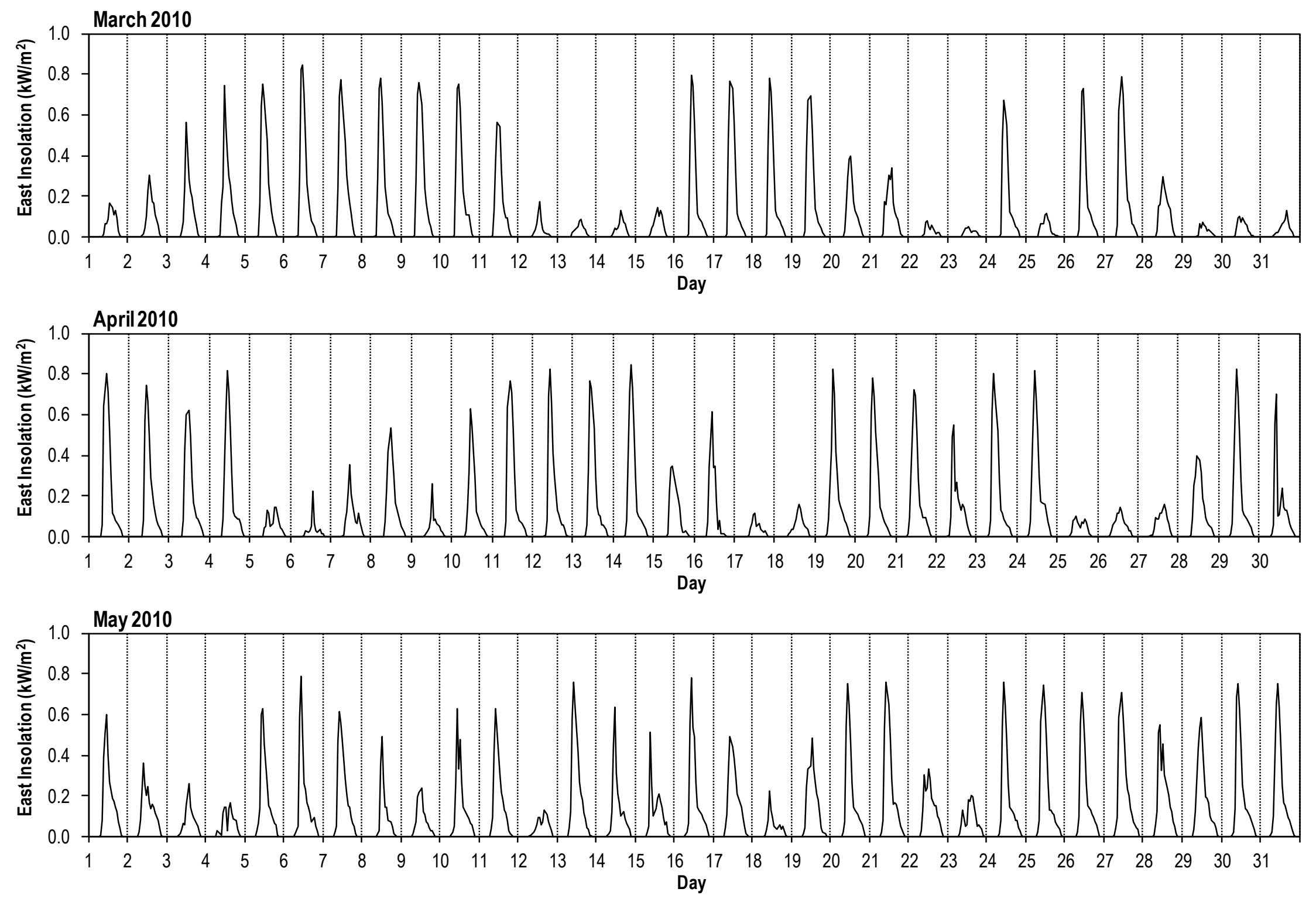

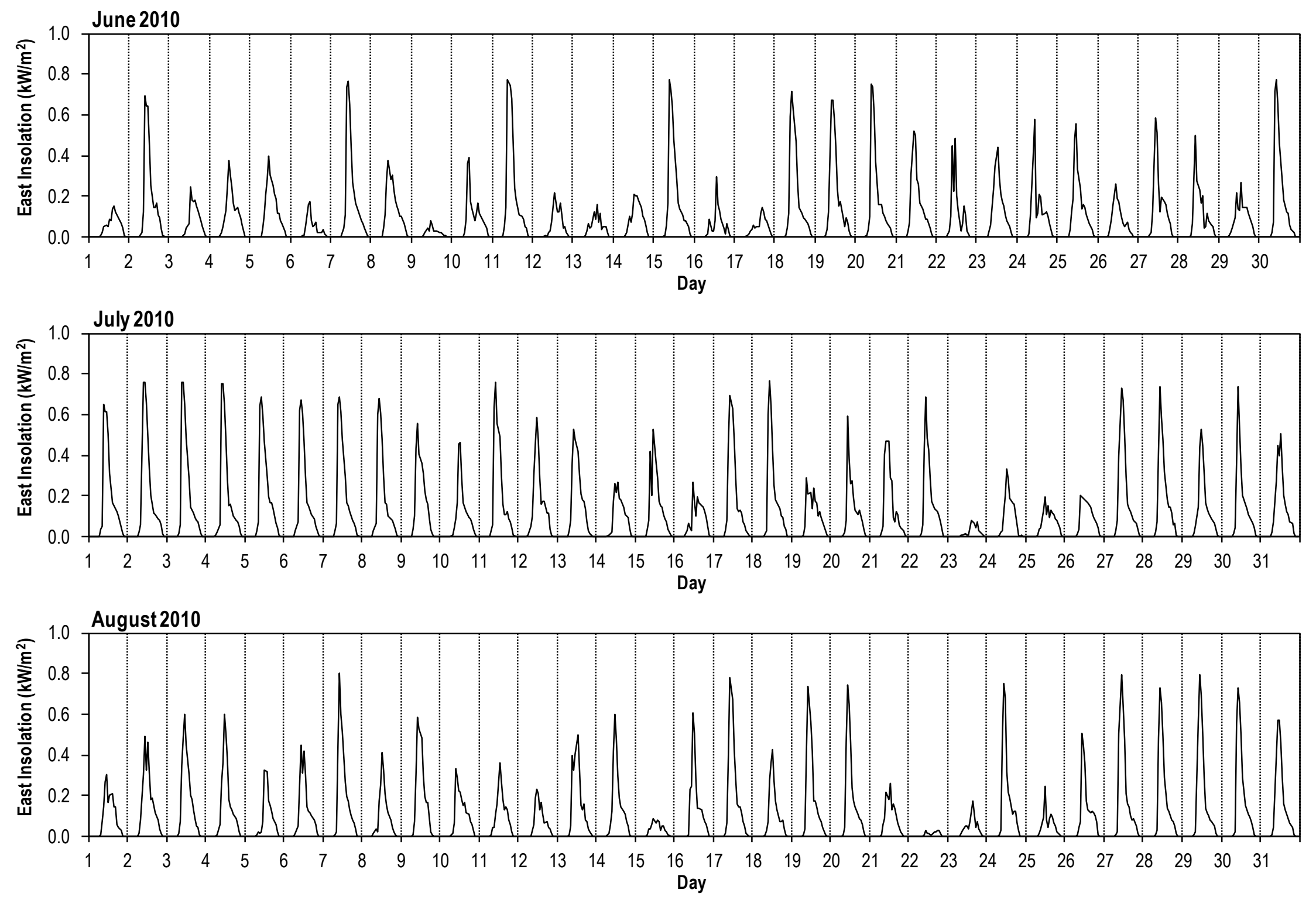
South insolation $\left(\mathrm{KW} / \mathrm{m}^{2}\right)$
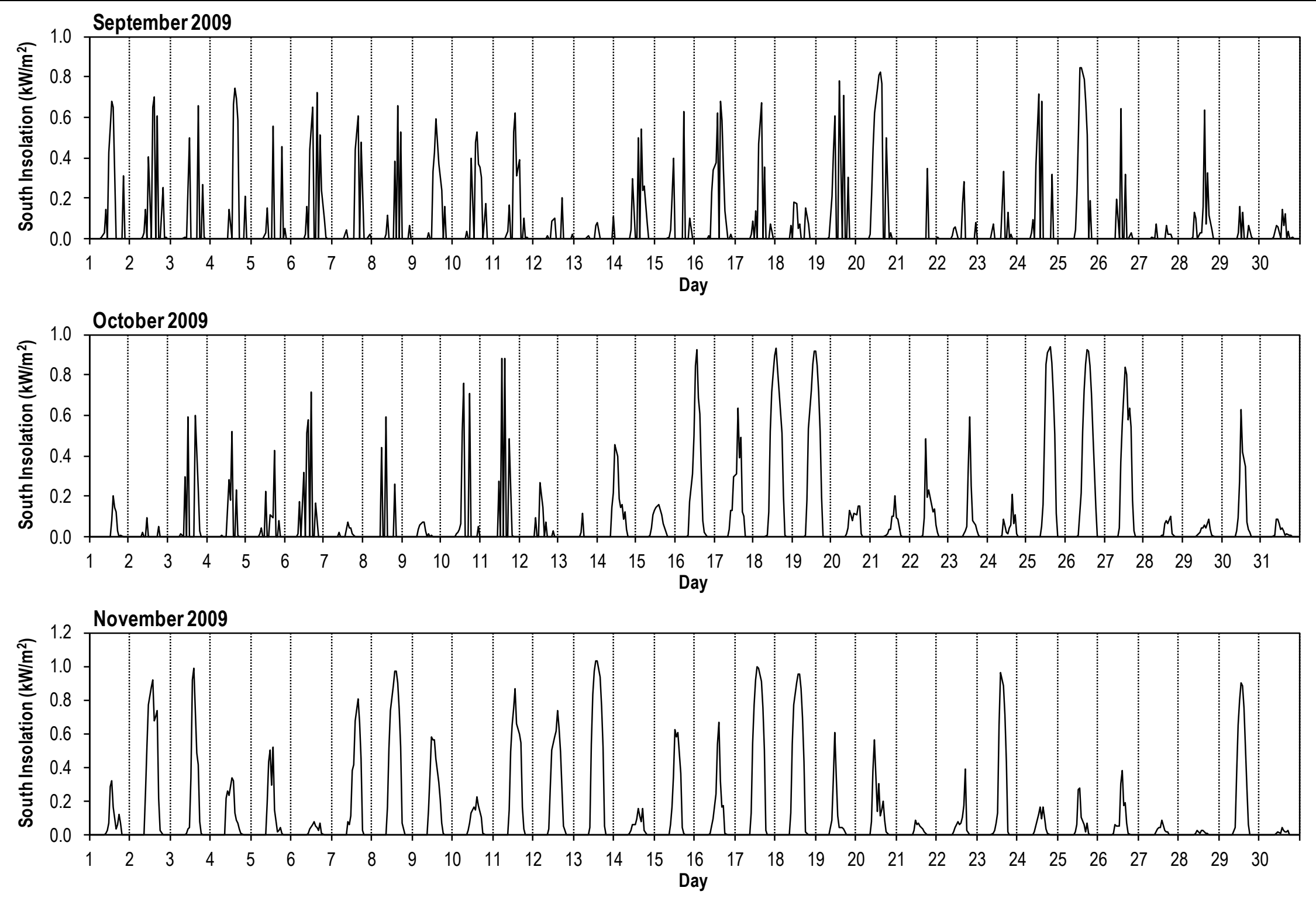

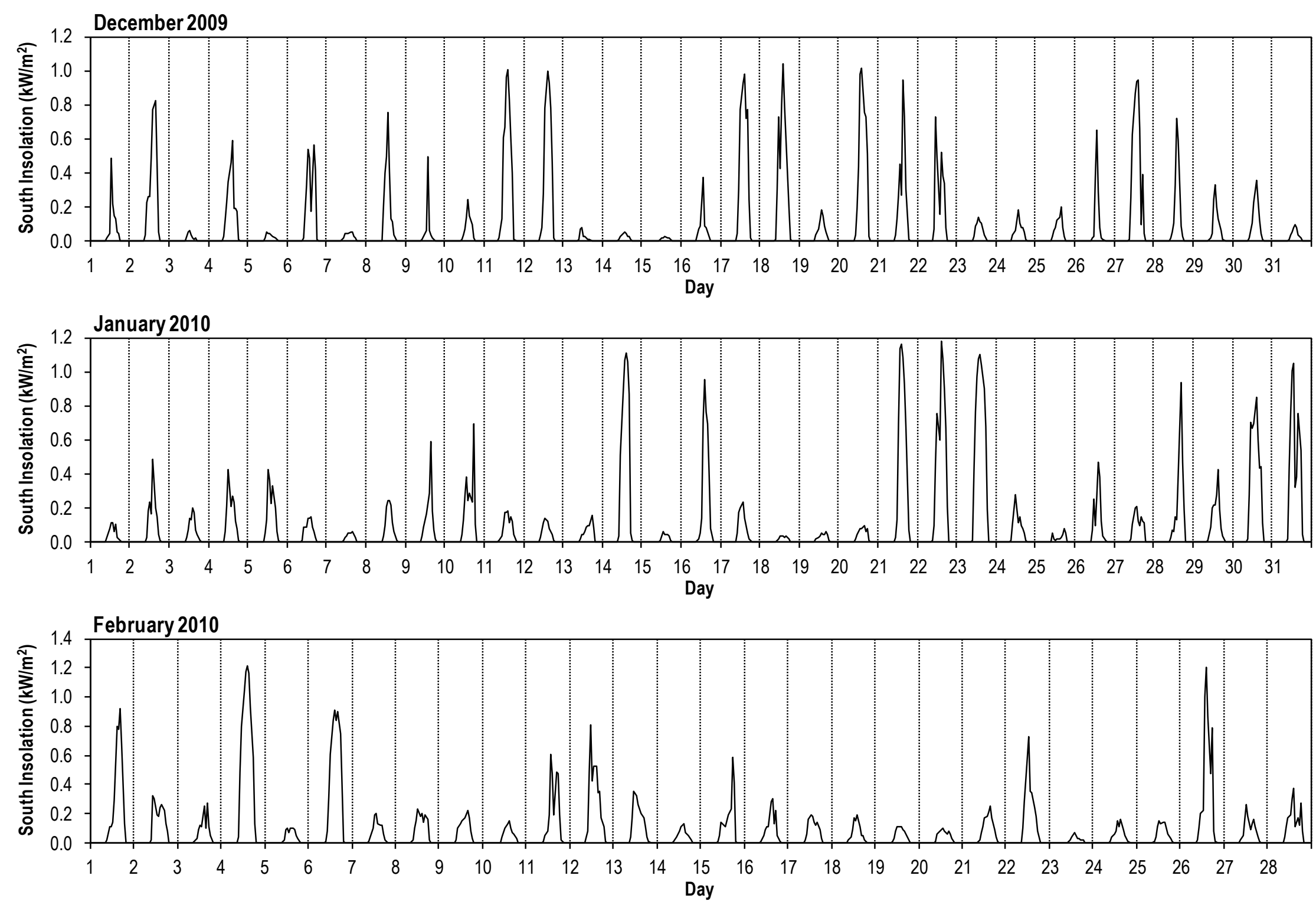

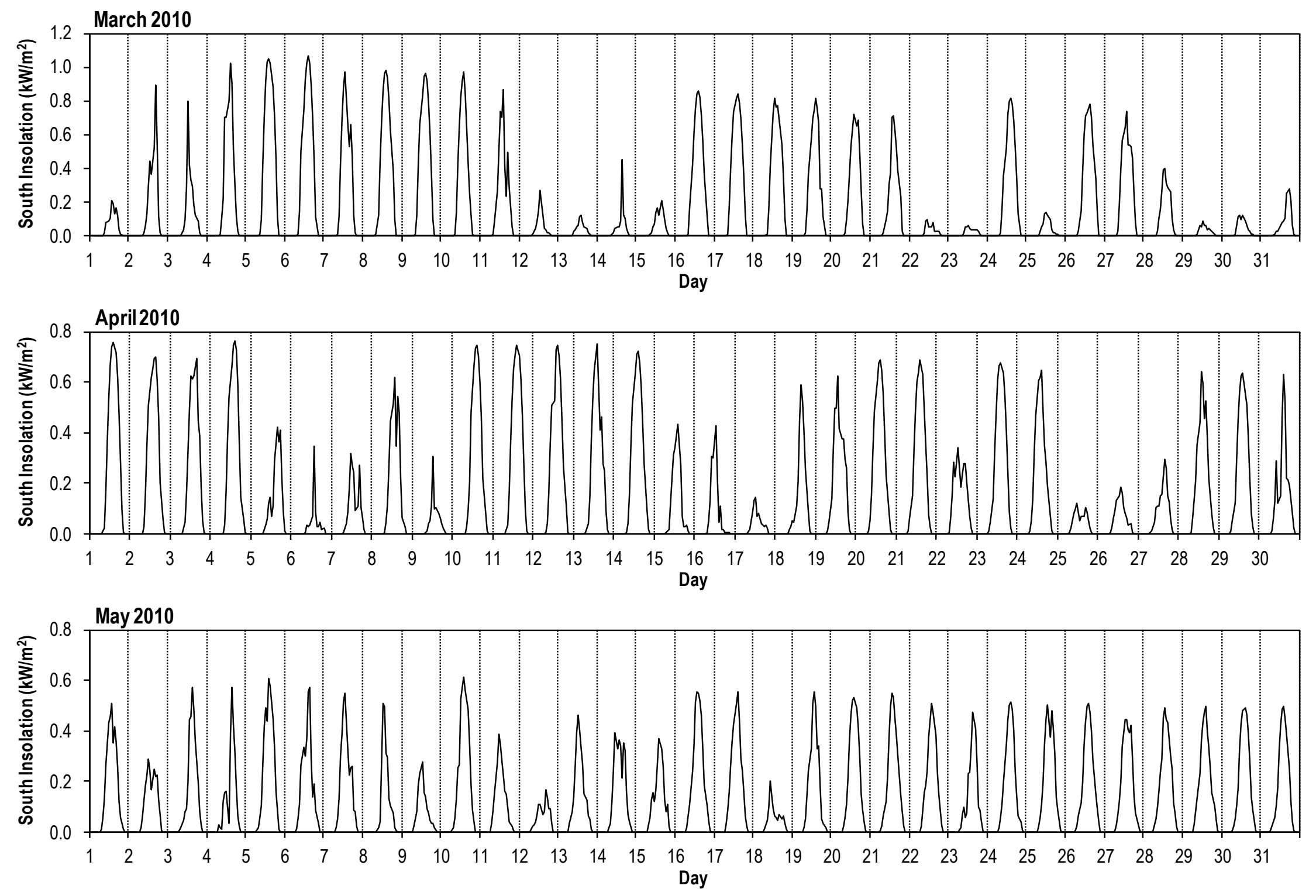
246
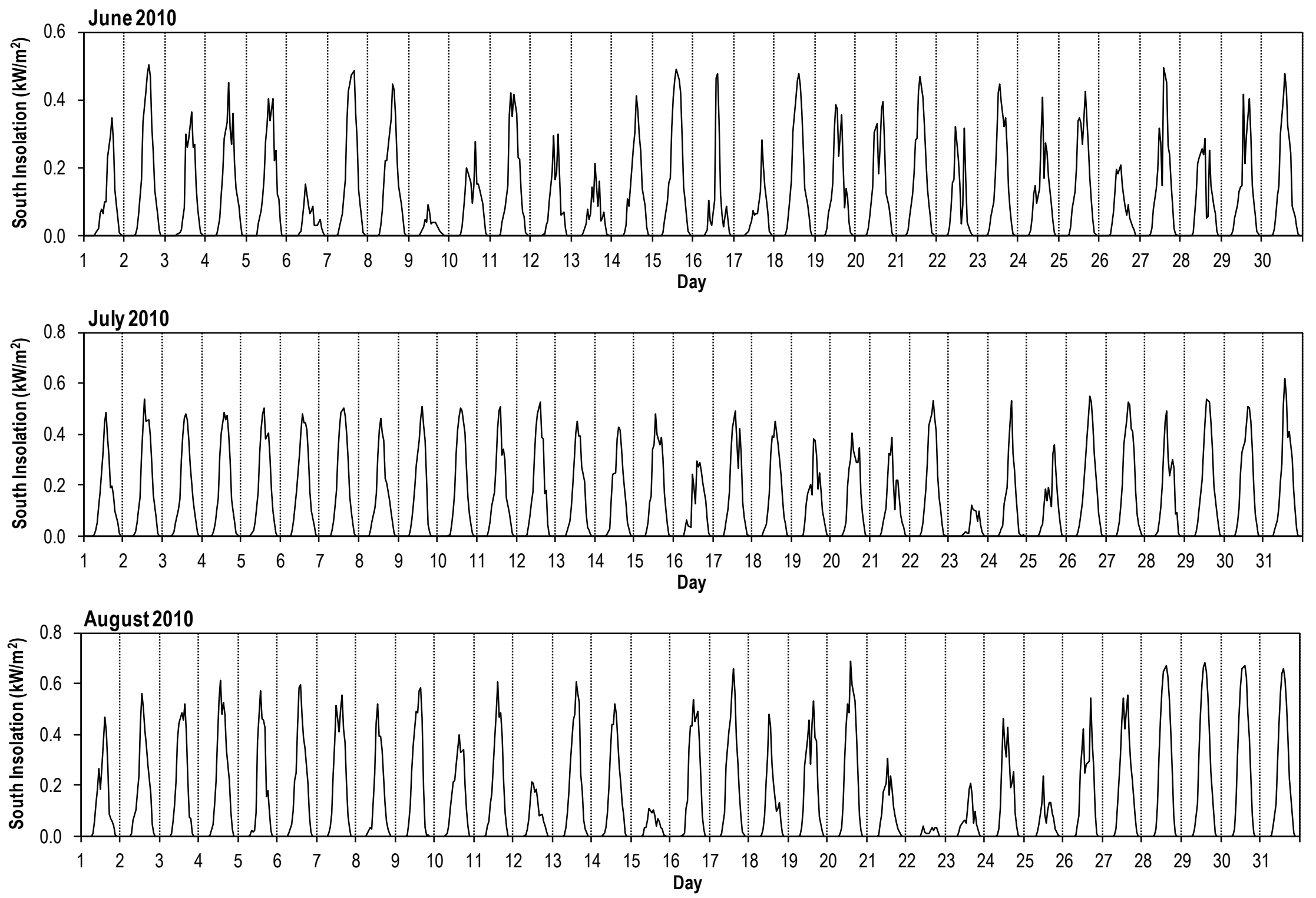


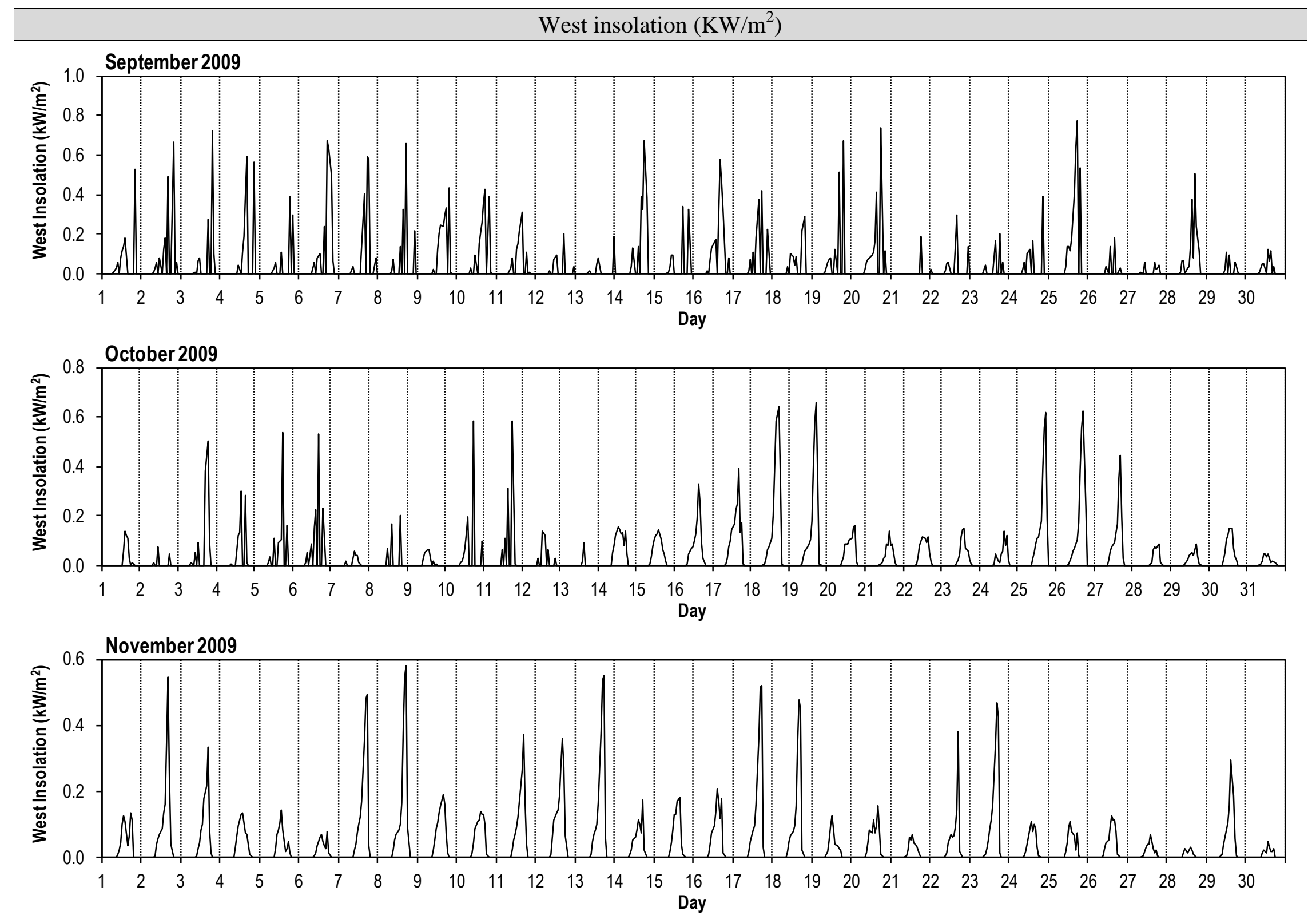



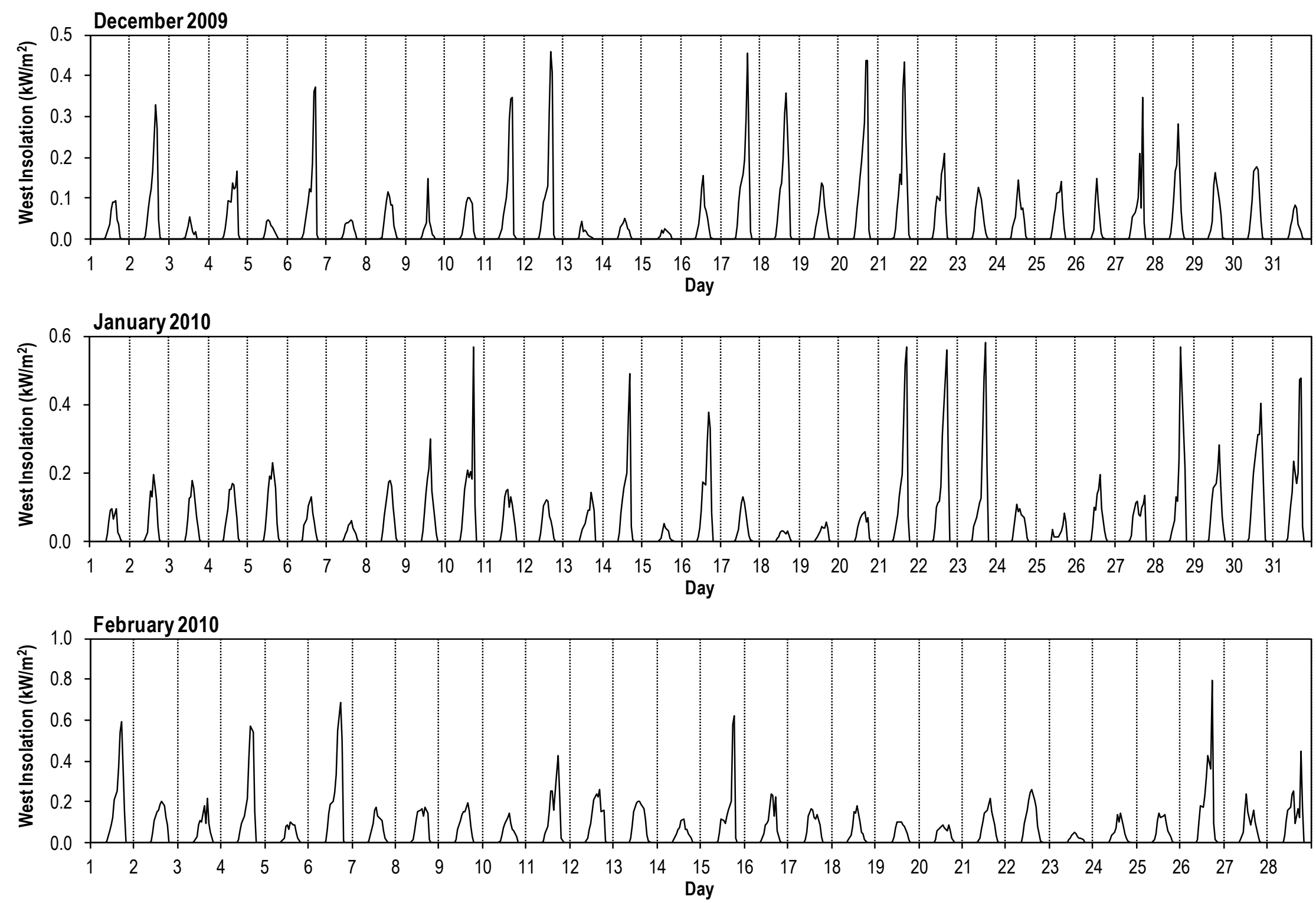

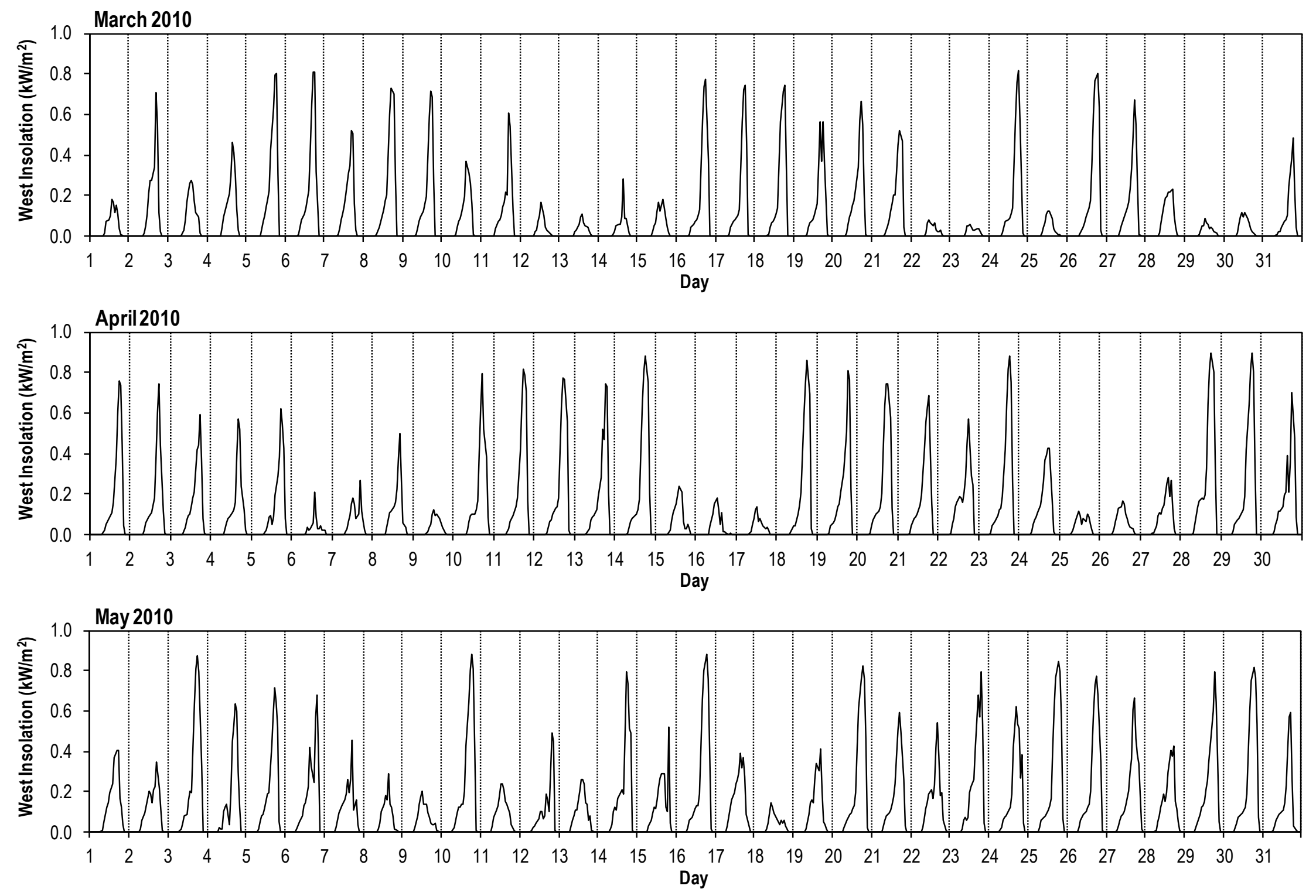

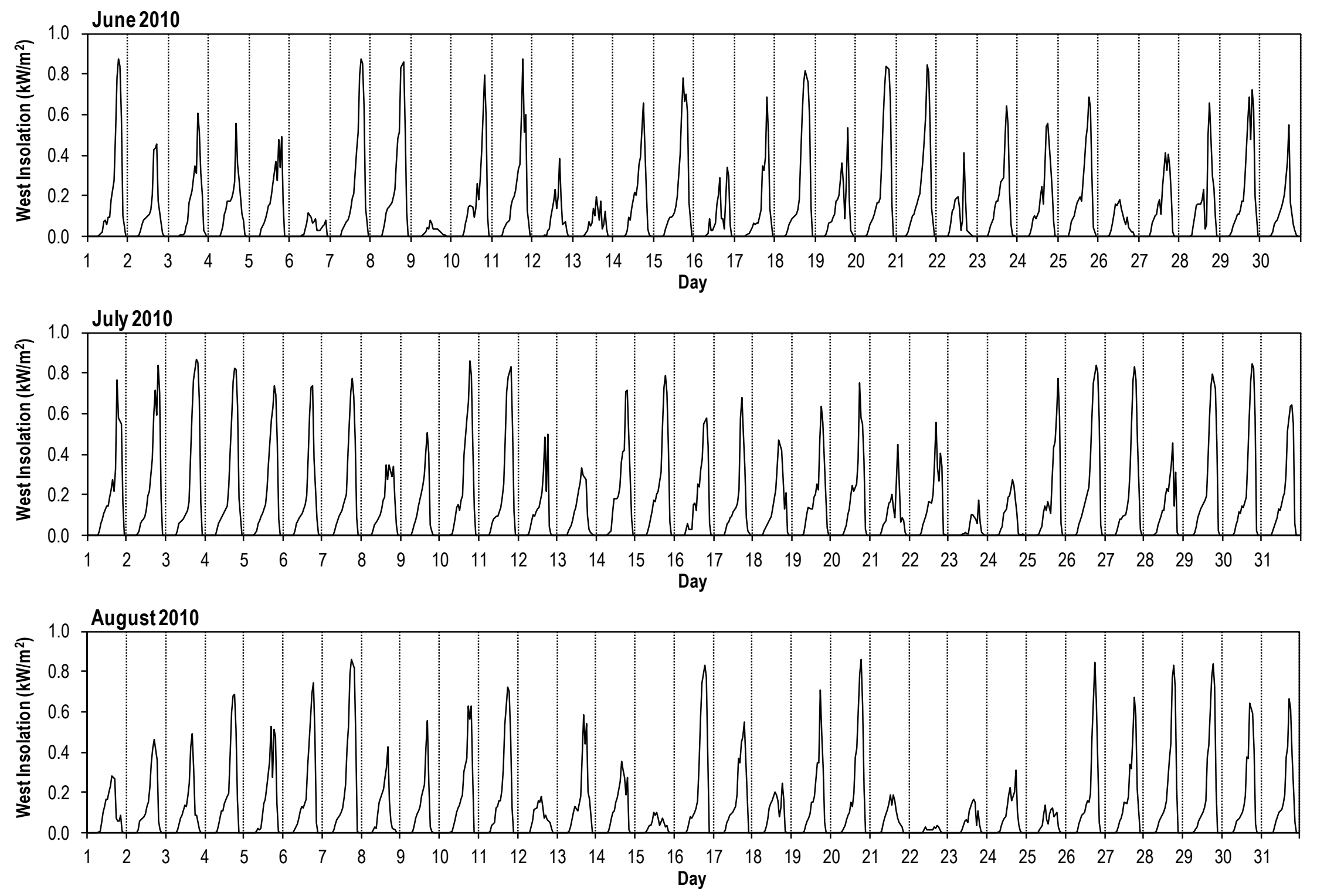


\section{Appendix F: Wind Speed}
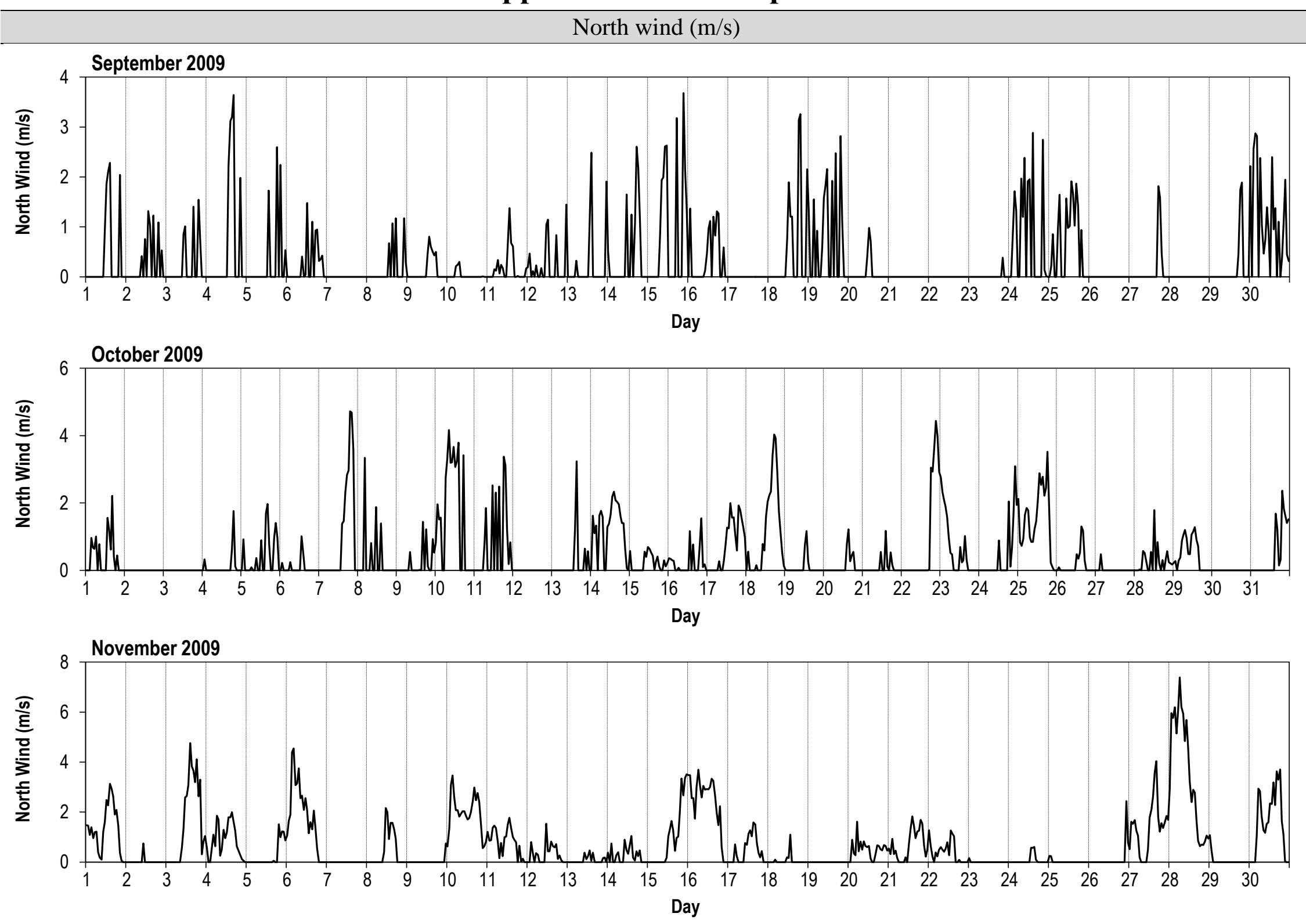

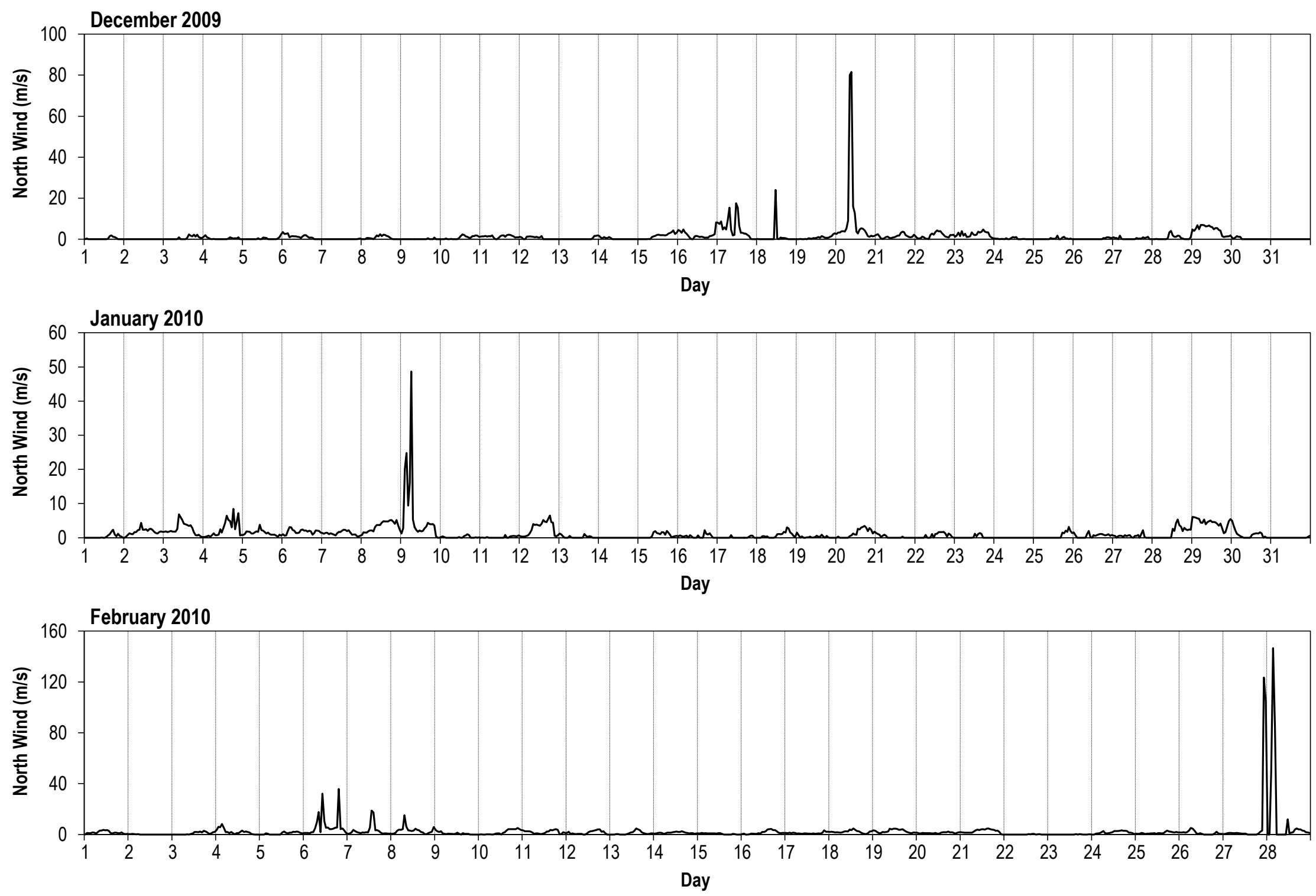

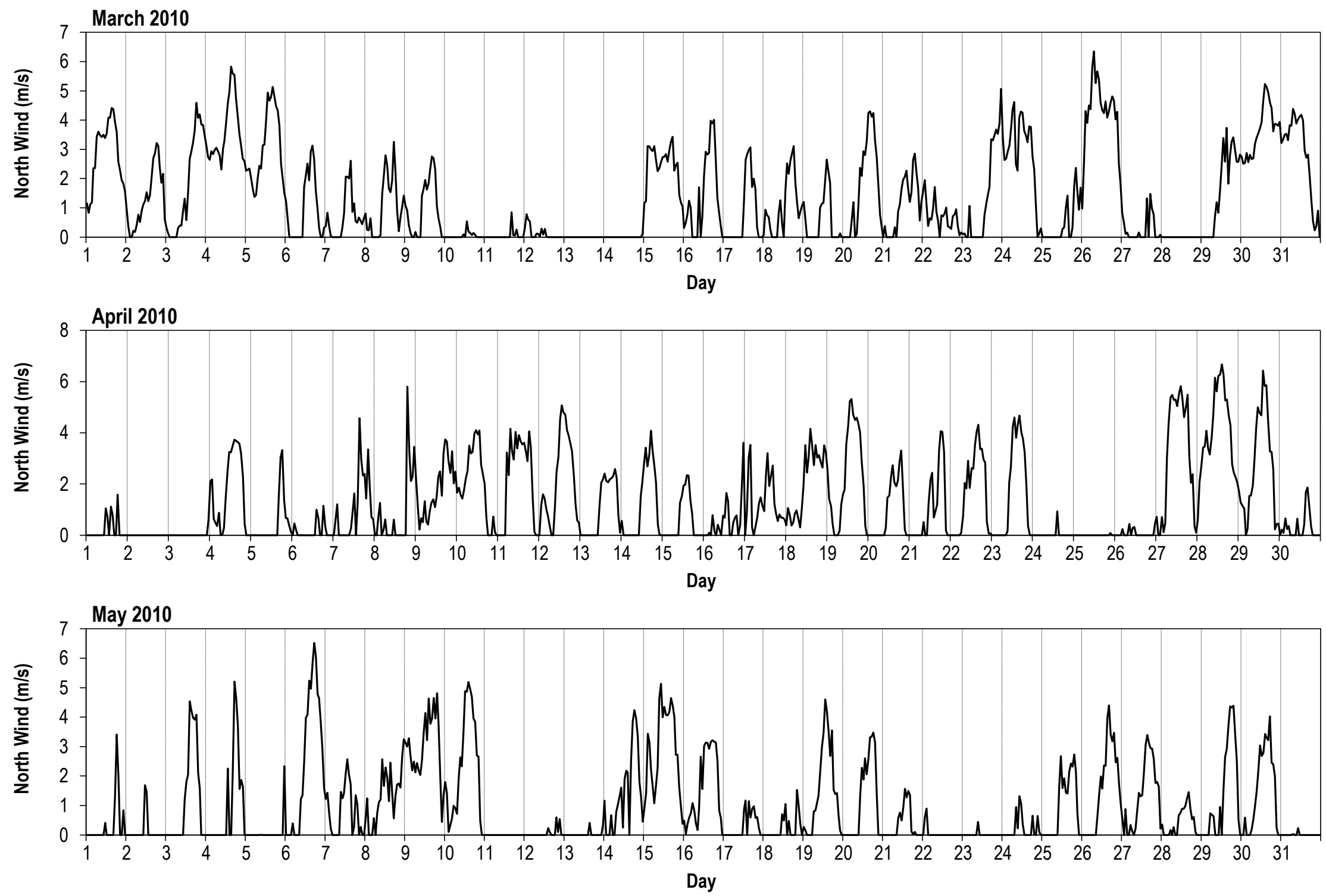

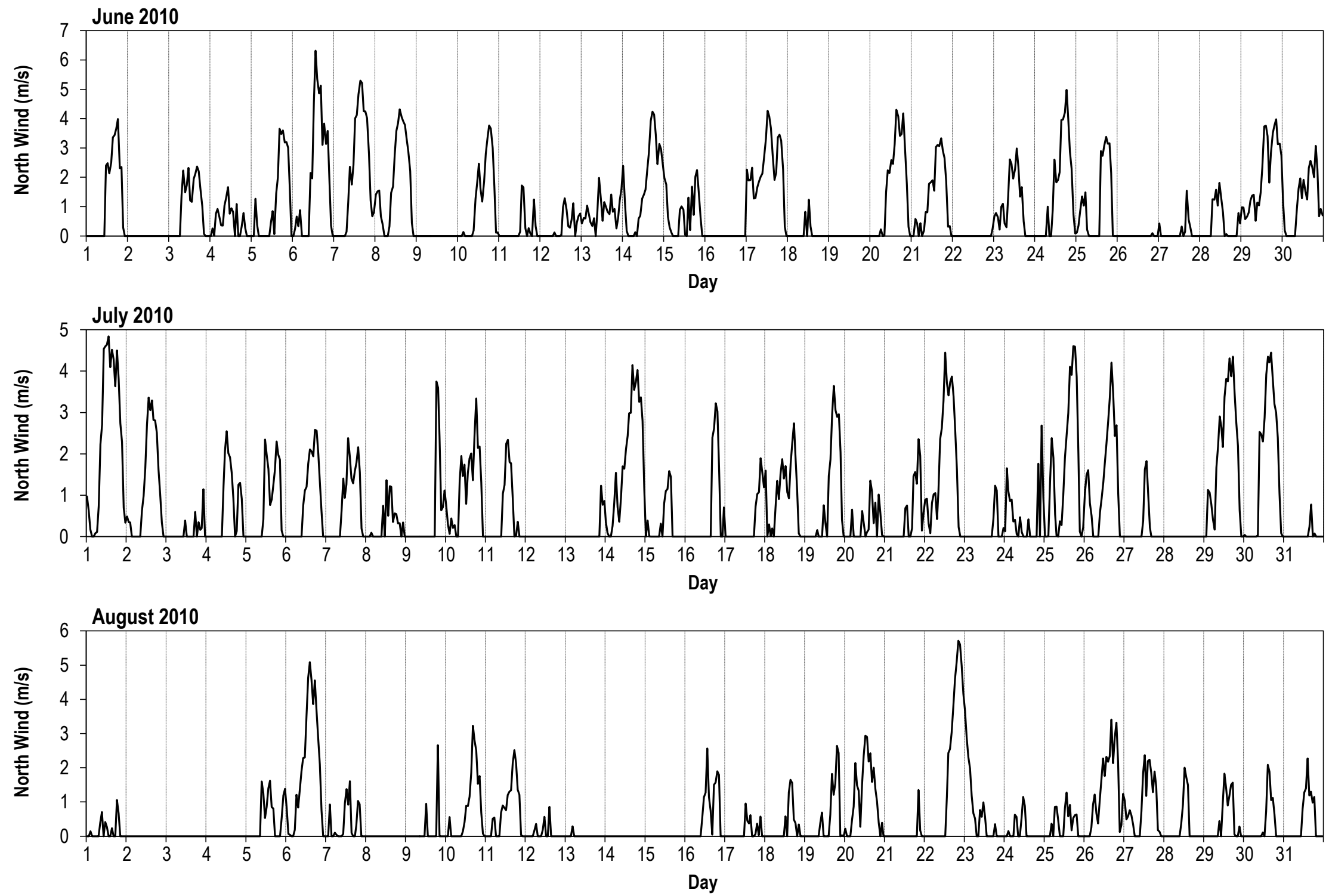

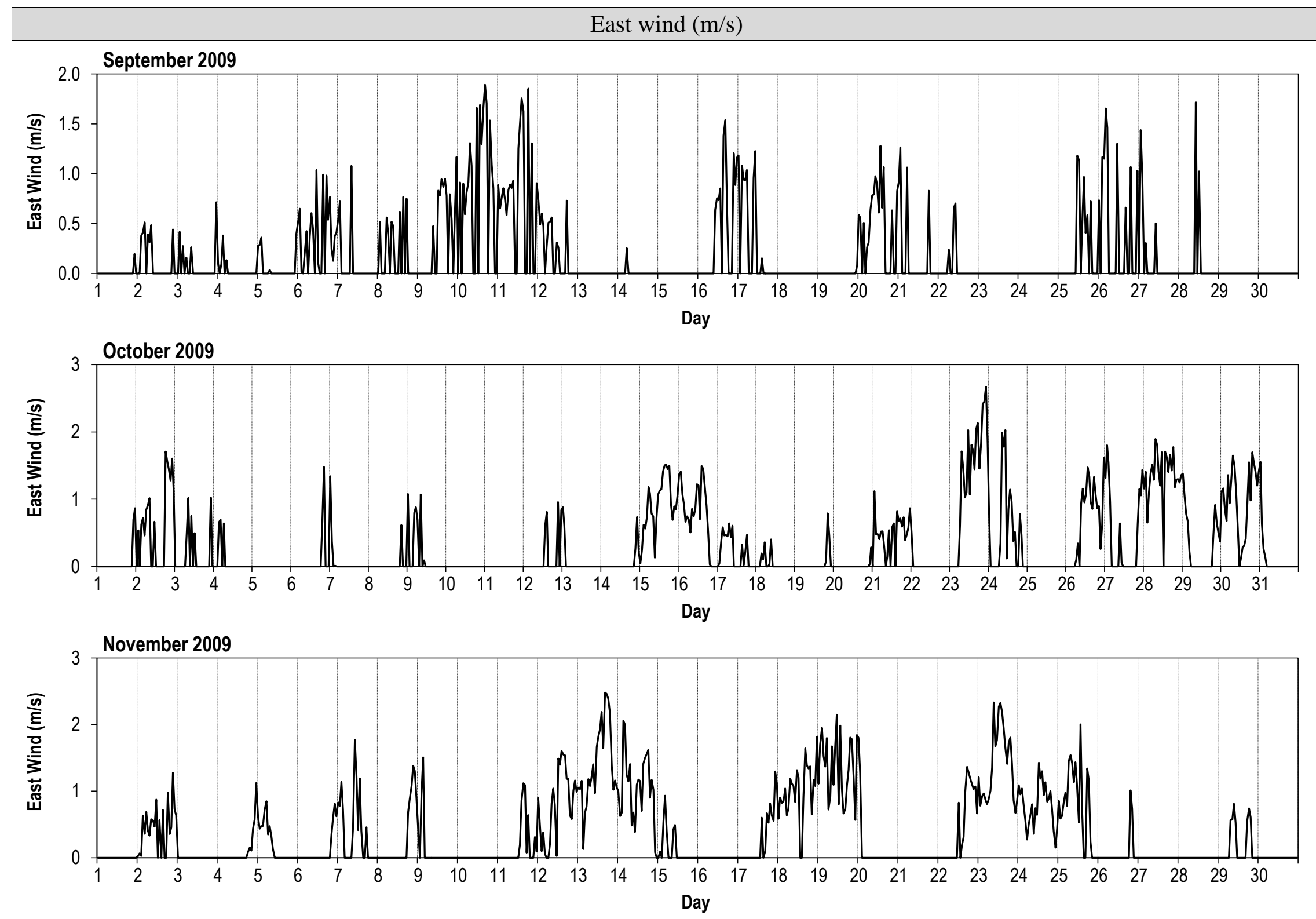

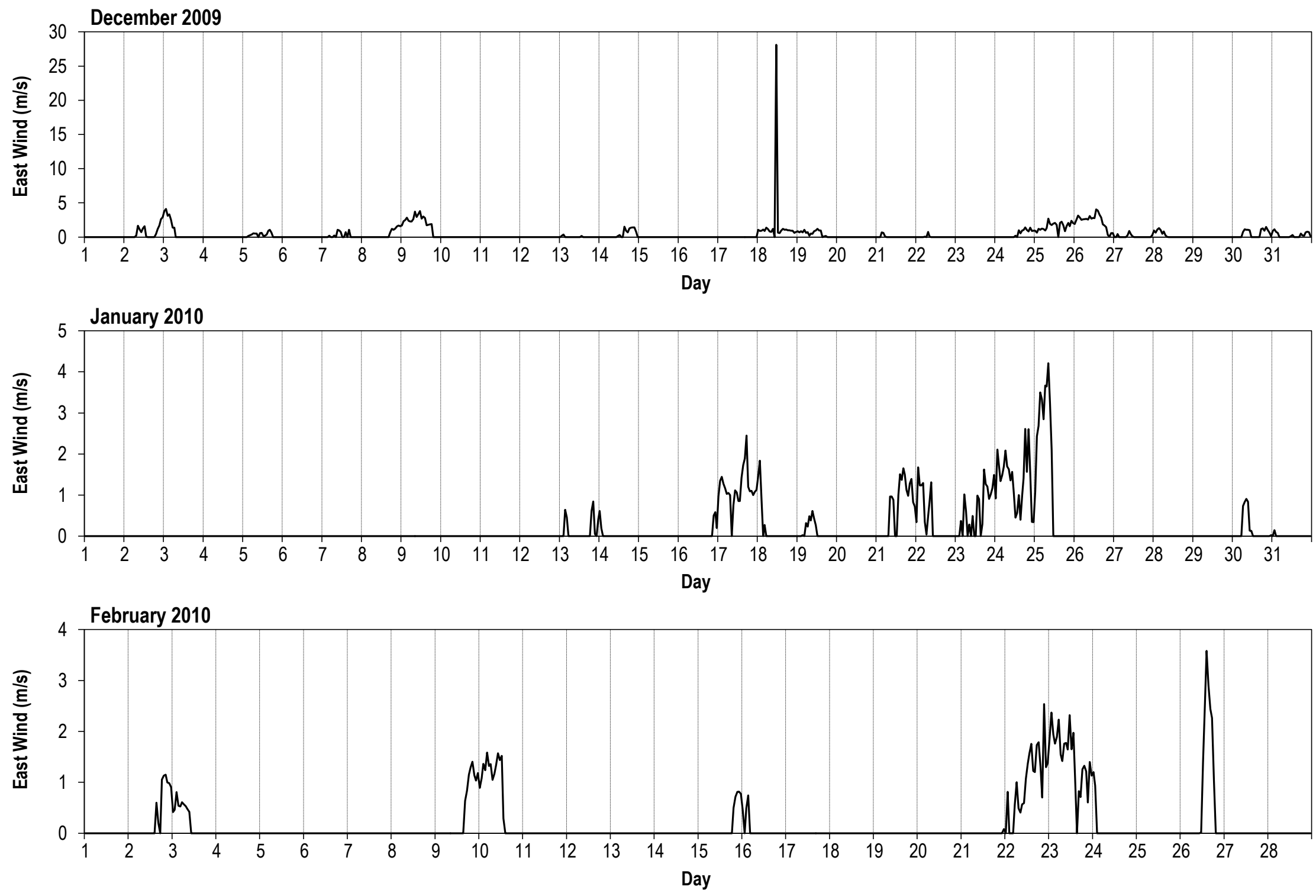

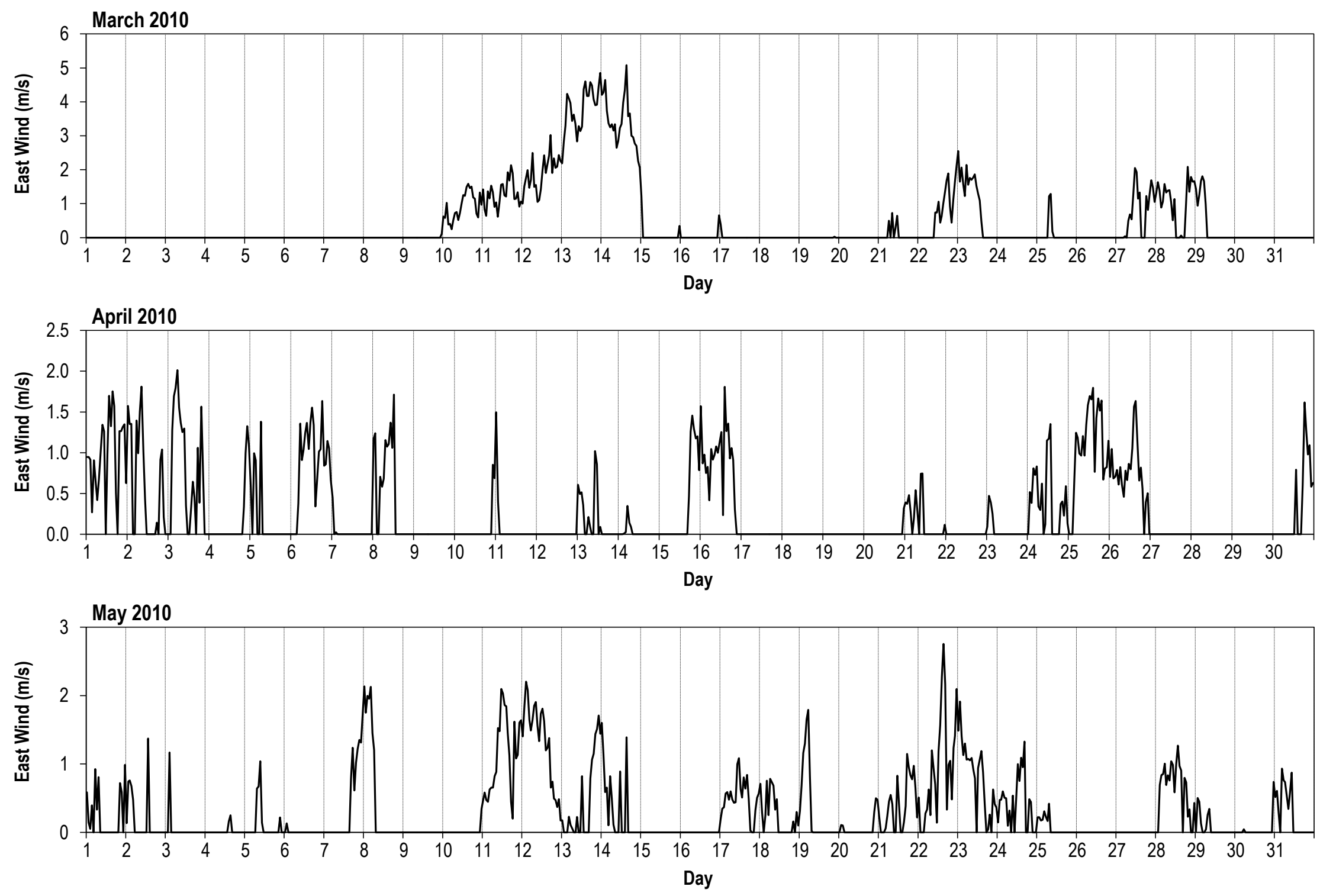

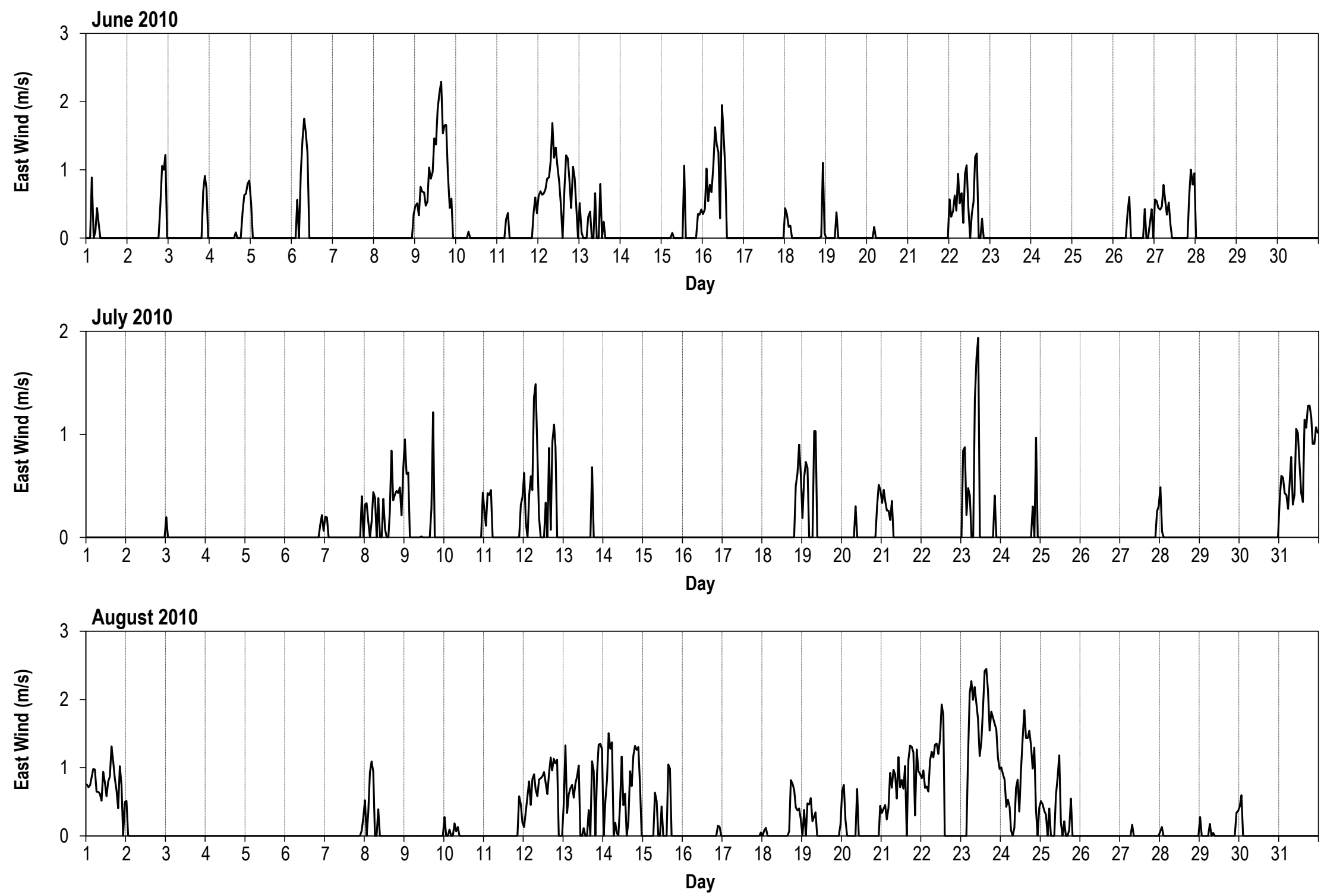

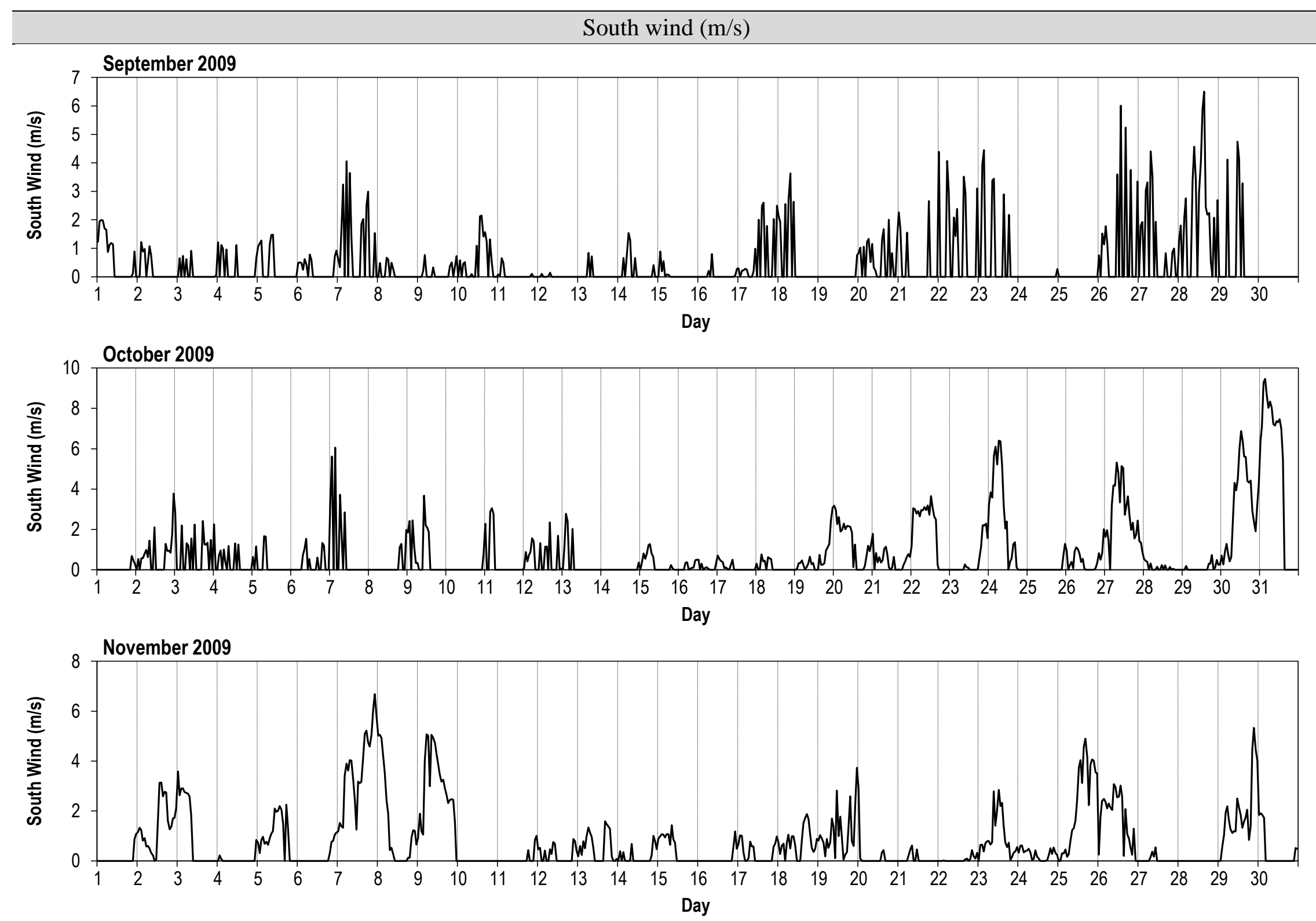

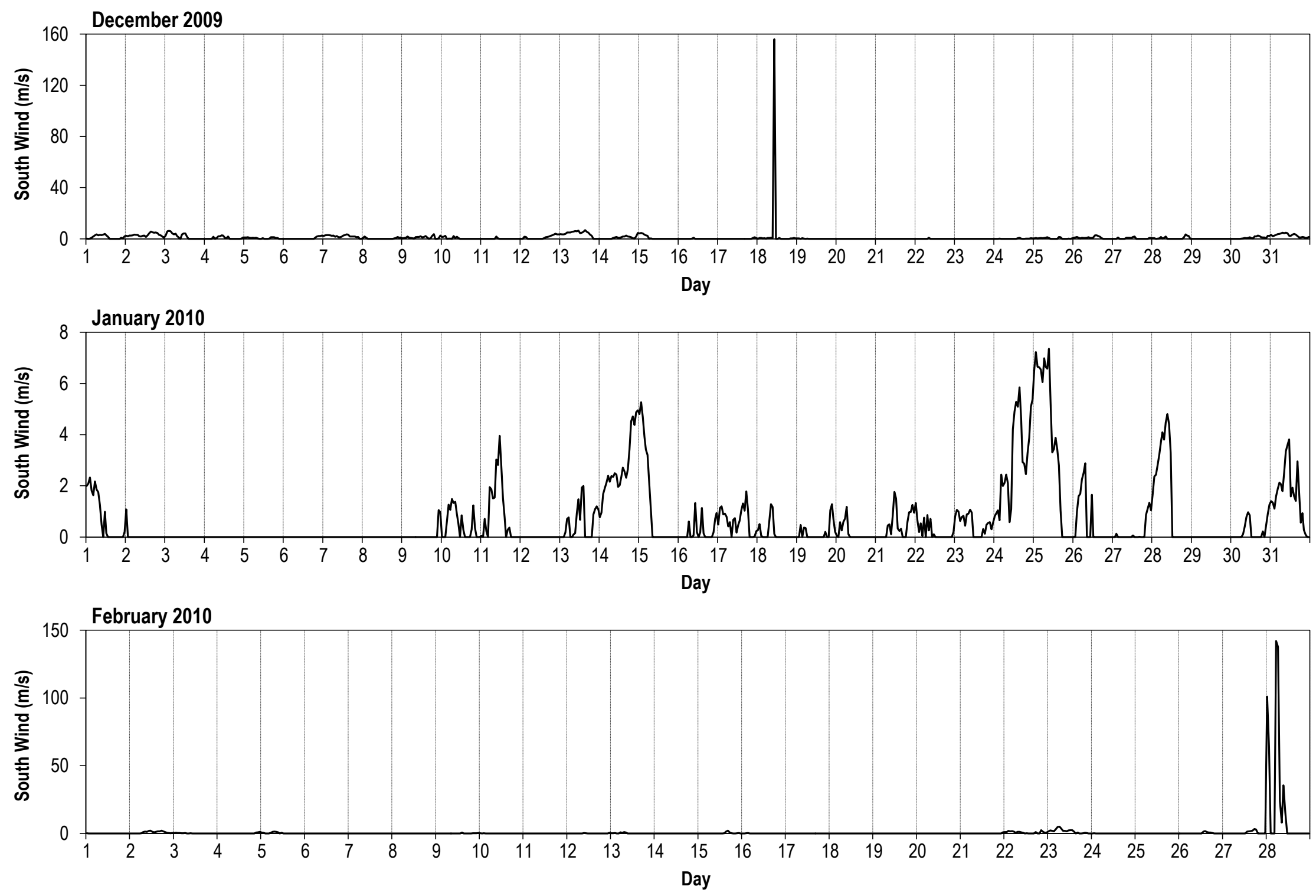

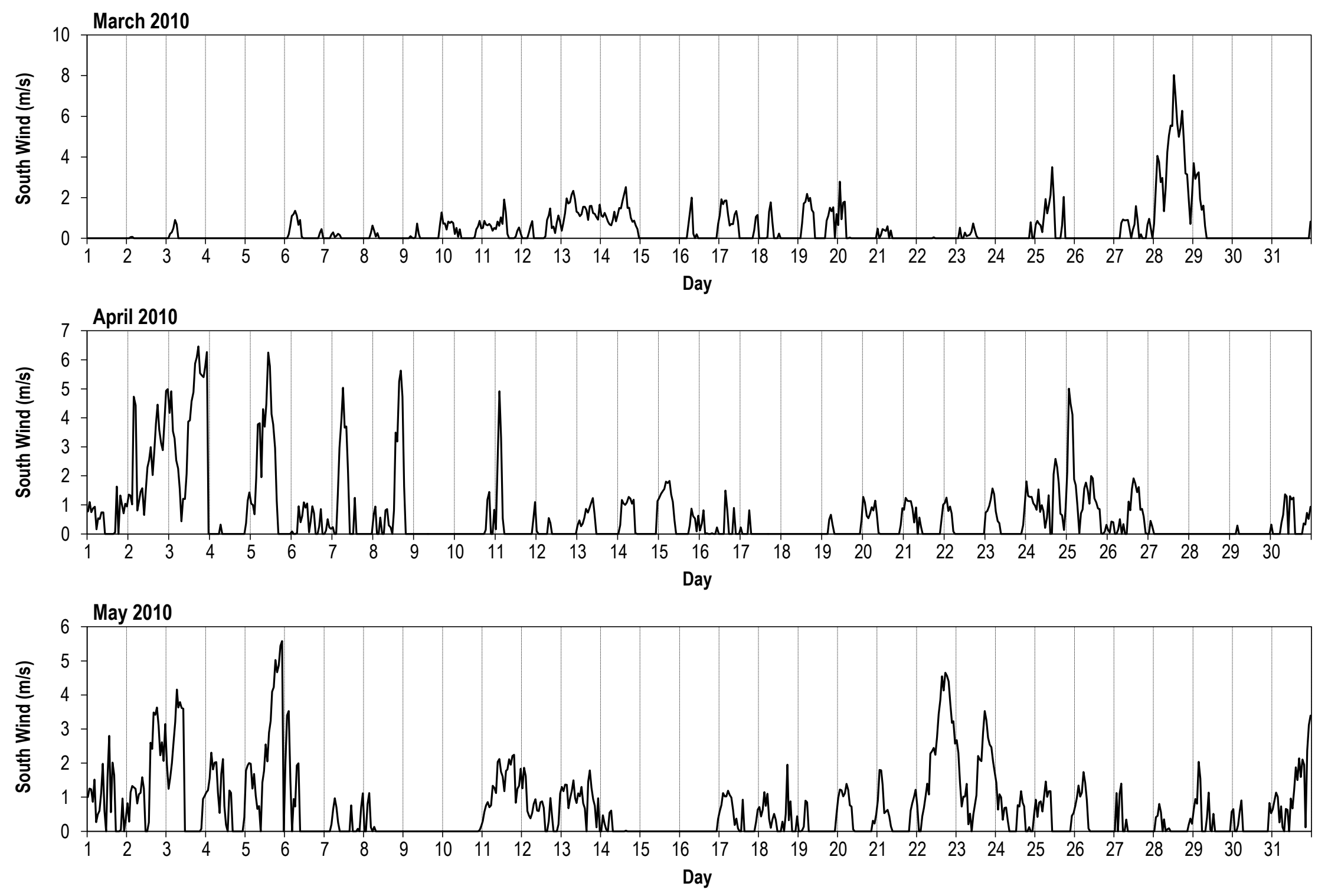

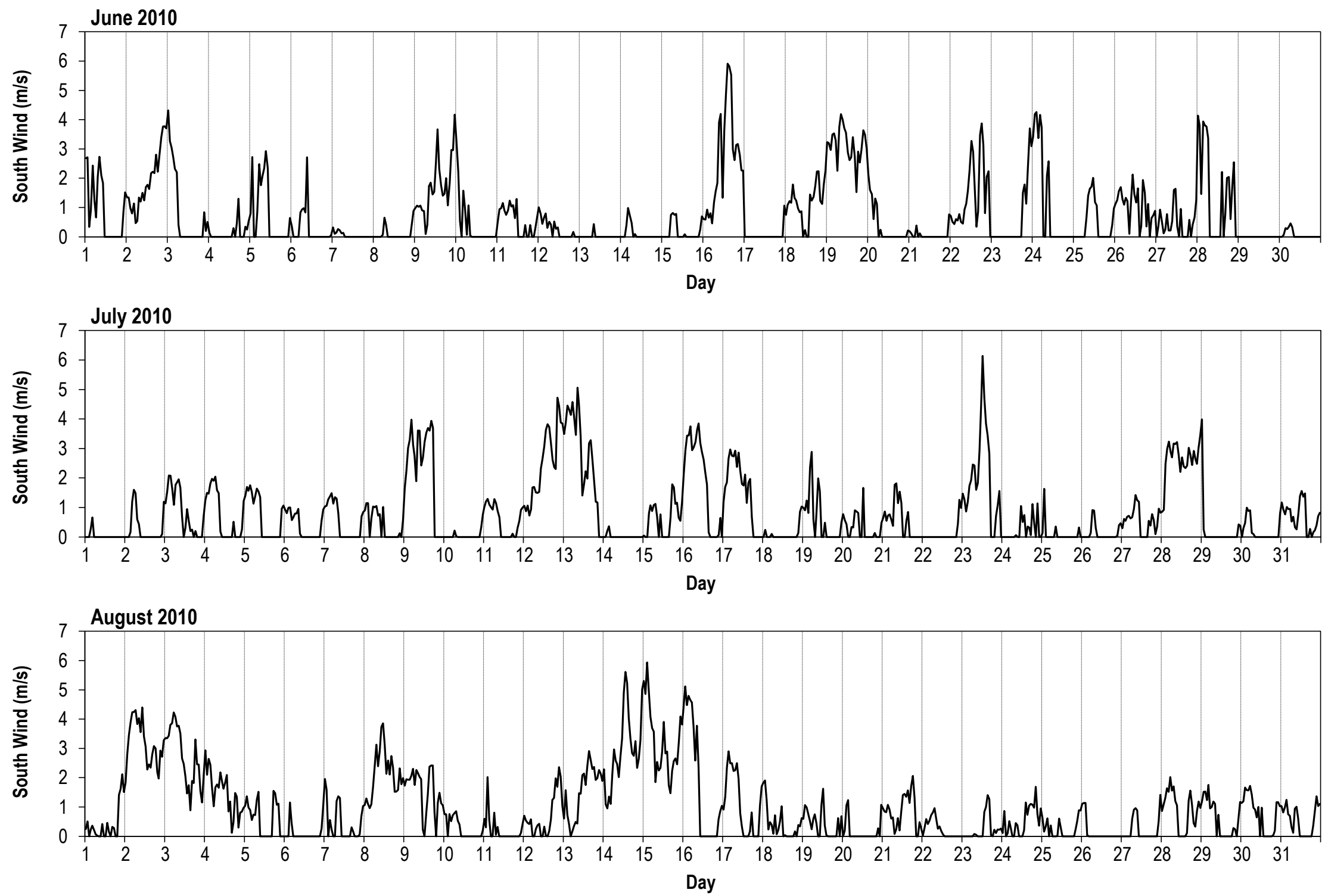

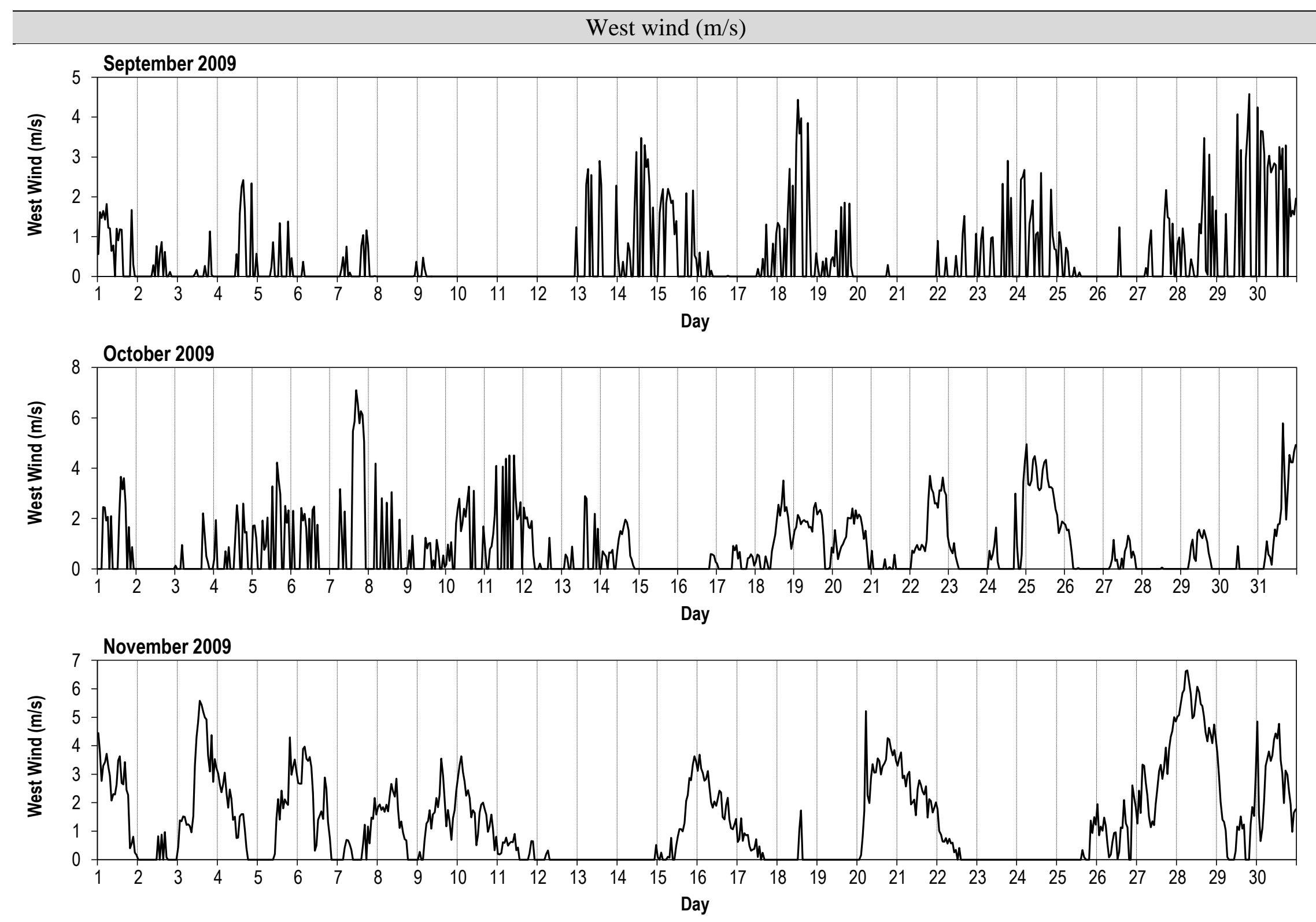

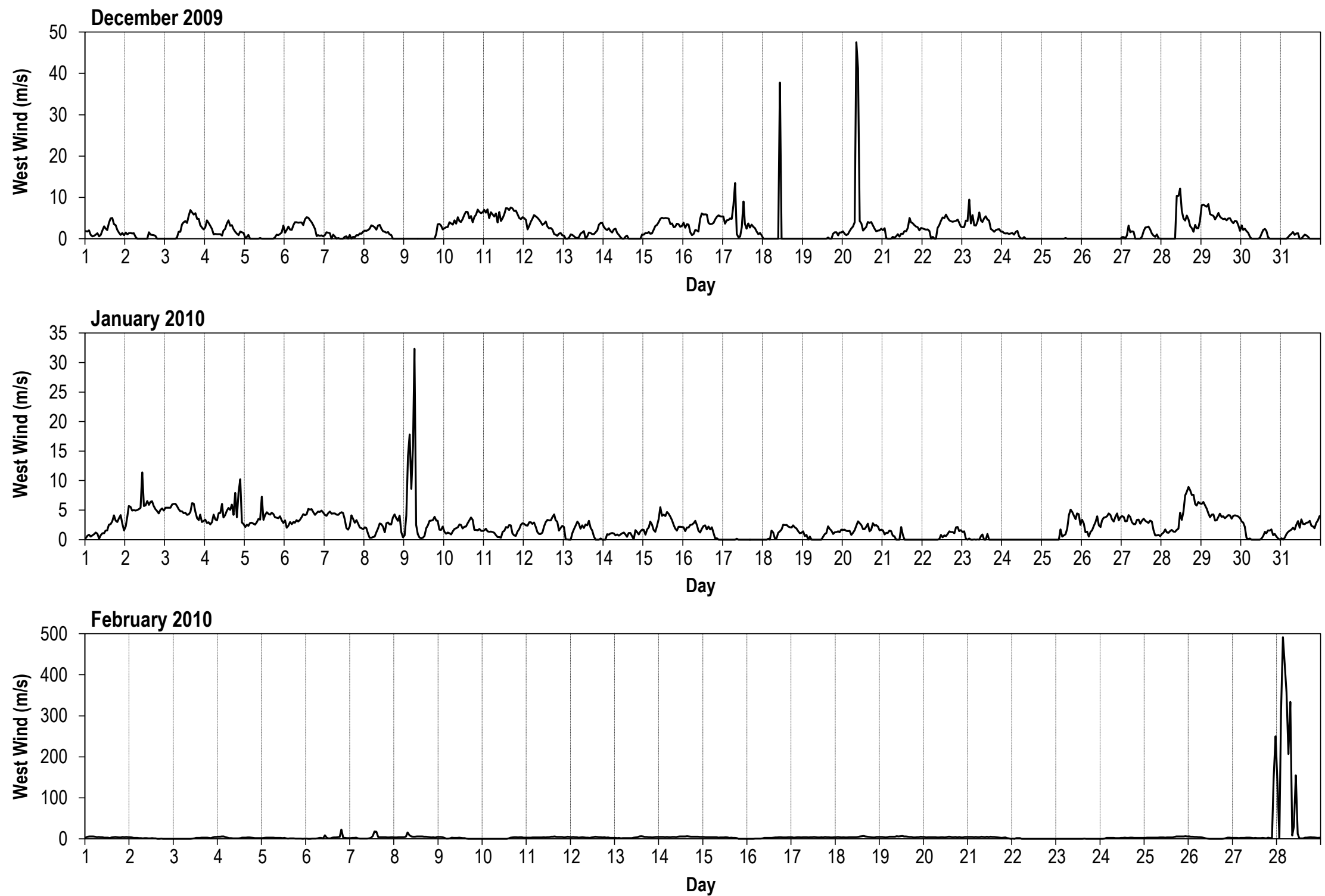

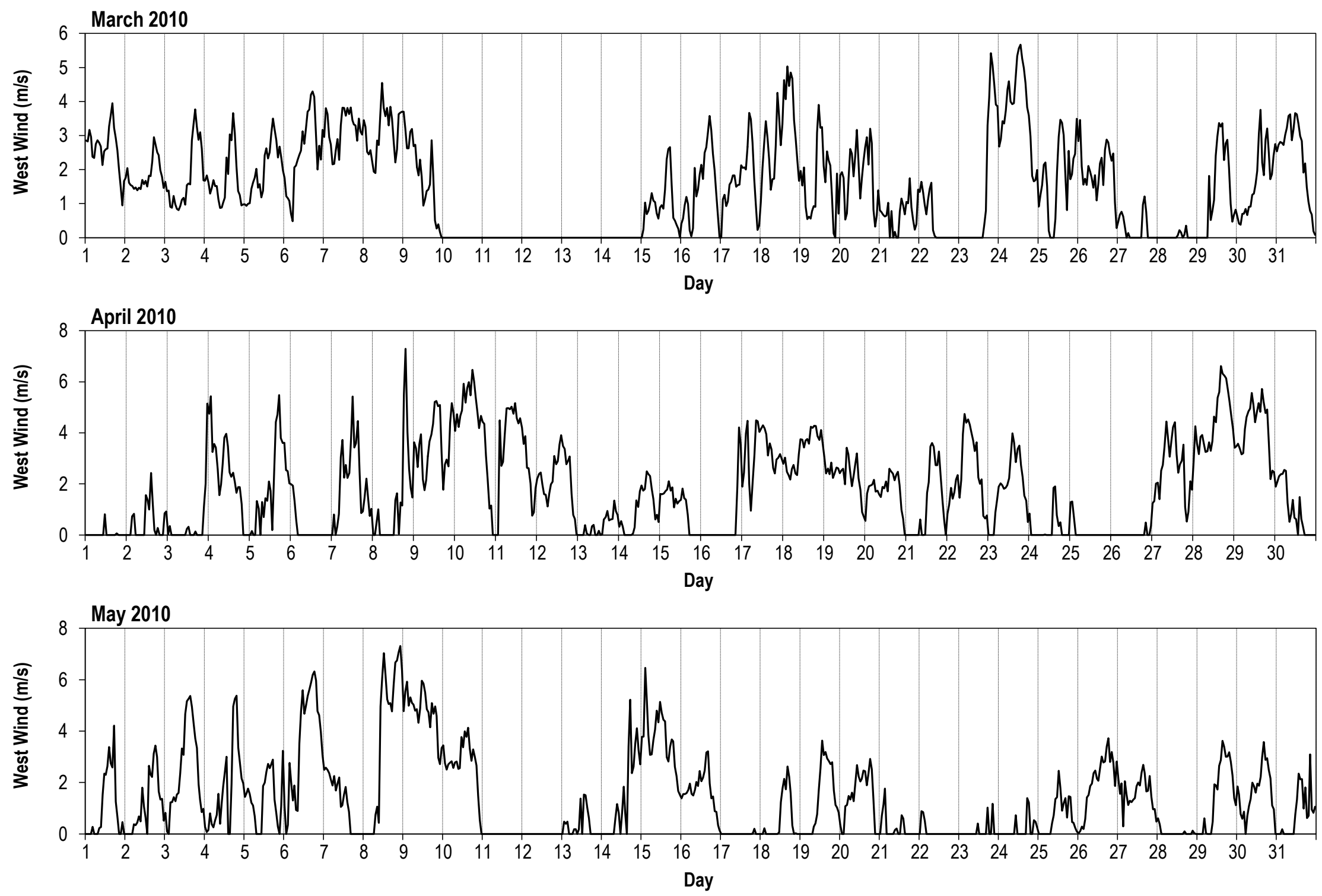

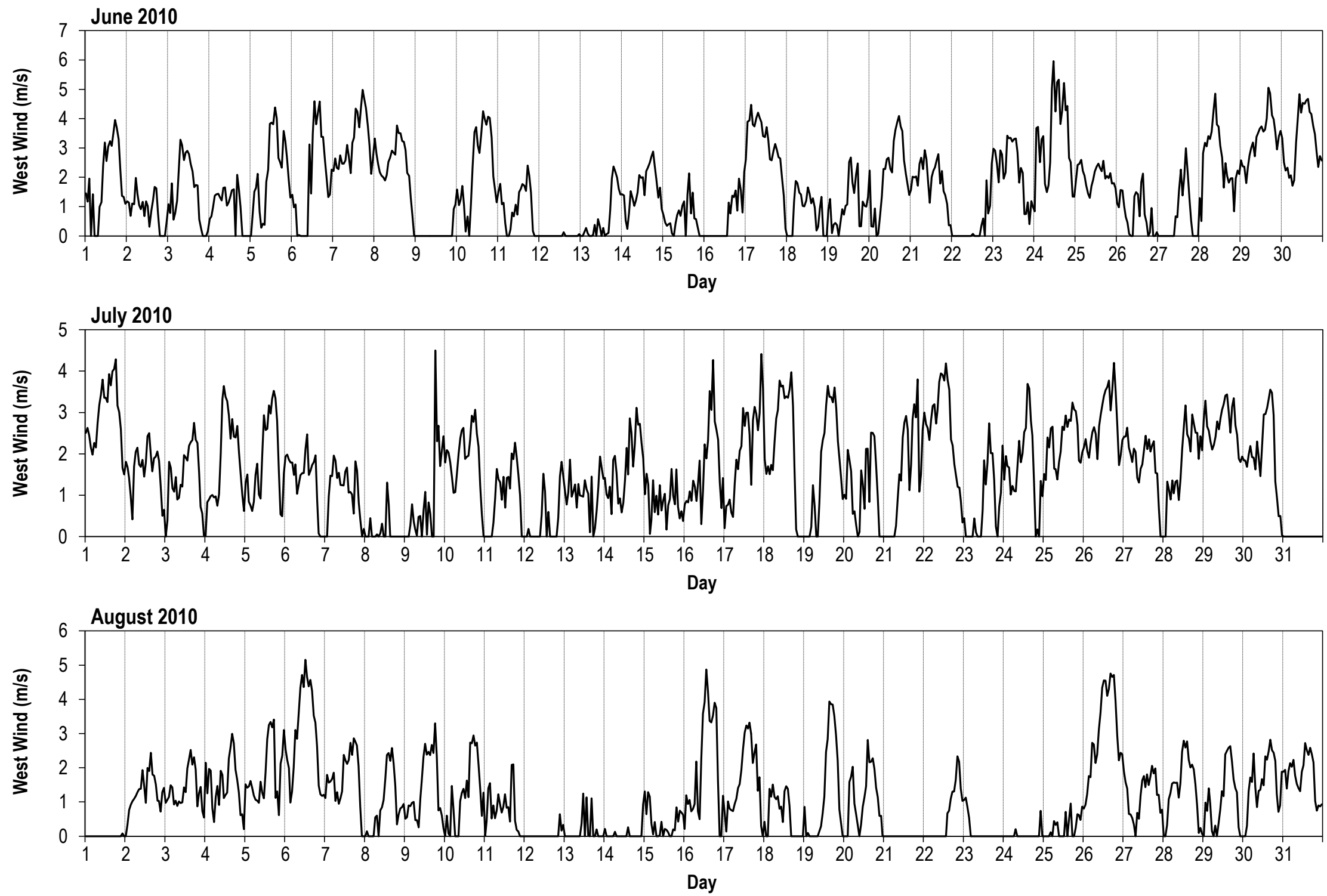


\section{Appendix G: Rain}
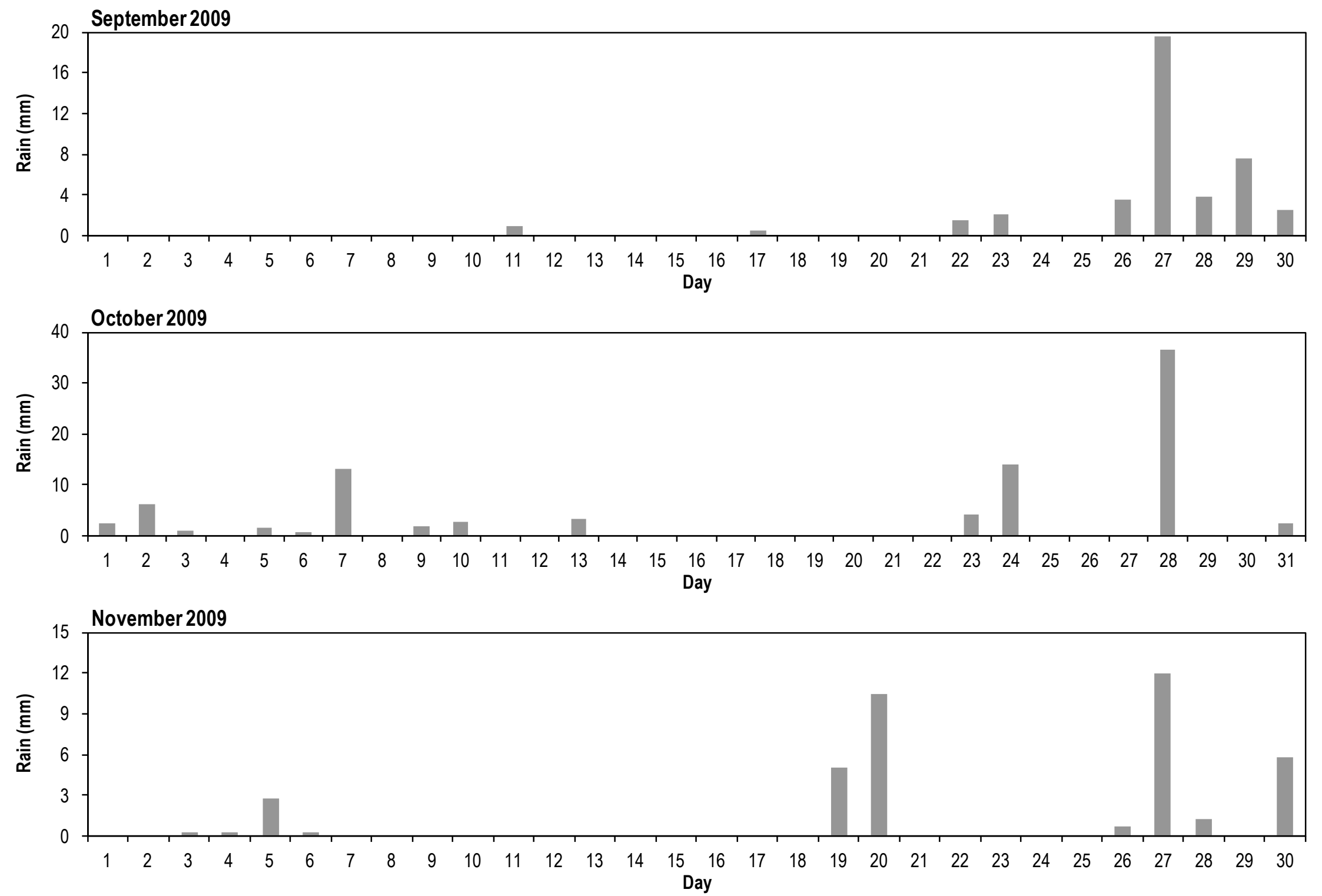

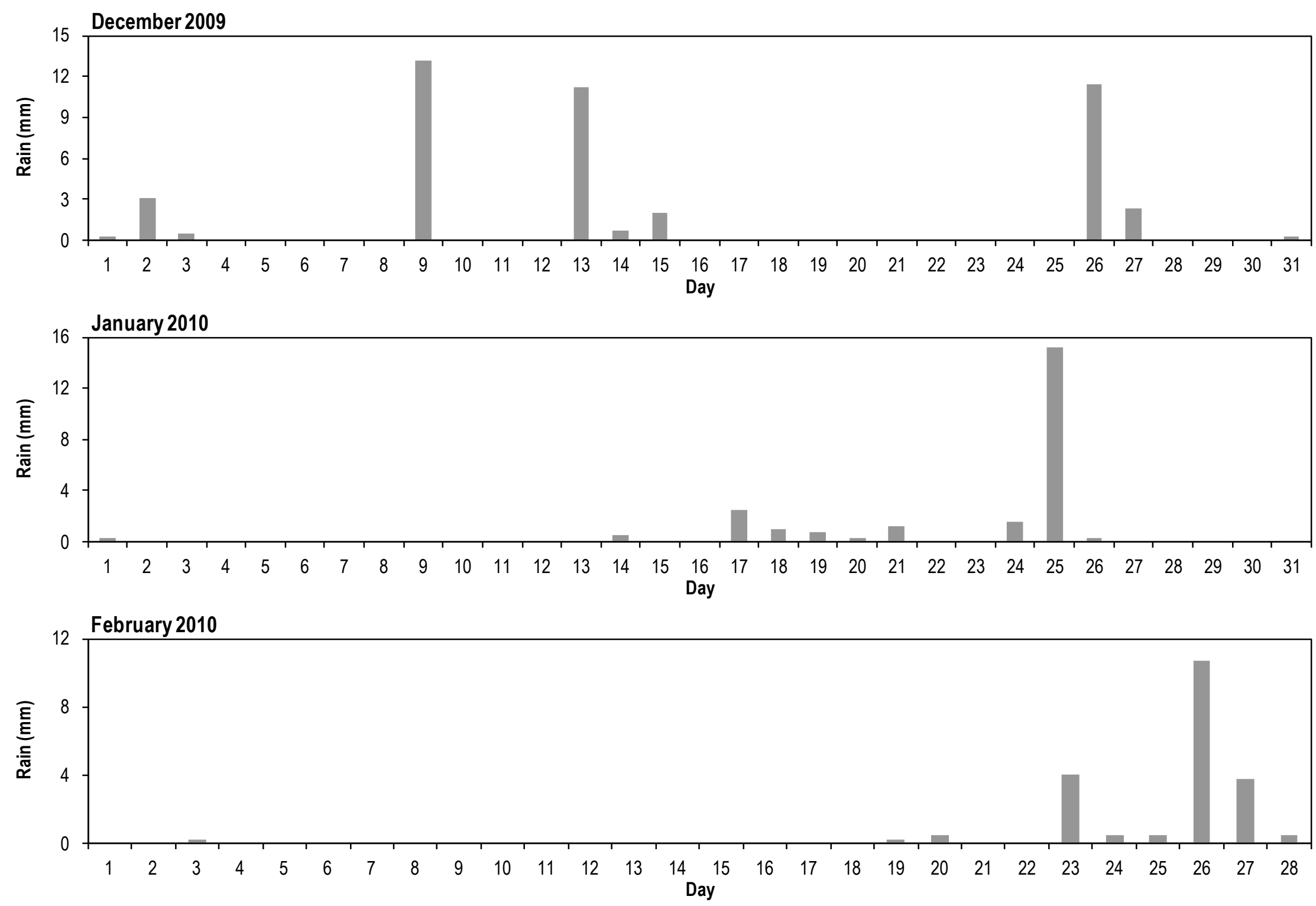

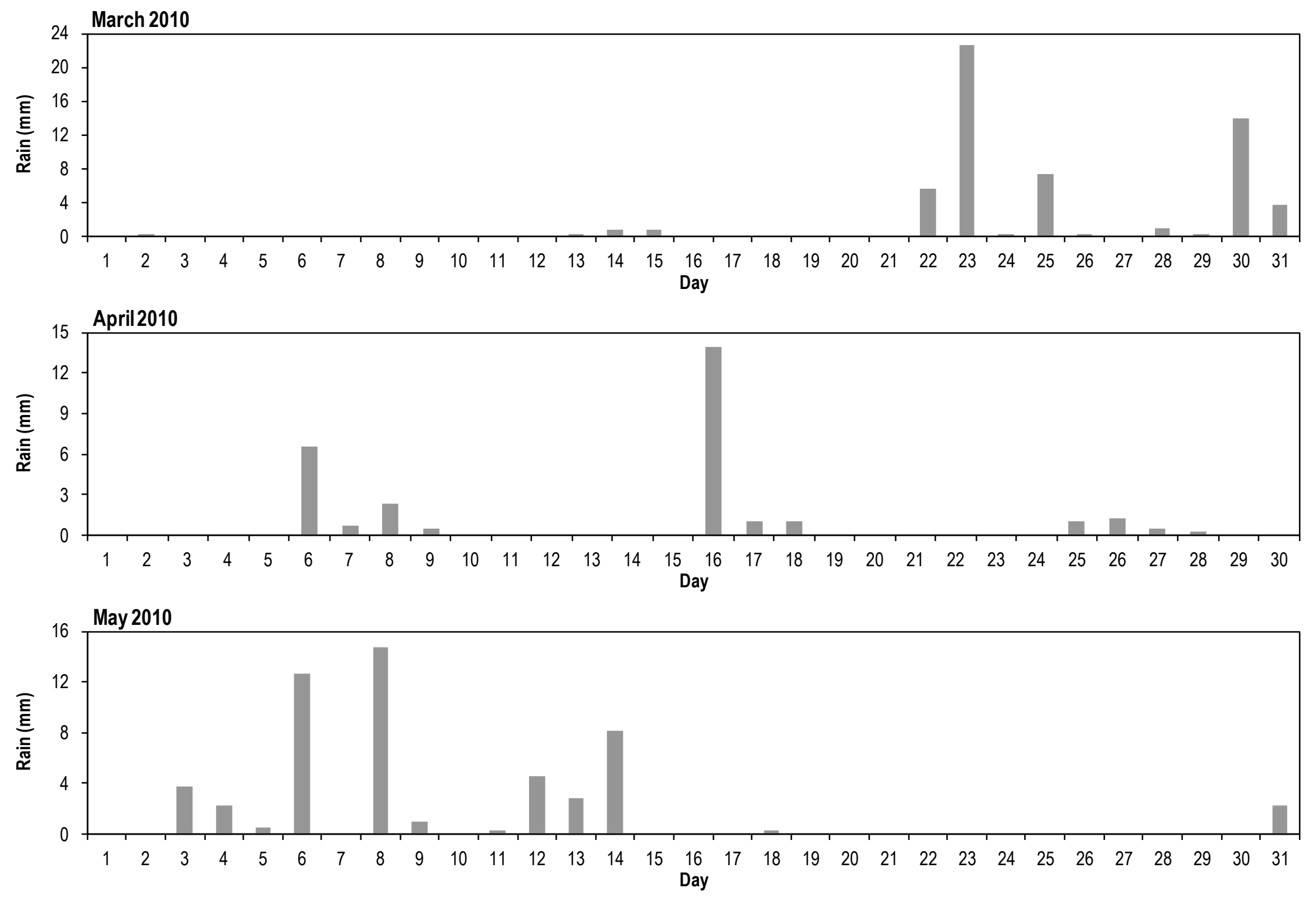

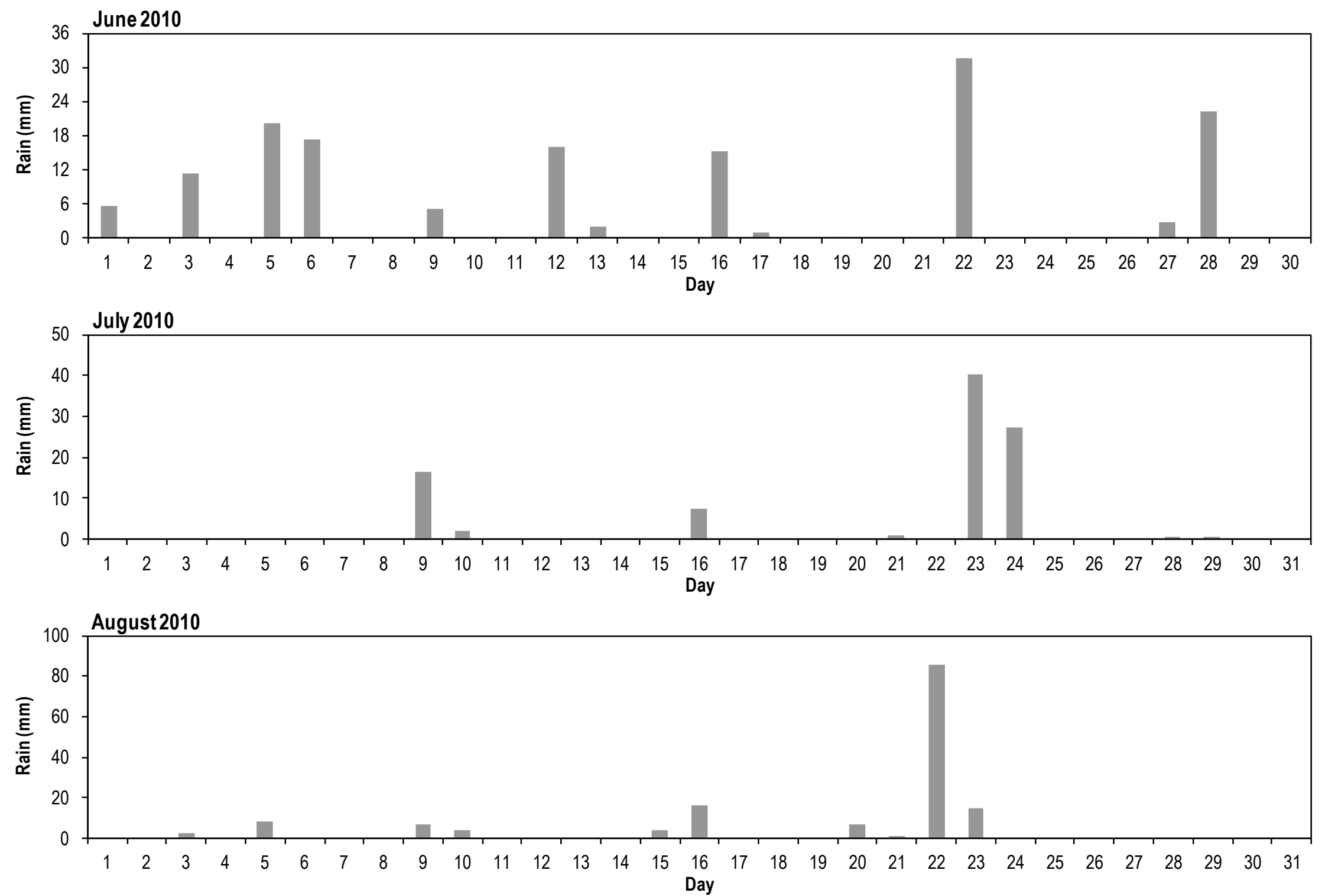


\section{Appendix H: Pressure Differential}

Pressure differential $(\mathrm{Pa})$ across the north side of the test hut
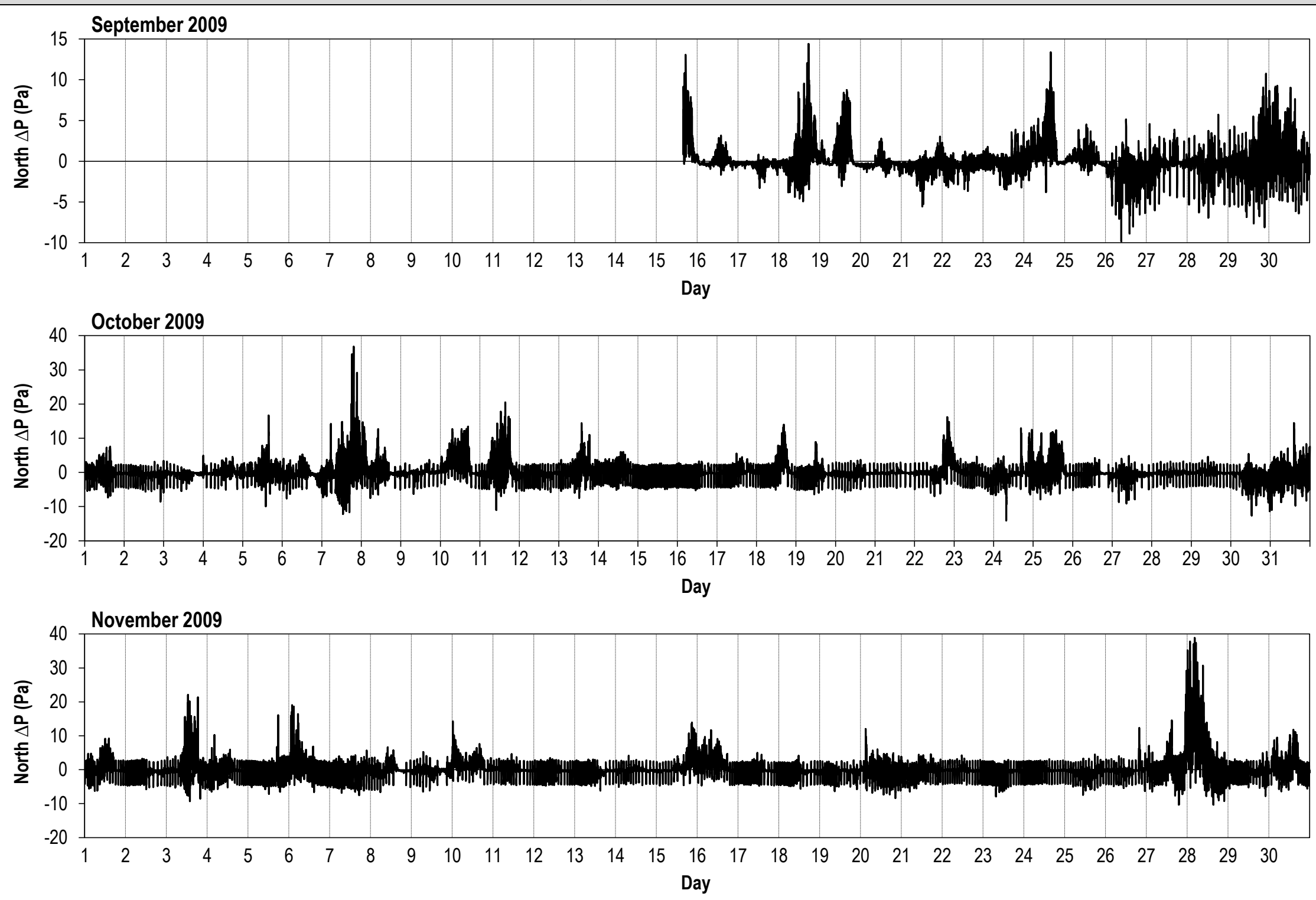

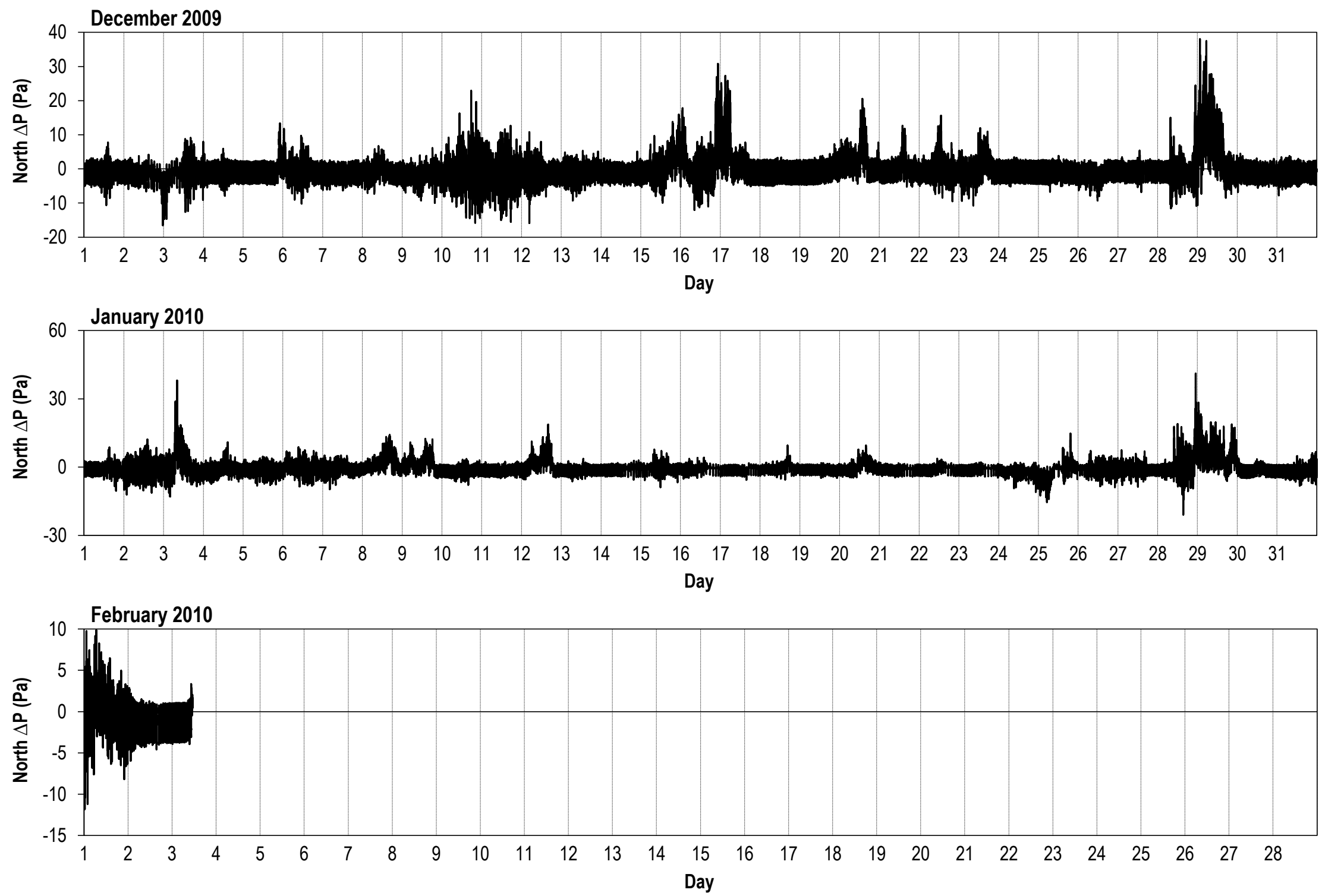


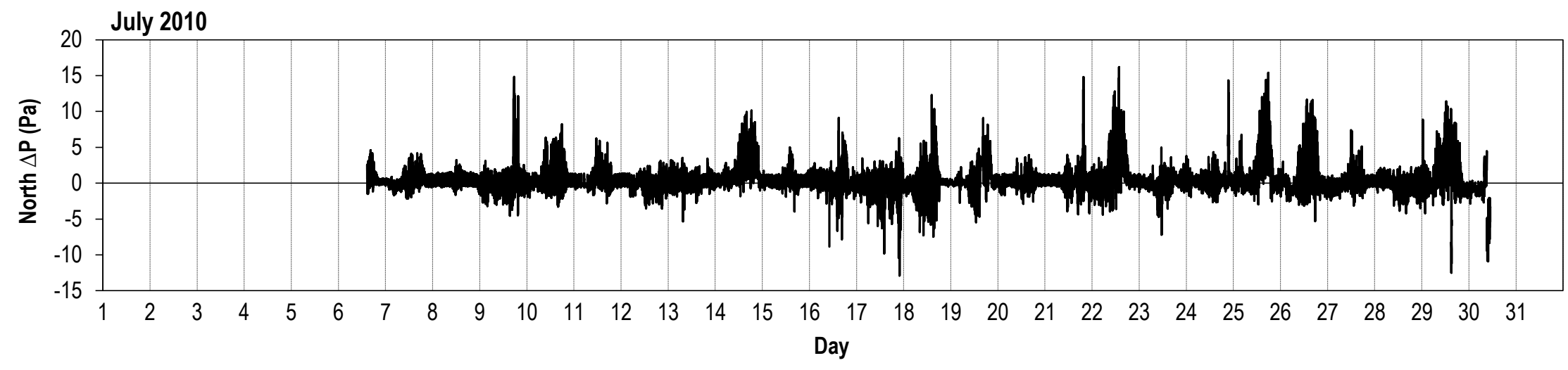

Notes:

a. Data missing due to equipment malfunction.

b. July measurements obtained while Syracuse University pressurized the test hut to characterize the air leakage of wall panels are not included in the graph above. 
Pressure differential $(\mathrm{Pa})$ across the east side of the test hut
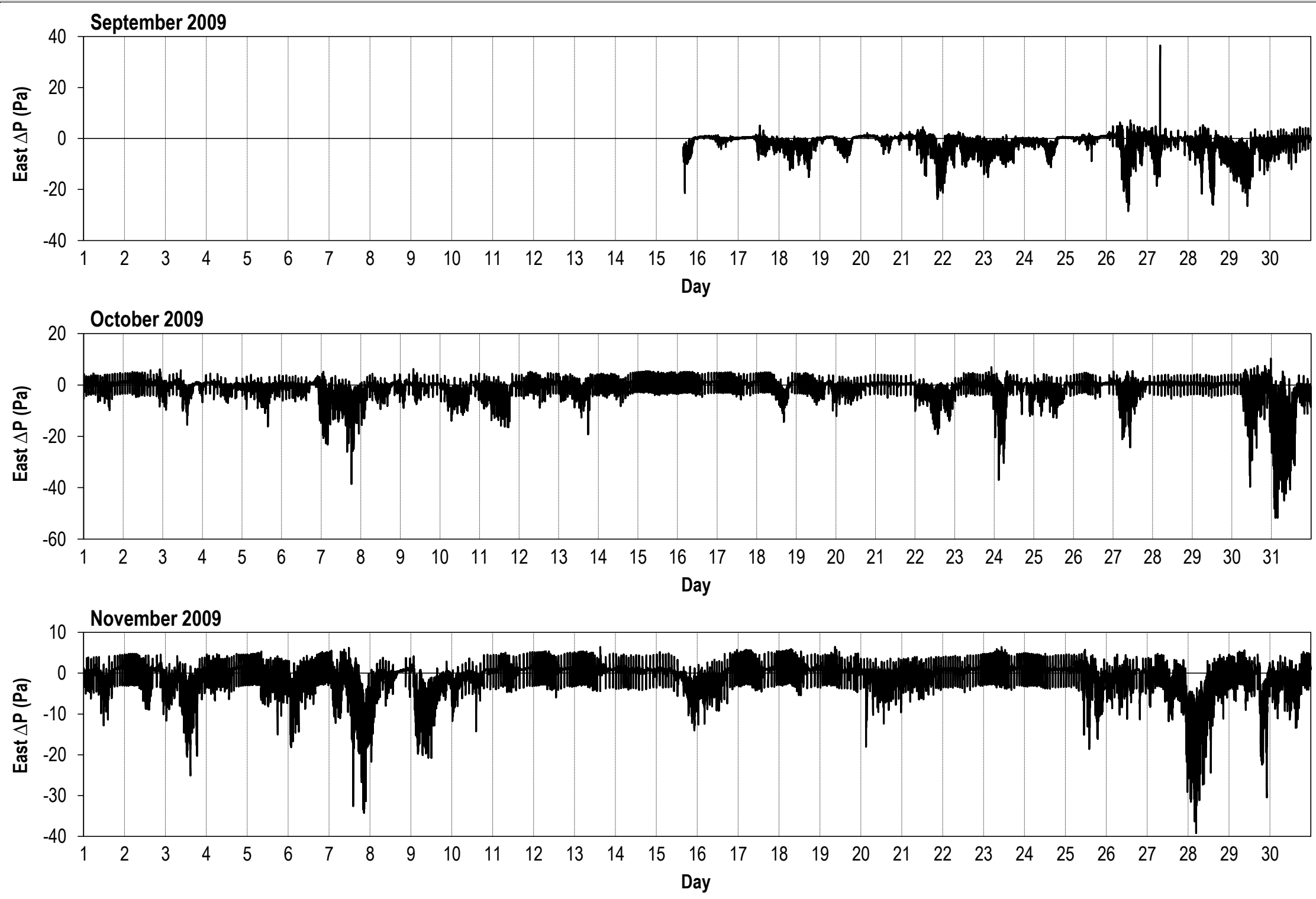

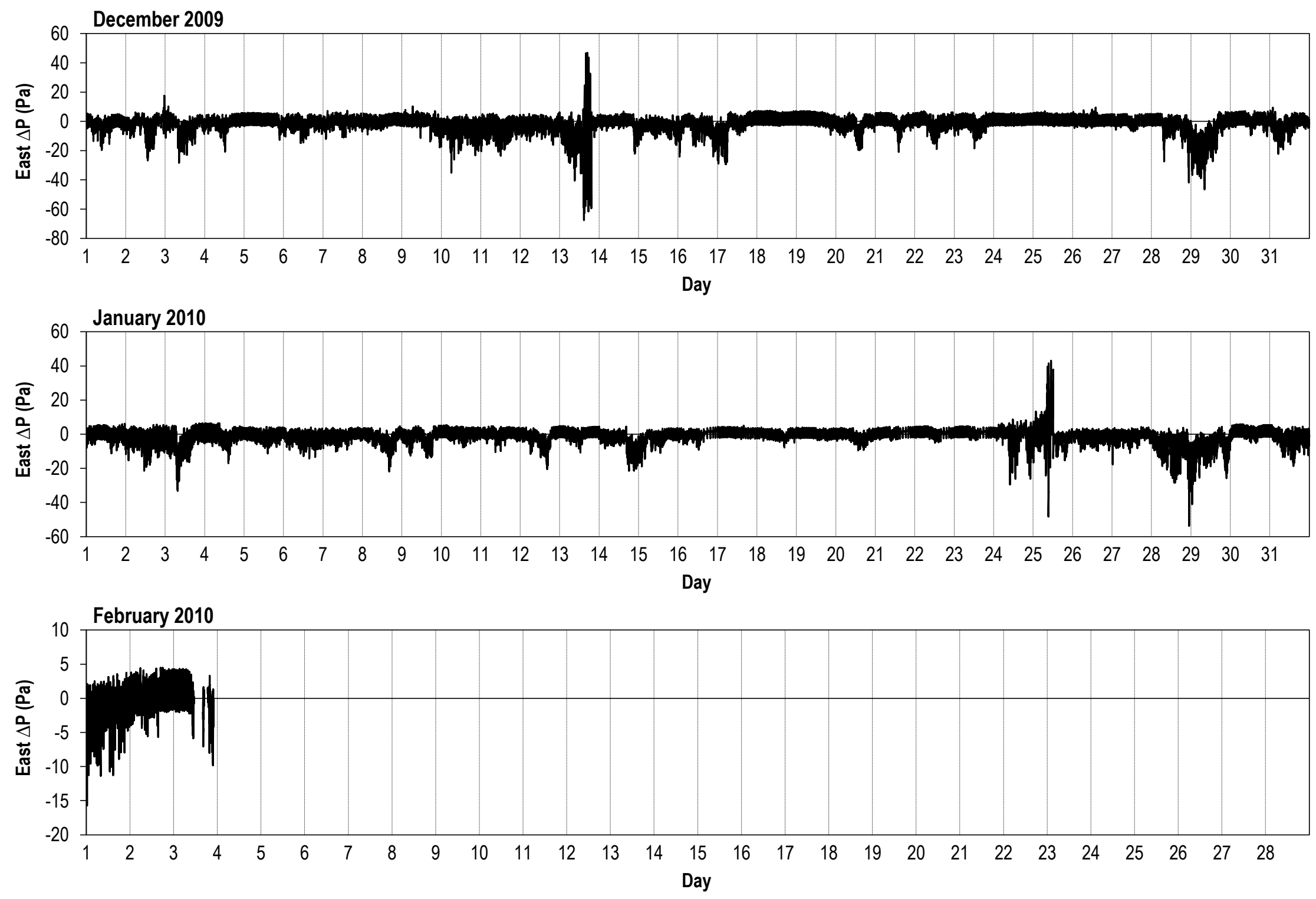

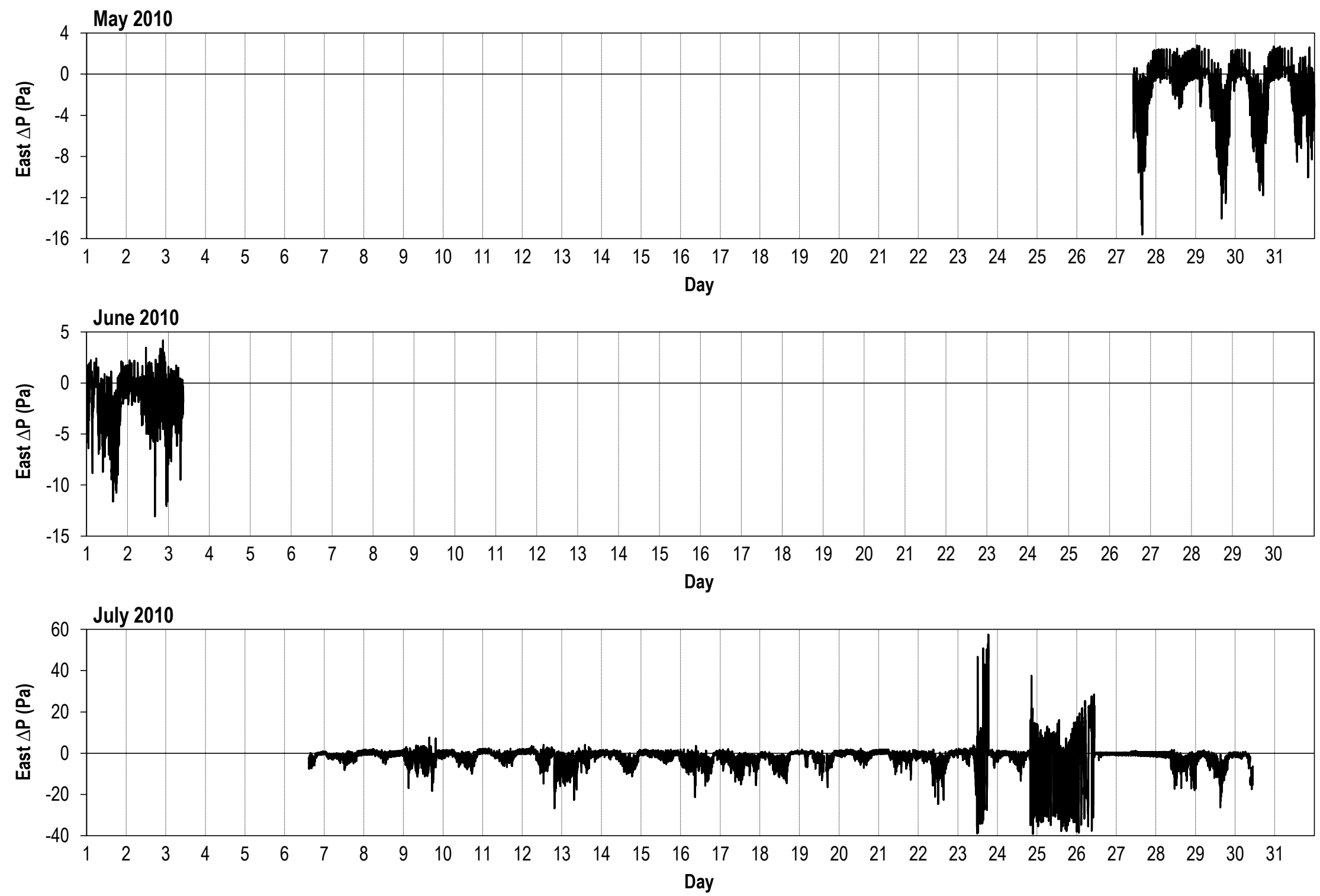

Notes:

a. Data missing due to equipment malfunction. 
b. July measurements obtained while Syracuse University pressurized the test hut to characterize the air leakage of wall panels are not included in the graph above.

Pressure differential $(\mathrm{Pa})$ across the south side of the test hut
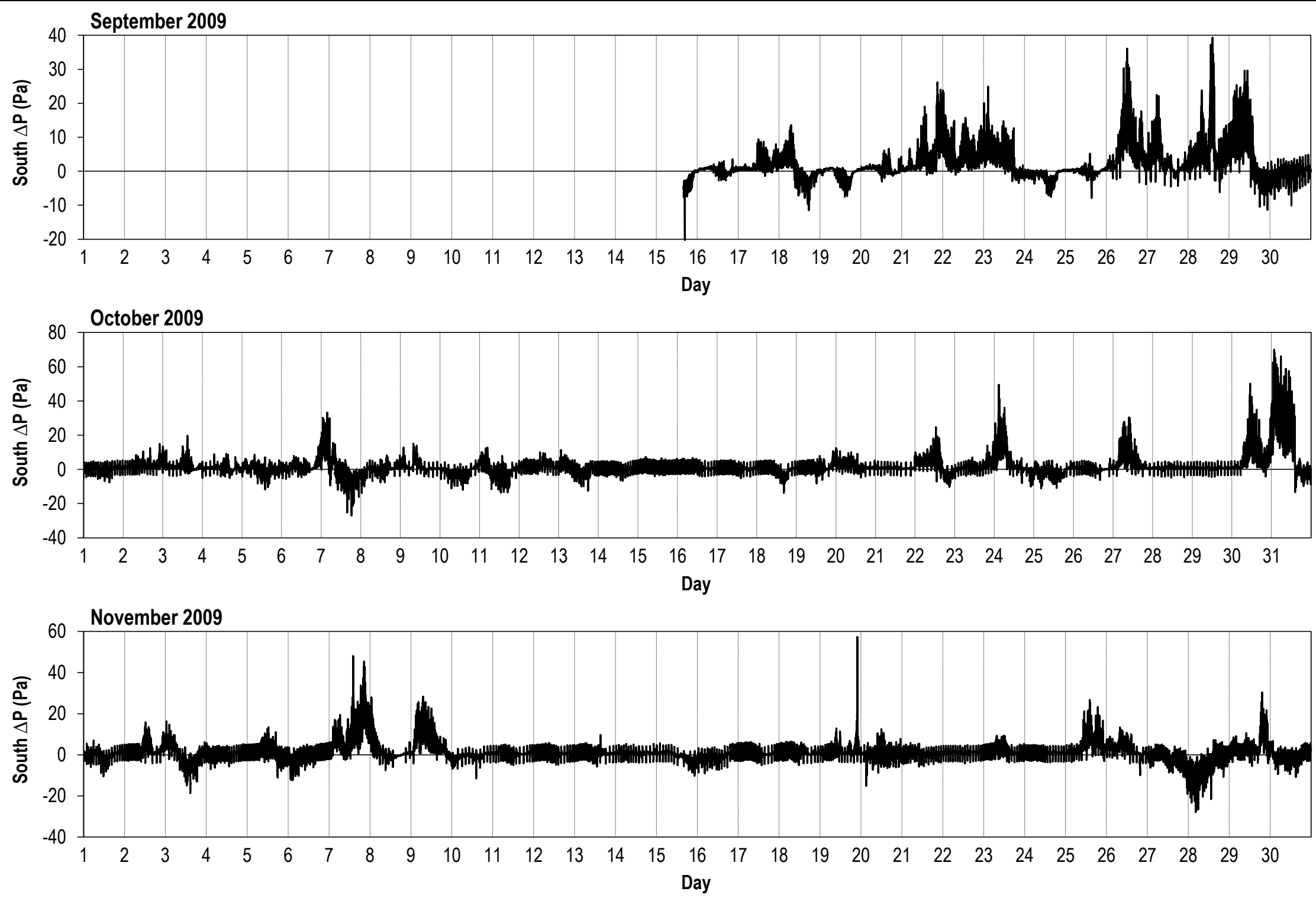

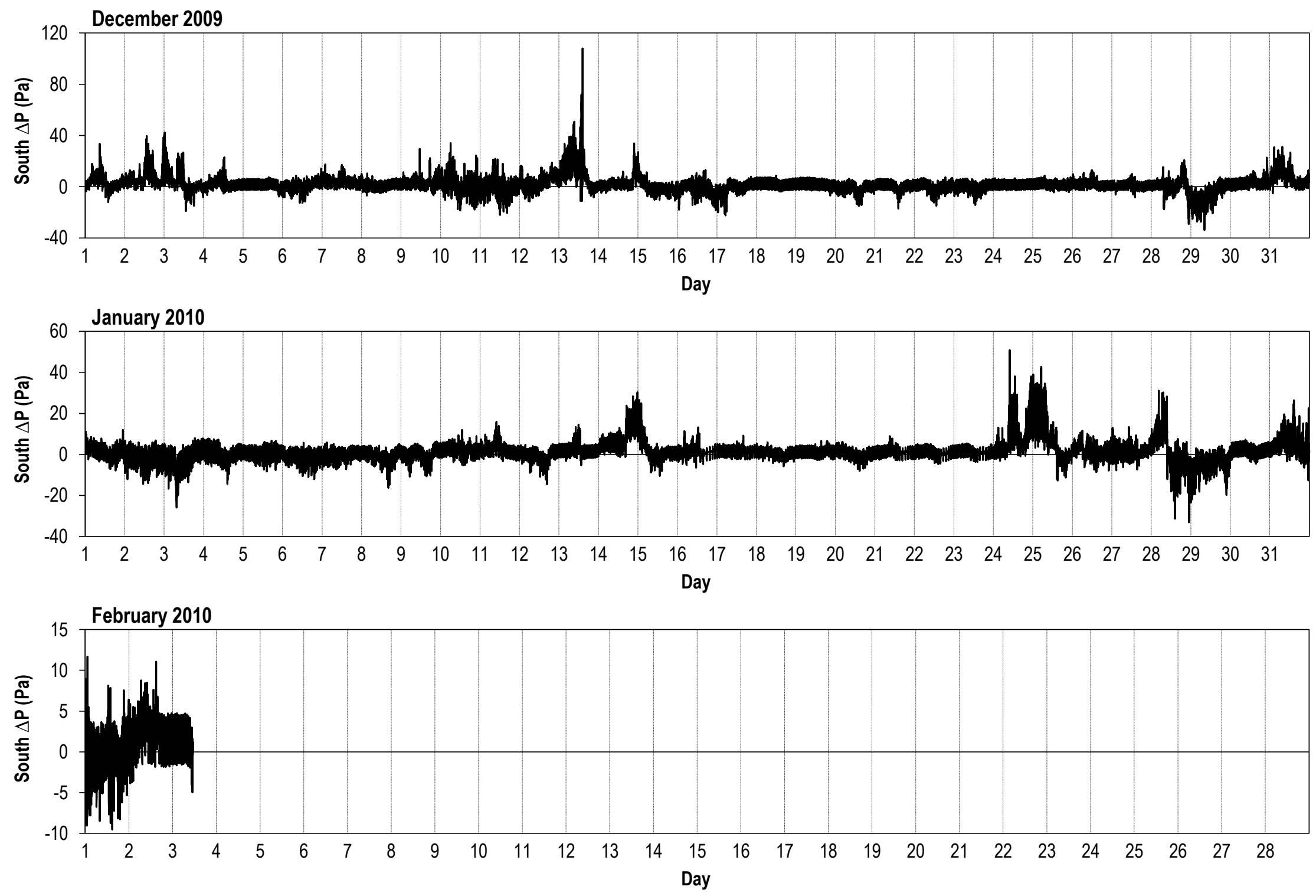

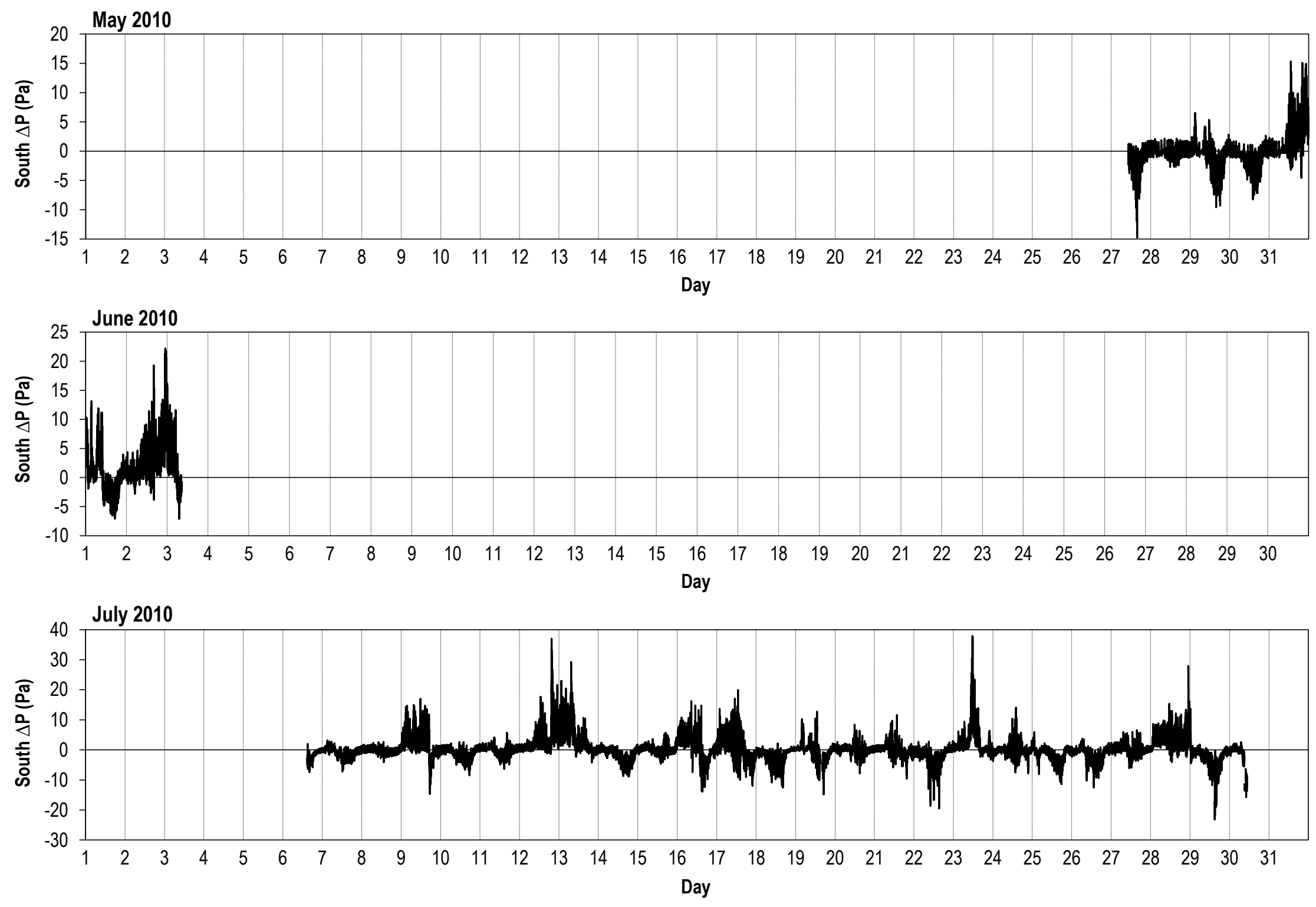

Notes:

a. Data missing due to equipment malfunction.

b. July measurements obtained while Syracuse University pressurized the test hut to characterize the air leakage of wall panels are not included in the graph above. 

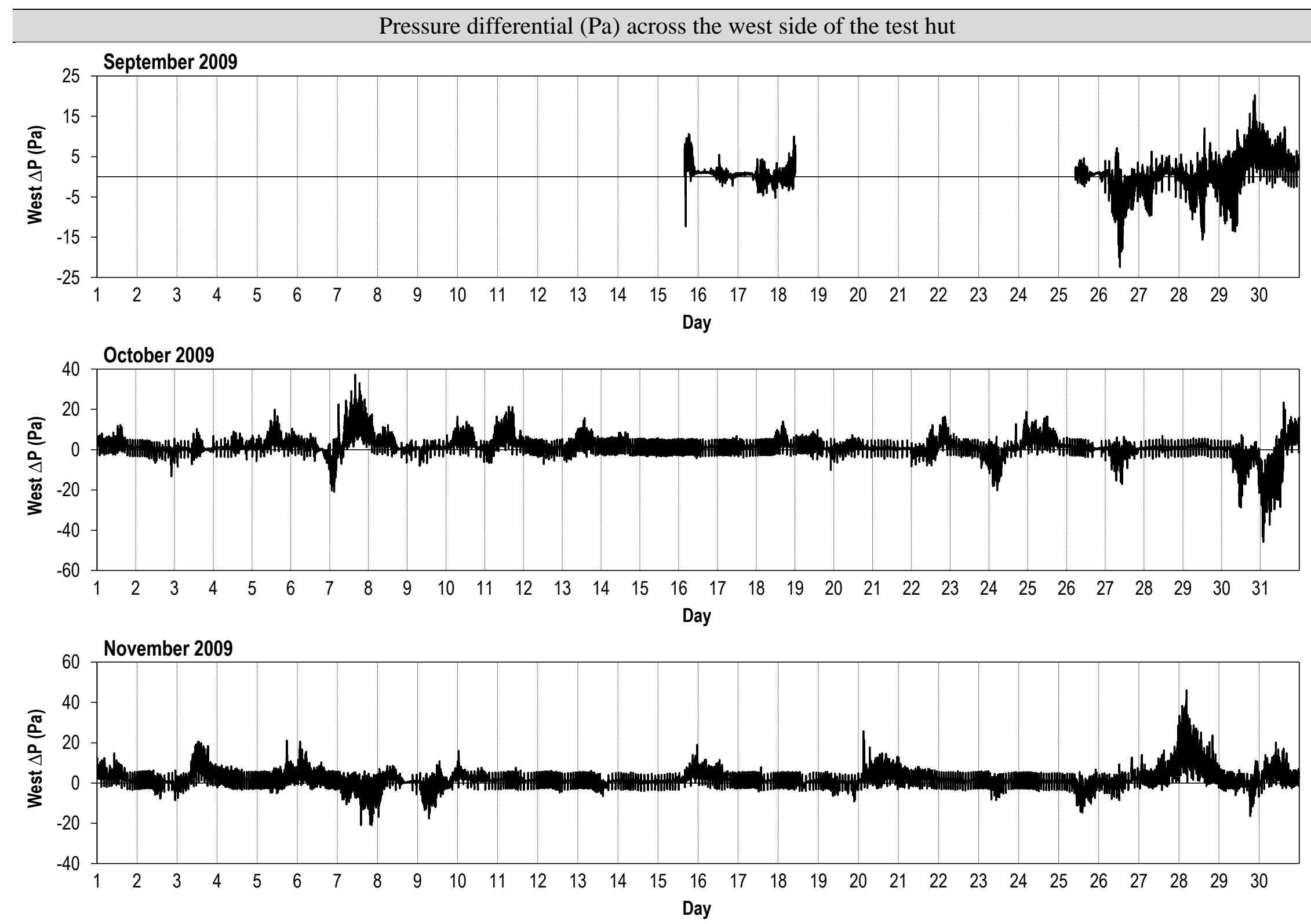

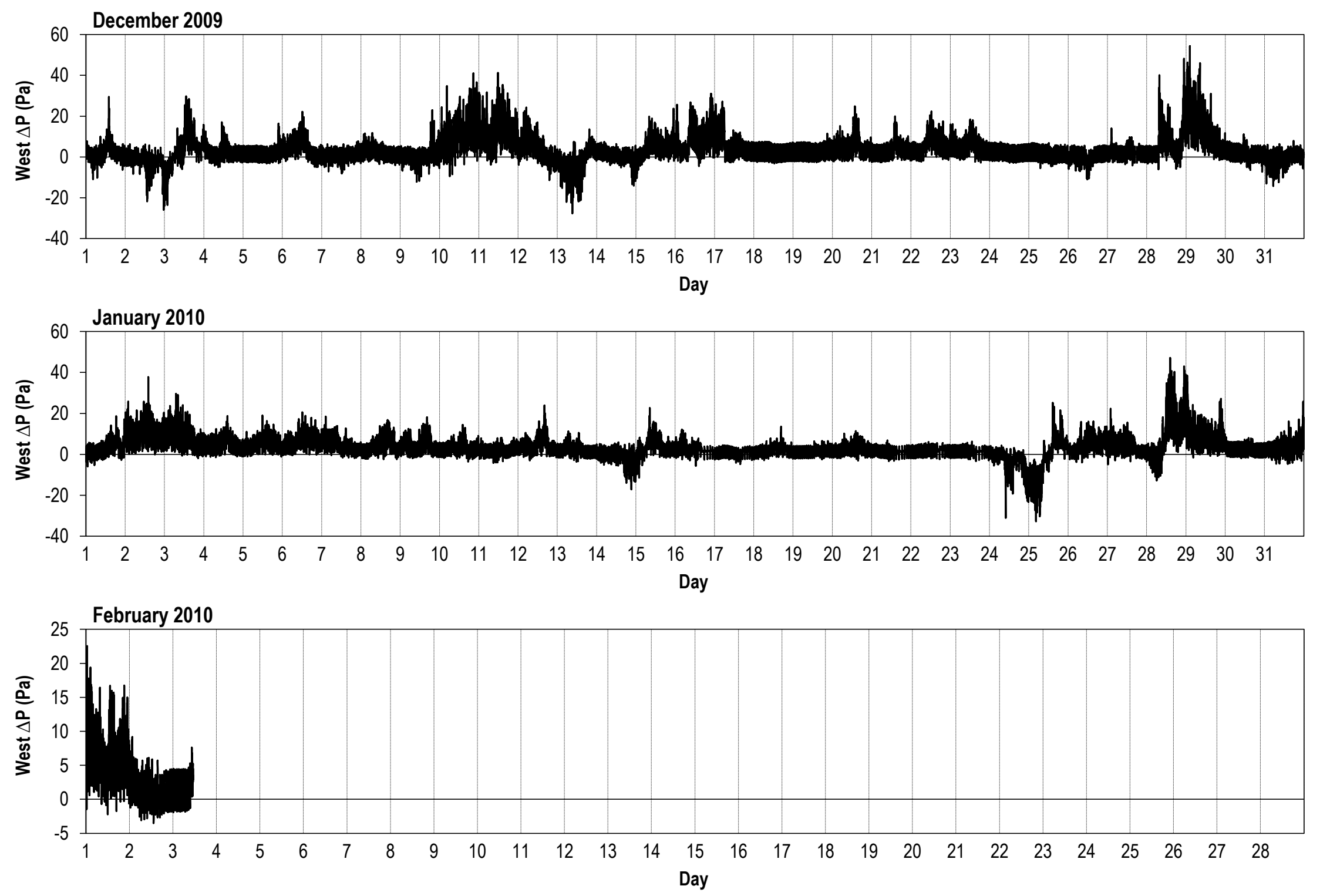

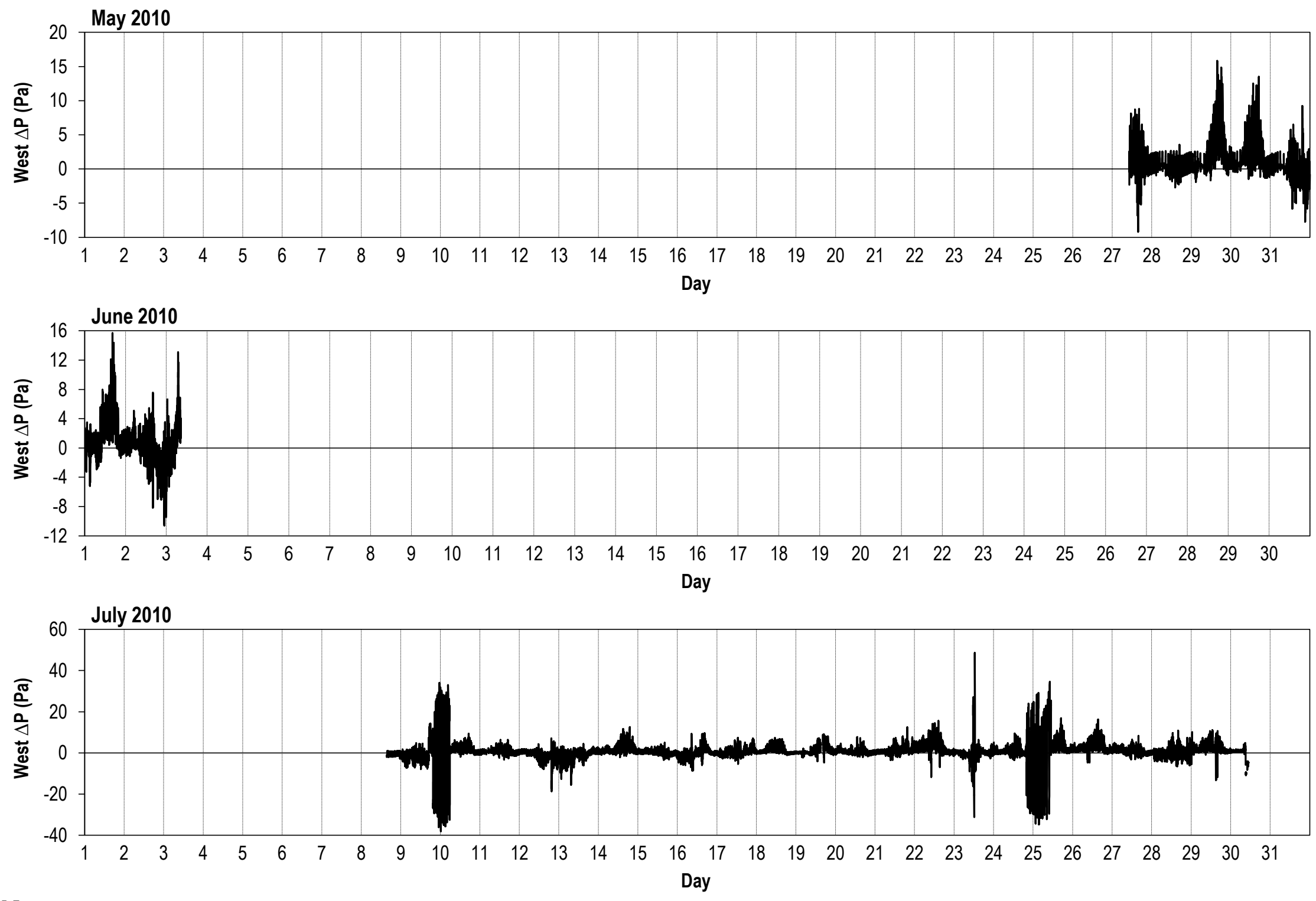

Notes:

a. Data missing due to equipment malfunction.

b. July measurements obtained while Syracuse University pressurized the test hut to characterize the air leakage of wall panels are not included in the graph above. 